\title{
Regarding a Persisting Puzzle in Olefin Metathesis with Ru complexes: Why are Transformations of Alkenes with a Small Substituent Z-Selective?
}

\author{
Sebastian Torker*, Ming Joo Koh, R. Kashif M. Khan, and Amir H. Hoveyda* \\ Department of Chemistry, Merkert Chemistry Center, Boston College \\ Chestnut Hill, Massachusettes 02467 \\ E-mail: amir.hoveyda@bc.edu, sebastian.torker@bc.edu
}

\section{SUPPORTING INFORMATION}

\section{Table of Contents}

1. HPLC chromatograms of ROCM products 11a-14c.........................................................................S4

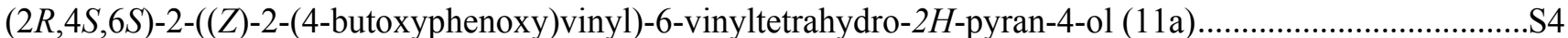

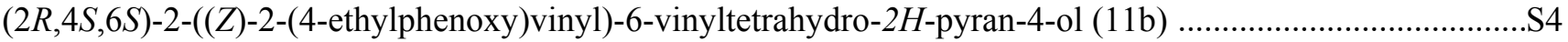

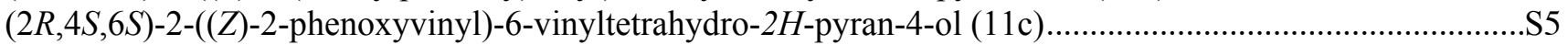

$(2 R, 4 S, 6 S)-2-((Z)-2-(4-b r o m o p h e n o x y) v i n y l)-6$-vinyltetrahydro-2H-pyran-4-ol (11d) ....................................

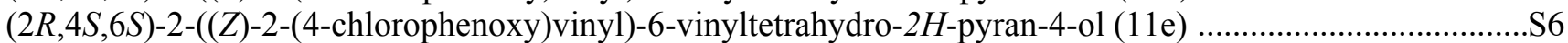

$(2 R, 4 S, 6 S)$-2-((Z)-2-(4-(trifluoromethyl)phenoxy)vinyl)-6-vinyltetrahydro-2H-pyran-4-ol (11f) .....................S6

$(3 \mathrm{a} S, 4 S, 6 R, 6 \mathrm{a} R)-4-((Z)-2$-butoxyvinyl)-6-vinyltetrahydro-1 $H$-cyclopenta[ $c]$ furan-1,3(3a $H)$ dione (12) ...........S7

$(1 R, 2 S, 3 R, 5 R)-3-((Z)-2$-butoxyvinyl)-5-vinylcyclopentane-1 2-diyl)dimethanol (13) ...................................S7

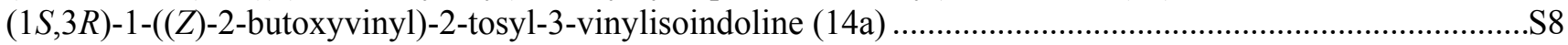

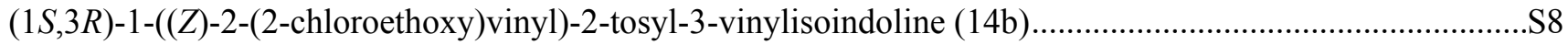

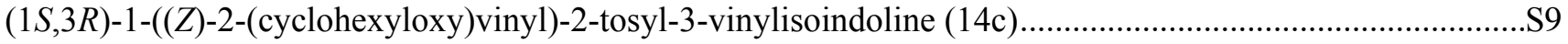

2. ${ }^{1} \mathrm{H}$ and ${ }^{13} \mathrm{C}$ NMR spectra of ROCM products.................................................................................. S10

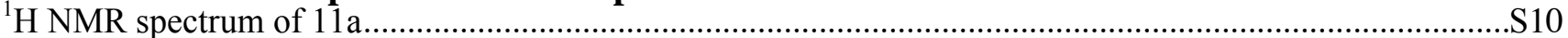

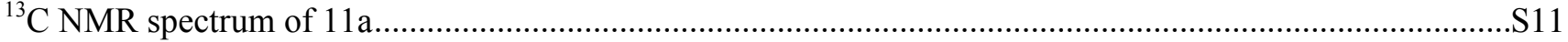

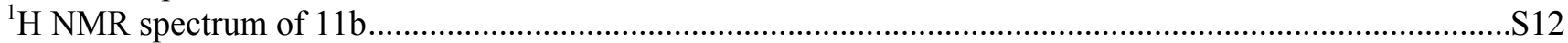

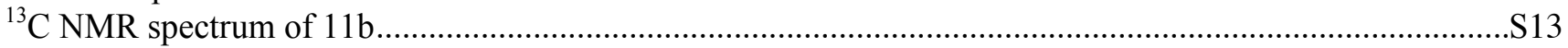

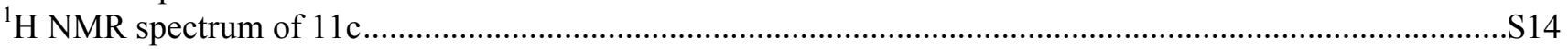




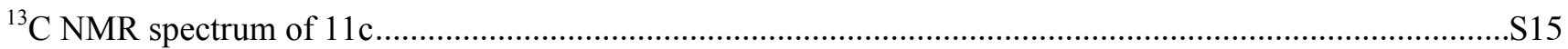

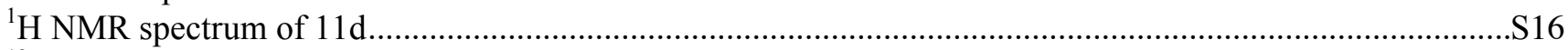

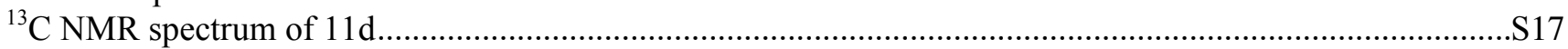

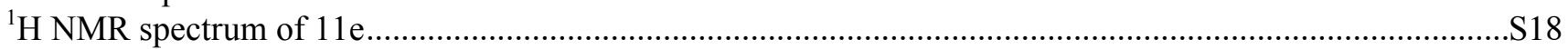

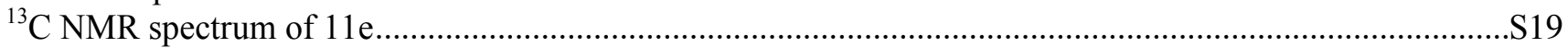

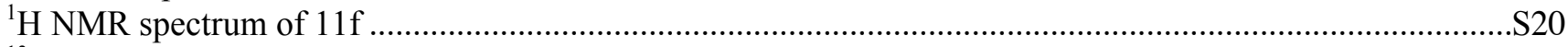

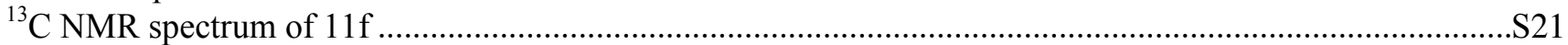

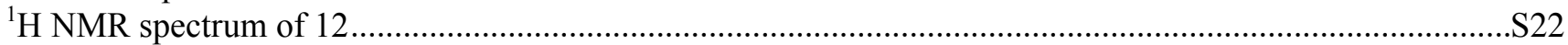

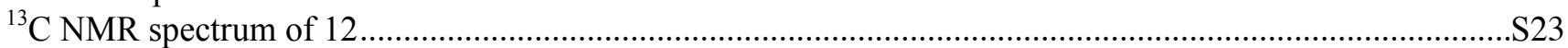

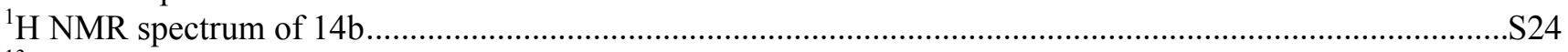

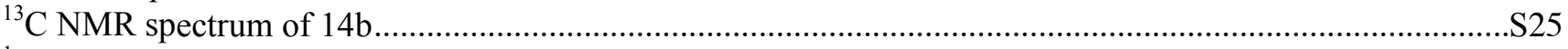

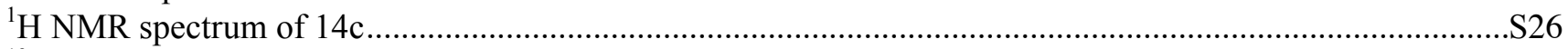

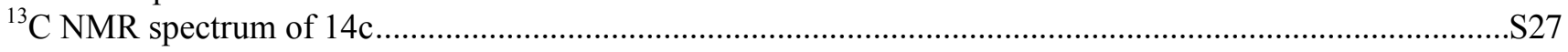

3. Density Functional Theory (DFT) calculations.................................................................. S28

Comparison of potential energy surfaces and free energy surfaces for ROCM promoted by $\mathrm{Ru}-4 \mathrm{f}$ and $\mathrm{Ru}-4 \mathrm{~b}$

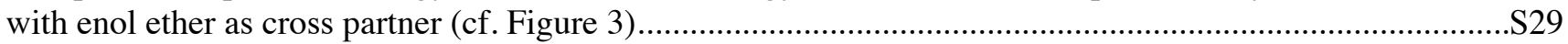

Potential energy surfaces for ROCM promoted by Ru-5b with enol ether as cross partner (cf. Figure 5)........S33

Potential energy surfaces for ROCM promoted by Ru-5b with styrene as cross partner ...............................S34

Assessment of Gibbs free energy surfaces as a function of density functional and solvation.........................S37

More detailed assessment of transition state for ligand association (ts0) ....................................................S38

4. Gibbs Free Energy Surfaces $(\Delta G)$ with BP86, $\omega$ B97XD and M06...................................... S40

Pathways EPhos, ZPhos, ENHC and ZNHC in Figure $3(\Delta \mathrm{G})$ with BP86/basis2 .......................................S40

Pathways EPhos, ZPhos, ENHC and ZNHC in Figure $3(\Delta \mathrm{G})$ with $\omega \mathrm{B} 97 \mathrm{XD} / \mathrm{basis} 2$....................................S42

Pathways EPhos, ZPhos, ENHC and ZNHC in Figure $3(\Delta \mathrm{G})$ with M06/basis2 .........................................S44

Pathways EPhos-model, ZPhos-model, ENHC-model and ZNHC-model $(\Delta \mathrm{G})$ with BP86/basis2 ..................S46

Pathways EPhos-model, ZPhos-model, ENHC-model and ZNHC-model $(\Delta \mathrm{G})$ with $\omega \mathrm{B} 97 \mathrm{XD} / \mathrm{basis} 2$............S48

Pathways EPhos-model, ZPhos-model, ENHC-model and ZNHC-model $(\Delta \mathrm{G})$ with M06/basis2 ...................S50

Pathways ERu-5b and ZRu-5b in Figure 5 and Figure S22 $(\Delta \mathrm{G})$ with BP86/basis2 ....................................S52

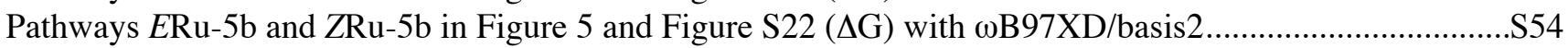

Pathways ERu-5b and ZRu-5b in Figure 5 and Figure $S 22(\Delta \mathrm{G})$ with M06/basis2 ...................................S56

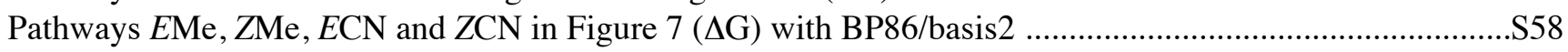

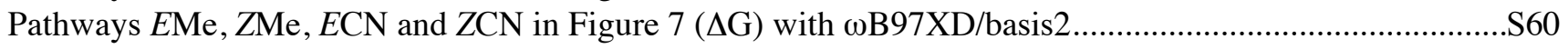

Pathways EMe, ZMe, ECN and ZCN in Figure $7(\Delta \mathrm{G})$ with M06/basis2 ....................................................S62

Pathways ESMe, ZSMe, EMe' and ZMe' in Figures 9-10 $(\Delta \mathrm{G})$ with BP86/basis2 ......................................S64

Pathways ESMe, ZSMe, EMe' and ZMe' in Figures 9-10 $(\Delta \mathrm{G})$ with $\omega \mathrm{B} 97 \mathrm{XD} /$ basis2 2.................................S66

Pathways ESMe, ZSMe, EMe' and ZMe' in Figures 9-10 $(\Delta \mathrm{G})$ with M06/basis2 .......................................S68

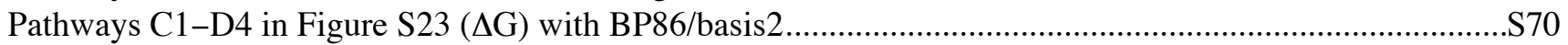

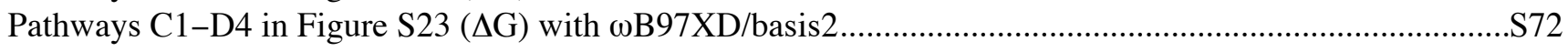

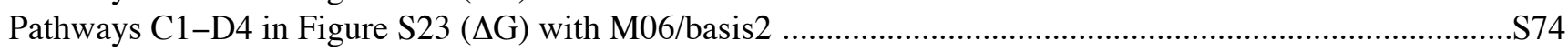

5. Gibbs Free Energy Surfaces $(\Delta G)$ after optimization with BP86/basis $1_{D C M}$............................... S76

6. Energies and Gibbs Free Energies with BP86.................................................................................... S87

Table S12. ROCM with catalyst Ru-4f and enol ether (pathways EPhos, ZPhos in Figure 3).......................S87

Table S13. ROCM with catalyst Ru-4b and enol ether (pathways ENHC, ZNHC in Figure 3).....................S88

Table S14. ROCM with catalyst Ru-4f-model and enol ether (pathways EPhos-model, ZPhos-model) ..........S89

Table S15. ROCM with catalyst Ru-4b-model and enol ether (pathways ENHC-model, ZNHC-model) ........S90

Table S16. ROCM with catalyst Ru-5b and enol ether (pathways A1 = ZRu-5b, AB1, B1 in Figure 5 and

Figure S22). 
Table S17. ROCM with catalyst Ru-5b and enol ether (pathways A2, AB2, B2 in Figure 5 and Figure S22).92 Table S18. ROCM with catalyst Ru-5b and enol ether (pathways A3, AB3, B3 in Figure 5 and Figure S22).93 Table S19. ROCM with catalyst Ru-5b and enol ether (pathways A4 $=E R u-5 b, A B 4$, B 4 in Figure 5 and Figure S22).

Table S20. CM between acrylonitrile and propene with catalyst Ru-4b (pathways EMe, ECN in Figure 7) ...S95 Table S21. CM between acrylonitrile and propene with catalyst Ru-4b (pathways ZMe, ZCN in Figure 7)....S96 Table S22. CM between vinyl sulfide and trans-dichloroethylene with catalyst Ru-4b (ESMe, ZSMe in Figure 9).

Table S23. Homodimerization of propene with catalyst Ru-4b (pathways EMe', ZMe' in Figure 10) ............S98 Table S24. ROCM with catalyst Ru-5b and styrene (pathways C1, CD1, D1 in Figure S23) ........................S99 Table S25. ROCM with catalyst Ru-5b and styrene (pathways C2, CD2, D2 in Figure S23) ......................S100 Table S26. ROCM with catalyst Ru-5b and styrene (pathways C3, CD3, D3 in Figure S23) ......................S101 Table S27. ROCM with catalyst Ru-5b and styrene (pathways C4, CD4, D4 in Figure S23) ......................S102

7. Energies and Gibbs Free Energies with $\omega$ B97XD and M06 .............................................. S103

Table S28. ROCM with catalyst Ru-4f and enol ether (pathways EPhos, ZPhos in Fig. 3) .........................S103

Table S29. ROCM with catalyst Ru-4b and enol ether (pathways ENHC, ZNHC in Figure 3)....................S104

Table S30. ROCM with catalyst Ru-4f-model and enol ether (pathways EPhos-model, ZPhos-model) ........S105 Table S31. ROCM with catalyst Ru-4b-model and enol ether (pathways ENHC-model, ZNHC-model) ......S106 Table S32. ROCM with catalyst Ru-5b and enol ether (pathways A1 $=Z \mathrm{Ru}-5 \mathrm{~b}, \mathrm{AB} 1, \mathrm{~B} 1$ in Figure 5 and Figure S22) ........................................................................................................................ 107 Table S33. ROCM with catalyst Ru-5b and enol ether (pathways A2, AB2, B2 in Figure 5 and Figure S22)

Table S34. ROCM with catalyst Ru-5b and enol ether (pathways A3, AB3, B3 in Figure 5 and Figure S22)

Table S35. ROCM with catalyst Ru-5b and enol ether (pathways A4 =ERu-5b, AB4, B4 in Figure 5 and Figure $\mathrm{S} 22$ ).

Table S36. CM between acrylonitrile and propene with catalyst Ru-4b (pathways EMe, ECN in Figure 7) .S115 Table S37. CM between acrylonitrile and propene with catalyst Ru-4b (pathways ZMe, ZCN in Figure 7)..S117 Table S38. CM between vinyl sulfide and trans-dichloroethylene with catalyst Ru-4b (ESMe, ZSMe in Figure 9)......

Table S39. Homodimerization of propene with catalyst Ru-4b (pathways EMe', ZMe' in Figure 10) ..........S121

Table S40. ROCM with catalyst Ru-5b and styrene (pathways C1, CD1, D1 in Figure S23) ......................S123

Table S41. ROCM with catalyst Ru-5b and styrene (pathways C2, CD2, D2 in Figure S23) ......................S125

Table S42. ROCM with catalyst Ru-5b and styrene (pathways C3, CD3, D3 in Figure S23) ......................S127

Table S43. ROCM with catalyst Ru-5b and styrene (pathways C4, CD4, D4 in Figure S23) ......................S129

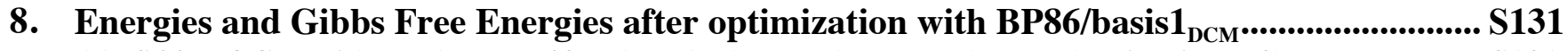
Table S44. ROCM with catalyst Ru-4f and enol ether (pathways EPhos, ZPhos in Figure 3)......................S131

Table S45. ROCM with catalyst Ru-4b and enol ether (pathways ENHC, ZNHC in Figure 3)....................S135

9. Images of transition states.

TSs for ROCM of 2 with Ru-4f and Ru-4b and enol ether (pathways EPhos, ZPhos, ENHC, ZNHC in Figure

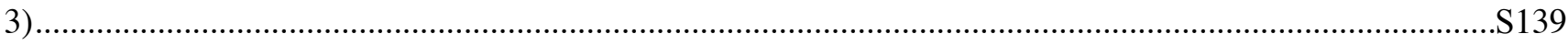

TSs for ROCM of 2 with catalyst Ru-5b and enol ether (pathways ERu-5b, ZRu-5b; A1-B4 in Figure S22)

TSs for nonproductive OM of enol ether with catalyst Ru-5b (pathways AB1-AB4 in Figure S22) ............S142

TSs for CM between propene and acrylonitrile with Ru-4b (pathways EMe, ZMe, ECN, ZCN in Figure 7).S143

TSs for CM between methyl vinyl sulfide and trans-dichloroethylene (pathways ESMe, ZSMe in Figure 9) and homodimerization of propene with catalyst Ru-4b (pathways EMe', ZMe' in Figure 10)......................S144

TSs for ROCM of 2 with catalyst Ru-5b and styrene (pathways C1-D4 in Figure S23)...........................S145

TSs for nonproductive OM of styrene with catalyst Ru-5b (pathways CD1-CD4 in Figure S23) ................S147 


\section{HPLC chromatograms of ROCM products 11a-14c}

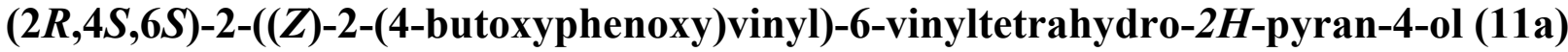

Table S1. HPLC chromatogram for enantiomerically enriched ROCM product 11a (Daicel Chiralpak OD-H column, 97:3 hexanes: $i-\mathrm{PrOH}, 0.5 \mathrm{~mL} / \mathrm{min}, 220 \mathrm{~nm}$ )
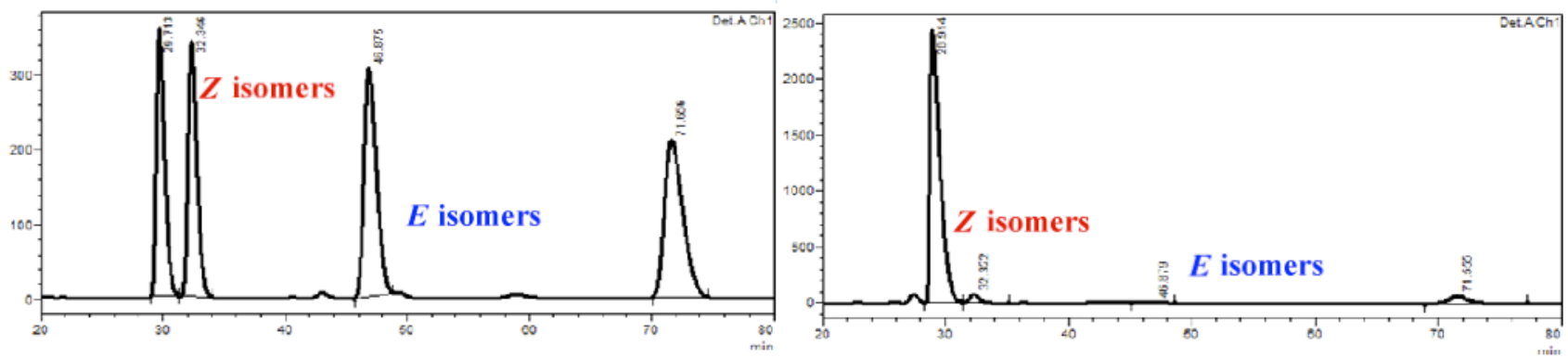

\begin{tabular}{|l|l|l|l|l|l|l|l|}
\hline Peak \# & $\begin{array}{l}\text { Retention } \\
\text { time (min) }\end{array}$ & Area & Area \% & Peak \# & $\begin{array}{l}\text { Retention } \\
\text { time (min) }\end{array}$ & Area & Area \% \\
\hline 1 & 29.71 & 17032013 & 21.93 & 1 & 28.91 & 140649946 & 94.39 \\
\hline 2 & 32.35 & 22706960 & 22.10 & 2 & 32.32 & 3844804 & 3.05 \\
\hline 3 & 46.88 & 21599199 & 27.81 & 3 & 46.88 & 408209 & 0.20 \\
\hline 4 & 71.66 & 21881097 & 28.17 & 4 & 71.56 & 6309277 & 2.37 \\
\hline
\end{tabular}

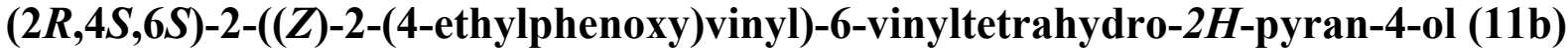

Table S2. HPLC chromatogram for enantiomerically enriched ROCM product 11b (Daicel Chiralpak OD-H column, 97:3 hexanes: $i$-PrOH, $0.5 \mathrm{~mL} / \mathrm{min}, 220 \mathrm{~nm}$ )
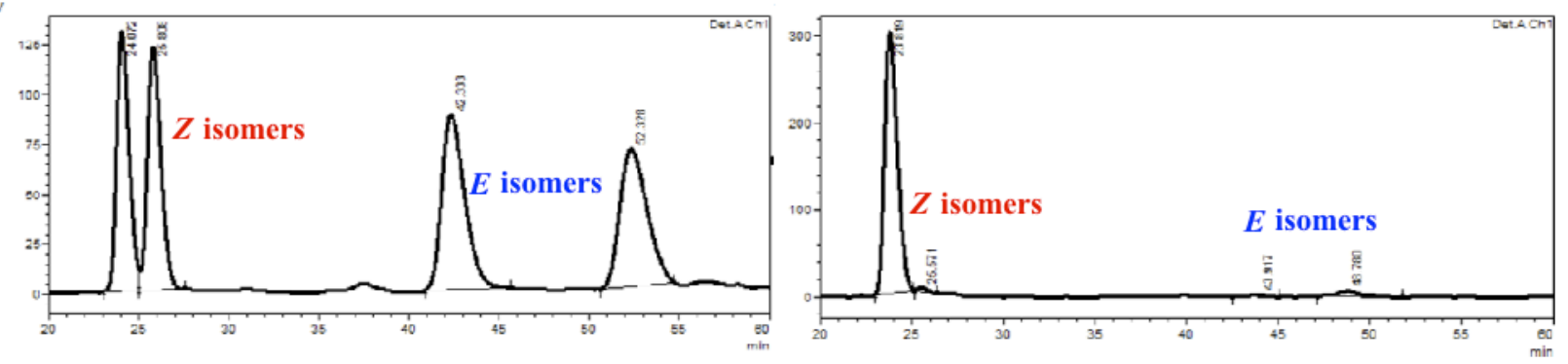

\begin{tabular}{|l|l|l|l|l|l|l|l|}
\hline Peak \# & $\begin{array}{l}\text { Retention } \\
\text { time (min) }\end{array}$ & Area & Area \% & Peak \# & $\begin{array}{l}\text { Retention } \\
\text { time (min) }\end{array}$ & Area & Area \% \\
\hline 1 & 24.07 & 6279887 & 22.76 & 1 & 23.82 & 300587 & 96.03 \\
\hline 2 & 25.81 & 6354881 & 23.03 & 2 & 25.57 & 4483 & 1.43 \\
\hline 3 & 42.33 & 7756172 & 28.11 & 3 & 43.92 & 1915 & 0.61 \\
\hline 4 & 52.33 & 7200278 & 26.10 & 4 & 48.78 & 6017 & 1.92 \\
\hline
\end{tabular}




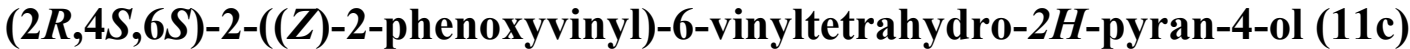

Table S3. HPLC chromatogram for enantiomerically enriched ROCM product 11c (Daicel Chiralpak OJ-H column, 95:5 hexanes: $i$-PrOH, $0.5 \mathrm{~mL} / \mathrm{min}, 220 \mathrm{~nm}$ )
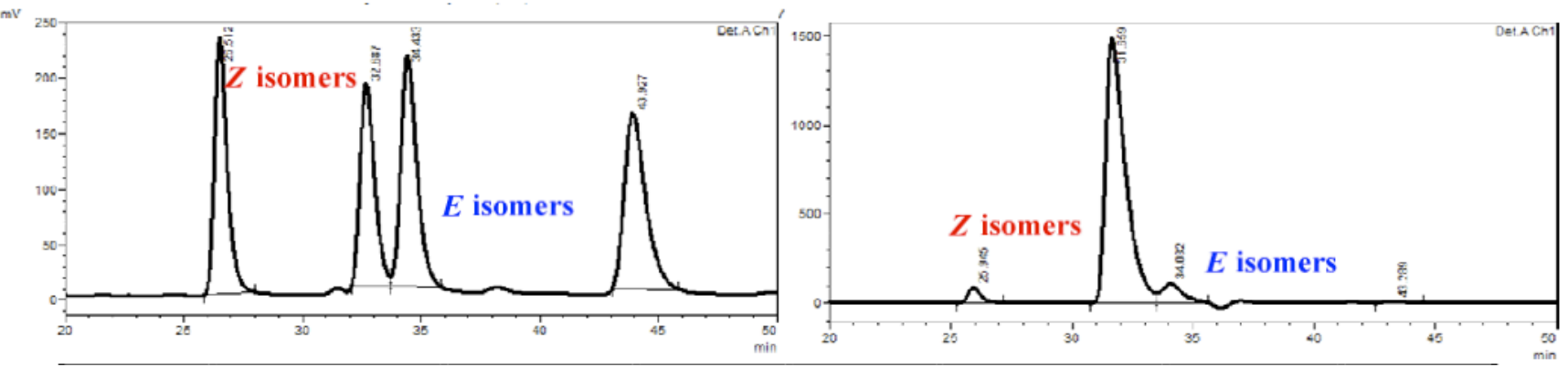

\begin{tabular}{|l|l|l|l|l|l|l|l|}
\hline Peak \# & $\begin{array}{l}\text { Retention } \\
\text { time (min) }\end{array}$ & Area & Area \% & Peak \# & $\begin{array}{l}\text { Retention } \\
\text { time (min) }\end{array}$ & Area & Area \% \\
\hline 1 & 26.51 & 8672147 & 23.86 & 1 & 26.53 & 1967223 & 3.28 \\
\hline 2 & 32.69 & 7869583 & 21.65 & 2 & 32.40 & 53904482 & 89.75 \\
\hline 3 & 34.43 & 9839102 & 27.07 & 3 & 34.43 & 4060752 & 6.76 \\
\hline 4 & 43.93 & 9970684 & 27.43 & 4 & 44.11 & 126739 & 0.21 \\
\hline
\end{tabular}

\section{$(2 R, 4 S, 6 S)-2-((Z)-2-(4-b r o m o p h e n o x y) v i n y l)-6-v i n y l t e t r a h y d r o-2 H-p y r a n-4-o l ~(11 d)$}

Table S4. HPLC chromatogram for enantiomerically enriched ROCM product 11d (Daicel Chiralpak OD-H column, 97:3 hexanes:i-PrOH, $0.5 \mathrm{~mL} / \mathrm{min}, 220 \mathrm{~nm}$ )
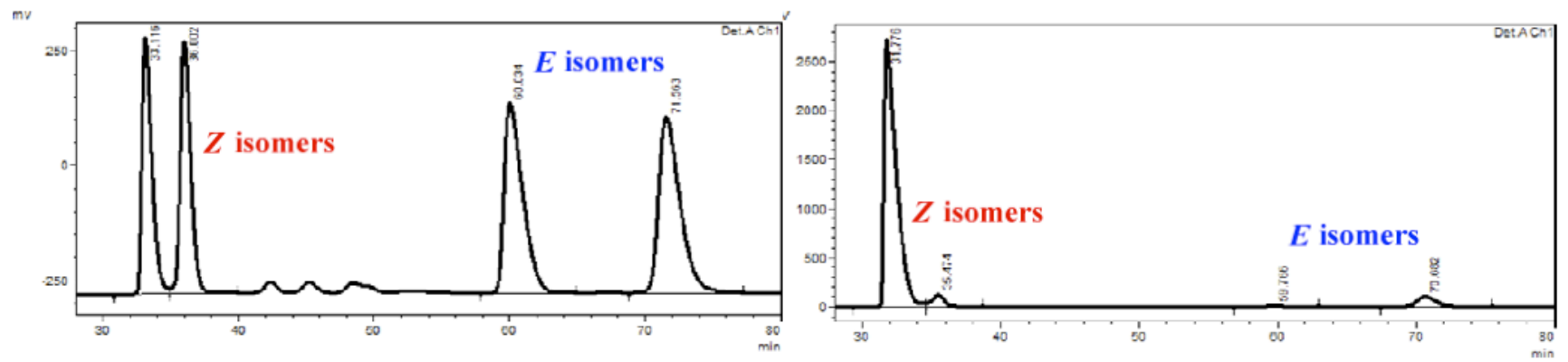

\begin{tabular}{|l|l|l|l|l|l|l|l|}
\hline Peak \# & $\begin{array}{l}\text { Retention } \\
\text { time (min) }\end{array}$ & Area & Area \% & Peak \# & $\begin{array}{l}\text { Retention } \\
\text { time (min) }\end{array}$ & Area & Area \% \\
\hline 1 & 31.78 & 167693945 & 23.86 & 1 & 31.78 & 167693945 & 90.62 \\
\hline 2 & 31.78 & 6935678 & 21.65 & 2 & 31.78 & 6935678 & 3.75 \\
\hline 3 & 59.77 & 669768 & 27.07 & 3 & 59.77 & 669768 & 0.36 \\
\hline 4 & 70.68 & 9752658 & 27.43 & 4 & 70.68 & 9752658 & 5.27 \\
\hline
\end{tabular}




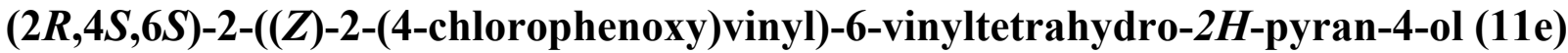

Table S5. HPLC chromatogram for enantiomerically enriched ROCM product 11e (Daicel Chiralpak OD-H column, 97:3 hexanes: $i$-PrOH, $0.5 \mathrm{~mL} / \mathrm{min}, 220 \mathrm{~nm}$ )
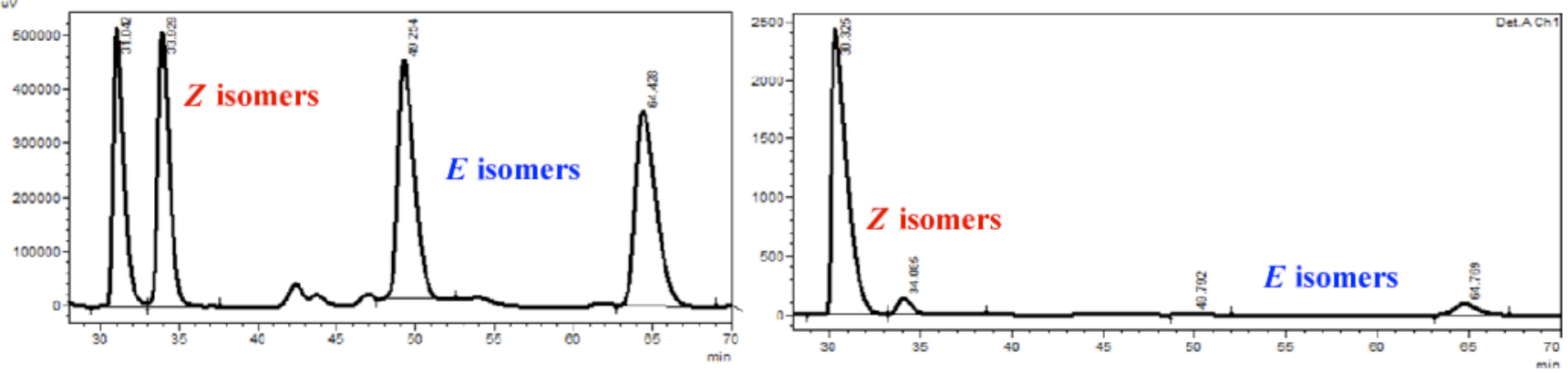

\begin{tabular}{|l|l|l|l|l|l|l|l|}
\hline Peak \# & $\begin{array}{l}\text { Retention } \\
\text { time (min) }\end{array}$ & Area & Area \% & Peak \# & $\begin{array}{l}\text { Retention } \\
\text { time (min) }\end{array}$ & Area & Area \% \\
\hline 1 & 31.04 & 24494013 & 21.40 & 1 & 30.33 & 140839691 & 89.77 \\
\hline 2 & 33.93 & 25722642 & 22.47 & 2 & 34.09 & 7572864 & 4.83 \\
\hline 3 & 49.25 & 31561739 & 27.57 & 3 & 49.79 & 541771 & 0.35 \\
\hline 4 & 64.43 & 32688385 & 28.56 & 4 & 64.77 & 7929359 & 5.05 \\
\hline
\end{tabular}

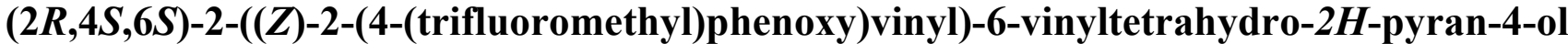

(11f)

Table S6. HPLC chromatogram for enantiomerically enriched ROCM product 11f (Daicel Chiralpak OD-H column, 97:3 hexanes: $i$-PrOH, $0.5 \mathrm{~mL} / \mathrm{min}, 220 \mathrm{~nm}$ )
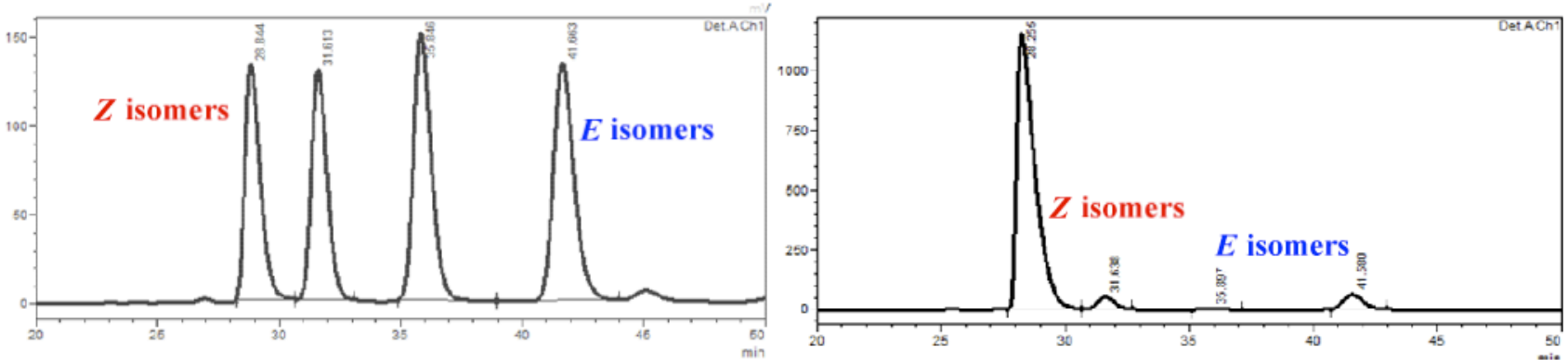

\begin{tabular}{|l|l|l|l|l|l|l|l|}
\hline Peak \# & $\begin{array}{l}\text { Retention } \\
\text { time (min) }\end{array}$ & Area & Area \% & Peak \# & $\begin{array}{l}\text { Retention } \\
\text { time (min) }\end{array}$ & Area & Area \% \\
\hline 1 & 28.84 & 5812515 & 21.47 & 1 & 28.26 & 58846886 & 90.82 \\
\hline 2 & 31.61 & 5835827 & 21.55 & 2 & 31.64 & 2371686 & 3.66 \\
\hline 3 & 35.85 & 7636024 & 28.20 & 3 & 35.90 & 263232 & 0.41 \\
\hline 4 & 41.66 & 7793665 & 28.78 & 4 & 41.58 & 3311592 & 5.11 \\
\hline
\end{tabular}




\section{(3aS,4S,6R,6a R)-4-((Z)-2-butoxyvinyl)-6-vinyltetrahydro-1H-cyclopenta[c]furan-} 1,3(3aH)dione (12)

Table S7. HPLC chromatogram for enantiomerically enriched ROCM product 12 (Daicel Chiralpak OD-H column, 97:3 hexanes:i-PrOH, $0.5 \mathrm{~mL} / \mathrm{min}, 220 \mathrm{~nm}$ )
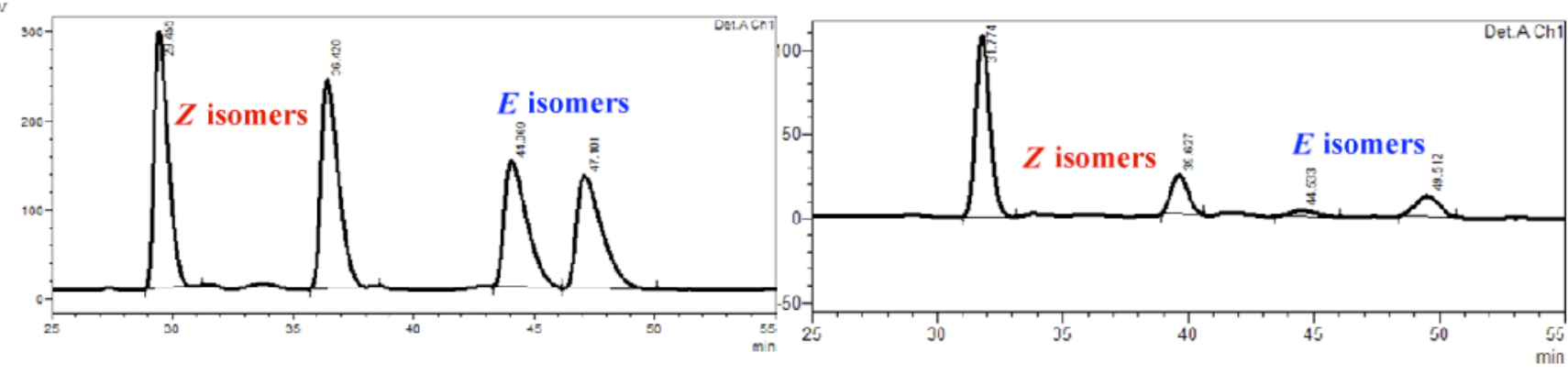

\begin{tabular}{|l|l|l|l|l|l|l|l|}
\hline Peak \# & $\begin{array}{l}\text { Retention } \\
\text { time (min) }\end{array}$ & Area & Area \% & Peak \# & $\begin{array}{l}\text { Retention } \\
\text { time (min) }\end{array}$ & Area & Area \% \\
\hline 1 & 29.46 & 11662014 & 27.92 & 1 & 31.77 & 4267929 & 66.85 \\
\hline 2 & 36.42 & 11667826 & 27.93 & 2 & 39.63 & 1064541 & 16.68 \\
\hline 3 & 44.07 & 9221034 & 22.08 & 3 & 44.53 & 254762 & 3.99 \\
\hline 4 & 47.10 & 9219179 & 22.07 & 4 & 49.51 & 796699 & 12.48 \\
\hline
\end{tabular}

\section{(1R,2S,3R,5R)-3-((Z)-2-butoxyvinyl)-5-vinylcyclopentane-1,2-diyl)dimethanol (13)}

Table S8. HPLC chromatogram for enantiomerically enriched ROCM product 13 (Daicel Chiralpak OD-H column, 97:3 hexanes: $i$-PrOH, $0.5 \mathrm{~mL} / \mathrm{min}, 220 \mathrm{~nm}$ )
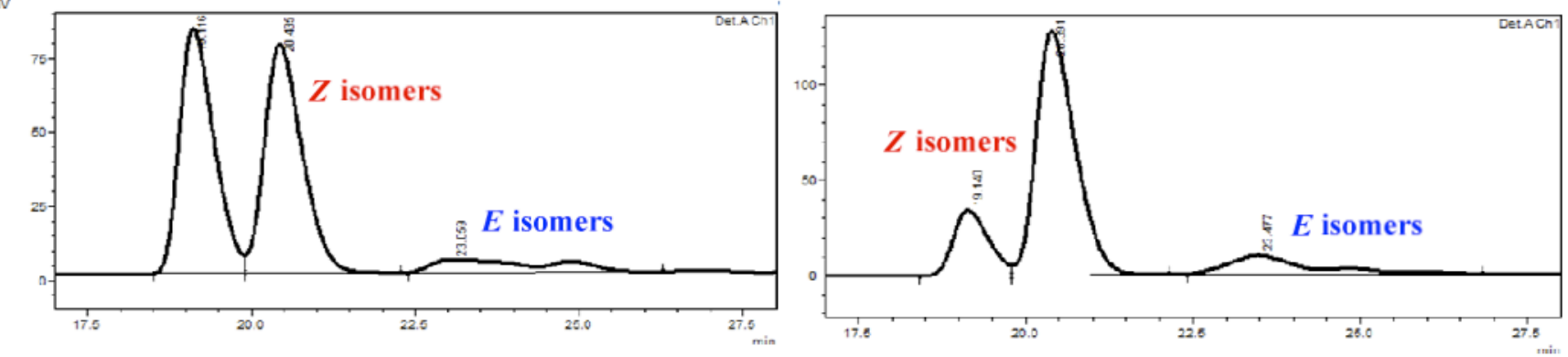

\begin{tabular}{|l|l|l|l|l|l|l|l|}
\hline Peak \# & $\begin{array}{l}\text { Retention } \\
\text { time (min) }\end{array}$ & Area & Area \% & Peak \# & $\begin{array}{l}\text { Retention } \\
\text { time (min) }\end{array}$ & Area & Area \% \\
\hline 1 & 19.12 & 3068312 & 45.57 & 1 & 19.14 & 1249574 & 17.05 \\
\hline 2 & 20.44 & 3126322 & 46.44 & 2 & 20.39 & 5227075 & 71.31 \\
\hline 3 & 23.06 & 538047 & 7.992 & 3 & 23.48 & 853845 & 11.65 \\
\hline
\end{tabular}




\section{(1S,3R)-1-((Z)-2-butoxyvinyl)-2-tosyl-3-vinylisoindoline (14a)}

Table 59. HPLC chromatogram for enantiomerically enriched ROCM product 14a (Daicel Chiralpak OZ-H column, 97:3 hexanes: $i$-PrOH, $0.5 \mathrm{~mL} / \mathrm{min}, 220 \mathrm{~nm}$ )
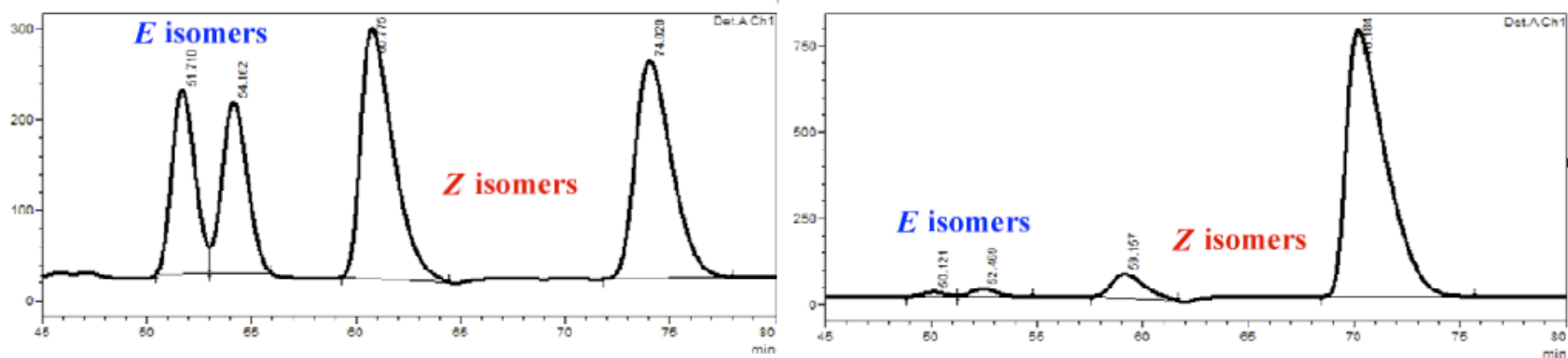

\begin{tabular}{|l|l|l|l|l|l|l|l|}
\hline Peak \# & $\begin{array}{l}\text { Retention } \\
\text { time (min) }\end{array}$ & Area & Area \% & Peak \# & $\begin{array}{l}\text { Retention } \\
\text { time (min) }\end{array}$ & Area & Area \% \\
\hline 1 & 51.71 & 15650694 & 17.27 & 1 & 50.12 & 981171 & 0.89 \\
\hline 2 & 54.16 & 15928943 & 17.58 & 2 & 52.47 & 1892357 & 1.72 \\
\hline 3 & 60.78 & 29905833 & 33.00 & 3 & 59.16 & 7740919 & 7.04 \\
\hline 4 & 74.02 & 29149806 & 32.16 & 4 & 70.18 & 99294178 & 90.34 \\
\hline
\end{tabular}

\section{(1S,3R)-1-((Z)-2-(2-chloroethoxy)vinyl)-2-tosyl-3-vinylisoindoline (14b)}

Table S10. HPLC chromatogram for enantiomerically enriched ROCM product 14b (Daicel Chiralpak OZ-H column, 95:5 hexanes: $i$-PrOH, $0.5 \mathrm{~mL} / \mathrm{min}, 220 \mathrm{~nm}$ )
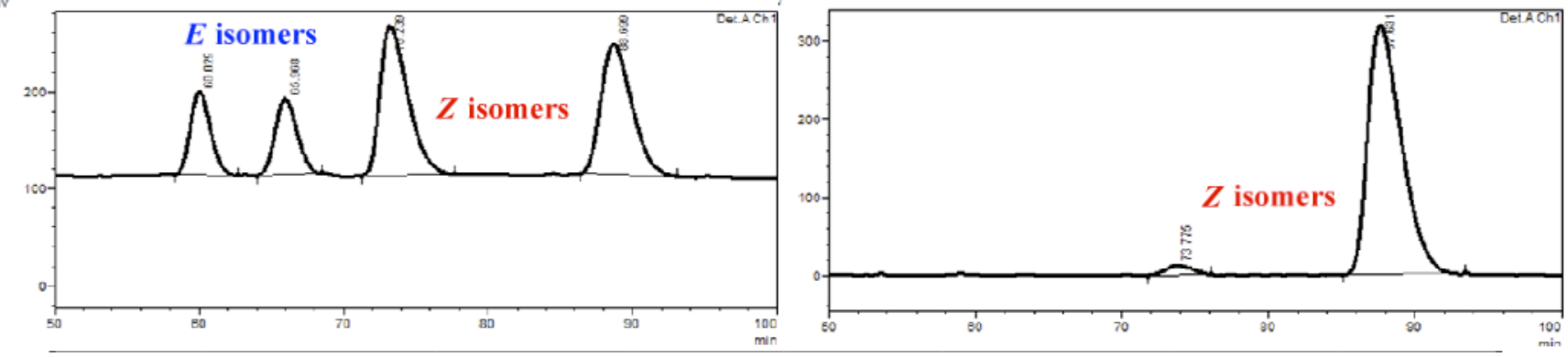

\begin{tabular}{|l|l|l|l|l|l|l|l|}
\hline Peak \# & $\begin{array}{l}\text { Retention } \\
\text { time (min) }\end{array}$ & Area & Area \% & Peak \# & $\begin{array}{l}\text { Retention } \\
\text { time (min) }\end{array}$ & Area & Area \% \\
\hline 1 & 60.03 & 8312603 & 14.54 & 1 & - & - & - \\
\hline 2 & 65.97 & 8274598 & 14.47 & 2 & - & - & - \\
\hline 3 & 73.24 & 20620512 & 36.06 & 3 & 73.78 & 1570301 & 2.99 \\
\hline 4 & 88.70 & 19984178 & 34.94 & 4 & 87.63 & 50928246 & 97.01 \\
\hline
\end{tabular}




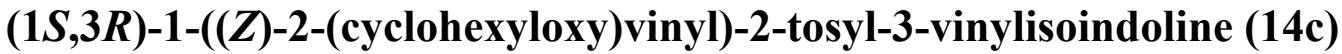

Table S11. HPLC chromatogram for enantiomerically enriched ROCM product 14c (Daicel Chiralpak OZ-H column, 95:5 hexanes: $i-\mathrm{PrOH}, 0.5 \mathrm{~mL} / \mathrm{min}, 220 \mathrm{~nm}$ )
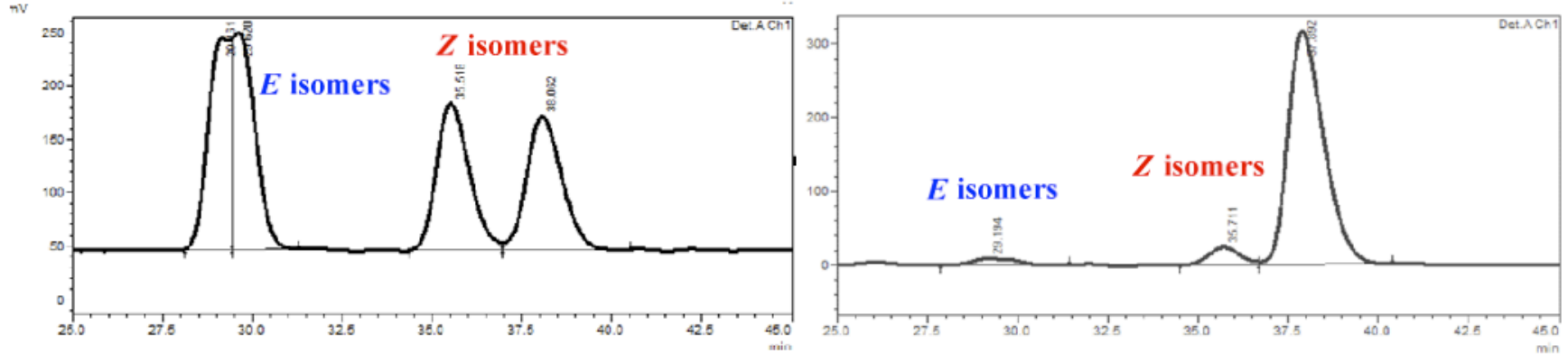

\begin{tabular}{|l|l|l|l|l|l|l|l|}
\hline Peak \# & $\begin{array}{l}\text { Retention } \\
\text { time (min) }\end{array}$ & Area & Area \% & Peak \# & $\begin{array}{l}\text { Retention } \\
\text { time (min) }\end{array}$ & Area & Area \% \\
\hline 1 & 29.16 & 8592404 & 24.89 & 1 & 29.19 & 765381 & 3.18 \\
\hline 2 & 29.62 & 8674604 & 25.12 & 2 & - & - & - \\
\hline 3 & 35.52 & 8753515 & 25.35 & 3 & 35.71 & 1471601 & 6.11 \\
\hline 4 & 38.06 & 8506483 & 24.64 & 4 & 37.89 & 21842920 & 90.71 \\
\hline
\end{tabular}




\section{2. ${ }^{1} \mathrm{H}$ and ${ }^{13} \mathrm{C}$ NMR spectra of ROCM products}

\section{${ }^{1} \mathrm{H}$ NMR spectrum of $11 \mathrm{a}$}

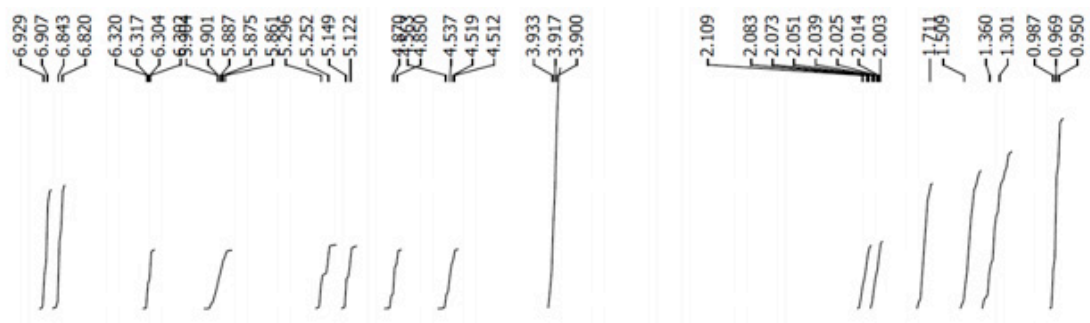<smiles>C=C[C@H]1C[C@@H](O)C[C@@H](/C=C\Oc2ccc(OCCC)cc2)O1</smiles>

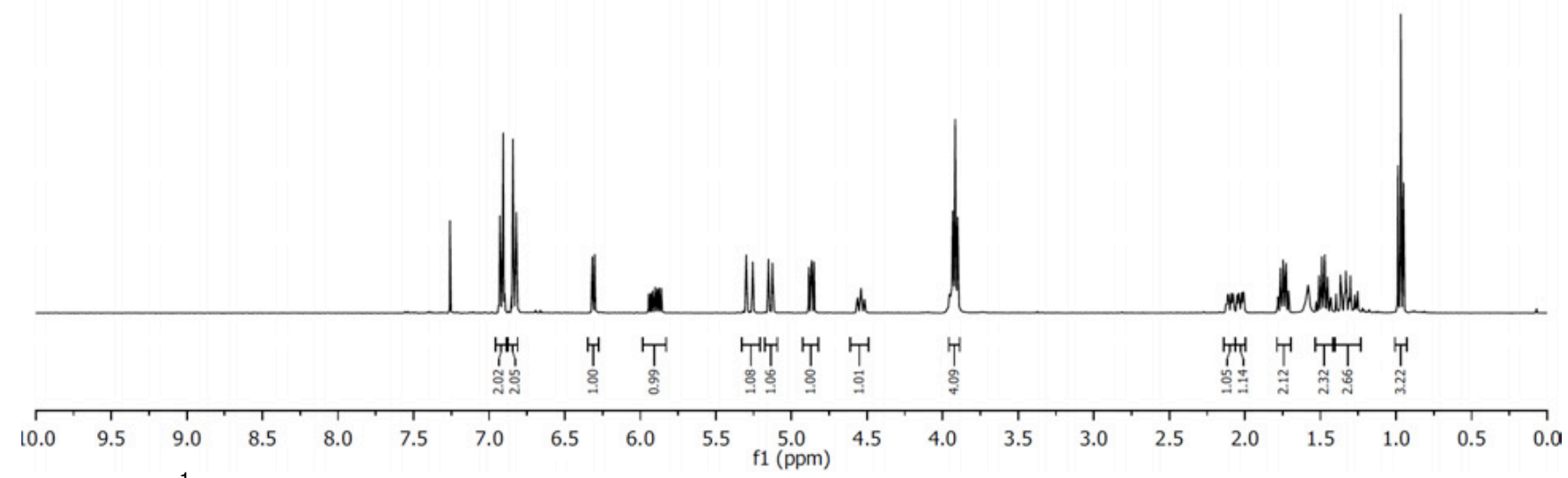

Figure S1. ${ }^{1} \mathrm{H}$ NMR spectrum of $11 \mathrm{a}$ in $\mathrm{CDCl}_{3}$. 


\section{${ }^{13} \mathrm{C}$ NMR spectrum of $11 \mathrm{a}$}

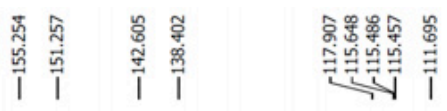

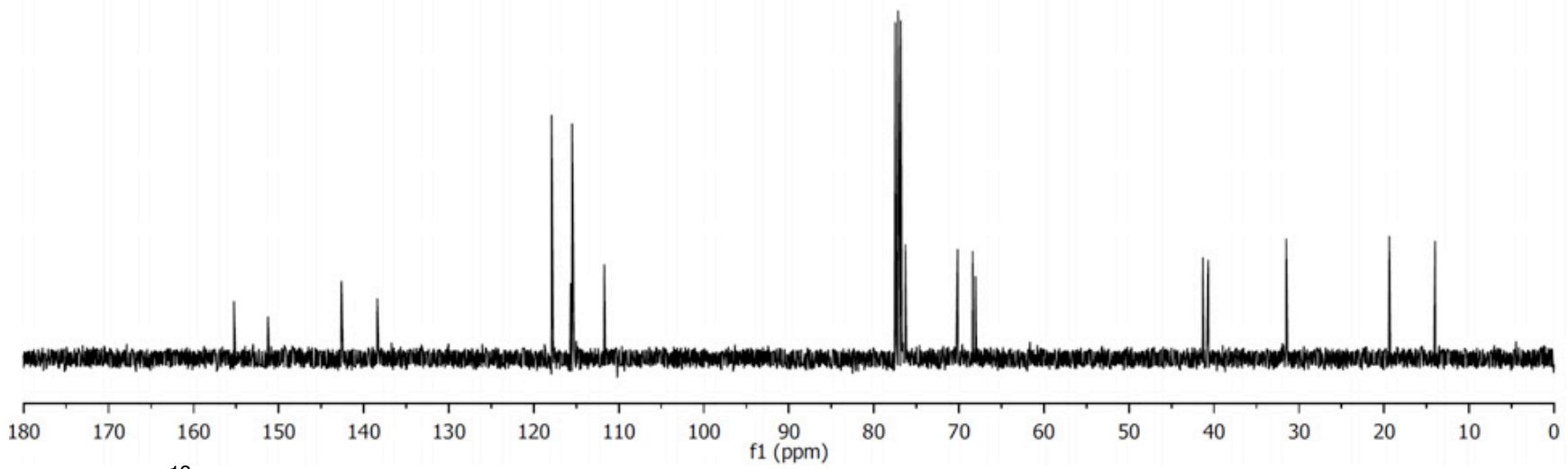

Figure S2. ${ }^{13} \mathrm{C}$ NMR spectrum of 11a in $\mathrm{CDCl}_{3}$. 


\section{${ }^{1} \mathrm{H}$ NMR spectrum of $11 \mathrm{~b}$}
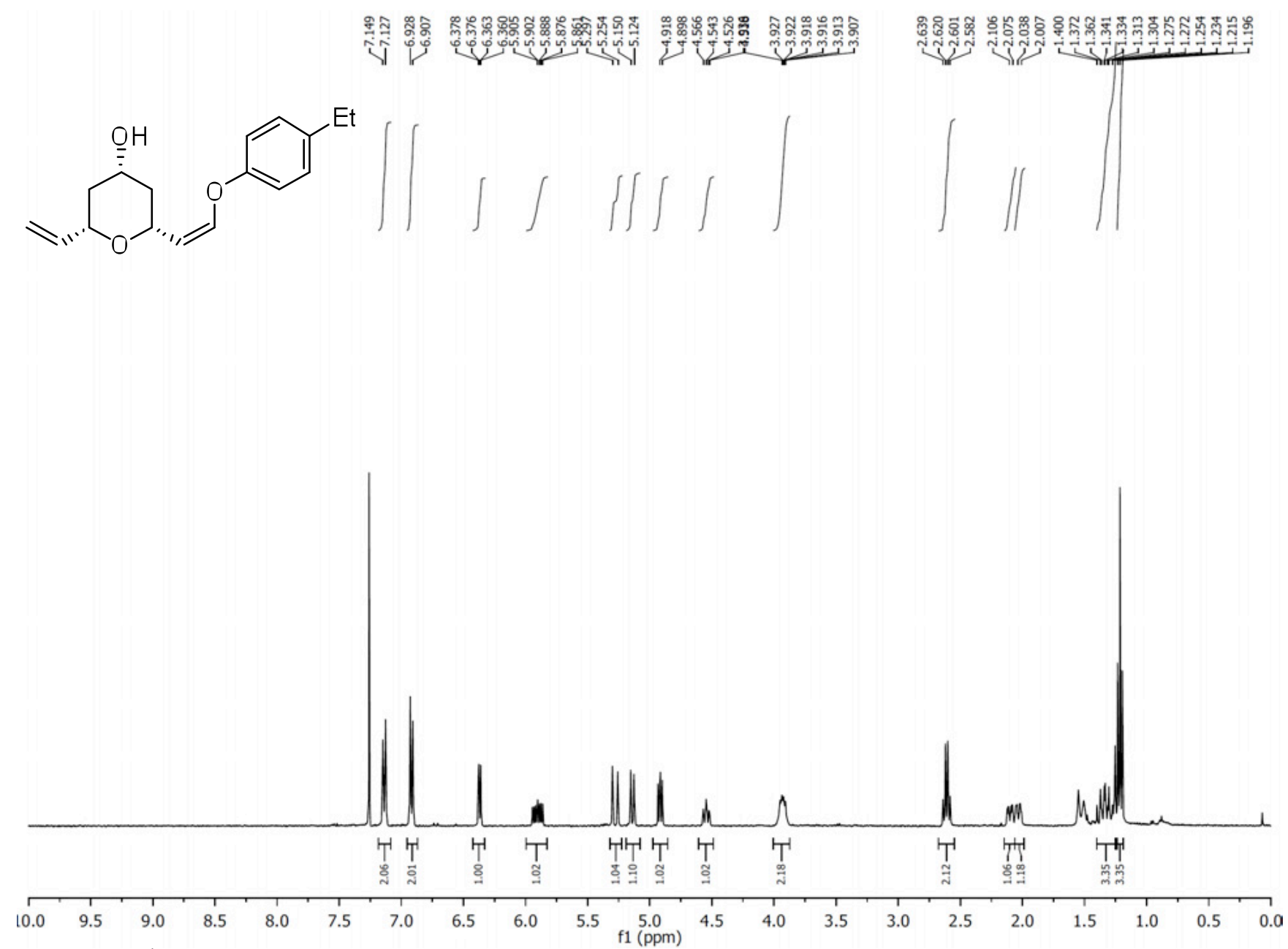

Figure S3. ${ }^{1} \mathrm{H}$ NMR spectrum of $11 \mathrm{~b}$ in $\mathrm{CDCl}_{3}$. 


\section{${ }^{13} \mathrm{C}$ NMR spectrum of $11 \mathrm{~b}$}

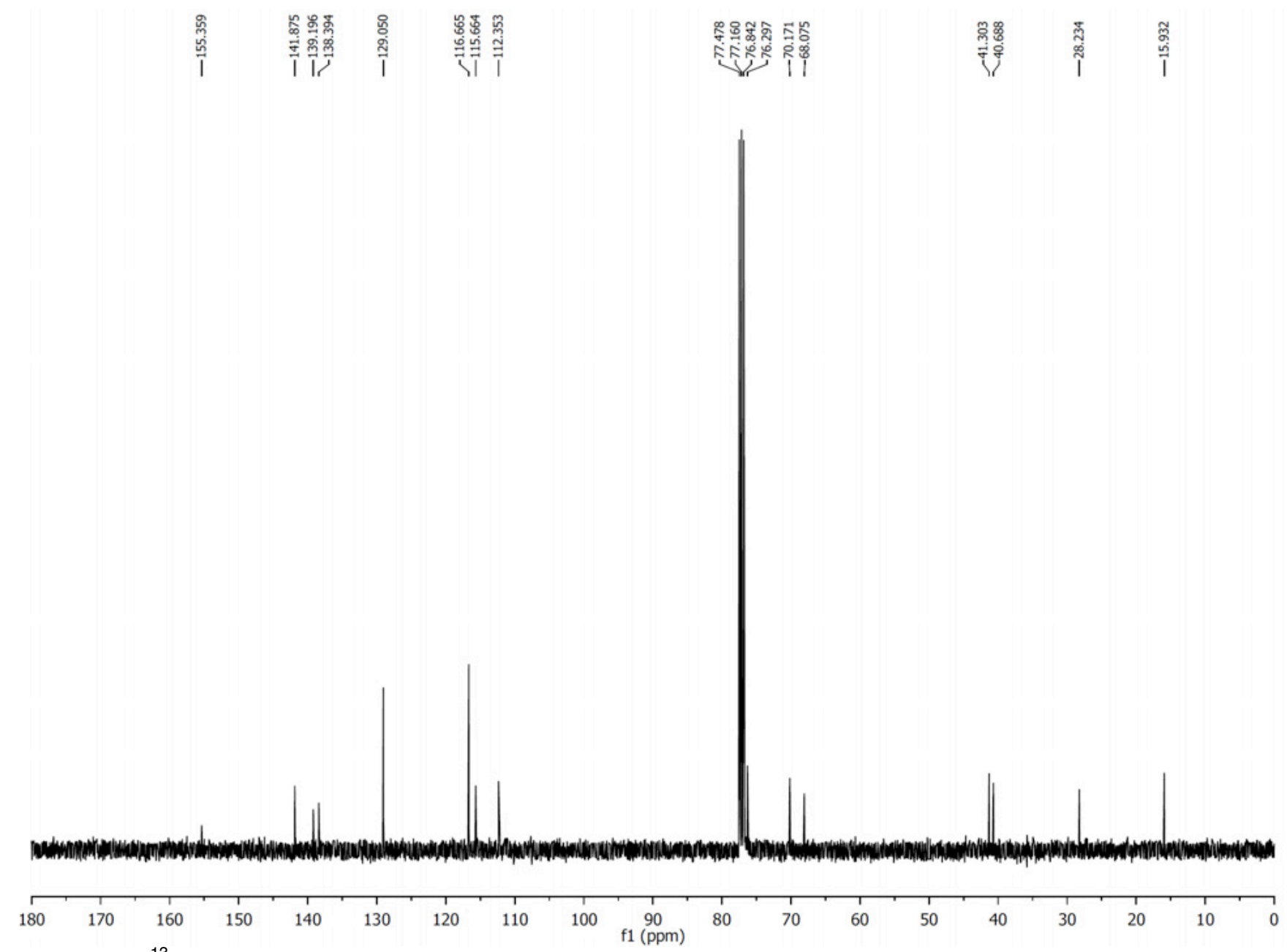

Figure S4. ${ }^{13} \mathrm{C}$ NMR spectrum of $11 \mathrm{~b}$ in $\mathrm{CDCl}_{3}$. 


\section{${ }^{1} \mathrm{H}$ NMR spectrum of $11 \mathrm{c}$}
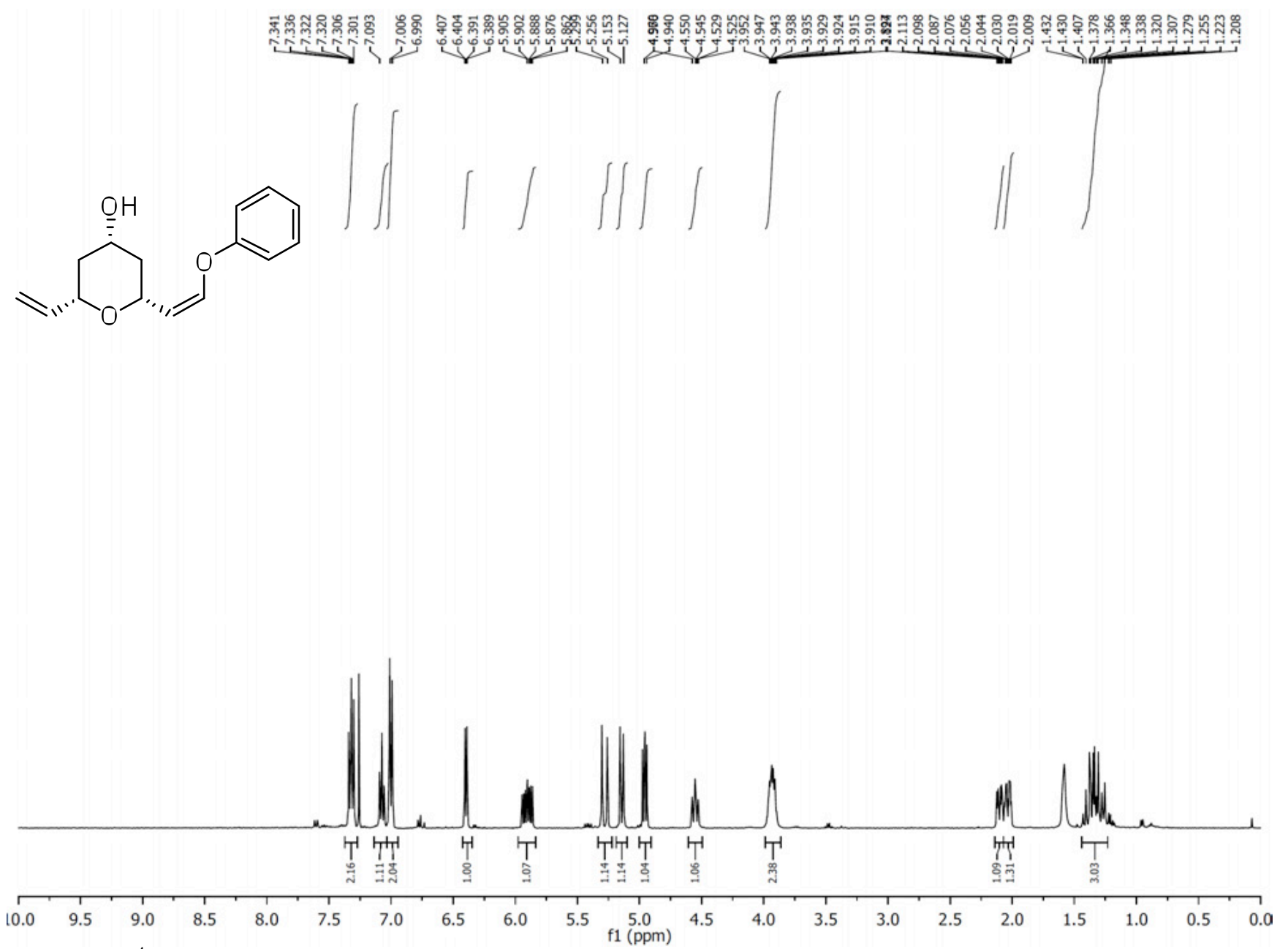

Figure S5. ${ }^{1} \mathrm{H}$ NMR spectrum of $11 \mathrm{c}$ in $\mathrm{CDCl}_{3}$. 


\section{${ }^{13} \mathrm{C}$ NMR spectrum of $11 \mathrm{c}$}

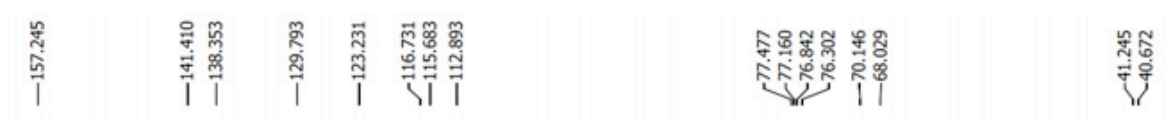

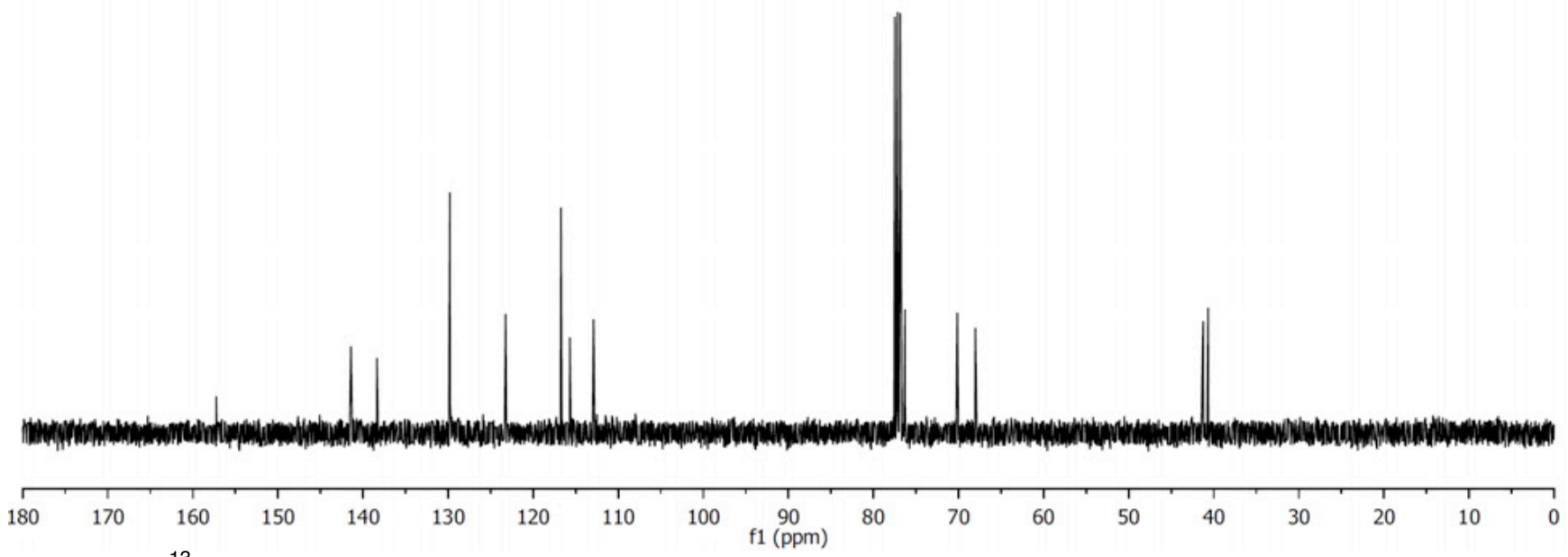

Figure S6. ${ }^{13} \mathrm{C}$ NMR spectrum of $11 \mathrm{c}$ in $\mathrm{CDCl}_{3}$. 


\section{${ }^{1} \mathrm{H}$ NMR spectrum of $11 \mathrm{~d}$}

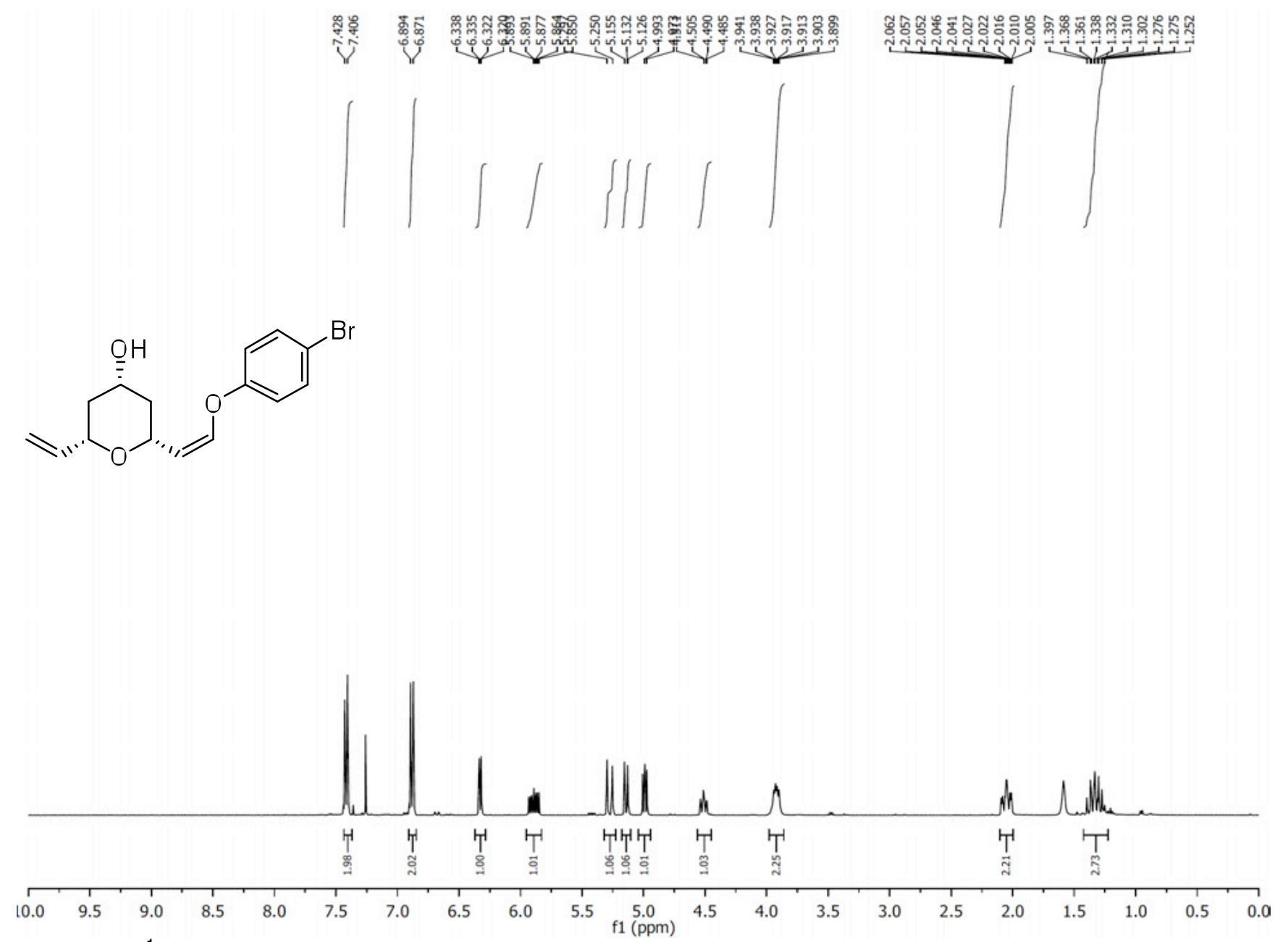

Figure S7. ${ }^{1} \mathrm{H}$ NMR spectrum of $11 \mathrm{~d}$ in $\mathrm{CDCl}_{3}$. 


\section{${ }^{13}$ C NMR spectrum of $11 d$}

\begin{tabular}{|c|c|c|}
\hline 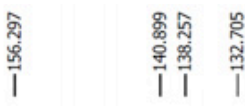 & 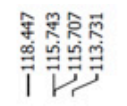 & 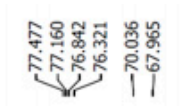 \\
\hline
\end{tabular}

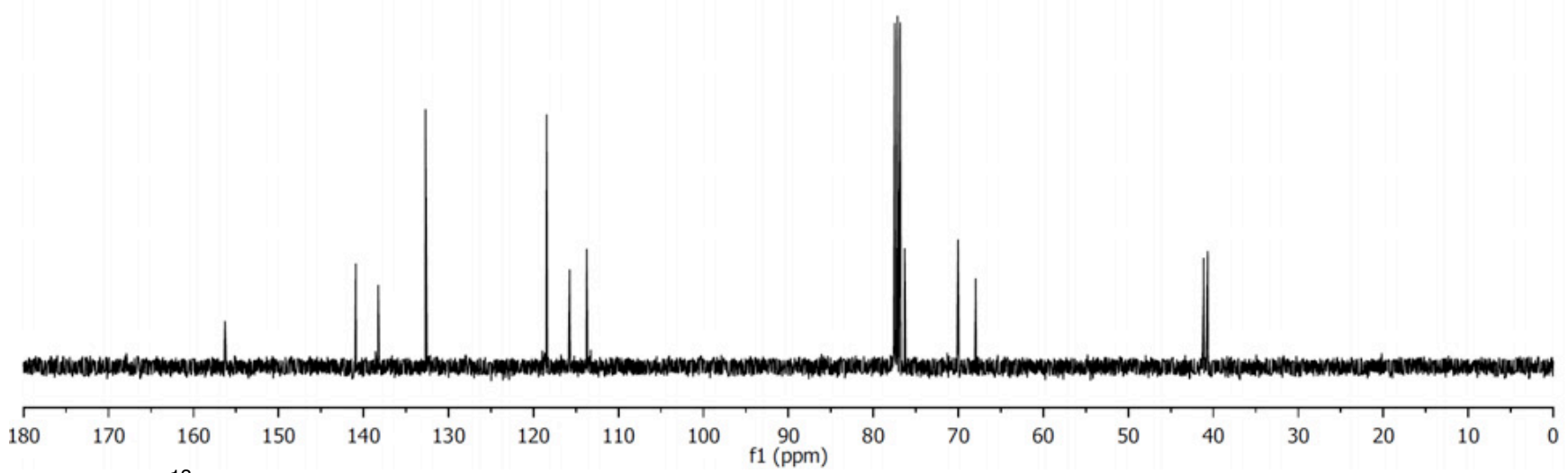

Figure S8. ${ }^{13} \mathrm{C}$ NMR spectrum of $11 \mathrm{~d}$ in $\mathrm{CDCl}_{3}$. 


\section{${ }^{1} \mathrm{H}$ NMR spectrum of $11 \mathrm{e}$}

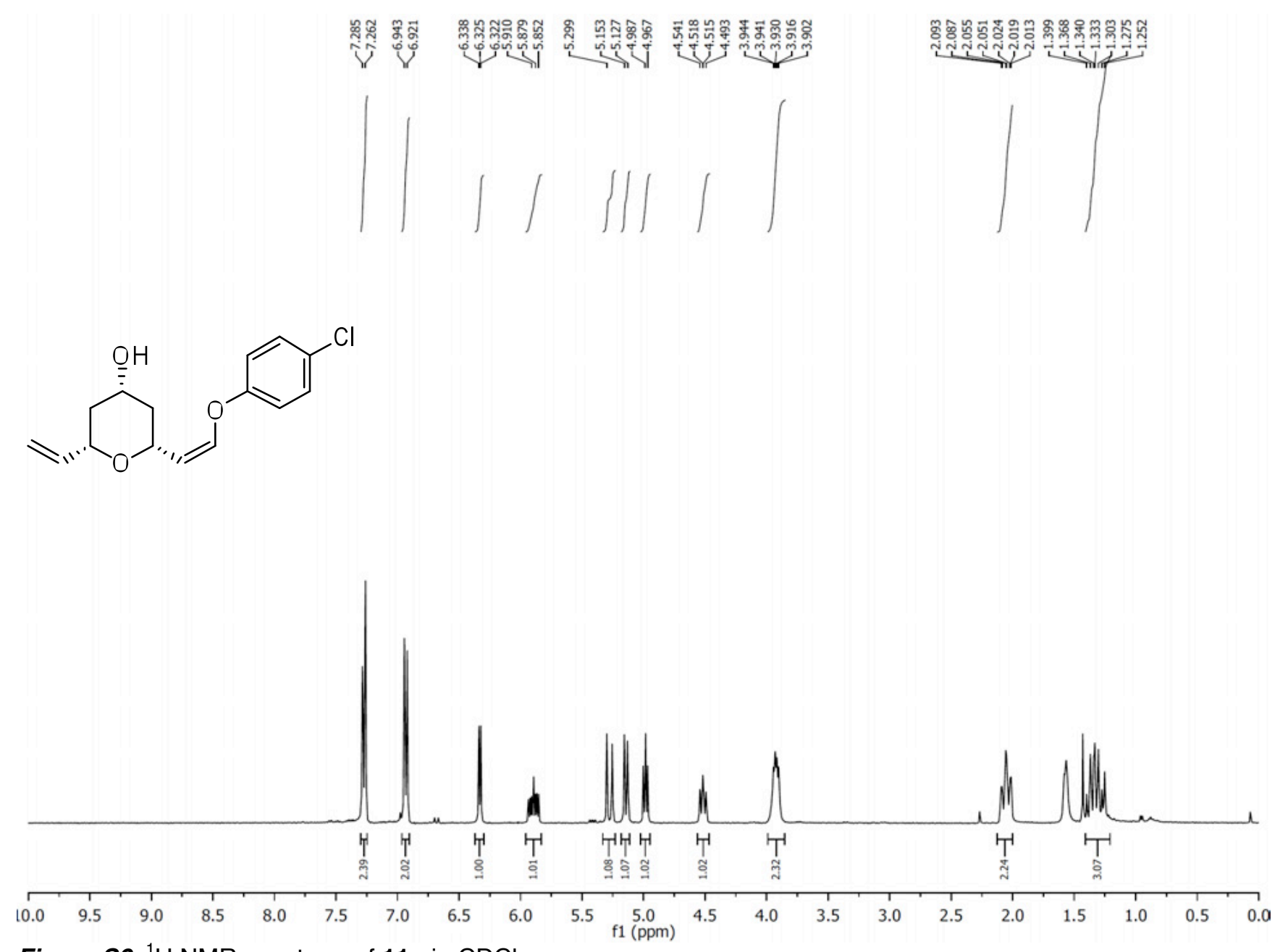

Figure S9. ${ }^{1} \mathrm{H}$ NMR spectrum of $11 \mathrm{e}$ in $\mathrm{CDCl}_{3}$. 


\section{${ }^{13} \mathrm{C}$ NMR spectrum of $11 \mathrm{e}$}

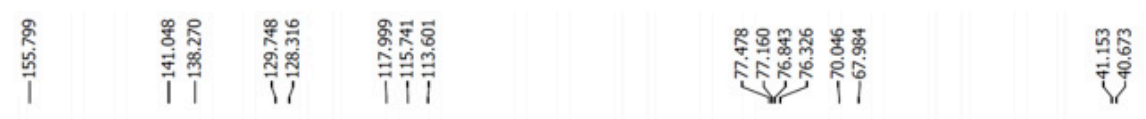

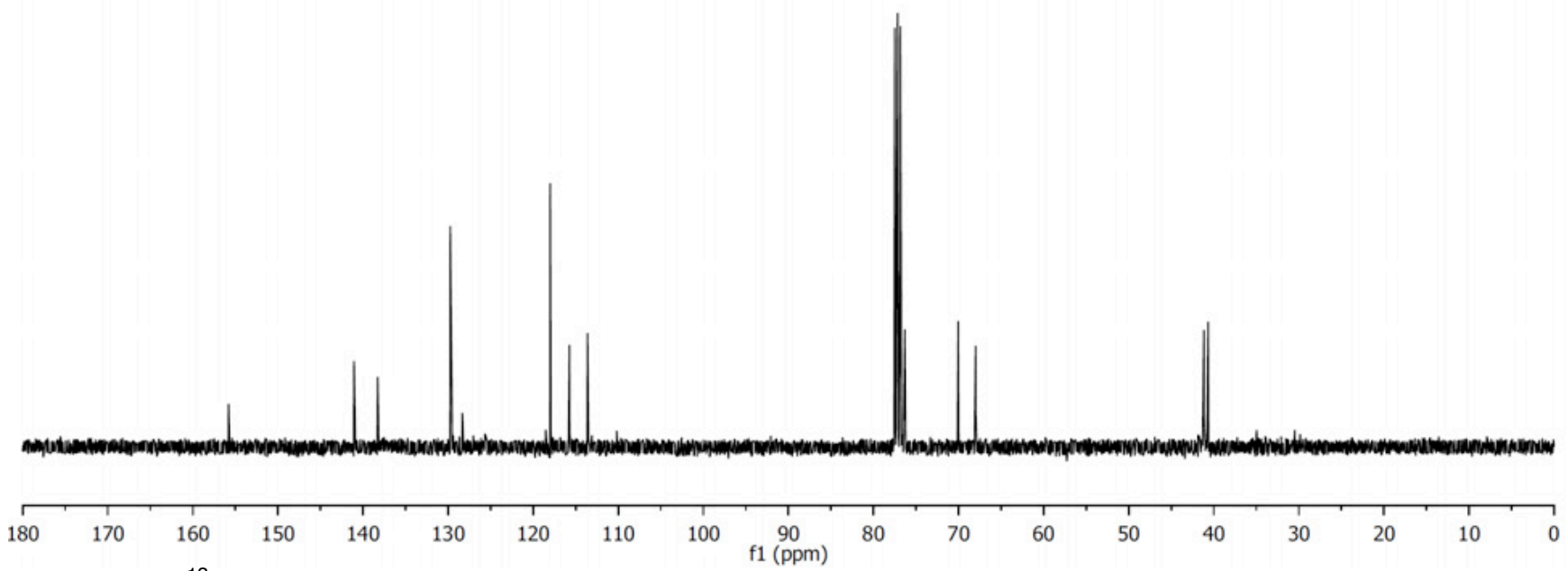

Figure S10. ${ }^{13} \mathrm{C}$ NMR spectrum of $11 \mathrm{e}$ in $\mathrm{CDCl}_{3}$. 


\section{${ }^{1} \mathrm{H}$ NMR spectrum of $11 \mathrm{f}$}

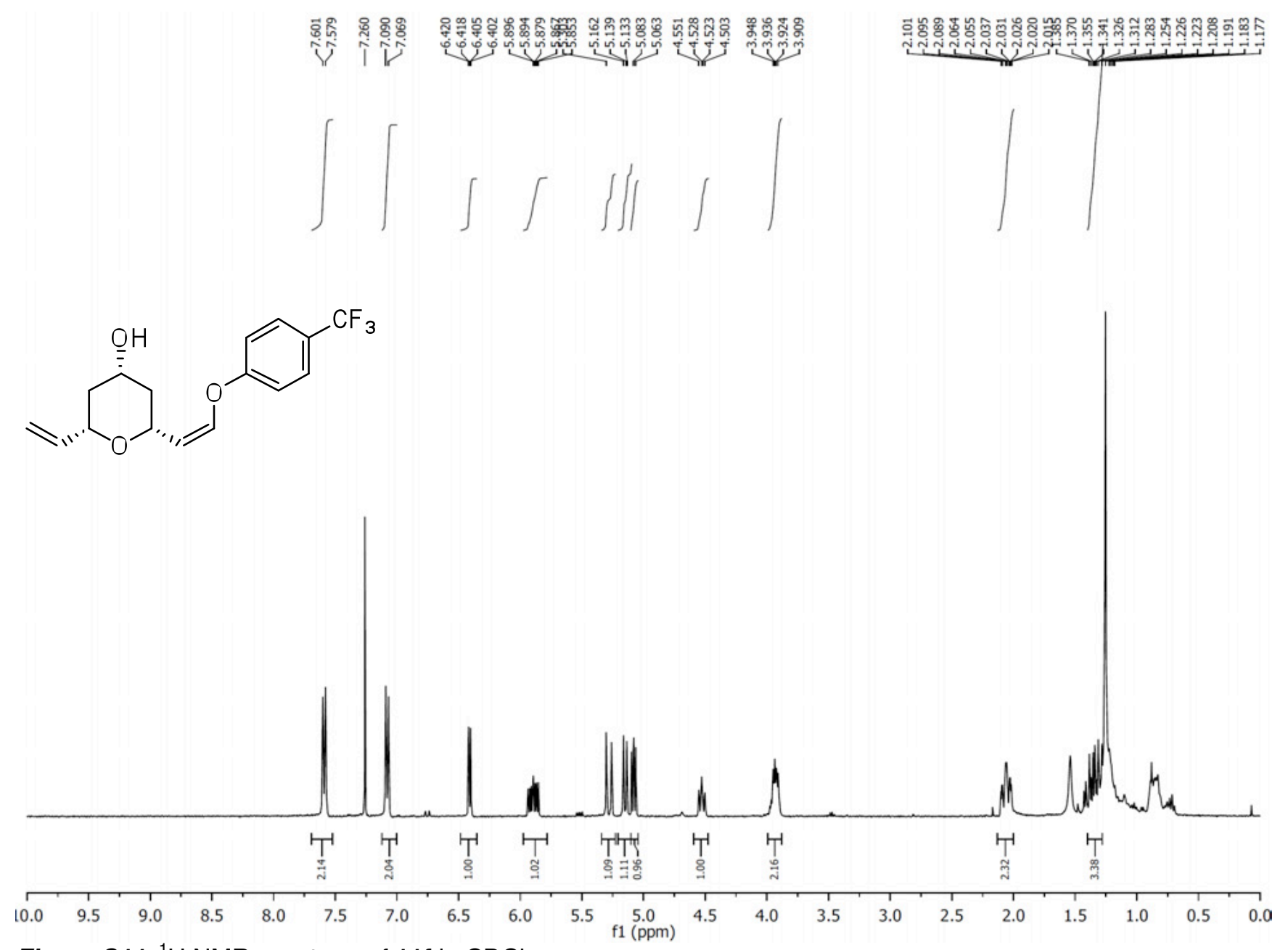

Figure S11. ${ }^{1} \mathrm{H}$ NMR spectrum of $11 \mathrm{f}$ in $\mathrm{CDCl}_{3}$. 


\section{${ }^{13} \mathrm{C}$ NMR spectrum of $11 \mathrm{f}$}

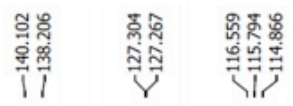

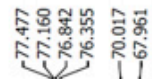

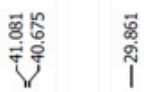

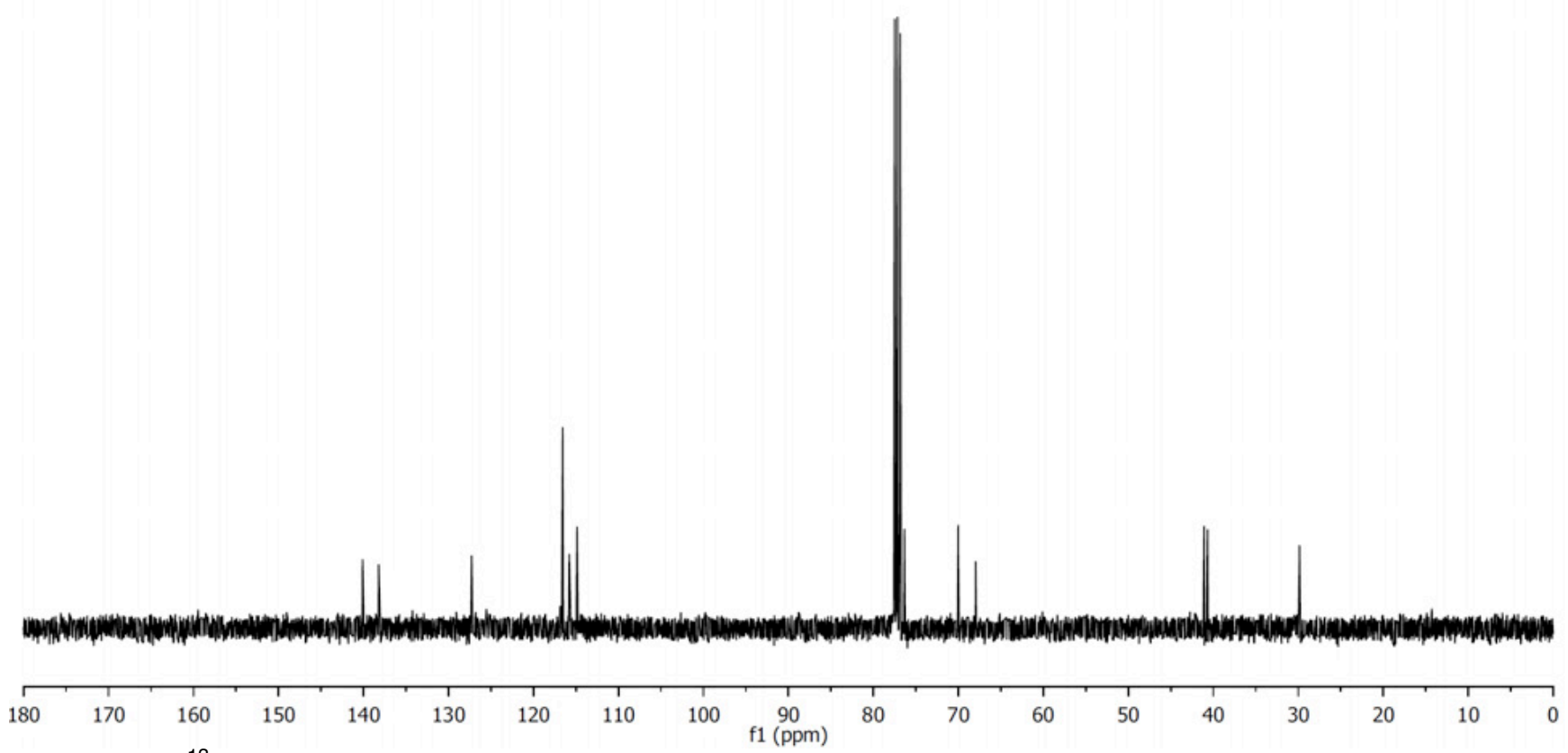

Figure S12. ${ }^{13} \mathrm{C}$ NMR spectrum of $11 \mathrm{f}$ in $\mathrm{CDCl}_{3}$. 


\section{${ }^{1}$ H NMR spectrum of 12}

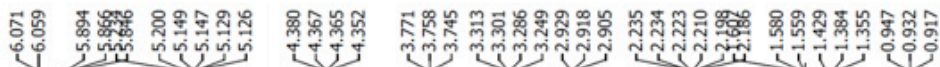

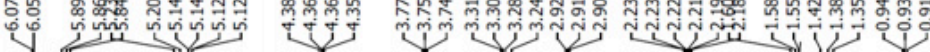<smiles>C=C[C@H]1C[C@H](/C=C\OCC)[C@H]2C(=O)OC(=O)[C@H]12</smiles>

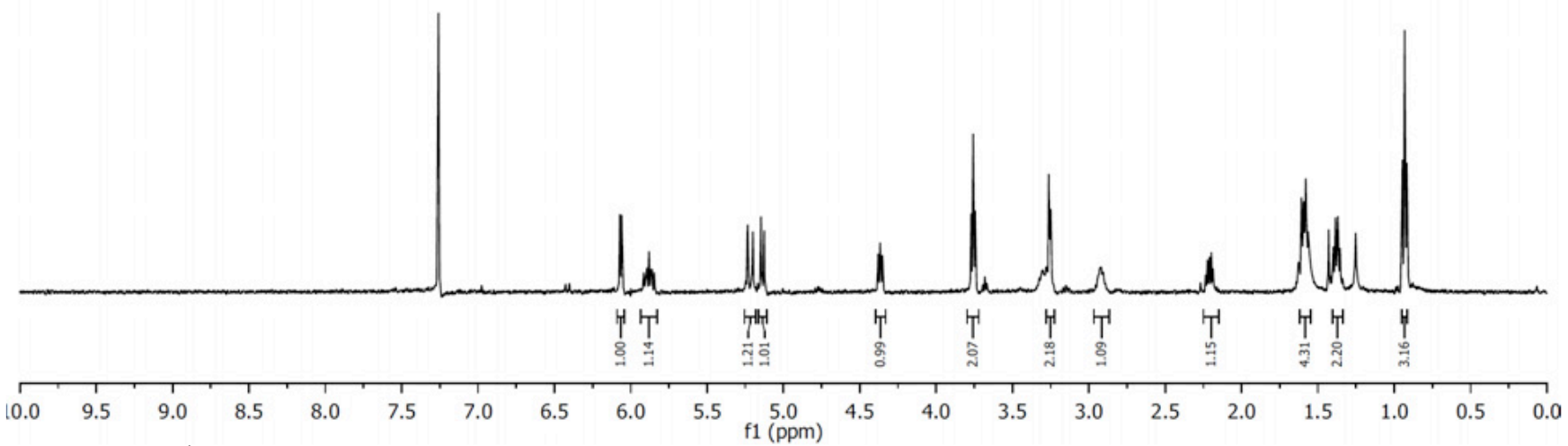

Figure S13. ${ }^{1} \mathrm{H}$ NMR spectrum of 12 in $\mathrm{CDCl}_{3}$. 


\section{${ }^{13} \mathrm{C}$ NMR spectrum of 12}

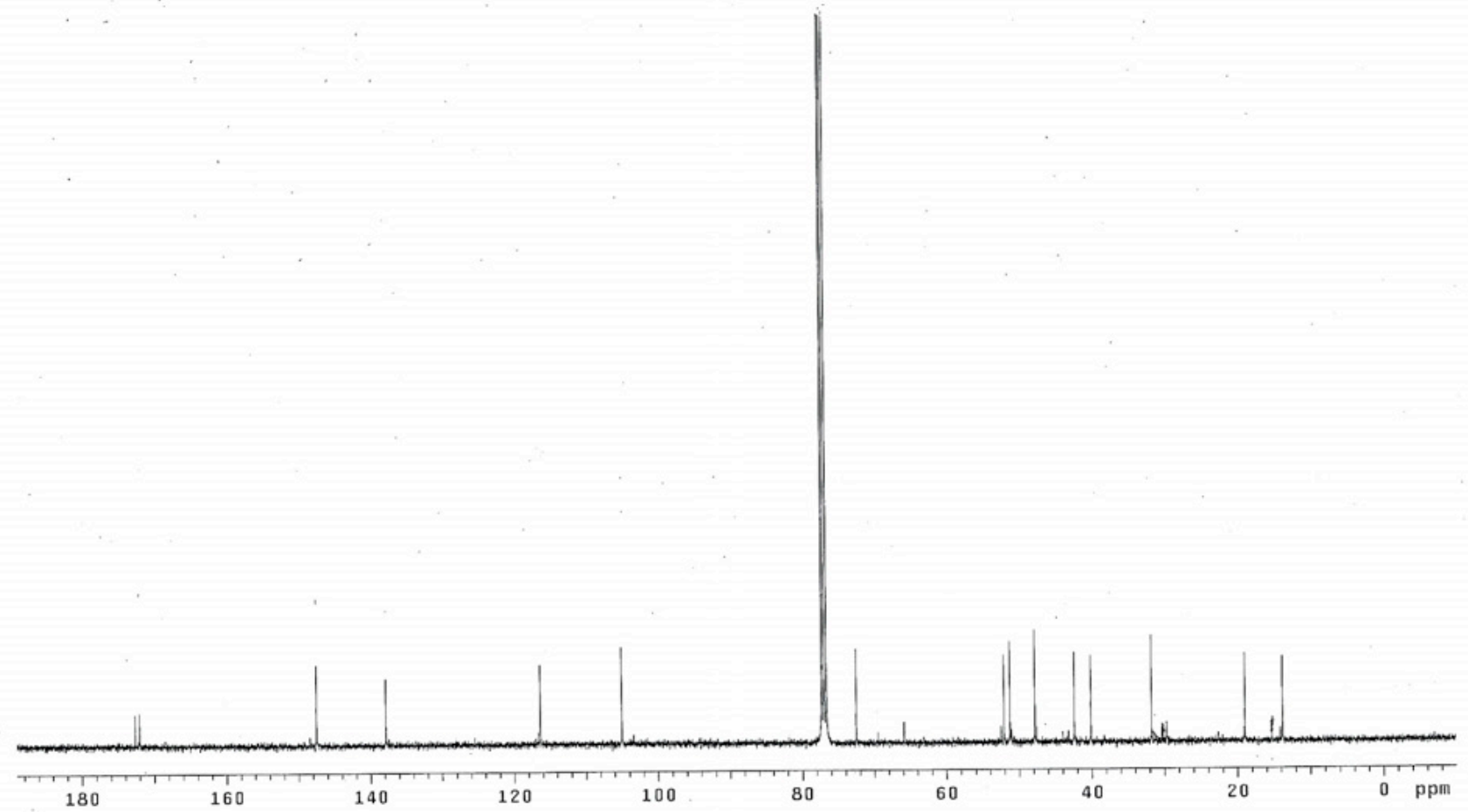

Figure S14. ${ }^{13} \mathrm{C}$ NMR spectrum of 12 in $\mathrm{CDCl}_{3}$. 


\section{${ }^{1} \mathrm{H}$ NMR spectrum of $14 \mathrm{~b}$}
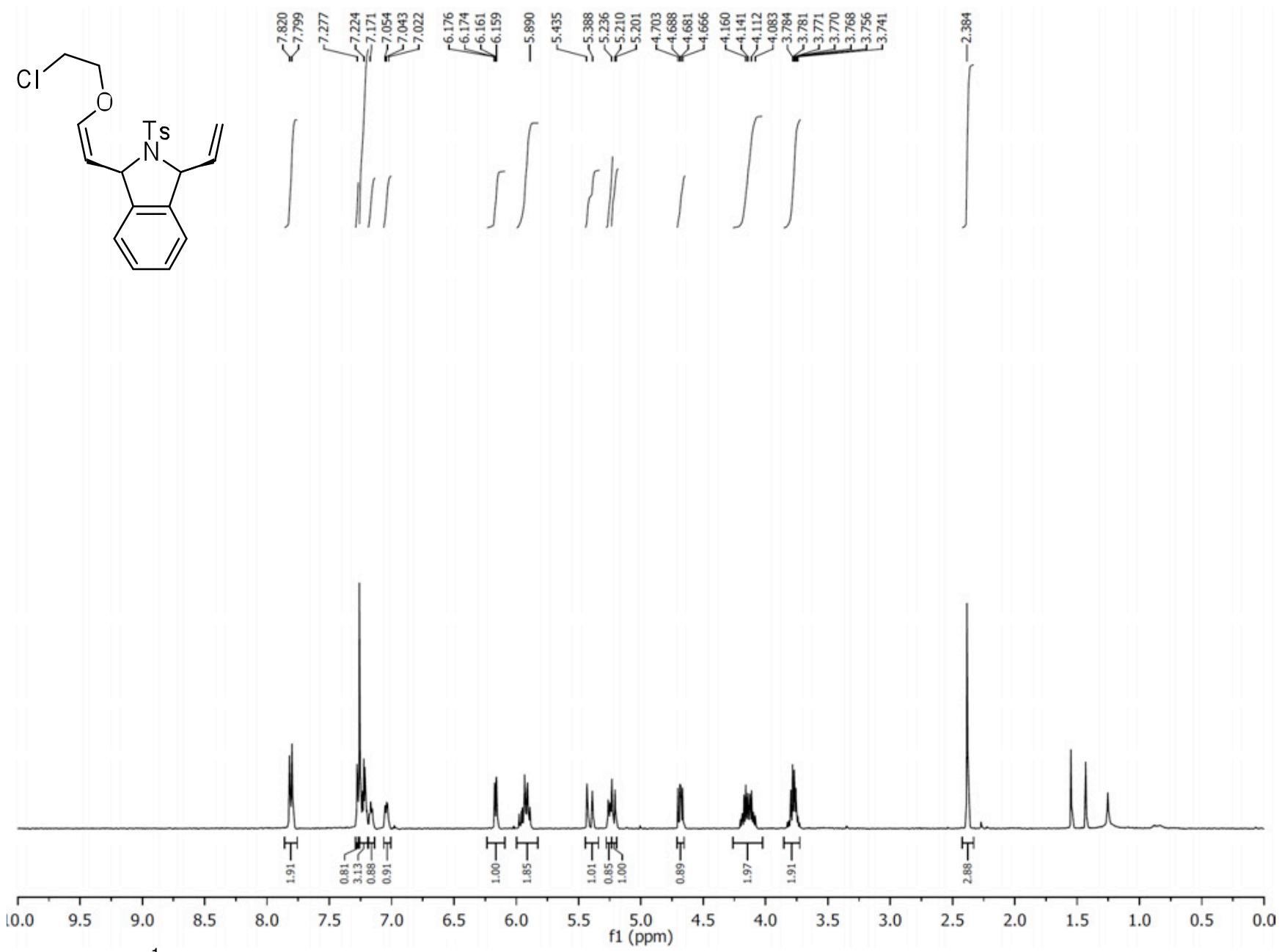

Figure S15. ${ }^{1} \mathrm{H}$ NMR spectrum of $14 \mathrm{~b}$ in $\mathrm{CDCl}_{3}$. 


\section{${ }^{13} \mathrm{C}$ NMR spectrum of $14 \mathrm{~b}$}

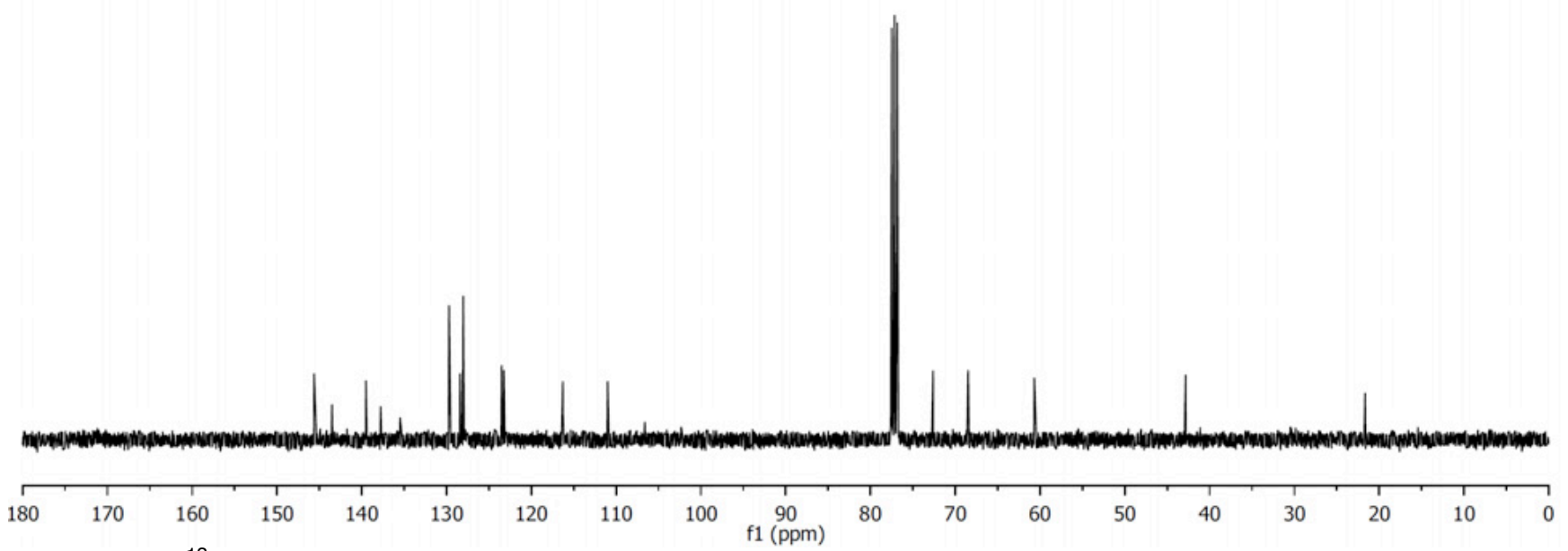

Figure $516 .{ }^{13} \mathrm{C}$ NMR spectrum of $14 \mathrm{~b}$ in $\mathrm{CDCl}_{3}$. 


\section{${ }^{1} \mathrm{H}$ NMR spectrum of $14 \mathrm{c}$}
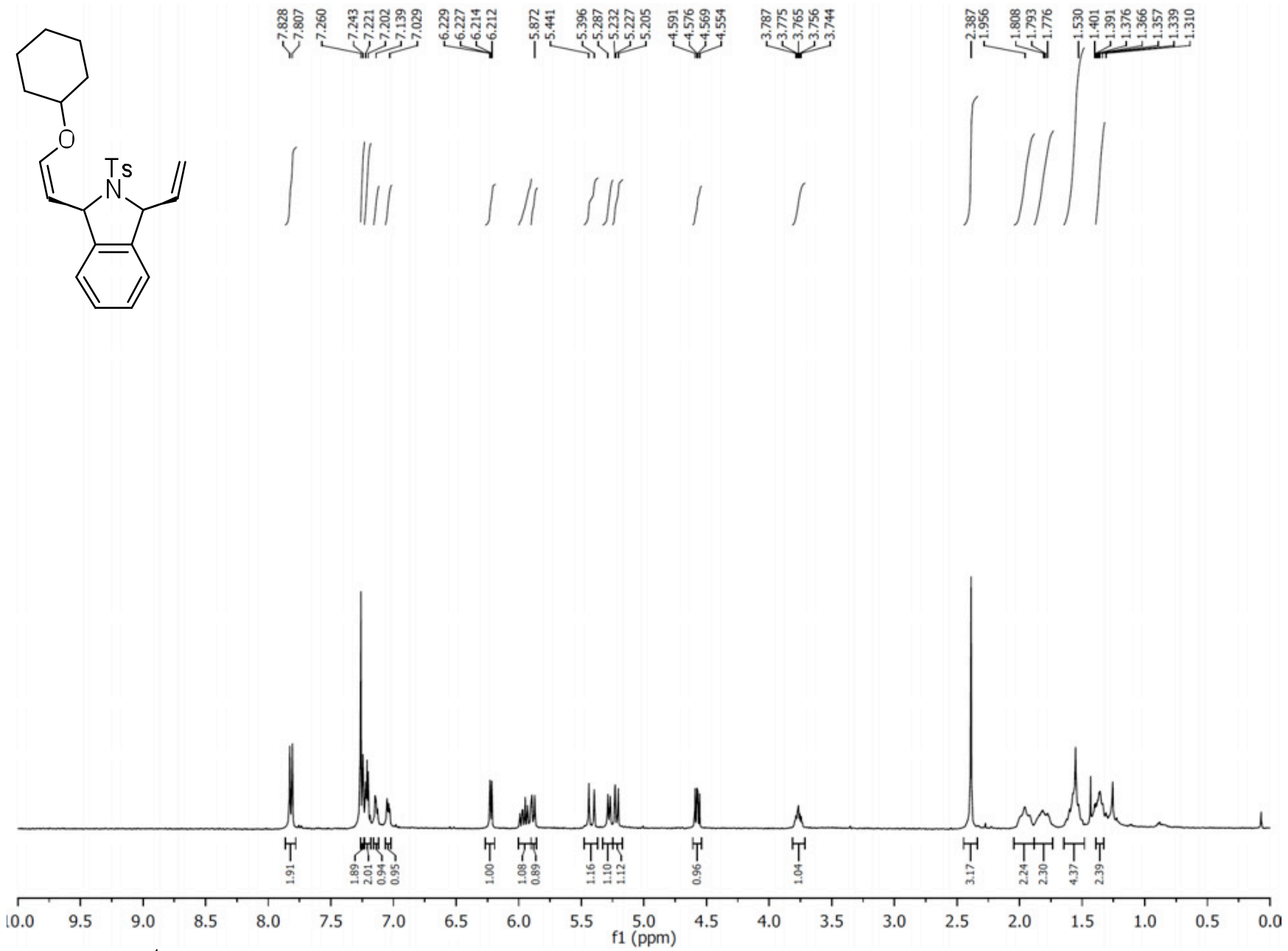

Figure S17. ${ }^{1} \mathrm{H}$ NMR spectrum of $14 \mathrm{c}$ in $\mathrm{CDCl}_{3}$. 


\section{${ }^{13} \mathrm{C}$ NMR spectrum of $14 \mathrm{c}$}

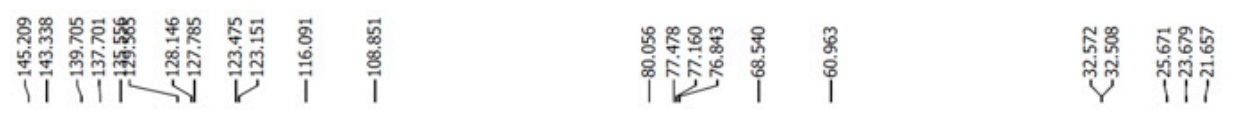

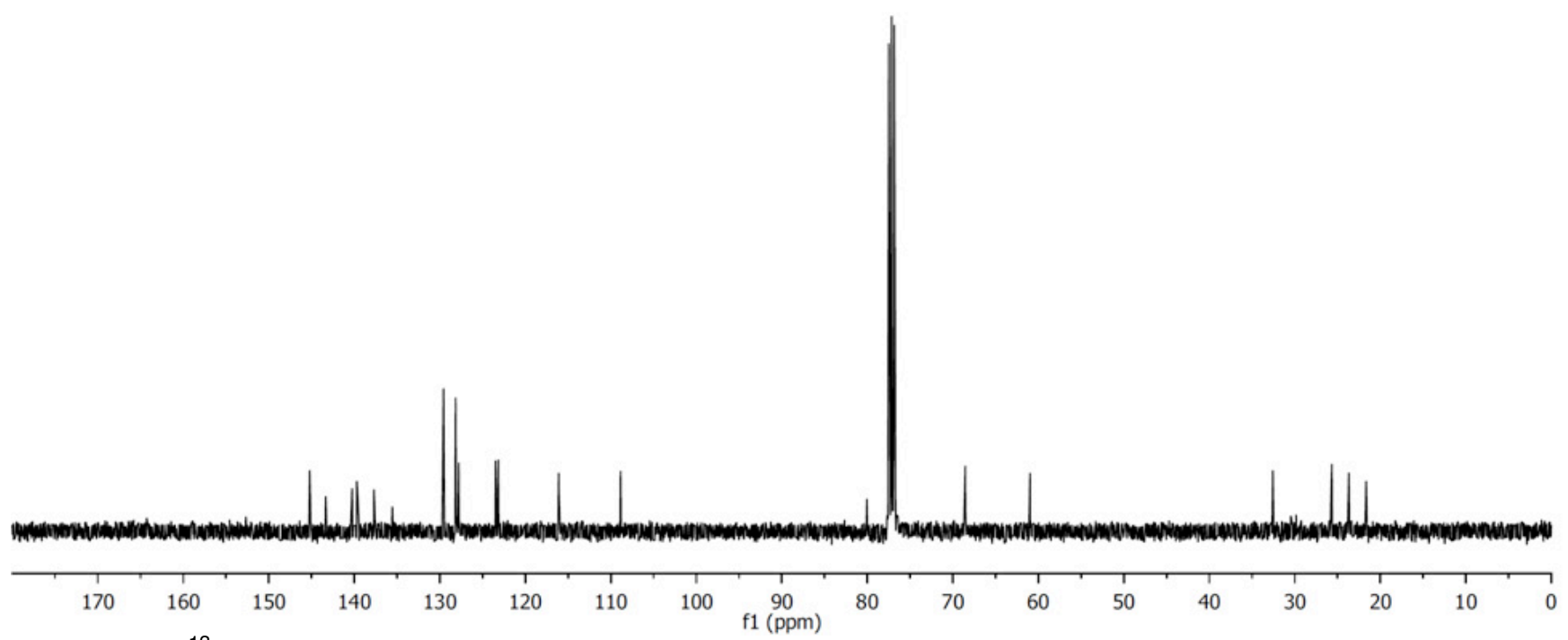

Figure S18. ${ }^{13} \mathrm{C}$ NMR spectrum of $14 \mathrm{c}$ in $\mathrm{CDCl}_{3}$. 


\section{Density Functional Theory (DFT) calculations}

DFT $^{1}$ computations were performed with the Gaussian 09 suite of programs. ${ }^{2}$ The following basis set (termed "basis1") was used for geometry optimizations and evaluation of thermal corrections to the Gibbs free energy at standard conditions (298.15 K, 1 atm): 6-31G(d,p) basis set for hydrogen and carbon atoms, including additional diffuse functions $(+)$ on heteroatoms (oxygen, nitrogen, phosphorous and chloride). A quasi-relativistic effective core potential (ECP) of the Stuttgart-Dresden type $^{3}$ was used for ruthenium and iodide (MWB28 and MWB46 keywords, respectively in Gaussian for basis set and ECP). The nature of all stationary points was checked through vibrational analysis. Geometries for the potential energy surfaces have been optimized with 2 different functionals: BP86 ${ }^{4}$ and $\omega \mathrm{B} 97 \mathrm{XD}^{5}$. Single point electronic energy $\left(\Delta \mathrm{E}_{\mathrm{sp}}\right)$ calculations applying functionals BP86, $\omega \mathrm{B} 97 \mathrm{XD}$, $\mathrm{M}^{6} 6^{6}$ in solution (benzene and dichloromethane) were performed on the gas phase geometries obtained with basis 1 through application of an integral equation formalism variant of the polarizable continuum model (IEFPCM) ${ }^{7}$ and the larger basis set termed "basis2": 6-311+G(2d,p) on $\mathrm{H}, \mathrm{C}, \mathrm{O}, \mathrm{N}, \mathrm{P}, \mathrm{Cl}$ and MWB28/MWB46 on ruthenium/iodide. The single point electronic energies $\left(\Delta \mathrm{E}_{\mathrm{sp}}\right)$ at the basis 2 level were corrected by addition of thermal corrections to the Gibbs free energy $\left(\Delta \mathrm{G}_{\text {corr }}\right)$ obtained at the corresponding basis1 level. In addition, we have investigated the relationship between ts0 and ts 2 in more detail. Therefore, we have re-optimized the potential energy surfaces in Figure 3 in the manuscript with BP86/basis1 in dichloromethane, and performed single point calculations with BP86-

(1) For a recent review on the application of DFT to complexes containing transition metals, see: Cramer, C. J.; Truhlar, D. G. Phys. Chem. Chem. Phys. 2009, 11, 10757.

(2) Frisch, M. J.; Trucks, G. W.; Schlegel, H. B.; Scuseria, G. E.; Robb, M. A.; Cheeseman, J. R.; Scalmani, G.; Barone, V.; Mennucci, B.; Petersson, G. A.; Nakatsuji, H.; Caricato, M.; Li, X.; Hratchian, H. P.; Izmaylov, A. F.; Bloino, J.; Zheng, G.; Sonnenberg, J. L.; Hada, M.; Ehara, M.; Toyota, K.; Fukuda, R.; Hasegawa, J.; Ishida, M.; Nakajima, T.; Honda, Y.; Kitao, O.; Nakai, H.; Vreven, T.; Montgomery, Jr., J. A.; Peralta, J. E.; Ogliaro, F.; Bearpark, M.; Heyd, J. J.; Brothers, E.; Kudin, K. N.; Staroverov, V. N.; Kobayashi, R.; Normand, J.; Raghavachari, K.; Rendell, A.; Burant, J. C.; Iyengar, S. S.; Tomasi, J.; Cossi, M.; Rega, N.; Millam, J. M.; Klene, M.; Knox, J. E.; Cross, J. B.; Bakken, V.; Adamo, C.; Jaramillo, J.; Gomperts, R.; Stratmann, R. E.; Yazyev, O.; Austin, A. J.; Cammi, R.; Pomelli, C.; Ochterski, J. W.; Martin, R. L.; Morokuma, K.; Zakrzewski, V. G.; Voth, G. A.; Salvador, P.; Dannenberg, J. J.; Dapprich, S.; Daniels, A. D.; Farkas, Ö.; Foresman, J. B.; Ortiz, J. V.; Cioslowski, J.; Fox, D. J. Gaussian 09, Revision D.01, Gaussian, Inc., Wallingford CT, 2009.

(3) Andrae, D.; Haeussermann, U.; Dolg, M.; Stoll, H.; Preuss, H. Theor. Chim. Acta 1990, 77, 123.

(4) (a) Becke, A. D. Phys. Rev. A 1988, 38, 3098. (b) Perdew, J. P.; Yue, W. Phys. Rev. B 1986, 33, 8800.

(5) Chai, J.-D.; Head-Gordon, M. Phys. Chem. Chem. Phys., 2008, 10, 6615.

(6) Zhao, Y.; Truhlar, D. G. Acc. Chem. Res. 2008, 41, 157.

(7) Scalmani, G.; Frisch, M. J. J. Chem. Phys. 2010, 132, 114110. 
D3BJ, PBE0-D3BJ ${ }^{8}$ (both of which include Grimme's D3 empirical dispersion with Becke-Johnson damping $)^{9}$, as well as $\omega \mathrm{B} 97 \mathrm{XD}$ and M06 with the even larger Def2QZVP ${ }^{10}$ basis set. The free energy surfaces with functionals BP86, $\omega$ B97XD, M06, BP86-D3BJ and PBE0-D3BJ (Section 4-5), tables of energies (Sections 6-8) and images of transition states (Section 9) are given below and the geometries obtained with $\omega \mathrm{B} 97 \mathrm{XD} /$ basis1 and BP86/basis $1_{\mathrm{DCM}}$ are provided in the separate "computed cartesian coordinates.xyz" file.

\section{Comparison of potential energy surfaces and free energy surfaces for ROCM promoted by $\mathrm{Ru}-4 \mathrm{f}$ and $\mathrm{Ru}-\mathbf{4 b}$ with enol ether as cross partner (cf. Figure 3 )}

In addition to Figures 3 and 4 in the manuscript, comparison of the electronic and free energy surfaces for ring-opening metathesis (ROM) of methyl vinyl ether and oxabicycle $\mathbf{2}$ with $\mathbf{R u}-\mathbf{4 f}$ and $\mathbf{R u}-\mathbf{4 b}$ are shown in Figure S19. Examination of the $\Delta \mathrm{E}$ surfaces (Figure S19) shows that olefin decoordination in case of the NHC based complex Ru-4b is less facile then mcb formation/breakage, as $\mathbf{t s} \mathbf{2}_{\mathrm{ZNHC}}$ lies significantly more below the corresponding $\mathbf{1 4 e}$ complex $(-11.3 \mathrm{kcal} / \mathrm{mol})$ compared to $\mathbf{t s} \mathbf{2}_{\text {ZPhos }}(-8.4 \mathrm{kcal} / \mathrm{mol})$, likely rendering olefin exchange (coordination/de-coordination) ratedetermining. For a more detailed investigation of the relationship between ts0 and ts 2 , we refer to the end of this section. Structural comparison of the cycloreversion transition states (ts2) and the intramolecular $\pi$ complexes (pc2) for complexes Ru-4f (Figure S20) and Ru-4b (Figure S21) reveals, that the same steric/stereoelectronic effects are likely operative in the reaction steps following olefin coordination, and that the main origin for a reversal in selectivity lies in a change of the kinetic profile, as discussed in sections 1 and 2 in the manuscript.

(8) For the use of the PBE0 functional with D3BJ dispersion, see: (a) Steinmetz, M.; Grimme, S. ChemistryOpen 2013, 2, 115-124. (b) Luo, S.; Zhao, Y.; Truhlar, D. G. Phys. Chem. Chem. Phys. 2011, 13, 13683-13689.

(9) Grimme, S.; Ehrlich, S.; and Goerigk, L. J. Comp. Chem. 2011, 32, 1456-1465.

(10) Weigend, F.; and Ahlrichs, R. Phys. Chem. Chem. Phys., 2005, 7, 3297-3305. 
a

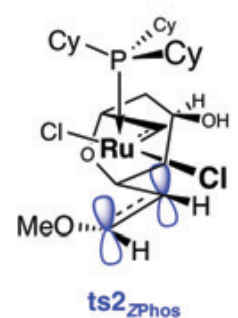

\section{ZPhos}

$\Delta \mathrm{E} / \Delta \mathrm{G}$

[kcal/mol]

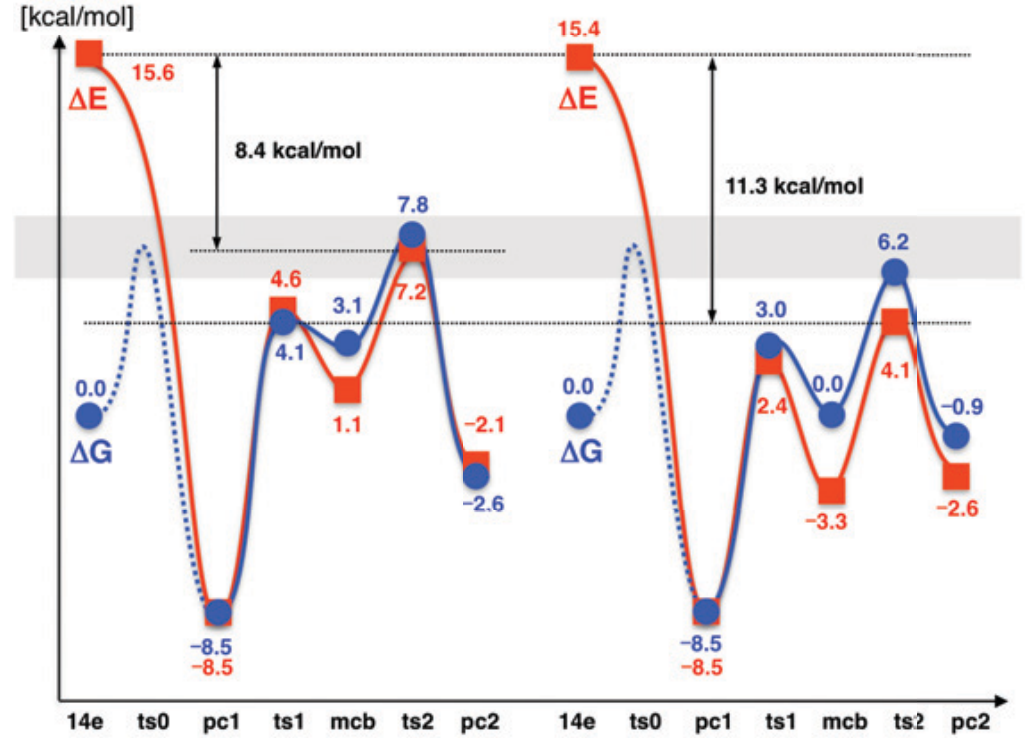

b

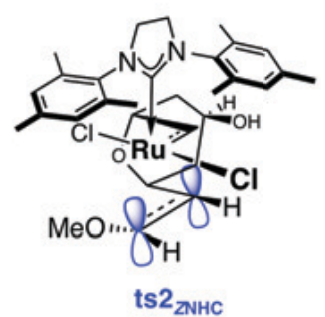

\section{ZNHC}


a structural aspects of ts $2_{E P h o s}(\longrightarrow$ Eproduct)
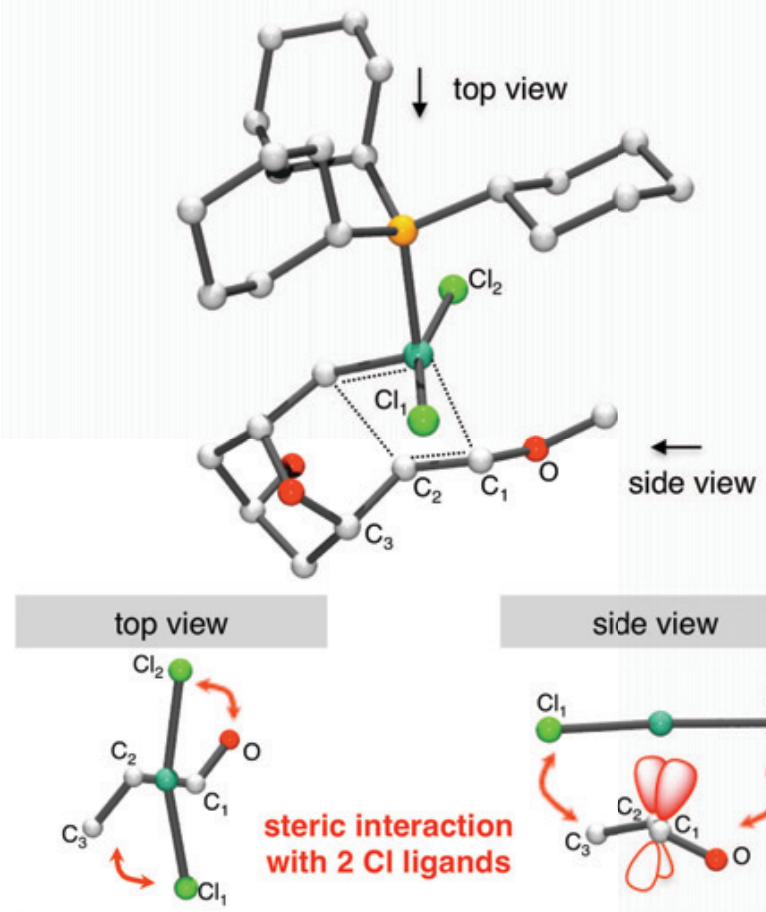

side view

overlap for incipient $\pi$ bond is weaker

c structural aspects of $\mathrm{pc}_{\mathrm{EPhos}}(\longrightarrow E$ product $)$
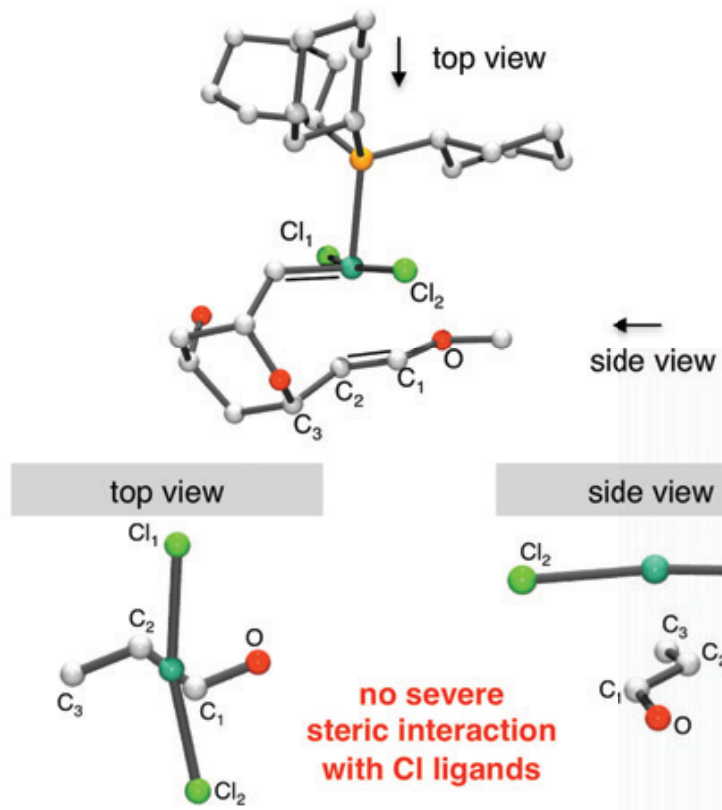

no severe
steric interaction
with $\mathrm{Cl}$ ligands
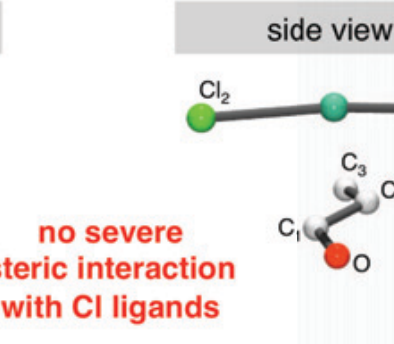

b structural aspects of ts $2_{\text {ZPhos }}(\longrightarrow Z$ product $)$
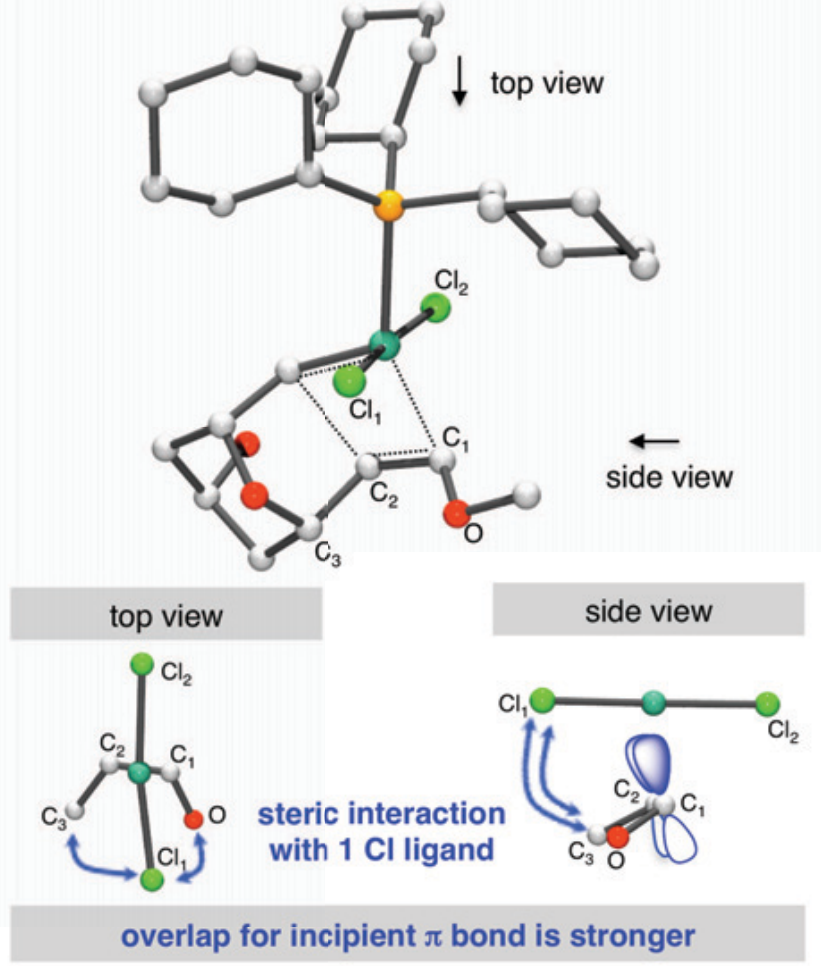

d structural aspects of $\mathrm{pc} 2$ ZPhos $(\longrightarrow Z$ product $)$
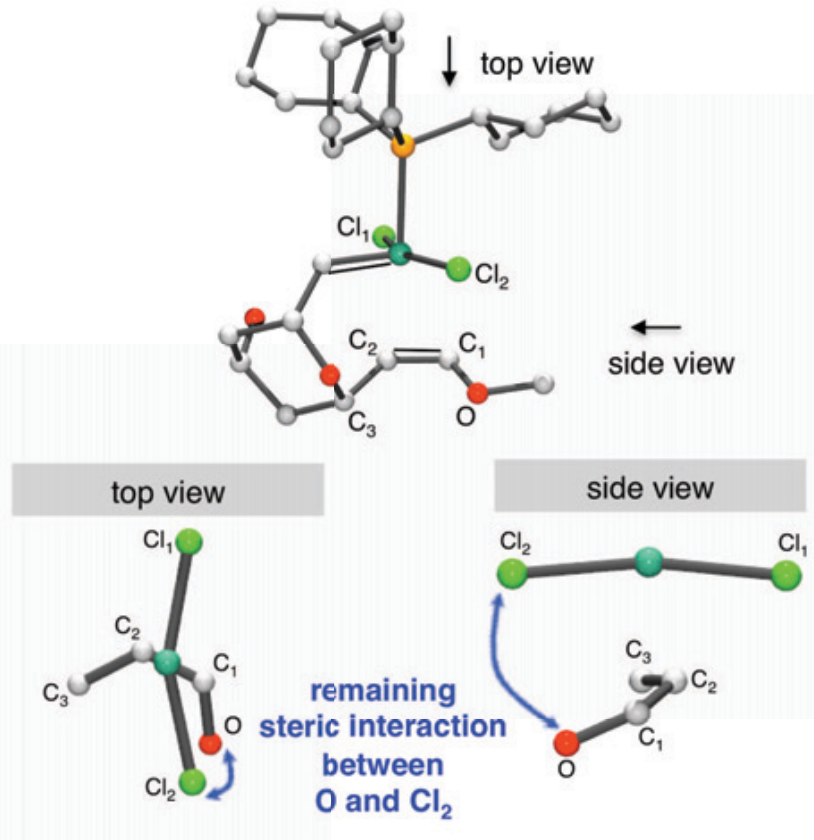

Figure S20. Analysis and comparison of cycloreversion transition states (ts2, top) and intramolecular $\pi$ complexes (pc2, bottom), and stereochemical model for $Z$ selectivity in ROCM reactions with achiral complex Ru-4f and enol ethers. 
a structural aspects of ts $2_{\mathrm{ENHC}}(\longrightarrow E$ product)

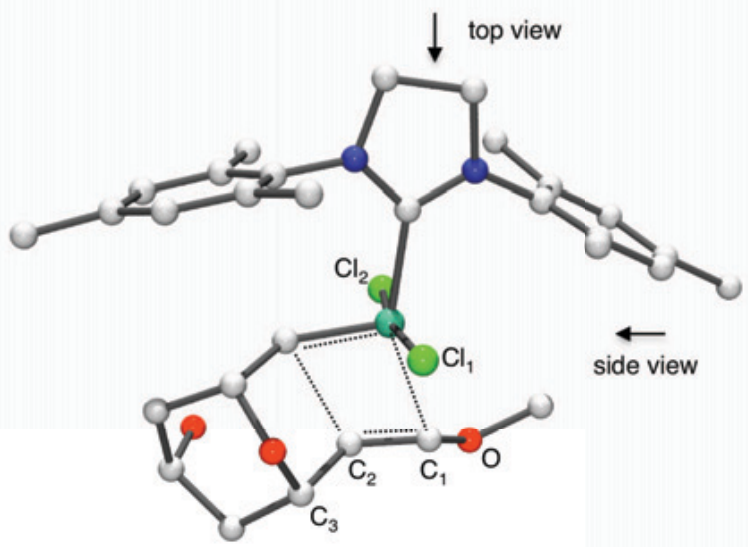

b structural aspects of ts $2_{\text {ZNHC }}(\longrightarrow Z$ product $)$
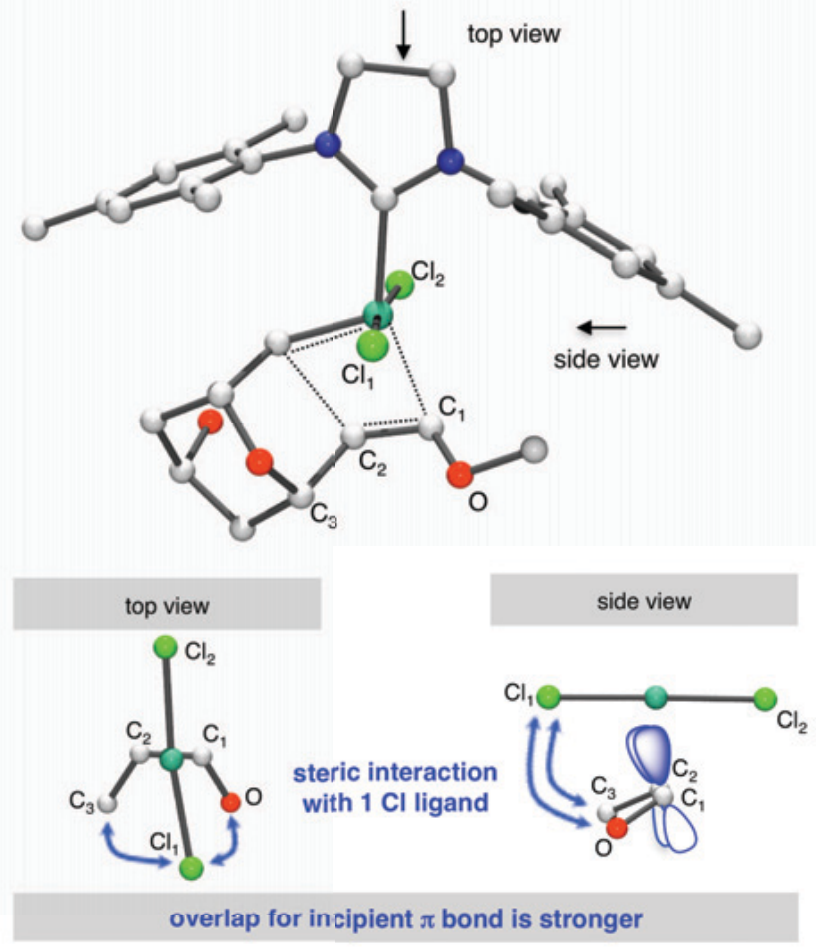

d structural aspects of $\mathrm{pc}_{2 \mathrm{ZNC}}(\longrightarrow Z$ product $)$

c structural aspects of $\mathrm{pc} 2_{\mathrm{ENHC}}$ ( $\longrightarrow E$ product)
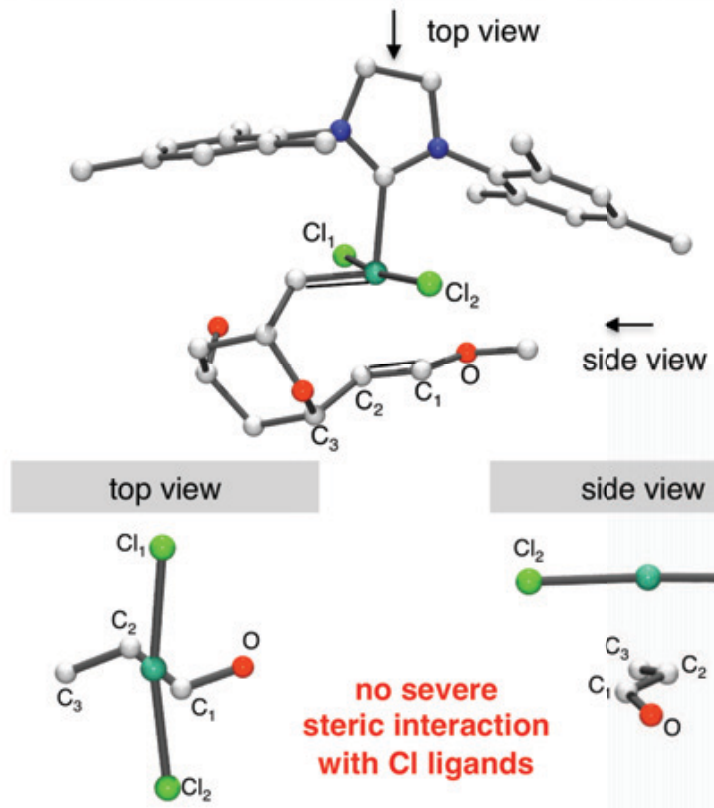

no severe steric interaction with $\mathrm{Cl}$ ligands side view
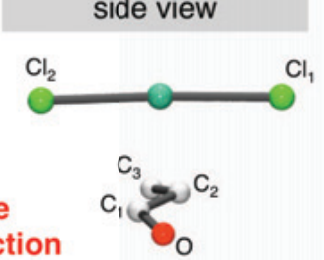

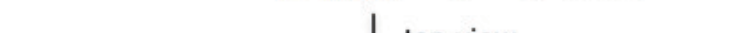

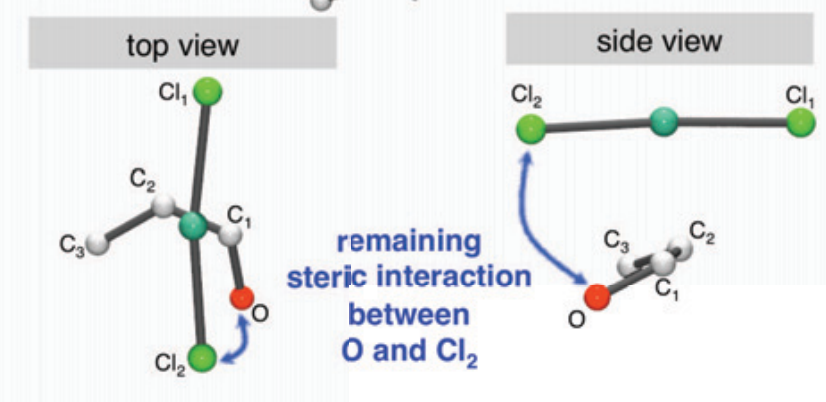

Figure S21. Analysis and comparison of cycloreversion transition states (ts2, top) and intramolecular $\pi$ complexes (pc2, bottom), and stereochemical model for $Z$ selectivity in ROCM reactions with achiral complex Ru-4b and enol ethers. 


\section{Potential energy surfaces for ROCM promoted by Ru-5b with enol ether as cross partner (cf. Figure 5)}

To derive the stereochemical model for the dramatic reversal of stereochemistry in ROCM reactions with Ru-5b (vs styrene; cf. Scheme 3a), we investigated all eight possible product-yielding sequences that proceed via intermediates where the mcb is positioned anti to the NHC ligand (Figure S22); thus, each of the four different possible stereoisomers may be formed through a Curtin-Hammett or a non-Curtin-Hammett pathway (Figure S22, top panel). The Curtin-Hammett routes, where the higher energy 5-endo carbene is involved in the ring-opening metathesis (ROM) stage, are labeled A1-A4, with A1 (= ZRu-5b in Figure 5) and A2 being associated with the formation of the $Z$ isomer and $\mathbf{A 3}$ and $\mathbf{A 4}(=E \mathbf{R u}-5 \mathrm{~b}$ in Figure 5$)$ generating the $E$-alkene. The same terminology applies to the complexes involved in the non-Curtin-Hammett pathways, B1-B4; these entities are derived from the lower energy Ru carbene 5-exo. For simplicity, only the Ru-alkene complexes that likely participate in the three most critical ROM sequences are presented (for a graphical representation of all pathways, see Figures S44-S52).

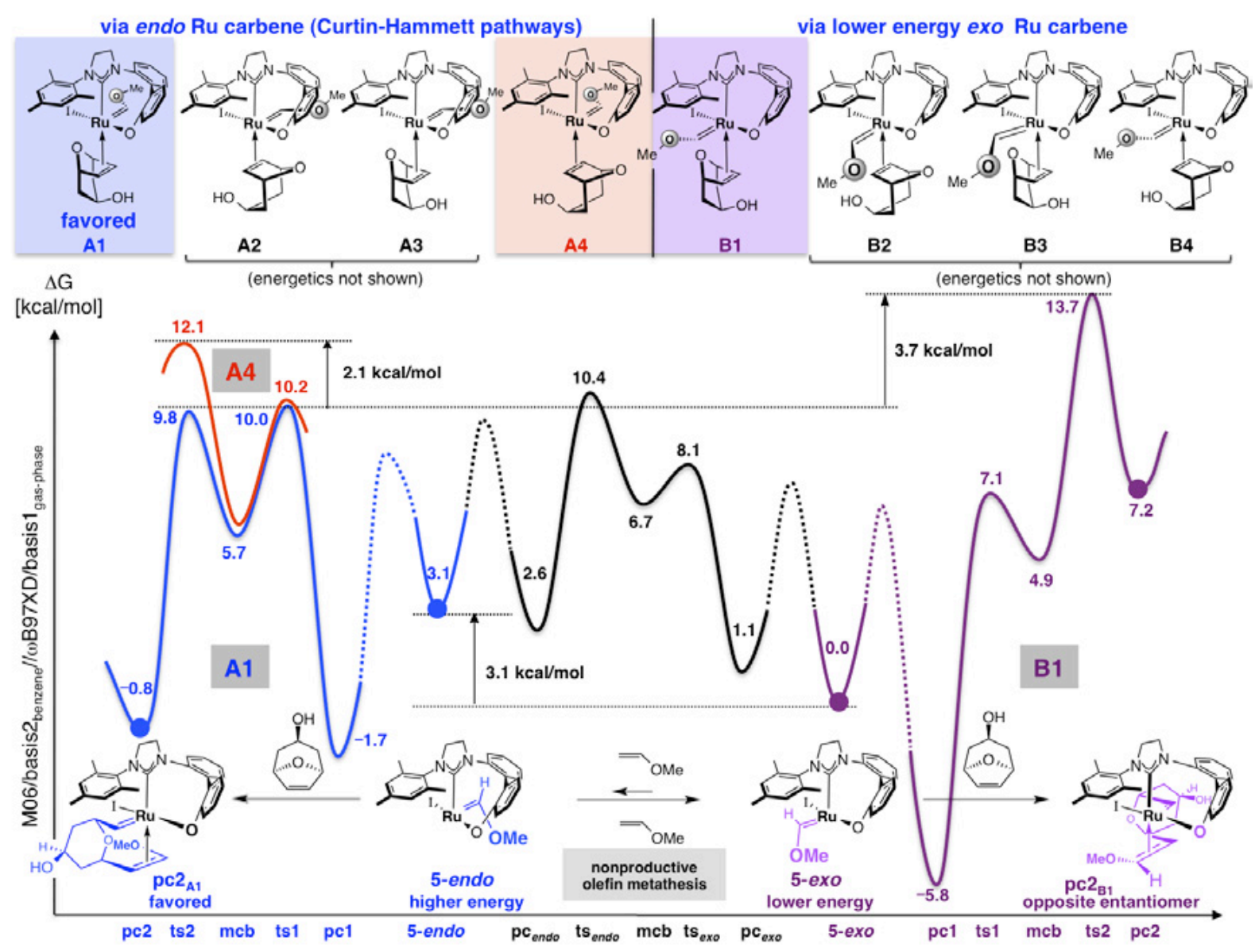

Figure S22. Calculations (DFT) allow for comparison of eight different anti-to-NHC pathways for ROCM of oxabicyclic alkene 2 and methyl vinyl ether promoted by complexes derived from Ru-5b. The energies of the three most critical pathways (A1, A4 and B1) are shown. For a complete list, see Section 4. 14e $=14$-electron complex, ts $=$ transition state, $\mathbf{p c}=\pi$ complex, $\mathbf{m c b}=$ metallacyclobutane 
Also shown in Figure S22 is the lowest energy route for nonproductive OM through which Fischer carbene diastereomers 5-exo and 5-endo interconvert. The latter process appears to be relatively facile in the case of enol ethers $\left(\mathrm{E}_{\mathrm{rel}} \mathbf{t s}_{\text {endo }}=10.4 \mathrm{kcal} / \mathrm{mol}\right)$, a requirement for the applicability of the originally proposed Curtin-Hammett kinetics. The most favorable mode of addition of

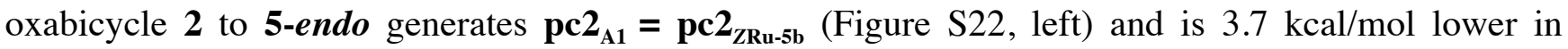
energy than the lowest pathway for ROM via 5-exo and pc2 $2_{\mathbf{B} 1}$ (B1; Figure S22, right). In this way, the opposite sense of enantioselectivity results (vs reactions with aryl-substituted alkenes that likely proceed through an exo carbene; $\mathrm{cf}$. Scheme $3 \mathrm{a}$ in manuscript). In the rate-limiting step of the pathway that might proceed via 5-exo (non-Curtin-Hammett route; $\mathrm{E}_{\mathrm{rel}}=13.7 \mathrm{kcal} / \mathrm{mol}$; $\mathbf{t s} \mathbf{2}_{\mathbf{B} 1}$ ) the oxabicyclic reactant can undergo ROM while positioned in a manner that allows steric repulsion with the NHC's biphenoxy linkage to be avoided. Nonetheless, the non-Curtin-Hammett sequences (B1-B4) are energetically disfavored because of the inherently low reactivity of the electronically/resonancestabilized Fischer carbene 5-exo. The number of energetically accessible reaction modes is therefore reduced by a factor of two. The most favorable Curtin-Hammett based pathways $\mathbf{A 1}(=\mathbf{Z R u - 5 b}$ in Figure 5) and A4 (= ERu-5b in Figure 5) have been compared in Figures 5 and 6 in the manuscript.

\section{Potential energy surfaces for ROCM promoted by Ru-5b with styrene as cross partner}

The corresponding DFT calculations for ROCM with styrene as cross partner are shown in Figure S23. The Curtin-Hammett routes, where the higher energy 5-endo carbene is involved in the ring-opening metathesis (ROM) stage, are labeled $\mathbf{C 1 - C 4}$, with $\mathbf{C 1}$ and $\mathbf{C 2}$ being associated with the formation of the $Z$ isomer and $\mathbf{C 3}$ and $\mathbf{C 4}$ generating the $E$-alkene. The same terminology applies to the complexes involved in the non-Curtin-Hammett pathways, D1-D4; these entities are derived from the lower energy Ru carbene 5-exo. In contrast to the results with enol ether, there is a clear preference for non-Curtin-Hammett pathways, i.e. mode of addition $\mathbf{D 3}$ is favored by $5.7 \mathrm{kcal} / \mathrm{mol}$ over pathway $\mathbf{C 2}$, the most favorable Curtin-Hammett based scenario. For simplicity, only the Ru-alkene complexes that likely participate in the three most critical ROM sequences are presented. In addition, a fundamental requirement for the applicability of Curtin-Hammett kinetics is not met, i.e. the equilibration of benzylidenes 5-endo and 5-exo through nonproductive OM with styrene $(13.9 \mathrm{kcal} / \mathrm{mol})$ is less favored than ring opening involving the cyclic substrate $2\left(\mathrm{E}_{\mathrm{rel}}=7.2 \mathrm{kcal} / \mathrm{mol}\right.$ for the rate limiting transition state $\mathbf{t s} \mathbf{1}_{\mathbf{D} 3}$ of the most favored mode of addition).

A close examination of critical transition state structures for cycloreversion (ts2) lends credence to the experimentally derived stereochemical model and, furthermore, offers insights into the kinetic nature for the formation of $E$ double bonds, despite the strong thermodynamic bias for generation of the same geometric isomer (Figure S24). In the favored non-Curtin-Hammett pathways via the lower energy carbene 5-exo (D3-D4) cycloreversion is rate-determining (in case of $\mathbf{t s} \mathbf{2}_{\mathbf{D} 4}$ ) or at least equally 


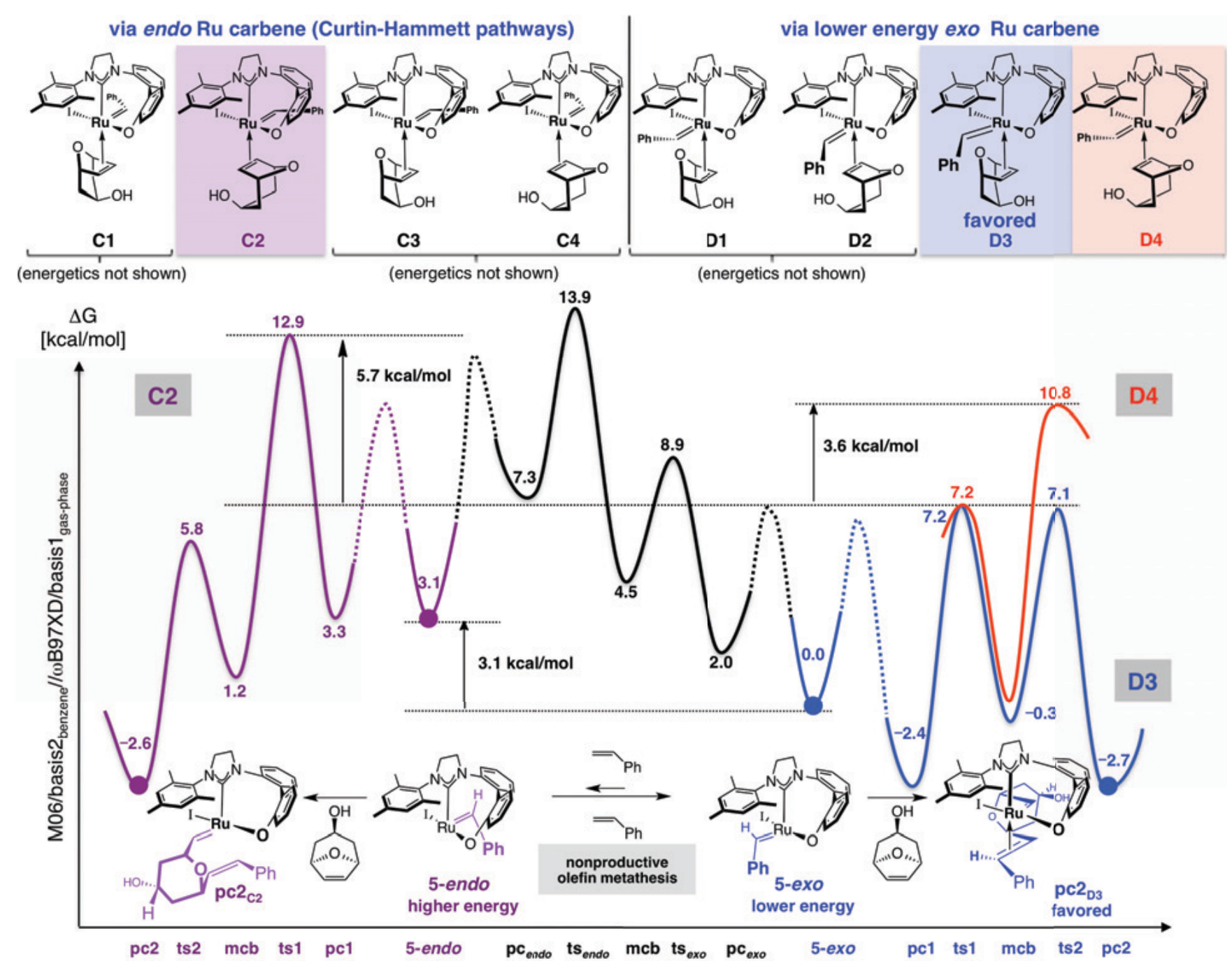

Figure S23. Calculations (DFT) allow for comparison of eight different anti-to-NHC pathways for ROCM of oxabicyclic alkene 2 and methyl vinyl ether promoted by complexes derived from Ru-5b. The energies of the three most critical pathways (C2, D3 and D4) are shown. For a complete list, see the Section 4. 14e $=14$-electron complex, ts $=$ transition state, $\mathbf{p c}=\pi$ complex, $\mathbf{m c b}=$ metallacyclobutane

favored compared to cycloaddition (in case of $\mathbf{t s} \mathbf{2}_{\mathrm{D} 3}$ ). Due to the short $\mathrm{C}^{\mathrm{B}}-\mathrm{C}^{\mathrm{C}}$ bond distances of the incipient double bond, minimization of steric repulsion between the phenyl substituent $(\mathrm{Ph})$ and the oxabicycle framework $\left(\mathrm{C}^{\mathrm{D}}\right)$ becomes the dominant factor, which prohibits any formation of $Z$ isomer (cf. $\mathbf{t s} \mathbf{2}_{\mathrm{D} 3}$, Figure $\mathrm{S} 24 \mathrm{a}$ ). The competitive transition state for formation of the $E$ product with opposite sense of enantioselectivity $\mathbf{t s} \mathbf{2}_{\mathbf{D} 4}$, Figure $\mathbf{S} 24 \mathrm{~b}$ ) suffers from two severe steric interactions (red arrows) and is disfavored relative to $\mathbf{t s} \mathbf{2}_{\mathbf{D} 3}$ by $3.4 \mathrm{kcal} / \mathrm{mol}$. Nonetheless, $\mathbf{t s} \mathbf{2}_{\mathbf{D} 4}$ is still energetically more accessible than both pathways through which any of the two $Z$ isomers is generated (energetics for D1 and D2 not shown in Figure S23). The most favored Curtin-Hammett based pathway C2 predicts predominant formation of $Z$ isomer, which appears to be in contradiction to the presence of severe steric interaction between the phenyl substituent and the oxabicycle moiety (ts $\mathbf{2}_{\mathrm{C} 2}$, Figure $\mathrm{S} 24 \mathrm{c}$ ). A 
reasonable explanation lies in the rate-limiting nature of cycloaddition transition state $\mathbf{t s}_{\mathbf{C} 2}\left(\mathrm{E}_{\text {rel }}=12.9\right.$ $\mathrm{kcal} / \mathrm{mol}$, Figure S23), wherein steric interaction between the phenyl substituent $(\mathrm{Ph})$ and the oxabicycle is not the dominant factor (long $\mathrm{C}^{\mathrm{B}}-\mathrm{C}^{\mathrm{C}}$ distance of $2.52 \AA$ ) compared to $\mathbf{t s} \mathbf{2}_{\mathrm{C} 2}\left(\mathrm{C}^{\mathrm{B}}-\mathrm{C}^{\mathrm{C}}=1.43\right.$ $\AA$ ), which is significantly lower in energy due to generation of a lower energy exo carbene $\left(E_{\text {rel }}=5.8\right.$ $\mathrm{kcal} / \mathrm{mol}$ ). Comparison of orbital interactions (lower portion of Figure S24) indicates that $E$ alkene isomers are preferentially generated despite the opposing, but comparatively small, stereoelectronic contribution.
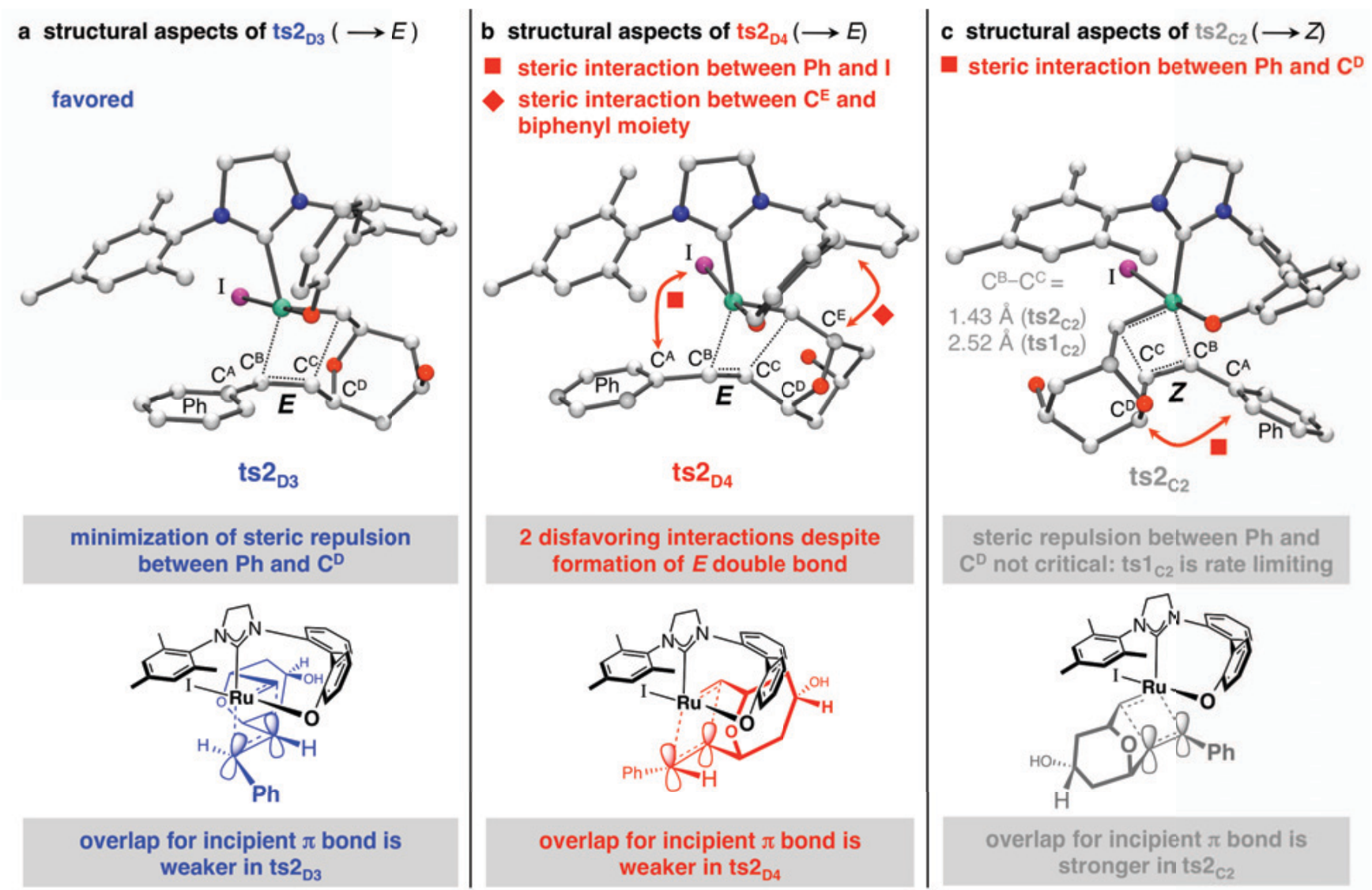

Figure S24. Analysis and comparison of cycloreversion transitions state structures (ts2) and stereochemical model for $E$ selectivity in ROCM reactions with chiral catalyst Ru-5b and styrene. 


\section{Assessment of Gibbs free energy surfaces as a function of density functional and solvation}

Assuming that the relative computational errors (around $1 \mathrm{kcal} / \mathrm{mol}$ ) could be as high as the energy difference that leads to moderate selectivity (83:17 corresponds to an energy difference of $1 \mathrm{kcal} / \mathrm{mol}$ at $25^{\circ} \mathrm{C}$ ) we tested the performance of several DFT functionals (BP86, $\omega$ B97XD and M06) under various conditions (gas-phase, benzene, dichloromethane). Graphical representation of all Gibbs free energy surfaces is provided in Section 4. The most important findings are summarized here:

(1) In ROCM reactions of $\mathbf{2}$ and enol ether promoted by either Ru-4f or Ru-4b (cf. Figure 3), the cycloreversion transition state (ts2) that leads to formation of the $Z$ (vs $E$ ) product is lower in all investigated instances in case of Ru-4b (Figures S26-S34), whereas it is slightly higher in energy in case of Ru-4f when modeled with density functionals BP86 and $\omega$ B97XD in dichloromethane (Figures S28 and S31).

(2) A similar trend is observed when the same calculations are carried out with significantly truncated model systems (Ru-4f-model and Ru-4b-model), suggesting that the steric bulk of either $\mathrm{PCy}_{3}$ or SIMes ligands does not constitute a major factor that influences the stereochemistry in the cycloreversion transition state ts2 (Figures S35-S43). Only when the ROCM reaction with $\mathbf{R u}-\mathbf{4 b}$-model is modeled with BP86 in dichloromethane is the pathway leading to generation of the $E$ product marginally lower in energy (Figure S37).

(3) Detailed examination of the free energy surfaces for the ROCM reaction of 2 and enol ether promoted by Ru-5b (cf. Figure 5 in the manuscript and Figure S22) at the various levels of theory (Figures S44-S52) shows that pathway $\mathbf{A 1}(=\mathbf{Z R u}-\mathbf{5 b})$ is favored in all instances. In certain cases, however, a change in rate-limiting transition state from $\mathbf{t s} \mathbf{2}_{\mathbf{A} \mathbf{1}}$ to $\mathbf{t s} \mathbf{1}_{\mathbf{A} \mathbf{1}}$ is observed (Figures S47, S48, S50, S51), particularly when modeled with $\omega$ B97XD or M06 in gas-phase (Figures S47 and S50) and to a lesser extent in benzene (Figures S48 and S51). Furthermore, the most favorable pathway for interconversion of the 5-exo and 5-endo carbene diastereomers is higher in energy than pathway A1 with density functional $\omega B$ 97XD (Figures S47-S49), but still below the other reaction pathways, which proceed through the more reactive 5-endo carbene diastereomer (A2-A4).

(4) Pathway $\mathbf{Z C N}$ is favored in $\mathrm{CM}$ of propene with acrylonitrile (cf. Figure 7 in manuscript) with all investigated levels of theory (Figures S53-S61). Furthermore, the nonproductive CM event, through which the ethylidene and the cyanomethylidene species interconvert, is energetically less costly than $\mathbf{t s} \mathbf{2}_{\mathrm{ZCN}}$.

(5) Pathway ZSMe in CM of vinyl sulfide with E-1,2-dichloroethylene (cf. Figure 9 in the manuscript) to generate the $Z$ product is clearly lower in energy than the reaction sequence that leads to the $E$ alkene product (ESMe) at all levels of theory (Figures S62-S70). 
(6) As already discussed in the text (cf. Figure 10 in the manuscript), the difference in energies between $\mathbf{t s} \mathbf{2}_{\mathrm{ZMe}}$, and $\mathbf{t s} \mathbf{2}_{\mathrm{EMe}}$, in homocoupling of propene is significantly smaller (Figures S62-S70), however, $\mathbf{t s} \mathbf{2}_{Z M e}$, is consistently lower in energy than ts $\mathbf{2}_{E M e}$, with density functionals BP86 and M06 and roughly equal in energy compared to ts $\mathbf{2}_{E M e}$, with $\omega$ B97XD (Figures S65-S67).

(7) Detailed examination of the free energy surfaces for the ROCM reaction of 2 and styrene promoted by Ru-5b (cf. Figure S23) at various levels of theory (Figures S71-S79) shows that pathway $\mathbf{D 3}$ is favored in all instances. In certain cases, however, a change in rate-limiting transition state from ts $\mathbf{2}_{\mathrm{D} 3}$ to ts $\mathbf{1}_{\mathrm{D} 3}$ is observed, particularly when modeled with $\omega \mathrm{B} 97 \mathrm{XD}$ or M06 in dichloromethane (Figures S76 and S79). Furthermore, the most favorable pathway for interconversion of the 5-exo and 5-endo carbene diastereomers is higher in energy than pathway D3 with all tested density functionals and solvents, strongly suggesting that CurtinHammett kinetics do not apply.

\section{More detailed assessment of transition state for ligand association (ts0)}

We have re-optimized the reactions shown in Figure 3 in the manuscript at the BP86/basis1 level in dichloromethane. The corresponding energy $(\Delta \mathrm{E})$ and free energy surfaces $(\Delta \mathrm{G})$ are shown in Figures S80-S81. The corresponding thermal corrections to the free energy $(\Delta \mathrm{G}=\Delta \mathrm{E}+\Delta \mathrm{Gcorr})$ are plotted in Figure S82. The transition states for olefin association (ts0) have been located, and furthermore, we performed constrained optimizations at larger olefin-Ru distances (ts0a-ts0d). Afterwards we evaluated $\Delta \mathrm{E}$ and $\Delta \mathrm{G}$ with 4 different density functionals including dispersion (BP86D3BJ, PBE0-D3BJ, wB97XD, and M06) with the Def2QZVP basis set in gas phase, benzene and dichloromethane (Figures S83-S88).

The following trends are observed:

(1) The magnitude of the thermal correction to the free energy ( $\Delta$ Gcorr) decreases from ts0 to ts0d (cf. Figure S82). While $\Delta$ Gcorr is relatively constant for the intermediates and transition states between pc1 and pc2, it drops significantly for ts0-ts0d (Figure S82).

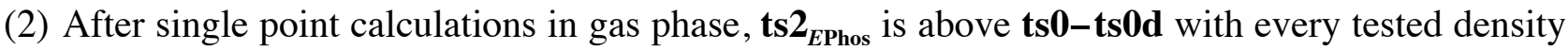
functional, whereas ts $\mathbf{2}_{\boldsymbol{E} \text { NHC }}$ is below ts0b with BP86-D3BJ/Def2QZVP and M06/Def2QZVP and marginally above ts0b with PBE0-D3BJ/Def2QZVP and $\omega B$ 97XD/Def2QZVP (cf. Figure S84). 
(3) Through the increase of solvent polarity to benzene (Figure S86) and DCM (Figure S88), $\mathbf{t s} \mathbf{2}_{\boldsymbol{E N H C}}$ is raised relative to ts0-ts0d. Nonetheless, $\mathbf{t s} \mathbf{2}_{\boldsymbol{E N H C}}$ is only $1.7,1.5$ and $0.7 \mathrm{kcal}$ above ts0b when modeled in benzene with BP86-D3BJ/Def2QZVP, PBE0-D3BJ/Def2QZVP and M06/Def2QZVP (Figure S86).

(4) Hence, one of the difficulties in determining the exact relationship between ts 2 and ts0-ts0d can be attributed to the accurate determination of the free energy corrections ( $\Delta$ Gcorr). $\Delta$ Gcorr ranges between 14.4 and $16.4 \mathrm{kcal} / \mathrm{mol}$ for $\mathbf{t s} \mathbf{2}_{E P h o s}, \mathbf{t s} \mathbf{2}_{\text {ZPhos }}, \mathbf{t s} \mathbf{2}_{E \mathrm{NHC}}$, and $\mathbf{t s} \mathbf{2}_{\text {ZNHC }}$, respectively, whereas this correction drops to 7.1-12.2 kcal/mol for ts0-ts0d (cf. Figure S82). The difference in $\Delta$ Gcorr between ts2 and ts0-ts0d (which is $>5 \mathrm{kcal} / \mathrm{mol}$ ) is hence far greater than the difference in $\Delta \mathrm{G}$ between ts2 and ts0-ts0d (typically less than $2 \mathrm{kcal} / \mathrm{mol}$ in benzene, cf. Figure S86).

(5) Even more striking differences between ts2 and ts0-ts0d are obtained when dispersion is excluded (Figures S89-S90). With BP86/Def2QZVP ts2 is approximately $10 \mathrm{kcal} / \mathrm{mol}$ above ts0 in gas phase and solution (for the results in benzene, see Figure S25 below; for the results in gas phase and DCM, see Figure S90). These results highlight the importance of including dispersion forces when modeling ligand association/dissociation reactions. Only when the latter factors are considered, leading to a more realistic representation, ${ }^{8,11}$ are the energies of ts 2 and ts0 close enough for a change in rate determining step to be considered.

With Grimme's D3-BJ dispersion

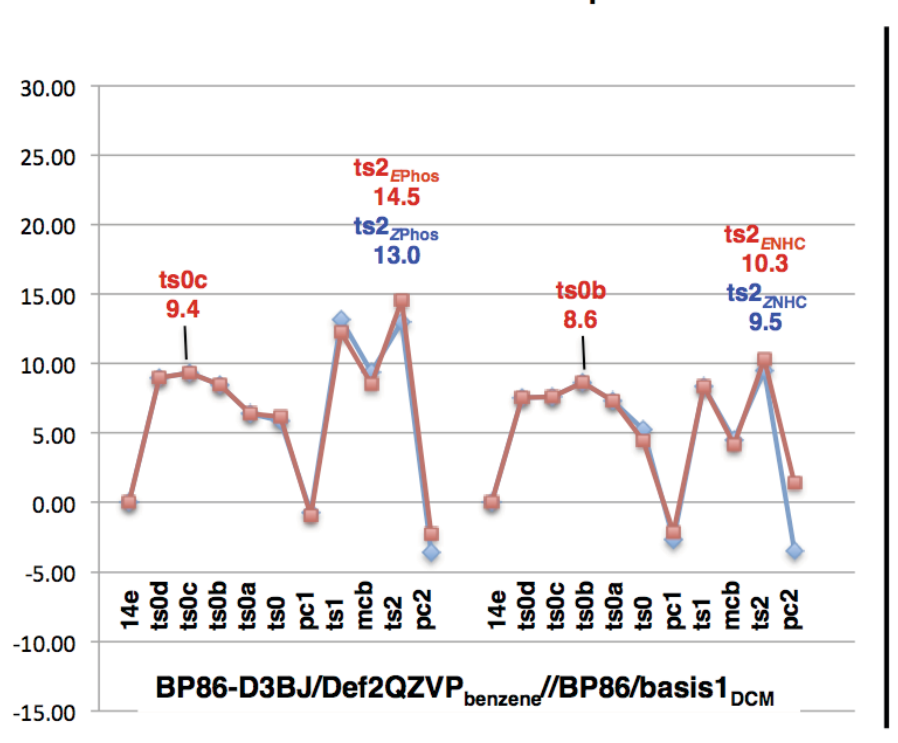

Without dispersion

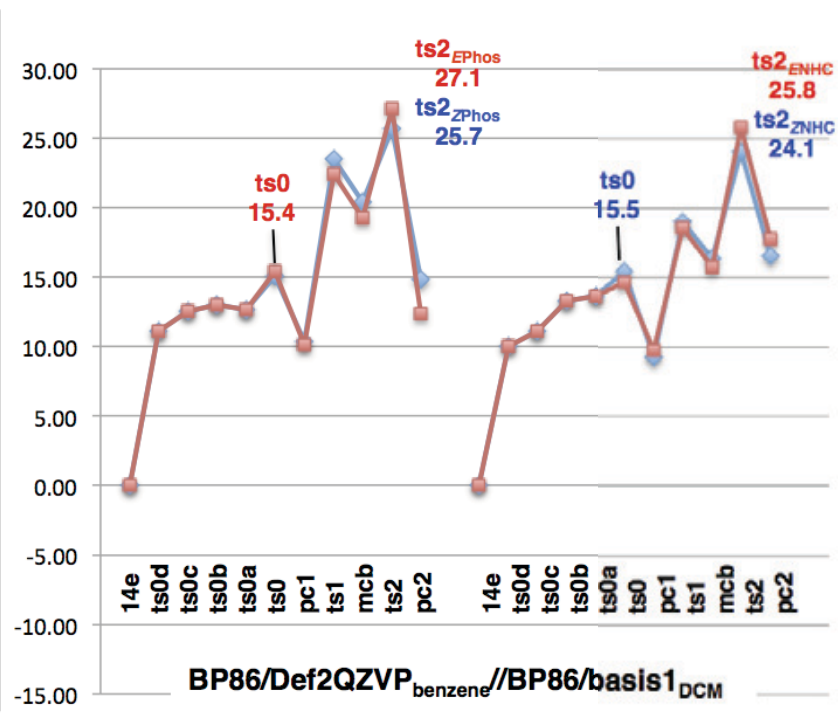

Figure S25. Comparison of single point free energies $\triangle G$ for the reactions shown in Figure 3 with BP86/ Def2QZVP in benzene after optimization with BP86/basis DCM $_{\text {; }}$ with D3BJ dispersion (left), without D3BJ dispersion (right) 


\section{Gibbs Free Energy Surfaces $(\Delta G)$ with BP86, $\omega$ B97XD and M06}

\section{Pathways EPhos, ZPhos, ENHC and ZNHC in Figure $3(\Delta G)$ with BP86/basis2}
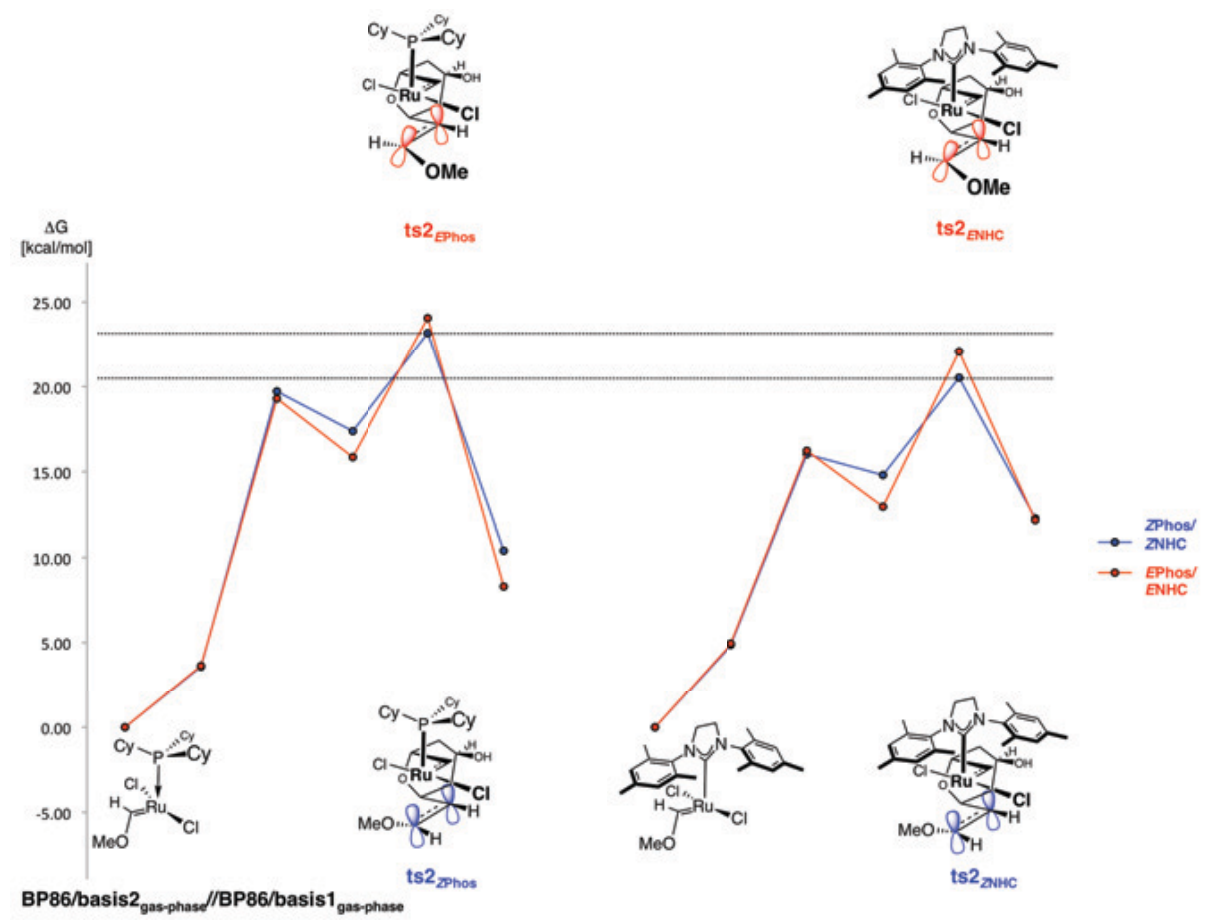

Figure S26. Reaction shown in Figure 3 at the BP86/basis2 $2_{\text {gas-phase }} / / \mathrm{BP} 86 /$ basis $1_{\text {gas-phase }}$ level

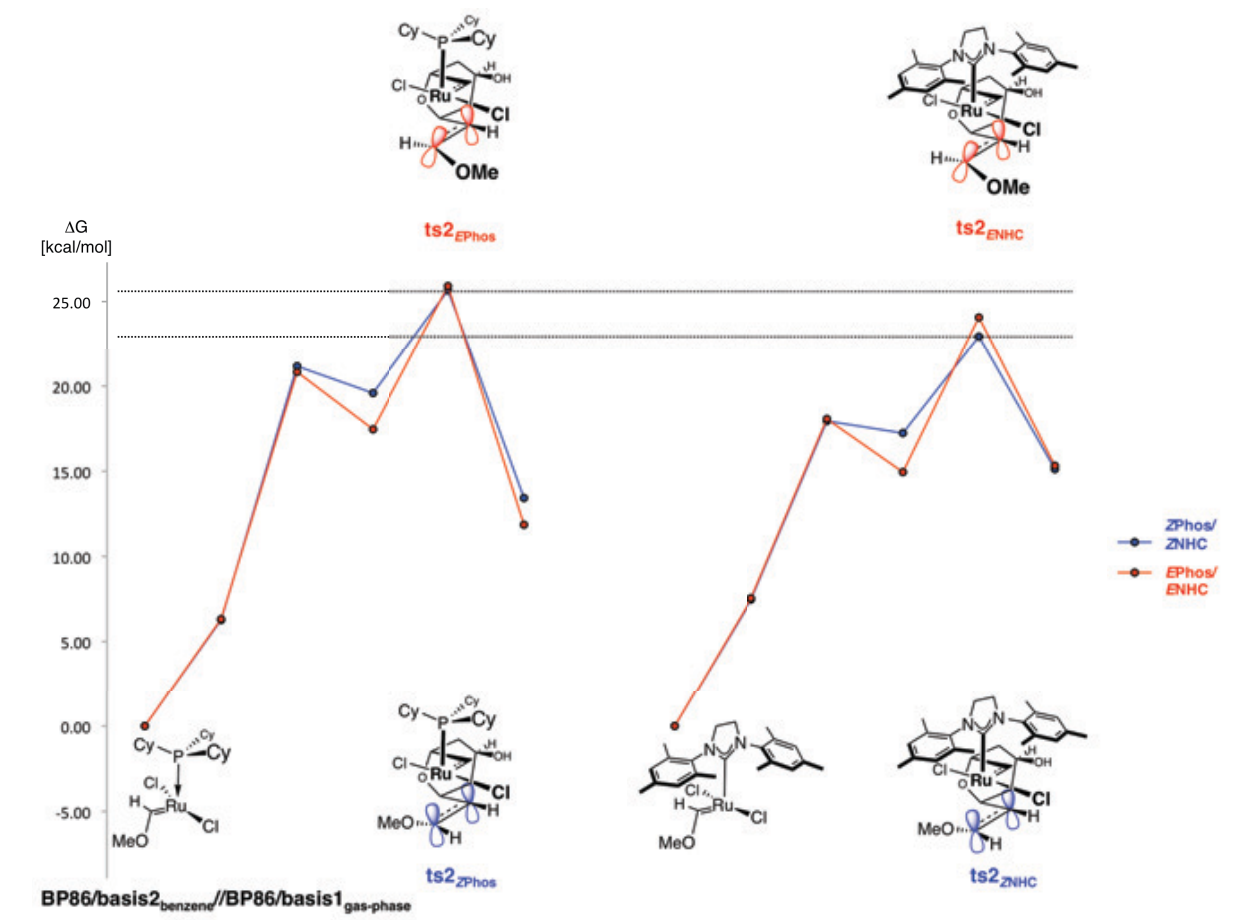

Figure S27. Reaction shown in Figure 3 at the BP86/basis $2_{\text {benzene }} / / \mathrm{BP} 86 / \mathrm{basis} 1_{\text {gas-phase }}$ level 


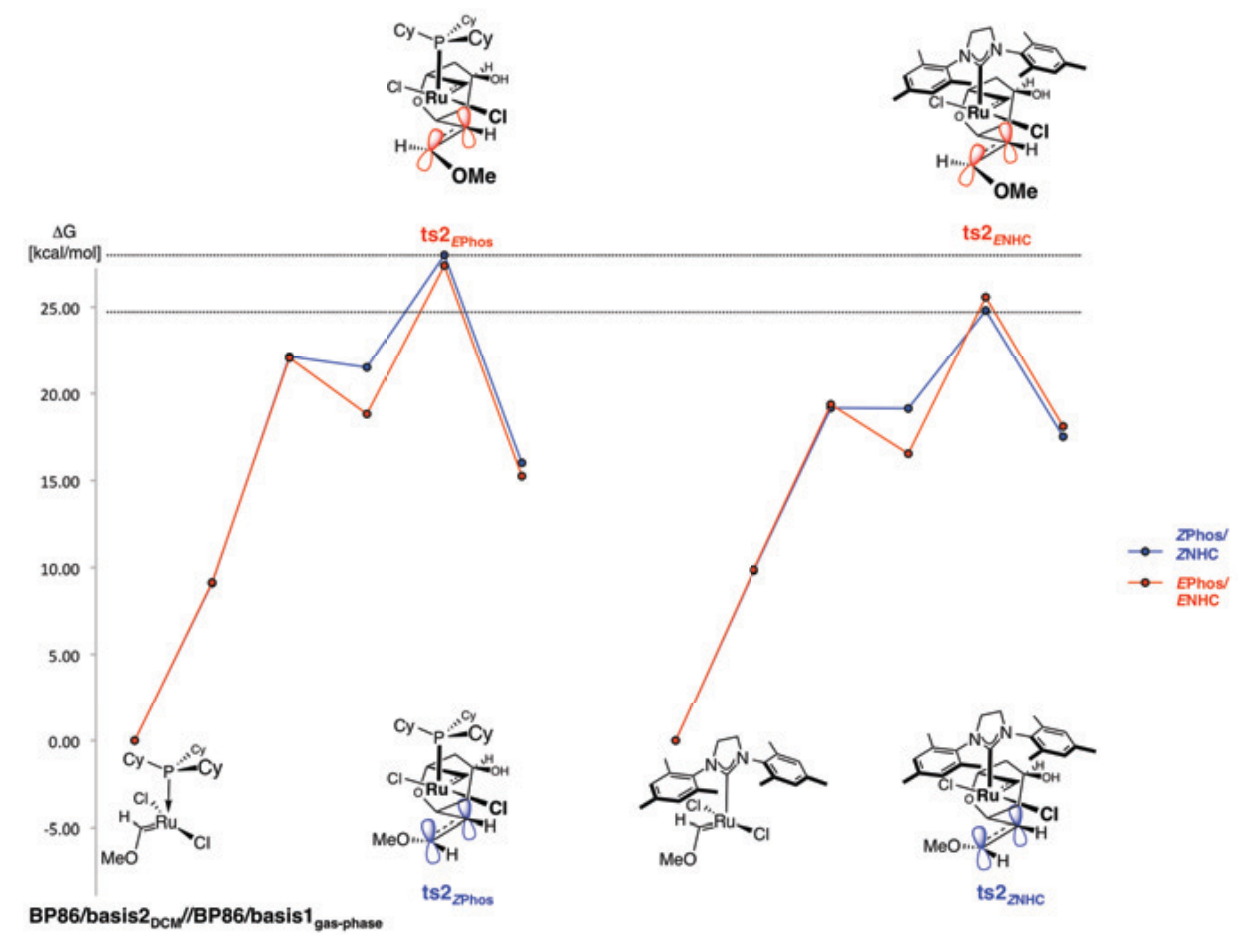

Figure S28. Reaction shown in Figure 3 at the BP86/basis $2_{\mathrm{DCM}} / / \mathrm{BP} 86 / \mathrm{basis} 1_{\text {gas-phase }}$ level 


\section{Pathways EPhos, ZPhos, ENHC and ZNHC in Figure $3(\Delta G)$ with $\omega$ B97XD/basis2}
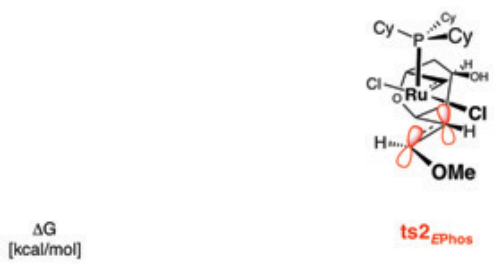

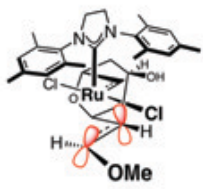

ts $2_{\text {eNHC }}$

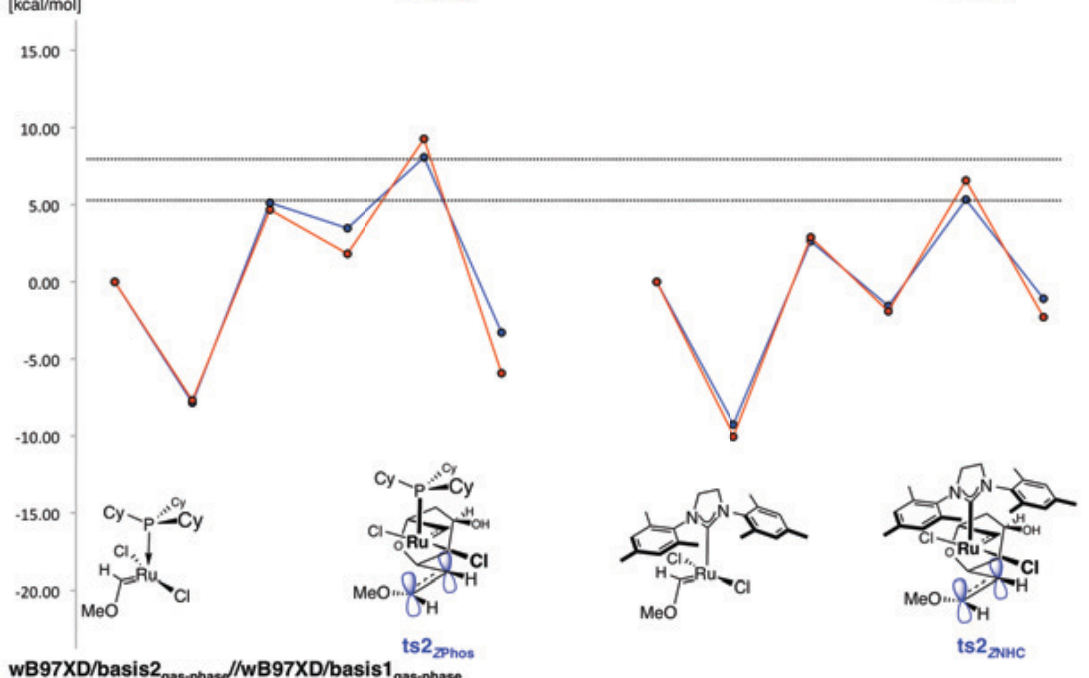

Figure S29. Reaction shown in Figure 3 at the $\omega \mathrm{B} 97 \mathrm{XD} / \mathrm{basis} 2_{\text {gas-phase }} / / \omega \mathrm{B} 97 \mathrm{XD} / \mathrm{basis} 1_{\text {gas-phase }}$ level

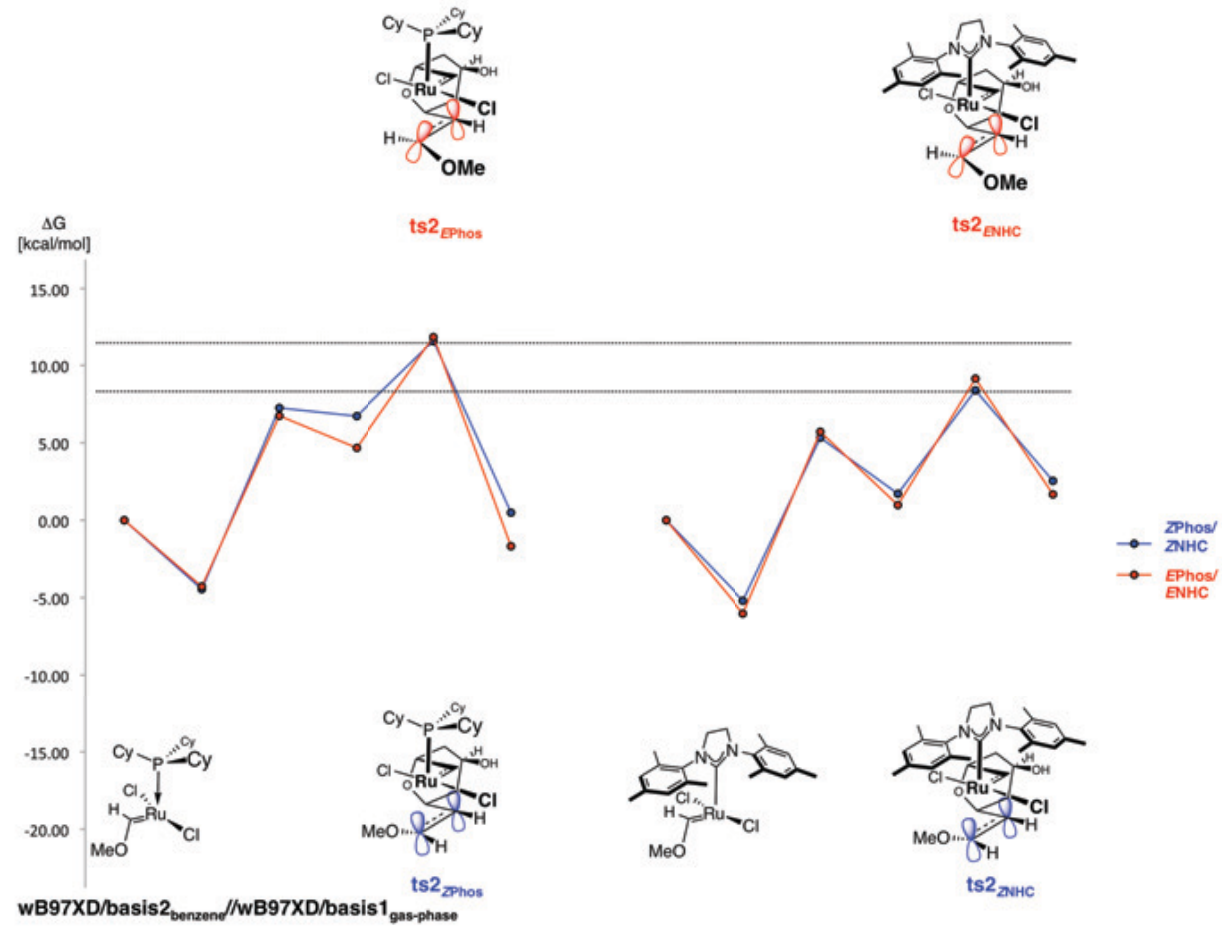

Figure S30. Reaction shown in Figure 3 at the $\omega \mathrm{B} 97 \mathrm{XD} / \mathrm{basis} 2_{\text {benzene }} / / \omega \mathrm{B} 97 \mathrm{XD} / \mathrm{basis} 1_{\text {gas-phase }}$ level 


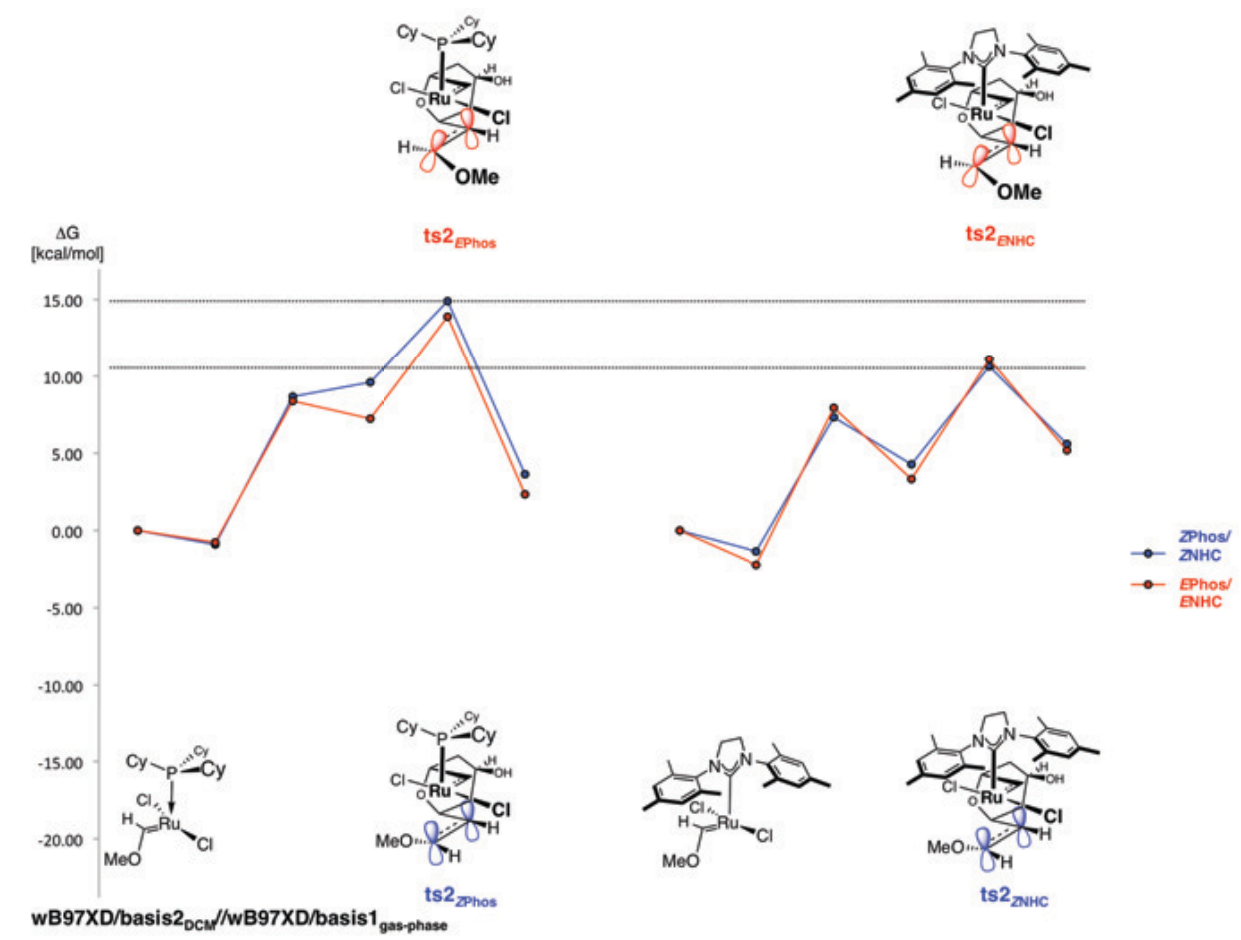

Figure S31. Reaction shown in Figure 3 at the $\omega \mathrm{B} 97 \mathrm{XD} / \mathrm{basis} 2_{\mathrm{DCM}} / / \omega \mathrm{B} 97 \mathrm{XD} / \mathrm{basis} 1_{\text {gas-phase }}$ level 


\section{Pathways EPhos, ZPhos, ENHC and ZNHC in Figure $3(\Delta G)$ with M06/basis2}

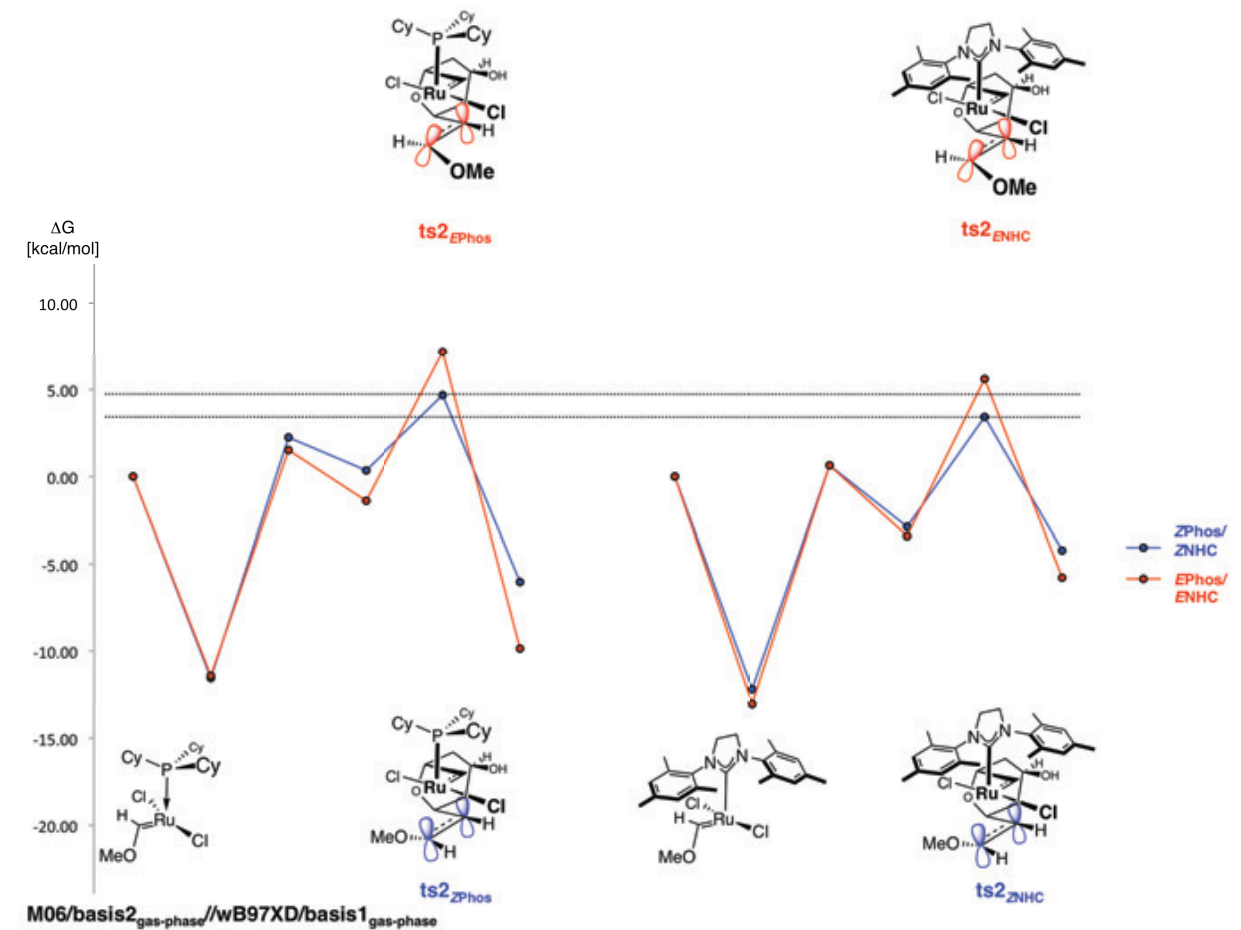

Figure S32. Reaction shown in Figure 3 at the M06/basis $2_{\text {gas-phase }} / / \omega B 97 X D /$ basis $1_{\text {gas-phase }}$ level

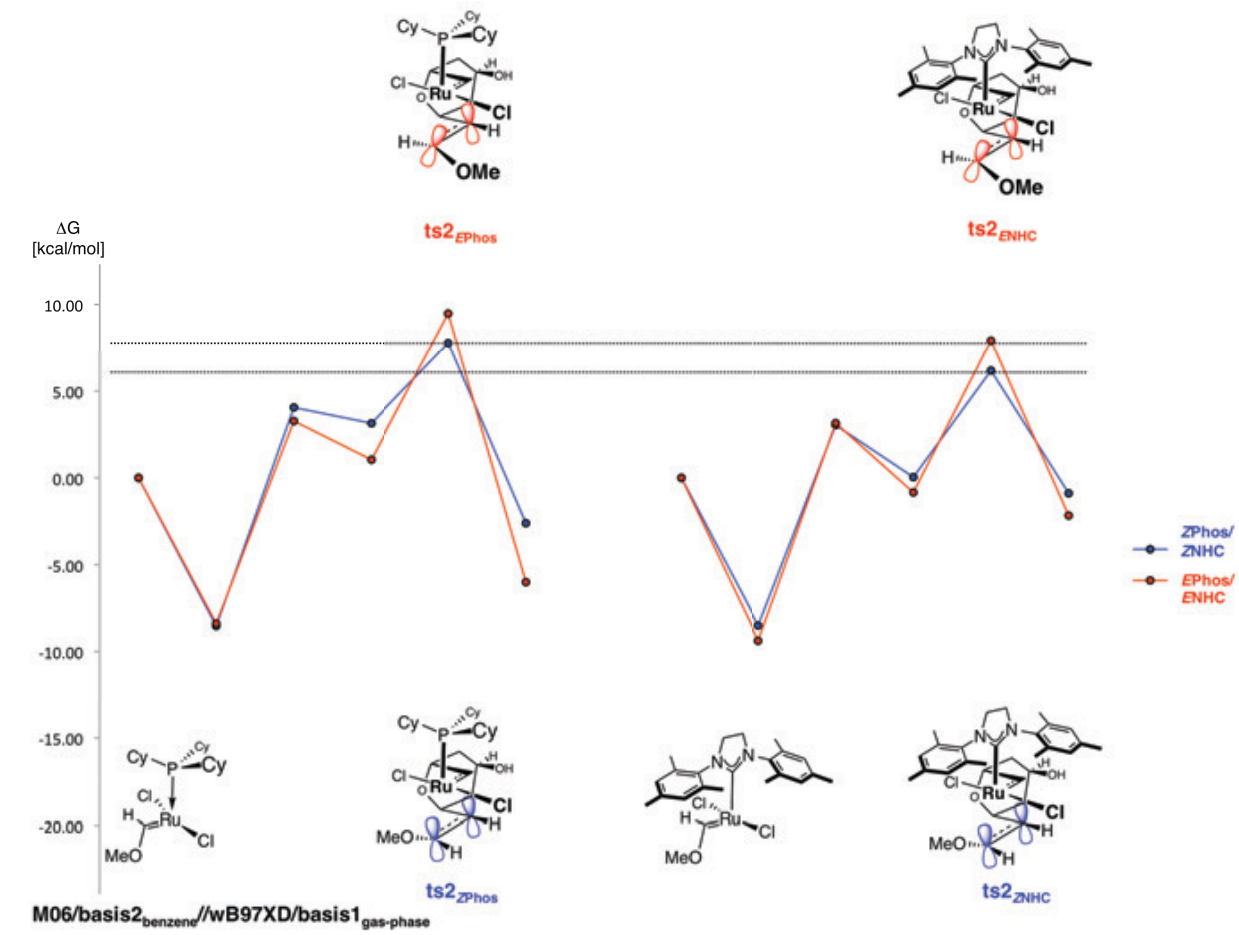

Figure S33. Reaction shown in Figure 3 at the M06/basis $2_{\text {benzene }} / / \omega B 97 X D / b a s i s 1_{\text {gas-phase }}$ level 


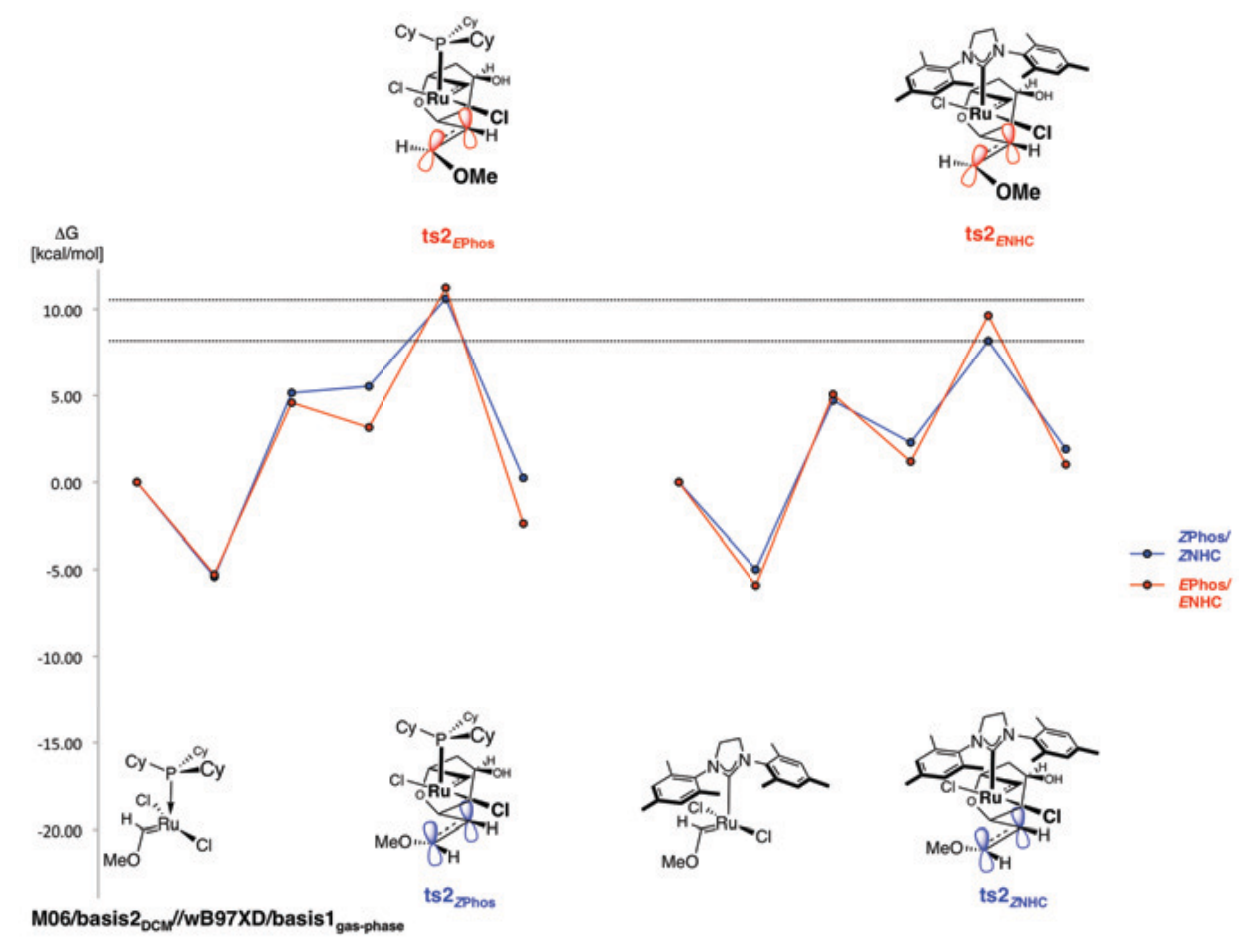

Figure S34. Reaction shown in Figure 3 at the M06/basis $2_{\mathrm{DCM}} / / \omega \mathrm{B} 97 \mathrm{XD} / \mathrm{basis} 1_{\text {gas-phase }}$ level 


\section{Pathways EPhos-model, ZPhos-model, ENHC-model and ZNHC-model $(\Delta \mathrm{G})$ with BP86/basis2}

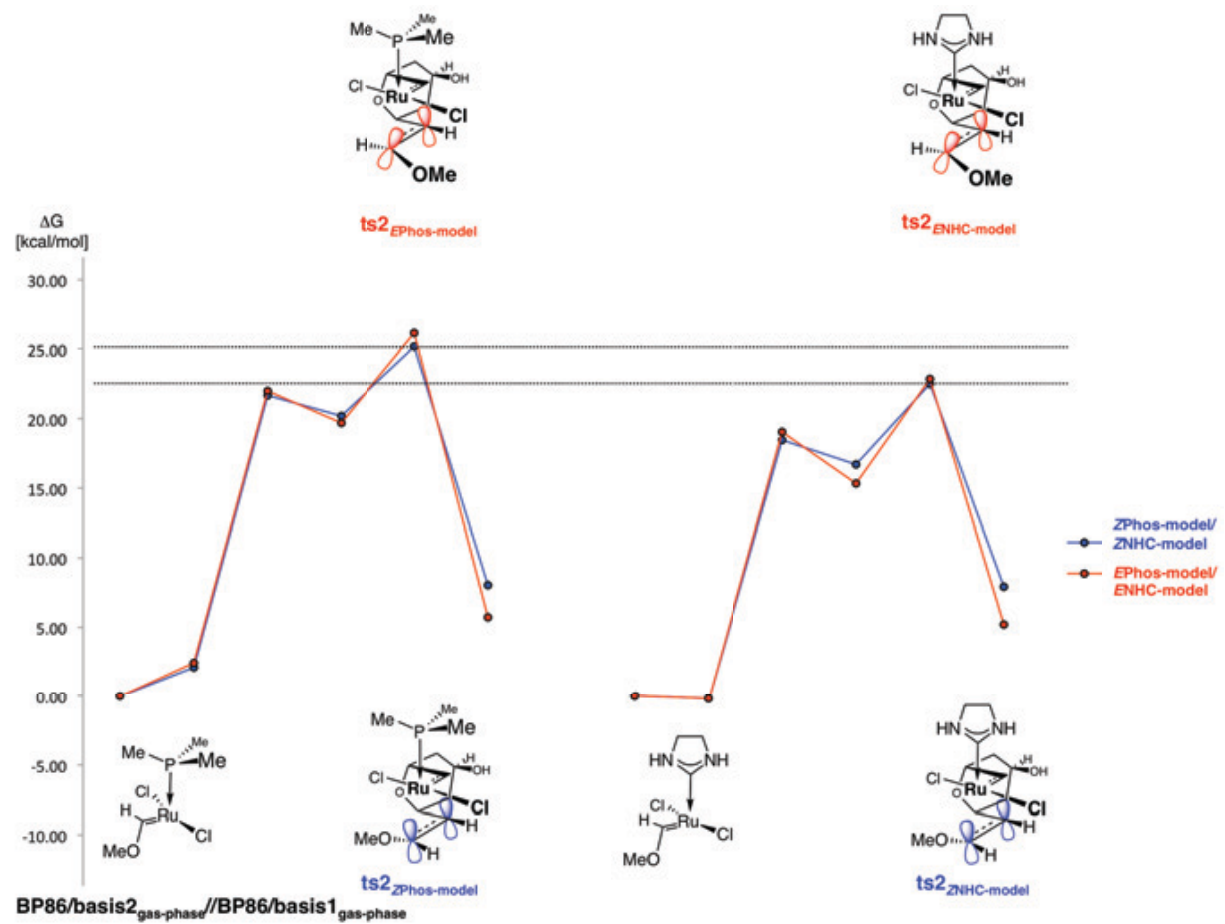

Figure S35. Reaction shown in Figure 3 with model ligands at the BP86/basis2 $2_{\text {gas-phase }} / / \mathrm{BP} 86 /$ basis $1_{\text {gas-phase }}$ level
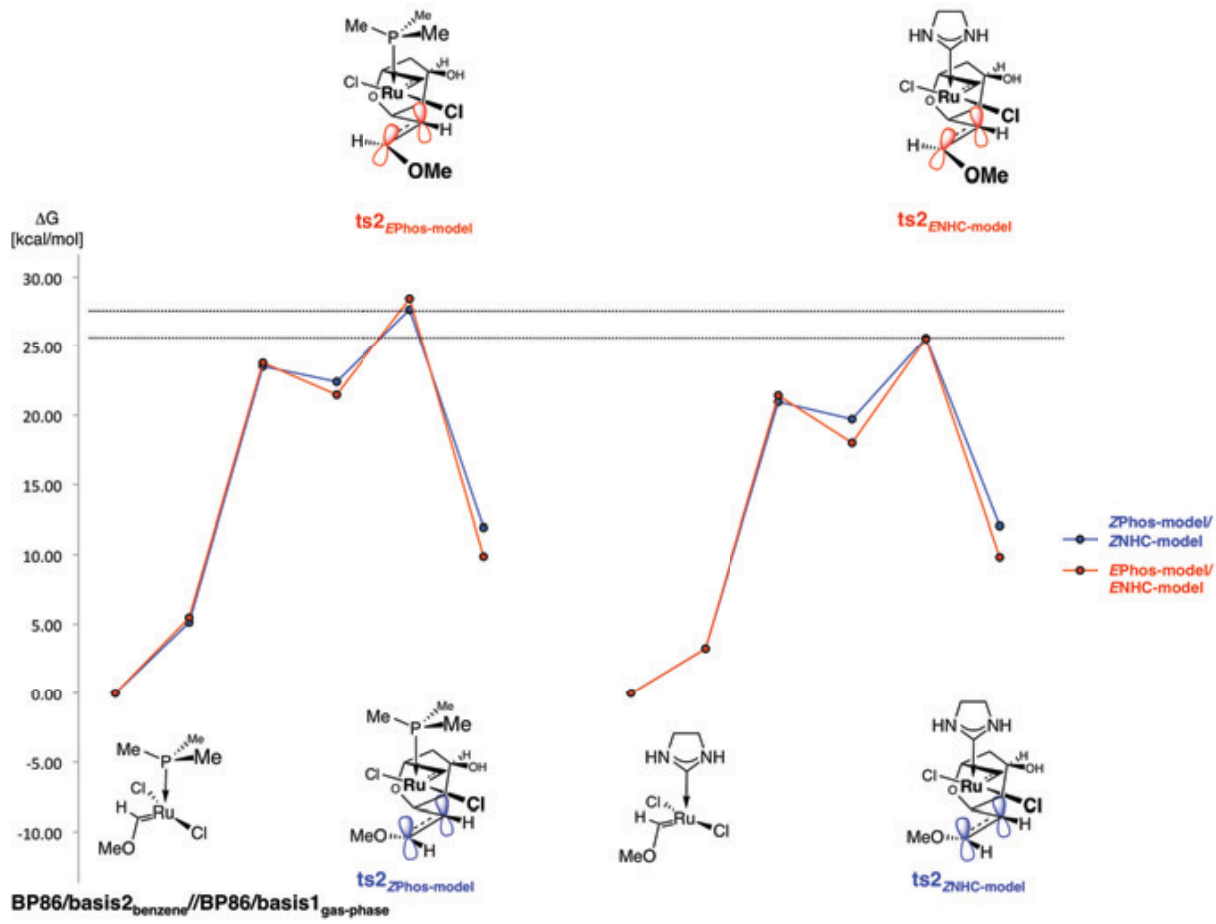

Figure S36. Reaction shown in Figure 3 with model ligands at the BP86/basis $2_{\text {benzene }} / / \mathrm{BP} 86 /$ basis $1_{\text {gas-phase }}$ level 


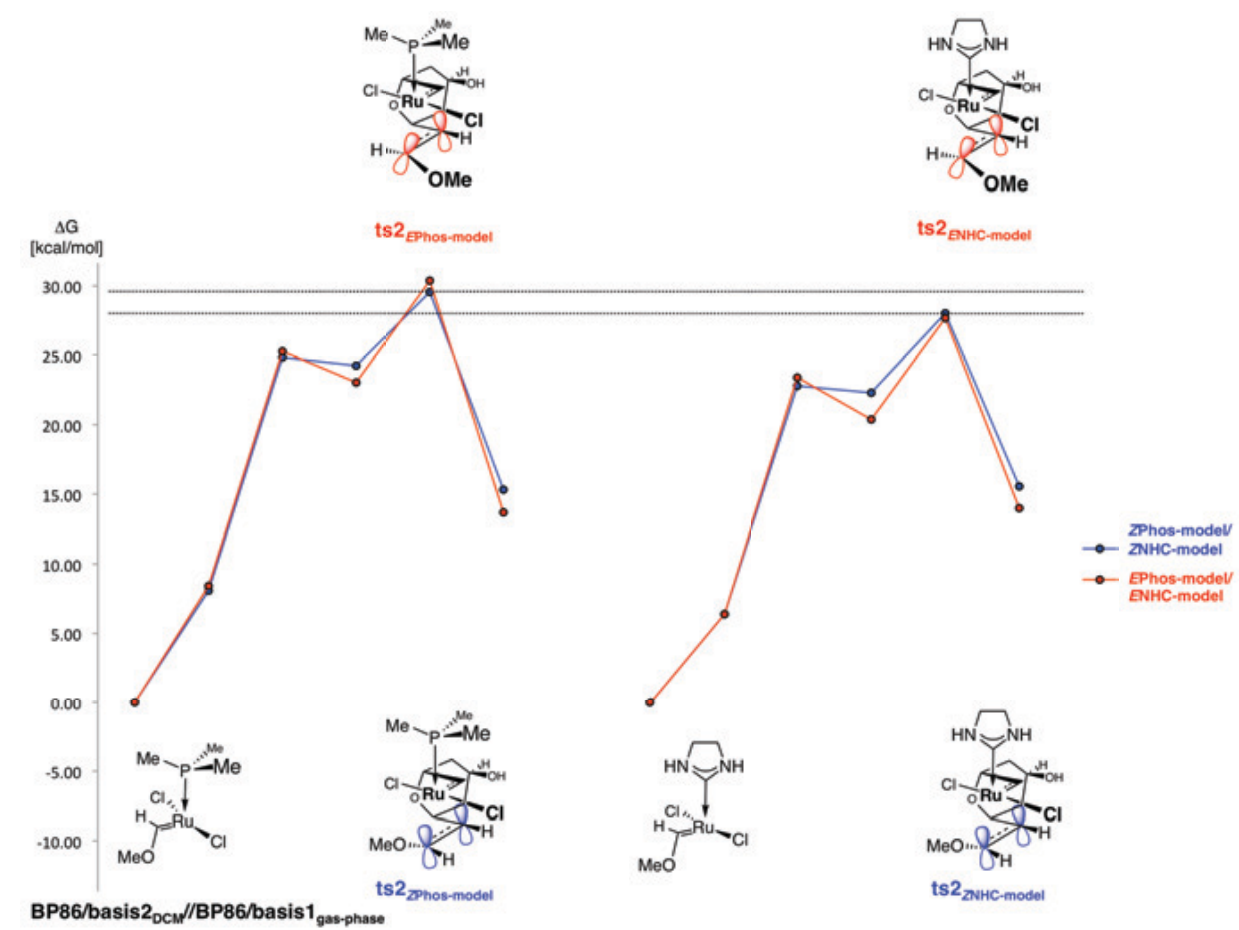

Figure S37. Reaction shown in Figure 3 with model ligands at the BP86/basis2 ${ }_{\mathrm{DCM}} / / \mathrm{BP} 86 / \mathrm{basis} 1_{\text {gas-phase }}$ level 


\section{Pathways EPhos-model, ZPhos-model, ENHC-model and ZNHC-model $(\Delta G)$ with $\omega$ B97XD/basis2}

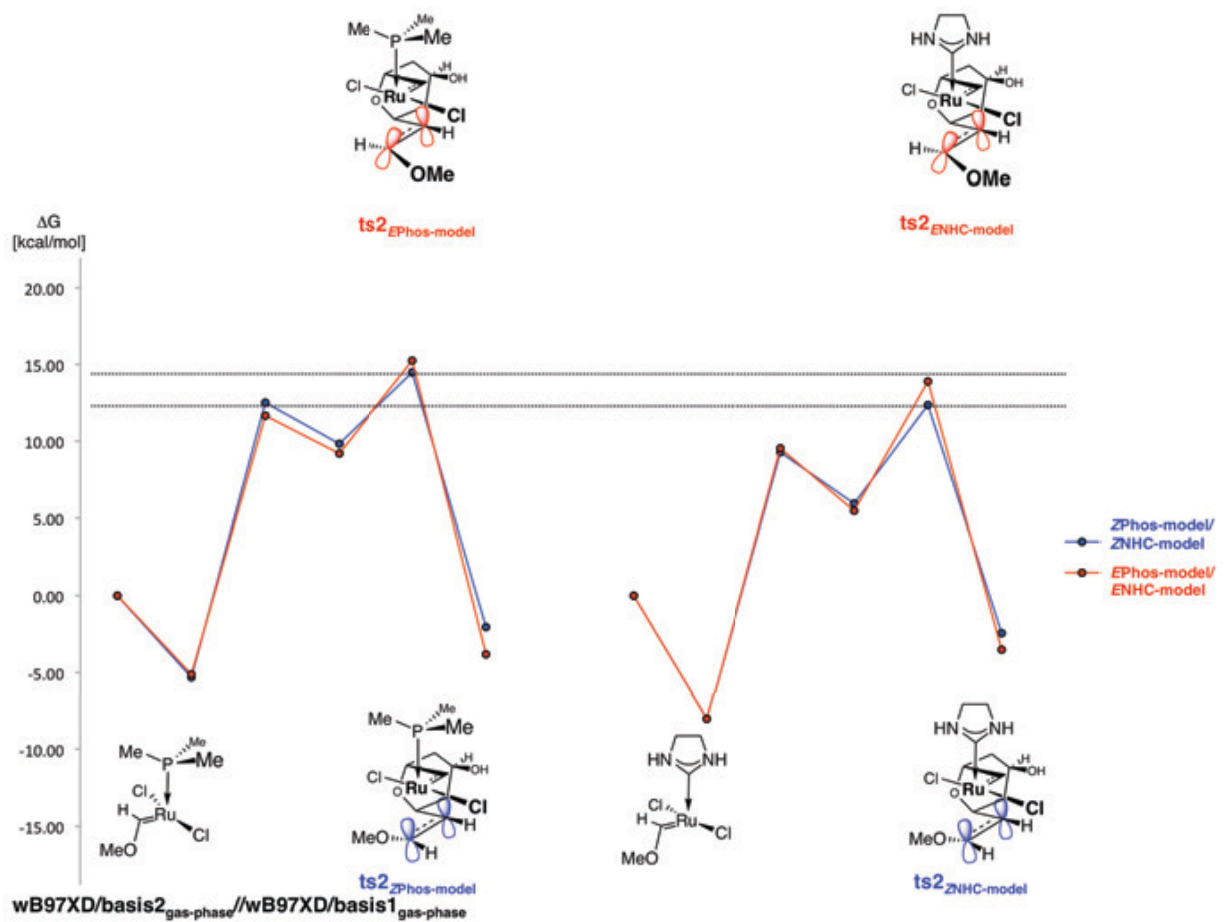

Figure S38. Reaction shown in Figure 3 with model ligands at the $\omega \mathrm{B} 97 \mathrm{XD} / \mathrm{basis} 2_{\text {gas-phase }} / / \omega \mathrm{B} 97 \mathrm{XD} / \mathrm{basis} 1_{\text {gas-phase }}$ level

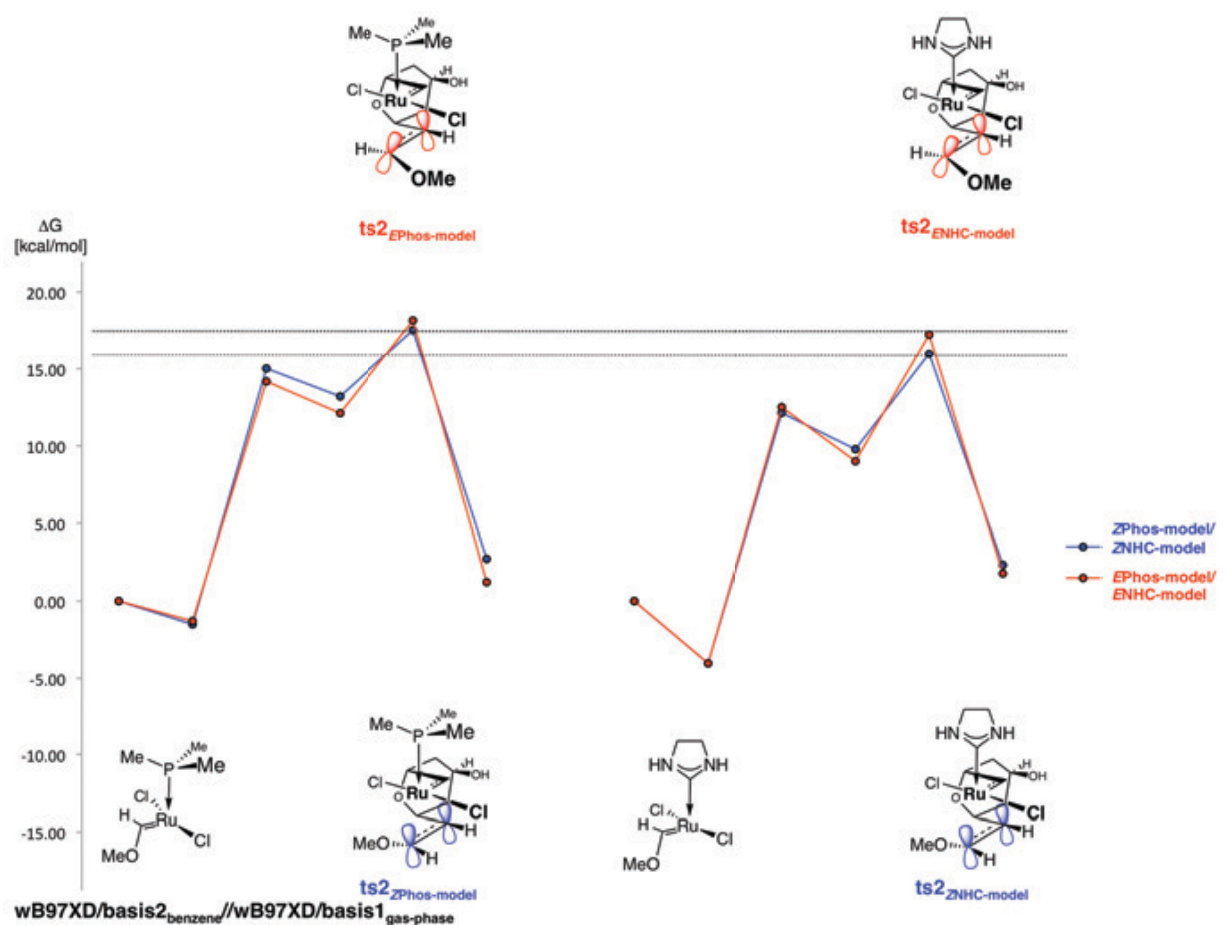

Figure S39. Reaction shown in Figure 3 with model ligands at the $\omega \mathrm{B} 97 \mathrm{XD} / \mathrm{basis} 2_{\text {benzene }} / / \omega \mathrm{B} 97 \mathrm{XD} / \mathrm{basis} 1_{\text {gas-phase }}$ level 


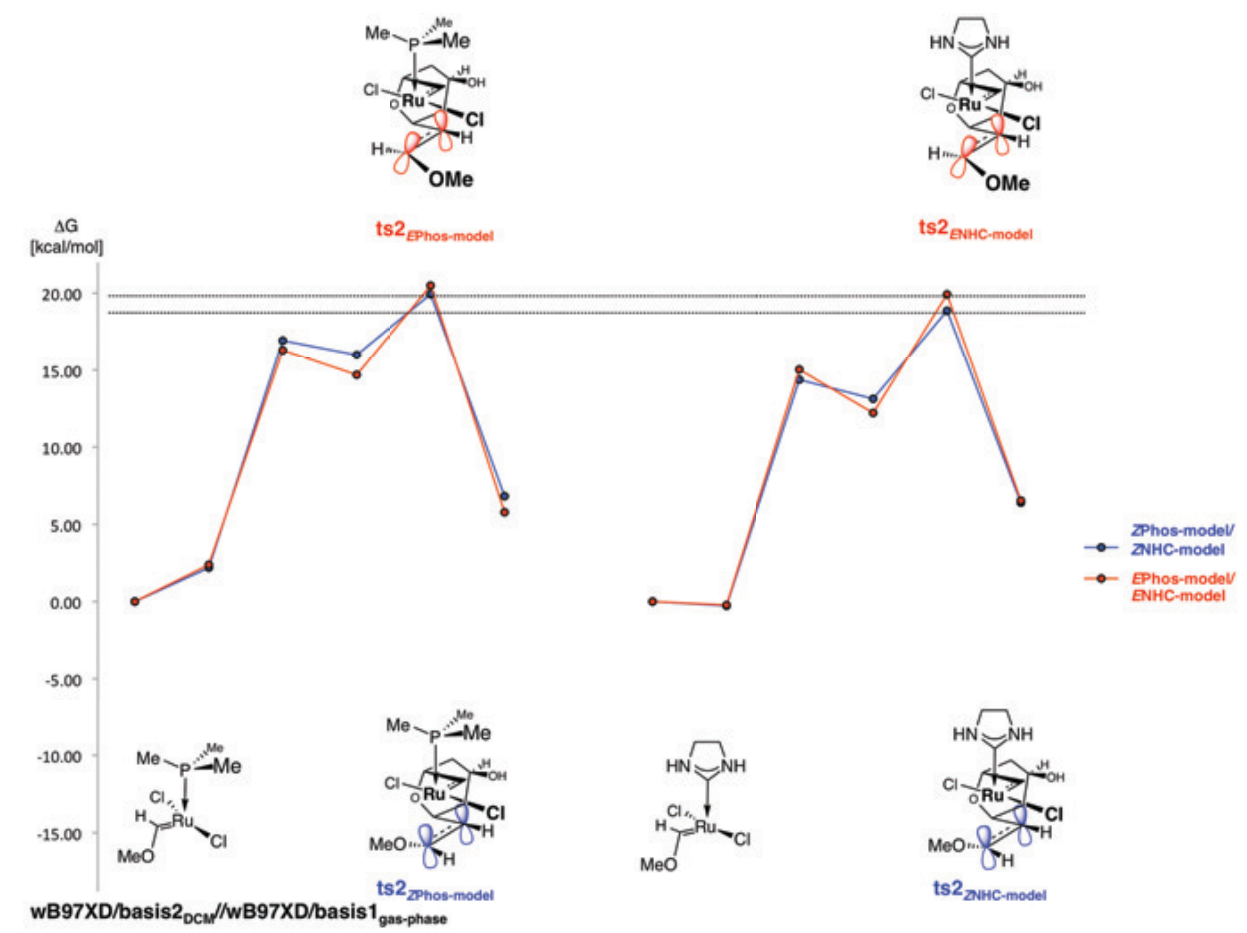

Figure S40. Reaction shown in Figure 3 with model ligands at the $\omega \mathrm{B} 97 \mathrm{XD} / \mathrm{basis} 2_{\mathrm{DCM}} / / \omega \mathrm{B} 97 \mathrm{XD} / \mathrm{basis} 1_{\text {gas-phase }}$ level 


\section{Pathways EPhos-model, ZPhos-model, ENHC-model and ZNHC-model $(\Delta G)$ with M06/basis2}

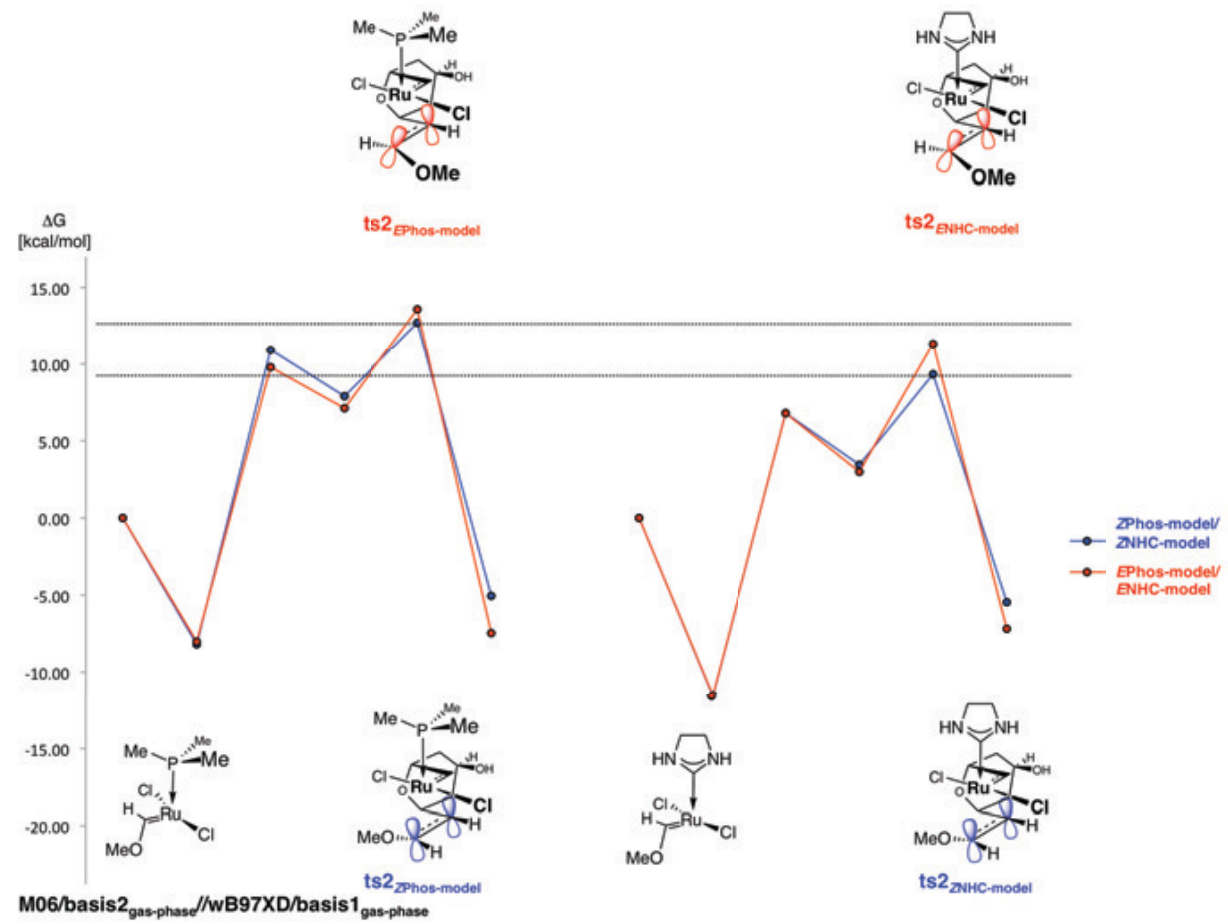

Figure S41. Reaction shown in Figure 3 with model ligands at the M06/basis $2_{\text {gas-phase }} / / \omega B 97 X D /$ basis $1_{\text {gas-phase }}$ level

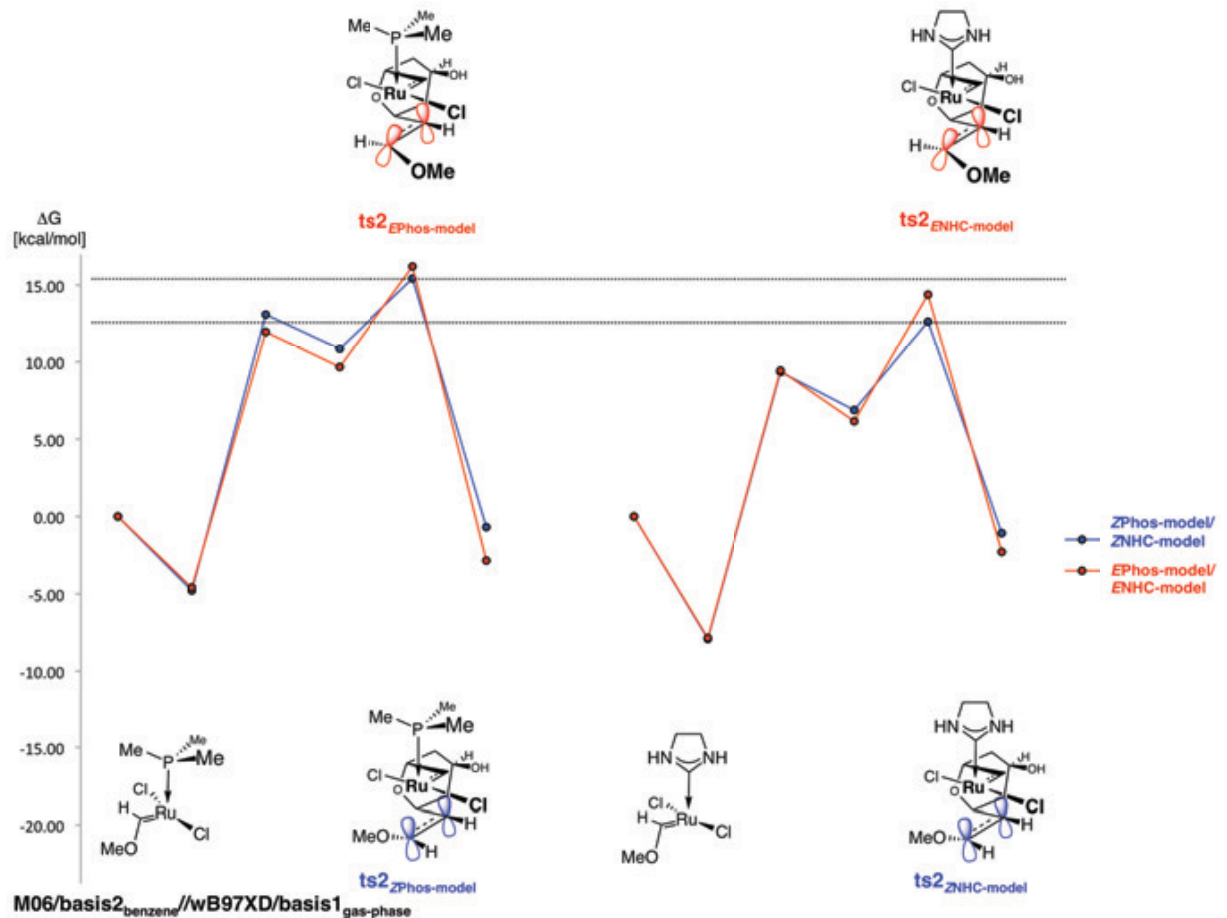

Figure S42. Reaction shown in Figure 3 with model ligands at the M06/basis $2_{\text {benzene }} / / \omega B 97 X D / b a s i s 1_{\text {gas-phase }}$ level 


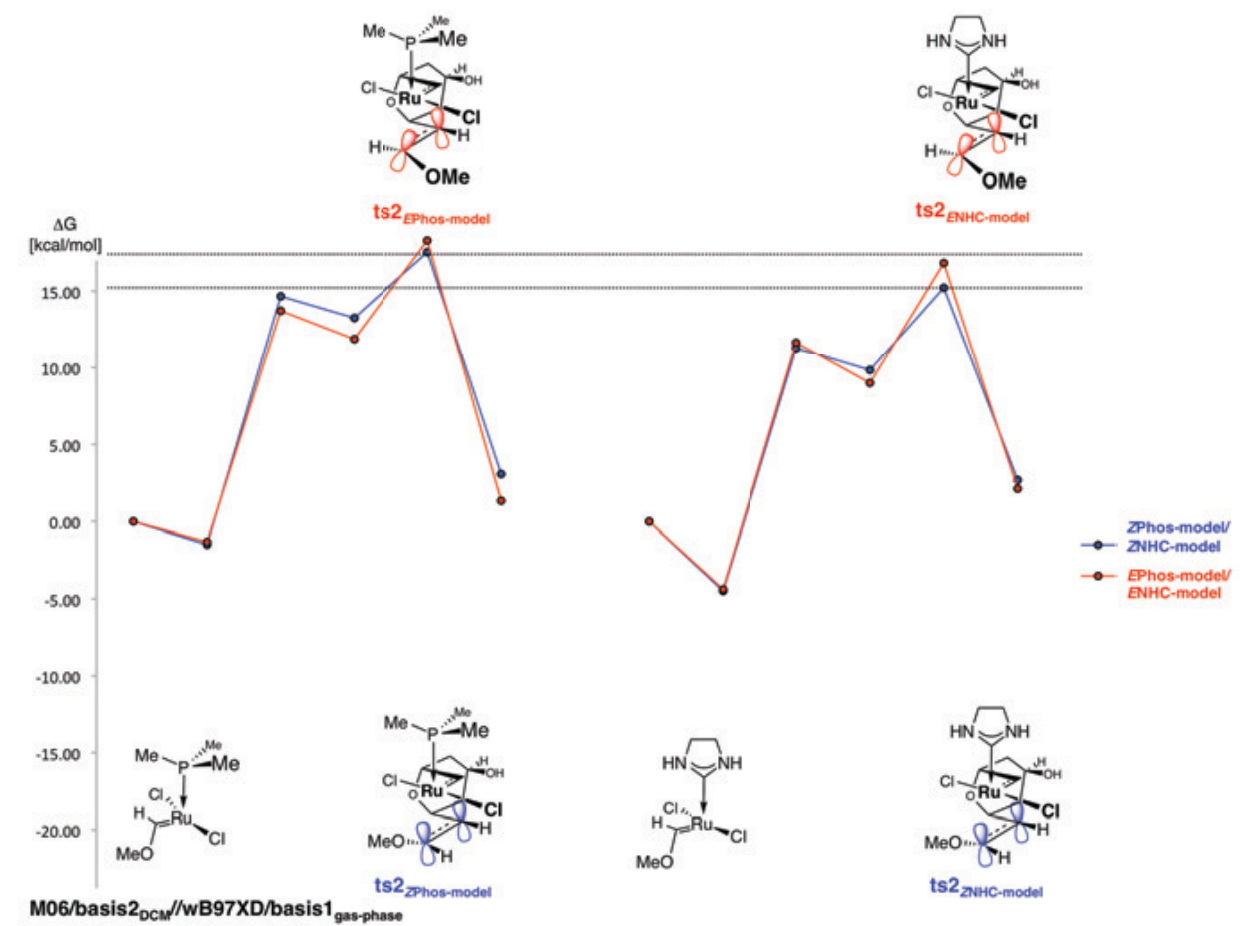

Figure S43. Reaction shown in Figure 3 with model ligands at the M06/basis $2_{\mathrm{DCM}} / / \omega \mathrm{B} 97 \mathrm{XD} /$ basis $_{\text {gas-phase }}$ level 


\section{Pathways ERu-5b and $Z R u-5 b$ in Figure 5 and Figure S22 $(\Delta G)$ with BP86/basis2}

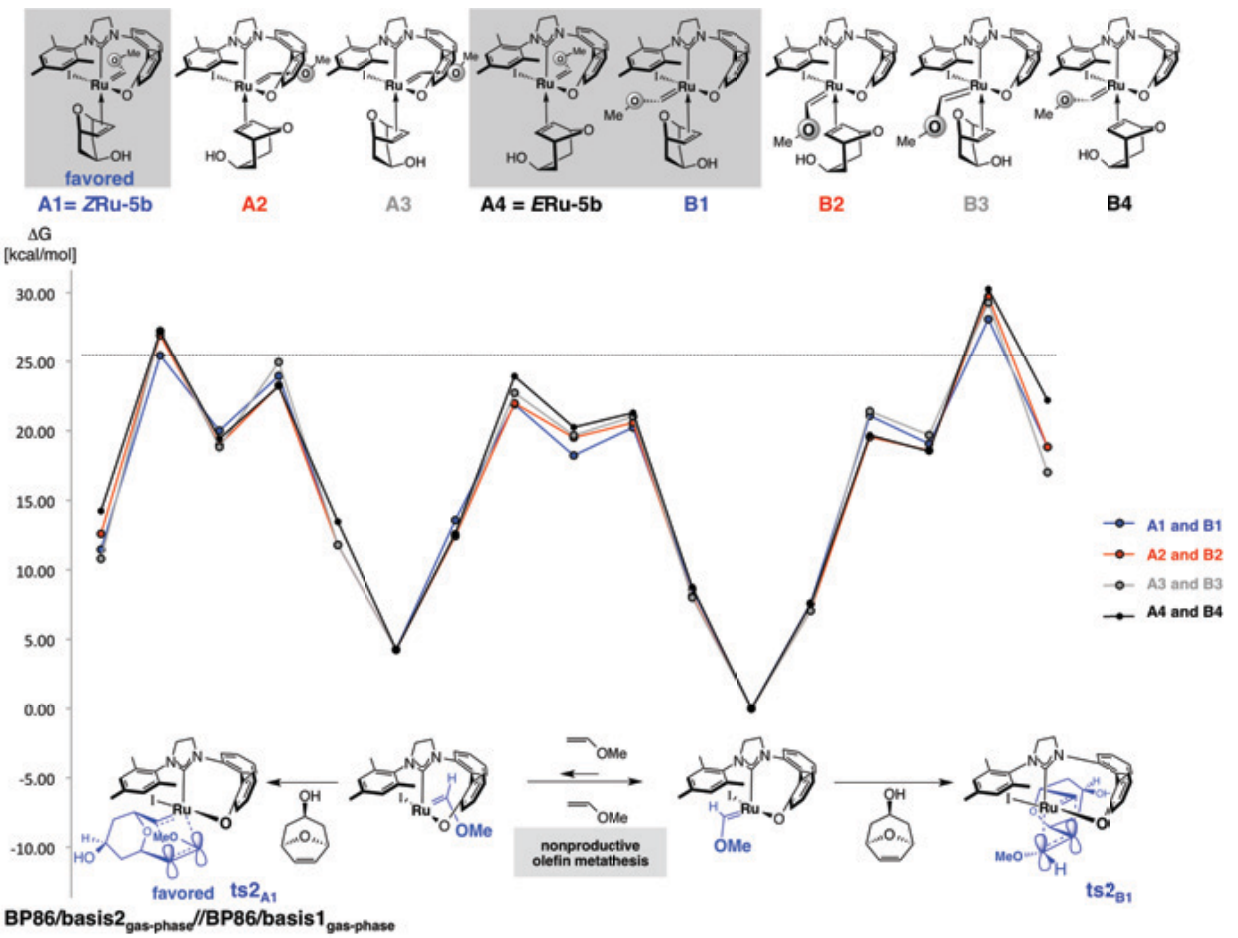

Figure S44. Reaction shown in Figure 5 and Figure S4 at the BP86/basis2 $2_{\text {gas-phase }} / / \mathrm{BP} 86 /$ basis $1_{\text {gas-phase }}$ level

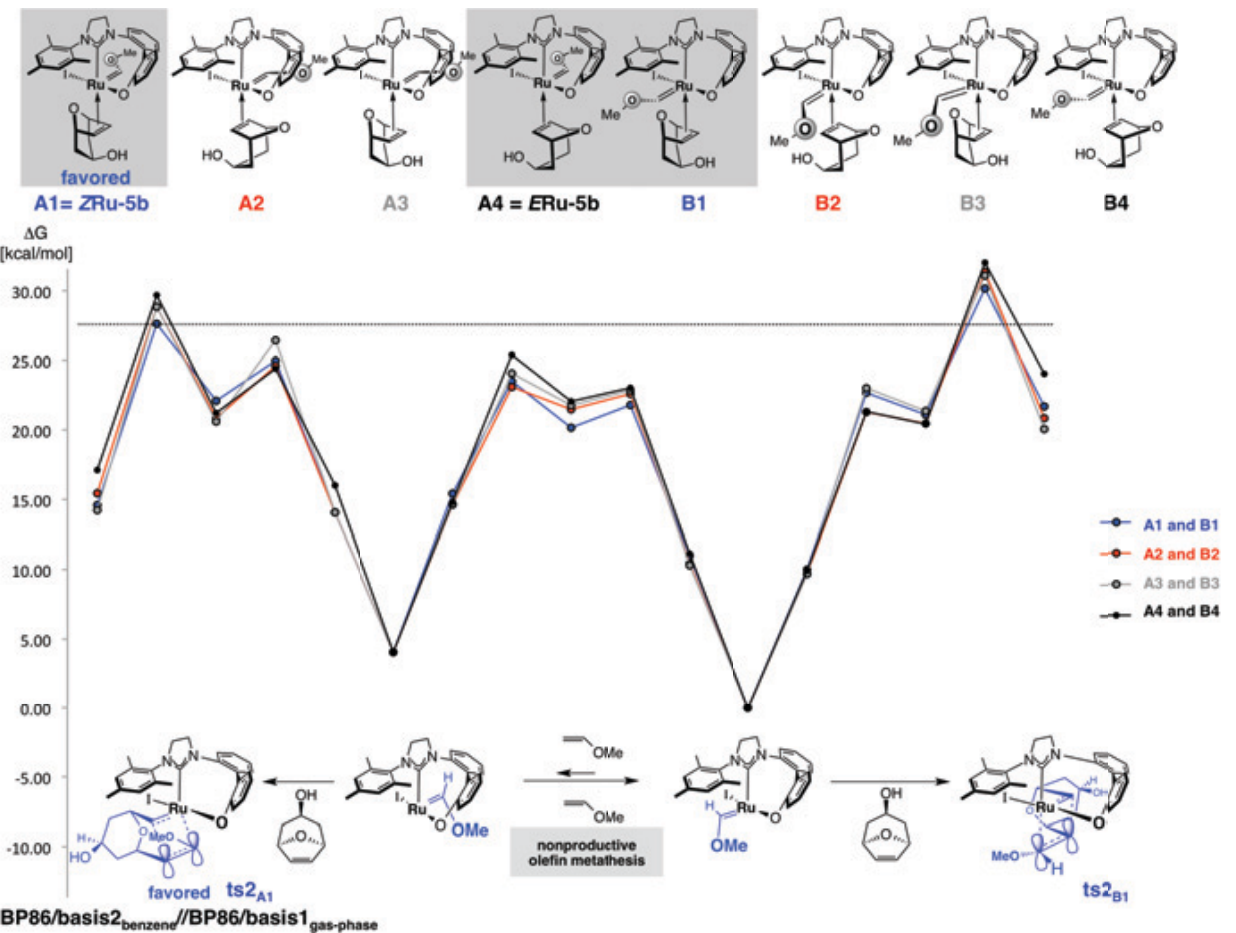

Figure S45. Reaction shown in Figure 5 and Figure S4 at the BP86/basis $2_{\text {benzene }} / / \mathrm{BP} 86 / \mathrm{basis} 1_{\text {gas-phase }}$ level 


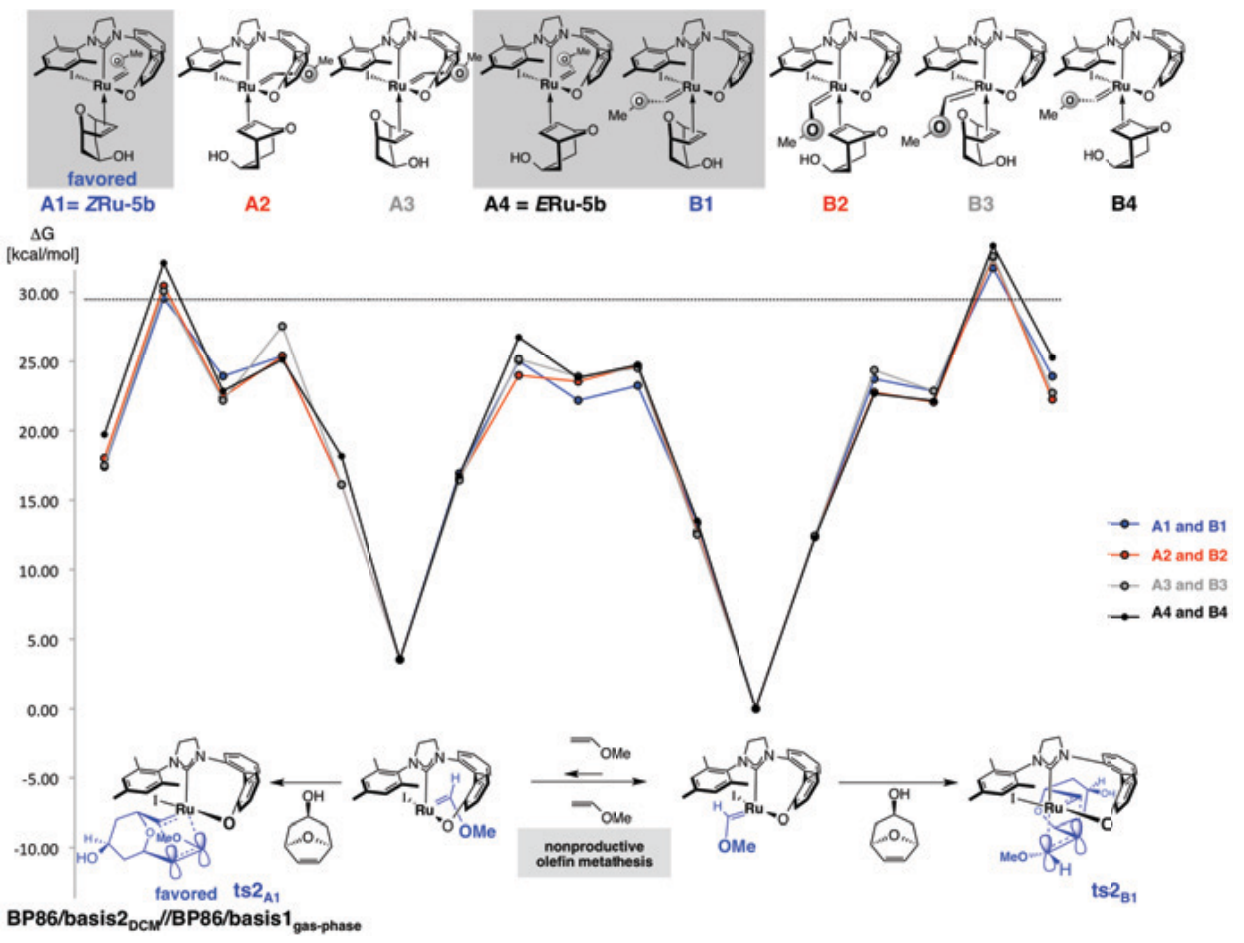

Figure S46. Reaction shown in Figure 5 and Figure S4 at the BP86/basis $2_{\mathrm{DCM}} / / \mathrm{BP} 86 / \mathrm{basis} 1_{\text {gas-phase }}$ level 


\section{Pathways ERu-5b and ZRu-5b in Figure 5 and Figure S22 $(\Delta G)$ with $\omega$ B97XD/basis2}

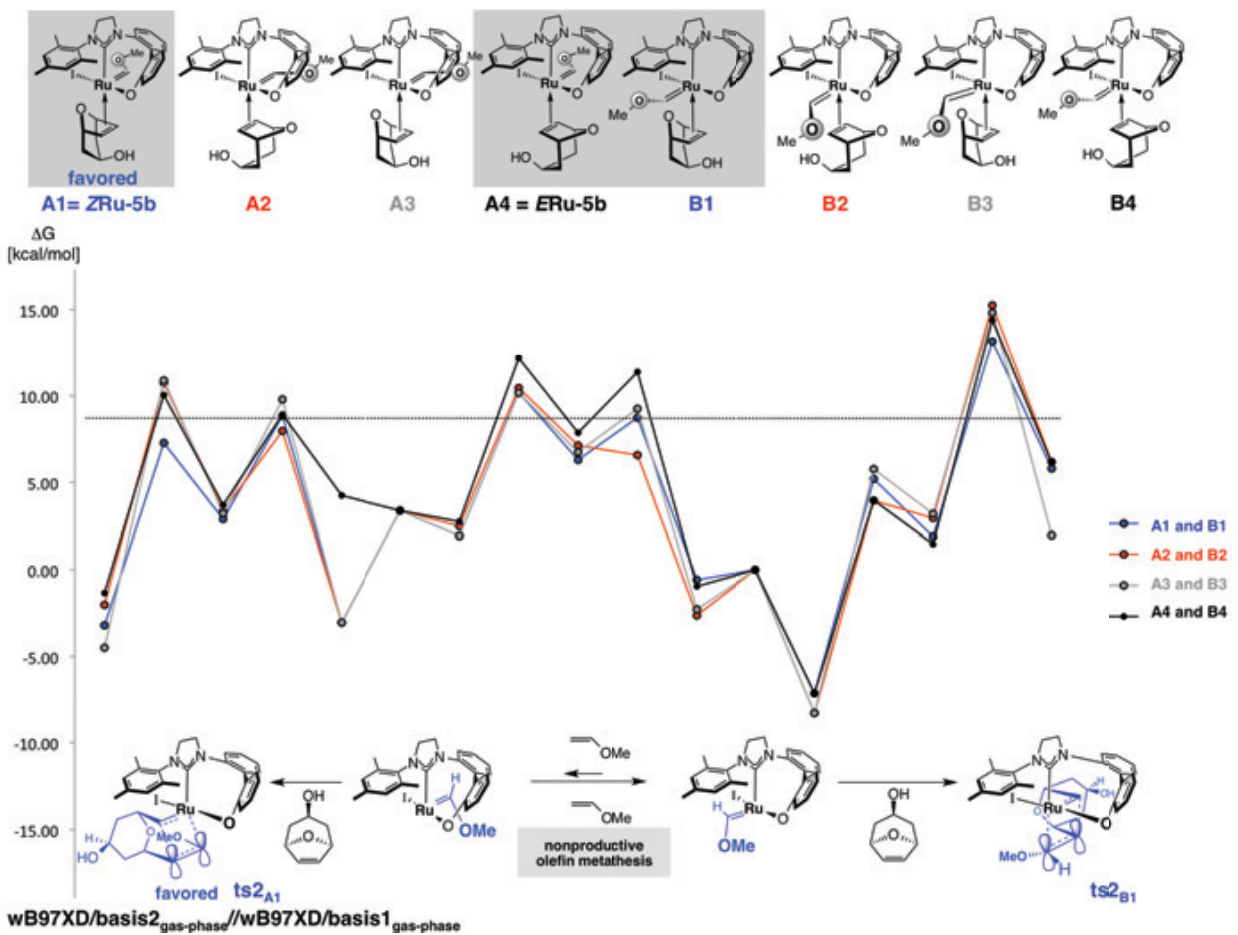

Figure S47. Reaction shown in Figure 5 and Figure S4 at the $\omega \mathrm{B} 97 \mathrm{XD} / \mathrm{basis} 2_{\text {gas-phase }} / / \omega \mathrm{B} 97 \mathrm{XD} / \mathrm{basis} 1_{\text {gas-phase }}$ level

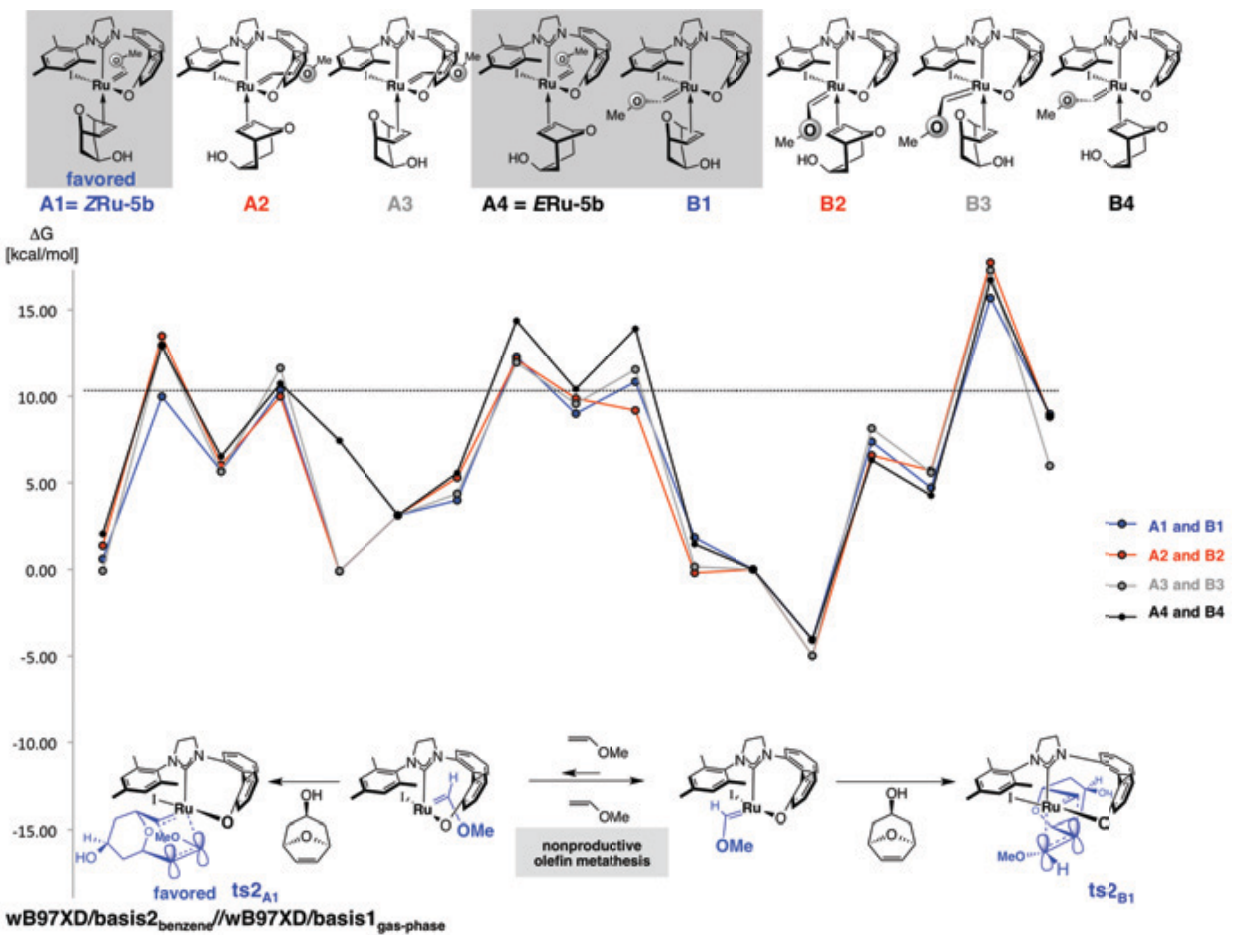

Figure S48. Reaction shown in Figure 5 and Figure S4 at the $\omega \mathrm{B} 97 \mathrm{XD} / \mathrm{basis} 2_{\text {benzene }} / / \omega \mathrm{B} 97 \mathrm{XD} / \mathrm{basis} 1_{\text {gas-phase }}$ level 


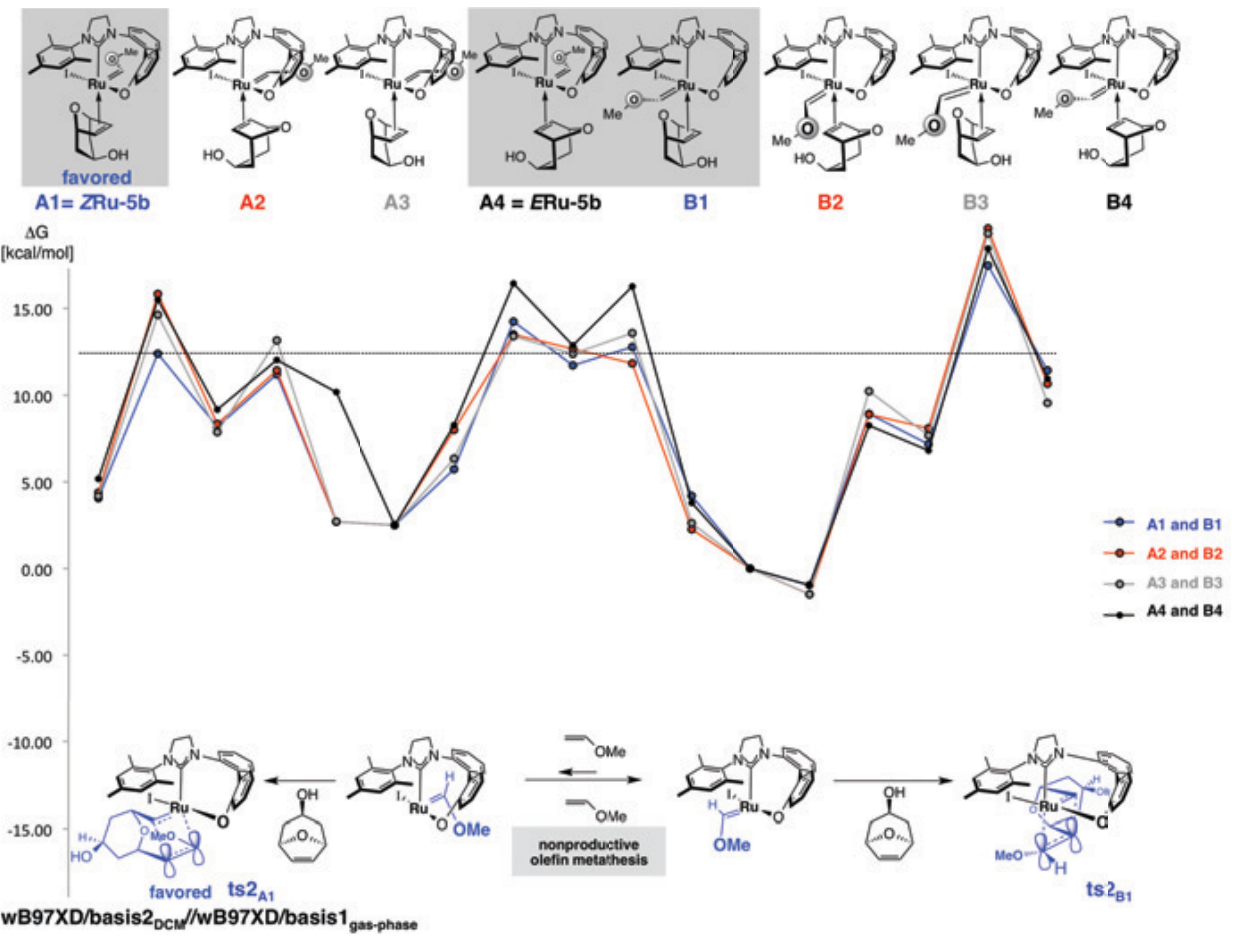

Figure S49. Reaction shown in Figure 5 and Figure S4 at the $\omega \mathrm{B} 97 \mathrm{XD} / \mathrm{basis} 2_{\mathrm{DCm}} / \omega \mathrm{B} 97 \mathrm{XD} / \mathrm{basis} 1_{\text {gas-phase }}$ level 


\section{Pathways $E$ Ru-5b and $Z R u-5 b$ in Figure 5 and Figure S22 $(\Delta G)$ with M06/basis2}

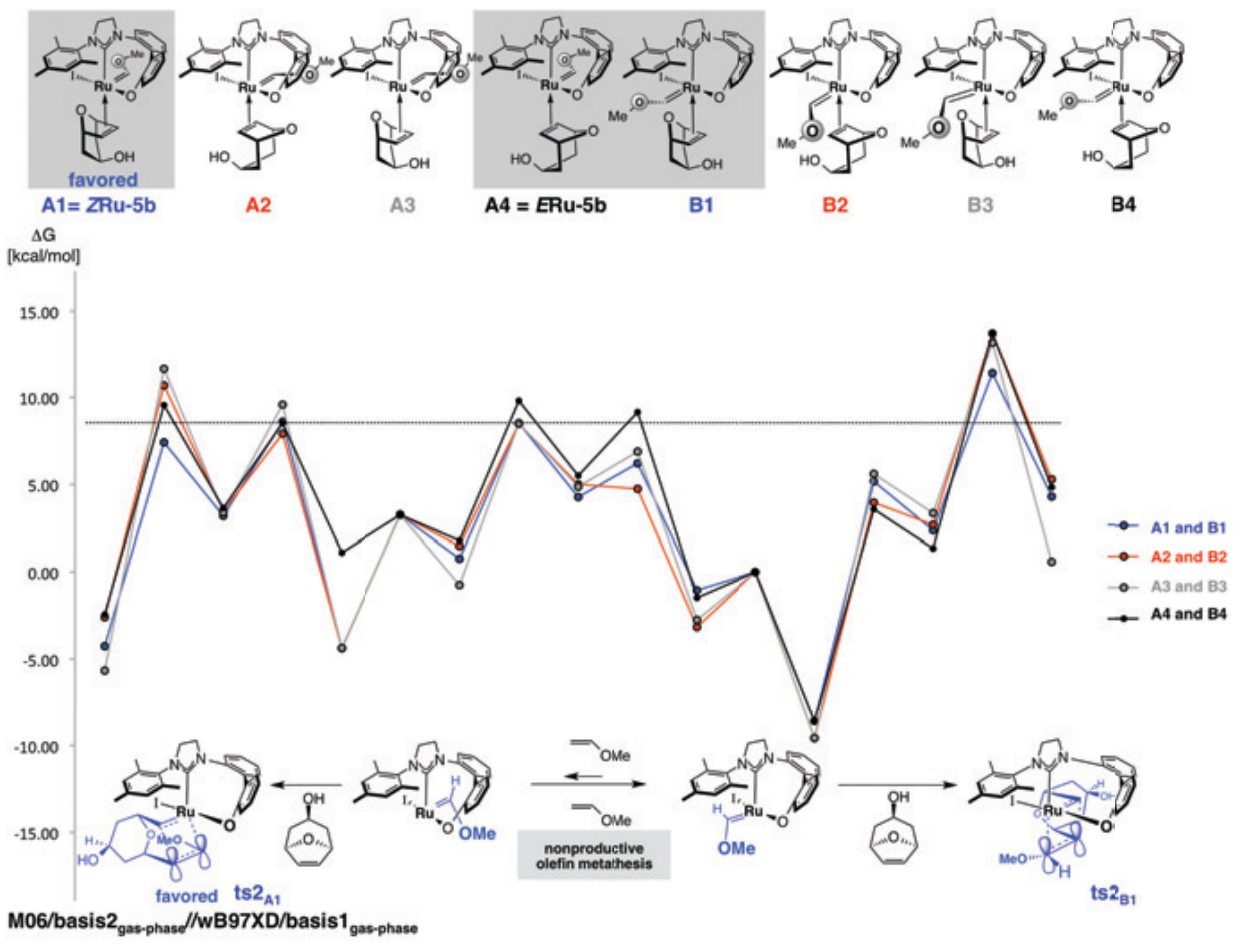

Figure S50. Reaction shown in Figure 5 and Figure S4 at the M06/basis2 ${ }_{\text {gas-phase }} / / \omega B 97 X D /$ basis $1_{\text {gas-phase }}$ level

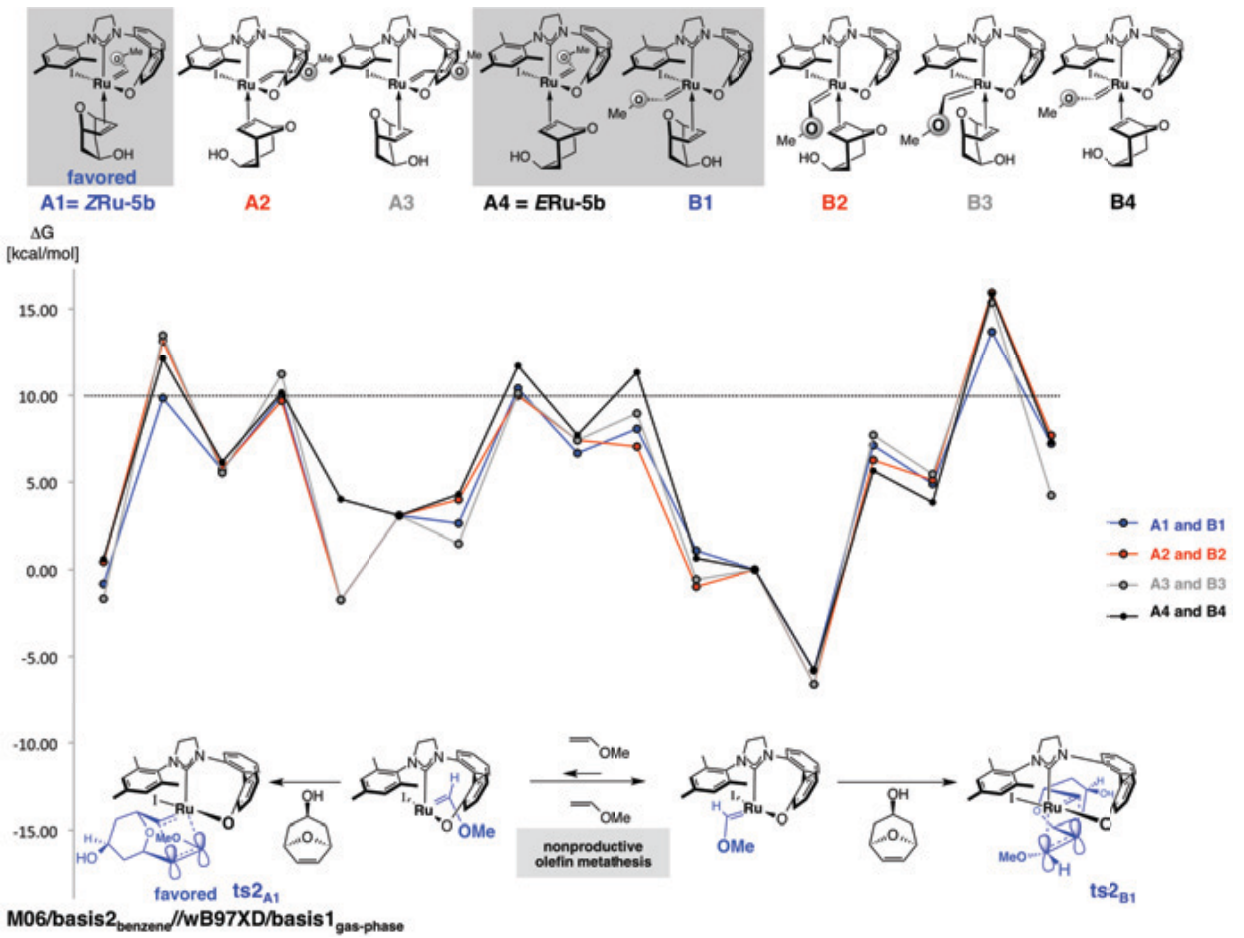

Figure S51. Reaction shown in Figure 5 and Figure S4 at the M06/basis $2_{\text {benzene }} / / \omega B 97 X D / b a s i s 1_{\text {gas-phase }}$ level 


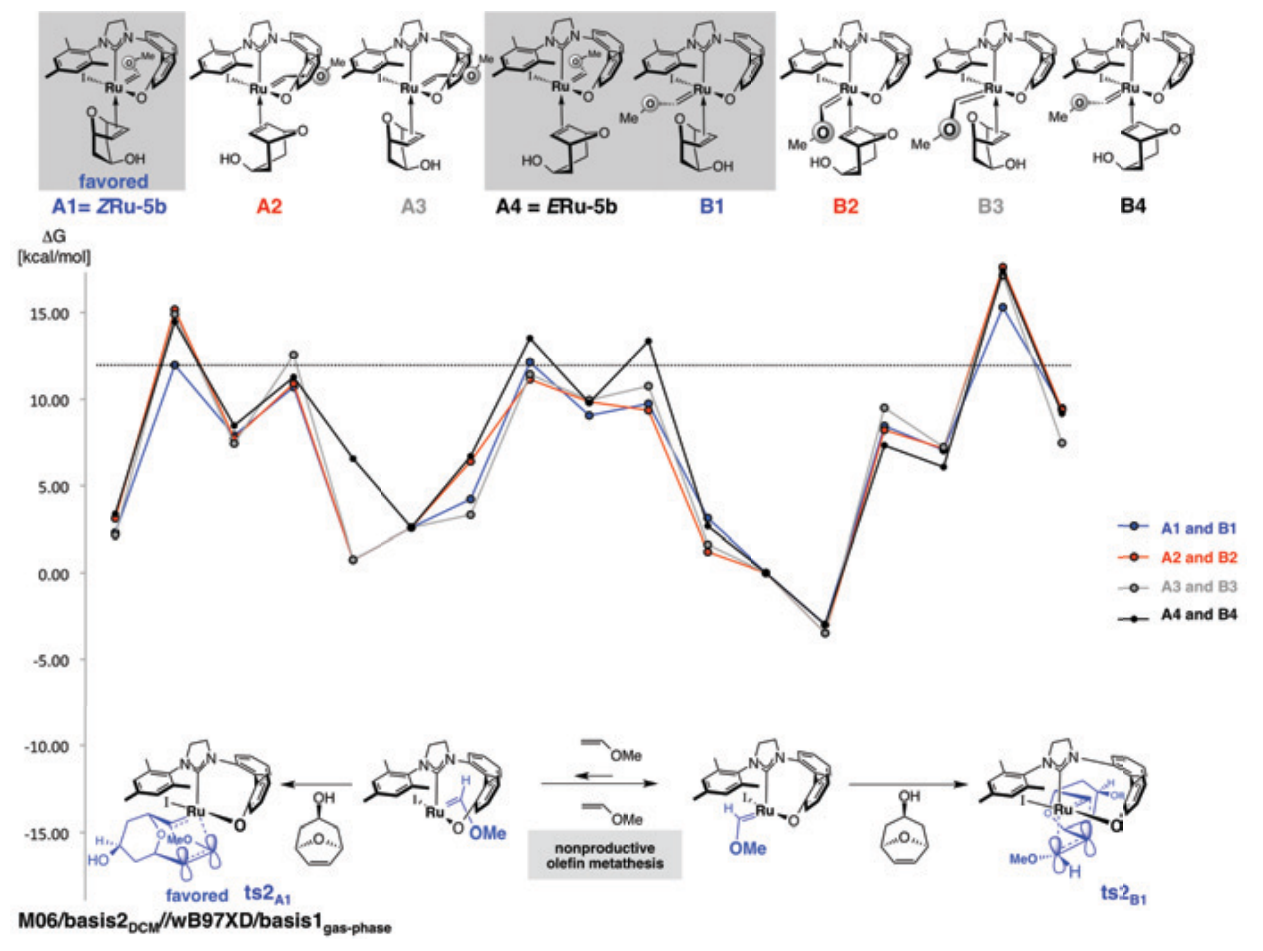

Figure S52. Reaction shown in Figure 5 and Figure $S 4$ at the M06/basis $2_{\mathrm{DCM}} / / \omega B 97 \mathrm{XD} / \mathrm{basis} 1_{\text {gas-phase }}$ level 


\section{Pathways $E M e, Z M e, E C N$ and $Z C N$ in Figure $7(\Delta G)$ with BP86/basis2}
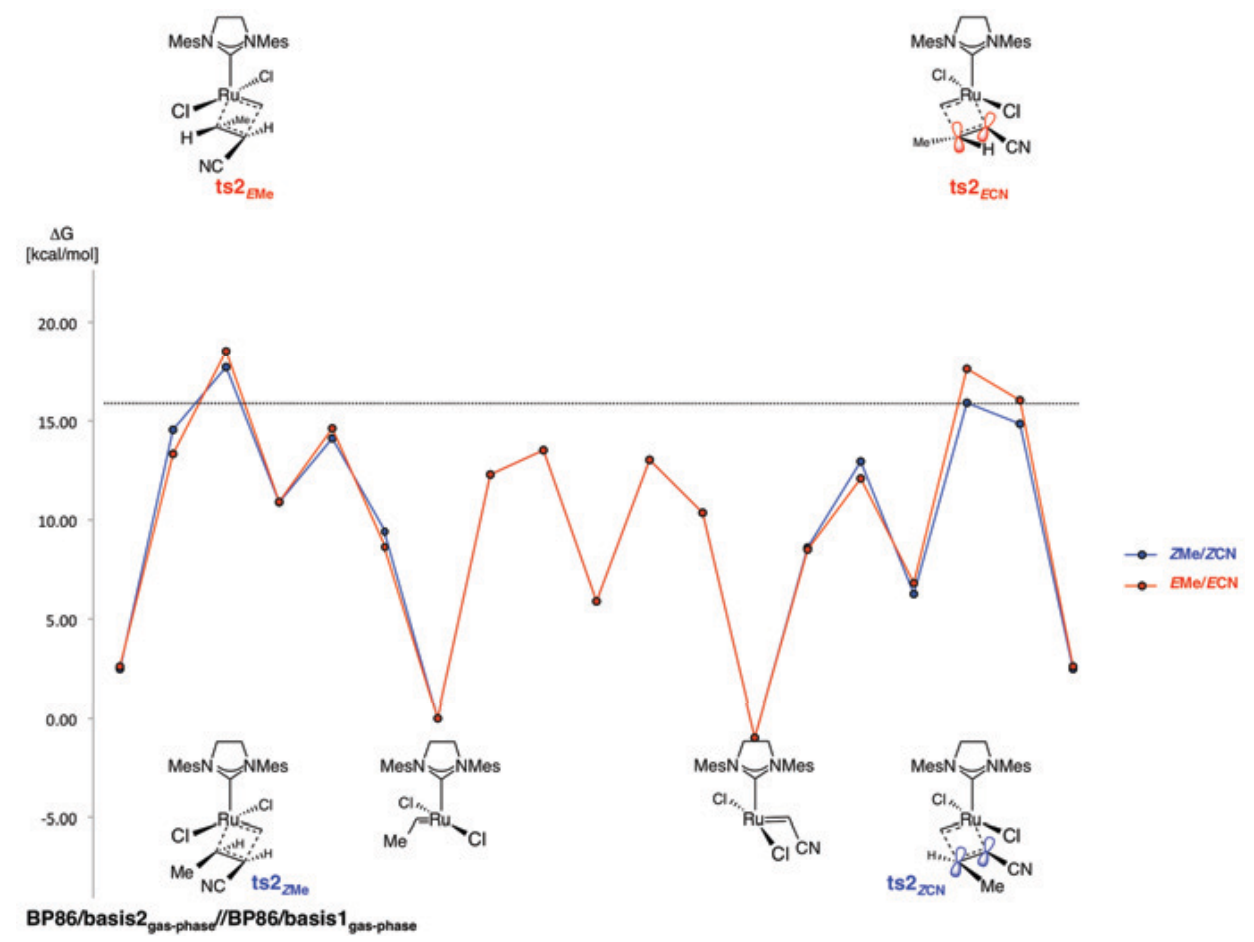

Figure S53. Reaction shown in Figure 7 at the BP86/basis $2_{\text {gas-phase }} / / \mathrm{BP} 86 / \mathrm{basis} 1_{\text {gas-phase }}$ level
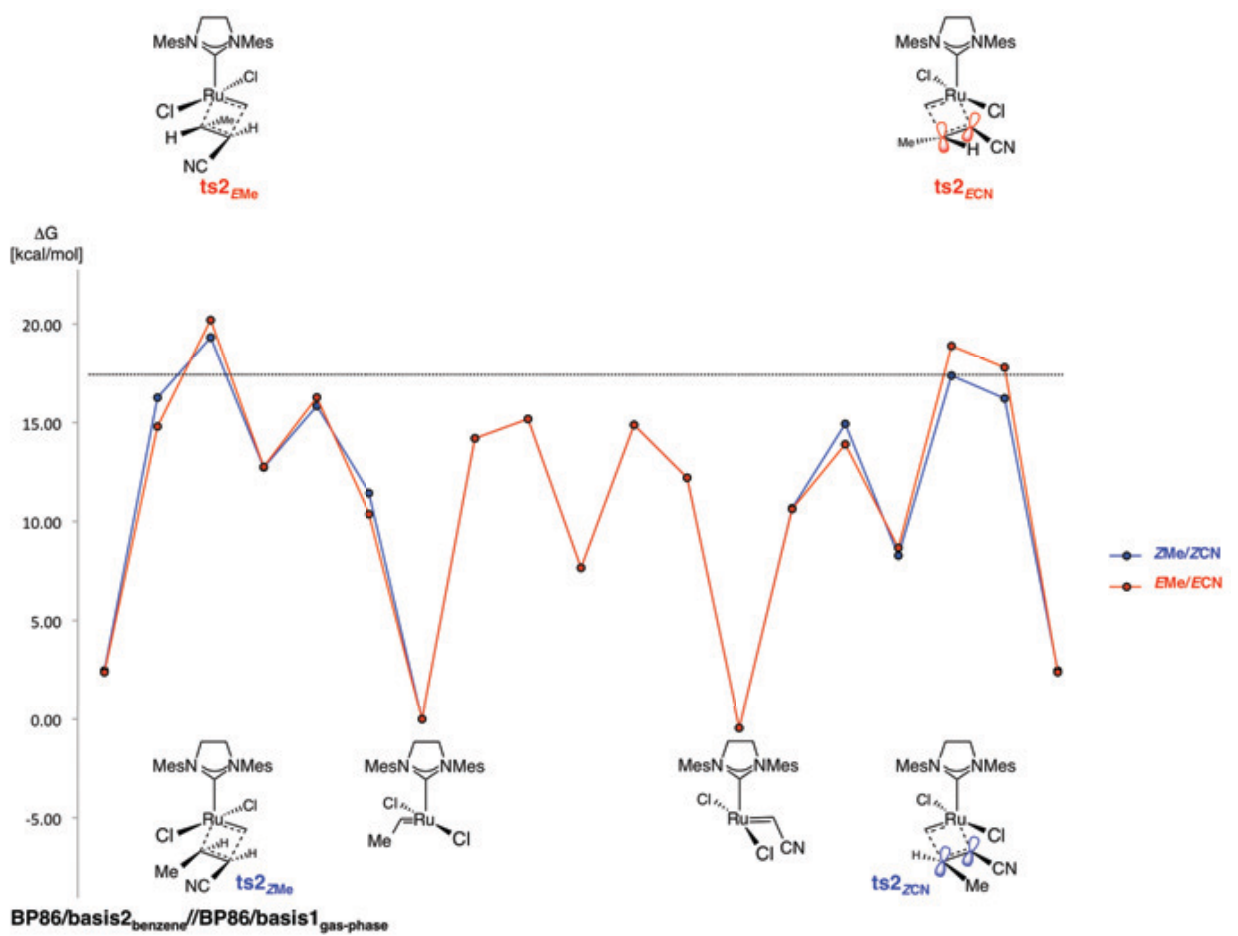

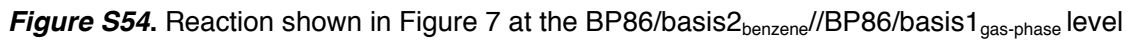



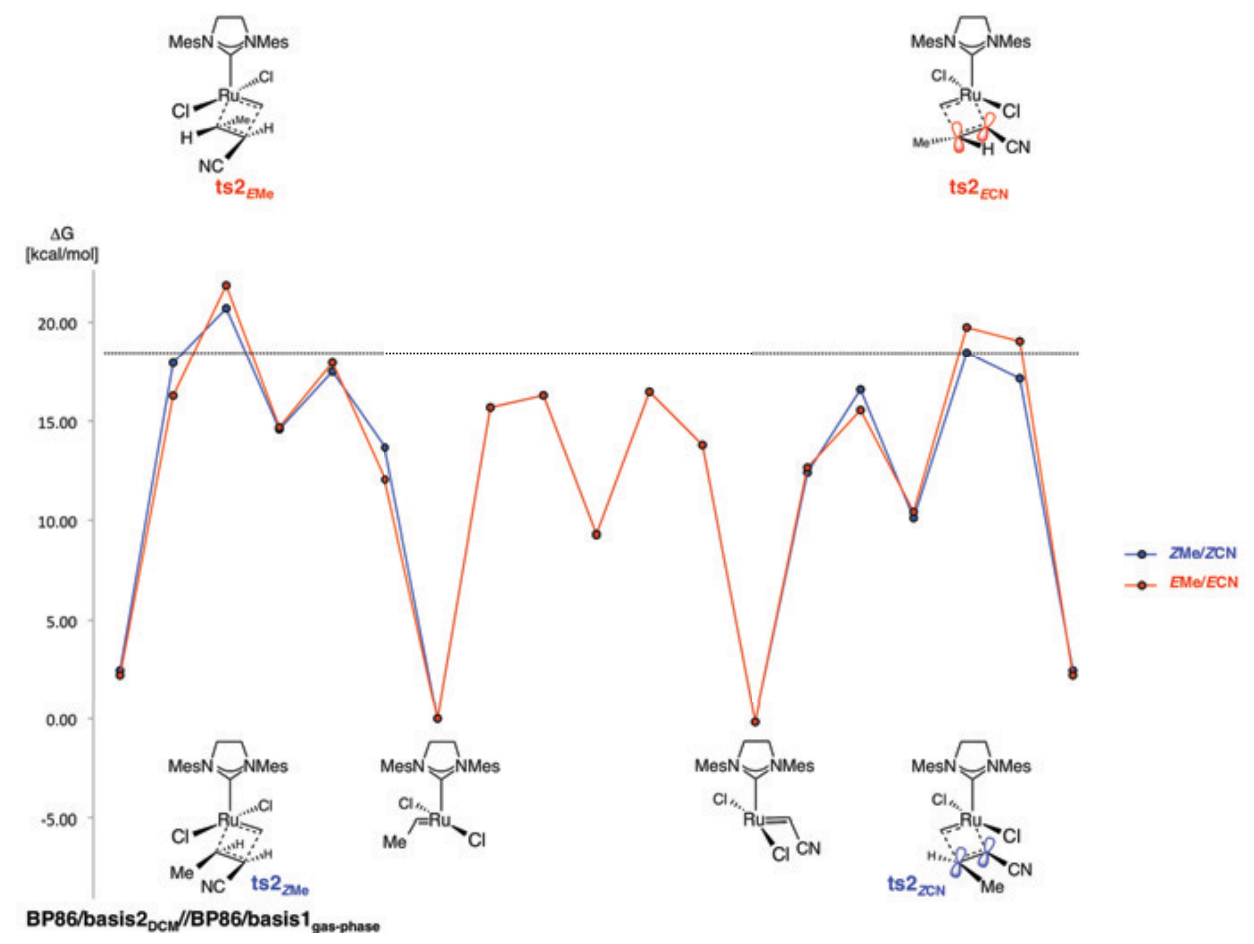

Figure S55. Reaction shown in Figure 7 at the BP86/basis $2_{\mathrm{DCM}} / / \mathrm{BP} 86 /$ basis $1_{\text {gas-phase }}$ level 


\section{Pathways $E M e, Z M e, E C N$ and $Z C N$ in Figure $7(\Delta G)$ with $\omega$ B97XD/basis2}
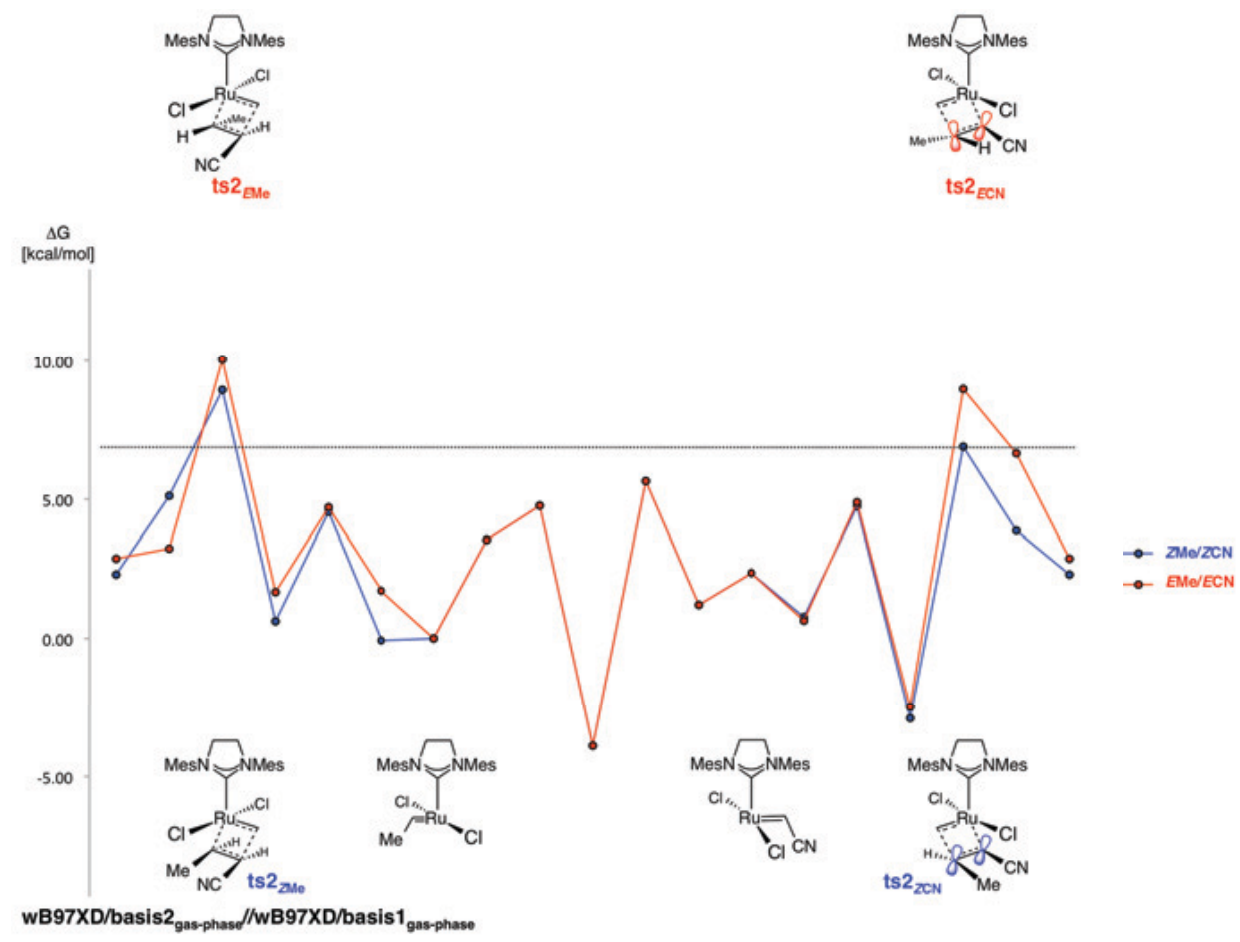

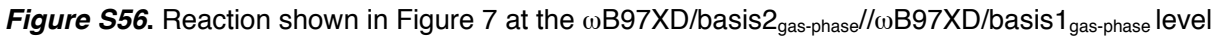
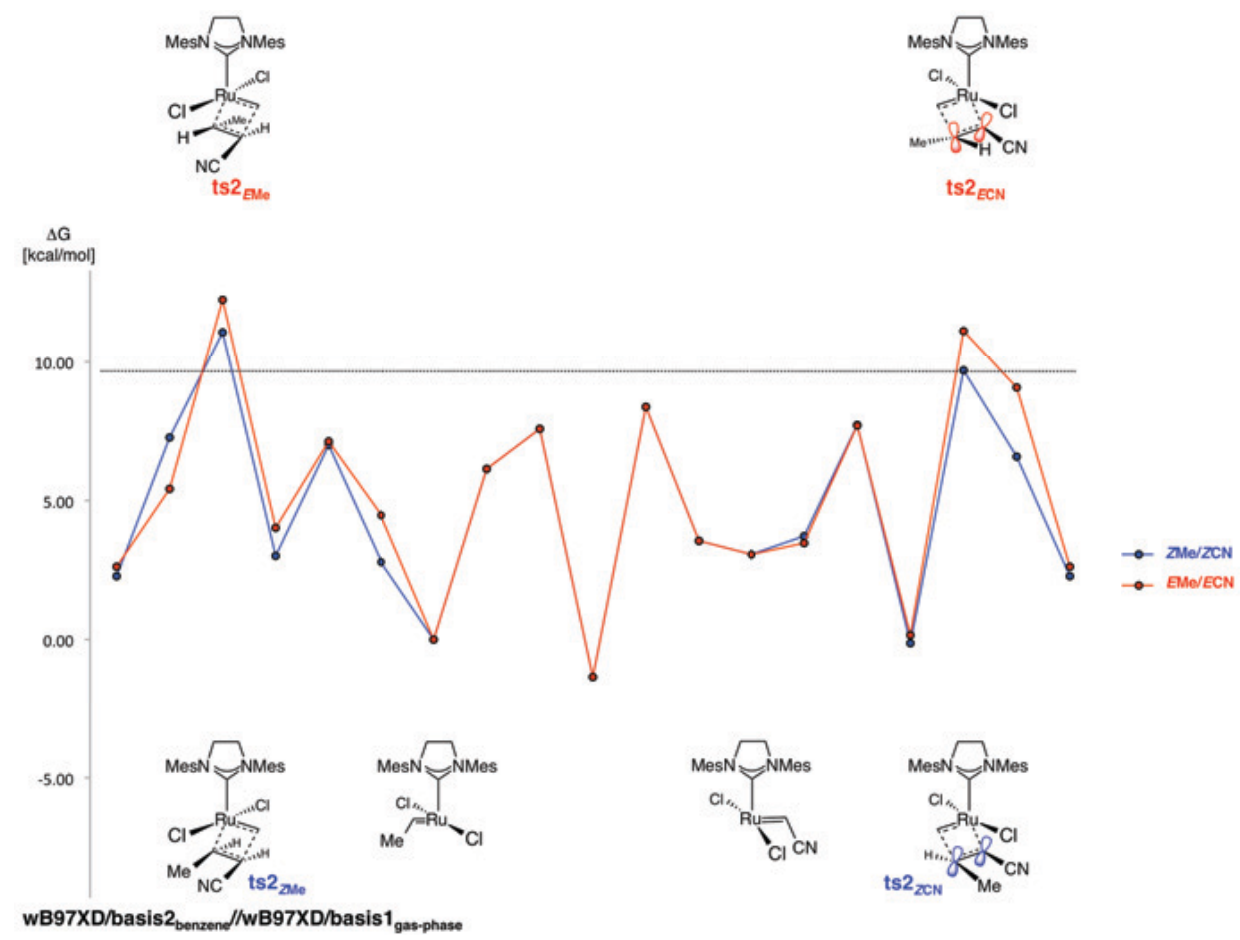

Figure S57. Reaction shown in Figure 7 at the $\omega \mathrm{B} 97 \mathrm{XD} / \mathrm{basis} 2_{\text {benaene }} / / \omega \mathrm{B} 97 \mathrm{XD} / \mathrm{basis} 1_{\text {gas-phase }}$ level 

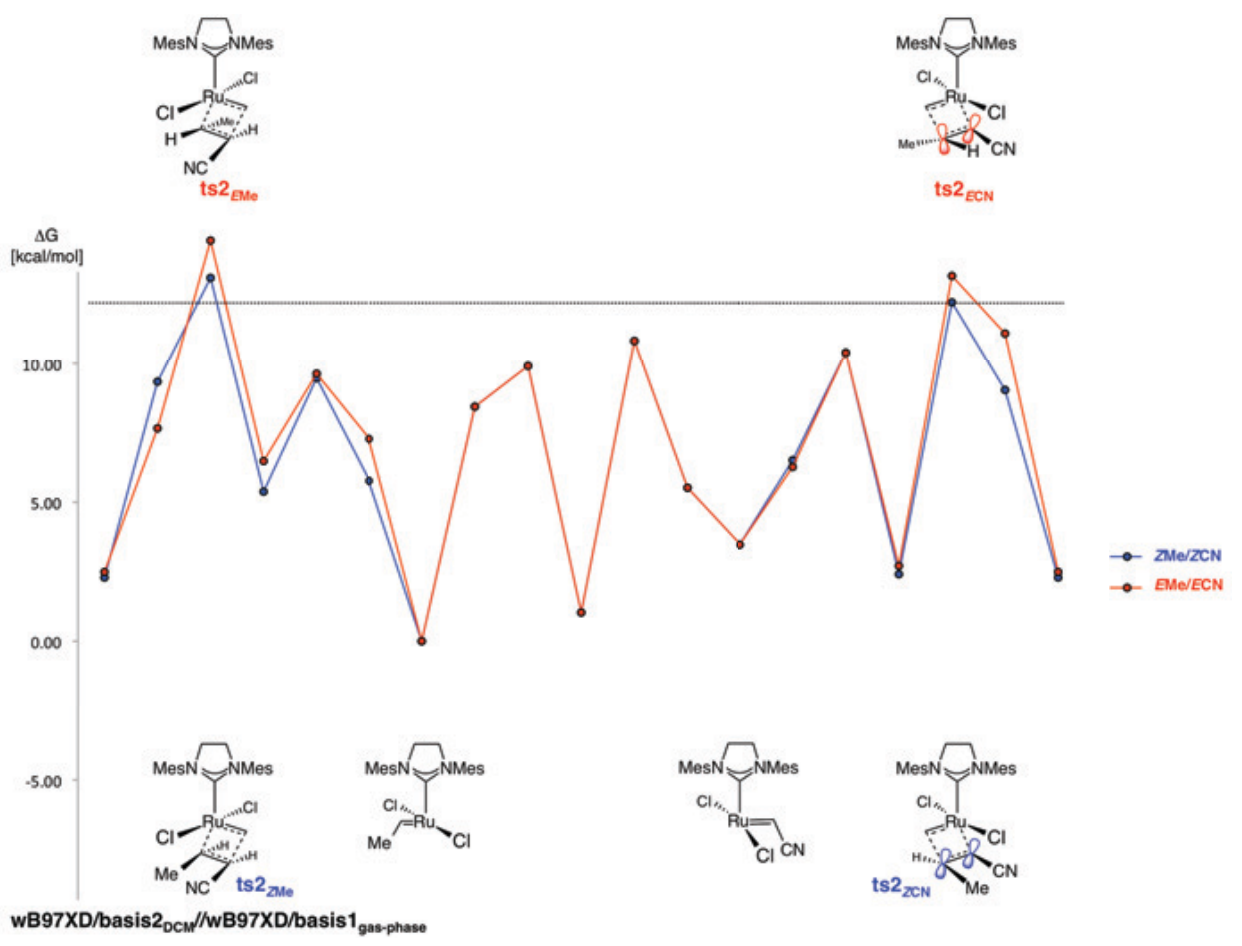

Figure S58. Reaction shown in Figure 7 at the $\omega \mathrm{B} 97 \mathrm{XD} / \mathrm{basis} 2_{\mathrm{DCM}} / / \omega \mathrm{B} 97 \mathrm{XD} / \mathrm{basis} 1_{\text {gas-phase }}$ level 


\section{Pathways $E M e, Z M e, E C N$ and $Z C N$ in Figure $7(\Delta G)$ with M06/basis2}
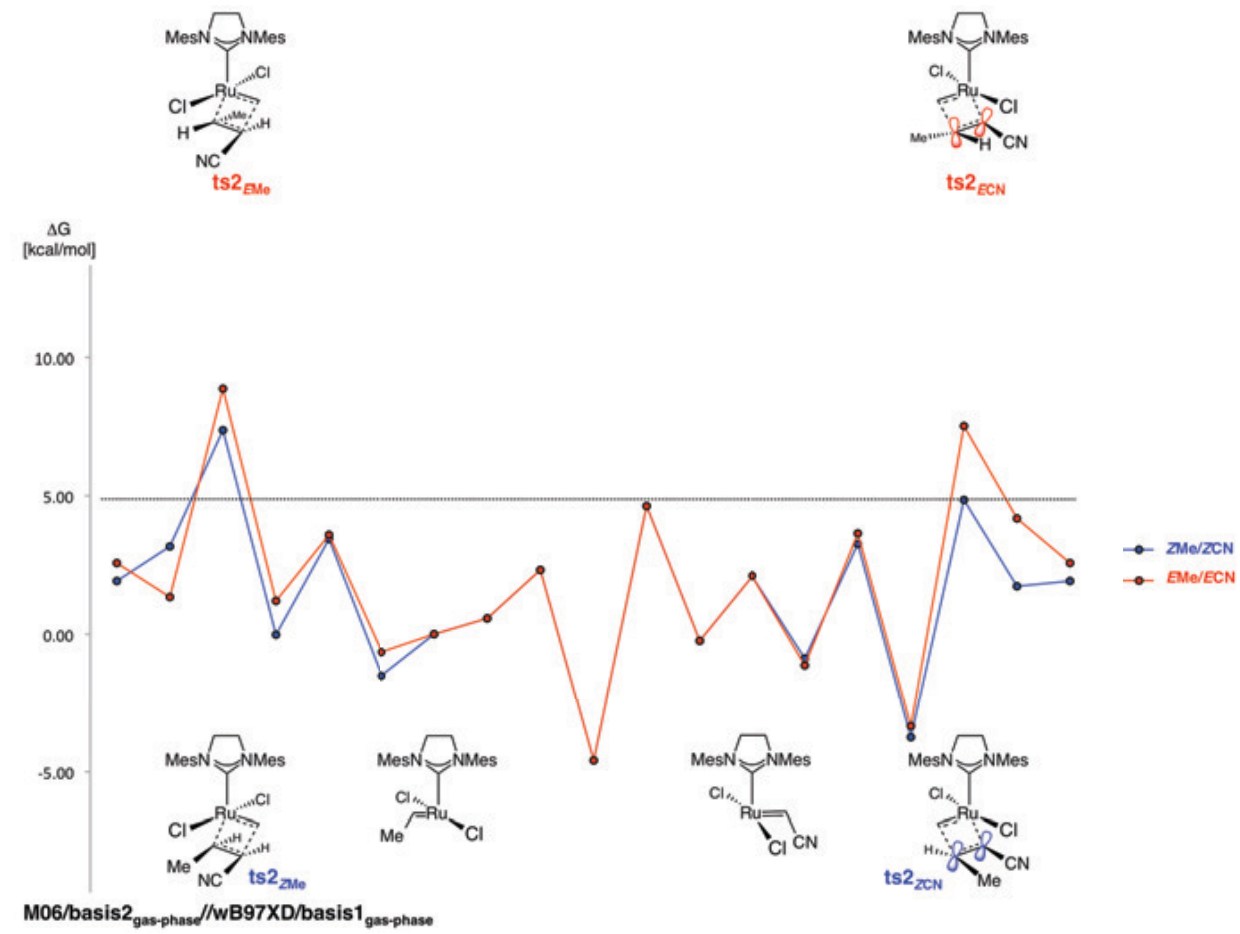

Figure S59. Reaction shown in Figure 7 at the M06/basis $2_{\text {gas-phase }} / / \omega \mathrm{B} 97 \mathrm{XD} / \mathrm{basis} 1_{\text {gas-phase }}$ level
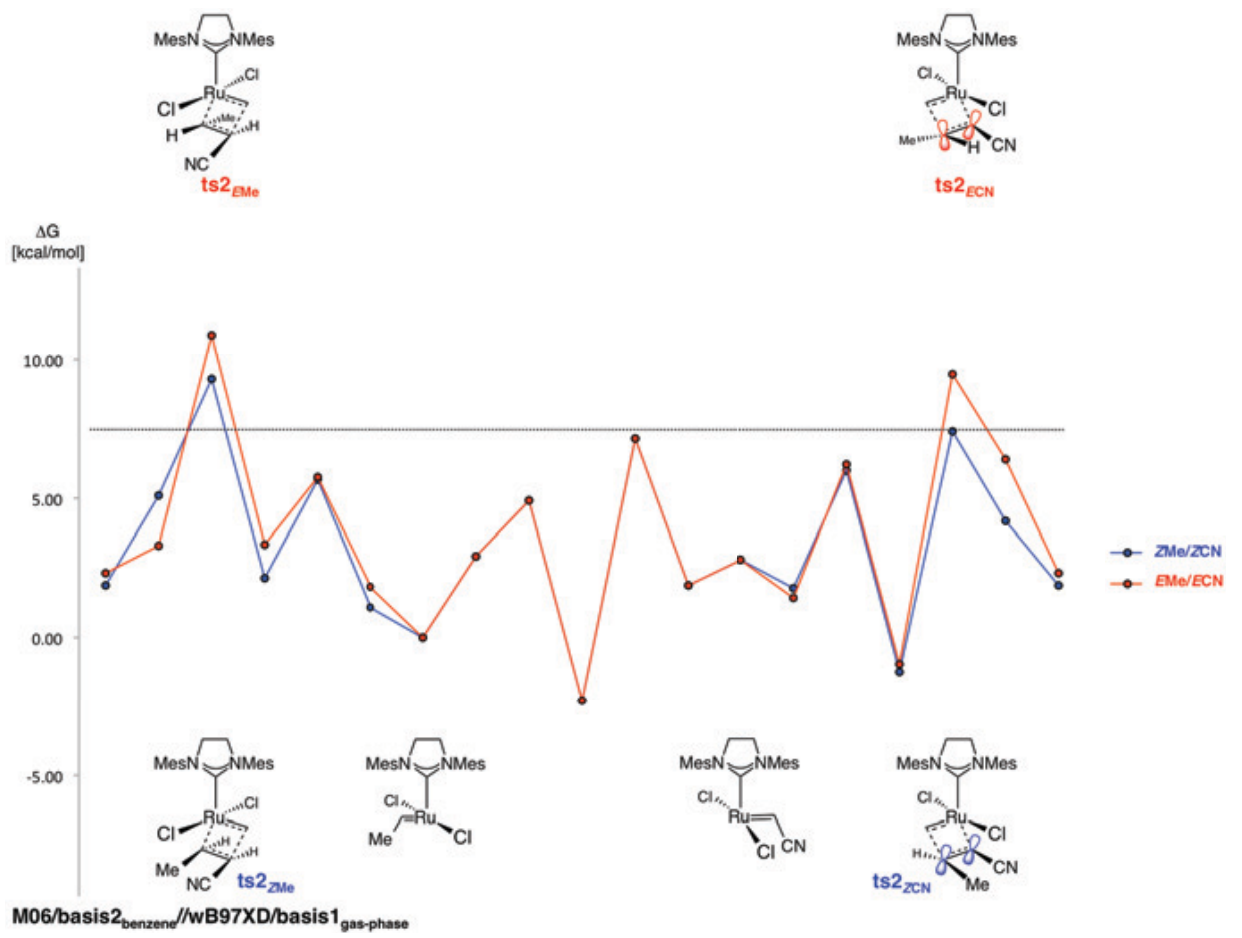

Figure S60. Reaction shown in Figure 7 at the M06/basis $2_{\text {benzene }} / / \omega B 97 X D / b a s i s 1_{\text {gas-phase }}$ level 

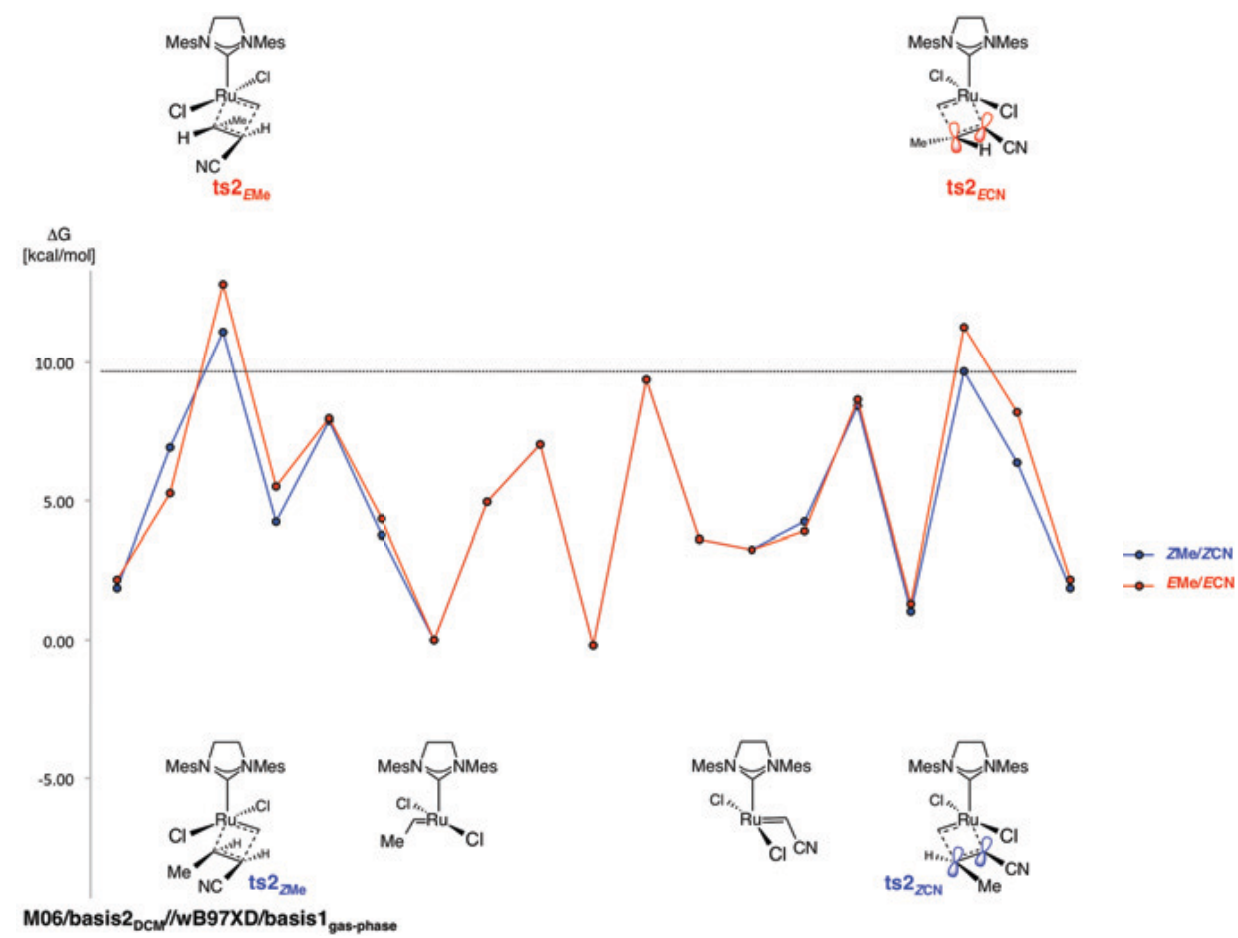

Figure S61. Reaction shown in Figure 7 at the M06/basis2 ${ }_{\mathrm{DCM}} / / \omega \mathrm{B} 97 \mathrm{XD} / \mathrm{basis} 1_{\text {gas-phase }}$ level 
Pathways ESMe, ZSMe, EMe' and ZMe' in Figures 9-10 $(\Delta G)$ with BP86/basis2
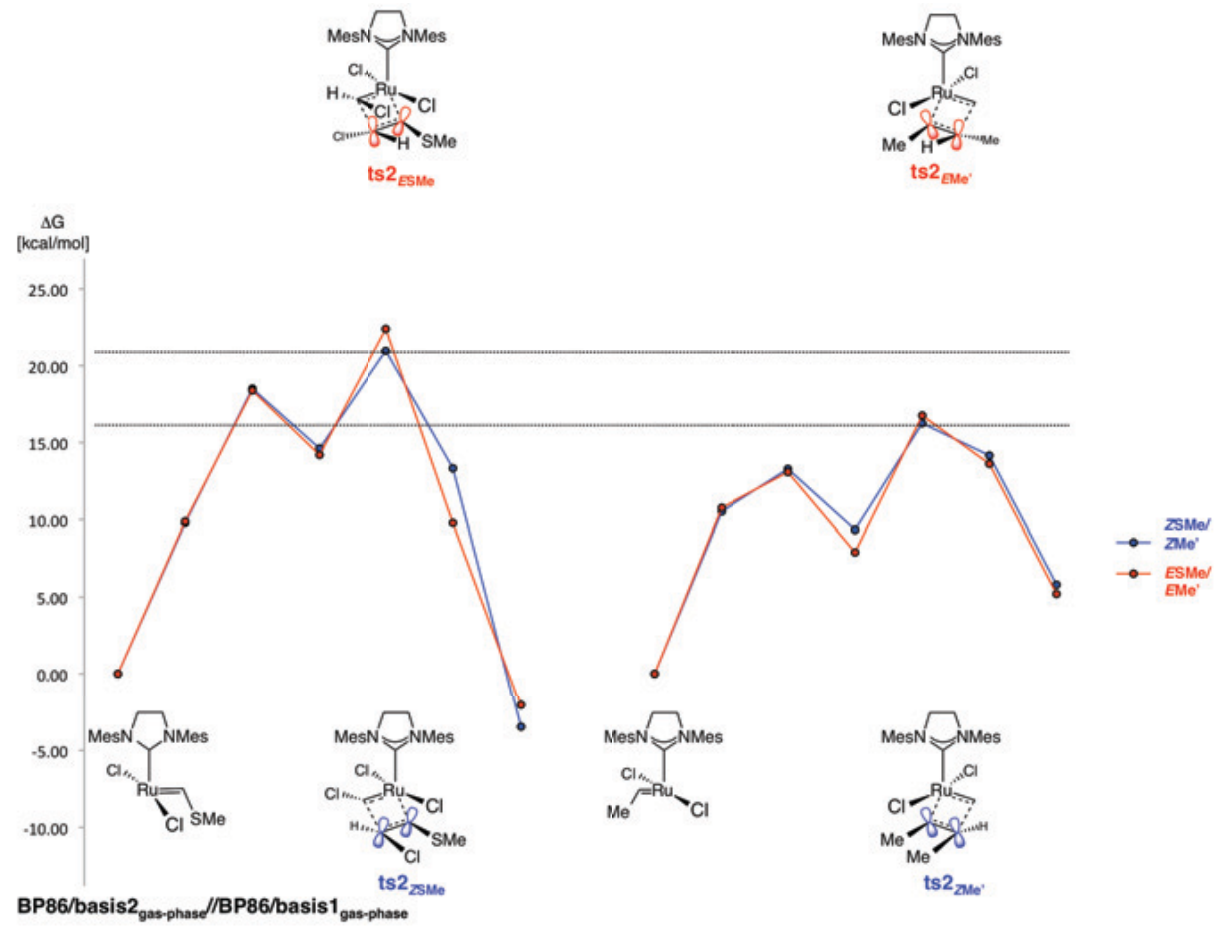

Figure S62. Reaction shown in Figures $9-10$ at the BP86/basis $2_{\text {gas-phase }} / / \mathrm{BP} 86 / \mathrm{basis} 1_{\text {gas-phase }}$ level
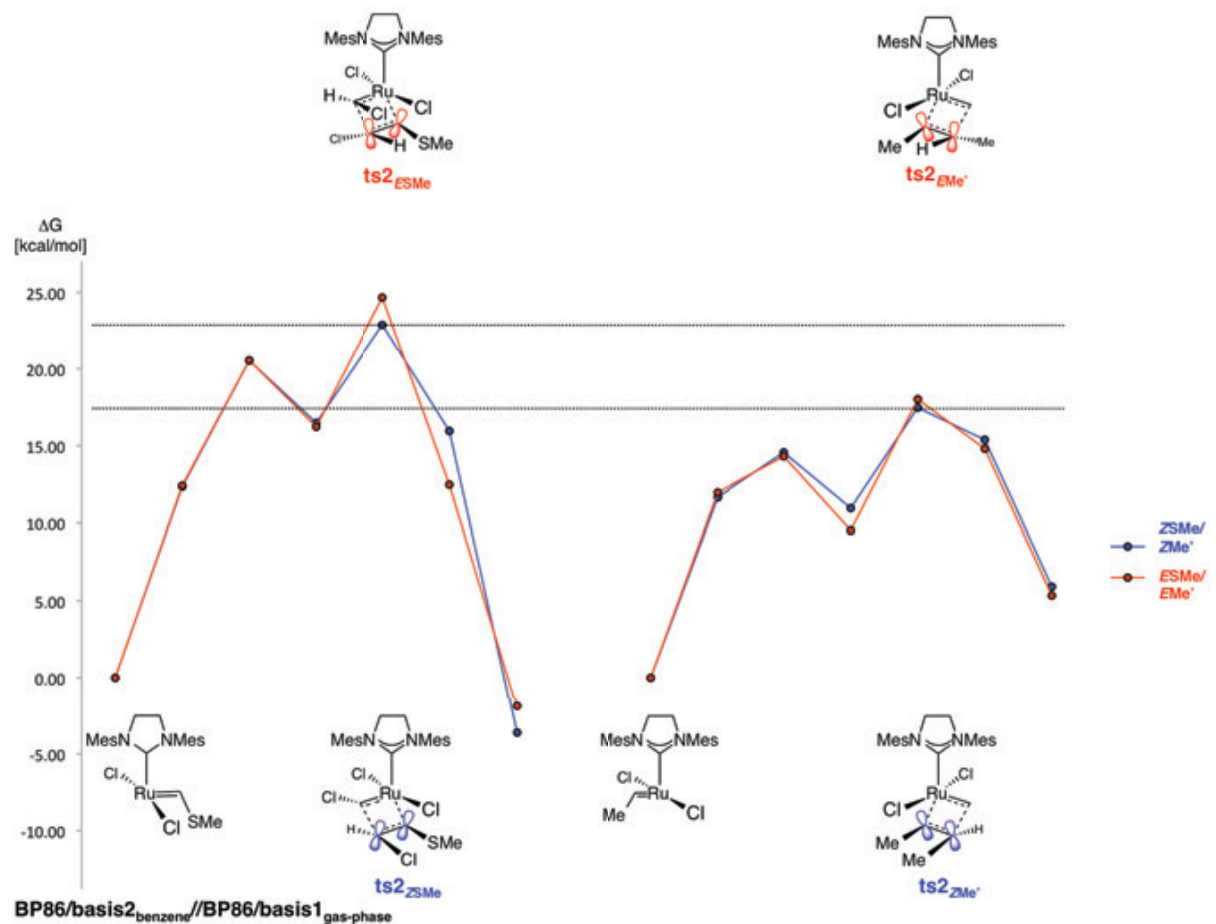

Figure S63. Reaction shown in Figures 9-10 at the BP86/basis $2_{\text {benzene }} / / \mathrm{BP} 86 / \mathrm{basis} 1_{\text {gas-phase }}$ level 

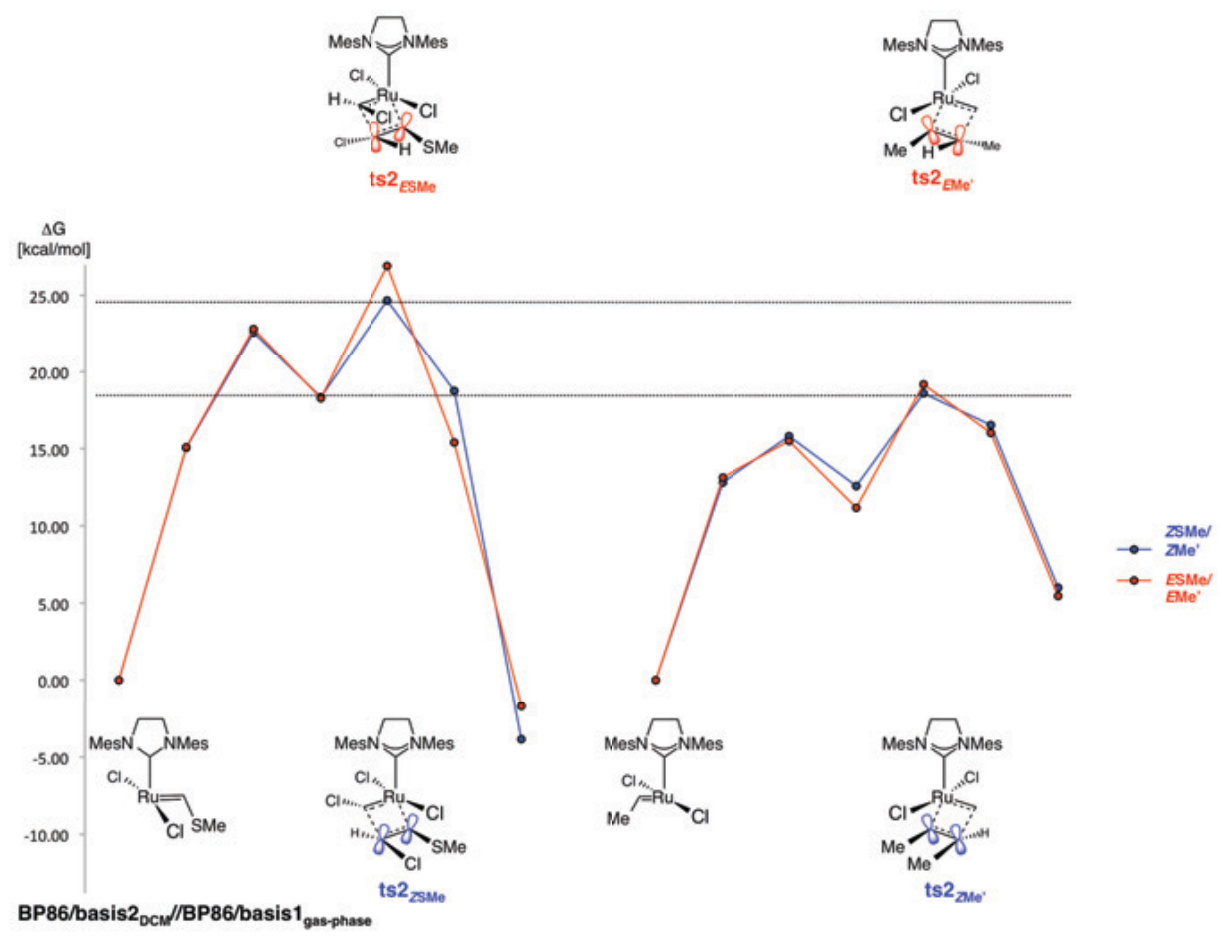

Figure S64. Reaction shown in Figures $9-10$ at the BP86/basis $2_{\mathrm{DCM}} / / \mathrm{BP} 86 / \mathrm{basis} 1_{\text {gas-phase }}$ level 


\section{Pathways ESMe, ZSMe, EMe' and ZMe' in Figures 9-10 $(\Delta$ G) with $\omega$ B97XD/basis2}
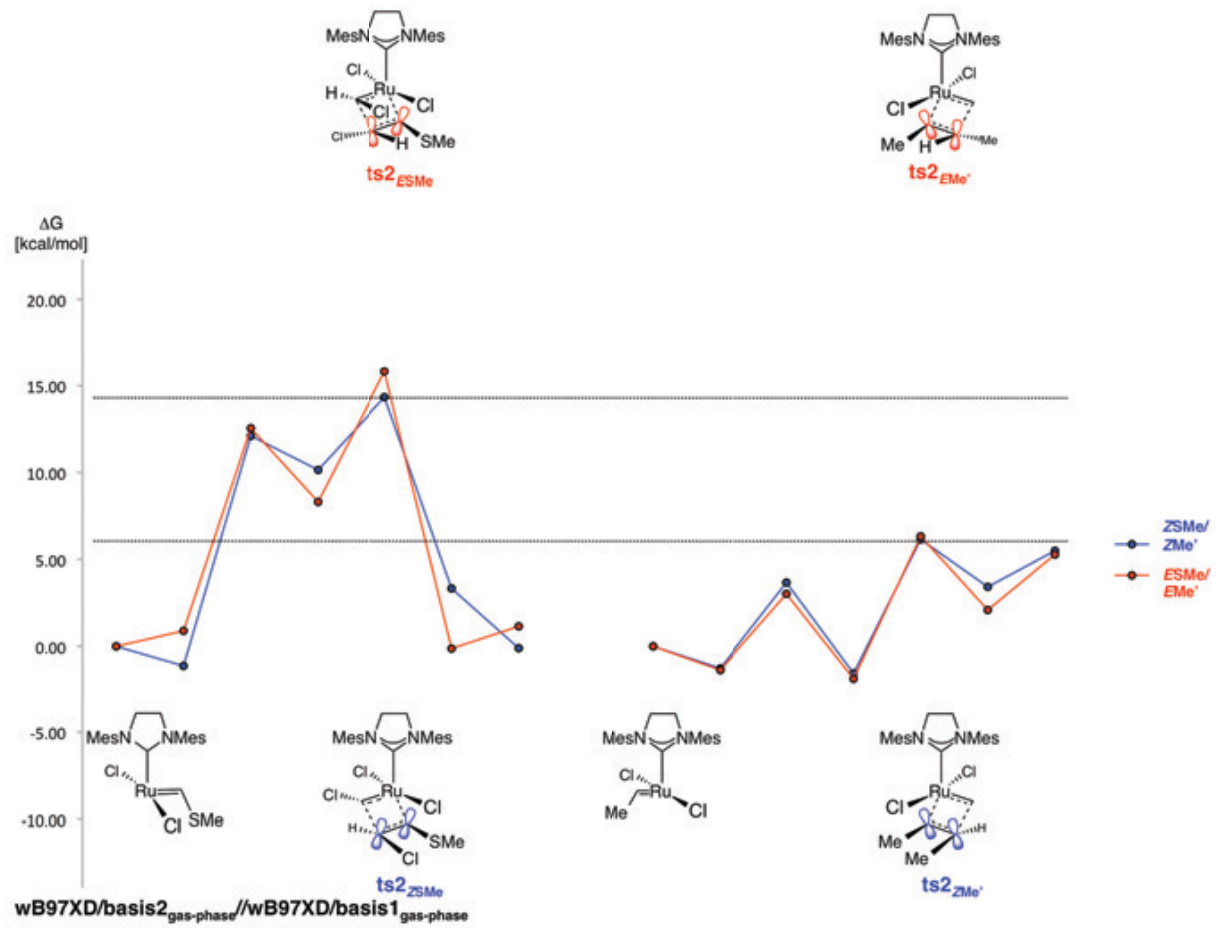

Figure S65. Reaction shown in Figures $9-10$ at the $\omega \mathrm{B} 97 \mathrm{XD} / \mathrm{basis} 2_{\text {gas-phase }} / / \omega \mathrm{B} 97 \mathrm{XD} / \mathrm{basis}_{1}$ gas-phase $_{\text {level }}$
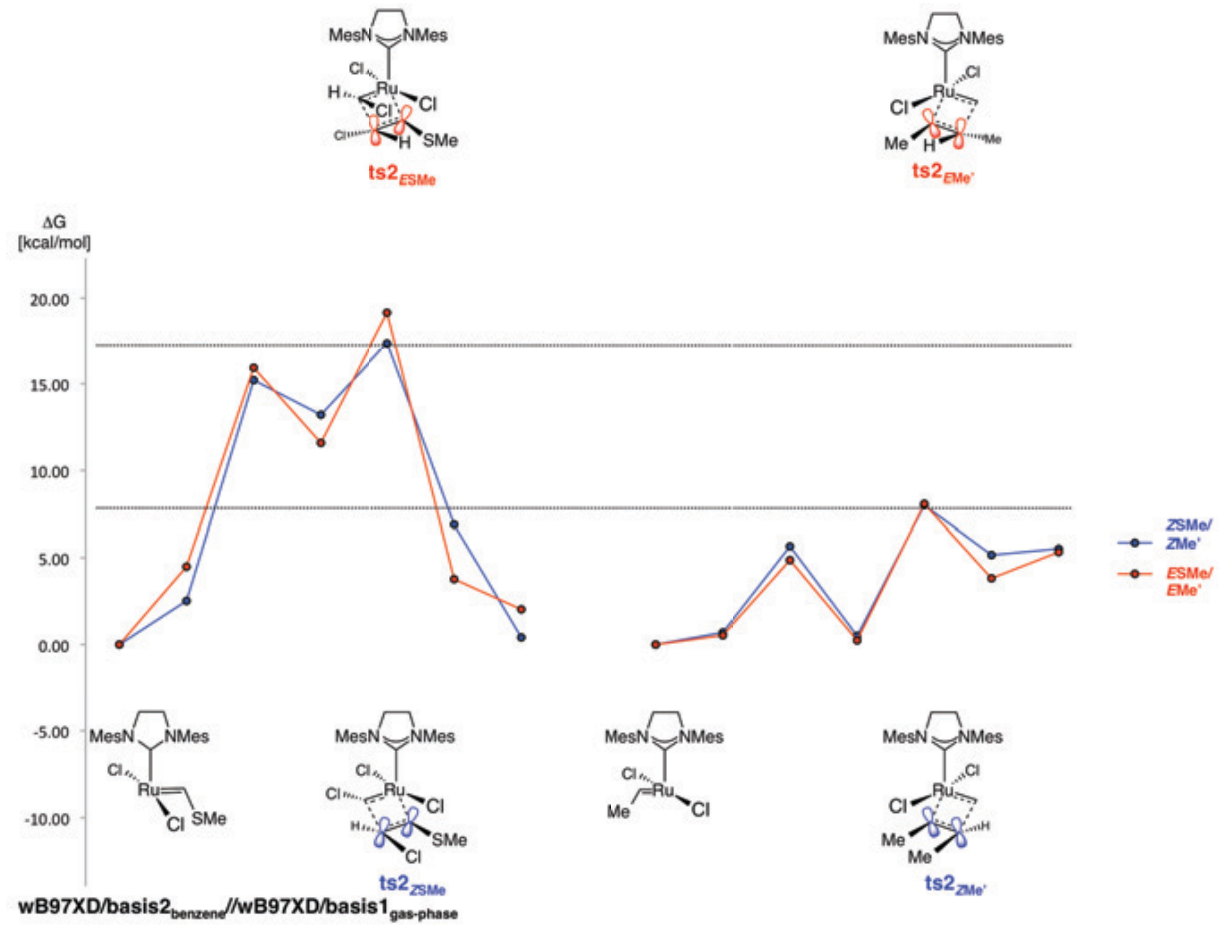

Figure S66. Reaction shown in Figures $9-10$ at the $\omega \mathrm{B} 97 \mathrm{XD} /$ basis $2_{\text {benzene }} / / \omega \mathrm{B} 97 \mathrm{XD} / \mathrm{basis} 1_{\text {gas-phase }}$ level 

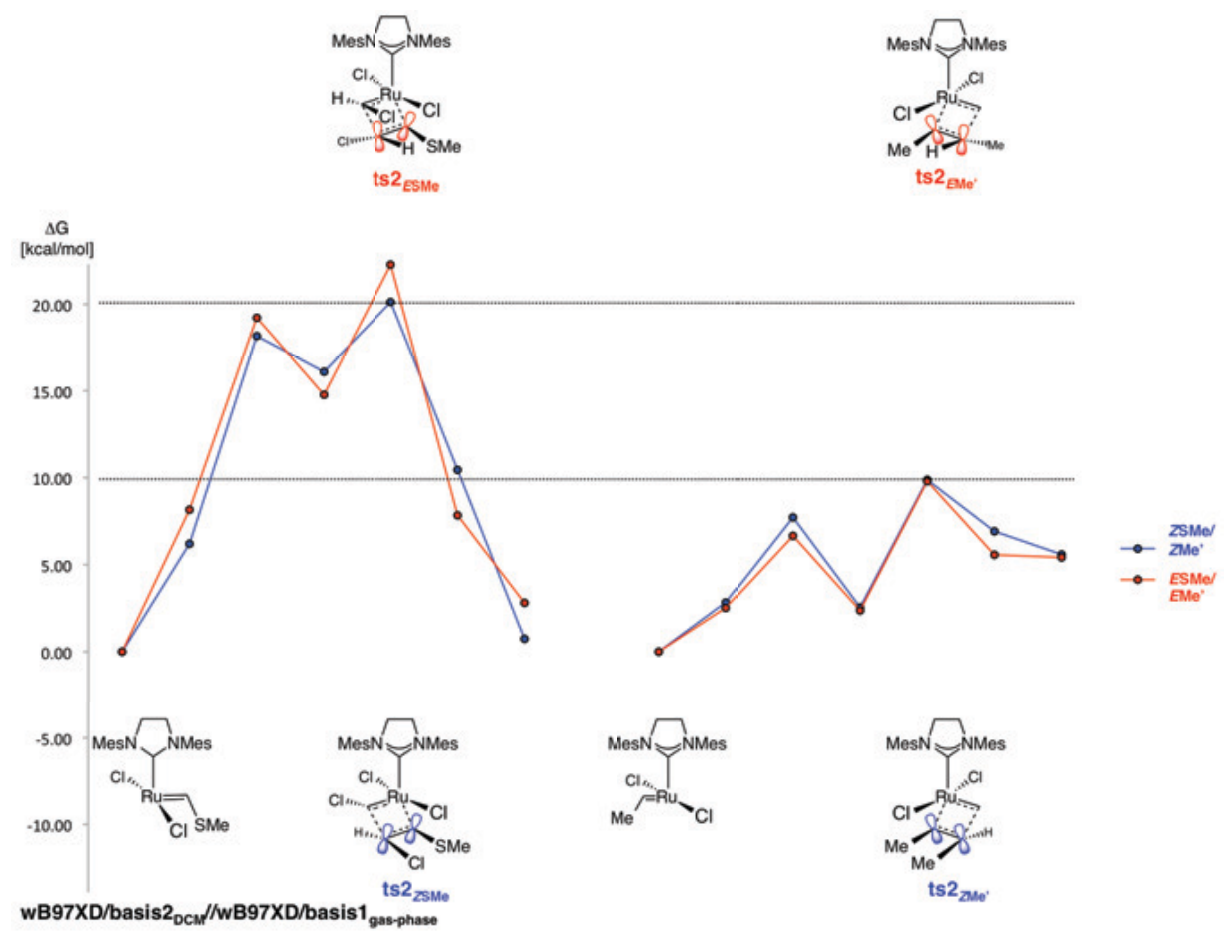

Figure S67. Reaction shown in Figures 9-10 at the $\omega \mathrm{B} 97 \mathrm{XD} / \mathrm{basis} 2_{\mathrm{DCM}} / / \omega \mathrm{B} 97 \mathrm{XD} / \mathrm{basis} 1_{\text {gas-phase }}$ level 
Pathways ESMe, ZSMe, EMe' and ZMe' in Figures 9-10 ( $\Delta$ G) with M06/basis2
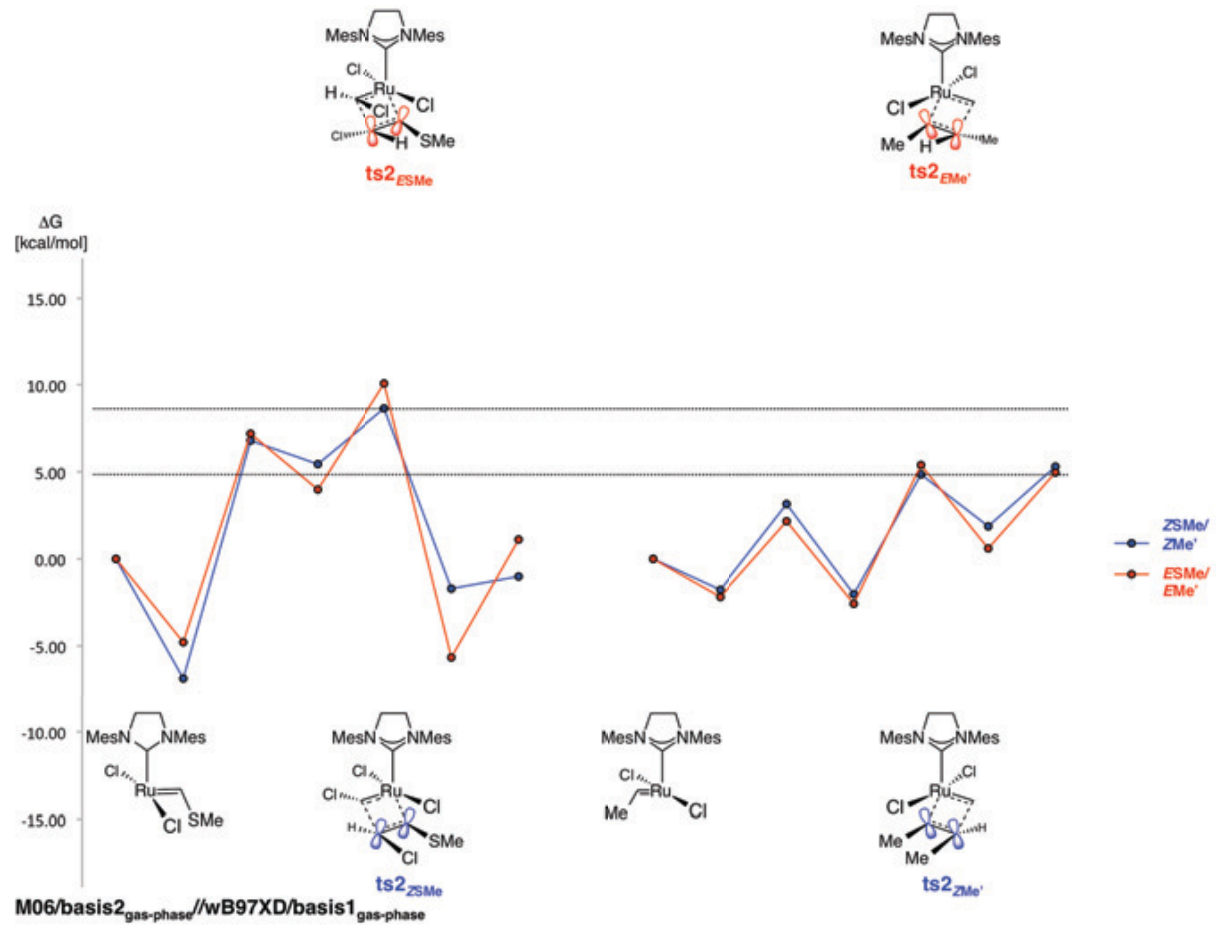

Figure S68. Reaction shown in Figures $9-10$ at the M06/basis $2_{\text {gas-phase }} / / \omega \mathrm{B} 97 \mathrm{XD} / \mathrm{basis} 1_{\text {gas-phase }}$ level
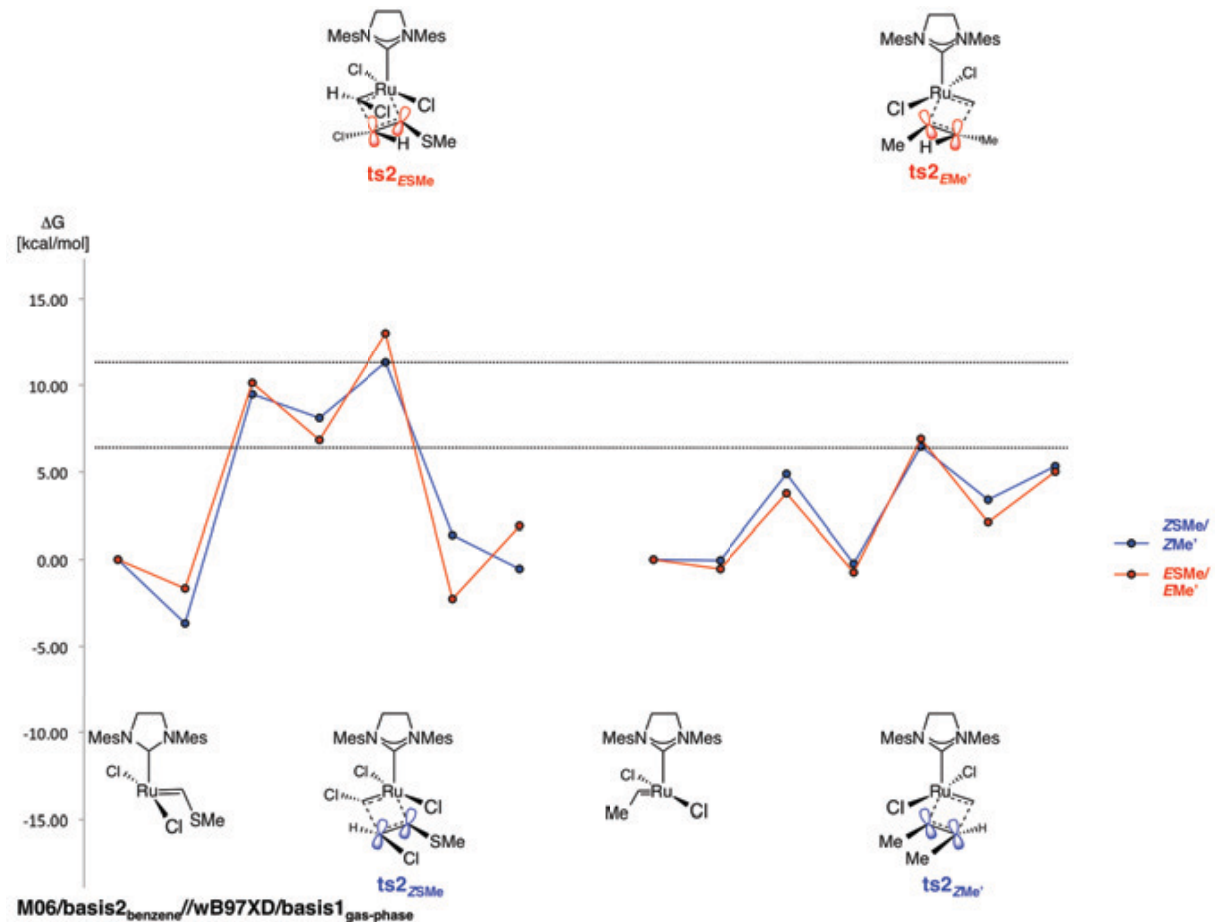

Figure S69. Reaction shown in Figures $9-10$ at the M06/basis $2_{\text {benzene }} / / \omega B 97 X D / b a s i s 1_{\text {gas-phase }}$ level 

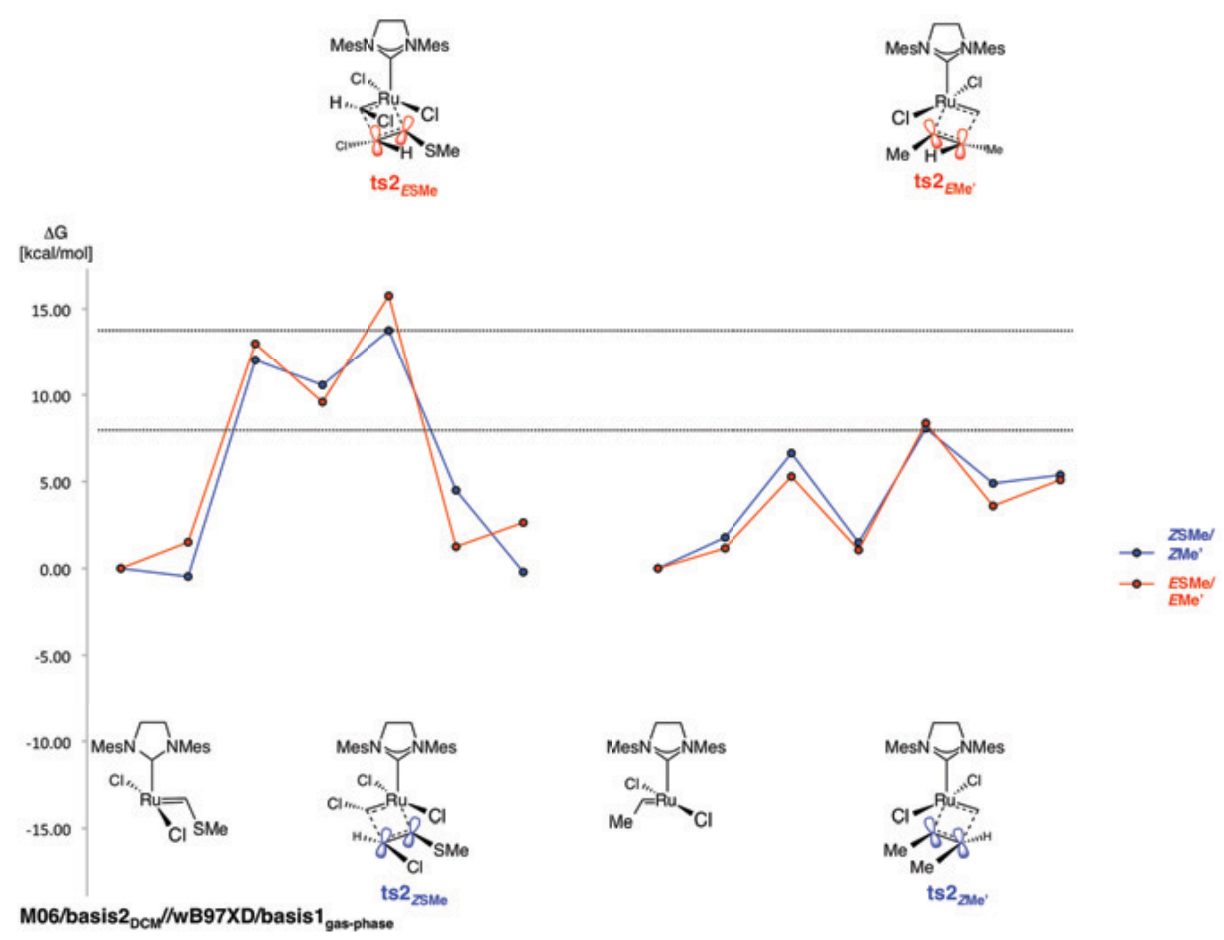

Figure S70. Reaction shown in Figures $9-10$ at the M06/basis $2_{\mathrm{DCM}} / / \omega \mathrm{B} 97 \mathrm{XD} / \mathrm{basis} 1_{\text {gas-phase }}$ level 


\section{Pathways C1-D4 in Figure S23 $(\Delta G)$ with BP86/basis2}
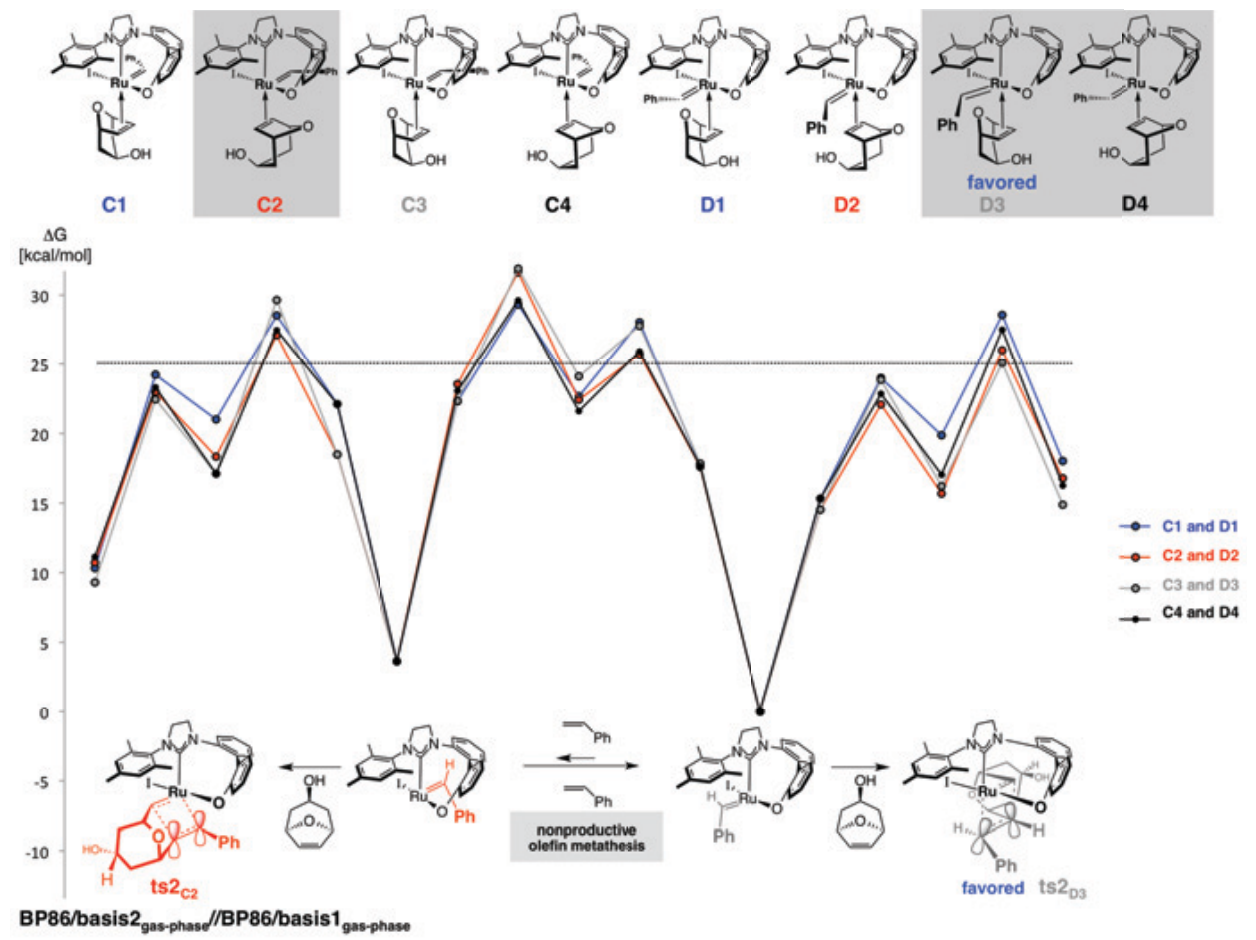

Figure S71. Reaction shown in Figure S5 at the BP86/basis2 $2_{\text {gas-phase }} / / \mathrm{BP} 86 /$ basis $1_{\text {gas-phase }}$ level
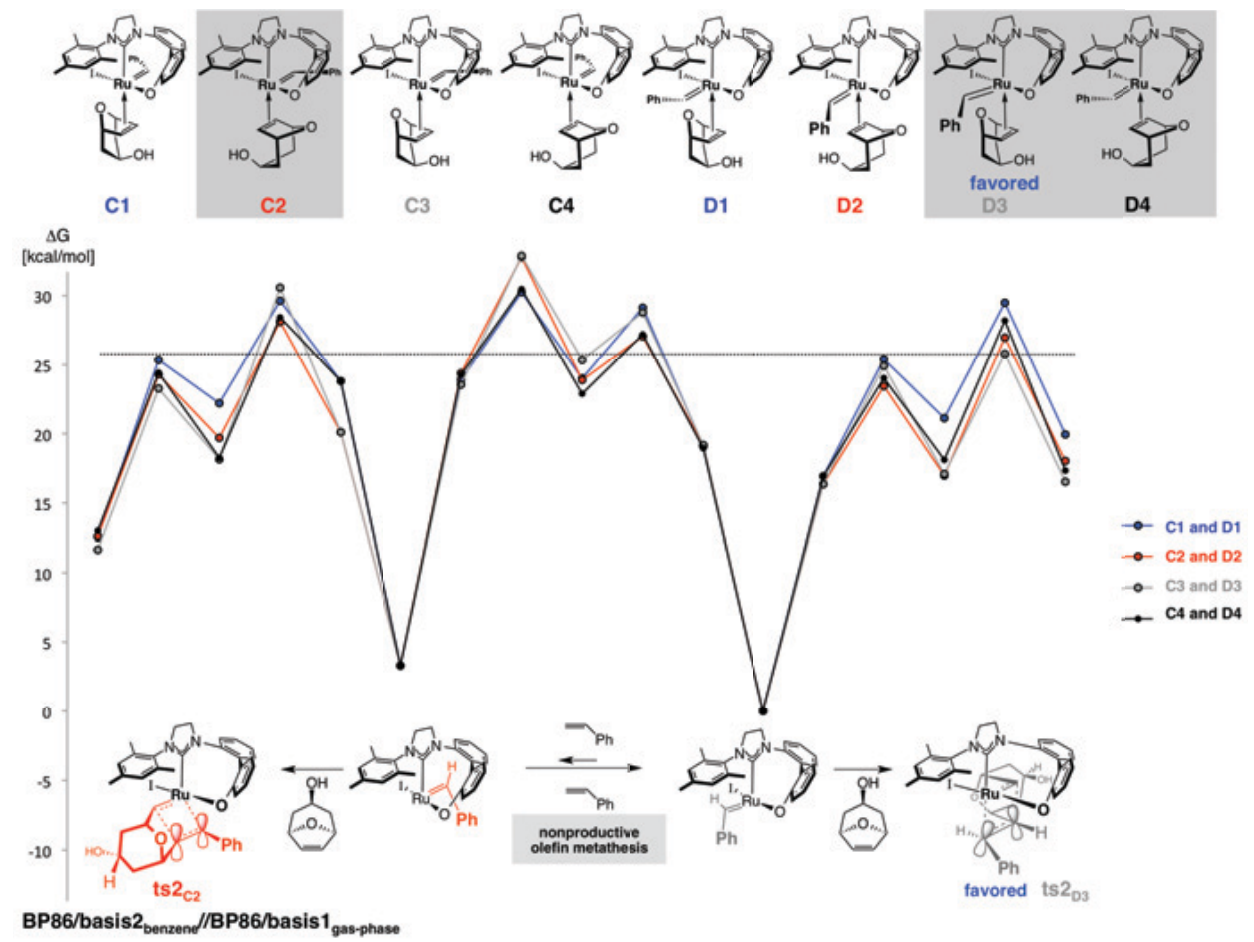

Figure S72. Reaction shown in Figure S5 at the BP86/basis $2_{\text {benzene }} / / \mathrm{BP} 86 / \mathrm{basis} 1_{\text {gas-phase }}$ level 

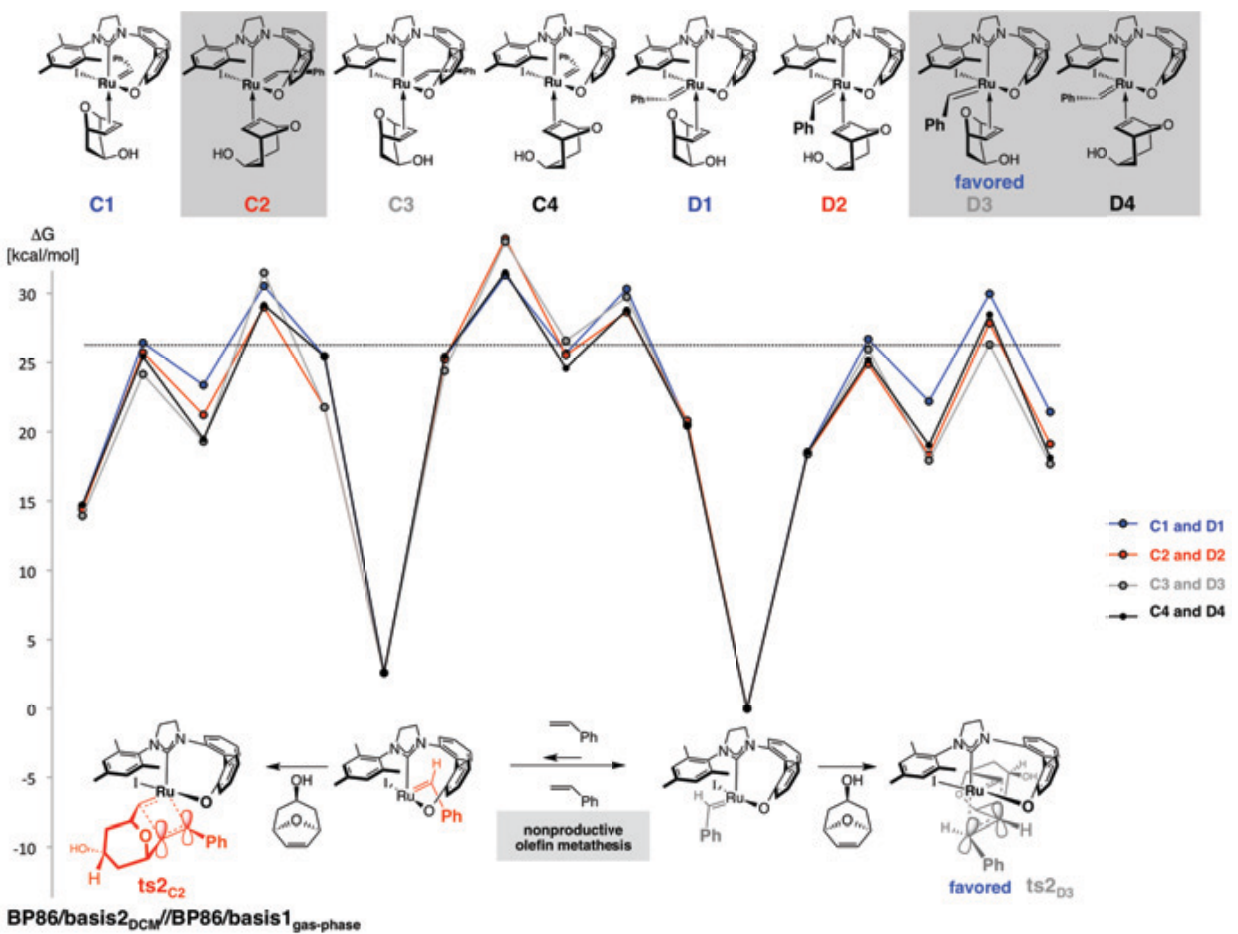

Figure S73. Reaction shown in Figure S5 at the BP86/basis2 $2_{\mathrm{DCM}} / / \mathrm{BP} 86 /$ basis $1_{\text {gas-phase }}$ level 


\section{Pathways C1-D4 in Figure S23 $(\Delta G)$ with $\omega$ B97XD/basis2}

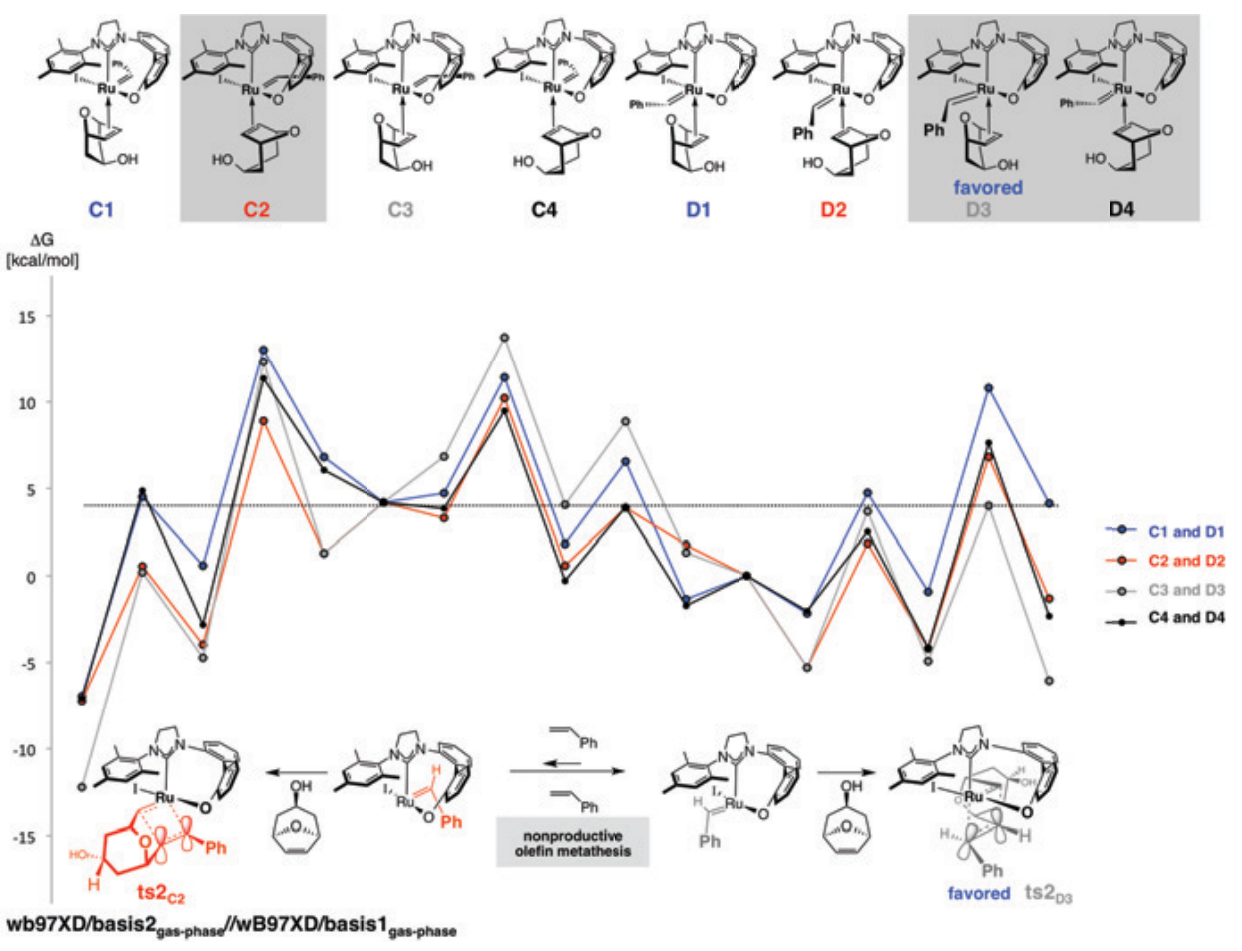

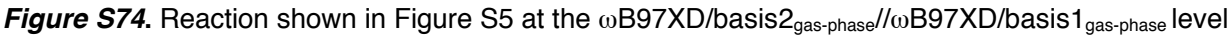
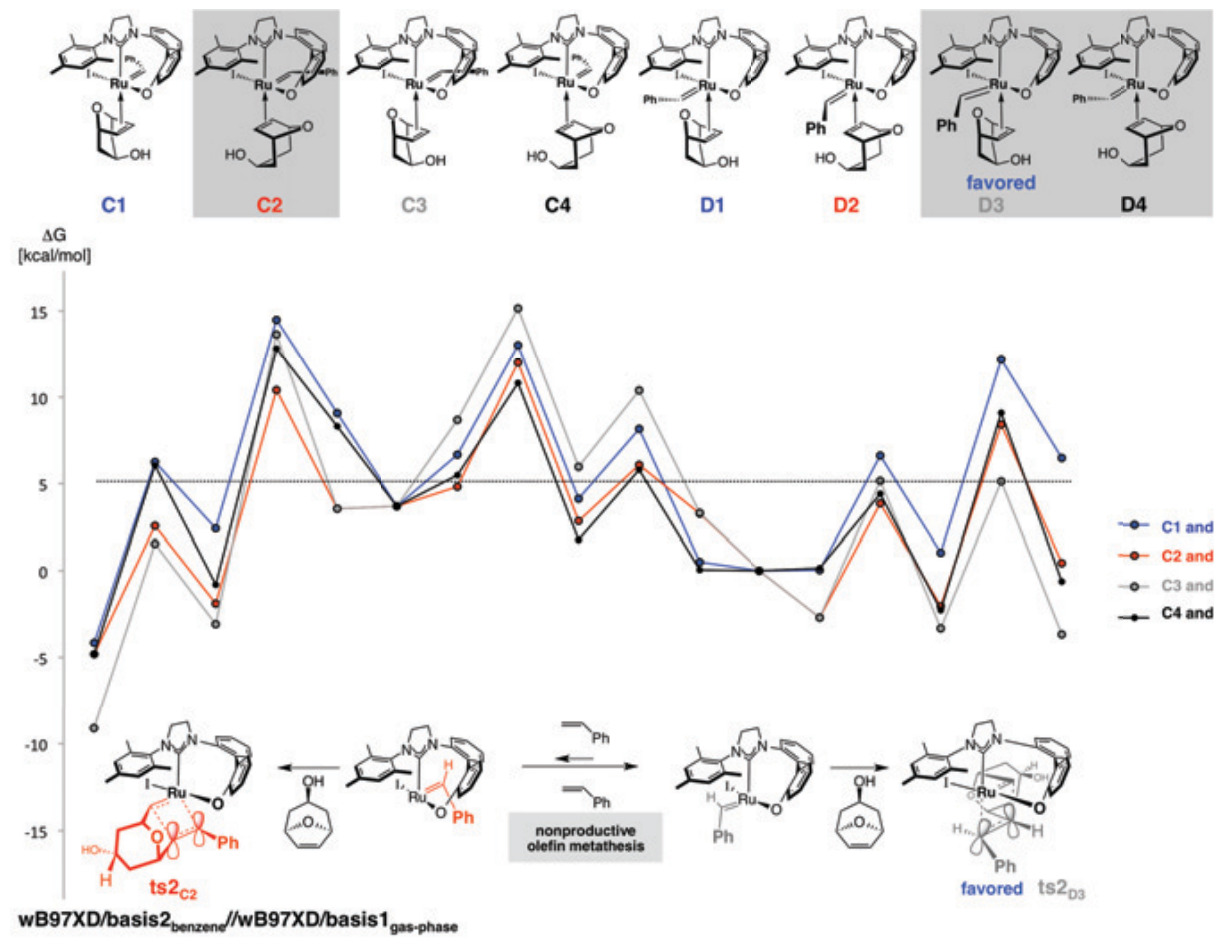

Figure S75. Reaction shown in Figure S5 at the $\omega \mathrm{B} 97 \mathrm{XD} / \mathrm{basis} 2_{\text {benzene }} / / \omega \mathrm{B} 97 \mathrm{XD} / \mathrm{basis} 1_{\text {gas-phase }}$ level 


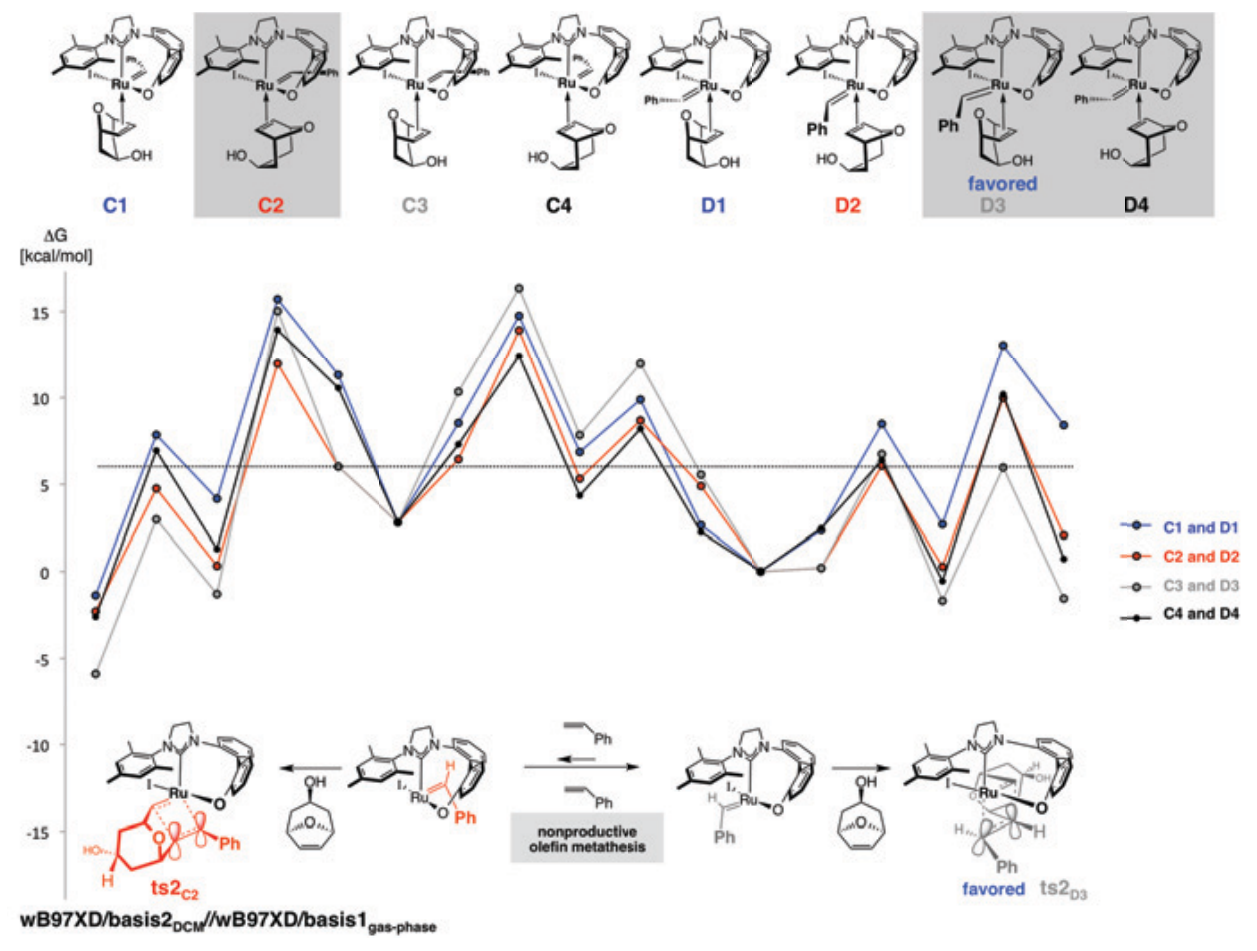

Figure S76. Reaction shown in Figure S5 at the $\omega \mathrm{B} 97 \mathrm{XD} / \mathrm{basis} 2_{\mathrm{DCM}} / / \omega \mathrm{B} 97 \mathrm{XD} / \mathrm{basis} 1_{\text {gas-phase }}$ level 


\section{Pathways C1-D4 in Figure S23 $(\Delta G)$ with M06/basis2}

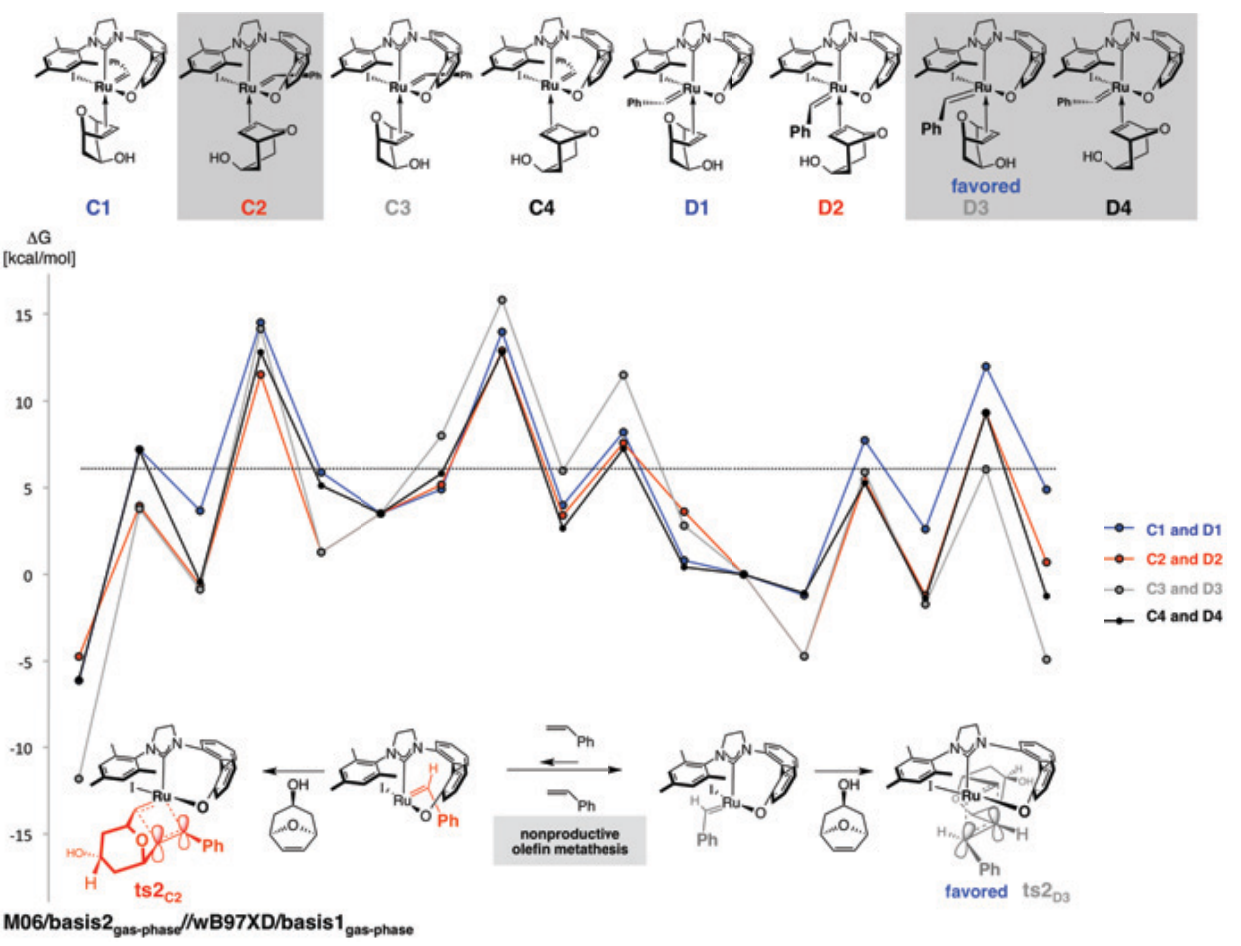

Figure S77. Reaction shown in Figure S5 at the M06/basis $2_{\text {gas-phase }} / / \omega B 97 X D / b a s i s 1_{\text {gas-phase }}$ level
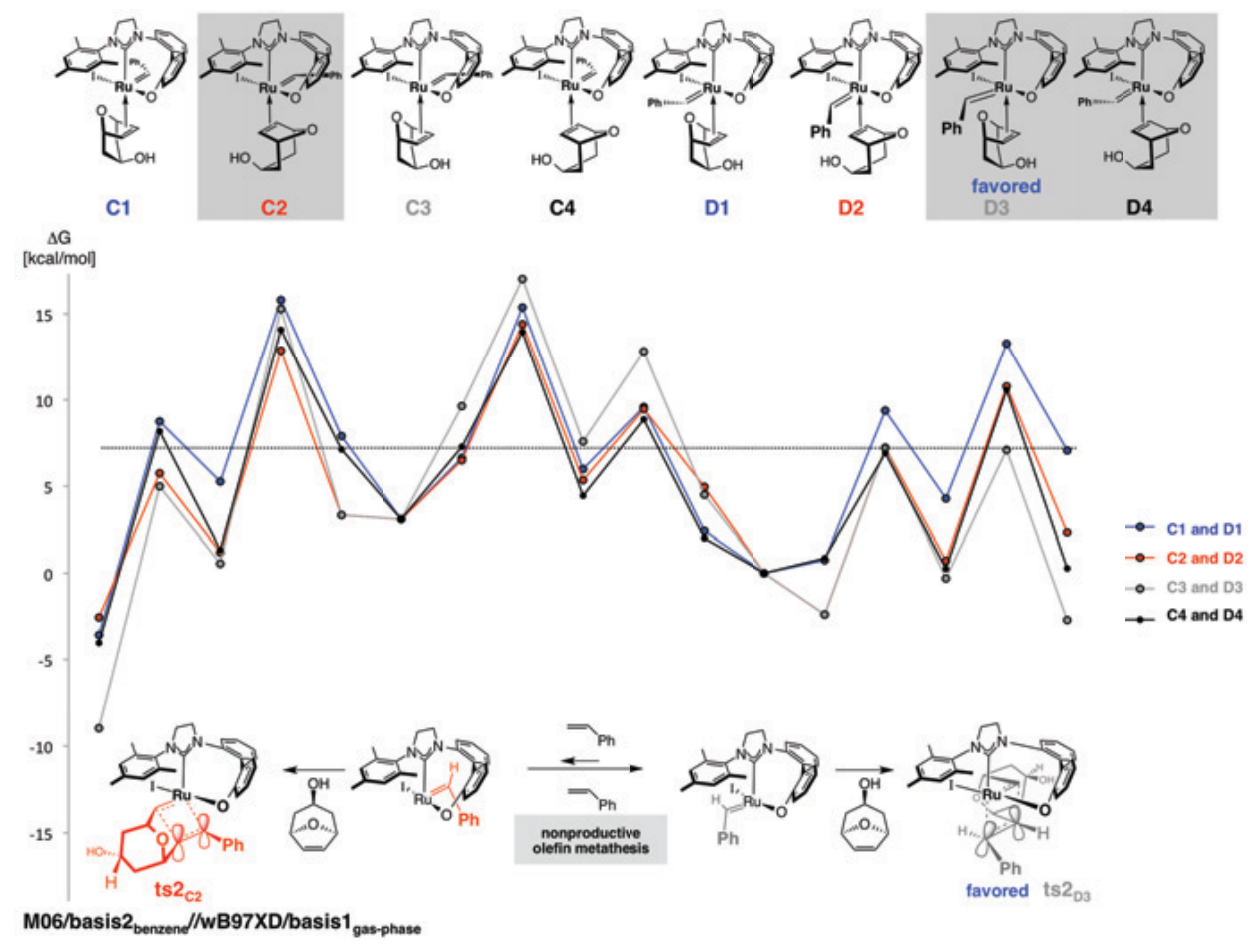

Figure S78. Reaction shown in Figure S5 at the M06/basis $2_{\text {benzene }} / / \omega \mathrm{B} 97 \mathrm{XD} / \mathrm{basis} 1_{\text {gas-phase }}$ level 


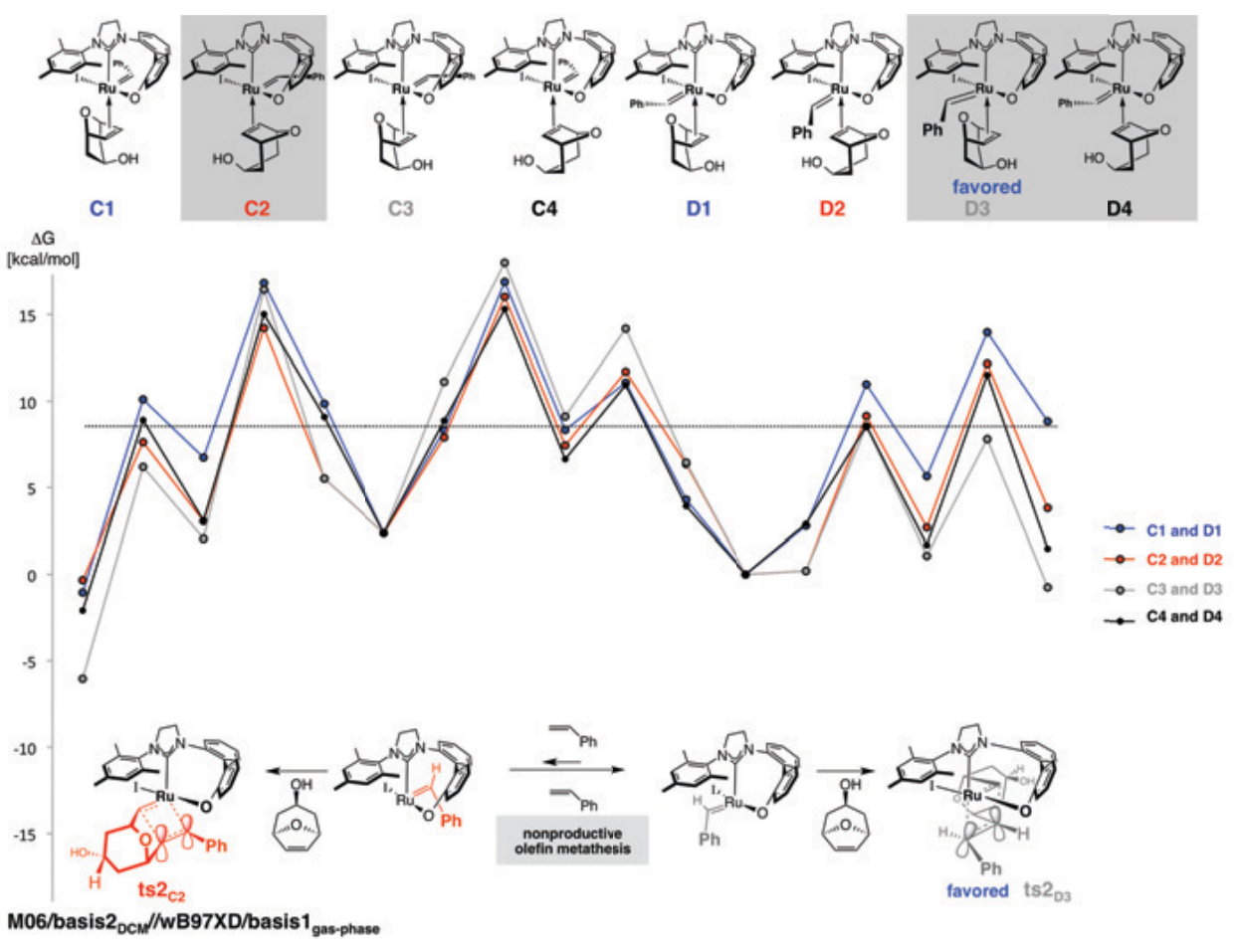

Figure S79. Reaction shown in Figure S5 at the M06/basis $2_{\mathrm{DCM}} / \omega \mathrm{\omega} 97 \mathrm{XD} / \mathrm{basis} 1_{\text {gas-phase }}$ level 


\section{Gibbs Free Energy Surfaces $(\Delta G)$ after optimization with BP86/basis $1_{D C M}$}

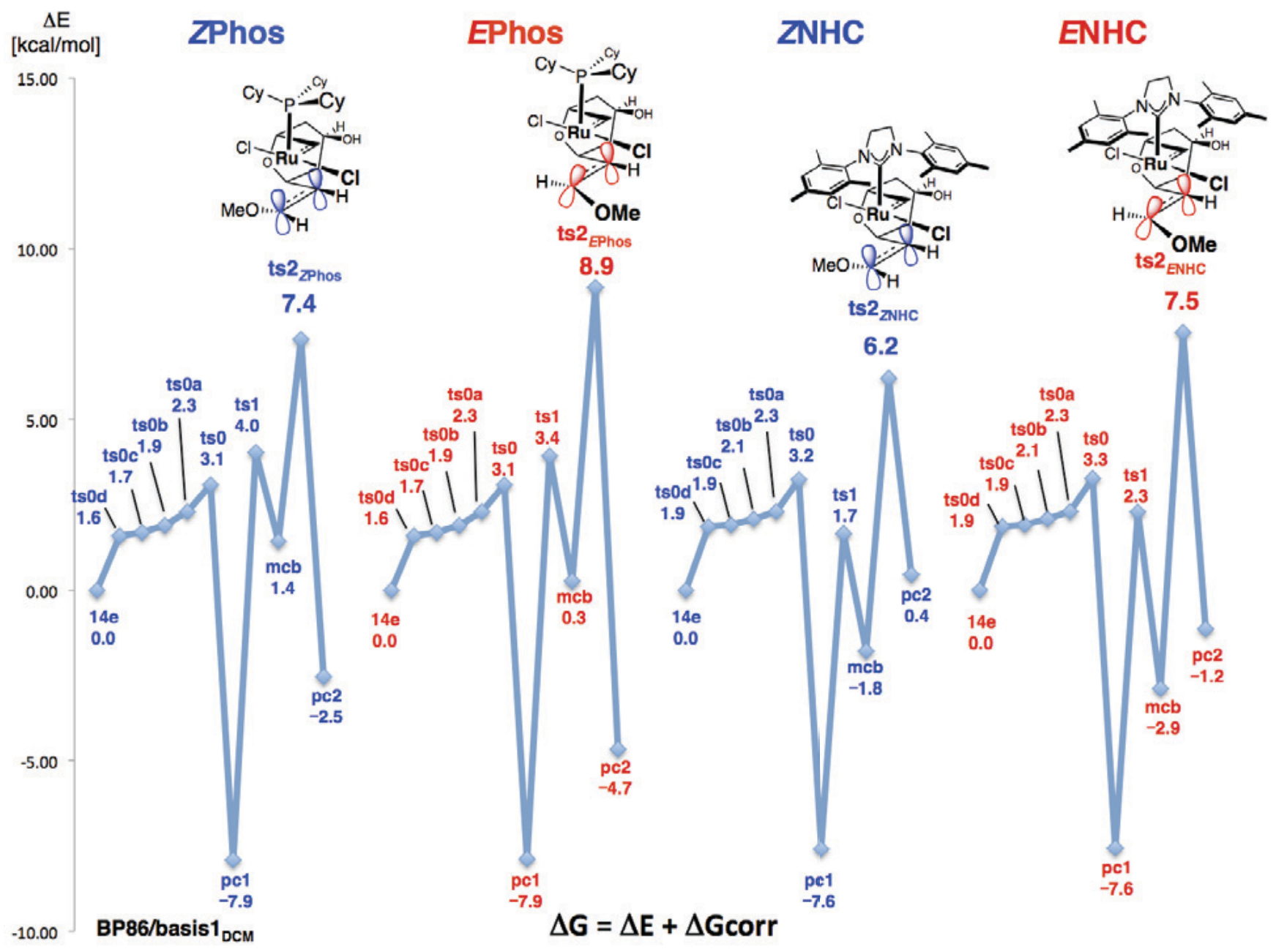

Figure $580 . \Delta \mathrm{E}$ of reaction shown in Figure 3 in manuscript at the BP86/basis $1_{\mathrm{DCM}}$ level 


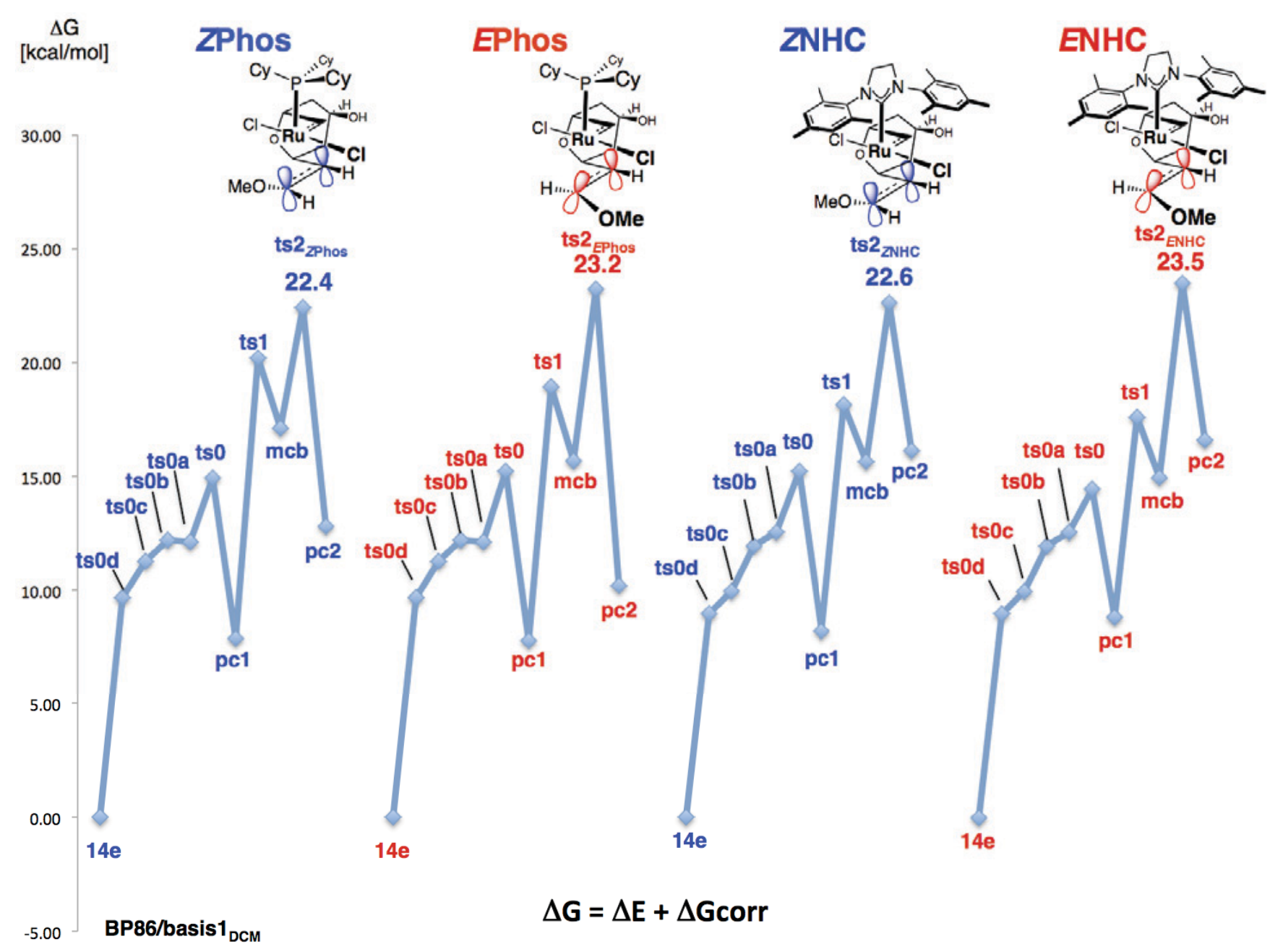

Figure S81. $\Delta \mathrm{G}$ of reaction shown in Figure 3 in manuscript at the BP86/basis $1_{\mathrm{DCM}}$ level 


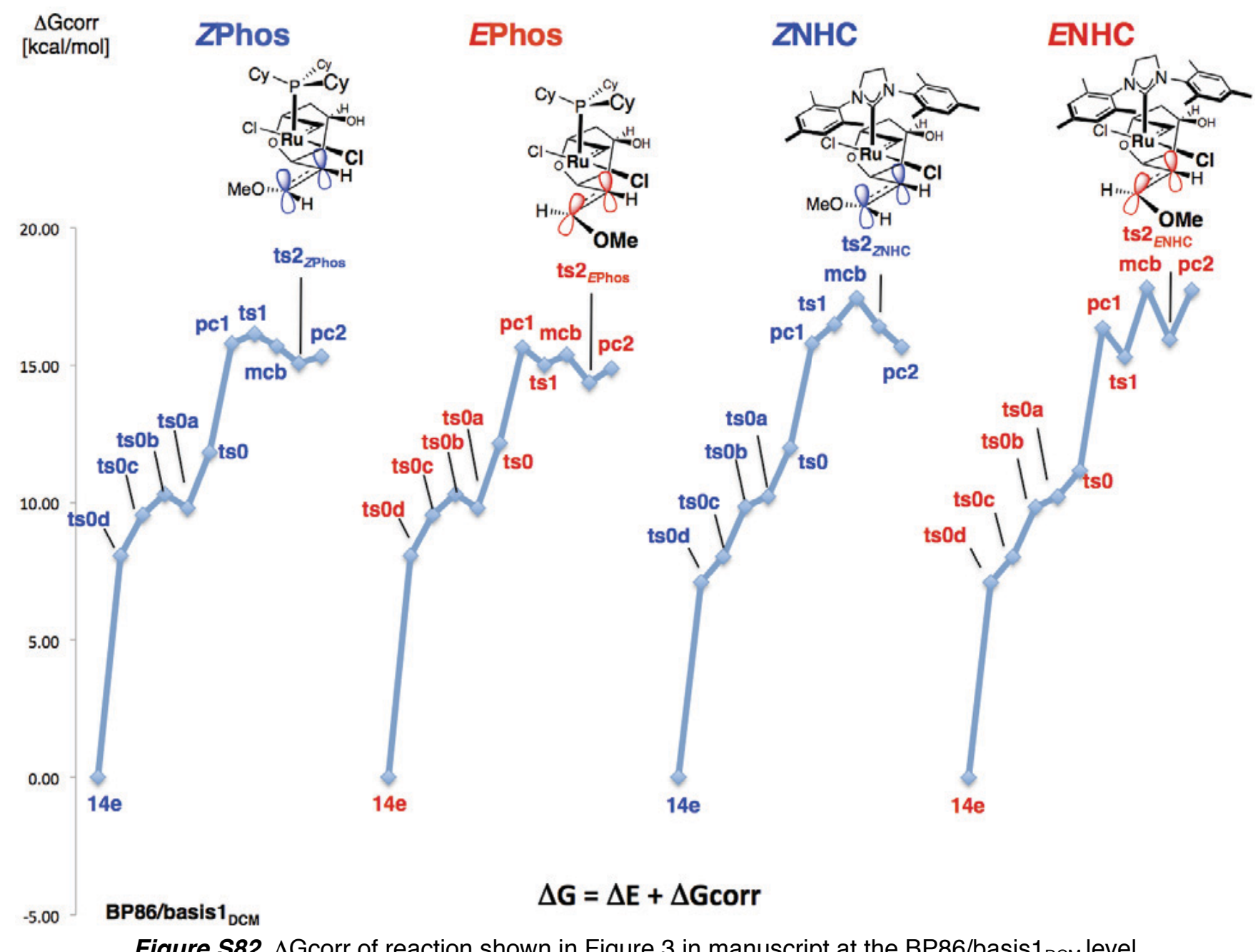

Figure S82. $\Delta$ Gcorr of reaction shown in Figure 3 in manuscript at the BP86/basis $1_{\mathrm{DCM}}$ level 


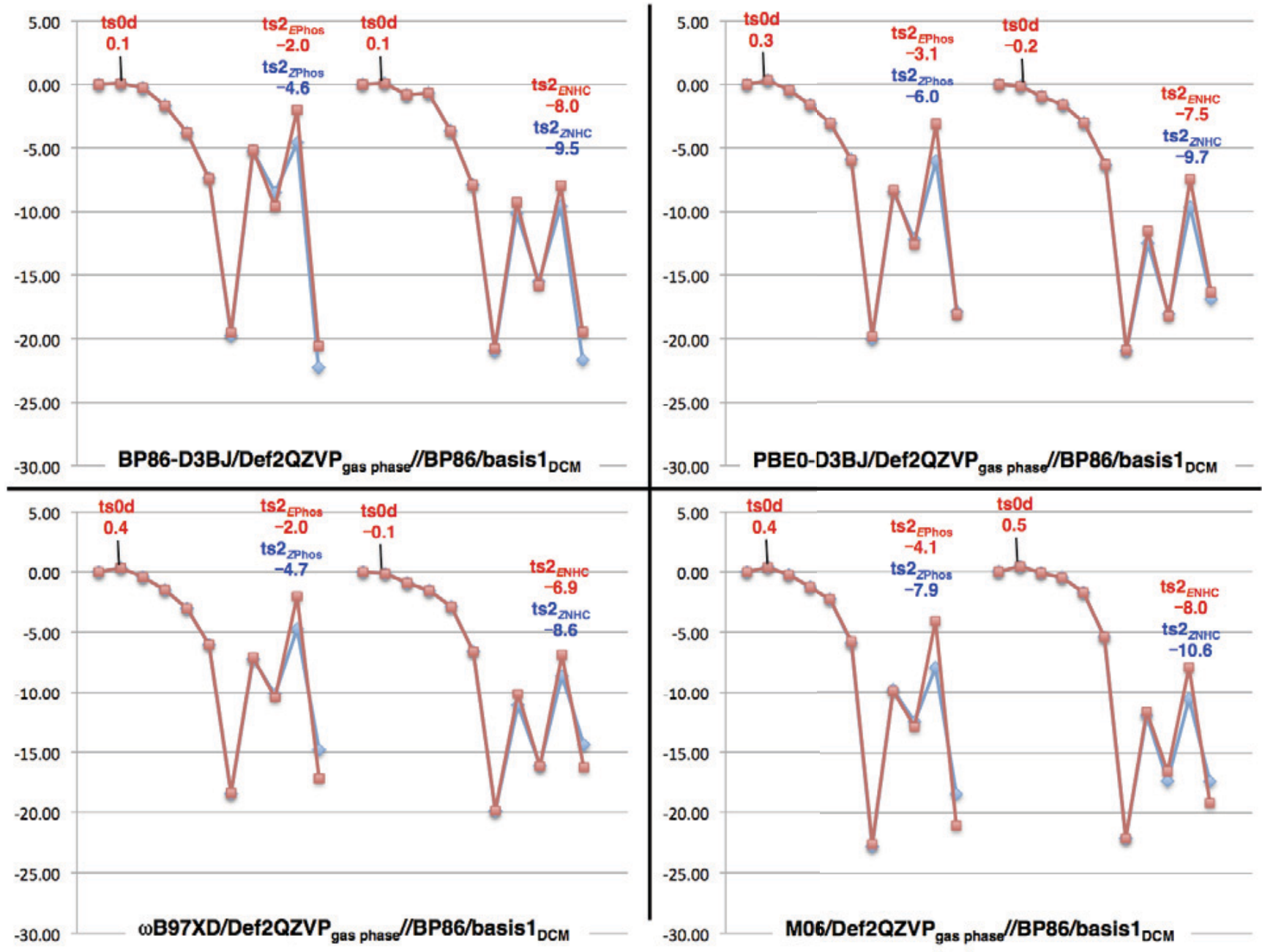

Figure S83. Single point $\Delta \mathrm{E}$ energies for reaction shown in Figure 3 in manuscript with various density functionals in gas phase after optimization with BP86/basis $1_{\mathrm{DCM}}$ 


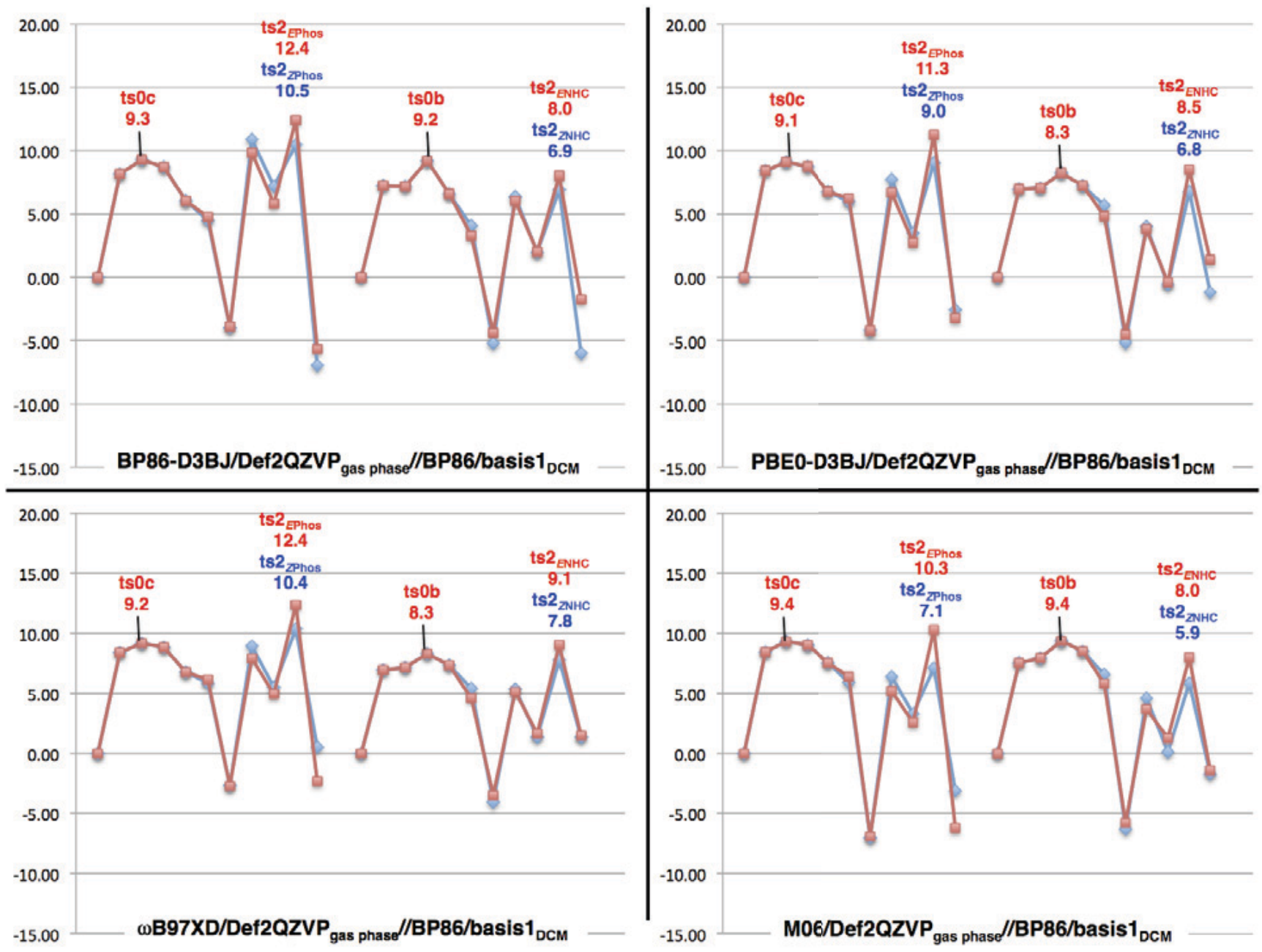

Figure S84. Single point $\Delta \mathrm{G}$ energies for reaction shown in Figure 3 in manuscript with various density functionals in gas phase after optimization with BP86/basis $1_{D C M}$ 

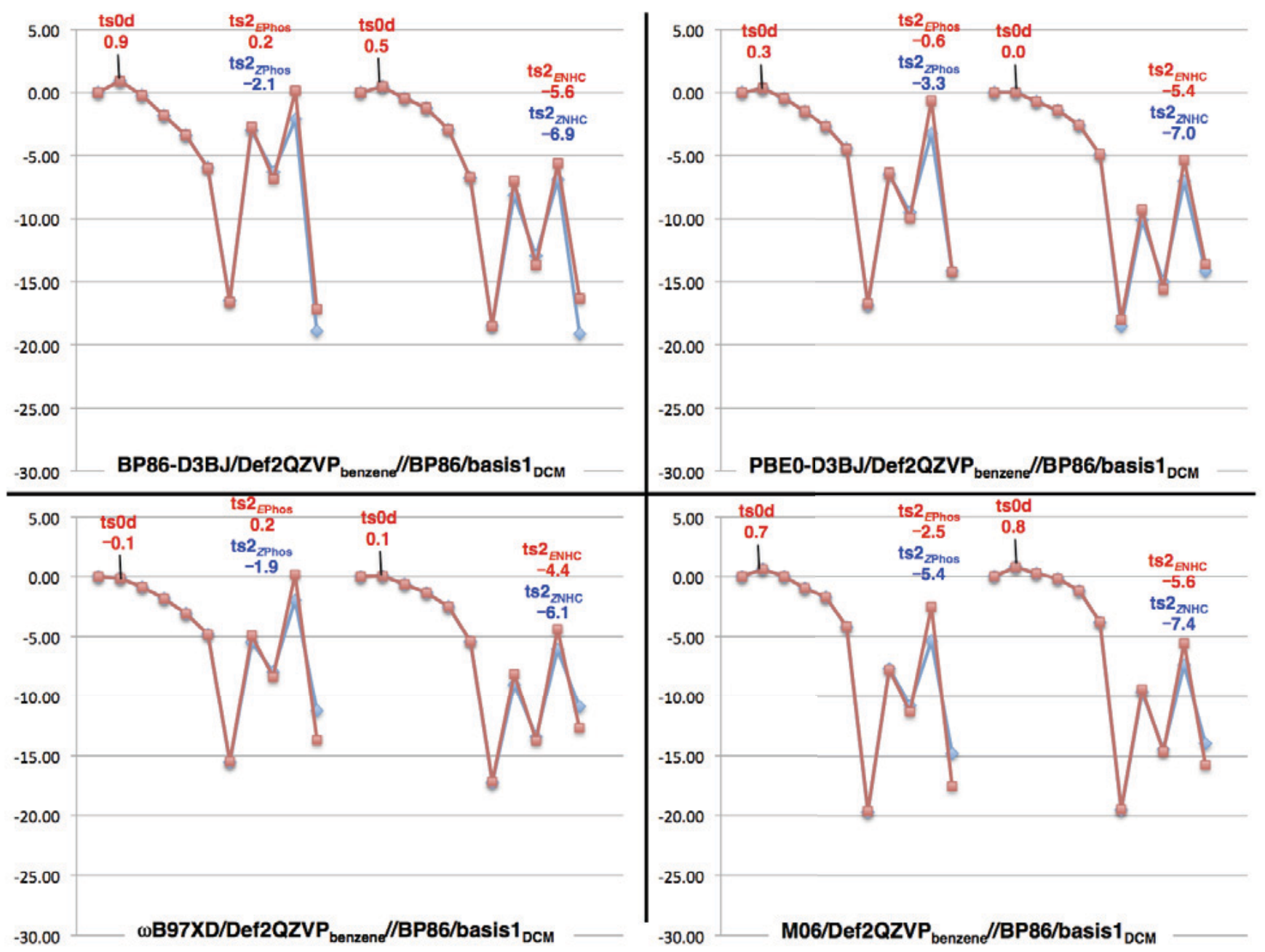

Figure S85. Single point $\Delta \mathrm{E}$ energies for reaction shown in Figure 3 in manuscript with various density functionals in benzene after optimization with BP86/basis $1_{\mathrm{DCM}}$ 


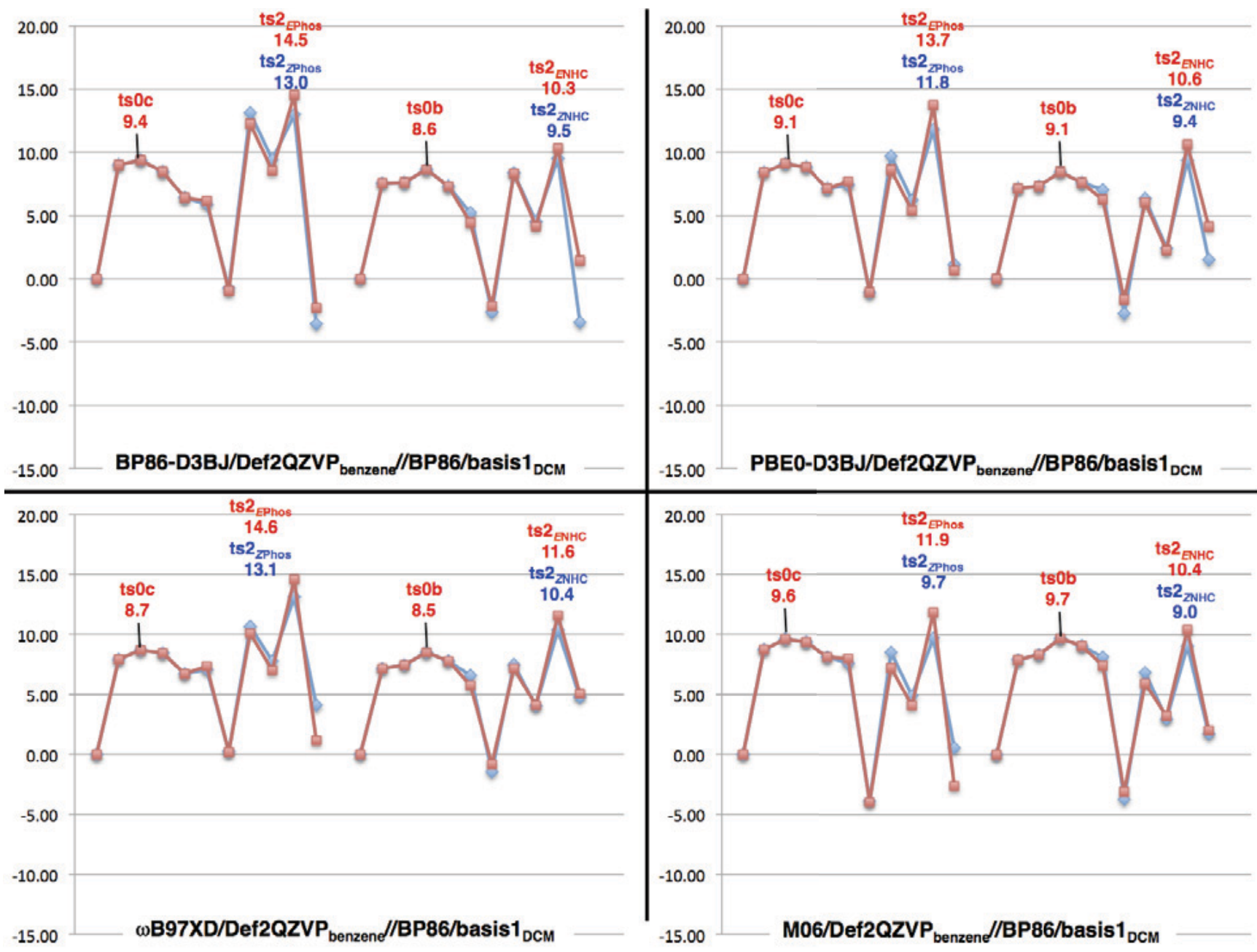

Figure S86. Single point $\Delta \mathrm{G}$ energies for reaction shown in Figure 3 in manuscript with various density functionals in benzene after optimization with BP86/basis $1_{\mathrm{DCM}}$ 

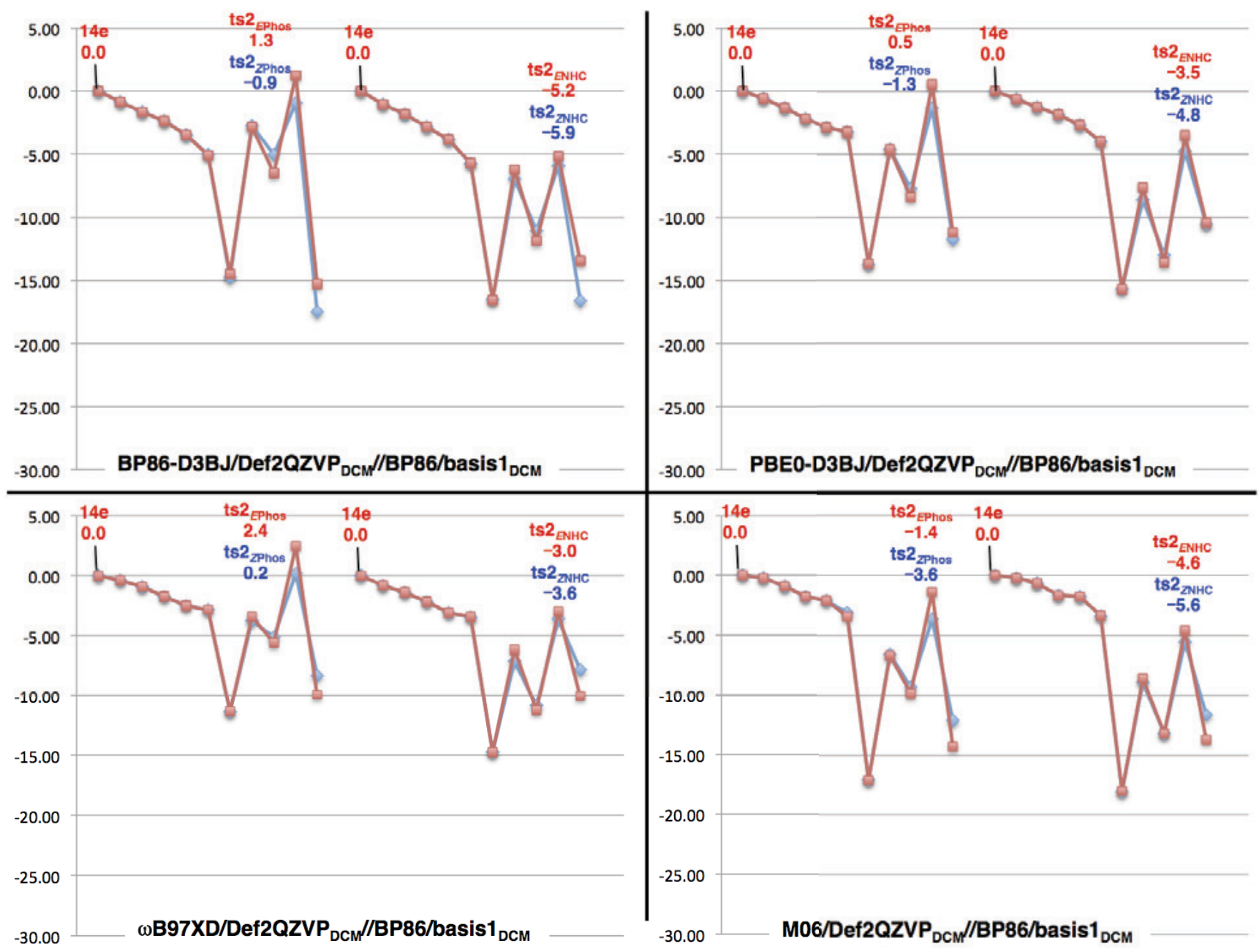

Figure S87. Single point $\Delta \mathrm{E}$ energies for reaction shown in Figure 3 in manuscript with various density functionals in DCM after optimization with BP86/basis $1_{\mathrm{DCM}}$ 


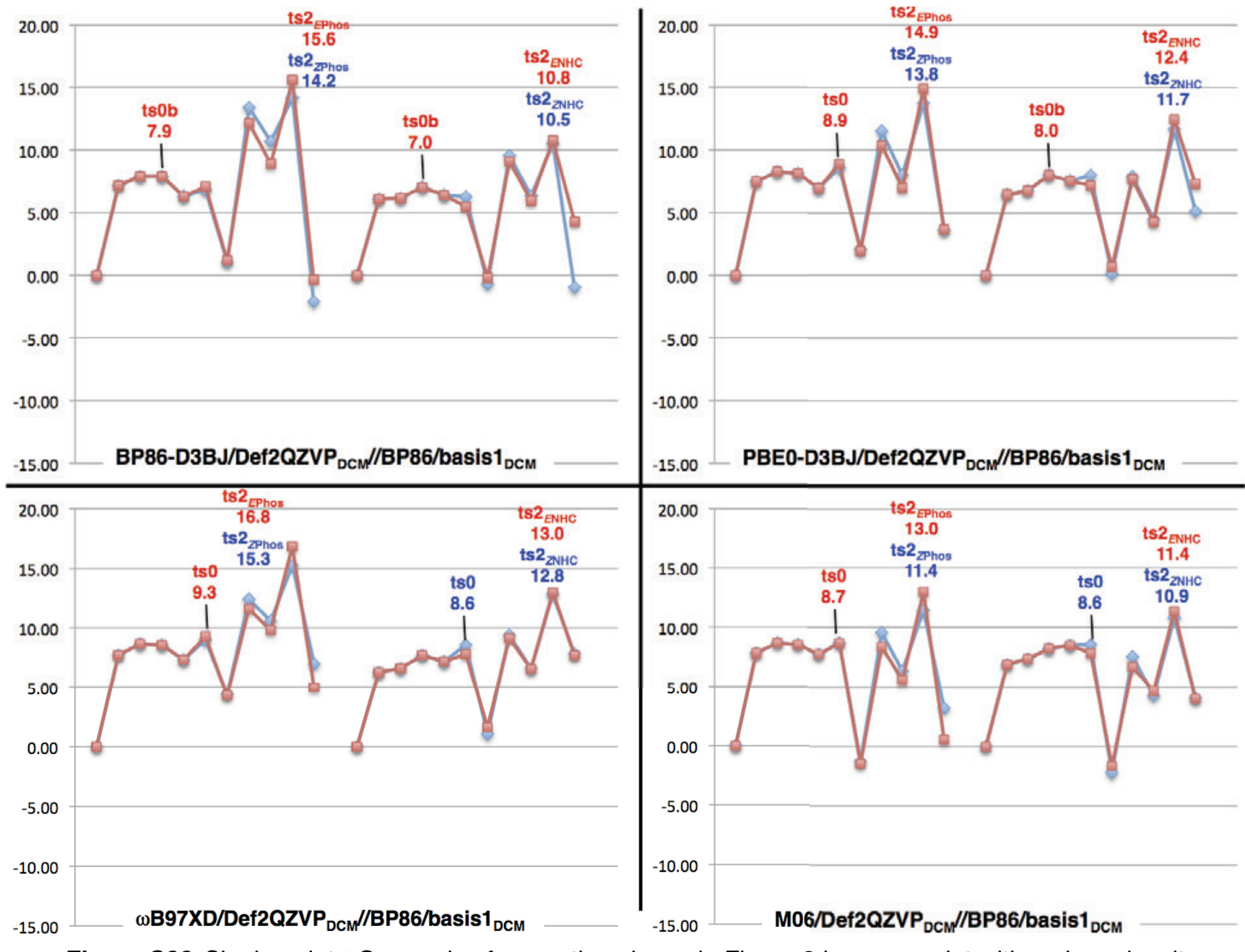

Figure S88. Single point $\Delta \mathrm{G}$ energies for reaction shown in Figure 3 in manuscript with various density functionals in DCM after optimization with BP86/basis $1_{\mathrm{DCM}}$ 
With dispersion
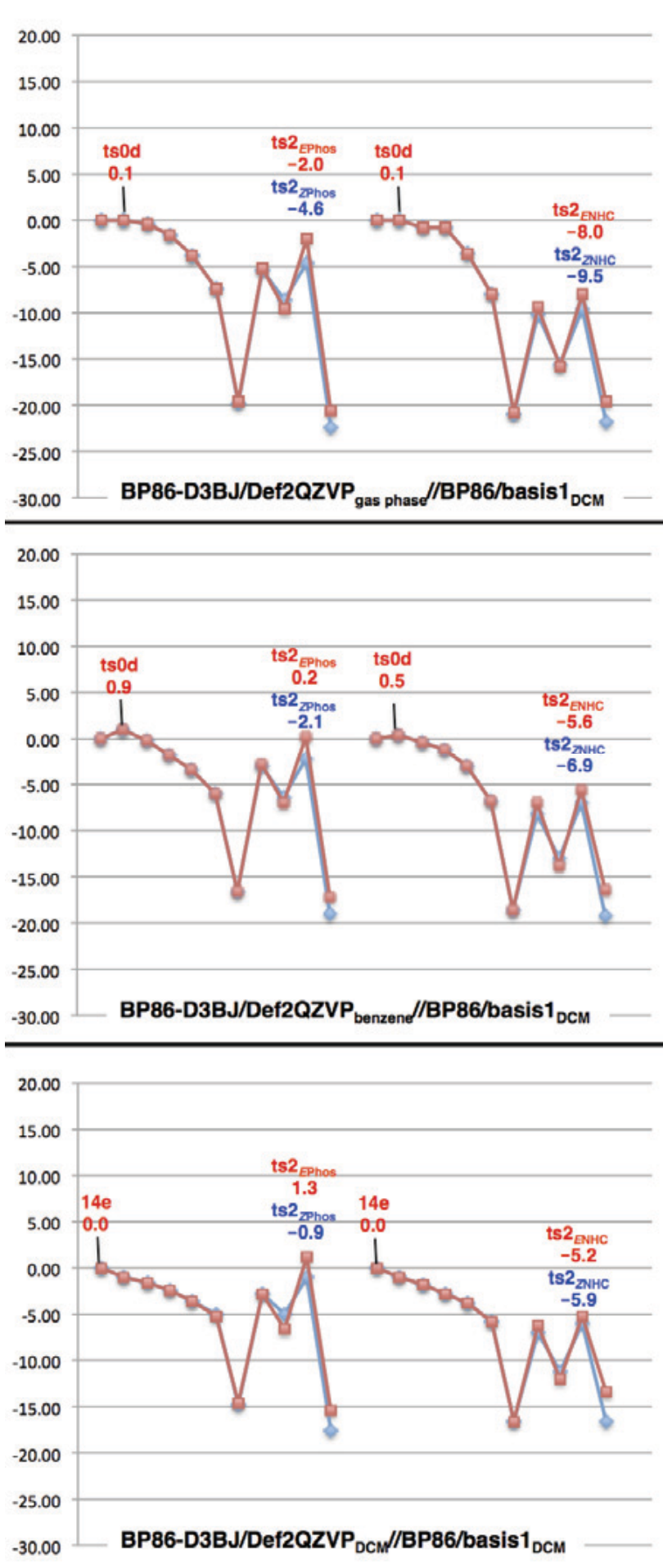

Without dispersion

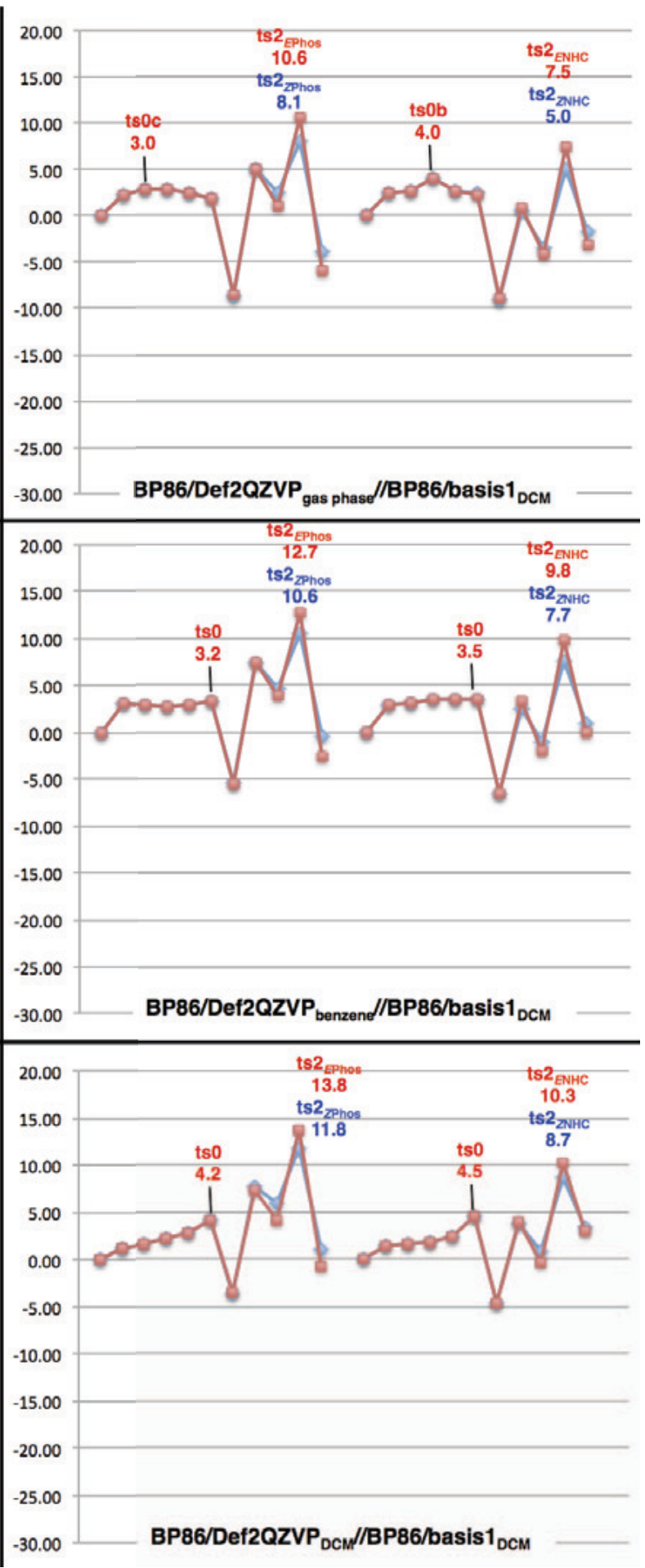

Figure S89. Single point $\triangle \mathrm{E}$ energies for reaction shown in Figure 3 with BP86/Def2QZVP after optimization with BP86/basis1 $1_{\mathrm{DCM}}$; with D3BJ dispersion (left), without D3BJ dispersion (right) 
With dispersion

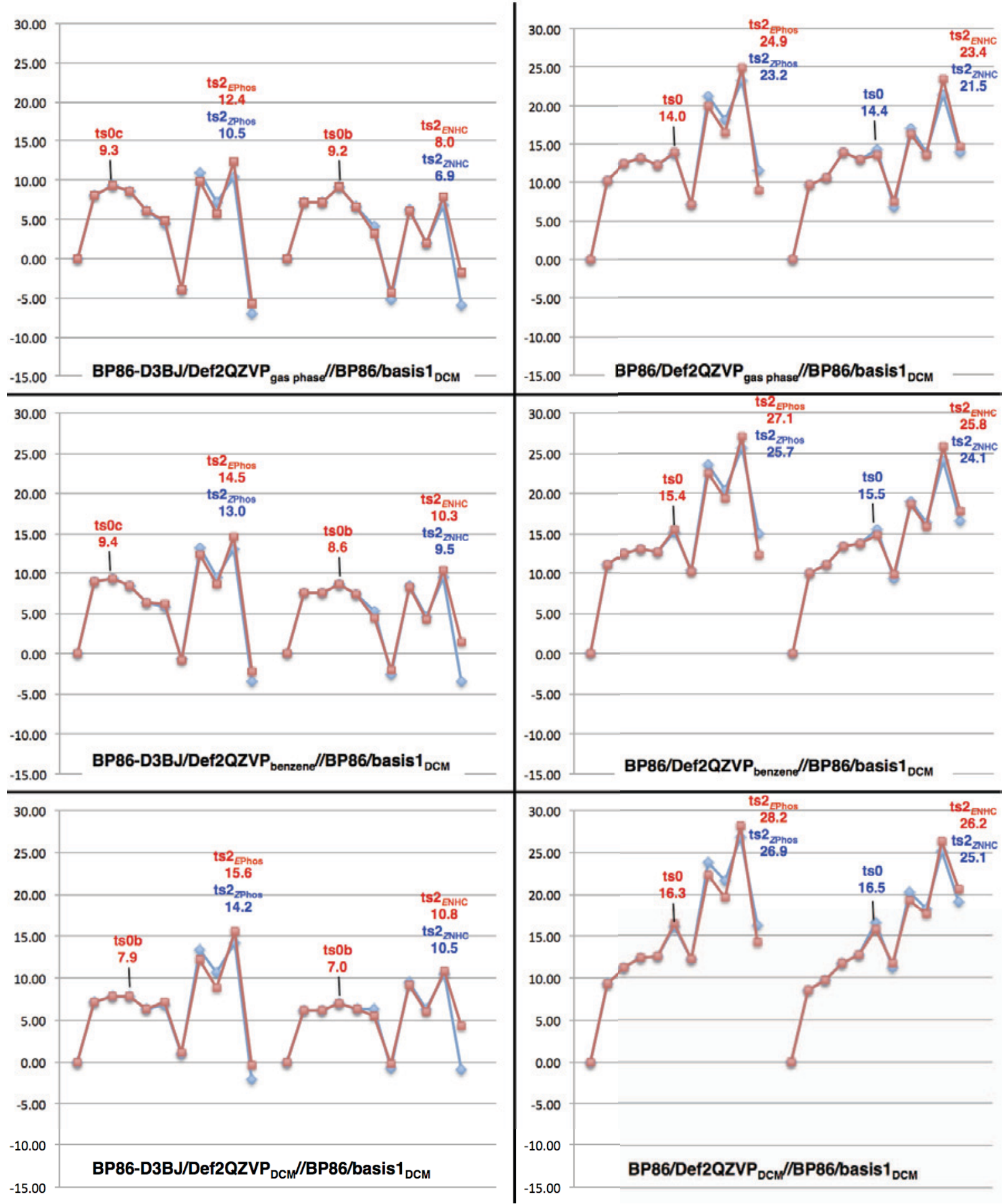

Figure S90. Single point $\Delta G$ energies for reaction shown in Figure 3 with BP86/Def2QZVP after optimization with BP86/basis $1_{\mathrm{DCM}}$; with D3BJ dispersion (left), without D3BJ dispersion (right) 


\section{Energies and Gibbs Free Energies with BP86}

\section{Table S12. ROCM with catalyst Ru-4f and enol ether (pathways EPhos, ZPhos in Figure} 3)

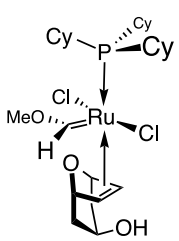

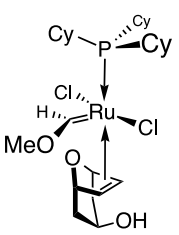

$$
\begin{array}{l|l}
\text { BP86/basis1 (gas-phase) } \\
\hline
\end{array}
$$

\begin{tabular}{|c|c|c|c|c|c|c|}
\hline pathway & & $\begin{array}{c}\mathbf{E} \\
\text { [hartree] }\end{array}$ & $\begin{array}{c}\Delta E \\
{[\mathrm{kcal} / \mathrm{mol}]}\end{array}$ & $\begin{array}{c}\mathbf{G} \\
{[\text { hartree] }}\end{array}$ & $\begin{array}{c}\Delta \mathbf{G} \\
{[\mathrm{kcal} / \mathrm{mol}]}\end{array}$ & $\begin{array}{c}\Delta \mathbf{G}_{\text {Corr }} \\
{[\mathrm{kcal} / \mathbf{m o l}]}\end{array}$ \\
\hline \multirow{6}{*}{ ZPhos } & $14 \mathrm{e}$ & -2639.77240817 & 0.00 & -2639.177766 & 0.00 & 0.00 \\
\hline & pc1 & -2639.79425054 & -13.71 & -2639.175761 & 1.26 & 14.96 \\
\hline & ts1 & -2639.76992353 & 1.56 & -2639.149895 & 17.49 & 15.93 \\
\hline & mcb & -2639.77614246 & -2.34 & -2639.155183 & 14.17 & 16.51 \\
\hline & ts2 & -2639.76746793 & 3.10 & -2639.146014 & 19.92 & 16.82 \\
\hline & pc2 & -2639.78589273 & -8.46 & -2639.165141 & 7.92 & 16.38 \\
\hline \multirow{6}{*}{ EPhos } & $14 e$ & -2639.77240817 & 0.00 & -2639.177766 & 0.00 & 0.00 \\
\hline & pc1 & -2639.79417073 & -13.66 & -2639.175663 & 1.32 & 14.98 \\
\hline & ts1 & -2639.77049151 & 1.20 & -2639.150849 & 16.89 & 15.69 \\
\hline & mcb & -2639.77713016 & -2.96 & -2639.157324 & 12.83 & 15.79 \\
\hline & ts2 & -2639.76358045 & 5.54 & -2639.144177 & 21.08 & 15.54 \\
\hline & pc2 & -2639.79113347 & -11.75 & -2639.168974 & 5.52 & 17.27 \\
\hline
\end{tabular}

BP86/basis2 (gas-phase)

\begin{tabular}{|c|c|c|c|c|c|c|c|c|}
\hline \multicolumn{3}{|c|}{ BP86/basis2 (gas-phase) } & \multicolumn{3}{|c|}{ BP86/basis2 (benzene) } & \multicolumn{3}{|c|}{ BP86/basis2 (dichloromethane) } \\
\hline $\begin{array}{c}\mathbf{E}_{\mathrm{sp}} \\
\text { [hartree] }\end{array}$ & $\begin{array}{c}\Delta \mathrm{E}_{\mathrm{sp}} \\
{[\mathrm{kcal} / \mathrm{mol}]}\end{array}$ & $\begin{array}{c}\Delta \mathbf{G}_{\mathrm{sp}} \\
{[\mathrm{kcal} / \mathrm{mol}]}\end{array}$ & $\begin{array}{c}\mathrm{E}_{\mathrm{sp}} \\
\text { [hartree] }\end{array}$ & $\begin{array}{c}\Delta \mathbf{E}_{\mathrm{sp}} \\
{[\mathrm{kcal} / \mathrm{mol}]}\end{array}$ & $\begin{array}{c}\Delta \mathbf{G}_{\mathrm{sp}} \\
{[\mathrm{kcal} / \mathrm{mol}]}\end{array}$ & $\begin{array}{c}\mathrm{E}_{\mathrm{sp}} \\
\text { [hartree] }\end{array}$ & $\begin{array}{c}\Delta \mathbf{E}_{\mathrm{sp}} \\
{[\mathrm{kcal} / \mathrm{mol}]}\end{array}$ & $\begin{array}{c}\Delta \mathbf{G}_{\mathrm{sp}} \\
{[\mathrm{kcal} / \mathrm{mol}]}\end{array}$ \\
\hline-2640.19729088 & 0.00 & 0.00 & -2640.20982208 & 0.00 & 0.00 & -2640.22277886 & 0.00 & 0.00 \\
\hline-2640.21543413 & -11.39 & 3.58 & -2640.22368132 & -8.70 & 6.27 & -2640.23212053 & -5.86 & 9.10 \\
\hline-2640.19125700 & 3.79 & 19.72 & -2640.20146761 & 5.24 & 21.17 & -2640.21288340 & 6.21 & 22.14 \\
\hline-2640.19590588 & 0.87 & 17.38 & -2640.20492826 & 3.07 & 19.59 & -2640.21482380 & 4.99 & 21.51 \\
\hline-2640.18717450 & 6.35 & 23.17 & -2640.19573921 & 8.84 & 25.66 & -2640.20500489 & 11.15 & 27.98 \\
\hline-2640.20682019 & -5.98 & 10.40 & -2640.21453573 & -2.96 & 13.43 & -2640.22337297 & -0.37 & 16.01 \\
\hline-2640.19729088 & 0.00 & 0.00 & -2640.20982208 & 0.00 & 0.00 & -2640.22277886 & 0.00 & 0.00 \\
\hline-2640.21537220 & -11.35 & 3.63 & -2640.22361256 & -8.65 & 6.32 & -2640.23208671 & -5.84 & 9.14 \\
\hline-2640.19153528 & 3.61 & 19.30 & -2640.20162954 & 5.14 & 20.83 & -2640.21263060 & 6.37 & 22.06 \\
\hline-2640.19719399 & 0.06 & 15.85 & -2640.20715804 & 1.67 & 17.46 & -2640.21794931 & 3.03 & 18.82 \\
\hline-2640.18370945 & 8.52 & 24.06 & -2640.19329331 & 10.37 & 25.91 & -2640.20390511 & 11.84 & 27.38 \\
\hline-2640.21158594 & -8.97 & 8.30 & -2640.21844126 & -5.41 & 11.86 & -2640.22598675 & -2.01 & 15.25 \\
\hline \multicolumn{9}{|c|}{ E .......... gas phase electronic energy in hartree with basis 1} \\
\hline \multicolumn{9}{|c|}{ G ......... sum of electronic and thermal free energies with basis 1} \\
\hline \multicolumn{9}{|c|}{$\Delta \mathrm{E} \ldots \ldots \ldots$ relative electronic energy in $\mathrm{kcal} / \mathrm{mol}$ with basis 1} \\
\hline \multicolumn{9}{|c|}{$\Delta \mathrm{G} \ldots \ldots \ldots$ relative free energy in $\mathrm{kcal} / \mathrm{mol}$ with basis 1} \\
\hline \multicolumn{9}{|c|}{$\Delta \mathrm{G}_{\text {corr }} \ldots$. gas phase thermal correction to free energy in $\mathrm{kcal} / \mathrm{mol}$ obtained with basis $1\left(\Delta \mathrm{G}=\Delta \mathrm{E}+\Delta \mathrm{G}_{\text {corr }}\right)$} \\
\hline \multicolumn{9}{|c|}{$\mathrm{E}_{\mathrm{sp}} \ldots \ldots \ldots$ single point electronic energy in gas phase or solution in hartree with basis 2} \\
\hline \multicolumn{9}{|c|}{$\Delta \mathrm{E}_{\mathrm{sp}} \ldots \ldots$. relative single point electronic energy in gas phase or solution in $\mathrm{kcal} / \mathrm{mol}$ with basis 2} \\
\hline$\Delta \mathrm{G}_{\mathrm{sp}} \ldots \ldots .$. relativ & & & & & & & & \\
\hline
\end{tabular}


Table S13. ROCM with catalyst Ru-4b and enol ether (pathways $E N H C, Z N H C$ in Figure 3)
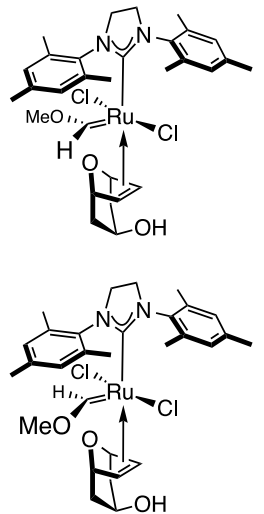

\begin{tabular}{|c|c|c|c|c|c|c|}
\hline \multirow[b]{2}{*}{ pathway } & & \multicolumn{5}{|c|}{ BP86/basis1 (gas-phase) } \\
\hline & & $\begin{array}{c}\mathbf{E} \\
\text { [hartree] }\end{array}$ & $\begin{array}{c}\Delta \mathrm{E} \\
{[\mathrm{kcal} / \mathrm{mol}]}\end{array}$ & $\begin{array}{c}\mathbf{G} \\
\text { [hartree] }\end{array}$ & $\begin{array}{c}\Delta \mathbf{G} \\
{[\mathrm{kcal} / \mathrm{mol}]}\end{array}$ & $\begin{array}{c}\Delta \mathbf{G}_{\mathrm{Corr}} \\
{[\mathrm{kcal} / \mathrm{mol}]}\end{array}$ \\
\hline \multirow{7}{*}{ ZNHC } & $14 \mathrm{e}$ & -2517.98784585 & 0.00 & -2517.460968 & 0.00 & 0.00 \\
\hline & pc1 & -2518.00749476 & -12.33 & -2517.455677 & 3.32 & 15.65 \\
\hline & ts1 & -2517.99027673 & -1.53 & -2517.437713 & 14.59 & 16.12 \\
\hline & mcb & -2517.99780722 & -6.25 & -2517.441199 & 12.41 & 18.66 \\
\hline & ts2 & -2517.98547021 & 1.49 & -2517.431971 & 18.20 & 16.71 \\
\hline & pc2 & -2517.99620732 & -5.25 & -2517.444887 & 10.09 & 15.34 \\
\hline & $14 \mathrm{e}$ & -2517.98784585 & 0.00 & -2517.460968 & 0.00 & 0.00 \\
\hline \multirow{5}{*}{$E \mathrm{NHC}$} & pc1 & -2518.00730369 & -12.21 & -2517.455506 & 3.43 & 15.64 \\
\hline & ts1 & -2517.98936596 & -0.95 & -2517.437502 & 14.73 & 15.68 \\
\hline & mcb & -2517.99870009 & -6.81 & -2517.443930 & 10.69 & 17.50 \\
\hline & ts2 & -2517.98169555 & 3.86 & -2517.429711 & 19.61 & 15.75 \\
\hline & pc2 & -2517.99954868 & -7.34 & -2517.445272 & 9.85 & 17.19 \\
\hline
\end{tabular}

BP86/basis2 (benzene)

\begin{tabular}{|c|c|c|c|c|c|c|c|c|}
\hline \multicolumn{3}{|c|}{ BP86/basis2 (gas-phase) } & \multicolumn{3}{|c|}{ BP86/basis2 (benzene) } & \multicolumn{3}{|c|}{ BP86/basis2 (dichloromethane) } \\
\hline $\begin{array}{c}\mathbf{E}_{\mathrm{sp}} \\
{[\text { hartree] }}\end{array}$ & $\begin{array}{c}\Delta \mathrm{E}_{\mathrm{sp}} \\
{[\mathrm{kcal} / \mathrm{mol}]}\end{array}$ & $\begin{array}{c}\Delta \mathbf{G}_{\mathrm{sp}} \\
{[\mathrm{kcal} / \mathrm{mol}]}\end{array}$ & $\begin{array}{c}\mathbf{E}_{\mathrm{sp}} \\
\text { [hartree] }\end{array}$ & $\begin{array}{c}\Delta \mathbf{E}_{\mathrm{sp}} \\
{[\mathrm{kcal} / \mathrm{mol}]}\end{array}$ & $\begin{array}{c}\Delta \mathbf{G}_{\mathrm{sp}} \\
{[\mathrm{kcal} / \mathrm{mol}]}\end{array}$ & $\begin{array}{c}\mathbf{E}_{\mathrm{sp}} \\
{[\text { hartree] }}\end{array}$ & $\begin{array}{c}\Delta \mathbf{E}_{\mathrm{sp}} \\
{[\mathrm{kcal} / \mathrm{mol}]}\end{array}$ & $\begin{array}{c}\Delta \mathbf{G}_{\mathrm{sp}} \\
{[\mathrm{kcal} / \mathrm{mol}]}\end{array}$ \\
\hline-2518.44138516 & 0.00 & 0.00 & -2518.45551070 & 0.00 & 0.00 & -2518.46985960 & 0.00 & 0.00 \\
\hline-2518.45856804 & -10.78 & 4.87 & -2518.46854979 & -8.18 & 7.47 & -2518.47914512 & -5.83 & 9.82 \\
\hline-2518.44152463 & -0.09 & 16.03 & -2518.45259082 & 1.83 & 17.95 & -2518.46500013 & 3.05 & 19.17 \\
\hline-2518.44753375 & -3.86 & 14.80 & -2518.45776398 & -1.41 & 17.24 & -2518.46908794 & 0.48 & 19.14 \\
\hline-2518.43529446 & 3.82 & 20.53 & -2518.44558321 & 6.23 & 22.93 & -2518.45696475 & 8.09 & 24.80 \\
\hline-2518.44628593 & -3.08 & 12.26 & -2518.45586354 & -0.22 & 15.12 & -2518.46637678 & 2.19 & 17.52 \\
\hline-2518.44138516 & 0.00 & 0.00 & -2518.45551070 & 0.00 & 0.00 & -2518.46985960 & 0.00 & 0.00 \\
\hline-2518.45841146 & -10.68 & 4.95 & -2518.46840350 & -8.09 & 7.55 & -2518.47904206 & -5.76 & 9.88 \\
\hline-2518.44050153 & 0.55 & 16.23 & -2518.45169791 & 2.39 & 18.07 & -2518.46397755 & 3.69 & 19.37 \\
\hline-2518.44866241 & -4.57 & 12.94 & -2518.45958440 & -2.56 & 14.95 & -2518.47139132 & -0.96 & 16.54 \\
\hline-2518.43130135 & 6.33 & 22.08 & -2518.44227400 & 8.31 & 24.06 & -2518.45422587 & 9.81 & 25.57 \\
\hline-2518.44941909 & -5.04 & 12.15 & -2518.45848783 & -1.87 & 15.32 & -2518.46839642 & 0.92 & 18.11 \\
\hline $\begin{array}{l}\mathrm{E} \ldots \ldots \ldots \ldots \text { gas } \\
\mathrm{G} \ldots \ldots \ldots \ldots \text { sum of } \\
\Delta \mathrm{E} \ldots \ldots \ldots \text { relativ } \\
\Delta \mathrm{G} \ldots \ldots \ldots \text { relativ } \\
\Delta \mathrm{G}_{\mathrm{corr}} \ldots \ldots \text { gas } \mathrm{fh} \\
\mathrm{E}_{\mathrm{sp}} \ldots \ldots \ldots \text { single } \\
\Delta \mathrm{E}_{\mathrm{sp}} \ldots \ldots \text { relativ } \\
\Delta \mathrm{G}_{\mathrm{sp}} \ldots \ldots \ldots \text { relativ }\end{array}$ & $\begin{array}{l}\text { electronic en } \\
\text { ectronic and } \\
\text { lectronic ener } \\
\text { tee energy in } \\
\text { thermal corr } \\
\text { int electronic } \\
\text { ingle point el } \\
\text { ingle point fre }\end{array}$ & $\begin{array}{l}\text { gy in hartree } \\
\text { rmal free ene } \\
\text { in kcal/mol } \\
\text { al/mol with b } \\
\text { tion to free en } \\
\text { ergy in gas pl } \\
\text { ronic energy } \\
\text { energy in gas }\end{array}$ & $\begin{array}{l}\text { th basis } 1 \\
\text { ies with basis } 1 \\
\text { h basis } 1 \\
\text { s } 1 \\
\text { gy in } \mathrm{kcal} / \mathrm{mol} \text { obt } \\
\text { e or solution in he } \\
\text { gas phase or solut }\end{array}$ & $\begin{array}{l}\text { ed with basis } \\
\text { in } \mathrm{kcal} / \mathrm{mol} \\
\mathrm{al} / \mathrm{mol}(\Delta \mathrm{G}\end{array}$ & $\begin{array}{l}(\Delta \mathrm{G}=\Delta \mathrm{E}+ \\
\mathrm{h} \text { basis } 2 \\
\mathrm{E}_{\mathrm{sp}} / \mathrm{b} \text { asis } 2+\end{array}$ & corr $/$ basis1) & & \\
\hline
\end{tabular}




\section{Table S14. ROCM with catalyst Ru-4f-model and enol ether (pathways EPhos-model, ZPhos-model)}
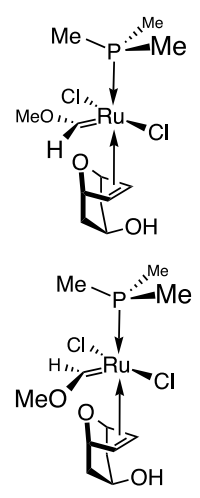

\begin{tabular}{|c|c|c|c|c|c|c|}
\hline & & \multicolumn{5}{|c|}{ BP86/basis1 (gas-phase) } \\
\hline \multicolumn{2}{|l|}{ pathway } & $\begin{array}{c}\mathbf{E} \\
\text { [hartree] }\end{array}$ & $\begin{array}{c}\Delta E \\
{[\mathrm{kcal} / \mathrm{mol}]}\end{array}$ & $\begin{array}{c}\mathbf{G} \\
{[\text { hartree] }}\end{array}$ & $\begin{array}{c}\Delta \mathbf{G} \\
{[\mathrm{kcal} / \mathrm{mol}]}\end{array}$ & $\begin{array}{c}\Delta \mathbf{G}_{\mathrm{Corr}} \\
{[\mathrm{kcal} / \mathrm{mol}]}\end{array}$ \\
\hline \multirow{6}{*}{ ZPhos-model } & $14 e$ & -2053.71121620 & 0.00 & -2053.460699 & 0.00 & 0.00 \\
\hline & pc1 & -2053.73640280 & -15.80 & -2053.461233 & -0.34 & 15.47 \\
\hline & ts1 & -2053.70607137 & 3.23 & -2053.429967 & 19.28 & 16.06 \\
\hline & mcb & -2053.71107885 & 0.09 & -2053.433545 & 17.04 & 16.95 \\
\hline & ts2 & -2053.70266921 & 5.36 & -2053.425591 & 22.03 & 16.67 \\
\hline & pc2 & -2053.72828460 & -10.71 & -2053.451393 & 5.84 & 16.55 \\
\hline \multirow{6}{*}{ EPhos-model } & $14 \mathrm{e}$ & -2053.71121620 & 0.00 & -2053.460699 & 0.00 & 0.00 \\
\hline & pc1 & -2053.73633423 & -15.76 & -2053.460674 & 0.02 & 15.78 \\
\hline & ts1 & -2053.70606778 & 3.23 & -2053.429785 & 19.40 & 16.17 \\
\hline & mcb & -2053.71205055 & -0.52 & -2053.434254 & 16.59 & 17.12 \\
\hline & ts2 & -2053.69956080 & 7.31 & -2053.423768 & 23.17 & 15.86 \\
\hline & pc2 & -2053.73261676 & -13.43 & -2053.455820 & 3.06 & 16.49 \\
\hline
\end{tabular}

BP86/basis2 (gas-phase)

\begin{tabular}{|c|c|c|c|c|c|c|c|c|}
\hline \multicolumn{3}{|c|}{ BP86/basis2 (gas-phase) } & \multicolumn{3}{|c|}{ BP86/basis2 (benzene) } & \multicolumn{3}{|c|}{ BP86/basis2 (dichloromethane) } \\
\hline $\begin{array}{c}\mathbf{E}_{\mathrm{sp}} \\
{[\text { hartree] }}\end{array}$ & $\begin{array}{c}\Delta \mathbf{E}_{\mathrm{sp}} \\
{[\mathrm{kcal} / \mathrm{mol}]}\end{array}$ & $\begin{array}{c}\Delta \mathbf{G}_{\mathrm{sp}} \\
{[\mathrm{kcal} / \mathrm{mol}]}\end{array}$ & $\begin{array}{c}\mathbf{E}_{\mathbf{s p}} \\
{[\text { hartree] }}\end{array}$ & $\begin{array}{c}\Delta \mathbf{E}_{\mathrm{sp}} \\
{[\mathrm{kcal} / \mathrm{mol}]}\end{array}$ & $\begin{array}{c}\Delta \mathbf{G}_{\mathrm{sp}} \\
{[\mathrm{kcal} / \mathrm{mol}]}\end{array}$ & $\begin{array}{c}\mathbf{E}_{\mathrm{sp}} \\
\text { [hartree] }\end{array}$ & $\begin{array}{c}\Delta \mathbf{E}_{\mathrm{sp}} \\
{[\mathrm{kcal} / \mathrm{mol}]}\end{array}$ & $\begin{array}{c}\Delta \mathbf{G}_{\mathrm{sp}} \\
{[\mathrm{kcal} / \mathrm{mol}]}\end{array}$ \\
\hline-2053.99966132 & 0.00 & 0.00 & -2054.01405885 & 0.00 & 0.00 & -2054.02827376 & 0.00 & 0.00 \\
\hline-2054.02096791 & -13.37 & 2.10 & -2054.03054867 & -10.35 & 5.12 & -2054.04003905 & -7.38 & 8.09 \\
\hline-2053.99069782 & 5.62 & 21.68 & -2054.00215742 & 7.47 & 23.52 & -2054.01426352 & 8.79 & 24.85 \\
\hline -2053.99449966 & 3.24 & 20.19 & -2054.00536555 & 5.46 & 22.41 & -2054.01663674 & 7.30 & 24.26 \\
\hline-2053.98608446 & 8.52 & 25.19 & -2053.99653799 & 10.99 & 27.66 & -2054.00772998 & 12.89 & 29.56 \\
\hline-2054.01321497 & -8.51 & 8.05 & -2054.02142005 & -4.62 & 11.93 & -2054.03024143 & -1.23 & 15.32 \\
\hline-2053.99966132 & 0.00 & 0.00 & -2054.01405885 & 0.00 & 0.00 & -2054.02827376 & 0.00 & 0.00 \\
\hline-2054.02093501 & -13.35 & 2.43 & -2054.03048840 & -10.31 & 5.47 & -2054.03999422 & -7.35 & 8.42 \\
\hline-2053.99037218 & 5.83 & 22.00 & -2054.00192779 & 7.61 & 23.78 & -2054.01374376 & 9.12 & 25.29 \\
\hline-2053.99556139 & 2.57 & 19.69 & -2054.00712925 & 4.35 & 21.47 & -2054.01883159 & 5.93 & 23.04 \\
\hline-2053.98324078 & 10.30 & 26.16 & -2053.99398014 & 12.60 & 28.46 & -2054.00514285 & 14.51 & 30.38 \\
\hline-2054.01681894 & -10.77 & 5.72 & -2054.02461882 & -6.63 & 9.86 & -2054.03274964 & -2.81 & 13.68 \\
\hline $\begin{array}{l}\mathrm{E} \ldots \ldots \ldots \ldots \text { gas } \\
\mathrm{G} \ldots \ldots \ldots \text { sum of } \\
\Delta \mathrm{E} \ldots \ldots \ldots \text { relativ } \\
\Delta \mathrm{G} \ldots \ldots \ldots \text { relative } \\
\Delta \mathrm{G}_{\mathrm{cor}} \ldots \ldots \text { gas ph} \\
\mathrm{E}_{\mathrm{sp}} \ldots \ldots \ldots \text { single } \\
\Delta \mathrm{E}_{\mathrm{sp}} \ldots \ldots \text { relativ } \\
\Delta \mathrm{G}_{\mathrm{sp}} \ldots \ldots \text { relativ }\end{array}$ & $\begin{array}{l}\text { electronic en } \\
\text { ectronic and } t \\
\text { lectronic ener } \\
\text { ree energy in } \\
\text { thermal corr } \\
\text { int electronic } \\
\text { ingle point ele } \\
\text { ingle point fre }\end{array}$ & $\begin{array}{l}\text { gy in hartree } \\
\text { rmal free ene } \\
\text { in kcal/mol } \\
\text { al } / \mathrm{mol} \text { with b } \\
\text { ion to free en } \\
\text { ergy in gas pl } \\
\text { ronic energy } \\
\text { energy in gas }\end{array}$ & $\begin{array}{l}\text { ith basis } 1 \\
\text { ies with basis } 1 \\
\text { th basis } 1 \\
\text { is } 1 \\
\text { gy in } \mathrm{kcal} / \mathrm{mol} \text { obta } \\
\text { se or solution in ha } \\
\text { gas phase or soluti } \\
\text { hase or solution in }\end{array}$ & $\begin{array}{l}\text { ed with basis } \\
\text { ee with basis } 2 \\
\text { in } \mathrm{kcal} / \mathrm{mol} \\
\mathrm{al} / \mathrm{mol}\left(\Delta \mathrm{G}_{\mathrm{sp}}\right.\end{array}$ & $\begin{array}{l}\Delta \mathrm{G}=\Delta \mathrm{E}+ \\
\mathrm{h} \text { basis } 2 \\
\mathrm{E}_{\mathrm{sp}} / \text { basis } 2+\end{array}$ & corr) & & \\
\hline
\end{tabular}




\section{Table S15. ROCM with catalyst Ru-4b-model and enol ether (pathways ENHC-model, ZNHC-model)}

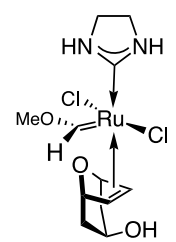<smiles>CO[C@H](Cl)C(Cl)(Cl)C1NCCN1</smiles>

\begin{tabular}{|c|c|c|c|c|c|c|}
\hline & & \multicolumn{5}{|c|}{ BP86/basis1 (gas-phase) } \\
\hline \multicolumn{2}{|l|}{ pathway } & $\begin{array}{c}\mathbf{E} \\
{[\text { hartree] }}\end{array}$ & $\begin{array}{c}\Delta \mathrm{E} \\
{[\mathrm{kcal} / \mathrm{mol}]}\end{array}$ & $\begin{array}{c}\mathbf{G} \\
{[\text { hartree] }}\end{array}$ & $\begin{array}{c}\Delta \mathbf{G} \\
{[\mathrm{kcal} / \mathrm{mol}]}\end{array}$ & $\begin{array}{c}\Delta \mathbf{G}_{\text {Corr }} \\
{[\mathrm{kcal} / \mathrm{mol}]}\end{array}$ \\
\hline \multirow{7}{*}{ ZNHC-model } & $14 \mathrm{e}$ & -1820.01906357 & 0.00 & -1819.782908 & 0.00 & 0.00 \\
\hline & pc1 & -1820.04555317 & -16.62 & -1819.785885 & -1.87 & 14.75 \\
\hline & ts1 & -1820.01675250 & 1.45 & -1819.755640 & 17.11 & 15.66 \\
\hline & mcb & -1820.02310421 & -2.54 & -1819.759751 & 14.53 & 17.07 \\
\hline & ts2 & -1820.01275098 & 3.96 & -1819.750611 & 20.27 & 16.31 \\
\hline & pc2 & -1820.03468190 & -9.80 & -1819.772919 & 6.27 & 16.07 \\
\hline & $14 \mathrm{e}$ & -1820.01906357 & 0.00 & -1819.782908 & 0.00 & 0.00 \\
\hline \multirow{5}{*}{ ENHC-model } & pc1 & -1820.04555318 & -16.62 & -1819.785884 & -1.87 & 14.76 \\
\hline & ts1 & -1820.01609923 & 1.86 & -1819.755043 & 17.49 & 15.63 \\
\hline & mcb & -1820.02512205 & -3.80 & -1819.761829 & 13.23 & 17.03 \\
\hline & ts2 & -1820.00974114 & 5.85 & -1819.749995 & 20.65 & 14.80 \\
\hline & pc2 & -1820.03950373 & -12.83 & -1819.777746 & 3.24 & 16.07 \\
\hline
\end{tabular}

BP86/basis2 (gas-phase)

BP86/basis2 (benzene)

\begin{tabular}{|c|c|c|c|c|c|c|c|c|}
\hline \multicolumn{3}{|c|}{ BP86/Dasisz (gas-pnase) } & \multicolumn{3}{|c|}{ BPdo/Dasisz (benzene) } & \multicolumn{3}{|c|}{ BPso/Dasis2 (acniorometnane) } \\
\hline $\begin{array}{c}\mathbf{E}_{\mathrm{sp}} \\
{[\text { hartree] }}\end{array}$ & $\begin{array}{c}\Delta \mathbf{E}_{\mathrm{sp}} \\
{[\mathrm{kcal} / \mathrm{mol}]}\end{array}$ & $\begin{array}{c}\Delta \mathbf{G}_{\mathrm{sp}} \\
{[\mathrm{kcal} / \mathrm{mol}]}\end{array}$ & $\begin{array}{c}\mathbf{E}_{\mathbf{s p}} \\
{[\text { hartree] }}\end{array}$ & $\begin{array}{c}\Delta \mathbf{E}_{\mathrm{sp}} \\
{[\mathrm{kcal} / \mathrm{mol}]}\end{array}$ & $\begin{array}{c}\Delta \mathbf{G}_{\mathrm{sp}} \\
{[\mathrm{kcal} / \mathrm{mol}]}\end{array}$ & $\begin{array}{c}\mathbf{E}_{\mathrm{sp}} \\
\text { [hartree] }\end{array}$ & $\begin{array}{c}\Delta \mathbf{E}_{\mathrm{sp}} \\
{[\mathrm{kcal} / \mathrm{mol}]}\end{array}$ & $\begin{array}{c}\Delta \mathbf{G}_{\mathrm{sp}} \\
{[\mathrm{kcal} / \mathrm{mol}]}\end{array}$ \\
\hline-1820.30370763 & 0.00 & 0.00 & -1820.31985398 & 0.00 & 0.00 & -1820.33508554 & 0.00 & 0.00 \\
\hline-1820.32750266 & -14.93 & -0.18 & -1820.33821206 & -11.52 & 3.23 & -1820.34841771 & -8.37 & 6.39 \\
\hline-1820.29924389 & 2.80 & 18.46 & -1820.31141712 & 5.29 & 20.95 & -1820.32370648 & 7.14 & 22.80 \\
\hline-1820.30429031 & -0.37 & 16.70 & -1820.31564947 & 2.64 & 19.71 & -1820.32673756 & 5.24 & 22.31 \\
\hline-1820.29381986 & 6.20 & 22.51 & -1820.30506789 & 9.28 & 25.58 & -1820.31640595 & 11.72 & 28.03 \\
\hline-1820.31668176 & -8.14 & 7.93 & -1820.32625840 & -4.02 & 12.05 & -1820.33591183 & -0.52 & 15.55 \\
\hline-1820.30370763 & 0.00 & 0.00 & -1820.31985398 & 0.00 & 0.00 & -1820.33508554 & 0.00 & 0.00 \\
\hline-1820.32750270 & -14.93 & -0.18 & -1820.33821214 & -11.52 & 3.24 & -1820.34841776 & -8.37 & 6.39 \\
\hline-1820.29825923 & 3.42 & 19.04 & -1820.31062109 & 5.79 & 21.42 & -1820.32269356 & 7.78 & 23.40 \\
\hline-1820.30641783 & -1.70 & 15.33 & -1820.31830374 & 0.97 & 18.00 & -1820.32974356 & 3.35 & 20.38 \\
\hline-1820.29086427 & 8.06 & 22.86 & -1820.30276119 & 10.73 & 25.53 & -1820.31456458 & 12.88 & 27.68 \\
\hline-1820.32101592 & -10.86 & 5.20 & -1820.32981150 & -6.25 & 9.82 & -1820.33839968 & -2.08 & 13.99 \\
\hline $\begin{array}{l}\mathrm{E} \ldots \ldots \ldots \ldots \text { gas pha } \\
\mathrm{G} \ldots \ldots \ldots \text { sum of } \\
\Delta \mathrm{E} \ldots \ldots \ldots \text { relative } \\
\Delta \mathrm{G} \ldots \ldots \ldots \text { relative } \\
\Delta \mathrm{G}_{\mathrm{corr}} \ldots \ldots \text { gas pha } \\
\mathrm{E}_{\mathrm{sp}} \ldots \ldots \ldots \text { single } \mathrm{p} \\
\Delta \mathrm{E}_{\mathrm{sp}} \ldots \ldots \text { relative } \\
\Delta \mathrm{G}_{\mathrm{sp}} \ldots \ldots . \text { relative }\end{array}$ & $\begin{array}{l}\text { e electronic en } \\
\text { lectronic and t } \\
\text { electronic ener } \\
\text { free energy in } 1 \\
\text { e thermal corre } \\
\text { oint electronic } \\
\text { single point ele } \\
\text { single point fre }\end{array}$ & $\begin{array}{l}\text { gy in hartree } \\
\text { rmal free ene } \\
\text { in kcal/mol v } \\
\text { al/mol with ba } \\
\text { tion to free en } \\
\text { ergy in gas ph } \\
\text { ronic energy } \\
\text { energy in gas }\end{array}$ & $\begin{array}{l}\text { ith basis } 1 \\
\text { ies with basis } 1 \\
\text { th basis } 1 \\
\text { is } 1 \\
\text { gy in } \mathrm{kcal} / \mathrm{mol} \text { obta } \\
\text { se or solution in ha } \\
\text { gas phase or soluti } \\
\text { hase or solution in }\end{array}$ & $\begin{array}{l}\text { ee with basis } \\
\text { in } \mathrm{kcal} / \mathrm{mol} \text {. } \\
\mathrm{al} / \mathrm{mol}\left(\Delta \mathrm{G}_{\mathrm{sp}}=\right.\end{array}$ & $\Delta \mathrm{G}=\Delta \mathrm{E}+$ & corr) & & \\
\hline
\end{tabular}


Table S16. ROCM with catalyst Ru-5b and enol ether (pathways A1 = ZRu-5b, AB1, B1 in Figure 5 and Figure S22)
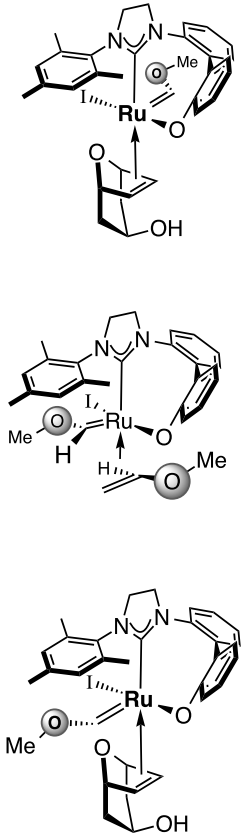

\begin{tabular}{|c|c|c|c|c|c|c|}
\hline & & \multicolumn{5}{|c|}{ BP86/basis1 (gas-phase) } \\
\hline \multicolumn{2}{|c|}{ pathway } & $\begin{array}{c}\mathbf{E} \\
\text { [hartree] }\end{array}$ & $\begin{array}{c}\Delta \mathbf{E} \\
{[\mathrm{kcal} / \mathrm{mol}]}\end{array}$ & $\begin{array}{c}\mathbf{G} \\
\text { [hartree] }\end{array}$ & $\begin{array}{c}\Delta \mathbf{G} \\
{[\mathrm{kcal} / \mathrm{mol}]}\end{array}$ & $\begin{array}{c}\Delta \mathbf{G}_{\text {Corr }} \\
{[\mathrm{kcal} / \mathrm{mol}]}\end{array}$ \\
\hline \multirow{5}{*}{ A1 } & pc2 & -1796.71325106 & -7.96 & -1796.169406 & 8.57 & 16.53 \\
\hline & ts2 & -1796.69137303 & 5.77 & -1796.146707 & 22.82 & 17.04 \\
\hline & mcb & -1796.70203339 & -0.92 & -1796.155189 & 17.49 & 18.41 \\
\hline & ts1 & -1796.69023199 & 6.49 & -1796.147232 & 22.49 & 16.00 \\
\hline & pc1 & -1796.70902869 & -5.31 & -1796.167327 & 9.88 & 15.18 \\
\hline \multirow{7}{*}{ AB1 } & 5-endo & -1796.69381668 & 4.24 & -1796.176350 & 4.21 & -0.02 \\
\hline & pc-endo & -1796.70944128 & -5.57 & -1796.167300 & 9.89 & 15.46 \\
\hline & ts-endo & -1796.69780257 & 1.74 & -1796.153580 & 18.50 & 16.77 \\
\hline & mcb & -1796.70594653 & -3.37 & -1796.159903 & 14.54 & 17.91 \\
\hline & ts-exo & -1796.69881702 & 1.10 & -1796.155904 & 17.05 & 15.94 \\
\hline & pc-exo & -1796.71665476 & -10.09 & -1796.175153 & 4.97 & 15.06 \\
\hline & 5-exo & -1796.70057147 & 0.00 & -1796.183067 & 0.00 & 0.00 \\
\hline \multirow{5}{*}{ B1 } & pc1 & -1796.71824815 & -11.09 & -1796.174274 & 5.52 & 16.61 \\
\hline & ts1 & -1796.69589839 & 2.93 & -1796.152125 & 19.42 & 16.48 \\
\hline & mcb & -1796.70211528 & -0.97 & -1796.156859 & 16.45 & 17.41 \\
\hline & ts2 & -1796.68631814 & 8.94 & -1796.142080 & 25.72 & 16.78 \\
\hline & pc2 & -1796.70079067 & -0.14 & -1796.157232 & 16.21 & 16.35 \\
\hline
\end{tabular}

\begin{tabular}{|c|c|c|c|c|c|c|c|c|}
\hline \multicolumn{3}{|c|}{ BP86/basis2 (gas-phase) } & \multicolumn{3}{|c|}{ BP86/basis2 (benzene) } & \multicolumn{3}{|c|}{ BP86/basis2 (dichloromethane) } \\
\hline $\begin{array}{c}\mathbf{E}_{\mathrm{sp}} \\
\text { [hartree] }\end{array}$ & $\begin{array}{c}\Delta \mathbf{E}_{\mathrm{sp}} \\
{[\mathrm{kcal} / \mathrm{mol}]}\end{array}$ & $\begin{array}{c}\Delta \mathbf{G}_{\mathrm{sp}} \\
{[\mathrm{kcal} / \mathrm{mol}]}\end{array}$ & $\begin{array}{c}\mathbf{E}_{\mathrm{sp}} \\
\text { [hartree] }\end{array}$ & $\begin{array}{c}\Delta \mathbf{E}_{\mathrm{sp}} \\
{[\mathrm{kcal} / \mathrm{mol}]}\end{array}$ & $\begin{array}{c}\Delta \mathbf{G}_{\mathrm{sp}} \\
{[\mathrm{kcal} / \mathrm{mol}]}\end{array}$ & $\begin{array}{c}\mathbf{E}_{\mathrm{sp}} \\
\text { [hartree] }\end{array}$ & $\begin{array}{c}\Delta \mathrm{E}_{\mathrm{sp}} \\
{[\mathrm{kcal} / \mathrm{mol}]}\end{array}$ & $\begin{array}{c}\Delta \mathbf{G}_{\mathrm{sp}} \\
{[\mathrm{kcal} / \mathrm{mol}]}\end{array}$ \\
\hline-1797.14839343 & -5.07 & 11.46 & -1797.15641828 & -1.94 & 14.59 & -1797.16507387 & 0.88 & 17.41 \\
\hline-1797.12694343 & 8.39 & 25.44 & -1797.13646908 & 10.58 & 27.62 & -1797.14652968 & 12.52 & 29.56 \\
\hline-1797.13776417 & 1.60 & 20.01 & -1797.14746152 & 3.68 & 22.09 & -1797.15768850 & 5.52 & 23.93 \\
\hline-1797.12763997 & 7.96 & 23.95 & -1797.13903689 & 8.96 & 24.96 & -1797.15153372 & 9.38 & 25.38 \\
\hline-1797.14572409 & -3.39 & 11.79 & -1797.15510165 & -1.12 & 14.07 & -1797.16499660 & 0.93 & 16.12 \\
\hline-1797.13349533 & 4.28 & 4.26 & -1797.14682340 & 4.08 & 4.06 & -1797.16081264 & 3.56 & 3.53 \\
\hline-1797.14332282 & -1.89 & 13.57 & -1797.15338861 & -0.04 & 15.42 & -1797.16416231 & 1.46 & 16.92 \\
\hline-1797.13213395 & 5.14 & 21.90 & -1797.14259073 & 6.73 & 23.50 & -1797.15330701 & 8.27 & 25.03 \\
\hline-1797.13982463 & 0.31 & 18.22 & -1797.14974293 & 2.25 & 20.16 & -1797.15968134 & 4.27 & 22.18 \\
\hline-1797.13347184 & 4.30 & 20.24 & -1797.14400816 & 5.85 & 21.79 & -1797.15484411 & 7.30 & 23.25 \\
\hline-1797.15058129 & -6.44 & 8.62 & -1797.15979125 & -4.06 & 11.00 & -1797.16916906 & -1.69 & 13.37 \\
\hline-1797.14031854 & 0.00 & 0.00 & -1797.15332350 & 0.00 & 0.00 & -1797.16648185 & 0.00 & 0.00 \\
\hline-1797.15470261 & -9.03 & 7.58 & -1797.16385936 & -6.61 & 10.00 & -1797.17326657 & -4.26 & 12.35 \\
\hline-1797.13298772 & 4.60 & 21.08 & -1797.14345991 & 6.19 & 22.67 & -1797.15493154 & 7.25 & 23.73 \\
\hline-1797.13770155 & 1.64 & 19.06 & -1797.14745665 & 3.68 & 21.10 & -1797.15779761 & 5.45 & 22.86 \\
\hline-1797.12229044 & 11.31 & 28.09 & -1797.13199128 & 13.39 & 30.16 & -1797.14258724 & 14.99 & 31.77 \\
\hline-1797.13636549 & 2.48 & 18.83 & -1797.14481791 & 5.34 & 21.69 & -1797.15441522 & 7.57 & 23.92 \\
\hline
\end{tabular}


Table S17. ROCM with catalyst Ru-5b and enol ether (pathways A2, AB2, B2 in Figure 5 and Figure S22)
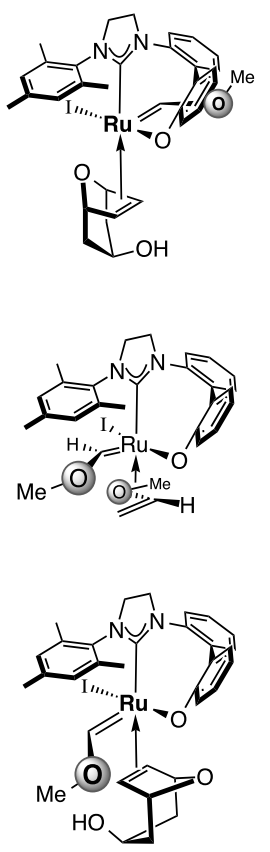

\begin{tabular}{|c|c|c|c|c|c|c|}
\hline & & \multicolumn{5}{|c|}{ BP86/basis1 (gas-phase) } \\
\hline \multicolumn{2}{|l|}{ pathway } & $\begin{array}{c}\mathbf{E} \\
\text { [hartree] }\end{array}$ & $\begin{array}{c}\Delta \mathrm{E} \\
{[\mathrm{kcal} / \mathrm{mol}]}\end{array}$ & $\begin{array}{c}\mathbf{G} \\
\text { [hartree] }\end{array}$ & $\begin{array}{c}\Delta \mathbf{G} \\
{[\mathrm{kcal} / \mathrm{mol}]}\end{array}$ & $\begin{array}{c}\Delta \mathbf{G}_{\text {Corr }} \\
{[\mathrm{kcal} / \mathrm{mol}]}\end{array}$ \\
\hline \multirow{5}{*}{ A2 } & pc2 & -1796.71056132 & -6.27 & -1796.166790 & 10.21 & 16.48 \\
\hline & ts2 & -1796.68754228 & 8.18 & -1796.144228 & 24.37 & 16.20 \\
\hline & mcb & -1796.70139429 & -0.52 & -1796.156226 & 16.84 & 17.36 \\
\hline & ts1 & -1796.69173563 & 5.54 & -1796.148575 & 21.64 & 16.10 \\
\hline & pc1 & -1796.70902868 & -5.31 & -1796.167327 & 9.88 & 15.18 \\
\hline \multirow{7}{*}{ AB2 } & 5-endo & -1796.69381668 & 4.24 & -1796.176350 & 4.21 & -0.02 \\
\hline & pc-endo & -1796.71105637 & -6.58 & -1796.168815 & 8.94 & 15.52 \\
\hline & ts-endo & -1796.69674465 & 2.40 & -1796.153233 & 18.72 & 16.32 \\
\hline & mcb & -1796.70444539 & -2.43 & -1796.158389 & 15.49 & 17.92 \\
\hline & ts-exo & -1796.70108613 & -0.32 & -1796.156221 & 16.85 & 17.17 \\
\hline & pc-exo & -1796.71779227 & -10.81 & -1796.176099 & 4.37 & 15.18 \\
\hline & 5-exo & -1796.70057147 & 0.00 & -1796.183067 & 0.00 & 0.00 \\
\hline \multirow{5}{*}{ B2 } & pc1 & -1796.71836005 & -11.16 & -1796.175397 & 4.81 & 15.98 \\
\hline & ts1 & -1796.69831099 & 1.42 & -1796.154939 & 17.65 & 16.23 \\
\hline & mcb & -1796.70236053 & -1.12 & -1796.157416 & 16.10 & 17.22 \\
\hline & ts2 & -1796.68289477 & 11.09 & -1796.139422 & 27.39 & 16.30 \\
\hline & pc2 & -1796.69824315 & 1.46 & -1796.156556 & 16.64 & 15.17 \\
\hline
\end{tabular}

\begin{tabular}{|c|c|c|c|c|c|c|c|c|}
\hline \multicolumn{3}{|c|}{ BP86/basis2 (gas-phase) } & \multicolumn{3}{|c|}{ BP86/basis2 (benzene) } & \multicolumn{3}{|c|}{ BP86/basis2 (dichloromethane) } \\
\hline $\begin{array}{c}\mathbf{E}_{\mathrm{sp}} \\
\text { [hartree] }\end{array}$ & $\begin{array}{c}\Delta \mathrm{E}_{\mathrm{sp}} \\
{[\mathrm{kcal} / \mathrm{mol}]}\end{array}$ & $\begin{array}{c}\Delta \mathbf{G}_{\mathrm{sp}} \\
{[\mathrm{kcal} / \mathrm{mol}]}\end{array}$ & $\begin{array}{c}\mathbf{E}_{\mathrm{sp}} \\
\text { [hartree] }\end{array}$ & $\begin{array}{c}\Delta \mathrm{E}_{\mathrm{sp}} \\
{[\mathrm{kcal} / \mathrm{mol}]}\end{array}$ & $\begin{array}{c}\Delta \mathbf{G}_{\mathrm{sp}} \\
{[\mathrm{kcal} / \mathrm{mol}]}\end{array}$ & $\begin{array}{c}\mathbf{E}_{\mathrm{sp}} \\
\text { [hartree] }\end{array}$ & $\begin{array}{c}\Delta \mathrm{E}_{\mathrm{sp}} \\
{[\mathrm{kcal} / \mathrm{mol}]}\end{array}$ & $\begin{array}{c}\Delta \mathbf{G}_{\mathrm{sp}} \\
{[\mathrm{kcal} / \mathrm{mol}]}\end{array}$ \\
\hline-1797.14651837 & -3.89 & 12.59 & -1797.15499166 & -1.05 & 15.44 & -1797.16403106 & 1.54 & 18.02 \\
\hline-1797.12323336 & 10.72 & 26.92 & -1797.13319266 & 12.63 & 28.83 & -1797.14372836 & 14.28 & 30.47 \\
\hline-1797.13770851 & 1.64 & 19.00 & -1797.14773987 & 3.50 & 20.86 & -1797.15839792 & 5.07 & 22.43 \\
\hline-1797.12891045 & 7.16 & 23.26 & -1797.13978715 & 8.49 & 24.59 & -1797.15171549 & 9.27 & 25.37 \\
\hline-1797.14572407 & -3.39 & 11.79 & -1797.15510162 & -1.12 & 14.07 & -1797.16499658 & 0.93 & 16.12 \\
\hline-1797.13349533 & 4.28 & 4.26 & -1797.14682340 & 4.08 & 4.06 & -1797.16081264 & 3.56 & 3.53 \\
\hline-1797.14526439 & -3.10 & 12.42 & -1797.15476188 & -0.90 & 14.62 & -1797.16482058 & 1.04 & 16.57 \\
\hline-1797.13130018 & 5.66 & 21.98 & -1797.14253947 & 6.77 & 23.09 & -1797.15426430 & 7.67 & 23.99 \\
\hline-1797.13777199 & 1.60 & 19.51 & -1797.14764461 & 3.56 & 21.48 & -1797.15751602 & 5.63 & 23.54 \\
\hline-1797.13488461 & 3.41 & 20.58 & -1797.14468987 & 5.42 & 22.59 & -1797.15450284 & 7.52 & 24.69 \\
\hline-1797.15156930 & -7.06 & 8.12 & -1797.16088411 & -4.74 & 10.43 & -1797.17040456 & -2.46 & 12.72 \\
\hline-1797.14031854 & 0.00 & 0.00 & -1797.15332350 & 0.00 & 0.00 & -1797.16648185 & 0.00 & 0.00 \\
\hline-1797.15453109 & -8.92 & 7.06 & -1797.16332648 & -6.28 & 9.70 & -1797.17212611 & -3.54 & 12.43 \\
\hline-1797.13503668 & 3.31 & 19.55 & -1797.14526465 & 5.06 & 21.29 & -1797.15607503 & 6.53 & 22.76 \\
\hline-1797.13814267 & 1.37 & 18.58 & -1797.14815163 & 3.25 & 20.46 & -1797.15877158 & 4.84 & 22.06 \\
\hline-1797.11893948 & 13.42 & 29.71 & -1797.12932038 & 15.06 & 31.36 & -1797.14060947 & 16.24 & 32.53 \\
\hline-1797.13449711 & 3.65 & 18.83 & -1797.14429155 & 5.67 & 20.84 & -1797.15520630 & 7.08 & 22.25 \\
\hline
\end{tabular}


Table S18. ROCM with catalyst Ru-5b and enol ether (pathways A3, AB3, B3 in Figure 5 and Figure S22)
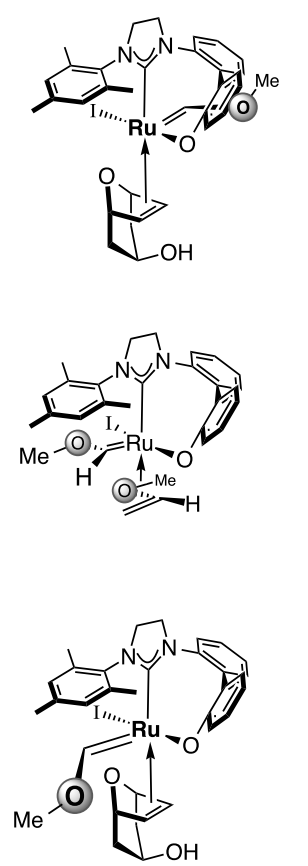

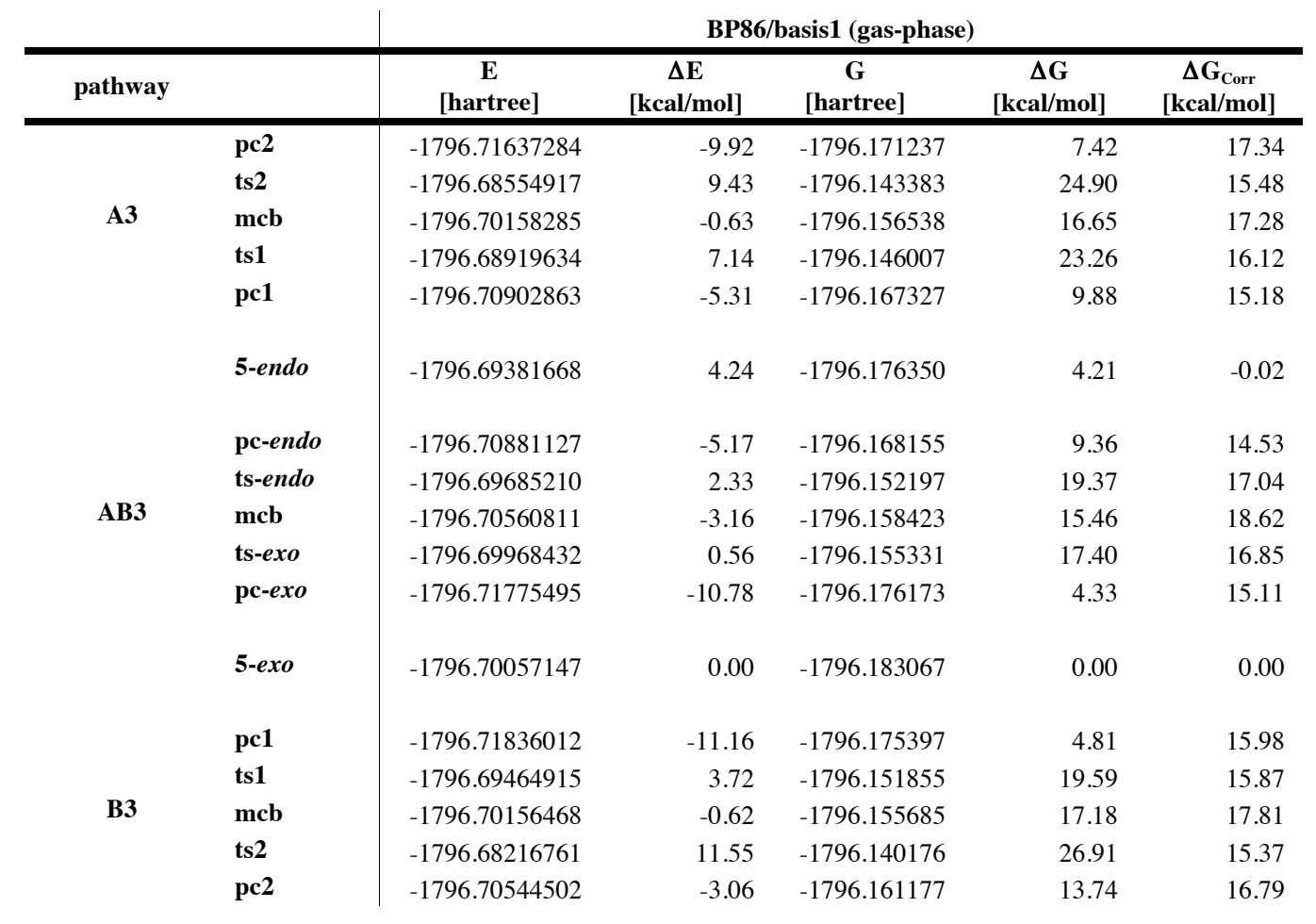

\begin{tabular}{|c|c|c|c|c|c|c|c|c|}
\hline \multicolumn{3}{|c|}{ BP86/basis2 (gas-phase) } & \multicolumn{3}{|c|}{ BP86/basis2 (benzene) } & \multicolumn{3}{|c|}{ BP86/basis2 (dichloromethane) } \\
\hline $\begin{array}{c}\mathbf{E}_{\mathrm{sp}} \\
\text { [hartree] }\end{array}$ & $\begin{array}{c}\Delta \mathrm{E}_{\mathrm{sp}} \\
{[\mathrm{kcal} / \mathrm{mol}]}\end{array}$ & $\begin{array}{c}\Delta \mathbf{G}_{\mathrm{sp}} \\
{[\mathrm{kcal} / \mathrm{mol}]}\end{array}$ & $\begin{array}{c}\mathbf{E}_{\mathrm{sp}} \\
\text { [hartree] }\end{array}$ & $\begin{array}{c}\Delta \mathrm{E}_{\mathrm{sp}} \\
{[\mathrm{kcal} / \mathrm{mol}]}\end{array}$ & $\begin{array}{c}\Delta \mathbf{G}_{\mathrm{sp}} \\
{[\mathrm{kcal} / \mathrm{mol}]}\end{array}$ & $\begin{array}{c}\mathbf{E}_{\mathrm{sp}} \\
\text { [hartree] }\end{array}$ & $\begin{array}{c}\Delta \mathrm{E}_{\mathrm{sp}} \\
{[\mathrm{kcal} / \mathrm{mol}]}\end{array}$ & $\begin{array}{c}\Delta \mathbf{G}_{\mathrm{sp}} \\
{[\mathrm{kcal} / \mathrm{mol}]}\end{array}$ \\
\hline-1797.15074347 & -6.54 & 10.80 & -1797.15828285 & -3.11 & 14.23 & -1797.16624703 & 0.15 & 17.49 \\
\hline-1797.12157065 & 11.76 & 27.24 & -1797.13202817 & 13.36 & 28.84 & -1797.14317002 & 14.63 & 30.10 \\
\hline-1797.13785486 & 1.55 & 18.83 & -1797.14803492 & 3.32 & 20.60 & -1797.15868201 & 4.89 & 22.18 \\
\hline-1797.12623653 & 8.84 & 24.95 & -1797.13689256 & 10.31 & 26.43 & -1797.14828575 & 11.42 & 27.54 \\
\hline-1797.14572408 & -3.39 & 11.79 & -1797.15510163 & -1.12 & 14.07 & -1797.16499660 & 0.93 & 16.12 \\
\hline-1797.13349533 & 4.28 & 4.26 & -1797.14682340 & 4.08 & 4.06 & -1797.16081264 & 3.56 & 3.53 \\
\hline-1797.14347239 & -1.98 & 12.55 & -1797.15309426 & 0.14 & 14.67 & -1797.16345508 & 1.90 & 16.43 \\
\hline-1797.13124700 & 5.69 & 22.73 & -1797.14213910 & 7.02 & 24.06 & -1797.15354302 & 8.12 & 25.16 \\
\hline-1797.13865237 & 1.05 & 19.67 & -1797.14831034 & 3.15 & 21.77 & -1797.15806809 & 5.28 & 23.90 \\
\hline-1797.13366611 & 4.17 & 21.02 & -1797.14377581 & 5.99 & 22.84 & -1797.15426907 & 7.66 & 24.51 \\
\hline-1797.15162528 & -7.10 & 8.01 & -1797.16097849 & -4.80 & 10.31 & -1797.17058466 & -2.57 & 12.53 \\
\hline-1797.14031854 & 0.00 & 0.00 & -1797.15332350 & 0.00 & 0.00 & -1797.16648185 & 0.00 & 0.00 \\
\hline-1797.15453105 & -8.92 & 7.06 & -1797.16332655 & -6.28 & 9.70 & -1797.17212612 & -3.54 & 12.43 \\
\hline-1797.13151151 & 5.53 & 21.40 & -1797.14195898 & 7.13 & 23.00 & -1797.15294555 & 8.49 & 24.36 \\
\hline-1797.13731282 & 1.89 & 19.69 & -1797.14766248 & 3.55 & 21.36 & -1797.15843141 & 5.05 & 22.86 \\
\hline-1797.11812262 & 13.93 & 29.29 & -1797.12824881 & 15.73 & 31.10 & -1797.13898090 & 17.26 & 32.62 \\
\hline-1797.13996769 & 0.22 & 17.01 & -1797.14812384 & 3.26 & 20.06 & -1797.15703565 & 5.93 & 22.72 \\
\hline
\end{tabular}


Table S19. ROCM with catalyst Ru-5b and enol ether (pathways A4 = ERu-5b, AB4, B4 in Figure 5 and Figure S22)

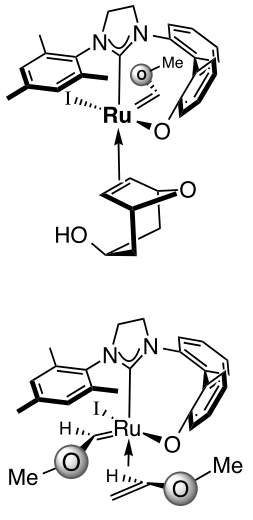

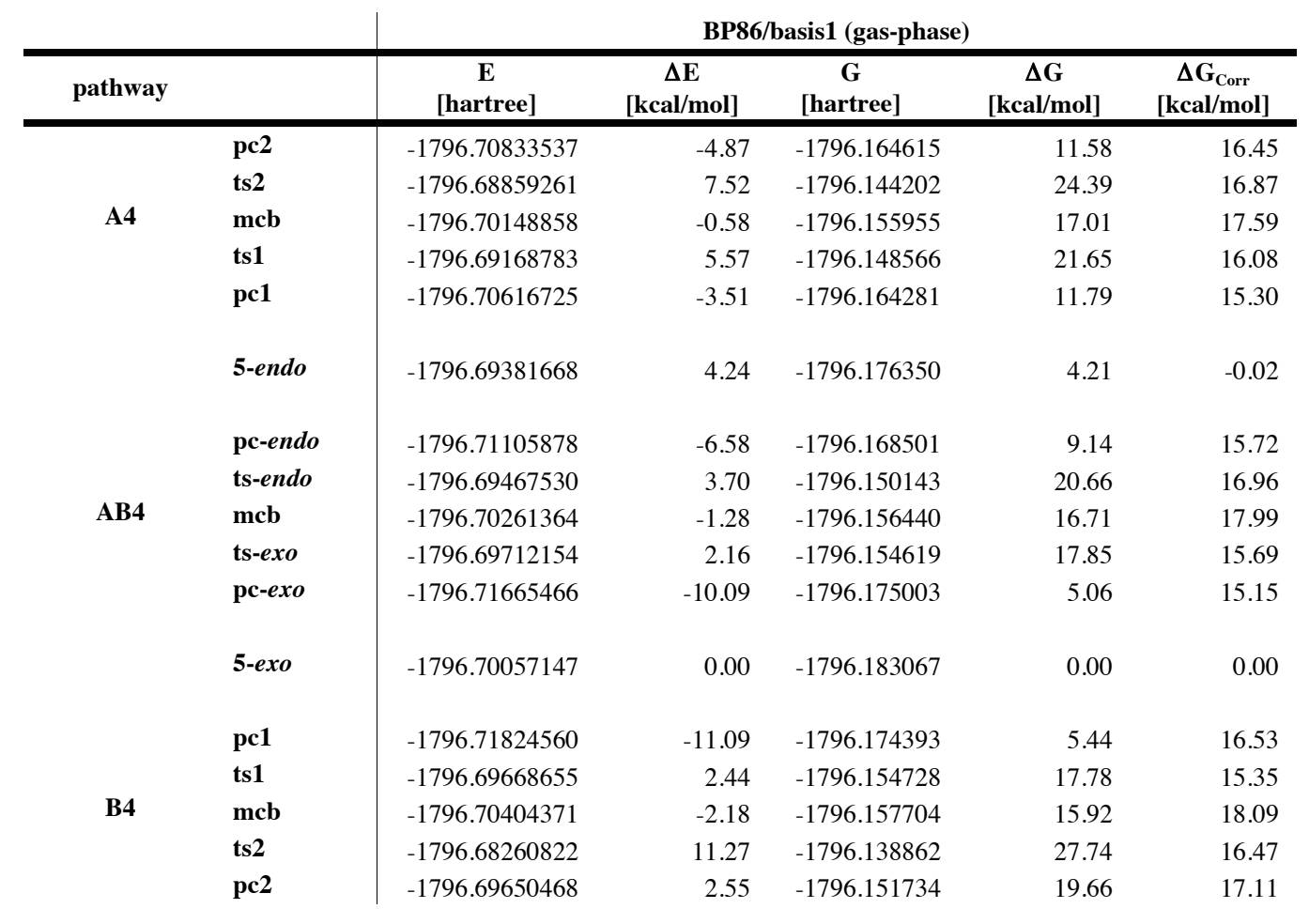

\begin{tabular}{|c|c|c|c|c|c|c|c|c|}
\hline \multicolumn{3}{|c|}{ BP86/basis2 (gas-phase) } & \multicolumn{3}{|c|}{ BP86/basis2 (benzene) } & \multicolumn{3}{|c|}{ BP86/basis2 (dichloromethane) } \\
\hline $\begin{array}{c}\mathbf{E}_{\mathrm{sp}} \\
\text { [hartree] }\end{array}$ & $\begin{array}{c}\Delta \mathrm{E}_{\mathrm{sp}} \\
{[\mathrm{kcal} / \mathrm{mol}]}\end{array}$ & $\begin{array}{c}\Delta \mathbf{G}_{\mathrm{sp}} \\
{[\mathrm{kcal} / \mathrm{mol}]}\end{array}$ & $\begin{array}{c}\mathbf{E}_{\mathrm{sp}} \\
\text { [hartree] }\end{array}$ & $\begin{array}{c}\Delta \mathbf{E}_{\mathrm{sp}} \\
{[\mathrm{kcal} / \mathrm{mol}]}\end{array}$ & $\begin{array}{c}\Delta \mathbf{G}_{\mathrm{sp}} \\
{[\mathrm{kcal} / \mathrm{mol}]}\end{array}$ & $\begin{array}{c}\mathbf{E}_{\mathrm{sp}} \\
\text { [hartree] }\end{array}$ & $\begin{array}{c}\Delta \mathrm{E}_{\mathrm{sp}} \\
{[\mathrm{kcal} / \mathrm{mol}]}\end{array}$ & $\begin{array}{c}\Delta \mathbf{G}_{\mathrm{sp}} \\
{[\mathrm{kcal} / \mathrm{mol}]}\end{array}$ \\
\hline-1797.14385343 & -2.22 & 14.23 & -1797.15227670 & 0.66 & 17.11 & -1797.16127863 & 3.27 & 19.72 \\
\hline-1797.12392809 & 10.29 & 27.16 & -1797.13291921 & 12.80 & 29.68 & -1797.14218409 & 15.25 & 32.12 \\
\hline-1797.13738998 & 1.84 & 19.43 & -1797.14753463 & 3.63 & 21.22 & -1797.15807532 & 5.28 & 22.86 \\
\hline-1797.12895297 & 7.13 & 23.21 & -1797.14005766 & 8.32 & 24.40 & -1797.15204837 & 9.06 & 25.13 \\
\hline-1797.14325670 & -1.84 & 13.46 & -1797.15219651 & 0.71 & 16.01 & -1797.16192544 & 2.86 & 18.16 \\
\hline-1797.13349533 & 4.28 & 4.26 & -1797.14682340 & 4.08 & 4.06 & -1797.16081264 & 3.56 & 3.53 \\
\hline-1797.14526963 & -3.11 & 12.61 & -1797.15476455 & -0.90 & 14.82 & -1797.16481661 & 1.04 & 16.77 \\
\hline-1797.12921141 & 6.97 & 23.93 & -1797.13990680 & 8.42 & 25.38 & -1797.15090632 & 9.77 & 26.73 \\
\hline-1797.13670982 & 2.26 & 20.25 & -1797.14685983 & 4.06 & 22.05 & -1797.15720711 & 5.82 & 23.81 \\
\hline-1797.13138227 & 5.61 & 21.29 & -1797.14164405 & 7.33 & 23.02 & -1797.15207055 & 9.04 & 24.73 \\
\hline-1797.15050406 & -6.39 & 8.76 & -1797.15971351 & -4.01 & 11.14 & -1797.16908259 & -1.63 & 13.52 \\
\hline-1797.14031854 & 0.00 & 0.00 & -1797.15332350 & 0.00 & 0.00 & -1797.16648185 & 0.00 & 0.00 \\
\hline-1797.15469792 & -9.02 & 7.51 & -1797.16385492 & -6.61 & 9.93 & -1797.17326058 & -4.25 & 12.28 \\
\hline-1797.13341386 & 4.33 & 19.68 & -1797.14380113 & 5.98 & 21.32 & -1797.15482885 & 7.31 & 22.66 \\
\hline-1797.13964348 & 0.42 & 18.52 & -1797.14961111 & 2.33 & 20.42 & -1797.16002536 & 4.05 & 22.15 \\
\hline-1797.11833226 & 13.80 & 30.26 & -1797.12852394 & 15.56 & 32.03 & -1797.13954158 & 16.91 & 33.37 \\
\hline-1797.13220898 & 5.09 & 22.20 & -1797.14229409 & 6.92 & 24.03 & -1797.15345862 & 8.17 & 25.28 \\
\hline
\end{tabular}


Table S20. CM between acrylonitrile and propene with catalyst $\mathrm{Ru}-4 \mathrm{~b}$ (pathways $E M e$, ECN in Figure 7)<smiles>CN1CCN(C)C1[Ge](Cl)(Cl)C=CC#N</smiles><smiles>CC=CC(Cl)(Cl)C1=NCCN1C</smiles>

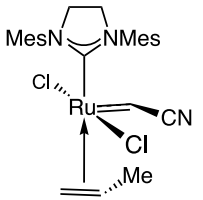

\begin{tabular}{|c|c|c|c|c|c|c|}
\hline \multirow{2}{*}{\multicolumn{2}{|c|}{ pathway }} & \multicolumn{5}{|c|}{ BP86/basis1 (gas-phase) } \\
\hline & & $\begin{array}{c}\mathbf{E} \\
\text { [hartree] }\end{array}$ & $\begin{array}{c}\Delta \mathrm{E} \\
{[\mathrm{kcal} / \mathrm{mol}]}\end{array}$ & $\begin{array}{c}\mathbf{G} \\
{[\text { hartree] }}\end{array}$ & $\begin{array}{c}\Delta \mathbf{G} \\
{[\mathrm{kcal} / \mathrm{mol}]}\end{array}$ & $\begin{array}{c}\Delta \mathbf{G}_{\mathrm{Corr}} \\
{[\mathrm{kcal} / \mathrm{mol}]}\end{array}$ \\
\hline \multirow{7}{*}{ EMe } & $14 \mathrm{e}$ & -2190.41508667 & 2.01 & -2189.993697 & 2.39 & 0.38 \\
\hline & pc2 & -2190.42444693 & -3.87 & -2189.980752 & 10.51 & 14.38 \\
\hline & ts2 & -2190.41797410 & 0.19 & -2189.972321 & 15.80 & 15.61 \\
\hline & mcb & -2190.43277716 & -9.10 & -2189.984440 & 8.20 & 17.29 \\
\hline & ts1 & -2190.42477229 & -4.07 & -2189.978440 & 11.96 & 16.04 \\
\hline & pc1 & -2190.43117312 & -8.09 & -2189.987180 & 6.48 & 14.57 \\
\hline & $14 e_{M e}$ & -2190.41828323 & 0.00 & -2189.997506 & 0.00 & 0.00 \\
\hline \multirow{5}{*}{$\mathrm{Me}-\mathrm{CN}$} & pc1 & -2190.42634823 & -5.06 & -2189.981805 & 9.85 & 14.91 \\
\hline & ts1 & -2190.42458561 & -3.95 & -2189.979984 & 11.00 & 14.95 \\
\hline & mcb & -2190.44121268 & -14.39 & -2189.992334 & 3.25 & 17.63 \\
\hline & ts2 & -2190.42611269 & -4.91 & -2189.980911 & 10.41 & 15.33 \\
\hline & pc2 & -2190.42968959 & -7.16 & -2189.985122 & 7.77 & 14.93 \\
\hline \multirow{7}{*}{$E C N$} & $14 e_{\mathrm{CN}}$ & -2190.41769415 & 0.37 & -2189.996569 & 0.59 & 0.22 \\
\hline & pc1 & -2190.43108391 & -8.03 & -2189.988232 & 5.82 & 13.85 \\
\hline & ts1 & -2190.42776761 & -5.95 & -2189.982698 & 9.29 & 15.24 \\
\hline & mcb & -2190.43980206 & -13.50 & -2189.991422 & 3.82 & 17.32 \\
\hline & ts2 & -2190.41947990 & -0.75 & -2189.973922 & 14.80 & 15.55 \\
\hline & pc2 & -2190.42080279 & -1.58 & -2189.976269 & 13.33 & 14.91 \\
\hline & $14 \mathrm{e}$ & -2190.41508667 & 2.01 & -2189.993697 & 2.39 & 0.38 \\
\hline
\end{tabular}

BP86/basis2 (gas-phase)

\begin{tabular}{|c|c|c|c|c|c|c|c|c|}
\hline \multicolumn{3}{|c|}{ BP86/basis2 (gas-phase) } & \multicolumn{3}{|c|}{ BP86/basis2 (benzene) } & \multicolumn{3}{|c|}{ BP86/basis2 (dichloromethane) } \\
\hline $\begin{array}{c}\mathbf{E}_{\mathrm{sp}} \\
\text { [hartree] }\end{array}$ & $\begin{array}{c}\Delta \mathrm{E}_{\mathrm{sp}} \\
{[\mathrm{kcal} / \mathrm{mol}]}\end{array}$ & $\begin{array}{c}\Delta \mathbf{G}_{\mathrm{sp}} \\
{[\mathrm{kcal} / \mathrm{mol}]}\end{array}$ & $\begin{array}{c}\mathbf{E}_{\mathrm{sp}} \\
\text { [hartree] }\end{array}$ & $\begin{array}{c}\Delta \mathrm{E}_{\mathrm{sp}} \\
{[\mathrm{kcal} / \mathrm{mol}]}\end{array}$ & $\begin{array}{c}\Delta \mathbf{G}_{\mathrm{sp}} \\
{[\mathrm{kcal} / \mathrm{mol}]}\end{array}$ & $\begin{array}{c}\mathbf{E}_{\mathrm{sp}} \\
\text { [hartree] }\end{array}$ & $\begin{array}{c}\Delta \mathrm{E}_{\mathrm{sp}} \\
{[\mathrm{kcal} / \mathrm{mol}]}\end{array}$ & $\begin{array}{c}\Delta \mathbf{G}_{\mathrm{sp}} \\
{[\mathrm{kcal} / \mathrm{mol}]}\end{array}$ \\
\hline-2190.77765359 & 2.21 & 2.60 & -2190.78988633 & 1.99 & 2.38 & -2190.80204808 & 1.84 & 2.23 \\
\hline-2190.78286506 & -1.06 & 13.33 & -2190.79238983 & 0.42 & 14.80 & -2190.80196015 & 1.90 & 16.28 \\
\hline-2190.77652950 & 2.92 & 18.53 & -2190.78574702 & 4.59 & 20.20 & -2190.79503186 & 6.25 & 21.86 \\
\hline-2190.79135796 & -6.38 & 10.91 & -2190.80029538 & -4.54 & 12.76 & -2190.80915625 & -2.62 & 14.68 \\
\hline-2190.78343538 & -1.41 & 14.62 & -2190.79269175 & 0.23 & 16.27 & -2190.80193365 & 1.92 & 17.95 \\
\hline-2190.79065311 & -5.94 & 8.63 & -2190.79975002 & -4.20 & 10.37 & -2190.80899418 & -2.51 & 12.05 \\
\hline-2190.78118309 & 0.00 & 0.00 & -2190.79306202 & 0.00 & 0.00 & -2190.80498646 & 0.00 & 0.00 \\
\hline-2190.78537101 & -2.63 & 12.29 & -2190.79419923 & -0.71 & 14.20 & -2190.80376554 & 0.77 & 15.68 \\
\hline-2190.78345700 & -1.43 & 13.52 & -2190.79269031 & 0.23 & 15.18 & -2190.80285039 & 1.34 & 16.29 \\
\hline-2190.79989603 & -11.74 & 5.89 & -2190.80896064 & -9.98 & 7.66 & -2190.81826004 & -8.33 & 9.30 \\
\hline-2190.78484833 & -2.30 & 13.03 & -2190.79376864 & -0.44 & 14.88 & -2190.80316495 & 1.14 & 16.47 \\
\hline-2190.78846506 & -4.57 & 10.36 & -2190.79739539 & -2.72 & 12.21 & -2190.80679715 & -1.14 & 13.79 \\
\hline-2190.78308819 & -1.20 & -0.98 & -2190.79412447 & -0.67 & -0.45 & -2190.80559919 & -0.38 & -0.17 \\
\hline-2190.78971308 & -5.35 & 8.50 & -2190.79819875 & -3.22 & 10.63 & -2190.80689743 & -1.20 & 12.65 \\
\hline-2190.78621547 & -3.16 & 12.09 & -2190.79519851 & -1.34 & 13.90 & -2190.80450011 & 0.31 & 15.55 \\
\hline-2190.79793178 & -10.51 & 6.81 & -2190.80685470 & -8.66 & 8.67 & -2190.81597611 & -6.90 & 10.42 \\
\hline-2190.77787436 & 2.08 & 17.63 & -2190.78775090 & 3.33 & 18.88 & -2190.79831183 & 4.19 & 19.74 \\
\hline-2190.77938316 & 1.13 & 16.04 & -2190.78847230 & 2.88 & 17.79 & -2190.79840085 & 4.13 & 19.04 \\
\hline-2190.77765359 & 2.21 & 2.60 & -2190.78988633 & 1.99 & 2.38 & -2190.80204808 & 1.84 & 2.23 \\
\hline
\end{tabular}


Table S21. CM between acrylonitrile and propene with catalyst $R u-4 b$ (pathways $Z M e$, ZCN in Figure 7)

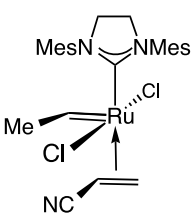

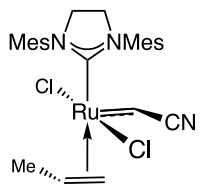<smiles>C=CC(Cl)(Cl)C1=NCCN1C</smiles>

$$
\begin{array}{r|r}
\text { BP86/basis1 (gas-phase) } \\
\hline
\end{array}
$$

\begin{tabular}{|c|c|c|c|c|c|c|}
\hline pathway & & $\begin{array}{c}\mathbf{E} \\
\text { [hartree] }\end{array}$ & $\begin{array}{c}\Delta \mathbf{E} \\
{[\mathrm{kcal} / \mathrm{mol}]}\end{array}$ & $\begin{array}{c}\mathbf{G} \\
\text { [hartree] }\end{array}$ & $\begin{array}{c}\Delta \mathbf{G} \\
{[\mathrm{kcal} / \mathrm{mol}]}\end{array}$ & $\begin{array}{c}\Delta \mathbf{G}_{\text {Corr }} \\
{[\mathrm{kcal} / \mathrm{mol}]}\end{array}$ \\
\hline \multirow{7}{*}{ ZMe } & $14 \mathrm{e}$ & -2190.41520737 & 1.93 & -2189.993795 & 2.33 & 0.40 \\
\hline & pc2 & -2190.42245391 & -2.62 & -2189.978913 & 11.67 & 14.28 \\
\hline & ts2 & -2190.41779061 & 0.31 & -2189.973602 & 15.00 & 14.69 \\
\hline & mcb & -2190.43256847 & -8.96 & -2189.984327 & 8.27 & 17.23 \\
\hline & ts1 & -2190.42510793 & -4.28 & -2189.979138 & 11.53 & 15.81 \\
\hline & pc1 & -2190.43141289 & -8.24 & -2189.986663 & 6.80 & 15.04 \\
\hline & $14 e_{\mathrm{Me}}$ & -2190.41828323 & 0.00 & -2189.997506 & 0.00 & 0.00 \\
\hline \multirow{5}{*}{$\mathrm{Me}-\mathrm{CN}$} & pc1 & -2190.42634823 & -5.06 & -2189.981805 & 9.85 & 14.91 \\
\hline & ts1 & -2190.42458561 & -3.95 & -2189.979984 & 11.00 & 14.95 \\
\hline & mcb & -2190.44121268 & -14.39 & -2189.992334 & 3.25 & 17.63 \\
\hline & ts 2 & -2190.42611269 & -4.91 & -2189.980911 & 10.41 & 15.33 \\
\hline & pc2 & -2190.42968959 & -7.16 & -2189.985122 & 7.77 & 14.93 \\
\hline \multirow{7}{*}{$Z C N$} & $14 e_{\mathrm{CN}}$ & -2190.41769415 & 0.37 & -2189.996569 & 0.59 & 0.22 \\
\hline & pc1 & -2190.43180107 & -8.48 & -2189.988089 & 5.91 & 14.39 \\
\hline & ts1 & -2190.42796860 & -6.08 & -2189.981261 & 10.19 & 16.27 \\
\hline & mcb & -2190.43899506 & -13.00 & -2189.992106 & 3.39 & 16.39 \\
\hline & ts2 & -2190.42084251 & -1.61 & -2189.976578 & 13.13 & 14.74 \\
\hline & pc2 & -2190.42150643 & -2.02 & -2189.978341 & 12.03 & 14.05 \\
\hline & $14 \mathrm{e}$ & -2190.41520737 & 1.93 & -2189.993795 & 2.33 & 0.40 \\
\hline
\end{tabular}

BP86/basis2 (gas-phase)

\begin{tabular}{|c|c|c|c|c|c|c|c|c|}
\hline \multicolumn{3}{|c|}{ BP86/basis2 (gas-phase) } & \multicolumn{3}{|c|}{ BP86/basis2 (benzene) } & \multicolumn{3}{|c|}{ BP86/basis2 (dichloromethane) } \\
\hline $\begin{array}{c}\mathbf{E}_{\mathrm{sp}} \\
\text { [hartree] }\end{array}$ & $\begin{array}{c}\Delta \mathrm{E}_{\mathrm{sp}} \\
{[\mathrm{kcal} / \mathrm{mol}]}\end{array}$ & $\begin{array}{c}\Delta \mathbf{G}_{\mathrm{sp}} \\
{[\mathrm{kcal} / \mathrm{mol}]}\end{array}$ & $\begin{array}{c}\mathbf{E}_{\mathrm{sp}} \\
\text { [hartree] }\end{array}$ & $\begin{array}{c}\Delta \mathbf{E}_{\mathrm{sp}} \\
{[\mathrm{kcal} / \mathrm{mol}]}\end{array}$ & $\begin{array}{c}\Delta \mathbf{G}_{\mathrm{sp}} \\
{[\mathrm{kcal} / \mathrm{mol}]}\end{array}$ & $\begin{array}{c}\mathbf{E}_{\mathrm{sp}} \\
\text { [hartree] }\end{array}$ & $\begin{array}{c}\Delta \mathbf{E}_{\mathrm{sp}} \\
{[\mathrm{kcal} / \mathrm{mol}]}\end{array}$ & $\begin{array}{c}\Delta \mathbf{G}_{\mathrm{sp}} \\
{[\mathrm{kcal} / \mathrm{mol}]}\end{array}$ \\
\hline-2190.77787725 & 2.07 & 2.47 & -2190.78976088 & 2.07 & 2.47 & -2190.80168499 & 2.07 & 2.47 \\
\hline-2190.78076307 & 0.26 & 14.55 & -2190.78990969 & 1.98 & 16.26 & -2190.79916834 & 3.65 & 17.94 \\
\hline-2190.77636336 & 3.02 & 17.72 & -2190.78569559 & 4.62 & 19.31 & -2190.79540657 & 6.01 & 20.70 \\
\hline-2190.79129223 & -6.34 & 10.89 & -2190.80023734 & -4.50 & 12.73 & -2190.80921673 & -2.65 & 14.58 \\
\hline-2190.78386636 & -1.68 & 14.12 & -2190.79300941 & 0.03 & 15.84 & -2190.80229407 & 1.69 & 17.50 \\
\hline-2190.79015946 & -5.63 & 9.41 & -2190.79881463 & -3.61 & 11.43 & -2190.80719604 & -1.39 & 13.66 \\
\hline-2190.78118309 & 0.00 & 0.00 & -2190.79306202 & 0.00 & 0.00 & -2190.80498646 & 0.00 & 0.00 \\
\hline-2190.78537101 & -2.63 & 12.29 & -2190.79419923 & -0.71 & 14.20 & -2190.80376554 & 0.77 & 15.68 \\
\hline-2190.78345700 & -1.43 & 13.52 & -2190.79269031 & 0.23 & 15.18 & -2190.80285039 & 1.34 & 16.29 \\
\hline-2190.79989603 & -11.74 & 5.89 & -2190.80896064 & -9.98 & 7.66 & -2190.81826004 & -8.33 & 9.30 \\
\hline-2190.78484833 & -2.30 & 13.03 & -2190.79376864 & -0.44 & 14.88 & -2190.80316495 & 1.14 & 16.47 \\
\hline-2190.78846506 & -4.57 & 10.36 & -2190.79739539 & -2.72 & 12.21 & -2190.80679715 & -1.14 & 13.79 \\
\hline-2190.78308819 & -1.20 & -0.98 & -2190.79412447 & -0.67 & -0.45 & -2190.80559919 & -0.38 & -0.17 \\
\hline-2190.79039151 & -5.78 & 8.61 & -2190.79899270 & -3.72 & 10.67 & -2190.80815819 & -1.99 & 12.40 \\
\hline-2190.78647976 & -3.32 & 12.95 & -2190.79520590 & -1.35 & 14.93 & -2190.80448403 & 0.32 & 16.59 \\
\hline-2190.79731758 & -10.12 & 6.26 & -2190.80598441 & -8.11 & 8.28 & -2190.81498754 & -6.28 & 10.11 \\
\hline-2190.77931445 & 1.17 & 15.91 & -2190.78885048 & 2.64 & 17.38 & -2190.79907289 & 3.71 & 18.45 \\
\hline-2190.77990460 & 0.80 & 14.85 & -2190.78960799 & 2.17 & 16.22 & -2190.80003951 & 3.10 & 17.15 \\
\hline-2190.77787725 & 2.07 & 2.47 & -2190.78976088 & 2.07 & 2.47 & -2190.80168499 & 2.07 & 2.47 \\
\hline
\end{tabular}




\section{Table S22. CM between vinyl sulfide and trans-dichloroethylene with catalyst $\mathrm{Ru}-4 \mathrm{~b}$ (ESMe, ZSMe in Figure 9)}

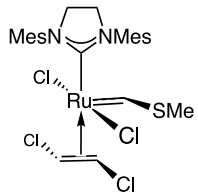

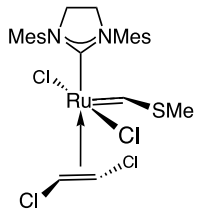

\begin{tabular}{|c|c|c|c|c|c|c|}
\hline \multirow[b]{2}{*}{ pathway } & & \multicolumn{5}{|c|}{ BP86/basis1 (gas-phase) } \\
\hline & & $\begin{array}{c}\mathbf{E} \\
\text { [hartree] }\end{array}$ & $\begin{array}{c}\Delta \mathbf{E} \\
{[\mathrm{kcal} / \mathrm{mol}]}\end{array}$ & $\begin{array}{c}\mathbf{G} \\
\text { [hartree] }\end{array}$ & $\begin{array}{c}\Delta \mathbf{G} \\
{[\mathrm{kcal} / \mathrm{mol}]}\end{array}$ & $\begin{array}{c}\Delta \mathbf{G}_{\mathrm{Corr}} \\
{[\mathrm{kcal} / \mathrm{mol}]}\end{array}$ \\
\hline \multirow{7}{*}{ ZSMe } & $14 \mathrm{e}$ & -3415.64269665 & 0.00 & -3415.241401 & 0.00 & 0.00 \\
\hline & pc1 & -3415.65435640 & -7.32 & -3415.227515 & 8.71 & 16.03 \\
\hline & ts1 & -3415.64211968 & 0.36 & -3415.215247 & 16.41 & 16.05 \\
\hline & mcb & -3415.64689508 & -2.63 & -3415.222189 & 12.06 & 14.69 \\
\hline & ts2 & -3415.63783439 & 3.05 & -3415.211596 & 18.70 & 15.65 \\
\hline & pc2 & -3415.65077453 & -5.07 & -3415.222831 & 11.65 & 16.72 \\
\hline & $14 \mathrm{e}$ & -3415.64439129 & -1.06 & -3415.245660 & -2.67 & -1.61 \\
\hline \multirow{7}{*}{ ESMe } & $14 e$ & -3415.64269665 & 0.00 & -3415.241401 & 0.00 & 0.00 \\
\hline & pc1 & -3415.65284234 & -6.37 & -3415.227264 & 8.87 & 15.24 \\
\hline & ts1 & -3415.64131688 & 0.87 & -3415.214985 & 16.58 & 15.71 \\
\hline & mcb & -3415.64831879 & -3.53 & -3415.222703 & 11.73 & 15.26 \\
\hline & ts2 & -3415.63645820 & 3.91 & -3415.209009 & 20.33 & 16.41 \\
\hline & pc2 & -3415.65727572 & -9.15 & -3415.228378 & 8.17 & 17.32 \\
\hline & $14 \mathrm{e}$ & -3415.64420225 & -0.94 & -3415.244678 & -2.06 & -1.11 \\
\hline
\end{tabular}

\begin{tabular}{|c|c|c|c|c|c|c|c|c|}
\hline \multicolumn{3}{|c|}{ BP86/basis2 (gas-phase) } & \multicolumn{3}{|c|}{ BP86/basis2 (benzene) } & \multicolumn{3}{|c|}{ BP86/basis2 (dichloromethane) } \\
\hline $\begin{array}{c}\mathbf{E}_{\mathrm{sp}} \\
\text { [hartree] }\end{array}$ & $\begin{array}{c}\Delta \mathbf{E}_{\mathrm{sp}} \\
{[\mathrm{kcal} / \mathrm{mol}]}\end{array}$ & $\begin{array}{c}\Delta \mathbf{G}_{\mathrm{sp}} \\
{[\mathrm{kcal} / \mathrm{mol}]}\end{array}$ & $\begin{array}{c}\mathbf{E}_{\mathrm{sp}} \\
\text { [hartree] }\end{array}$ & $\begin{array}{c}\Delta \mathbf{E}_{\mathrm{sp}} \\
{[\mathrm{kcal} / \mathrm{mol}]}\end{array}$ & $\begin{array}{c}\Delta \mathbf{G}_{\mathrm{sp}} \\
{[\mathrm{kcal} / \mathrm{mol}]}\end{array}$ & $\begin{array}{c}\mathbf{E}_{\mathrm{sp}} \\
\text { [hartree] }\end{array}$ & $\begin{array}{c}\Delta \mathbf{E}_{\mathrm{sp}} \\
{[\mathrm{kcal} / \mathrm{mol}]}\end{array}$ & $\begin{array}{c}\Delta \mathbf{G}_{\text {sp }} \\
{[\mathrm{kcal} / \mathrm{mol}]}\end{array}$ \\
\hline-3416.08024862 & 0.00 & 0.00 & -3416.09152066 & 0.00 & 0.00 & -3416.10337581 & 0.00 & 0.00 \\
\hline-3416.09020991 & -6.25 & 9.78 & -3416.09738362 & -3.68 & 12.35 & -3416.10493883 & -0.98 & 15.05 \\
\hline-3416.07632070 & 2.46 & 18.51 & -3416.08438066 & 4.48 & 20.53 & -3416.09296316 & 6.53 & 22.58 \\
\hline-3416.08036284 & -0.07 & 14.62 & -3416.08865186 & 1.80 & 16.49 & -3416.09756486 & 3.65 & 18.34 \\
\hline-3416.07176121 & 5.33 & 20.98 & -3416.08000675 & 7.23 & 22.88 & -3416.08899797 & 9.02 & 24.67 \\
\hline-3416.08565948 & -3.40 & 13.33 & -3416.09273892 & -0.76 & 15.96 & -3416.10013805 & 2.03 & 18.75 \\
\hline-3416.08320257 & -1.85 & -3.46 & -3416.09470223 & -2.00 & -3.61 & -3416.10692506 & -2.23 & -3.84 \\
\hline-3416.08024862 & 0.00 & 0.00 & -3416.09152066 & 0.00 & 0.00 & -3416.10337581 & 0.00 & 0.00 \\
\hline-3416.08879406 & -5.36 & 9.88 & -3416.09601780 & -2.82 & 12.42 & -3416.10361338 & -0.15 & 15.09 \\
\hline-3416.07597990 & 2.68 & 18.39 & -3416.08380685 & 4.84 & 20.55 & -3416.09206338 & 7.10 & 22.81 \\
\hline-3416.08194345 & -1.06 & 14.20 & -3416.08997962 & 0.97 & 16.23 & -3416.09857731 & 3.01 & 18.27 \\
\hline-3416.07069687 & 5.99 & 22.41 & -3416.07835768 & 8.26 & 24.67 & -3416.08664298 & 10.50 & 26.91 \\
\hline-3416.09227245 & -7.55 & 9.78 & -3416.09923019 & -4.84 & 12.48 & -3416.10644137 & -1.92 & 15.40 \\
\hline-3416.08173534 & -0.93 & -2.04 & -3416.09272232 & -0.75 & -1.87 & -3416.10430349 & -0.58 & -1.69 \\
\hline $\begin{array}{l}\mathrm{E} \ldots \ldots \ldots \ldots \text { gas } \\
\mathrm{G} \ldots \ldots \ldots \text { sum of } \\
\Delta \mathrm{E} \ldots \ldots \ldots \text { relativ } \\
\Delta \mathrm{G} \ldots \ldots \ldots \text { relativ } \\
\Delta \mathrm{G}_{\mathrm{cor}} \ldots \ldots \text { gas ph} \\
\mathrm{E}_{\mathrm{sp}} \ldots \ldots \ldots \text { single } \\
\Delta \mathrm{E}_{\mathrm{sp}} \ldots \ldots \text { relativ } \\
\Delta \mathrm{G}_{\mathrm{sp}} \ldots \ldots \text { relativ }\end{array}$ & $\begin{array}{l}\text { e electronic en } \\
\text { lectronic and } t \\
\text { electronic ener } \\
\text { ree energy in } 1 \\
\text { e thermal corre } \\
\text { int electronic } \\
\text { ingle point ele } \\
\text { single point fre }\end{array}$ & $\begin{array}{l}\text { gy in hartree } \\
\text { ermal free ener } \\
\text { y in kcal/mol y } \\
\text { al/mol with ba } \\
\text { tion to free en } \\
\text { nergy in gas ph } \\
\text { tronic energy } \mathrm{i} \\
\text { energy in gas }\end{array}$ & $\begin{array}{l}\text { ith basis } 1 \\
\text { ies with basis } 1 \\
\text { th basis } 1 \\
\text { is } 1 \\
\text { gy in kcal/mol obta } \\
\text { se or solution in ha } \\
\text { gas phase or soluti } \\
\text { hase or solution in } 1\end{array}$ & $\begin{array}{l}\text { ned with basis } \\
\text { ree with basis2 } \\
\text { in } \mathrm{kcal} / \mathrm{mol} \mathrm{vol}\left(\Delta \mathrm{G}_{\mathrm{sp}}\right.\end{array}$ & $\begin{array}{l}(\Delta \mathrm{G}=\Delta \mathrm{E}+\Delta \\
\mathrm{h} \text { basis } 2 \\
\mathrm{E}_{\mathrm{sp}} / \text { basis } 2+\end{array}$ & (corr) & & \\
\hline
\end{tabular}


Table S23. Homodimerization of propene with catalyst Ru-4b (pathways EMe', ZMe' in Figure 10)<smiles>CC=C[Ge](Cl)(CC)C(C)(Cl)C1=NCCN1C</smiles>

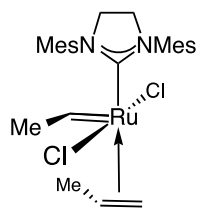

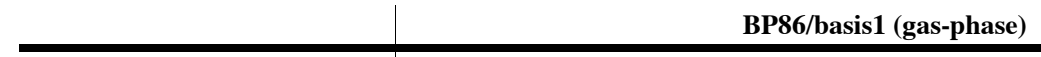

\begin{tabular}{|c|c|c|c|c|c|c|}
\hline pathway & & $\begin{array}{c}\mathbf{E} \\
\text { [hartree] }\end{array}$ & $\begin{array}{c}\Delta \mathbf{E} \\
{[\mathrm{kcal} / \mathrm{mol}]}\end{array}$ & $\begin{array}{c}\mathbf{G} \\
{[\text { hartree] }}\end{array}$ & $\begin{array}{c}\Delta \mathbf{G} \\
{[\mathrm{kcal} / \mathrm{mol}]}\end{array}$ & $\begin{array}{c}\Delta \mathbf{G}_{\text {Corr }} \\
{[\mathrm{kcal} / \mathrm{mol}]}\end{array}$ \\
\hline \multirow{7}{*}{ ZMe' } & $14 \mathrm{e}$ & -2137.48924659 & 0.00 & -2137.039223 & 0.00 & 0.00 \\
\hline & pc1 & -2137.50243876 & -8.28 & -2137.028699 & 6.60 & 14.88 \\
\hline & ts1 & -2137.50115230 & -7.47 & -2137.024328 & 9.35 & 16.82 \\
\hline & mcb & -2137.51048429 & -13.33 & -2137.030861 & 5.25 & 18.57 \\
\hline & ts2 & -2137.49483286 & -3.51 & -2137.019588 & 12.32 & 15.83 \\
\hline & pc2 & -2137.49636017 & -4.46 & -2137.023002 & 10.18 & 14.64 \\
\hline & $14 \mathrm{e}$ & -2137.48074411 & 5.34 & -2137.030346 & 5.57 & 0.24 \\
\hline \multirow{7}{*}{ EMe' } & $14 \mathrm{e}$ & -2137.48924659 & 0.00 & -2137.039223 & 0.00 & 0.00 \\
\hline & pc1 & -2137.50217467 & -8.11 & -2137.028271 & 6.87 & 14.98 \\
\hline & ts1 & -2137.50174728 & -7.84 & -2137.024699 & 9.11 & 16.96 \\
\hline & mcb & -2137.51241561 & -14.54 & -2137.033312 & 3.71 & 18.25 \\
\hline & ts2 & -2137.49459268 & -3.35 & -2137.019064 & 12.65 & 16.00 \\
\hline & pc2 & -2137.49721814 & -5.00 & -2137.023894 & 9.62 & 14.62 \\
\hline & $14 \mathrm{e}$ & -2137.48269753 & 4.11 & -2137.031375 & 4.92 & 0.82 \\
\hline
\end{tabular}

\begin{tabular}{|c|c|c|c|c|c|c|c|c|}
\hline \multicolumn{3}{|c|}{ BP86/basis2 (gas-phase) } & \multicolumn{3}{|c|}{ BP86/basis2 (benzene) } & \multicolumn{3}{|c|}{ BP86/basis2 (dichloromethane) } \\
\hline $\begin{array}{c}\mathbf{E}_{\mathrm{sp}} \\
\text { [hartree] }\end{array}$ & $\begin{array}{c}\Delta \mathbf{E}_{\mathrm{sp}} \\
{[\mathrm{kcal} / \mathrm{mol}]}\end{array}$ & $\begin{array}{c}\Delta \mathbf{G}_{\mathrm{sp}} \\
{[\mathrm{kcal} / \mathrm{mol}]}\end{array}$ & $\begin{array}{c}\mathbf{E}_{\mathrm{sp}} \\
\text { [hartree] }\end{array}$ & $\begin{array}{c}\Delta \mathbf{E}_{\mathrm{sp}} \\
{[\mathrm{kcal} / \mathrm{mol}]}\end{array}$ & $\begin{array}{c}\Delta \mathbf{G}_{\text {sp }} \\
{[\mathrm{kcal} / \mathrm{mol}]}\end{array}$ & $\begin{array}{c}\mathbf{E}_{\mathrm{sp}} \\
\text { [hartree] }\end{array}$ & $\begin{array}{c}\Delta \mathbf{E}_{\mathrm{sp}} \\
{[\mathrm{kcal} / \mathrm{mol}]}\end{array}$ & $\begin{array}{c}\Delta \mathbf{G}_{\text {sp }} \\
{[\mathrm{kcal} / \mathrm{mol}]}\end{array}$ \\
\hline-2137.83801771 & 0.00 & 0.00 & -2137.84711502 & 0.00 & 0.00 & -2137.85668245 & 0.00 & 0.00 \\
\hline-2137.84495707 & -4.35 & 10.53 & -2137.85225257 & -3.22 & 11.66 & -2137.85999027 & -2.08 & 12.81 \\
\hline-2137.84363678 & -3.53 & 13.29 & -2137.85069452 & -2.25 & 14.57 & -2137.85828492 & -1.01 & 15.81 \\
\hline-2137.85271981 & -9.23 & 9.35 & -2137.85924771 & -7.61 & 10.96 & -2137.86623754 & -6.00 & 12.58 \\
\hline-2137.83732428 & 0.44 & 16.26 & -2137.84448970 & 1.65 & 17.47 & -2137.85227237 & 2.77 & 18.59 \\
\hline-2137.83878851 & -0.48 & 14.16 & -2137.84592199 & 0.75 & 15.39 & -2137.85367766 & 1.89 & 16.53 \\
\hline-2137.82913575 & 5.57 & 5.81 & -2137.83807563 & 5.67 & 5.91 & -2137.84749192 & 5.77 & 6.00 \\
\hline-2137.83801771 & 0.00 & 0.00 & -2137.84711502 & 0.00 & 0.00 & -2137.85668245 & 0.00 & 0.00 \\
\hline-2137.84473410 & -4.21 & 10.77 & -2137.85191880 & -3.01 & 11.97 & -2137.85963204 & -1.85 & 13.13 \\
\hline-2137.84419562 & -3.88 & 13.08 & -2137.85131481 & -2.64 & 14.32 & -2137.85900409 & -1.46 & 15.50 \\
\hline-2137.85451383 & -10.35 & 7.90 & -2137.86100902 & -8.72 & 9.53 & -2137.86796591 & -7.08 & 11.17 \\
\hline-2137.83681646 & 0.75 & 16.76 & -2137.84390282 & 2.02 & 18.02 & -2137.85163696 & 3.17 & 19.17 \\
\hline-2137.83961959 & -1.01 & 13.62 & -2137.84681442 & 0.19 & 14.81 & -2137.85445710 & 1.40 & 16.02 \\
\hline-2137.83101273 & 4.40 & 5.21 & -2137.83990140 & 4.53 & 5.34 & -2137.84927545 & 4.65 & 5.46 \\
\hline \multicolumn{9}{|c|}{ E ......... gas phase electronic energy in hartree with basis 1} \\
\hline \multicolumn{9}{|c|}{ G ......... sum of electronic and thermal free energies with basis 1} \\
\hline \multicolumn{9}{|c|}{$\Delta \mathrm{E} \ldots \ldots \ldots$ relative electronic energy in $\mathrm{kcal} / \mathrm{mol}$ with basis 1} \\
\hline \multicolumn{9}{|c|}{$\Delta \mathrm{G}$........ relative free energy in $\mathrm{kcal} / \mathrm{mol}$ with basis 1} \\
\hline \multicolumn{9}{|c|}{$\Delta \mathrm{G}_{\text {corr }} \ldots$. gas phase thermal correction to free energy in $\mathrm{kcal} / \mathrm{mol}$ obtained with basis $1\left(\Delta \mathrm{G}=\Delta \mathrm{E}+\Delta \mathrm{G}_{\text {corr }}\right)$} \\
\hline \multicolumn{9}{|c|}{$\mathrm{E}_{\mathrm{sp}} \ldots \ldots \ldots$ single point electronic energy in gas phase or solution in hartree with basis 2} \\
\hline \multicolumn{9}{|c|}{$\Delta \mathrm{E}_{\mathrm{sp}} \ldots \ldots$ relative single point electronic energy in gas phase or solution in $\mathrm{kcal} / \mathrm{mol}$ with basis 2} \\
\hline & & & & & & rr (basis1) & & \\
\hline
\end{tabular}




\section{Table S24. ROCM with catalyst Ru-5b and styrene (pathways C1, CD1, D1 in Figure S23)}

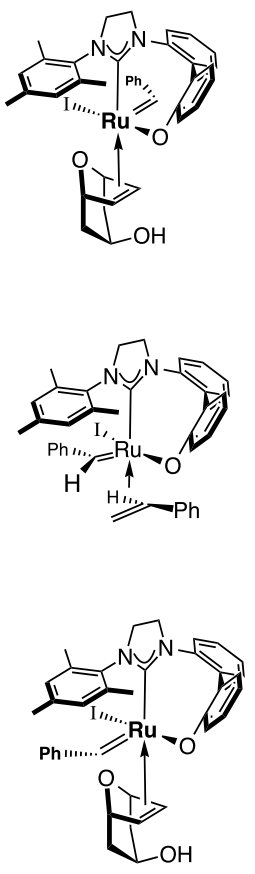

\begin{tabular}{|c|c|c|c|c|c|c|}
\hline & & \multicolumn{5}{|c|}{ BP86/basis1 (gas-phase) } \\
\hline pathway & & $\begin{array}{c}\Delta \mathbf{E} \\
{[\text { [hartree] }}\end{array}$ & $\begin{array}{c}\Delta \mathbf{E} \\
{[\mathrm{kcal} / \mathrm{mol}]}\end{array}$ & $\begin{array}{c}\Delta \mathbf{G} \\
{[\text { hartree] }}\end{array}$ & $\begin{array}{c}\Delta \mathbf{G} \\
{[\mathrm{kcal} / \mathrm{mol}]}\end{array}$ & $\begin{array}{c}\Delta \mathbf{G}_{\text {Corr }} \\
{[\mathrm{kcal} / \mathrm{mol}]}\end{array}$ \\
\hline \multirow{5}{*}{ C1 } & pc2 & -1913.23501090 & -9.56 & -1912.646513 & 7.51 & 17.07 \\
\hline & ts2 & -1913.21129724 & 5.32 & -1912.623458 & 21.98 & 16.66 \\
\hline & mcb & -1913.21925307 & 0.33 & -1912.628445 & 18.85 & 18.52 \\
\hline & ts1 & -1913.20299812 & 10.53 & -1912.615874 & 26.73 & 16.21 \\
\hline & pc1 & -1913.21385014 & 3.72 & -1912.625773 & 20.52 & 16.81 \\
\hline \multirow{7}{*}{ CD1 } & 5-endo & -1913.21319288 & 4.13 & -1912.652596 & 3.69 & -0.44 \\
\hline & pc-endo & -1913.21779861 & 1.24 & -1912.628803 & 18.62 & 17.38 \\
\hline & ts-endo & -1913.20679340 & 8.14 & -1912.617043 & 26.00 & 17.86 \\
\hline & mcb & -1913.21890666 & 0.54 & -1912.627291 & 19.57 & 19.03 \\
\hline & ts-exo & -1913.20910492 & 6.69 & -1912.619396 & 24.52 & 17.83 \\
\hline & pc-exo & -1913.22305586 & -2.06 & -1912.635727 & 14.28 & 16.34 \\
\hline & 5-exo & -1913.21977129 & 0.00 & -1912.658478 & 0.00 & 0.00 \\
\hline \multirow{5}{*}{ D1 } & pc1 & -1913.22307035 & -2.07 & -1912.637406 & 13.22 & 15.29 \\
\hline & ts1 & -1913.21201333 & 4.87 & -1912.623449 & 21.98 & 17.11 \\
\hline & mcb & -1913.22050629 & -0.46 & -1912.630494 & 17.56 & 18.02 \\
\hline & ts2 & -1913.20531411 & 9.07 & -1912.616327 & 26.45 & 17.38 \\
\hline & pc2 & -1913.22137079 & -1.00 & -1912.633587 & 15.62 & 16.62 \\
\hline
\end{tabular}

\begin{tabular}{|c|c|c|c|c|c|c|c|c|}
\hline \multicolumn{3}{|c|}{ BP86/basis2 (gas-phase) } & \multicolumn{3}{|c|}{ BP86/basis2 (benzene) } & \multicolumn{3}{|c|}{ BP86/basis2 (dichloromethane) } \\
\hline $\begin{array}{c}\Delta \mathbf{E} \\
\text { [hartree] }\end{array}$ & $\begin{array}{c}\Delta \mathbf{E} \\
{[\mathrm{kcal} / \mathrm{mol}]}\end{array}$ & $\begin{array}{c}\Delta \mathbf{G} \\
{[\mathrm{kcal} / \mathrm{mol}]}\end{array}$ & $\begin{array}{c}\Delta \mathbf{E} \\
{[\text { hartree] }}\end{array}$ & $\begin{array}{c}\Delta \mathbf{E} \\
{[\mathrm{kcal} / \mathrm{mol}]}\end{array}$ & $\begin{array}{c}\Delta \mathbf{G} \\
{[\mathrm{kcal} / \mathrm{mol}]}\end{array}$ & $\begin{array}{c}\Delta \mathbf{E} \\
{[\text { hartree] }}\end{array}$ & $\begin{array}{c}\Delta \mathbf{E} \\
{[\mathrm{kcal} / \mathrm{mol}]}\end{array}$ & $\begin{array}{c}\Delta \mathbf{G} \\
{[\mathrm{kcal} / \mathrm{mol}]}\end{array}$ \\
\hline-1913.69525731 & -6.74 & 10.33 & -1913.70283548 & -4.53 & 12.54 & -1913.71100659 & -2.41 & 14.67 \\
\hline-1913.67245795 & 7.57 & 24.23 & -1913.68174766 & 8.71 & 25.36 & -1913.69161576 & 9.76 & 26.42 \\
\hline-1913.68055673 & 2.49 & 21.01 & -1913.68973376 & 3.69 & 22.21 & -1913.69944905 & 4.85 & 23.37 \\
\hline-1913.66488132 & 12.32 & 28.53 & -1913.67422549 & 13.43 & 29.63 & -1913.68433756 & 14.33 & 30.54 \\
\hline-1913.67606361 & 5.31 & 22.11 & -1913.68444889 & 7.01 & 23.82 & -1913.69342248 & 8.63 & 25.44 \\
\hline-1913.67803679 & 4.07 & 3.63 & -1913.68966283 & 3.74 & 3.30 & -1913.70232255 & 3.04 & 2.61 \\
\hline-1913.67666589 & 4.93 & 22.31 & -1913.68521612 & 6.53 & 23.91 & -1913.69448924 & 7.96 & 25.34 \\
\hline-1913.66625206 & 11.46 & 29.32 & -1913.67587134 & 12.39 & 30.25 & -1913.68573882 & 13.45 & 31.31 \\
\hline-1913.67871880 & 3.64 & 22.67 & -1913.68768040 & 4.98 & 24.01 & -1913.69660446 & 6.63 & 25.66 \\
\hline-1913.66825355 & 10.21 & 28.04 & -1913.67761319 & 11.30 & 29.13 & -1913.68726314 & 12.49 & 30.32 \\
\hline-1913.68230792 & 1.39 & 17.73 & -1913.69125168 & 2.74 & 19.08 & -1913.70048477 & 4.20 & 20.53 \\
\hline-1913.68451938 & 0.00 & 0.00 & -1913.69562059 & 0.00 & 0.00 & -1913.70717307 & 0.00 & 0.00 \\
\hline-1913.68448237 & 0.02 & 15.32 & -1913.69301968 & 1.63 & 16.93 & -1913.70201131 & 3.24 & 18.53 \\
\hline-1913.67354646 & 6.89 & 24.00 & -1913.68240014 & 8.30 & 25.41 & -1913.69193024 & 9.57 & 26.68 \\
\hline-1913.68156756 & 1.85 & 19.87 & -1913.69065988 & 3.11 & 21.13 & -1913.70052840 & 4.17 & 22.19 \\
\hline-1913.66667728 & 11.20 & 28.57 & -1913.67631230 & 12.12 & 29.49 & -1913.68710674 & 12.59 & 29.97 \\
\hline-1913.68228661 & 1.40 & 18.02 & -1913.69031326 & 3.33 & 19.95 & -1913.69950843 & 4.81 & 21.43 \\
\hline
\end{tabular}


Table S25. ROCM with catalyst Ru-5b and styrene (pathways C2, CD2, D2 in Figure S23)

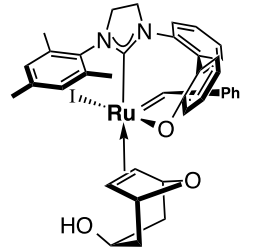

$$
\begin{array}{c|c}
\text { BP86/basis1 (gas-phase) } \\
\hline \text { pathay }
\end{array}
$$

\begin{tabular}{|c|c|c|c|c|c|c|}
\hline pathway & & $\begin{array}{c}\Delta \mathbf{E} \\
\text { [hartree] }\end{array}$ & $\begin{array}{c}\Delta \mathbf{E} \\
{[\mathrm{kcal} / \mathrm{mol}]}\end{array}$ & $\begin{array}{c}\Delta \mathbf{G} \\
\text { [hartree] }\end{array}$ & $\begin{array}{c}\Delta \mathbf{G} \\
{[\mathrm{kcal} / \mathbf{m o l}]}\end{array}$ & $\begin{array}{c}\Delta \mathbf{G}_{\text {Corr }} \\
{[\mathbf{k c a l} / \mathbf{m o l}]}\end{array}$ \\
\hline \multirow{5}{*}{$\mathrm{C} 2$} & pc2 & -1913.23221765 & -7.81 & -1912.644824 & 8.57 & 16.38 \\
\hline & ts2 & -1913.21488395 & 3.07 & -1912.625795 & 20.51 & 17.44 \\
\hline & mcb & -1913.22397905 & -2.64 & -1912.633112 & 15.92 & 18.56 \\
\hline & ts1 & -1913.20452989 & 9.56 & -1912.618529 & 25.07 & 15.50 \\
\hline & pc1 & -1913.21853608 & 0.78 & -1912.632038 & 16.59 & 15.82 \\
\hline \multirow{7}{*}{ CD2 } & 5-endo & -1913.21319288 & 4.13 & -1912.652596 & 3.69 & -0.44 \\
\hline & pc-endo & -1913.21464096 & 3.22 & -1912.626905 & 19.81 & 16.59 \\
\hline & ts-endo & -1913.20363987 & 10.12 & -1912.613514 & 28.22 & 18.09 \\
\hline & mcb & -1913.21960562 & 0.10 & -1912.627758 & 19.28 & 19.17 \\
\hline & ts-exo & -1913.21225696 & 4.72 & -1912.622735 & 22.43 & 17.71 \\
\hline & pc-exo & -1913.22281279 & -1.91 & -1912.635634 & 14.33 & 16.24 \\
\hline & 5-exo & -1913.21977129 & 0.00 & -1912.658478 & 0.00 & 0.00 \\
\hline \multirow{5}{*}{ D2 } & pe1 & -1913.22572262 & -3.73 & -1912.638556 & 12.50 & 16.24 \\
\hline & ts1 & -1913.21473368 & 3.16 & -1912.626947 & 19.79 & 16.62 \\
\hline & mcb & -1913.22412818 & -2.73 & -1912.637452 & 13.19 & 15.93 \\
\hline & ts2 & -1913.20812940 & 7.31 & -1912.621043 & 23.49 & 16.19 \\
\hline & pc2 & -1913.22198685 & -1.39 & -1912.635368 & 14.50 & 15.89 \\
\hline
\end{tabular}

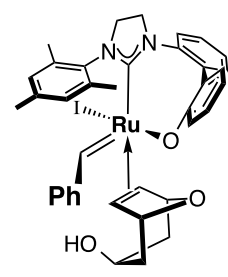

pc2 $\quad-1913.22198685$

BP86/basis2 (dichloromethane)

\begin{tabular}{|c|c|c|c|c|c|c|c|c|}
\hline \multicolumn{3}{|c|}{ BP86/basis2 (gas-phase) } & \multicolumn{3}{|c|}{ BP86/basis2 (benzene) } & \multicolumn{3}{|c|}{ BP86/basis2 (dichloromethane) } \\
\hline $\begin{array}{c}\Delta \mathrm{E} \\
{[\text { hartree] }}\end{array}$ & $\begin{array}{c}\Delta E \\
{[\mathrm{kcal} / \mathrm{mol}]}\end{array}$ & $\begin{array}{c}\Delta \mathbf{G} \\
{[\mathrm{kcal} / \mathrm{mol}]}\end{array}$ & $\begin{array}{c}\Delta \mathbf{E} \\
{[\text { hartree] }}\end{array}$ & $\begin{array}{c}\Delta E \\
{[\mathrm{kcal} / \mathrm{mol}]}\end{array}$ & $\begin{array}{c}\Delta \mathbf{G} \\
{[\mathrm{kcal} / \mathrm{mol}]}\end{array}$ & $\begin{array}{c}\Delta \mathbf{E} \\
\text { [hartree] }\end{array}$ & $\begin{array}{c}\Delta \mathbf{E} \\
{[\mathrm{kcal} / \mathrm{mol}]}\end{array}$ & $\begin{array}{c}\Delta \mathbf{G} \\
{[\mathrm{kcal} / \mathrm{mol}]}\end{array}$ \\
\hline-1913.69354150 & -5.66 & 10.72 & -1913.70164301 & -3.78 & 12.60 & -1913.71031431 & -1.97 & 14.41 \\
\hline-1913.67569404 & 5.54 & 22.98 & -1913.68471112 & 6.85 & 24.29 & -1913.69398165 & 8.28 & 25.72 \\
\hline-1913.68488473 & -0.23 & 18.33 & -1913.69380426 & 1.14 & 19.70 & -1913.70294338 & 2.65 & 21.21 \\
\hline-1913.66602905 & 11.60 & 27.11 & -1913.67551345 & 12.62 & 28.12 & -1913.68567462 & 13.49 & 28.99 \\
\hline-1913.68026482 & 2.67 & 18.49 & -1913.68877579 & 4.30 & 20.11 & -1913.69770223 & 5.94 & 21.76 \\
\hline-1913.67803679 & 4.07 & 3.63 & -1913.68966283 & 3.74 & 3.30 & -1913.70232255 & 3.04 & 2.61 \\
\hline-1913.67343372 & 6.96 & 23.55 & -1913.68320483 & 7.79 & 24.38 & -1913.69342363 & 8.63 & 25.22 \\
\hline-1913.66292444 & 13.55 & 31.64 & -1913.67221138 & 14.69 & 32.78 & -1913.68189408 & 15.86 & 33.96 \\
\hline-1913.67934732 & 3.25 & 22.42 & -1913.68811092 & 4.71 & 23.89 & -1913.69706158 & 6.35 & 25.52 \\
\hline-1913.67175696 & 8.01 & 25.72 & -1913.68072847 & 9.34 & 27.06 & -1913.68974575 & 10.94 & 28.65 \\
\hline-1913.68235533 & 1.36 & 17.60 & -1913.69096290 & 2.92 & 19.17 & -1913.69991091 & 4.56 & 20.80 \\
\hline-1913.68451938 & 0.00 & 0.00 & -1913.69562059 & 0.00 & 0.00 & -1913.70717307 & 0.00 & 0.00 \\
\hline-1913.68723341 & -1.70 & 14.53 & -1913.69542249 & 0.12 & 16.36 & -1913.70375259 & 2.15 & 18.38 \\
\hline-1913.67581579 & 5.46 & 22.09 & -1913.68474166 & 6.83 & 23.45 & -1913.69399648 & 8.27 & 24.89 \\
\hline-1913.68491048 & -0.25 & 15.68 & -1913.69395054 & 1.05 & 16.98 & -1913.70333736 & 2.41 & 18.33 \\
\hline-1913.66884435 & 9.84 & 26.02 & -1913.67842858 & 10.79 & 26.97 & -1913.68860903 & 11.65 & 27.83 \\
\hline-1913.68310078 & 0.89 & 16.78 & -1913.69221585 & 2.14 & 18.03 & -1913.70204003 & 3.22 & 19.11 \\
\hline
\end{tabular}


Table S26. ROCM with catalyst Ru-5b and styrene (pathways C3, CD3, D3 in Figure S23)

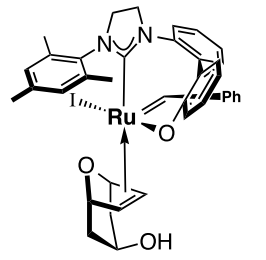

\begin{tabular}{|c|c|c|c|c|c|c|}
\hline \multirow[b]{2}{*}{ pathway } & & \multicolumn{5}{|c|}{ BP86/basis1 (gas-phase) } \\
\hline & & $\begin{array}{c}\Delta \mathbf{E} \\
\text { [hartree] }\end{array}$ & $\begin{array}{c}\Delta E \\
{[\mathrm{kcal} / \mathrm{mol}]}\end{array}$ & $\begin{array}{c}\Delta \mathbf{G} \\
{[\text { hartree] }}\end{array}$ & $\begin{array}{c}\Delta \mathbf{G} \\
{[\mathrm{kcal} / \mathrm{mol}]}\end{array}$ & $\begin{array}{c}\Delta \mathbf{G}_{\mathrm{Corr}} \\
{[\mathrm{kcal} / \mathrm{mol}]}\end{array}$ \\
\hline \multirow{5}{*}{$\mathbf{C 3}$} & pc2 & -1913.23960597 & -12.45 & -1912.648454 & 6.29 & 18.74 \\
\hline & ts2 & -1913.21390843 & 3.68 & -1912.625764 & 20.53 & 16.85 \\
\hline & mcb & -1913.22483669 & -3.18 & -1912.634304 & 15.17 & 18.35 \\
\hline & ts1 & -1913.20110523 & 11.71 & -1912.614200 & 27.78 & 16.07 \\
\hline & pc1 & -1913.21853609 & 0.78 & -1912.632038 & 16.59 & 15.82 \\
\hline \multirow{7}{*}{ CD3 } & 5-endo & -1913.21319288 & 4.13 & -1912.652596 & 3.69 & -0.44 \\
\hline & pc-endo & -1913.21402592 & 3.61 & -1912.627923 & 19.17 & 15.57 \\
\hline & ts-endo & -1913.20210261 & 11.09 & -1912.612729 & 28.71 & 17.62 \\
\hline & mcb & -1913.21655750 & 2.02 & -1912.624908 & 21.07 & 19.05 \\
\hline & ts-exo & -1913.20793742 & 7.43 & -1912.619510 & 24.45 & 17.03 \\
\hline & pc-exo & -1913.22137706 & -1.01 & -1912.635311 & 14.54 & 15.55 \\
\hline & 5-exo & -1913.21977129 & 0.00 & -1912.658478 & 0.00 & 0.00 \\
\hline \multirow{5}{*}{ D3 } & pc1 & -1913.22572262 & -3.73 & -1912.638556 & 12.50 & 16.24 \\
\hline & ts1 & -1913.21024238 & 5.98 & -1912.623816 & 21.75 & 15.77 \\
\hline & mcb & -1913.22448054 & -2.96 & -1912.636007 & 14.10 & 17.06 \\
\hline & ts2 & -1913.20893206 & 6.80 & -1912.622132 & 22.81 & 16.01 \\
\hline & pc2 & -1913.22920803 & -5.92 & -1912.639925 & 11.64 & 17.56 \\
\hline
\end{tabular}

\begin{tabular}{|c|c|c|c|c|c|c|c|c|}
\hline \multicolumn{3}{|c|}{ BP86/basis2 (gas-phase) } & \multicolumn{3}{|c|}{ BP86/basis2 (benzene) } & \multicolumn{3}{|c|}{ BP86/basis2 (dichloromethane) } \\
\hline $\begin{array}{c}\Delta \mathbf{E} \\
{[\text { hartree] }}\end{array}$ & $\begin{array}{c}\Delta \mathbf{E} \\
{[\mathrm{kcal} / \mathrm{mol}]}\end{array}$ & $\begin{array}{c}\Delta \mathbf{G} \\
{[\mathrm{kcal} / \mathrm{mol}]}\end{array}$ & $\begin{array}{c}\Delta \mathbf{E} \\
{[\text { hartree] }}\end{array}$ & $\begin{array}{c}\Delta \mathbf{E} \\
{[\mathrm{kcal} / \mathrm{mol}]}\end{array}$ & $\begin{array}{c}\Delta \mathbf{G} \\
{[\mathrm{kcal} / \mathrm{mol}]}\end{array}$ & $\begin{array}{c}\Delta \mathbf{E} \\
{[\text { hartree] }}\end{array}$ & $\begin{array}{c}\Delta \mathbf{E} \\
{[\mathrm{kcal} / \mathrm{mol}]}\end{array}$ & $\begin{array}{c}\Delta \mathbf{G} \\
{[\mathrm{kcal} / \mathrm{mol}]}\end{array}$ \\
\hline-1913.69955652 & -9.44 & 9.30 & -1913.70690212 & -7.08 & 11.66 & -1913.71481414 & -4.79 & 13.94 \\
\hline-1913.67558668 & 5.61 & 22.45 & -1913.68536506 & 6.44 & 23.28 & -1913.69554075 & 7.30 & 24.15 \\
\hline-1913.68648800 & -1.24 & 17.11 & -1913.69593646 & -0.20 & 18.15 & -1913.70567877 & 0.94 & 19.29 \\
\hline-1913.66289242 & 13.57 & 29.64 & -1913.67250471 & 14.51 & 30.58 & -1913.68259849 & 15.42 & 31.49 \\
\hline-1913.68026486 & 2.67 & 18.49 & -1913.68877583 & 4.30 & 20.11 & -1913.69770225 & 5.94 & 21.76 \\
\hline-1913.67803679 & 4.07 & 3.63 & -1913.68966283 & 3.74 & 3.30 & -1913.70232255 & 3.04 & 2.61 \\
\hline-1913.67372200 & 6.78 & 22.34 & -1913.68288048 & 7.99 & 23.56 & -1913.69309198 & 8.84 & 24.40 \\
\hline-1913.66175911 & 14.28 & 31.90 & -1913.67129775 & 15.26 & 32.88 & -1913.68150676 & 16.11 & 33.73 \\
\hline-1913.67644623 & 5.07 & 24.11 & -1913.68557913 & 6.30 & 25.35 & -1913.69520652 & 7.51 & 26.56 \\
\hline-1913.66738327 & 10.75 & 27.78 & -1913.67686734 & 11.77 & 28.79 & -1913.68690602 & 12.72 & 29.74 \\
\hline-1913.68085876 & 2.30 & 17.84 & -1913.68983128 & 3.63 & 19.18 & -1913.69937723 & 4.89 & 20.44 \\
\hline-1913.68451938 & 0.00 & 0.00 & -1913.69562059 & 0.00 & 0.00 & -1913.70717307 & 0.00 & 0.00 \\
\hline-1913.68723342 & -1.70 & 14.53 & -1913.69542259 & 0.12 & 16.36 & -1913.70375281 & 2.15 & 18.38 \\
\hline-1913.67163259 & 8.09 & 23.86 & -1913.68104212 & 9.15 & 24.92 & -1913.69096579 & 10.17 & 25.94 \\
\hline-1913.68586110 & -0.84 & 16.21 & -1913.69557182 & 0.03 & 17.09 & -1913.70577366 & 0.88 & 17.93 \\
\hline-1913.67003813 & 9.09 & 25.09 & -1913.68001767 & 9.79 & 25.80 & -1913.69079186 & 10.28 & 26.29 \\
\hline-1913.68879405 & -2.68 & 14.88 & -1913.69726237 & -1.03 & 16.53 & -1913.70699793 & 0.11 & 17.67 \\
\hline
\end{tabular}


Table S27. ROCM with catalyst Ru-5b and styrene (pathways C4, CD4, D4 in Figure S23)

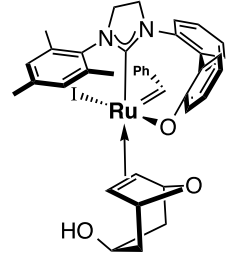

$$
\begin{array}{l|r} 
& \text { BP86/basis1 (gas-phase) }
\end{array}
$$

\begin{tabular}{|c|c|c|c|c|c|c|}
\hline pathway & & $\begin{array}{c}\Delta \mathbf{E} \\
{[\text { hartree] }}\end{array}$ & $\begin{array}{c}\Delta \mathbf{E} \\
{[\mathrm{kcal} / \mathrm{mol}]}\end{array}$ & $\begin{array}{c}\Delta \mathbf{G} \\
{[\text { hartree] }}\end{array}$ & $\begin{array}{c}\Delta \mathbf{G} \\
{[\mathrm{kcal} / \mathrm{mol}]}\end{array}$ & $\begin{array}{c}\Delta \mathbf{G}_{\mathrm{Corr}} \\
{[\mathrm{kcal} / \mathrm{mol}]}\end{array}$ \\
\hline \multirow{5}{*}{ C4 } & pc2 & -1913.23213498 & -7.76 & -1912.644322 & 8.88 & 16.64 \\
\hline & ts2 & -1913.21093979 & 5.54 & -1912.624918 & 21.06 & 15.52 \\
\hline & mcb & -1913.22327484 & -2.20 & -1912.634214 & 15.23 & 17.42 \\
\hline & ts1 & -1913.20397271 & 9.91 & -1912.617651 & 25.62 & 15.71 \\
\hline & pc1 & -1913.21385013 & 3.72 & -1912.625773 & 20.52 & 16.81 \\
\hline \multirow{7}{*}{ CD4 } & 5-endo & -1913.21319288 & 4.13 & -1912.652596 & 3.69 & -0.44 \\
\hline & pc-endo & -1913.21586889 & 2.45 & -1912.627421 & 19.49 & 17.04 \\
\hline & ts-endo & -1913.20479501 & 9.40 & -1912.617175 & 25.92 & 16.52 \\
\hline & mcb & -1913.22057216 & -0.50 & -1912.629365 & 18.27 & 18.77 \\
\hline & ts-exo & -1913.21132549 & 5.30 & -1912.622861 & 22.35 & 17.05 \\
\hline & pc-exo & -1913.22208553 & -1.45 & -1912.636176 & 13.99 & 15.45 \\
\hline & 5-exo & -1913.21977129 & 0.00 & -1912.658478 & 0.00 & 0.00 \\
\hline \multirow{5}{*}{ D4 } & pc1 & -1913.22307036 & -2.07 & -1912.637406 & 13.22 & 15.29 \\
\hline & ts1 & -1913.21242688 & 4.61 & -1912.625307 & 20.82 & 16.21 \\
\hline & mcb & -1913.22553069 & -3.61 & -1912.634901 & 14.79 & 18.41 \\
\hline & ts2 & -1913.20666577 & 8.22 & -1912.618250 & 25.24 & 17.02 \\
\hline & pc2 & -1913.22432578 & -2.86 & -1912.636558 & 13.76 & 16.61 \\
\hline
\end{tabular}

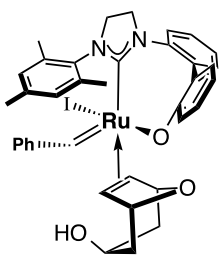

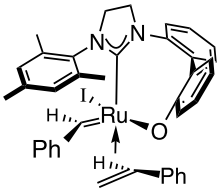

pc2

$-1913.22432578$

BP86/basis2 (dichloromethane)

\begin{tabular}{|c|c|c|c|c|c|c|c|c|}
\hline \multicolumn{3}{|c|}{ BP86/basis2 (gas-phase) } & \multicolumn{3}{|c|}{ BP86/basis2 (benzene) } & \multicolumn{3}{|c|}{ BP86/basis2 (dichloromethane) } \\
\hline $\begin{array}{c}\Delta \mathbf{E} \\
\text { [hartree] }\end{array}$ & $\begin{array}{c}\Delta \mathbf{E} \\
{[\mathrm{kcal} / \mathrm{mol}]}\end{array}$ & $\begin{array}{c}\Delta \mathbf{G} \\
{[\mathrm{kcal} / \mathrm{mol}]}\end{array}$ & $\begin{array}{c}\Delta \mathbf{E} \\
\text { [hartree] }\end{array}$ & $\begin{array}{c}\Delta \mathbf{E} \\
{[\mathrm{kcal} / \mathrm{mol}]}\end{array}$ & $\begin{array}{c}\Delta \mathbf{G} \\
{[\mathrm{kcal} / \mathrm{mol}]}\end{array}$ & $\begin{array}{c}\Delta \mathrm{E} \\
\text { [hartree] }\end{array}$ & $\begin{array}{c}\Delta \mathbf{E} \\
{[\mathrm{kcal} / \mathrm{mol}]}\end{array}$ & $\begin{array}{c}\Delta \mathbf{G} \\
{[\mathrm{kcal} / \mathrm{mol}]}\end{array}$ \\
\hline-1913.69330122 & -5.51 & 11.13 & -1913.70141678 & -3.64 & 13.00 & -1913.71023306 & -1.92 & 14.72 \\
\hline-1913.67209896 & 7.79 & 23.31 & -1913.68144096 & 8.90 & 24.42 & -1913.69138734 & 9.91 & 25.42 \\
\hline-1913.68503628 & -0.32 & 17.10 & -1913.69428878 & 0.84 & 18.26 & -1913.70390776 & 2.05 & 19.47 \\
\hline-1913.66579382 & 11.75 & 27.46 & -1913.67533865 & 12.73 & 28.43 & -1913.68575335 & 13.44 & 29.15 \\
\hline-1913.67606361 & 5.31 & 22.11 & -1913.68444887 & 7.01 & 23.82 & -1913.69342246 & 8.63 & 25.44 \\
\hline-1913.67803679 & 4.07 & 3.63 & -1913.68966283 & 3.74 & 3.30 & -1913.70232255 & 3.04 & 2.61 \\
\hline-1913.67490403 & 6.03 & 23.07 & -1913.68410550 & 7.23 & 24.27 & -1913.69377059 & 8.41 & 25.45 \\
\hline-1913.66366058 & 13.09 & 29.61 & -1913.67338834 & 13.95 & 30.47 & -1913.68329244 & 14.99 & 31.51 \\
\hline-1913.67999746 & 2.84 & 21.61 & -1913.68904890 & 4.12 & 22.90 & -1913.69791618 & 5.81 & 24.58 \\
\hline-1913.67036973 & 8.88 & 25.93 & -1913.67945694 & 10.14 & 27.19 & -1913.68845112 & 11.75 & 28.80 \\
\hline-1913.68101319 & 2.20 & 17.65 & -1913.69003568 & 3.50 & 18.95 & -1913.69929223 & 4.95 & 20.39 \\
\hline-1913.68451938 & 0.00 & 0.00 & -1913.69562059 & 0.00 & 0.00 & -1913.70717307 & 0.00 & 0.00 \\
\hline-1913.68448238 & 0.02 & 15.32 & -1913.69301968 & 1.63 & 16.93 & -1913.70201130 & 3.24 & 18.53 \\
\hline-1913.67392897 & 6.65 & 22.85 & -1913.68314926 & 7.83 & 24.03 & -1913.69288884 & 8.96 & 25.17 \\
\hline-1913.68670585 & -1.37 & 17.04 & -1913.69607895 & -0.29 & 18.12 & -1913.70619446 & 0.61 & 19.02 \\
\hline-1913.66781017 & 10.49 & 27.50 & -1913.67780655 & 11.18 & 28.20 & -1913.68893533 & 11.44 & 28.46 \\
\hline-1913.68508857 & -0.36 & 16.26 & -1913.69446516 & 0.73 & 17.34 & -1913.70478586 & 1.50 & 18.11 \\
\hline
\end{tabular}




\section{Energies and Gibbs Free Energies with $\omega$ B97XD and M06}

Table S28. ROCM with catalyst Ru-4f and enol ether (pathways EPhos, ZPhos in Fig. 3) $\omega \mathrm{B} 97 \mathrm{XD} / \mathrm{basis1}$ (gas-phase)

\begin{tabular}{|c|c|c|c|c|c|c|c|}
\hline \multirow{3}{*}{$C y-P .{ }^{c y}-C y$} & \multicolumn{2}{|l|}{ pathway } & $\begin{array}{c}\mathbf{E} \\
\text { [hartree] }\end{array}$ & $\begin{array}{c}\Delta \mathbf{E} \\
{[\mathrm{kcal} / \mathrm{mol}]}\end{array}$ & $\begin{array}{c}\mathbf{G} \\
{[\text { hartree] }}\end{array}$ & $\begin{array}{c}\Delta \mathbf{G} \\
{[\mathrm{kcal} / \mathrm{mol}]}\end{array}$ & $\begin{array}{c}\Delta \mathbf{G}_{\text {Corr }} \\
{[\mathrm{kcal} / \mathrm{mol}]}\end{array}$ \\
\hline & & $14 e$ & -2639.25285447 & 0.00 & -2638.622891 & 0.00 & 0.00 \\
\hline & & pc1 & -2639.29262257 & -24.95 & -2638.637792 & -9.35 & 15.60 \\
\hline & & ts1 & -2639.27092410 & -11.34 & -2638.616912 & 3.75 & 15.09 \\
\hline & ZPhos & mcb & -2639.27859053 & -16.15 & -2638.620545 & 1.47 & 17.62 \\
\hline & & ts2 & -2639.26955675 & -10.48 & -2638.613787 & 5.71 & 16.19 \\
\hline & & pc2 & -2639.28450021 & -19.86 & -2638.630466 & -4.75 & 15.10 \\
\hline & & $14 \mathrm{e}$ & -2639.25285447 & 0.00 & -2638.622891 & 0.00 & 0.00 \\
\hline & & pc1 & -2639.29253555 & -24.90 & -2638.637537 & -9.19 & 15.71 \\
\hline & & ts1 & -2639.27078609 & -11.25 & -2638.617842 & 3.17 & 14.42 \\
\hline $\mathrm{eO}_{\mathrm{O}}$ & EPhos & mcb & -2639.27764483 & -15.56 & -2638.623048 & -0.10 & 15.46 \\
\hline & & ts2 & -2639.26593124 & -8.21 & -2638.611341 & 7.25 & 15.45 \\
\hline & & pc2 & -2639.29045976 & -23.60 & -2638.635520 & -7.92 & 15.67 \\
\hline
\end{tabular}

$\omega \mathrm{B97XD/basis2}$ (gas-phase)

\begin{tabular}{|c|c|c|c|c|c|c|c|c|}
\hline $\begin{array}{c}\mathbf{E}_{\mathrm{sp}} \\
\text { [hartree] }\end{array}$ & $\begin{array}{c}\Delta \mathbf{E}_{\mathrm{sp}} \\
{[\mathrm{kcal} / \mathrm{mol}]}\end{array}$ & $\begin{array}{c}\Delta \mathbf{G}_{\mathrm{sp}} \\
{[\mathrm{kcal} / \mathrm{mol}]}\end{array}$ & $\begin{array}{c}\mathbf{E}_{\mathrm{sp}} \\
\text { [hartree] }\end{array}$ & $\begin{array}{c}\Delta \mathbf{E}_{\mathrm{sp}} \\
{[\mathrm{kcal} / \mathrm{mol}]}\end{array}$ & $\begin{array}{c}\Delta \mathbf{G}_{\mathrm{sp}} \\
{[\mathrm{kcal} / \mathrm{mol}]}\end{array}$ & $\begin{array}{c}\mathrm{E}_{\mathrm{sp}} \\
\text { [hartree] }\end{array}$ & $\begin{array}{c}\Delta \mathrm{E}_{\mathrm{sp}} \\
{[\mathrm{kcal} / \mathrm{mol}]}\end{array}$ & $\begin{array}{c}\Delta \mathbf{G}_{\mathrm{sp}} \\
{[\mathrm{kcal} / \mathrm{mol}]}\end{array}$ \\
\hline-2639.64195001 & 0.00 & 0.00 & -2639.65681947 & 0.00 & 0.00 & -2639.67196577 & 0.00 & 0.00 \\
\hline-2639.67932357 & -23.45 & -7.85 & -2639.68877102 & -20.05 & -4.45 & -2639.69828654 & -16.52 & -0.91 \\
\hline-2639.65782547 & -9.96 & 5.13 & -2639.66932057 & -7.84 & 7.25 & -2639.68220259 & -6.42 & 8.67 \\
\hline-2639.66448577 & -14.14 & 3.48 & -2639.67419629 & -10.90 & 6.72 & -2639.68475086 & -8.02 & 9.60 \\
\hline-2639.65487674 & -8.11 & 8.08 & -2639.66415186 & -4.60 & 11.59 & -2639.67402894 & -1.29 & 14.90 \\
\hline-2639.67126546 & -18.40 & -3.29 & -2639.68011386 & -14.62 & 0.49 & -2639.69023158 & -11.46 & 3.64 \\
\hline-2639.64195001 & 0.00 & 0.00 & -2639.65681947 & 0.00 & 0.00 & -2639.67196577 & 0.00 & 0.00 \\
\hline-2639.67924627 & -23.40 & -7.69 & -2639.68867741 & -19.99 & -4.28 & -2639.69823146 & -16.48 & -0.77 \\
\hline-2639.65747980 & -9.75 & 4.68 & -2639.66906146 & -7.68 & 6.74 & -2639.68159797 & -6.04 & 8.38 \\
\hline-2639.66367801 & -13.63 & 1.82 & -2639.67400586 & -10.78 & 4.67 & -2639.68505933 & -8.22 & 7.24 \\
\hline-2639.65179550 & -6.18 & 9.28 & -2639.66255323 & -3.60 & 11.86 & -2639.67445326 & -1.56 & 13.89 \\
\hline-2639.67637086 & -21.60 & -5.93 & -2639.68447255 & -17.35 & -1.68 & -2639.69320739 & -13.33 & 2.34 \\
\hline \multicolumn{3}{|c|}{ M06/basis2 (gas-phase) } & \multicolumn{3}{|c|}{ M06/basis2 (benzene) } & \multicolumn{3}{|c|}{ M06/basis2 (dichloromethane) } \\
\hline $\begin{array}{c}\mathbf{E}_{\mathrm{sp}} \\
{[\text { hartree] }}\end{array}$ & $\begin{array}{c}\Delta \mathrm{E}_{\mathrm{sp}} \\
{[\mathrm{kcal} / \mathrm{mol}]}\end{array}$ & $\begin{array}{c}\Delta \mathbf{G}_{\mathrm{sp}} \\
{[\mathrm{kcal} / \mathrm{mol}]}\end{array}$ & $\begin{array}{c}\mathbf{E}_{\mathrm{sp}} \\
\text { [hartree] }\end{array}$ & $\begin{array}{c}\Delta \mathrm{E}_{\mathrm{sp}} \\
{[\mathrm{kcal} / \mathrm{mol}]}\end{array}$ & $\begin{array}{c}\Delta \mathbf{G}_{\mathrm{sp}} \\
{[\mathrm{kcal} / \mathrm{mol}]}\end{array}$ & $\begin{array}{c}\mathbf{E}_{\mathrm{sp}} \\
\text { [hartree] }\end{array}$ & $\begin{array}{c}\Delta \mathrm{E}_{\mathrm{sp}} \\
{[\mathrm{kcal} / \mathrm{mol}]}\end{array}$ & $\begin{array}{c}\Delta \mathbf{G}_{\mathrm{sp}} \\
{[\mathrm{kcal} / \mathrm{mol}]}\end{array}$ \\
\hline-2638.95849120 & 0.00 & 0.00 & -2638.97213215 & 0.00 & 0.00 & -2638.98600942 & 0.00 & 0.00 \\
\hline-2639.00178418 & -27.17 & -11.56 & -2639.01059194 & -24.13 & -8.53 & -2639.01951257 & -21.02 & -5.42 \\
\hline-2638.97894653 & -12.84 & 2.25 & -2638.98973394 & -11.05 & 4.05 & -2639.00183048 & -9.93 & 5.16 \\
\hline-2638.98600975 & -17.27 & 0.35 & -2638.99521380 & -14.48 & 3.14 & -2639.00526843 & -12.09 & 5.54 \\
\hline-2638.97682450 & -11.50 & 4.69 & -2638.98554453 & -8.42 & 7.78 & -2638.99490005 & -5.58 & 10.61 \\
\hline-2638.99216477 & -21.13 & -6.03 & -2639.00036452 & -17.72 & -2.61 & -2639.00968816 & -14.86 & 0.25 \\
\hline-2638.95849120 & 0.00 & 0.00 & -2638.97213215 & 0.00 & 0.00 & -2638.98600942 & 0.00 & 0.00 \\
\hline-2639.00173063 & -27.13 & -11.42 & -2639.01052150 & -24.09 & -8.38 & -2639.01947710 & -21.00 & -5.29 \\
\hline-2638.97904608 & -12.90 & 1.52 & -2638.98989928 & -11.15 & 3.27 & -2639.00167517 & -9.83 & 4.59 \\
\hline-2638.98535263 & -16.86 & -1.40 & -2638.99511013 & -14.42 & 1.04 & -2639.00560477 & -12.30 & 3.16 \\
\hline-2638.97164673 & -8.26 & 7.20 & -2638.98165750 & -5.98 & 9.48 & -2638.99272613 & -4.21 & 11.24 \\
\hline-2638.99917487 & -25.53 & -9.86 & -2639.00667565 & -21.68 & -6.00 & -2639.01479610 & -18.06 & -2.39 \\
\hline
\end{tabular}


Table S29. ROCM with catalyst Ru-4b and enol ether (pathways ENHC, ZNHC in Figure 3)
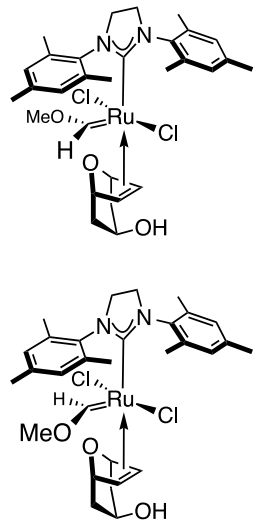

\begin{tabular}{|c|c|c|c|c|c|c|}
\hline & & \multicolumn{5}{|c|}{ wB97XD/basis1 (gas-phase) } \\
\hline \multicolumn{2}{|l|}{ pathway } & $\begin{array}{c}\mathbf{E} \\
\text { [hartree] }\end{array}$ & $\begin{array}{c}\Delta \mathrm{E} \\
{[\mathrm{kcal} / \mathrm{mol}]}\end{array}$ & $\begin{array}{c}\mathbf{G} \\
\text { [hartree] }\end{array}$ & $\begin{array}{c}\Delta \mathbf{G} \\
{[\mathrm{kcal} / \mathrm{mol}]}\end{array}$ & $\begin{array}{c}\Delta \mathbf{G}_{\text {Corr }} \\
{[\mathrm{kcal} / \mathrm{mol}]}\end{array}$ \\
\hline \multirow{6}{*}{ ZNHC } & $14 e$ & -2517.38909398 & 0.00 & -2516.828146 & 0.00 & 0.00 \\
\hline & pc1 & -2517.43008997 & -25.73 & -2516.844531 & -10.28 & 15.44 \\
\hline & ts1 & -2517.41177692 & -14.23 & -2516.825150 & 1.88 & 16.11 \\
\hline & mcb & -2517.42360322 & -21.65 & -2516.832805 & -2.92 & 18.73 \\
\hline & ts2 & -2517.41136043 & -13.97 & -2516.822416 & 3.60 & 17.57 \\
\hline & pc2 & -2517.42063212 & -19.79 & -2516.832287 & -2.60 & 17.19 \\
\hline \multirow{6}{*}{ ENHC } & $14 \mathrm{e}$ & -2517.38909398 & 0.00 & -2516.828146 & 0.00 & 0.00 \\
\hline & pc1 & -2517.43006141 & -25.71 & -2516.845889 & -11.13 & 14.57 \\
\hline & ts1 & -2517.41036186 & -13.35 & -2516.824888 & 2.04 & 15.39 \\
\hline & mcb & -2517.42225962 & -20.81 & -2516.833326 & -3.25 & 17.56 \\
\hline & ts2 & -2517.40859083 & -12.23 & -2516.820559 & 4.76 & 17.00 \\
\hline & pc2 & -2517.42406228 & -21.94 & -2516.834404 & -3.93 & 18.02 \\
\hline
\end{tabular}

$\omega \mathrm{B97XD/basis2}$ (gas-phase)

\begin{tabular}{|c|c|c|c|c|c|c|c|c|}
\hline \multicolumn{3}{|c|}{ 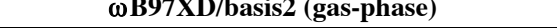 } & \multicolumn{3}{|c|}{ Фву/ху/Dasisz (Denzene) } & \multicolumn{3}{|c|}{ 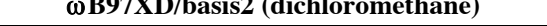 } \\
\hline $\begin{array}{c}\mathbf{E}_{\mathrm{sp}} \\
\text { [hartree] }\end{array}$ & $\begin{array}{c}\Delta \mathbf{E}_{\mathrm{sp}} \\
{[\mathrm{kcal} / \mathrm{mol}]}\end{array}$ & $\begin{array}{c}\Delta \mathbf{G}_{\text {sp }} \\
{[\mathrm{kcal} / \mathrm{mol}]}\end{array}$ & $\begin{array}{c}\mathbf{E}_{\mathrm{sp}} \\
\text { [hartree] }\end{array}$ & $\begin{array}{c}\Delta \mathbf{E}_{\mathrm{sp}} \\
{[\mathrm{kcal} / \mathrm{mol}]}\end{array}$ & $\begin{array}{c}\Delta \mathbf{G}_{\mathrm{sp}} \\
{[\mathrm{kcal} / \mathrm{mol}]}\end{array}$ & $\begin{array}{c}\mathbf{E}_{\mathrm{sp}} \\
\text { [hartree] }\end{array}$ & $\begin{array}{c}\Delta \mathbf{E}_{\mathrm{sp}} \\
{[\mathrm{kcal} / \mathrm{mol}]}\end{array}$ & $\begin{array}{c}\Delta \mathbf{G}_{\mathrm{sp}} \\
{[\mathrm{kcal} / \mathrm{mol}]}\end{array}$ \\
\hline-2517.80533773 & 0.00 & 0.00 & -2517.82195840 & 0.00 & 0.00 & -2517.83863692 & 0.00 & 0.00 \\
\hline-2517.84471712 & -24.71 & -9.27 & -2517.85485956 & -20.65 & -5.20 & -2517.86541321 & -16.80 & -1.36 \\
\hline-2517.82681589 & -13.48 & 2.64 & -2517.83913734 & -10.78 & 5.33 & -2517.85263608 & -8.78 & 7.33 \\
\hline-2517.83766998 & -20.29 & -1.56 & -2517.84908421 & -17.02 & 1.71 & -2517.86166848 & -14.45 & 4.28 \\
\hline-2517.82482721 & -12.23 & 5.34 & -2517.83658516 & -9.18 & 8.39 & -2517.84969040 & -6.94 & 10.63 \\
\hline-2517.83447358 & -18.28 & -1.09 & -2517.84530758 & -14.65 & 2.54 & -2517.85710811 & -11.59 & 5.60 \\
\hline-2517.80533773 & 0.00 & 0.00 & -2517.82195840 & 0.00 & 0.00 & -2517.83863692 & 0.00 & 0.00 \\
\hline-2517.84457811 & -24.62 & -10.05 & -2517.85479728 & -20.61 & -6.03 & -2517.86541607 & -16.80 & -2.23 \\
\hline-2517.82526248 & -12.50 & 2.89 & -2517.83738485 & -9.68 & 5.71 & -2517.85050248 & -7.45 & 7.94 \\
\hline-2517.83636369 & -19.47 & -1.91 & -2517.84838415 & -16.58 & 0.98 & -2517.86132144 & -14.23 & 3.33 \\
\hline-2517.82194084 & -10.42 & 6.58 & -2517.83447349 & -7.85 & 9.14 & -2517.84802465 & -5.89 & 11.10 \\
\hline-2517.83770130 & -20.31 & -2.29 & -2517.84800687 & -16.35 & 1.67 & -2517.85907272 & -12.82 & 5.19 \\
\hline
\end{tabular}

\begin{tabular}{|c|c|c|c|c|c|c|c|c|}
\hline \multicolumn{3}{|c|}{ M06/basis2 (gas-phase) } & \multicolumn{3}{|c|}{ M06/basis2 (benzene) } & \multicolumn{3}{|c|}{ M06/basis2 (dichloromethane) } \\
\hline $\begin{array}{c}\mathbf{E}_{\mathrm{sp}} \\
\text { [hartree] }\end{array}$ & $\begin{array}{c}\Delta \mathrm{E}_{\mathrm{sp}} \\
{[\mathrm{kcal} / \mathrm{mol}]}\end{array}$ & $\begin{array}{c}\Delta \mathbf{G}_{\mathrm{sp}} \\
{[\mathrm{kcal} / \mathrm{mol}]}\end{array}$ & $\begin{array}{c}\mathbf{E}_{\mathrm{sp}} \\
\text { [hartree] }\end{array}$ & $\begin{array}{c}\Delta \mathrm{E}_{\mathrm{sp}} \\
{[\mathrm{kcal} / \mathrm{mol}]}\end{array}$ & $\begin{array}{c}\Delta \mathbf{G}_{\mathrm{sp}} \\
{[\mathrm{kcal} / \mathrm{mol}]}\end{array}$ & $\begin{array}{c}\mathbf{E}_{\mathrm{sp}} \\
\text { [hartree] }\end{array}$ & $\begin{array}{c}\Delta \mathbf{E}_{\mathrm{sp}} \\
{[\mathrm{kcal} / \mathrm{mol}]}\end{array}$ & $\begin{array}{c}\Delta \mathbf{G}_{\text {sp }} \\
{[\mathrm{kcal} / \mathrm{mol}]}\end{array}$ \\
\hline-2517.09251291 & 0.00 & 0.00 & -2517.10775698 & 0.00 & 0.00 & -2517.12310616 & 0.00 & 0.00 \\
\hline-2517.13657162 & -27.65 & -12.20 & -2517.14590584 & -23.94 & -8.50 & -2517.15568778 & -20.45 & -5.00 \\
\hline-2517.11717856 & -15.48 & 0.64 & -2517.12861239 & -13.09 & 3.03 & -2517.14126082 & -11.39 & 4.72 \\
\hline-2517.12695090 & -21.61 & -2.88 & -2517.13754053 & -18.69 & 0.04 & -2517.14931388 & -16.45 & 2.29 \\
\hline-2517.11504870 & -14.14 & 3.43 & -2517.12590387 & -11.39 & 6.18 & -2517.13809578 & -9.41 & 8.16 \\
\hline-2517.12663047 & -21.41 & -4.22 & -2517.13657383 & -18.08 & -0.89 & -2517.14745800 & -15.28 & 1.91 \\
\hline-2517.09251291 & 0.00 & 0.00 & -2517.10775698 & 0.00 & 0.00 & -2517.12310616 & 0.00 & 0.00 \\
\hline-2517.13652080 & -27.62 & -13.04 & -2517.14592494 & -23.95 & -9.38 & -2517.15576586 & -20.49 & -5.92 \\
\hline-2517.11601816 & -14.75 & 0.64 & -2517.12726215 & -12.24 & 3.15 & -2517.13954772 & -10.32 & 5.07 \\
\hline-2517.12592973 & -20.97 & -3.41 & -2517.13708199 & -18.40 & -0.84 & -2517.14920323 & -16.38 & 1.19 \\
\hline-2517.11063657 & -11.37 & 5.62 & -2517.12221964 & -9.08 & 7.92 & -2517.13483867 & -7.36 & 9.63 \\
\hline-2517.13043262 & -23.79 & -5.78 & -2517.13992494 & -20.19 & -2.17 & -2517.15018984 & -17.00 & 1.02 \\
\hline
\end{tabular}




\section{Table S30. ROCM with catalyst Ru-4f-model and enol ether (pathways EPhos-model, ZPhos-model)}

\begin{tabular}{|c|c|c|c|c|c|c|c|}
\hline & & & & $\omega \mathrm{B97}$ & /basis1 (gas-phas & & \\
\hline & pathway & & $\begin{array}{c}\mathbf{E} \\
\text { [hartree] }\end{array}$ & $\begin{array}{c}\Delta \mathbf{E} \\
{[\mathrm{kcal} / \mathrm{mol}]}\end{array}$ & $\begin{array}{c}\mathbf{G} \\
{[\text { hartree] }}\end{array}$ & $\begin{array}{c}\Delta \mathbf{G} \\
{[\mathrm{kcal} / \mathrm{mol}]}\end{array}$ & $\begin{array}{c}\Delta \mathbf{G}_{\text {Corr }} \\
{[\mathrm{kcal} / \mathrm{mol}]}\end{array}$ \\
\hline & & $14 \mathrm{e}$ & -2053.28505376 & 0.00 & -2053.01895600 & 0.00 & 0.00 \\
\hline$-\mathrm{Me}$ & & pc1 & -2053.32246333 & -23.47 & -2053.03020300 & -7.06 & 16.42 \\
\hline & & ts1 & -2053.29662284 & -7.26 & -2053.00146500 & 10.98 & 18.24 \\
\hline & ZPhos-model & mcb & -2053.30223093 & -10.78 & -2053.00673100 & 7.67 & 18.45 \\
\hline & & ts2 & -2053.29410057 & -5.68 & -2052.99975100 & 12.05 & 17.73 \\
\hline & & pc2 & -2053.31736273 & -20.27 & -2053.02416700 & -3.27 & 17.00 \\
\hline${ }^{\mathrm{Me}} \mathrm{Me}$ & & $14 \mathrm{e}$ & -2053.28505376 & 0.00 & -2053.01895600 & 0.00 & 0.00 \\
\hline & & pc1 & -2053.32237640 & -23.42 & -2053.02985900 & -6.84 & 16.58 \\
\hline$H_{1 .}$ & & ts1 & -2053.29599613 & -6.87 & -2053.00313100 & 9.93 & 16.80 \\
\hline & EPhos-model & mcb & -2053.30169548 & -10.44 & -2053.00773000 & 7.04 & 17.49 \\
\hline & & ts2 & -2053.29109084 & -3.79 & -2052.99839300 & 12.90 & 16.69 \\
\hline $\mathrm{O}$ & & pc2 & -2053.32080808 & -22.44 & -2053.02791600 & -5.62 & 16.81 \\
\hline
\end{tabular}

\begin{tabular}{|c|c|c|c|c|c|c|c|c|}
\hline \multicolumn{3}{|c|}{$\omega$ B97XD/basis2 (gas-phase) } & \multicolumn{3}{|c|}{$\omega \mathrm{B97XD/basis2} \mathrm{(benzene)}$} & \multicolumn{3}{|c|}{$\omega \mathrm{B97XD/basis2} \mathrm{(dichloromethane)}$} \\
\hline $\begin{array}{c}\mathbf{E}_{\mathrm{sp}} \\
\text { [hartree] }\end{array}$ & $\begin{array}{c}\Delta \mathbf{E}_{\mathrm{sp}} \\
{[\mathrm{kcal} / \mathrm{mol}]}\end{array}$ & $\begin{array}{c}\Delta \mathbf{G}_{\mathrm{sp}} \\
{[\mathrm{kcal} / \mathrm{mol}]}\end{array}$ & $\begin{array}{c}\mathbf{E}_{\mathrm{sp}} \\
\text { [hartree] }\end{array}$ & $\begin{array}{c}\Delta \mathbf{E}_{\mathrm{sp}} \\
{[\mathrm{kcal} / \mathrm{mol}]}\end{array}$ & $\begin{array}{c}\Delta \mathbf{G}_{\text {sp }} \\
{[\mathrm{kcal} / \mathrm{mol}]}\end{array}$ & $\begin{array}{c}\mathbf{E}_{\mathrm{sp}} \\
\text { [hartree] }\end{array}$ & $\begin{array}{c}\Delta \mathbf{E}_{\mathrm{sp}} \\
{[\mathrm{kcal} / \mathrm{mol}]}\end{array}$ & $\begin{array}{c}\Delta \mathbf{G}_{\mathrm{sp}} \\
{[\mathrm{kcal} / \mathbf{m o l}]}\end{array}$ \\
\hline-2053.55240484 & 0.00 & 0.00 & -2053.56947083 & 0.00 & 0.00 & -2053.58613149 & 0.00 & 0.00 \\
\hline-2053.58702023 & -21.72 & -5.30 & -2053.59803703 & -17.93 & -1.51 & -2053.60880837 & -14.23 & 2.19 \\
\hline-2053.56153663 & -5.73 & 12.51 & -2053.57459270 & -3.21 & 15.02 & -2053.58821768 & -1.31 & 16.93 \\
\hline-2053.56614376 & -8.62 & 9.83 & -2053.57782709 & -5.24 & 13.21 & -2053.59004261 & -2.45 & 16.00 \\
\hline-2053.55759115 & -3.25 & 14.47 & -2053.56978599 & -0.20 & 17.53 & -2053.58261397 & 2.21 & 19.94 \\
\hline-2053.58275816 & -19.05 & -2.04 & -2053.59222652 & -14.28 & 2.72 & -2053.60236124 & -10.18 & 6.82 \\
\hline-2053.55240484 & 0.00 & 0.00 & -2053.56947083 & 0.00 & 0.00 & -2053.58613149 & 0.00 & 0.00 \\
\hline-2053.58696416 & -21.69 & -5.11 & -2053.59795546 & -17.87 & -1.30 & -2053.60875278 & -14.20 & 2.38 \\
\hline-2053.56059493 & -5.14 & 11.66 & -2053.57364471 & -2.62 & 14.18 & -2053.58692352 & -0.50 & 16.30 \\
\hline-2053.56562459 & -8.30 & 9.19 & -2053.57802621 & -5.37 & 12.12 & -2053.59059227 & -2.80 & 14.69 \\
\hline-2053.55472299 & -1.45 & 15.24 & -2053.56713219 & 1.47 & 18.16 & -2053.58007243 & 3.80 & 20.49 \\
\hline-2053.58527240 & -20.62 & -3.81 & -2053.59431722 & -15.59 & 1.22 & -2053.60371553 & -11.03 & 5.78 \\
\hline \multicolumn{3}{|c|}{ M06/basis2 (gas-phase) } & \multicolumn{3}{|c|}{ M06/basis2 (benzene) } & \multicolumn{3}{|c|}{ M06/basis2 (dichloromethane) } \\
\hline $\begin{array}{c}\mathbf{E}_{\mathrm{sp}} \\
{[\text { hartree] }}\end{array}$ & $\begin{array}{c}\Delta \mathbf{E}_{\mathrm{sp}} \\
{[\mathrm{kcal} / \mathrm{mol}]}\end{array}$ & $\begin{array}{c}\Delta \mathbf{G}_{\mathrm{sp}} \\
{[\mathrm{kcal} / \mathrm{mol}]}\end{array}$ & $\begin{array}{c}\mathbf{E}_{\mathrm{sp}} \\
\text { [hartree] }\end{array}$ & $\begin{array}{c}\Delta \mathbf{E}_{\mathrm{sp}} \\
{[\mathrm{kcal} / \mathrm{mol}]}\end{array}$ & $\begin{array}{c}\Delta \mathbf{G}_{\text {sp }} \\
{[\mathrm{kcal} / \mathrm{mol}]}\end{array}$ & $\begin{array}{c}\mathbf{E}_{\mathrm{sp}} \\
\text { [hartree] }\end{array}$ & $\begin{array}{c}\Delta \mathbf{E}_{\mathrm{sp}} \\
{[\mathrm{kcal} / \mathrm{mol}]}\end{array}$ & $\begin{array}{c}\Delta \mathbf{G}_{\mathrm{sp}} \\
{[\mathrm{kcal} / \mathrm{mol}]}\end{array}$ \\
\hline-2053.18682252 & 0.00 & 0.00 & -2053.20251905 & 0.00 & 0.00 & -2053.21786972 & 0.00 & 0.00 \\
\hline-2053.22603913 & -24.61 & -8.19 & -2053.23630950 & -21.20 & -4.79 & -2053.24639946 & -17.90 & -1.49 \\
\hline-2053.19847760 & -7.31 & 10.92 & -2053.21072359 & -5.15 & 13.09 & -2053.22355754 & -3.57 & 14.67 \\
\hline-2053.20364101 & -10.55 & 7.90 & -2053.21463037 & -7.60 & 10.85 & -2053.22614348 & -5.19 & 13.26 \\
\hline-2053.19482197 & -5.02 & 12.71 & -2053.20618298 & -2.30 & 15.43 & -2053.21818001 & -0.19 & 17.53 \\
\hline-2053.22196451 & -22.05 & -5.05 & -2053.23071526 & -17.69 & -0.69 & -2053.24007633 & -13.93 & 3.07 \\
\hline-2053.18682252 & 0.00 & 0.00 & -2053.20251905 & 0.00 & 0.00 & -2053.21786972 & 0.00 & 0.00 \\
\hline-2053.22601152 & -24.59 & -8.01 & -2053.23625735 & -21.17 & -4.59 & -2053.24637112 & -17.88 & -1.31 \\
\hline-2053.19799468 & -7.01 & 9.79 & -2053.21024018 & -4.85 & 11.95 & -2053.22277021 & -3.08 & 13.72 \\
\hline-2053.20335118 & -10.37 & 7.12 & -2053.21498478 & -7.82 & 9.66 & -2053.22680706 & -5.61 & 11.88 \\
\hline-2053.19177054 & -3.10 & 13.59 & -2053.20328764 & -0.48 & 16.21 & -2053.21532957 & 1.59 & 18.29 \\
\hline-2053.22551057 & -24.28 & -7.46 & -2053.23385328 & -19.66 & -2.85 & -2053.24253209 & -15.48 & 1.34 \\
\hline
\end{tabular}




\section{Table S31. ROCM with catalyst Ru-4b-model and enol ether (pathways ENHC-model, ZNHC-model)}

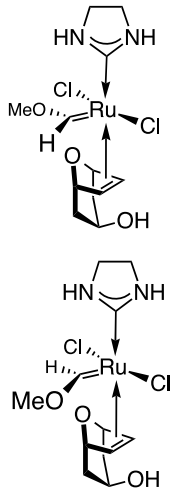

\begin{tabular}{|c|c|c|c|c|c|c|}
\hline & & \multicolumn{5}{|c|}{$\omega \mathrm{B97XD} /$ basis1 (gas-phase) } \\
\hline \multicolumn{2}{|l|}{ pathway } & $\begin{array}{c}\mathbf{E} \\
{[\text { hartree] }}\end{array}$ & $\begin{array}{c}\Delta \mathbf{E} \\
{[\mathrm{kcal} / \mathrm{mol}]}\end{array}$ & $\begin{array}{c}\mathbf{G} \\
{[\text { hartree] }}\end{array}$ & $\begin{array}{c}\Delta \mathbf{G} \\
{[\mathrm{kcal} / \mathrm{mol}]}\end{array}$ & $\begin{array}{c}\Delta \mathbf{G}_{\mathrm{Corr}} \\
{[\mathrm{kcal} / \mathrm{mol}]}\end{array}$ \\
\hline \multirow{6}{*}{ ZNHC-model } & $14 \mathrm{e}$ & -1819.58155109 & 0.00 & -1819.32992800 & 0.00 & 0.00 \\
\hline & pc1 & -1819.61937971 & -23.74 & -1819.34473900 & -9.29 & 14.44 \\
\hline & ts1 & -1819.59465159 & -8.22 & -1819.31584900 & 8.83 & 17.06 \\
\hline & mcb & -1819.60191222 & -12.78 & -1819.32221900 & 4.84 & 17.61 \\
\hline & ts2 & -1819.59107630 & -5.98 & -1819.31267200 & 10.83 & 16.81 \\
\hline & pc2 & -1819.61230044 & -19.30 & -1819.33528600 & -3.36 & 15.93 \\
\hline \multirow{6}{*}{ ENHC-model } & $14 e$ & -1819.58155109 & 0.00 & -1819.32992800 & 0.00 & 0.00 \\
\hline & pc1 & -1819.61933909 & -23.71 & -1819.34480500 & -9.34 & 14.38 \\
\hline & ts1 & -1819.59414088 & -7.90 & -1819.31580300 & 8.86 & 16.76 \\
\hline & mcb & -1819.60215581 & -12.93 & -1819.32298400 & 4.36 & 17.29 \\
\hline & ts2 & -1819.58788058 & -3.97 & -1819.31031200 & 12.31 & 16.28 \\
\hline & pc2 & -1819.61621996 & -21.76 & -1819.33765100 & -4.85 & 16.91 \\
\hline
\end{tabular}

$\omega \mathrm{B97XD/basis2}$ (gas-phase)

\begin{tabular}{|c|c|c|c|c|c|c|c|c|}
\hline & & \\
\hline $\begin{array}{c}\mathbf{E}_{\mathrm{sp}} \\
\text { [hartree] }\end{array}$ & $\begin{array}{c}\Delta \mathbf{E}_{\mathrm{sp}} \\
{[\mathrm{kcal} / \mathrm{mol}]}\end{array}$ & $\begin{array}{c}\Delta \mathbf{G}_{\mathrm{sp}} \\
{[\mathrm{kcal} / \mathrm{mol}]}\end{array}$ & $\begin{array}{c}\mathbf{E}_{\mathrm{sp}} \\
\text { [hartree] }\end{array}$ & $\begin{array}{c}\Delta \mathbf{E}_{\mathrm{sp}} \\
{[\mathrm{kcal} / \mathrm{mol}]}\end{array}$ & $\begin{array}{c}\Delta \mathbf{G}_{\mathrm{sp}} \\
{[\mathrm{kcal} / \mathrm{mol}]}\end{array}$ & $\begin{array}{c}\mathbf{E}_{\mathrm{sp}} \\
\text { [hartree] }\end{array}$ & $\begin{array}{c}\Delta \mathrm{E}_{\mathrm{sp}} \\
{[\mathrm{kcal} / \mathrm{mol}]}\end{array}$ & $\begin{array}{c}\Delta \mathbf{G}_{\mathrm{sp}} \\
{[\mathrm{kcal} / \mathrm{mol}]}\end{array}$ \\
\hline-1819.84506188 & 0.00 & 0.00 & -1819.86385352 & 0.00 & 0.00 & -1819.88148230 & 0.00 & 0.00 \\
\hline-1819.88086253 & -22.47 & -8.02 & -1819.89328985 & -18.47 & -4.03 & -1819.90493271 & -14.72 & -0.27 \\
\hline-1819.85746022 & -7.78 & 9.28 & -1819.87168629 & -4.92 & 12.14 & -1819.88576090 & -2.68 & 14.37 \\
\hline-1819.86365952 & -11.67 & 5.94 & -1819.87633098 & -7.83 & 9.78 & -1819.88863189 & -4.49 & 13.13 \\
\hline-1819.85216283 & -4.46 & 12.35 & -1819.86516087 & -0.82 & 15.99 & -1819.87820009 & 2.06 & 18.87 \\
\hline-1819.87433449 & -18.37 & -2.44 & -1819.88552854 & -13.60 & 2.33 & -1819.89667799 & -9.54 & 6.40 \\
\hline-1819.84506188 & 0.00 & 0.00 & -1819.86385352 & 0.00 & 0.00 & -1819.88148230 & 0.00 & 0.00 \\
\hline-1819.88079311 & -22.42 & -8.04 & -1819.89318292 & -18.40 & -4.03 & -1819.90475493 & -14.60 & -0.23 \\
\hline-1819.85658187 & -7.23 & 9.53 & -1819.87062735 & -4.25 & 12.51 & -1819.88424859 & -1.74 & 15.03 \\
\hline-1819.86389011 & -11.81 & 5.47 & -1819.87704060 & -8.28 & 9.01 & -1819.88957018 & -5.08 & 12.21 \\
\hline-1819.84888198 & -2.40 & 13.88 & -1819.86233514 & 0.95 & 17.23 & -1819.87568240 & 3.64 & 19.92 \\
\hline-1819.87760279 & -20.42 & -3.51 & -1819.88795778 & -15.13 & 1.78 & -1819.89801031 & -10.37 & 6.54 \\
\hline \multicolumn{3}{|c|}{ M06/basis2 (gas-phase) } & \multicolumn{3}{|c|}{ M06/basis2 (benzene) } & \multicolumn{3}{|c|}{ M06/basis2 (dichloromethane) } \\
\hline $\begin{array}{c}\mathbf{E}_{\mathrm{sp}} \\
\text { [hartree] }\end{array}$ & $\begin{array}{c}\Delta \mathbf{E}_{\mathrm{sp}} \\
{[\mathrm{kcal} / \mathrm{mol}]}\end{array}$ & $\begin{array}{c}\Delta \mathbf{G}_{\mathrm{sp}} \\
{[\mathrm{kcal} / \mathrm{mol}]}\end{array}$ & $\begin{array}{c}\mathbf{E}_{\mathrm{sp}} \\
\text { [hartree] }\end{array}$ & $\begin{array}{c}\Delta \mathbf{E}_{\mathrm{sp}} \\
{[\mathrm{kcal} / \mathrm{mol}]}\end{array}$ & $\begin{array}{c}\Delta \mathbf{G}_{\mathrm{sp}} \\
{[\mathrm{kcal} / \mathrm{mol}]}\end{array}$ & $\begin{array}{c}\mathbf{E}_{\mathrm{sp}} \\
\text { [hartree] }\end{array}$ & $\begin{array}{c}\Delta \mathrm{E}_{\mathrm{sp}} \\
{[\mathrm{kcal} / \mathrm{mol}]}\end{array}$ & $\begin{array}{c}\Delta \mathbf{G}_{\mathrm{sp}} \\
{[\mathrm{kcal} / \mathrm{mol}]}\end{array}$ \\
\hline-1819.47244673 & 0.00 & 0.00 & -1819.48984489 & 0.00 & 0.00 & -1819.50623393 & 0.00 & 0.00 \\
\hline-1819.51388271 & -26.00 & -11.56 & -1819.52547514 & -22.36 & -7.91 & -1819.53640033 & -18.93 & -4.49 \\
\hline-1819.48879620 & -10.26 & 6.80 & -1819.50214429 & -7.72 & 9.34 & -1819.51543404 & -5.77 & 11.28 \\
\hline-1819.49501986 & -14.16 & 3.45 & -1819.50695786 & -10.74 & 6.88 & -1819.51862112 & -7.77 & 9.84 \\
\hline-1819.48436737 & -7.48 & 9.33 & -1819.49650880 & -4.18 & 12.62 & -1819.50875802 & -1.58 & 15.22 \\
\hline-1819.50653752 & -21.39 & -5.46 & -1819.51694732 & -17.01 & -1.07 & -1819.52735071 & -13.25 & 2.68 \\
\hline-1819.47244673 & 0.00 & 0.00 & -1819.48984489 & 0.00 & 0.00 & -1819.50623393 & 0.00 & 0.00 \\
\hline-1819.51371312 & -25.90 & -11.52 & -1819.52526680 & -22.23 & -7.85 & -1819.53612202 & -18.76 & -4.38 \\
\hline-1819.48833666 & -9.97 & 6.79 & -1819.50151824 & -7.33 & 9.44 & -1819.51438694 & -5.12 & 11.65 \\
\hline-1819.49522847 & -14.30 & 2.99 & -1819.50758548 & -11.13 & 6.15 & -1819.51944154 & -8.29 & 9.00 \\
\hline-1819.48033751 & -4.95 & 11.33 & -1819.49286677 & -1.90 & 14.38 & -1819.50536582 & 0.54 & 16.83 \\
\hline-1819.51083348 & -24.09 & -7.18 & -1819.52044124 & -19.20 & -2.29 & -1819.52980169 & -14.79 & 2.12 \\
\hline
\end{tabular}


Table S32. ROCM with catalyst Ru-5b and enol ether (pathways A1 = ZRu-5b, AB1, B1 in Figure 5 and Figure S22)
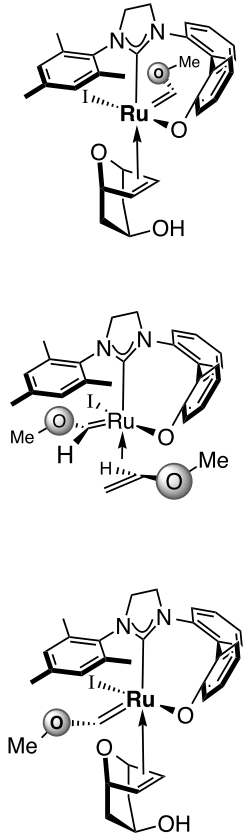

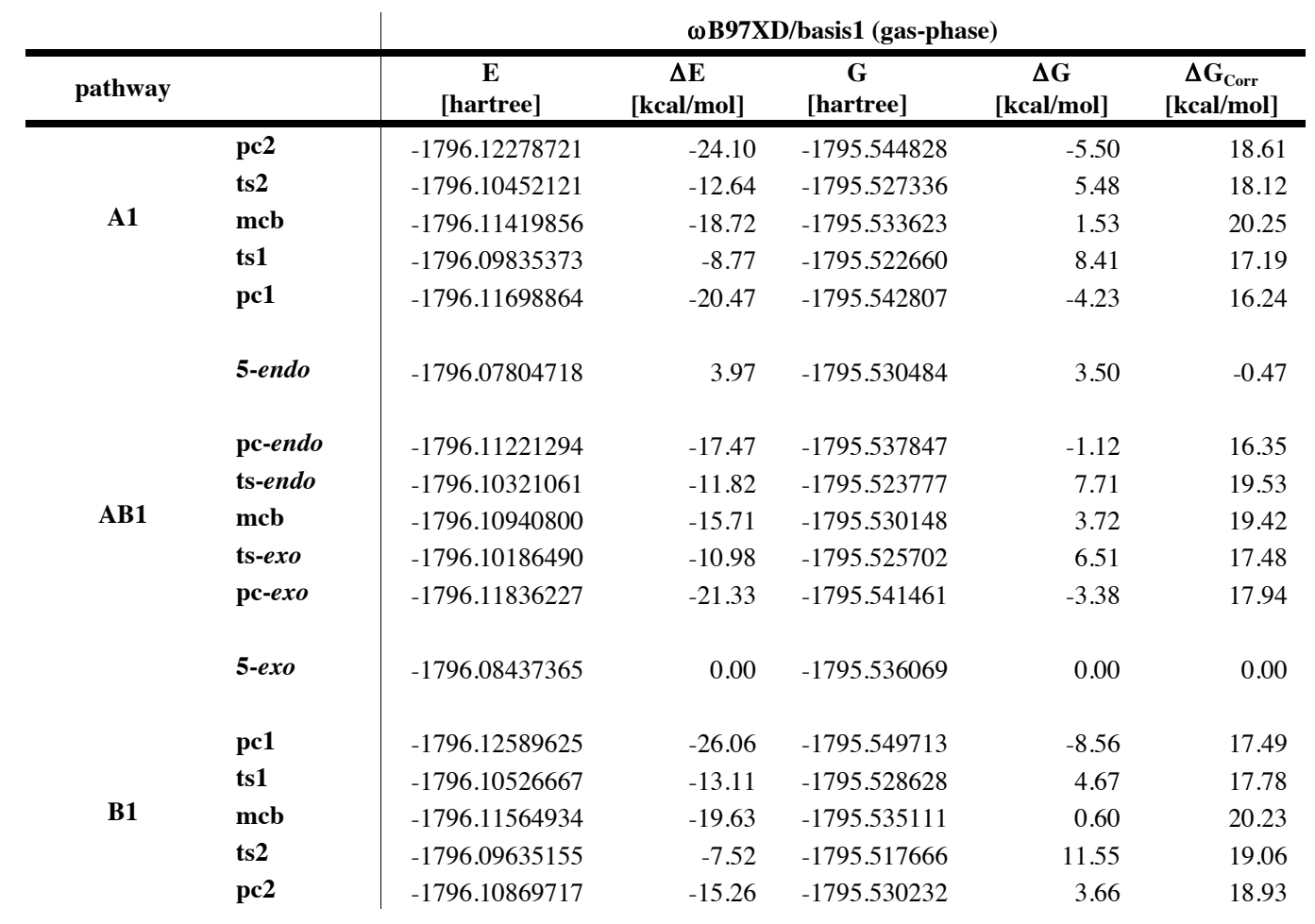

\begin{tabular}{|c|c|c|c|c|c|c|c|c|}
\hline \multicolumn{3}{|c|}{ wB97XD/basis2 (gas-phase) } & \multicolumn{3}{|c|}{ wB97XD/basis2 (benzene) } & \multicolumn{3}{|c|}{ wB97XD/basis2 (dichloromethane) } \\
\hline $\begin{array}{c}\mathbf{E}_{\mathrm{sp}} \\
\text { [hartree] }\end{array}$ & $\begin{array}{c}\Delta \mathrm{E}_{\mathrm{sp}} \\
{[\mathrm{kcal} / \mathrm{mol}]}\end{array}$ & $\begin{array}{c}\Delta \mathbf{G}_{\mathrm{sp}} \\
{[\mathrm{kcal} / \mathrm{mol}]}\end{array}$ & $\begin{array}{c}\mathrm{E}_{\mathrm{sp}} \\
\text { [hartree] }\end{array}$ & $\begin{array}{c}\Delta \mathrm{E}_{\mathrm{sp}} \\
{[\mathrm{kcal} / \mathrm{mol}]}\end{array}$ & $\begin{array}{c}\Delta \mathbf{G}_{\mathrm{sp}} \\
{[\mathrm{kcal} / \mathrm{mol}]}\end{array}$ & $\begin{array}{c}\mathbf{E}_{\mathrm{sp}} \\
\text { [hartree] }\end{array}$ & $\begin{array}{c}\Delta \mathrm{E}_{\mathrm{sp}} \\
{[\mathrm{kcal} / \mathrm{mol}]}\end{array}$ & $\begin{array}{c}\Delta \mathbf{G}_{\mathrm{sp}} \\
{[\mathrm{kcal} / \mathrm{mol}]}\end{array}$ \\
\hline-1796.52092798 & -21.81 & -3.20 & -1796.52992673 & -18.01 & 0.60 & -1796.53959482 & -14.55 & 4.06 \\
\hline-1796.50343873 & -10.84 & 7.28 & -1796.51420645 & -8.15 & 9.98 & -1796.52554887 & -5.73 & 12.39 \\
\hline-1796.51383088 & -17.36 & 2.89 & -1796.52443813 & -14.57 & 5.68 & -1796.53565046 & -12.07 & 8.18 \\
\hline-1796.49944753 & -8.33 & 8.85 & -1796.51211534 & -6.83 & 10.35 & -1796.52595684 & -5.99 & 11.20 \\
\hline-1796.51689041 & -19.28 & -3.04 & -1796.52727181 & -16.34 & -0.11 & -1796.53798476 & -13.54 & 2.70 \\
\hline-1796.48004259 & 3.84 & 3.38 & -1796.49551056 & 3.59 & 3.12 & -1796.51169149 & 2.96 & 2.50 \\
\hline-1796.50913774 & -14.41 & 1.94 & -1796.52092844 & -12.36 & 3.99 & -1796.53334377 & -10.63 & 5.73 \\
\hline-1796.50105639 & -9.34 & 10.19 & -1796.51276576 & -7.24 & 12.29 & -1796.52485050 & -5.30 & 14.24 \\
\hline-1796.50708007 & -13.12 & 6.30 & -1796.51785411 & -10.43 & 8.99 & -1796.52869527 & -7.71 & 11.72 \\
\hline-1796.50009647 & -8.74 & 8.74 & -1796.51180398 & -6.64 & 10.84 & -1796.52390150 & -4.70 & 12.78 \\
\hline-1796.51568941 & -18.53 & -0.58 & -1796.52688796 & -16.10 & 1.84 & -1796.53832881 & -13.75 & 4.19 \\
\hline-1796.48616691 & 0.00 & 0.00 & -1796.50122636 & 0.00 & 0.00 & -1796.51641073 & 0.00 & 0.00 \\
\hline-1796.52538398 & -24.61 & -7.12 & -1796.53556807 & -21.55 & -4.06 & -1796.54582546 & -18.46 & -0.96 \\
\hline-1796.50620632 & -12.57 & 5.20 & -1796.51781775 & -10.41 & 7.37 & -1796.53052977 & -8.86 & 8.92 \\
\hline-1796.51538584 & -18.34 & 1.89 & -1796.52595963 & -15.52 & 4.71 & -1796.53722488 & -13.06 & 7.17 \\
\hline-1796.49557837 & -5.91 & 13.16 & -1796.50664032 & -3.40 & 15.67 & -1796.51894659 & -1.59 & 17.47 \\
\hline-1796.50708989 & -13.13 & 5.80 & -1796.51709041 & -9.95 & 8.97 & -1796.52835845 & -7.50 & 11.43 \\
\hline
\end{tabular}




\begin{tabular}{|c|c|c|c|c|c|c|c|c|}
\hline \multicolumn{3}{|c|}{ M06/basis2 (gas-phase) } & \multicolumn{3}{|c|}{ M06/basis2 (benzene) } & \multicolumn{3}{|c|}{ M06/basis2 (dichloromethane) } \\
\hline $\begin{array}{c}\mathbf{E}_{\mathrm{sp}} \\
\text { [hartree] }\end{array}$ & $\begin{array}{c}\Delta \mathbf{E}_{\mathrm{sp}} \\
{[\mathrm{kcal} / \mathbf{m o l}]}\end{array}$ & $\begin{array}{c}\Delta \mathbf{G}_{\mathrm{sp}} \\
{[\mathrm{kcal} / \mathrm{mol}]}\end{array}$ & $\begin{array}{c}\mathbf{E}_{\mathrm{sp}} \\
{[\text { hartree] }}\end{array}$ & $\begin{array}{c}\Delta \mathbf{E}_{\mathrm{sp}} \\
{[\mathrm{kcal} / \mathrm{mol}]}\end{array}$ & $\begin{array}{c}\Delta \mathbf{G}_{\mathrm{sp}} \\
{[\mathrm{kcal} / \mathrm{mol}]}\end{array}$ & $\begin{array}{c}\mathbf{E}_{\mathrm{sp}} \\
{[\text { hartree] }}\end{array}$ & $\begin{array}{c}\Delta \mathbf{E}_{\mathrm{sp}} \\
{[\mathrm{kcal} / \mathbf{m o l}]}\end{array}$ & $\begin{array}{c}\Delta \mathbf{G}_{\mathrm{sp}} \\
{[\mathrm{kcal} / \mathrm{mol}]}\end{array}$ \\
\hline-1795.76316586 & -22.86 & -4.26 & -1795.77125476 & -19.43 & -0.82 & -1795.78004075 & -16.32 & 2.29 \\
\hline-1795.74379918 & -10.71 & 7.41 & -1795.75348741 & -8.28 & 9.84 & -1795.76381853 & -6.14 & 11.98 \\
\hline-1795.75391711 & -17.06 & 3.19 & -1795.76348438 & -14.56 & 5.69 & -1795.77370881 & -12.35 & 7.90 \\
\hline-1795.74040672 & -8.58 & 8.60 & -1795.75177777 & -7.21 & 9.98 & -1795.76437293 & -6.49 & 10.70 \\
\hline-1795.75957782 & -20.61 & -4.37 & -1795.76893307 & -17.98 & -1.74 & -1795.77871349 & -15.49 & 0.75 \\
\hline-1795.72079829 & 3.72 & 3.26 & -1795.73463513 & 3.55 & 3.08 & -1795.74917466 & 3.05 & 2.58 \\
\hline-1795.75159578 & -15.60 & 0.75 & -1795.76216170 & -13.73 & 2.63 & -1795.77336003 & -12.13 & 4.22 \\
\hline-1795.74428651 & -11.02 & 8.52 & -1795.75484008 & -9.13 & 10.40 & -1795.76581650 & -7.40 & 12.14 \\
\hline-1795.75087523 & -15.15 & 4.27 & -1795.76063676 & -12.77 & 6.66 & -1795.77055448 & -10.37 & 9.06 \\
\hline-1795.74469691 & -11.27 & 6.21 & -1795.75529800 & -9.42 & 8.06 & -1795.76636273 & -7.74 & 9.74 \\
\hline-1795.75699737 & -18.99 & -1.05 & -1795.76714575 & -16.85 & 1.09 & -1795.77764519 & -14.82 & 3.13 \\
\hline-1795.72672918 & 0.00 & 0.00 & -1795.74028747 & 0.00 & 0.00 & -1795.75403119 & 0.00 & 0.00 \\
\hline-1795.76829587 & -26.08 & -8.59 & -1795.77740822 & -23.29 & -5.80 & -1795.786667970 & -20.49 & -2.99 \\
\hline-1795.74681717 & -12.61 & 5.17 & -1795.75729118 & -10.67 & 7.11 & -1795.76890117 & -9.33 & 8.45 \\
\hline-1795.75518100 & -17.85 & 2.37 & -1795.76473377 & -15.34 & 4.89 & -1795.77501492 & -13.17 & 7.06 \\
\hline-1795.73896603 & -7.68 & 11.39 & -1795.74888209 & -5.39 & 13.67 & -1795.76002946 & -3.76 & 15.30 \\
\hline-1795.75004060 & -14.63 & 4.30 & -1795.75896226 & -11.72 & 7.21 & -1795.76908792 & -9.45 & 9.48 \\
\hline $\begin{array}{l}\mathrm{E} \ldots \ldots \ldots \ldots \text { gas ph } \\
\mathrm{G} \ldots \ldots \ldots \ldots \text { sum o } \\
\Delta \mathrm{E} \ldots \ldots \ldots \text { relativ } \\
\Delta \mathrm{G} \ldots \ldots \ldots \text { relativ } \\
\Delta \mathrm{G}_{\mathrm{corr}} \ldots \ldots \text { gas ph } \\
\mathrm{E}_{\mathrm{sp}} \ldots \ldots \ldots \text { single } \\
\Delta \mathrm{E}_{\mathrm{sp}} \ldots \ldots \text { relativ } \\
\Delta \mathrm{G}_{\mathrm{sp}} \ldots \ldots . \text { relativ }\end{array}$ & $\begin{array}{l}\text { e electronic en } \\
\text { lectronic and } t \\
\text { electronic ener } \\
\text { ree energy in } 1 \\
\text { e thermal corre } \\
\text { int electronic } \\
\text { ingle point ele } \\
\text { single point fre }\end{array}$ & $\begin{array}{l}\text { gy in hartree } \\
\text { ermal free ener } \\
\text { y in kcal/mol y } \\
\text { al/mol with ba } \\
\text { tion to free en } \\
\text { tergy in gas ph } \\
\text { tronic energy } \mathrm{i} \\
\text { energy in gas }\end{array}$ & $\begin{array}{l}\text { ith basis } 1 \\
\text { ies with basis } 1 \\
\text { th basis } 1 \\
\text { is } 1 \\
\text { gy in } \mathrm{kcal} / \mathrm{mol} \text { obt } \\
\text { se or solution in ha } \\
\text { gas phase or solut }\end{array}$ & $\begin{array}{l}\text { ned with basis } \\
\text { ree with basis? } \\
\text { in } \mathrm{kcal} / \mathrm{mol}\end{array}$ & $\begin{array}{l}(\Delta \mathrm{G}=\Delta \mathrm{E}+\Delta \\
\mathrm{h} \text { basis2 }\end{array}$ & corr) & & \\
\hline
\end{tabular}


Table S33. ROCM with catalyst Ru-5b and enol ether (pathways A2, AB2, B2 in Figure 5 and Figure S22)
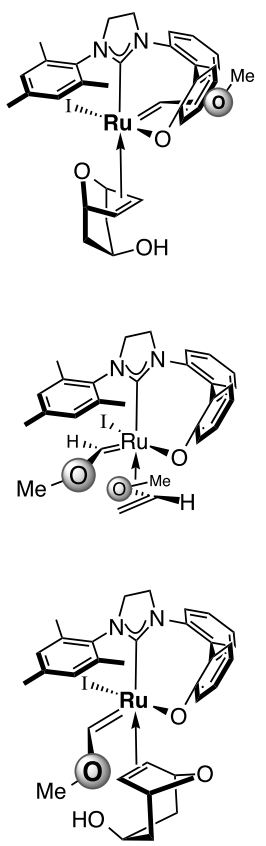

\begin{tabular}{|c|c|c|c|c|c|c|}
\hline & & \multicolumn{5}{|c|}{ wB97XD/basis1 (gas-phase) } \\
\hline \multicolumn{2}{|c|}{ pathway } & $\begin{array}{c}\mathbf{E} \\
\text { [hartree] }\end{array}$ & $\begin{array}{c}\Delta \mathrm{E} \\
{[\mathrm{kcal} / \mathrm{mol}]}\end{array}$ & $\begin{array}{c}\mathbf{G} \\
\text { [hartree] }\end{array}$ & $\begin{array}{c}\Delta \mathbf{G} \\
{[\mathrm{kcal} / \mathrm{mol}]}\end{array}$ & $\begin{array}{c}\Delta \mathbf{G}_{\mathrm{Corr}} \\
{[\mathrm{kcal} / \mathrm{mol}]}\end{array}$ \\
\hline \multirow{5}{*}{ A2 } & pc2 & -1796.11853578 & -21.44 & -1795.541796 & -3.59 & 17.84 \\
\hline & ts2 & -1796.10060080 & -10.18 & -1795.521610 & 9.07 & 19.26 \\
\hline & mcb & -1796.11032488 & -16.28 & -1795.532007 & 2.55 & 18.83 \\
\hline & ts1 & -1796.10025094 & -9.96 & -1795.524393 & 7.33 & 17.29 \\
\hline & pc1 & -1796.11698864 & -20.47 & -1795.542804 & -4.23 & 16.24 \\
\hline \multirow{7}{*}{ AB2 } & 5-endo & -1796.07804718 & 3.97 & -1795.530484 & 3.50 & -0.47 \\
\hline & pc-endo & -1796.11458133 & -18.96 & -1795.536605 & -0.34 & 18.62 \\
\hline & ts-endo & -1796.10019567 & -9.93 & -1795.522992 & 8.21 & 18.13 \\
\hline & mcb & -1796.10748161 & -14.50 & -1795.529069 & 4.39 & 18.89 \\
\hline & ts-exo & -1796.10632235 & -13.77 & -1795.529910 & 3.86 & 17.64 \\
\hline & pc-exo & -1796.12198051 & -23.60 & -1795.545333 & -5.81 & 17.79 \\
\hline & 5-exo & -1796.08437365 & 0.00 & -1795.536069 & 0.00 & 0.00 \\
\hline \multirow{5}{*}{ B2 } & pc1 & -1796.12607958 & -26.17 & -1795.551693 & -9.80 & 16.37 \\
\hline & ts1 & -1796.10888846 & -15.38 & -1795.531044 & 3.15 & 18.54 \\
\hline & mcb & -1796.11186873 & -17.25 & -1795.533028 & 1.91 & 19.16 \\
\hline & ts2 & -1796.09250806 & -5.10 & -1795.514255 & 13.69 & 18.79 \\
\hline & pc2 & -1796.10652884 & -13.90 & -1795.528218 & 4.93 & 18.83 \\
\hline
\end{tabular}

\begin{tabular}{|c|c|c|c|c|c|c|c|c|}
\hline \multicolumn{3}{|c|}{ 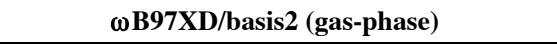 } & \multicolumn{3}{|c|}{ wB97XD/basis2 (benzene) } & \multicolumn{3}{|c|}{$\omega$ B97XD/basis2 (dichloromethane) } \\
\hline $\begin{array}{c}\mathbf{E}_{\mathrm{sp}} \\
\text { [hartree] }\end{array}$ & $\begin{array}{c}\Delta \mathrm{E}_{\mathrm{sp}} \\
{[\mathrm{kcal} / \mathrm{mol}]}\end{array}$ & $\begin{array}{c}\Delta \mathbf{G}_{\mathrm{sp}} \\
{[\mathrm{kcal} / \mathrm{mol}]}\end{array}$ & $\begin{array}{c}\mathbf{E}_{\mathrm{sp}} \\
\text { [hartree] }\end{array}$ & $\begin{array}{c}\Delta \mathbf{E}_{\mathrm{sp}} \\
{[\mathrm{kcal} / \mathrm{mol}]}\end{array}$ & $\begin{array}{c}\Delta \mathbf{G}_{\mathrm{sp}} \\
{[\mathrm{kcal} / \mathrm{mol}]}\end{array}$ & $\begin{array}{c}\mathbf{E}_{\mathrm{sp}} \\
\text { [hartree] }\end{array}$ & $\begin{array}{c}\Delta \mathrm{E}_{\mathrm{sp}} \\
{[\mathrm{kcal} / \mathrm{mol}]}\end{array}$ & $\begin{array}{c}\Delta \mathbf{G}_{\mathrm{sp}} \\
{[\mathrm{kcal} / \mathrm{mol}]}\end{array}$ \\
\hline-1796.51781379 & -19.86 & -2.02 & -1796.52747045 & -16.47 & 1.37 & -1796.53787367 & -13.47 & 4.38 \\
\hline-1796.49973765 & -8.52 & 10.74 & -1796.51043086 & -5.78 & 13.48 & -1796.52186990 & -3.43 & 15.83 \\
\hline-1796.51079347 & -15.45 & 3.38 & -1796.52163299 & -12.81 & 6.03 & -1796.53312657 & -10.49 & 8.34 \\
\hline-1796.50101235 & -9.32 & 7.97 & -1796.51288228 & -7.31 & 9.98 & -1796.52575696 & -5.86 & 11.43 \\
\hline-1796.51689039 & -19.28 & -3.04 & -1796.52727178 & -16.34 & -0.10 & -1796.53798472 & -13.54 & 2.70 \\
\hline-1796.48004259 & 3.84 & 3.38 & -1796.49551056 & 3.59 & 3.12 & -1796.51169149 & 2.96 & 2.50 \\
\hline-1796.51187478 & -16.13 & 2.49 & -1796.52246345 & -13.33 & 5.29 & -1796.53332902 & -10.62 & 8.00 \\
\hline-1796.49841937 & -7.69 & 10.45 & -1796.51079746 & -6.01 & 12.13 & -1796.52378914 & -4.63 & 13.50 \\
\hline-1796.50490533 & -11.76 & 7.13 & -1796.51559943 & -9.02 & 9.87 & -1796.52633183 & -6.23 & 12.67 \\
\hline-1796.50378256 & -11.05 & 6.58 & -1796.51470032 & -8.46 & 9.18 & -1796.52566860 & -5.81 & 11.83 \\
\hline-1796.51869654 & -20.41 & -2.63 & -1796.52988928 & -17.99 & -0.20 & -1796.54114509 & -15.52 & 2.26 \\
\hline-1796.48616691 & 0.00 & 0.00 & -1796.50122636 & 0.00 & 0.00 & -1796.51641073 & 0.00 & 0.00 \\
\hline-1796.52546181 & -24.66 & -8.29 & -1796.53525071 & -21.35 & -4.98 & -1796.54487195 & -17.86 & -1.49 \\
\hline-1796.50940099 & -14.58 & 3.96 & -1796.52028289 & -11.96 & 6.58 & -1796.53178946 & -9.65 & 8.89 \\
\hline-1796.51199522 & -16.21 & 2.95 & -1796.52267249 & -13.46 & 5.70 & -1796.53404683 & -11.07 & 8.09 \\
\hline-1796.49180879 & -3.54 & 15.25 & -1796.50294000 & -1.08 & 17.72 & -1796.51512469 & 0.81 & 19.60 \\
\hline-1796.50632927 & -12.65 & 6.18 & -1796.51720022 & -10.02 & 8.81 & -1796.52942189 & -8.16 & 10.66 \\
\hline
\end{tabular}




\begin{tabular}{|c|c|c|c|c|c|c|c|c|}
\hline \multicolumn{3}{|c|}{ M06/basis2 (gas-phase) } & \multicolumn{3}{|c|}{ M06/basis2 (benzene) } & \multicolumn{3}{|c|}{ M06/basis2 (dichloromethane) } \\
\hline $\begin{array}{c}\mathbf{E}_{\mathrm{sp}} \\
\text { [hartree] }\end{array}$ & $\begin{array}{c}\Delta \mathbf{E}_{\mathrm{sp}} \\
{[\mathrm{kcal} / \mathrm{mol}]}\end{array}$ & $\begin{array}{c}\Delta \mathbf{G}_{\mathrm{sp}} \\
{[\mathrm{kcal} / \mathrm{mol}]}\end{array}$ & $\begin{array}{c}\mathbf{E}_{\mathrm{sp}} \\
\text { [hartree] }\end{array}$ & $\begin{array}{c}\Delta \mathbf{E}_{\mathrm{sp}} \\
{[\mathrm{kcal} / \mathrm{mol}]}\end{array}$ & $\begin{array}{c}\Delta \mathbf{G}_{\text {sp }} \\
{[\mathrm{kcal} / \mathrm{mol}]}\end{array}$ & $\begin{array}{c}\mathbf{E}_{\mathrm{sp}} \\
\text { [hartree] }\end{array}$ & $\begin{array}{c}\Delta \mathbf{E}_{\mathrm{sp}} \\
{[\mathrm{kcal} / \mathrm{mol}]}\end{array}$ & $\begin{array}{c}\Delta \mathbf{G}_{\mathrm{sp}} \\
{[\mathrm{kcal} / \mathrm{mol}]}\end{array}$ \\
\hline-1795.75930631 & -20.44 & -2.60 & -1795.76802333 & -17.40 & 0.44 & -1795.77751540 & -14.74 & 3.11 \\
\hline-1795.74038984 & -8.57 & 10.68 & -1795.75005700 & -6.13 & 13.13 & -1795.76053673 & -4.08 & 15.17 \\
\hline-1795.75123794 & -15.38 & 3.45 & -1795.76109510 & -13.06 & 5.78 & -1795.77170847 & -11.09 & 7.74 \\
\hline-1795.74168255 & -9.38 & 7.91 & -1795.75241164 & -7.61 & 9.68 & -1795.76420962 & -6.39 & 10.90 \\
\hline-1795.75957783 & -20.61 & -4.37 & -1795.76893306 & -17.98 & -1.74 & -1795.77871348 & -15.49 & 0.75 \\
\hline-1795.72079829 & 3.72 & 3.26 & -1795.73463513 & 3.55 & 3.08 & -1795.74917466 & 3.05 & 2.58 \\
\hline-1795.75404102 & -17.14 & 1.48 & -1795.76359885 & -14.63 & 3.99 & -1795.77350726 & -12.22 & 6.40 \\
\hline-1795.74211109 & -9.65 & 8.48 & -1795.75329710 & -8.16 & 9.97 & -1795.76516403 & -6.99 & 11.15 \\
\hline-1795.74887484 & -13.90 & 5.00 & -1795.75857990 & -11.48 & 7.41 & -1795.76844198 & -9.04 & 9.85 \\
\hline-1795.74728050 & -12.90 & 4.74 & -1795.75717257 & -10.60 & 7.04 & -1795.76721516 & -8.27 & 9.36 \\
\hline-1795.76010358 & -20.94 & -3.16 & -1795.77019315 & -18.77 & -0.98 & -1795.78044344 & -16.57 & 1.21 \\
\hline-1795.72672918 & 0.00 & 0.00 & -1795.74028747 & 0.00 & 0.00 & -1795.75403119 & 0.00 & 0.00 \\
\hline-1795.76806850 & -25.94 & -9.57 & -1795.77688901 & -22.97 & -6.60 & -1795.78565621 & -19.85 & -3.48 \\
\hline-1795.74996168 & -14.58 & 3.96 & -1795.75985408 & -12.28 & 6.26 & -1795.77047292 & -10.32 & 8.22 \\
\hline-1795.75297353 & -16.47 & 2.69 & -1795.76268083 & -14.05 & 5.11 & -1795.77317358 & -12.01 & 7.15 \\
\hline-1795.73483330 & -5.09 & 13.71 & -1795.74483614 & -2.85 & 15.94 & -1795.75591205 & -1.18 & 17.61 \\
\hline-1795.74830249 & -13.54 & 5.29 & -1795.75805771 & -11.15 & 7.68 & -1795.76907756 & -9.44 & 9.39 \\
\hline $\begin{array}{l}\mathrm{E} \ldots \ldots \ldots \ldots \text { gas pha } \\
\mathrm{G} \ldots \ldots \ldots \text {. } \ldots \text { sum of } \\
\Delta \mathrm{E} \ldots \ldots \ldots \text { relative } \\
\Delta \mathrm{G} \ldots \ldots \ldots \text { relative } \\
\Delta \mathrm{G}_{\text {corr }} \ldots \ldots \text { gas pha } \\
\mathrm{E}_{\mathrm{sp}} \ldots \ldots \ldots \text { single } \\
\Delta \mathrm{E}_{\mathrm{sp}} \ldots \ldots \text { relative } \\
\Delta \mathrm{G}_{\mathrm{s}} \ldots \ldots . \text { relative }\end{array}$ & $\begin{array}{l}\text { electronic en } \\
\text { lectronic and t } \\
\text { lectronic ener } \\
\text { ree energy in } 1 \\
\text { e thermal corre } \\
\text { int electronic } \\
\text { ingle point ele } \\
\text { ingle point fre }\end{array}$ & $\begin{array}{l}\text { gy in hartree } \\
\text { rmal free ener } \\
\text { in kcal/mol w } \\
\text { al/mol with ba } \\
\text { tion to free ene } \\
\text { ergy in gas ph } \\
\text { ronic energy i } \\
\text { energy in gas }\end{array}$ & $\begin{array}{l}\text { th basis } 1 \\
\text { ies with basis } 1 \\
\text { th basis } 1 \\
\text { is } 1 \\
\text { gy in } \mathrm{kcal} / \mathrm{mol} \text { obta } \\
\text { se or solution in ha } \\
\text { gas phase or soluti } \\
\text { hase or solution in } 1\end{array}$ & $\begin{array}{l}\text { ned with basis } \\
\text { ree with basis2 } \\
\text { in } \mathrm{kcal} / \mathrm{mol} \\
\mathrm{al} / \mathrm{mol}\left(\Delta \mathrm{G}_{\mathrm{sp}}\right.\end{array}$ & $\begin{array}{l}(\Delta \mathrm{G}=\Delta \mathrm{E}+\Delta \\
\mathrm{h} \text { basis2 } \\
\Delta \mathrm{E}_{\mathrm{s}} / \mathrm{b} \text { asis } 2+\Delta\end{array}$ & corr) & & \\
\hline
\end{tabular}


Table S34. ROCM with catalyst Ru-5b and enol ether (pathways A3, AB3, B3 in Figure 5 and Figure S22)
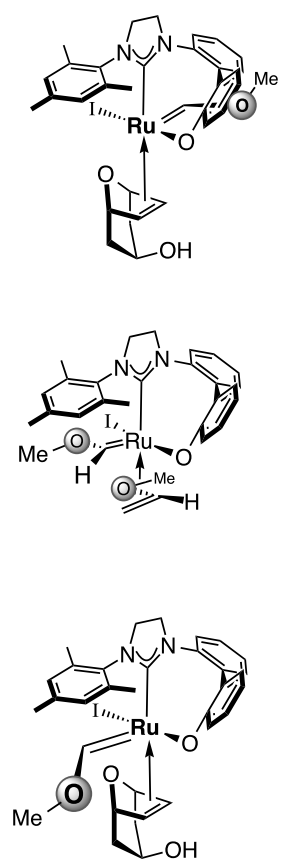

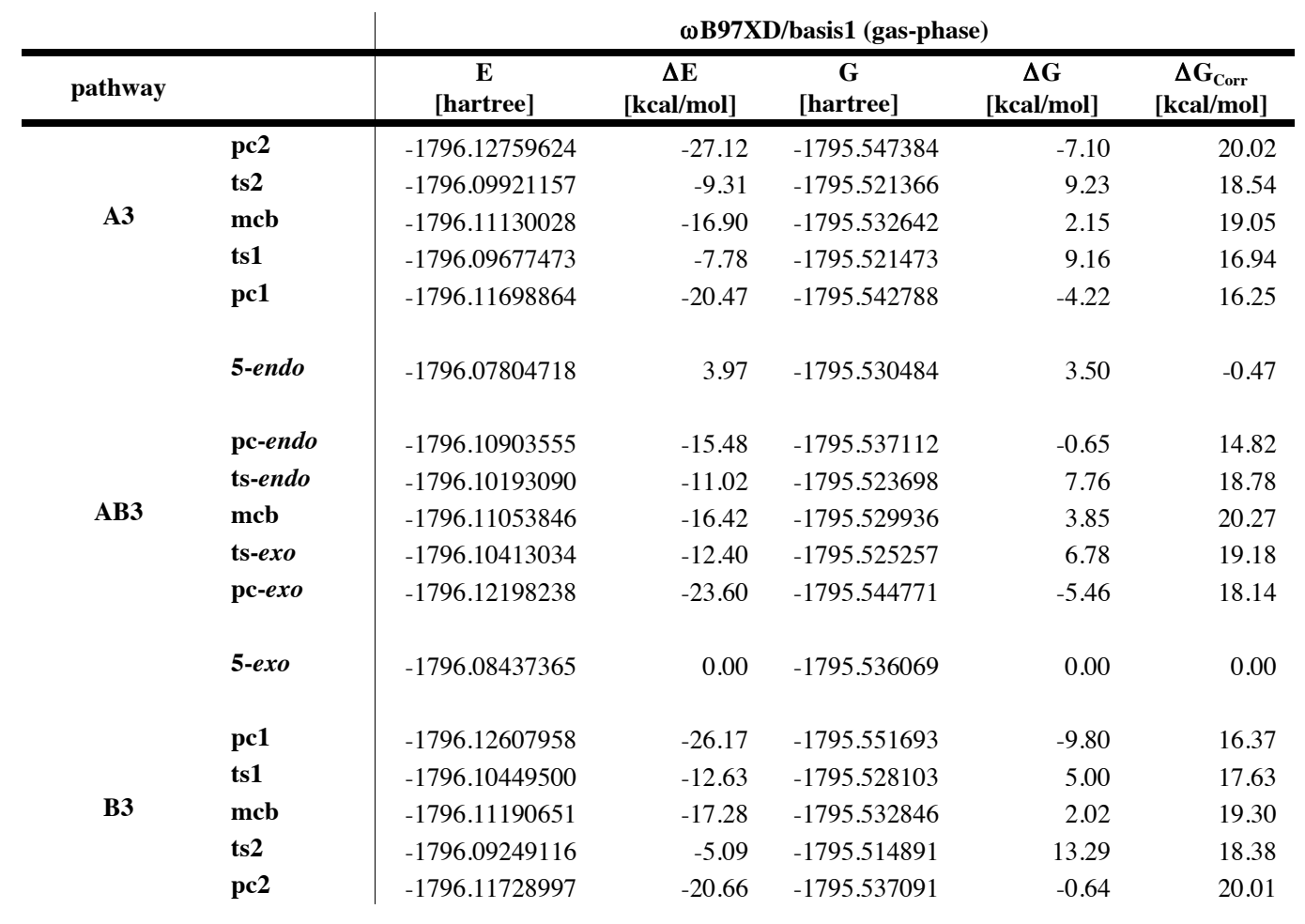

\begin{tabular}{|c|c|c|c|c|c|c|c|c|}
\hline \multicolumn{3}{|c|}{ 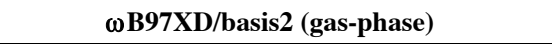 } & \multicolumn{3}{|c|}{ wB97XD/basis2 (benzene) } & \multicolumn{3}{|c|}{ wB97XD/basis2 (dichloromethane) } \\
\hline $\begin{array}{c}\mathbf{E}_{\mathrm{sp}} \\
\text { [hartree] }\end{array}$ & $\begin{array}{c}\Delta \mathrm{E}_{\mathrm{sp}} \\
{[\mathrm{kcal} / \mathrm{mol}]}\end{array}$ & $\begin{array}{c}\Delta \mathbf{G}_{\mathrm{sp}} \\
{[\mathrm{kcal} / \mathrm{mol}]}\end{array}$ & $\begin{array}{c}\mathbf{E}_{\mathrm{sp}} \\
\text { [hartree] }\end{array}$ & $\begin{array}{c}\Delta \mathrm{E}_{\mathrm{sp}} \\
{[\mathrm{kcal} / \mathrm{mol}]}\end{array}$ & $\begin{array}{c}\Delta \mathbf{G}_{\mathrm{sp}} \\
{[\mathrm{kcal} / \mathrm{mol}]}\end{array}$ & $\begin{array}{c}\mathbf{E}_{\mathrm{sp}} \\
\text { [hartree] }\end{array}$ & $\begin{array}{c}\Delta \mathrm{E}_{\mathrm{sp}} \\
{[\mathrm{kcal} / \mathrm{mol}]}\end{array}$ & $\begin{array}{c}\Delta \mathbf{G}_{\mathrm{sp}} \\
{[\mathrm{kcal} / \mathrm{mol}]}\end{array}$ \\
\hline-1796.52523039 & -24.51 & -4.49 & -1796.53326095 & -20.10 & -0.08 & -1796.54166299 & -15.85 & 4.18 \\
\hline-1796.49835573 & -7.65 & 10.89 & -1796.51014212 & -5.59 & 12.94 & -1796.52263207 & -3.90 & 14.63 \\
\hline-1796.51139122 & -15.83 & 3.22 & -1796.52258826 & -13.40 & 5.64 & -1796.53421866 & -11.17 & 7.87 \\
\hline-1796.49756199 & -7.15 & 9.79 & -1796.50969358 & -5.31 & 11.63 & -1796.52242876 & -3.78 & 13.16 \\
\hline-1796.51689043 & -19.28 & -3.03 & -1796.52727183 & -16.34 & -0.09 & -1796.53798478 & -13.54 & 2.71 \\
\hline-1796.48004259 & 3.84 & 3.38 & -1796.49551056 & 3.59 & 3.12 & -1796.51169149 & 2.96 & 2.50 \\
\hline-1796.50668908 & -12.88 & 1.94 & -1796.51790208 & -10.46 & 4.36 & -1796.52993230 & -8.48 & 6.34 \\
\hline-1796.49988220 & -8.61 & 10.17 & -1796.51211147 & -6.83 & 11.95 & -1796.52498909 & -5.38 & 13.40 \\
\hline-1796.50770802 & -13.52 & 6.75 & -1796.51828360 & -10.70 & 9.56 & -1796.52897493 & -7.88 & 12.38 \\
\hline-1796.50199545 & -9.93 & 9.25 & -1796.51339393 & -7.64 & 11.55 & -1796.52534138 & -5.60 & 13.58 \\
\hline-1796.51870862 & -20.42 & -2.28 & -1796.52989832 & -17.99 & 0.15 & -1796.54114145 & -15.52 & 2.62 \\
\hline-1796.48616691 & 0.00 & 0.00 & -1796.50122636 & 0.00 & 0.00 & -1796.51641073 & 0.00 & 0.00 \\
\hline-1796.52546184 & -24.66 & -8.29 & -1796.53525074 & -21.35 & -4.98 & -1796.54487197 & -17.86 & -1.49 \\
\hline-1796.50505190 & -11.85 & 5.77 & -1796.51634413 & -9.49 & 8.14 & -1796.52820346 & -7.40 & 10.23 \\
\hline-1796.51182943 & -16.10 & 3.20 & -1796.52306860 & -13.71 & 5.59 & -1796.53492982 & -11.62 & 7.68 \\
\hline-1796.49183784 & -3.56 & 14.82 & -1796.50297979 & -1.10 & 17.28 & -1796.51493362 & 0.93 & 19.31 \\
\hline-1796.51491880 & -18.04 & 1.97 & -1796.52359440 & -14.04 & 5.98 & -1796.53309047 & -10.47 & 9.55 \\
\hline
\end{tabular}




\begin{tabular}{|c|c|c|c|c|c|c|c|c|}
\hline \multicolumn{3}{|c|}{ M06/basis2 (gas-phase) } & \multicolumn{3}{|c|}{ M06/basis2 (benzene) } & \multicolumn{3}{|c|}{ M06/basis2 (dichloromethane) } \\
\hline $\begin{array}{c}\mathbf{E}_{\mathrm{sp}} \\
\text { [hartree] }\end{array}$ & $\begin{array}{c}\Delta \mathbf{E}_{\mathrm{sp}} \\
{[\mathrm{kcal} / \mathrm{mol}]}\end{array}$ & $\begin{array}{c}\Delta \mathbf{G}_{\mathrm{sp}} \\
{[\mathrm{kcal} / \mathrm{mol}]}\end{array}$ & $\begin{array}{c}\mathbf{E}_{\mathrm{sp}} \\
\text { [hartree] }\end{array}$ & $\begin{array}{c}\Delta \mathbf{E}_{\mathrm{sp}} \\
{[\mathrm{kcal} / \mathrm{mol}]}\end{array}$ & $\begin{array}{c}\Delta \mathbf{G}_{\text {sp }} \\
{[\mathrm{kcal} / \mathrm{mol}]}\end{array}$ & $\begin{array}{c}\mathbf{E}_{\mathrm{sp}} \\
\text { [hartree] }\end{array}$ & $\begin{array}{c}\Delta \mathbf{E}_{\mathrm{sp}} \\
{[\mathrm{kcal} / \mathrm{mol}]}\end{array}$ & $\begin{array}{c}\Delta \mathbf{G}_{\mathrm{sp}} \\
{[\mathrm{kcal} / \mathrm{mol}]}\end{array}$ \\
\hline-1795.76765615 & -25.68 & -5.66 & -1795.77485452 & -21.69 & -1.67 & -1795.78249500 & -17.86 & 2.16 \\
\hline-1795.73772157 & -6.90 & 11.64 & -1795.74838116 & -5.08 & 13.46 & -1795.75982034 & -3.63 & 14.90 \\
\hline-1795.75167567 & -15.65 & 3.39 & -1795.76183327 & -13.52 & 5.53 & -1795.77253179 & -11.61 & 7.44 \\
\hline-1795.73846408 & -7.36 & 9.58 & -1795.74939591 & -5.72 & 11.23 & -1795.76103232 & -4.39 & 12.55 \\
\hline-1795.75957796 & -20.61 & -4.36 & -1795.76893320 & -17.98 & -1.73 & -1795.77871363 & -15.49 & 0.76 \\
\hline-1795.72079829 & 3.72 & 3.26 & -1795.73463513 & 3.55 & 3.08 & -1795.74917466 & 3.05 & 2.58 \\
\hline-1795.75154030 & -15.57 & -0.75 & -1795.76154560 & -13.34 & 1.48 & -1795.77235469 & -11.50 & 3.32 \\
\hline-1795.74311904 & -10.28 & 8.50 & -1795.75408661 & -8.66 & 10.12 & -1795.76572826 & -7.34 & 11.44 \\
\hline-1795.75128090 & -15.41 & 4.86 & -1795.76078915 & -12.86 & 7.40 & -1795.77048041 & -10.32 & 9.95 \\
\hline-1795.74632982 & -12.30 & 6.88 & -1795.75659688 & -10.23 & 8.95 & -1795.76745571 & -8.42 & 10.76 \\
\hline-1795.76001949 & -20.89 & -2.75 & -1795.77010794 & -18.71 & -0.57 & -1795.78034820 & -16.51 & 1.63 \\
\hline-1795.72672918 & 0.00 & 0.00 & -1795.74028747 & 0.00 & 0.00 & -1795.75403119 & 0.00 & 0.00 \\
\hline-1795.76806856 & -25.94 & -9.57 & -1795.77688906 & -22.97 & -6.60 & -1795.78565626 & -19.85 & -3.48 \\
\hline-1795.74589517 & -12.03 & 5.60 & -1795.75610721 & -9.93 & 7.70 & -1795.76698193 & -8.13 & 9.50 \\
\hline-1795.75213887 & -15.94 & 3.35 & -1795.76235081 & -13.84 & 5.45 & -1795.77326797 & -12.07 & 7.23 \\
\hline-1795.73503115 & -5.21 & 13.17 & -1795.74509984 & -3.02 & 15.36 & -1795.75602446 & -1.25 & 17.13 \\
\hline-1795.75769621 & -19.43 & 0.58 & -1795.76544521 & -15.79 & 4.23 & -1795.77401582 & -12.54 & 7.47 \\
\hline $\begin{array}{l}\mathrm{E} \ldots \ldots \ldots \ldots \text { gas pha } \\
\mathrm{G} \ldots \ldots \ldots \text { sum of } \\
\Delta \mathrm{E} \ldots \ldots \ldots \text { relative } \\
\Delta \mathrm{G} \ldots \ldots \ldots \text { relative } \\
\Delta \mathrm{G}_{\mathrm{corr}} \ldots \ldots \text { gas pha } \\
\mathrm{E}_{\mathrm{sp}} \ldots \ldots \ldots \text { single } \\
\Delta \mathrm{E}_{\mathrm{sp}} \ldots \ldots \text { relative } \\
\Delta \mathrm{G}_{\mathrm{sp}} \ldots \ldots \ldots \text { relative }\end{array}$ & $\begin{array}{l}\text { electronic en } \\
\text { lectronic and } t \\
\text { lectronic ener } \\
\text { ree energy in } 1 \\
\text { e thermal corre } \\
\text { int electronic } \\
\text { ingle point ele } \\
\text { ingle point fre }\end{array}$ & $\begin{array}{l}\text { gy in hartree } \\
\text { rmal free ener } \\
\text { in kcal/mol w } \\
\text { al/mol with ba } \\
\text { tion to free ene } \\
\text { ergy in gas ph } \\
\text { ronic energy i } \\
\text { energy in gas }\end{array}$ & $\begin{array}{l}\text { th basis } 1 \\
\text { ies with basis } 1 \\
\text { th basis } 1 \\
\text { is } 1 \\
\text { gy in kcal/mol obta } \\
\text { se or solution in har } \\
\text { gas phase or soluti } \\
\text { hase or solution in } 1\end{array}$ & $\begin{array}{l}\text { ned with basis } \\
\text { ree with basis2 } \\
\text { in } \mathrm{kcal} / \mathrm{mol} \mathrm{m} \\
\mathrm{al} / \mathrm{mol}\left(\Delta \mathrm{G}_{\mathrm{sp}}=\right.\end{array}$ & $\begin{array}{l}(\Delta \mathrm{G}=\Delta \mathrm{E}+\Delta \\
\mathrm{h} \text { basis2 } \\
\mathrm{E}_{\mathrm{sp}} / \text { basis } 2+\end{array}$ & corr) & & \\
\hline
\end{tabular}




\section{Table S35. ROCM with catalyst Ru-5b and enol ether (pathways A4 $=E R u-5 b, A B 4, B 4$ in Figure 5 and Figure S22)}

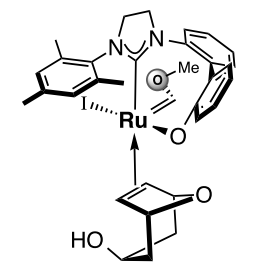

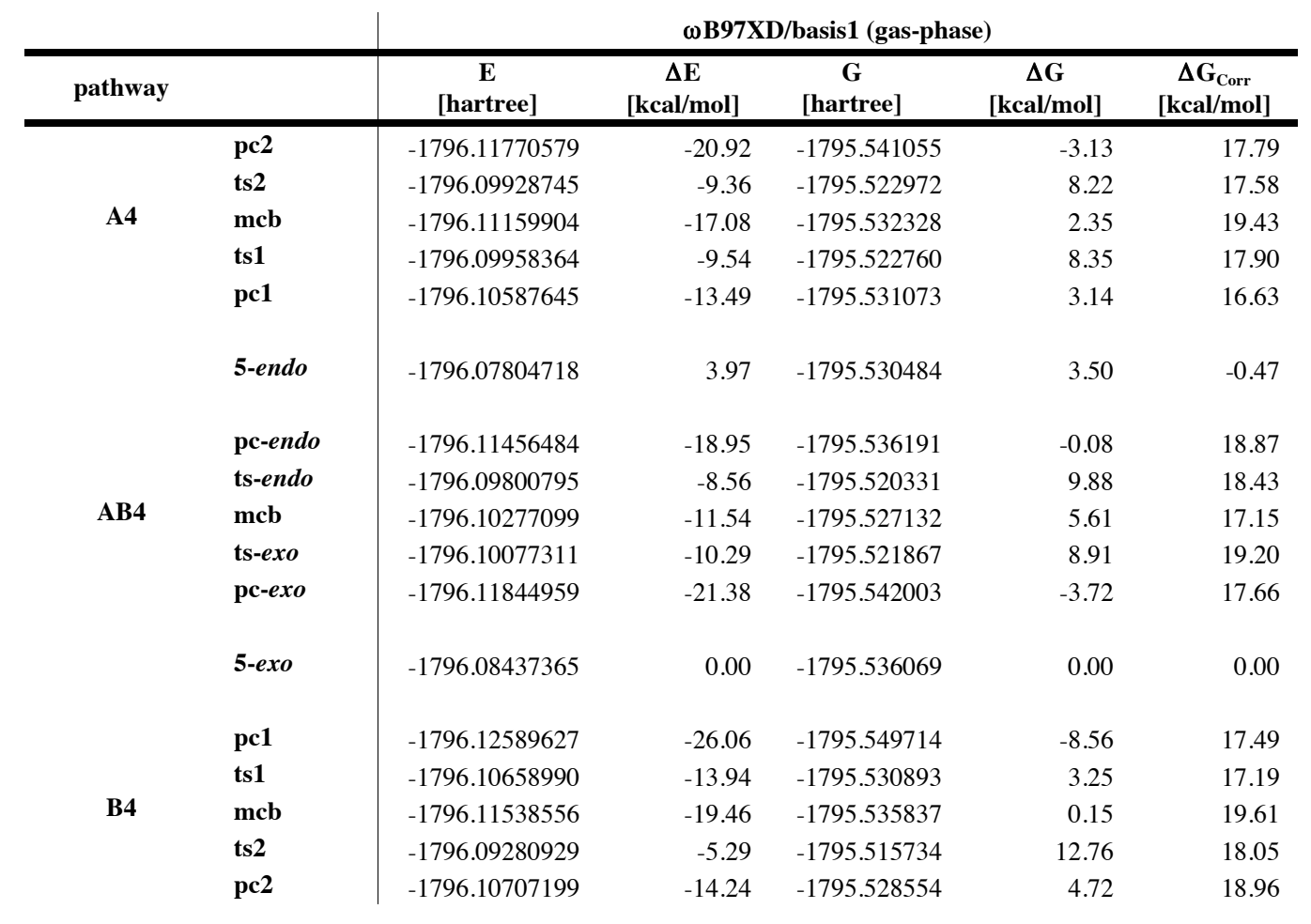

\begin{tabular}{|c|c|c|c|c|c|c|c|c|}
\hline \multicolumn{3}{|c|}{$\omega \mathrm{B} 97 X D /$ basis2 (gas-phase) } & \multicolumn{3}{|c|}{ wB97XD/basis2 (benzene) } & \multicolumn{3}{|c|}{ wB97XD/basis2 (dichloromethane) } \\
\hline $\begin{array}{c}\mathbf{E}_{\mathrm{sp}} \\
\text { [hartree] }\end{array}$ & $\begin{array}{c}\Delta \mathrm{E}_{\mathrm{sp}} \\
{[\mathrm{kcal} / \mathrm{mol}]}\end{array}$ & $\begin{array}{c}\Delta \mathbf{G}_{\mathrm{sp}} \\
{[\mathrm{kcal} / \mathrm{mol}]}\end{array}$ & $\begin{array}{c}\mathbf{E}_{\mathrm{sp}} \\
\text { [hartree] }\end{array}$ & $\begin{array}{c}\Delta \mathrm{E}_{\mathrm{sp}} \\
{[\mathrm{kcal} / \mathrm{mol}]}\end{array}$ & $\begin{array}{c}\Delta \mathbf{G}_{\mathrm{sp}} \\
{[\mathrm{kcal} / \mathrm{mol}]}\end{array}$ & $\begin{array}{c}\mathbf{E}_{\mathrm{sp}} \\
\text { [hartree] }\end{array}$ & $\begin{array}{c}\Delta \mathrm{E}_{\mathrm{sp}} \\
{[\mathrm{kcal} / \mathrm{mol}]}\end{array}$ & $\begin{array}{c}\Delta \mathbf{G}_{\mathrm{sp}} \\
{[\mathrm{kcal} / \mathrm{mol}]}\end{array}$ \\
\hline-1796.51665183 & -19.13 & -1.34 & -1796.52630401 & -15.74 & 2.05 & -1796.53652074 & -12.62 & 5.17 \\
\hline-1796.49817967 & -7.54 & 10.04 & -1796.50868334 & -4.68 & 12.90 & -1796.51971359 & -2.07 & 15.50 \\
\hline-1796.51123653 & -15.73 & 3.70 & -1796.52180760 & -12.91 & 6.52 & -1796.53275903 & -10.26 & 9.17 \\
\hline-1796.50046594 & -8.97 & 8.92 & -1796.51263887 & -7.16 & 10.73 & -1796.52575230 & -5.86 & 12.03 \\
\hline-1796.50588639 & -12.37 & 4.25 & -1796.51589232 & -9.20 & 7.43 & -1796.52668790 & -6.45 & 10.18 \\
\hline-1796.48004259 & 3.84 & 3.38 & -1796.49551056 & 3.59 & 3.12 & -1796.51169149 & 2.96 & 2.50 \\
\hline-1796.51185655 & -16.12 & 2.75 & -1796.52244231 & -13.31 & 5.56 & -1796.53330565 & -10.60 & 8.27 \\
\hline-1796.49612994 & -6.25 & 12.18 & -1796.50771810 & -4.07 & 14.36 & -1796.51959849 & -2.00 & 16.43 \\
\hline-1796.50095552 & -9.28 & 7.87 & -1796.51196879 & -6.74 & 10.41 & -1796.52323861 & -4.28 & 12.87 \\
\hline-1796.49863227 & -7.82 & 11.38 & -1796.50967642 & -5.30 & 13.90 & -1796.52109453 & -2.94 & 16.26 \\
\hline-1796.51582523 & -18.61 & -0.95 & -1796.52703930 & -16.20 & 1.46 & -1796.53851399 & -13.87 & 3.79 \\
\hline-1796.48616691 & 0.00 & 0.00 & -1796.50122636 & 0.00 & 0.00 & -1796.51641073 & 0.00 & 0.00 \\
\hline-1796.52538408 & -24.61 & -7.12 & -1796.53556817 & -21.55 & -4.06 & -1796.54582556 & -18.46 & -0.96 \\
\hline-1796.50722415 & -13.21 & 3.98 & -1796.51856711 & -10.88 & 6.31 & -1796.53065557 & -8.94 & 8.25 \\
\hline-1796.51506532 & -18.13 & 1.47 & -1796.52568457 & -15.35 & 4.26 & -1796.53680465 & -12.80 & 6.81 \\
\hline-1796.49200369 & -3.66 & 14.39 & -1796.50335428 & -1.34 & 16.72 & -1796.51580107 & 0.38 & 18.44 \\
\hline-1796.50660767 & -12.83 & 6.13 & -1796.51730386 & -10.09 & 8.87 & -1796.52917142 & -8.01 & 10.95 \\
\hline
\end{tabular}




\begin{tabular}{|c|c|c|c|c|c|c|c|c|}
\hline \multicolumn{3}{|c|}{ M06/basis2 (gas-phase) } & \multicolumn{3}{|c|}{ M06/basis2 (benzene) } & \multicolumn{3}{|c|}{ M06/basis2 (dichloromethane) } \\
\hline $\begin{array}{c}\mathbf{E}_{\mathrm{sp}} \\
{[\text { hartree] }}\end{array}$ & $\begin{array}{c}\Delta \mathbf{E}_{\mathrm{sp}} \\
{[\mathrm{kcal} / \mathrm{mol}]}\end{array}$ & $\begin{array}{c}\Delta \mathbf{G}_{\mathrm{sp}} \\
{[\mathrm{kcal} / \mathrm{mol}]}\end{array}$ & $\begin{array}{c}\mathbf{E}_{\mathrm{sp}} \\
\text { [hartree] }\end{array}$ & $\begin{array}{c}\Delta \mathbf{E}_{\mathrm{sp}} \\
{[\mathrm{kcal} / \mathrm{mol}]}\end{array}$ & $\begin{array}{c}\Delta \mathbf{G}_{\mathrm{sp}} \\
{[\mathrm{kcal} / \mathrm{mol}]}\end{array}$ & $\begin{array}{c}\mathbf{E}_{\mathrm{sp}} \\
\text { [hartree] }\end{array}$ & $\begin{array}{c}\Delta \mathbf{E}_{\mathrm{sp}} \\
{[\mathrm{kcal} / \mathrm{mol}]}\end{array}$ & $\begin{array}{c}\Delta \mathbf{G}_{\mathrm{sp}} \\
{[\mathrm{kcal} / \mathrm{mol}]}\end{array}$ \\
\hline-1795.75897018 & -20.23 & -2.44 & -1795.76767868 & -17.19 & 0.60 & -1795.77699910 & -14.41 & 3.37 \\
\hline-1795.73952327 & -8.03 & 9.55 & -1795.74896323 & -5.44 & 12.13 & -1795.75900396 & -3.12 & 14.46 \\
\hline-1795.75190046 & -15.80 & 3.64 & -1795.76145909 & -13.29 & 6.15 & -1795.77149868 & -10.96 & 8.47 \\
\hline-1795.74168044 & -9.38 & 8.51 & -1795.75262109 & -7.74 & 10.16 & -1795.76456873 & -6.61 & 11.28 \\
\hline-1795.75146917 & -15.52 & 1.10 & -1795.76039317 & -12.62 & 4.01 & -1795.77008273 & -10.07 & 6.56 \\
\hline-1795.72079829 & 3.72 & 3.26 & -1795.73463513 & 3.55 & 3.08 & -1795.74917466 & 3.05 & 2.58 \\
\hline-1795.75396683 & -17.09 & 1.78 & -1795.76352361 & -14.58 & 4.29 & -1795.77343111 & -12.17 & 6.70 \\
\hline-1795.74048247 & -8.63 & 9.80 & -1795.75099221 & -6.72 & 11.71 & -1795.76187400 & -4.92 & 13.51 \\
\hline-1795.74530206 & -11.65 & 5.50 & -1795.75535561 & -9.46 & 7.70 & -1795.76576735 & -7.36 & 9.79 \\
\hline-1795.74275584 & -10.06 & 9.15 & -1795.75283243 & -7.87 & 11.33 & -1795.76336422 & -5.86 & 13.35 \\
\hline-1795.75722859 & -19.14 & -1.48 & -1795.76738623 & -17.00 & 0.65 & -1795.77791126 & -14.98 & 2.67 \\
\hline-1795.72672918 & 0.00 & 0.00 & -1795.74028747 & 0.00 & 0.00 & -1795.75403119 & 0.00 & 0.00 \\
\hline-1795.76829594 & -26.08 & -8.59 & -1795.77740831 & -23.29 & -5.80 & -1795.78667979 & -20.49 & -2.99 \\
\hline-1795.74842034 & -13.61 & 3.58 & -1795.75867722 & -11.54 & 5.65 & -1795.76974385 & -9.86 & 7.33 \\
\hline-1795.75584166 & -18.27 & 1.34 & -1795.76542563 & -15.77 & 3.83 & -1795.77558159 & -13.52 & 6.08 \\
\hline-1795.73362890 & -4.33 & 13.72 & -1795.74381563 & -2.21 & 15.84 & -1795.75509117 & -0.67 & 17.39 \\
\hline-1795.74924685 & -14.13 & 4.83 & -1795.75887000 & -11.66 & 7.30 & -1795.76963214 & -9.79 & 9.17 \\
\hline $\begin{array}{l}\mathrm{E} \ldots \ldots \ldots \ldots \text { gas ph } \\
\mathrm{G} \ldots \ldots \ldots \text { sum of } \\
\Delta \mathrm{E} \ldots \ldots \ldots \text { relativ } \\
\Delta \mathrm{G} \ldots \ldots \ldots \text { relativ } \\
\Delta \mathrm{G}_{\mathrm{corr}} \ldots \ldots \text { gas ph } \\
\mathrm{E}_{\mathrm{sp}} \ldots \ldots \ldots \text { single } \\
\Delta \mathrm{E}_{\mathrm{sp}} \ldots \ldots \text { relativ } \\
\Delta \mathrm{G}_{\mathrm{sp}} \ldots \ldots \text { relativ }\end{array}$ & $\begin{array}{l}\text { electronic en } \\
\text { lectronic and tl } \\
\text { lectronic ener } \\
\text { ree energy in } k \\
\text { thermal corre } \\
\text { int electronic } \\
\text { ingle point ele } \\
\text { ingle point fre }\end{array}$ & $\begin{array}{l}\text { gy in hartree } \\
\text { rmal free ener } \\
\text { / in kcal/mol w } \\
\text { al/mol with ba } \\
\text { tion to free ene } \\
\text { ergy in gas ph } \\
\text { ronic energy i } \\
\text { energy in gas }\end{array}$ & $\begin{array}{l}\text { th basis } 1 \\
\text { ies with basis } 1 \\
\text { th basis } 1 \\
\text { is } 1 \\
\text { gy in } \mathrm{kcal} / \mathrm{mol} \text { obt } \\
\text { se or solution in ha } \\
\text { gas phase or solut } \\
\text { ase or solution in }\end{array}$ & $\begin{array}{l}\text { ned with basis } \\
\text { ee with basis2 } \\
\text { in } \mathrm{kcal} / \mathrm{mol} \\
\mathrm{al} / \mathrm{mol}\left(\Delta \mathrm{G}_{\mathrm{s}}=\right.\end{array}$ & $\begin{array}{l}(\Delta \mathrm{G}=\Delta \mathrm{E}+\Delta \\
\mathrm{h} \text { basis } 2 \\
\mathrm{E}_{\mathrm{s}} / \text { basis } 2+\end{array}$ & orr) & & \\
\hline
\end{tabular}


Table S36. CM between acrylonitrile and propene with catalyst $\mathrm{Ru}-4 \mathrm{~b}$ (pathways $E M e$, ECN in Figure 7)<smiles>C[C@H](Cl)[C@@](Cl)(C=CC#N)C1CCN(C)C1</smiles>

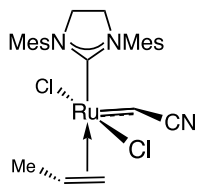

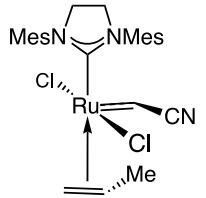

\begin{tabular}{|c|c|c|c|c|c|c|}
\hline & & \multicolumn{5}{|c|}{$\omega$ B97XD/basis1 (gas-phase) } \\
\hline \multicolumn{2}{|l|}{ pathway } & $\begin{array}{c}\mathbf{E} \\
\text { [hartree] }\end{array}$ & $\begin{array}{c}\Delta \mathrm{E} \\
{[\mathrm{kcal} / \mathrm{mol}]}\end{array}$ & $\begin{array}{c}\mathbf{G} \\
{[\text { hartree] }}\end{array}$ & $\begin{array}{c}\Delta \mathbf{G} \\
{[\mathrm{kcal} / \mathrm{mol}]}\end{array}$ & $\begin{array}{c}\Delta \mathbf{G}_{\mathrm{Corr}} \\
{[\mathrm{kcal} / \mathrm{mol}]}\end{array}$ \\
\hline \multirow{7}{*}{$E M e$} & $14 \mathrm{e}$ & -2189.89666470 & 3.44 & -2189.451306 & 2.77 & -0.67 \\
\hline & pc2 & -2189.92334396 & -13.30 & -2189.454279 & 0.90 & 14.21 \\
\hline & ts2 & -2189.91603074 & -8.71 & -2189.443060 & 7.94 & 16.66 \\
\hline & mcb & -2189.93173124 & -18.57 & -2189.456188 & -0.30 & 18.27 \\
\hline & ts1 & -2189.92427313 & -13.89 & -2189.451373 & 2.73 & 16.61 \\
\hline & pc1 & -2189.92868489 & -16.65 & -2189.455921 & -0.13 & 16.53 \\
\hline & $14 \mathrm{e}_{\mathrm{Me}}$ & -2189.90214360 & 0.00 & -2189.455716 & 0.00 & 0.00 \\
\hline \multirow{5}{*}{$\mathrm{Me}-\mathrm{CN}$} & pc1 & -2189.92763775 & -16.00 & -2189.452912 & 1.76 & 17.76 \\
\hline & ts1 & -2189.92526632 & -14.51 & -2189.450843 & 3.06 & 17.57 \\
\hline & mcb & -2189.94123520 & -24.53 & -2189.464880 & -5.75 & 18.78 \\
\hline & ts2 & -2189.92534844 & -14.56 & -2189.450121 & 3.51 & 18.07 \\
\hline & pc2 & -2189.92945369 & -17.14 & -2189.457470 & -1.10 & 16.04 \\
\hline \multirow{7}{*}{$E C N$} & $14 \mathrm{e}_{\mathrm{CN}}$ & -2189.89663961 & 3.45 & -2189.449681 & 3.79 & 0.33 \\
\hline & pc1 & -2189.92978658 & -17.35 & -2189.458688 & -1.86 & 15.48 \\
\hline & ts1 & -2189.92475184 & -14.19 & -2189.451621 & 2.57 & 16.76 \\
\hline & mcb & -2189.93823746 & -22.65 & -2189.463111 & -4.64 & 18.01 \\
\hline & ts2 & -2189.91977687 & -11.07 & -2189.445093 & 6.67 & 17.73 \\
\hline & pc2 & -2189.92132848 & -12.04 & -2189.448875 & 4.29 & 16.33 \\
\hline & $14 e$ & -2189.89666470 & 3.44 & -2189.451306 & 2.77 & -0.67 \\
\hline
\end{tabular}

$\omega \mathrm{B97XD/basis2}$ (gas-phase)

\begin{tabular}{|c|c|c|c|c|c|c|c|c|}
\hline \multicolumn{3}{|c|}{ wB97XD/basis2 (gas-phase) } & \multicolumn{3}{|c|}{$\omega \mathrm{B} 97 \mathrm{XD} / \mathrm{basis} 2$ (benzene) } & \multicolumn{3}{|c|}{ wB97XD/basis2 (dichloromethane) } \\
\hline $\begin{array}{c}\mathbf{E}_{\mathrm{sp}} \\
\text { [hartree] }\end{array}$ & $\begin{array}{c}\Delta \mathrm{E}_{\mathrm{sp}} \\
{[\mathrm{kcal} / \mathrm{mol}]}\end{array}$ & $\begin{array}{c}\Delta \mathbf{G}_{\mathrm{sp}} \\
{[\mathrm{kcal} / \mathrm{mol}]}\end{array}$ & $\begin{array}{c}\mathbf{E}_{\mathrm{sp}} \\
\text { [hartree] }\end{array}$ & $\begin{array}{c}\Delta \mathrm{E}_{\mathrm{sp}} \\
{[\mathrm{kcal} / \mathrm{mol}]}\end{array}$ & $\begin{array}{c}\Delta \mathbf{G}_{\mathrm{sp}} \\
{[\mathrm{kcal} / \mathrm{mol}]}\end{array}$ & $\begin{array}{c}\mathbf{E}_{\mathrm{sp}} \\
\text { [hartree] }\end{array}$ & $\begin{array}{c}\Delta \mathrm{E}_{\mathrm{sp}} \\
{[\mathrm{kcal} / \mathrm{mol}]}\end{array}$ & $\begin{array}{c}\Delta \mathbf{G}_{\mathrm{sp}} \\
{[\mathrm{kcal} / \mathrm{mol}]}\end{array}$ \\
\hline-2190.23013283 & 3.54 & 2.87 & -2190.24443041 & 3.29 & 2.62 & -2190.25849963 & 3.16 & 2.49 \\
\hline-2190.25327348 & -10.98 & 3.23 & -2190.26367394 & -8.79 & 5.42 & -2190.27395431 & -6.54 & 7.66 \\
\hline-2190.24632103 & -6.62 & 10.04 & -2190.25667615 & -4.40 & 12.26 & -2190.26707018 & -2.22 & 14.44 \\
\hline-2190.26223791 & -16.60 & 1.67 & -2190.27237268 & -14.25 & 4.02 & -2190.28232486 & -11.79 & 6.48 \\
\hline-2190.25474036 & -11.90 & 4.71 & -2190.26478352 & -9.48 & 7.13 & -2190.27465154 & -6.98 & 9.63 \\
\hline-2190.25936917 & -14.80 & 1.72 & -2190.26888276 & -12.06 & 4.47 & -2190.27826257 & -9.24 & 7.28 \\
\hline-2190.23577761 & 0.00 & 0.00 & -2190.24967120 & 0.00 & 0.00 & -2190.26353088 & 0.00 & 0.00 \\
\hline-2190.25843920 & -14.22 & 3.54 & -2190.26818438 & -11.62 & 6.14 & -2190.27838435 & -9.32 & 8.44 \\
\hline-2190.25616528 & -12.79 & 4.77 & -2190.26559076 & -9.99 & 7.58 & -2190.27573897 & -7.66 & 9.91 \\
\hline-2190.27190070 & -22.67 & -3.89 & -2190.28174721 & -20.13 & -1.35 & -2190.29181763 & -17.75 & 1.03 \\
\hline-2190.25557272 & -12.42 & 5.65 & -2190.26513886 & -9.71 & 8.37 & -2190.27507747 & -7.25 & 10.83 \\
\hline-2190.25939749 & -14.82 & 1.21 & -2190.26956887 & -12.49 & 3.55 & -2190.28028418 & -10.51 & 5.52 \\
\hline-2190.23255137 & 2.02 & 2.36 & -2190.24533120 & 2.72 & 3.06 & -2190.25852586 & 3.14 & 3.47 \\
\hline-2190.25942846 & -14.84 & 0.64 & -2190.26880445 & -12.01 & 3.47 & -2190.27821241 & -9.21 & 6.27 \\
\hline-2190.25468625 & -11.87 & 4.89 & -2190.26410174 & -9.06 & 7.70 & -2190.27365392 & -6.35 & 10.40 \\
\hline-2190.26842676 & -20.49 & -2.48 & -2190.27811499 & -17.85 & 0.16 & -2190.28791739 & -15.30 & 2.71 \\
\hline-2190.24974428 & -8.76 & 8.97 & -2190.26019268 & -6.60 & 11.13 & -2190.27080030 & -4.56 & 13.17 \\
\hline-2190.25120035 & -9.68 & 6.65 & -2190.26123986 & -7.26 & 9.07 & -2190.27187107 & -5.23 & 11.10 \\
\hline-2190.23013283 & 3.54 & 2.87 & -2190.24443041 & 3.29 & 2.62 & -2190.25849963 & 3.16 & 2.49 \\
\hline
\end{tabular}




\begin{tabular}{|c|c|c|c|c|c|c|c|c|}
\hline \multicolumn{3}{|c|}{ M06/basis2 (gas-phase) } & \multicolumn{3}{|c|}{ M06/basis2 (benzene) } & \multicolumn{3}{|c|}{ M06/basis2 (dichloromethane) } \\
\hline $\begin{array}{c}\mathbf{E}_{\mathrm{sp}} \\
{[\text { hartree] }}\end{array}$ & $\begin{array}{c}\Delta \mathbf{E}_{\mathrm{sp}} \\
{[\mathrm{kcal} / \mathrm{mol}]}\end{array}$ & $\begin{array}{c}\Delta \mathbf{G}_{\mathrm{sp}} \\
{[\mathrm{kcal} / \mathrm{mol}]}\end{array}$ & $\begin{array}{c}\mathbf{E}_{\mathrm{sp}} \\
\text { [hartree] }\end{array}$ & $\begin{array}{c}\Delta \mathbf{E}_{\mathrm{sp}} \\
{[\mathrm{kcal} / \mathrm{mol}]}\end{array}$ & $\begin{array}{c}\Delta \mathbf{G}_{\mathrm{sp}} \\
{[\mathrm{kcal} / \mathrm{mol}]}\end{array}$ & $\begin{array}{c}\mathbf{E}_{\mathrm{sp}} \\
\text { [hartree] }\end{array}$ & $\begin{array}{c}\Delta \mathbf{E}_{\mathrm{sp}} \\
{[\mathrm{kcal} / \mathrm{mol}]}\end{array}$ & $\begin{array}{c}\Delta \mathbf{G}_{\text {sp }} \\
{[\mathrm{kcal} / \mathrm{mol}]}\end{array}$ \\
\hline-2189.61802137 & 3.24 & 2.57 & -2189.63120496 & 2.99 & 2.32 & -2189.64417359 & 2.84 & 2.17 \\
\hline-2189.64367754 & -12.86 & 1.34 & -2189.65334251 & -10.91 & 3.30 & -2189.66293676 & -8.93 & 5.27 \\
\hline-2189.63558843 & -7.79 & 8.87 & -2189.64517236 & -5.78 & 10.88 & -2189.65483047 & -3.85 & 12.81 \\
\hline-2189.65038089 & -17.07 & 1.20 & -2189.65975996 & -14.93 & 3.34 & -2189.66903562 & -12.76 & 5.51 \\
\hline-2189.64393414 & -13.02 & 3.59 & -2189.65325456 & -10.85 & 5.76 & -2189.66246750 & -8.64 & 7.97 \\
\hline-2189.65055249 & -17.18 & -0.65 & -2189.65938677 & -14.70 & 1.83 & -2189.66810714 & -12.18 & 4.35 \\
\hline-2189.62317982 & 0.00 & 0.00 & -2189.63596323 & 0.00 & 0.00 & -2189.64869828 & 0.00 & 0.00 \\
\hline-2189.65056484 & -17.18 & 0.57 & -2189.65960454 & -14.84 & 2.92 & -2189.66908687 & -12.79 & 4.96 \\
\hline-2189.64747596 & -15.25 & 2.32 & -2189.65611393 & -12.64 & 4.92 & -2189.66549010 & -10.54 & 7.03 \\
\hline-2189.66041612 & -23.37 & -4.59 & -2189.66953851 & -21.07 & -2.29 & -2189.67893388 & -18.97 & -0.19 \\
\hline-2189.64460382 & -13.44 & 4.63 & -2189.65337605 & -10.93 & 7.15 & -2189.66255813 & -8.70 & 9.37 \\
\hline-2189.64911626 & -16.28 & -0.24 & -2189.65852984 & -14.16 & 1.88 & -2189.66849283 & -12.42 & 3.62 \\
\hline-2189.62035484 & 1.77 & 2.11 & -2189.63203463 & 2.47 & 2.80 & -2189.64405525 & 2.91 & 3.25 \\
\hline-2189.64965291 & -16.61 & -1.13 & -2189.65836085 & -14.05 & 1.43 & -2189.66715264 & -11.58 & 3.90 \\
\hline-2189.64407891 & -13.11 & 3.64 & -2189.65275812 & -10.54 & 6.22 & -2189.66162068 & -8.11 & 8.65 \\
\hline-2189.65722237 & -21.36 & -3.35 & -2189.66618506 & -18.96 & -0.96 & -2189.67531827 & -16.70 & 1.30 \\
\hline-2189.63945923 & -10.22 & 7.52 & -2189.64912743 & -8.26 & 9.47 & -2189.65900609 & -6.47 & 11.26 \\
\hline-2189.64254071 & -12.15 & 4.18 & -2189.65179363 & -9.93 & 6.40 & -2189.66166467 & -8.14 & 8.19 \\
\hline-2189.61802137 & 3.24 & 2.57 & -2189.63120496 & 2.99 & 2.32 & -2189.64417359 & 2.84 & 2.17 \\
\hline $\begin{array}{l}\mathrm{E} \ldots \ldots \ldots \ldots \text { gas } \\
\mathrm{G} \ldots \ldots \ldots \text { sum of } \\
\Delta \mathrm{E} \ldots \ldots \ldots \text { relativ } \\
\Delta \mathrm{G} \ldots \ldots \ldots \text { relativ } \\
\Delta \mathrm{G}_{\mathrm{cor}} \ldots \ldots \text { gas ph } \\
\mathrm{E}_{\mathrm{sp}} \ldots \ldots \ldots \text { single } \\
\Delta \mathrm{E}_{\mathrm{sp}} \ldots \ldots \text { relativ } \\
\Delta \mathrm{G}_{\mathrm{sp}} \ldots \ldots \text { relativ }\end{array}$ & $\begin{array}{l}\text { e electronic en } \\
\text { lectronic and } t \\
\text { lectronic ener } \\
\text { ree energy in } 1 \\
\text { e thermal corr } \\
\text { int electronic }\end{array}$ & $\begin{array}{l}\text { gy in hartree } \\
\text { ermal free ener } \\
\text { y in } \mathrm{kcal} / \mathrm{mol} \\
\mathrm{al} / \mathrm{mol} \text { with ba } \\
\text { tion to free en } \\
\text { eergy in gas ph }\end{array}$ & $\begin{array}{l}\text { ith basis } 1 \\
\text { gies with basis } 1 \\
\text { ith basis } 1 \\
\text { is } 1 \\
\text { rgy in } \mathrm{kcal} / \mathrm{mol} \text { obt } \\
\text { se or solution in ha }\end{array}$ & $\begin{array}{l}\text { ned with basis } \\
\text { ree with basis } 2\end{array}$ & $(\Delta \mathrm{G}=\Delta \mathrm{E}+\Delta$ & $\left.\mathrm{J}_{\text {corr }}\right)$ & & \\
\hline
\end{tabular}


Table S37. CM between acrylonitrile and propene with catalyst $\mathrm{Ru}-4 \mathrm{~b}$ (pathways $Z M e$, ZCN in Figure 7)<smiles>CC[C@](Cl)(C=CC#N)C1=NCCN1C</smiles>

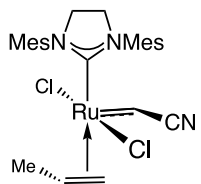<smiles>CC(C)[C@](Cl)(CC#N)C1=NCCN1C</smiles>

\begin{tabular}{|c|c|c|c|c|c|c|}
\hline & & \multicolumn{5}{|c|}{$\omega \mathrm{B97XD/basis1} \mathrm{(gas-phase)}$} \\
\hline \multicolumn{2}{|l|}{ pathway } & $\begin{array}{c}\mathbf{E} \\
\text { [hartree] }\end{array}$ & $\begin{array}{c}\Delta E \\
{[\mathrm{kcal} / \mathrm{mol}]}\end{array}$ & $\begin{array}{c}\mathbf{G} \\
{[\text { hartree] }}\end{array}$ & $\begin{array}{c}\Delta \mathbf{G} \\
{[\mathrm{kcal} / \mathrm{mol}]}\end{array}$ & $\begin{array}{c}\Delta \mathbf{G}_{\text {Corr }} \\
{[\mathrm{kcal} / \mathrm{mol}]}\end{array}$ \\
\hline \multirow{7}{*}{ ZMe } & $14 e$ & -2189.89714676 & 3.14 & -2189.452154 & 2.24 & -0.90 \\
\hline & pc2 & -2189.92188307 & -12.39 & -2189.451492 & 2.65 & 15.04 \\
\hline & ts2 & -2189.91674867 & -9.16 & -2189.444862 & 6.81 & 15.98 \\
\hline & mcb & -2189.93257338 & -19.09 & -2189.457688 & -1.24 & 17.86 \\
\hline & ts1 & -2189.92476573 & -14.20 & -2189.451595 & 2.59 & 16.78 \\
\hline & pc1 & -2189.92879313 & -16.72 & -2189.459559 & -2.41 & 14.31 \\
\hline & $14 \mathrm{e}_{\mathrm{Me}}$ & -2189.90214360 & 0.00 & -2189.455716 & 0.00 & 0.00 \\
\hline \multirow{5}{*}{$\mathrm{Me}-\mathrm{CN}$} & pc1 & -2189.92763775 & -16.00 & -2189.452912 & 1.76 & 17.76 \\
\hline & ts1 & -2189.92526632 & -14.51 & -2189.450843 & 3.06 & 17.57 \\
\hline & mcb & -2189.94123520 & -24.53 & -2189.464880 & -5.75 & 18.78 \\
\hline & ts2 & -2189.92534844 & -14.56 & -2189.450121 & 3.51 & 18.07 \\
\hline & pc2 & -2189.92945369 & -17.14 & -2189.457470 & -1.10 & 16.04 \\
\hline \multirow{7}{*}{$Z C N$} & $14 \mathrm{e}_{\mathrm{CN}}$ & -2189.89663961 & 3.45 & -2189.449681 & 3.79 & 0.33 \\
\hline & pc1 & -2189.93066796 & -17.90 & -2189.458480 & -1.73 & 16.16 \\
\hline & ts1 & -2189.92506932 & -14.39 & -2189.451731 & 2.50 & 16.89 \\
\hline & mcb & -2189.93830572 & -22.69 & -2189.463753 & -5.04 & 17.65 \\
\hline & ts2 & -2189.92168049 & -12.26 & -2189.448337 & 4.63 & 16.89 \\
\hline & pc2 & -2189.92323962 & -13.24 & -2189.453464 & 1.41 & 14.65 \\
\hline & $14 \mathrm{e}$ & -2189.89714676 & 3.14 & -2189.452154 & 2.24 & -0.90 \\
\hline
\end{tabular}

$\omega B 97 X D / b a s i s 2$ (gas-phase)

\begin{tabular}{|c|c|c|c|c|c|c|c|c|}
\hline \multicolumn{3}{|c|}{ ФВ97ХD/basis2 (gas-phase) } & \multicolumn{3}{|c|}{ ФБ97ХD/Dasis 2 (benzene) } & \multicolumn{3}{|c|}{$\omega \mathrm{B} 97 \times \mathrm{XD} / \mathrm{basis} 2$ (dichloromethane) } \\
\hline $\begin{array}{c}\mathbf{E}_{\mathrm{sp}} \\
\text { [hartree] }\end{array}$ & $\begin{array}{c}\Delta \mathrm{E}_{\mathrm{sp}} \\
{[\mathrm{kcal} / \mathrm{mol}]}\end{array}$ & $\begin{array}{c}\Delta \mathbf{G}_{\mathrm{sp}} \\
{[\mathrm{kcal} / \mathrm{mol}]}\end{array}$ & $\begin{array}{c}\mathbf{E}_{\mathrm{sp}} \\
\text { [hartree] }\end{array}$ & $\begin{array}{c}\Delta \mathbf{E}_{\mathrm{sp}} \\
{[\mathrm{kcal} / \mathrm{mol}]}\end{array}$ & $\begin{array}{c}\Delta \mathbf{G}_{\mathrm{sp}} \\
{[\mathrm{kcal} / \mathrm{mol}]}\end{array}$ & $\begin{array}{c}\mathbf{E}_{\mathrm{sp}} \\
\text { [hartree] }\end{array}$ & $\begin{array}{c}\Delta \mathbf{E}_{\mathrm{sp}} \\
{[\mathrm{kcal} / \mathrm{mol}]}\end{array}$ & $\begin{array}{c}\Delta \mathbf{G}_{\mathrm{sp}} \\
{[\mathrm{kcal} / \mathrm{mol}]}\end{array}$ \\
\hline-2190.23067062 & 3.20 & 2.30 & -2190.24460951 & 3.18 & 2.28 & -2190.25844827 & 3.19 & 2.29 \\
\hline-2190.25158156 & -9.92 & 5.12 & -2190.26204634 & -7.77 & 7.27 & -2190.27260449 & -5.69 & 9.34 \\
\hline-2190.24698621 & -7.03 & 8.94 & -2190.25747137 & -4.89 & 11.08 & -2190.26811125 & -2.87 & 13.10 \\
\hline-2190.26324581 & -17.24 & 0.62 & -2190.27333301 & -14.85 & 3.01 & -2190.28341879 & -12.48 & 5.38 \\
\hline-2190.25526663 & -12.23 & 4.55 & -2190.26525542 & -9.78 & 7.00 & -2190.27518254 & -7.31 & 9.47 \\
\hline-2190.25869710 & -14.38 & -0.07 & -2190.26802830 & -11.52 & 2.79 & -2190.27713835 & -8.54 & 5.77 \\
\hline-2190.23577761 & 0.00 & 0.00 & -2190.24967120 & 0.00 & 0.00 & -2190.26353088 & 0.00 & 0.00 \\
\hline-2190.25843920 & -14.22 & 3.54 & -2190.26818438 & -11.62 & 6.14 & -2190.27838435 & -9.32 & 8.44 \\
\hline-2190.25616528 & -12.79 & 4.77 & -2190.26559076 & -9.99 & 7.58 & -2190.27573897 & -7.66 & 9.91 \\
\hline-2190.27190070 & -22.67 & -3.89 & -2190.28174721 & -20.13 & -1.35 & -2190.29181763 & -17.75 & 1.03 \\
\hline-2190.25557272 & -12.42 & 5.65 & -2190.26513886 & -9.71 & 8.37 & -2190.27507747 & -7.25 & 10.83 \\
\hline-2190.25939749 & -14.82 & 1.21 & -2190.26956887 & -12.49 & 3.55 & -2190.28028418 & -10.51 & 5.52 \\
\hline-2190.23255137 & 2.02 & 2.36 & -2190.24533120 & 2.72 & 3.06 & -2190.25852586 & 3.14 & 3.47 \\
\hline-2190.26031246 & -15.40 & 0.77 & -2190.26950606 & -12.45 & 3.72 & -2190.27891783 & -9.66 & 6.51 \\
\hline-2190.25511591 & -12.13 & 4.75 & -2190.26427430 & -9.16 & 7.72 & -2190.27384831 & -6.47 & 10.41 \\
\hline-2190.26850601 & -20.54 & -2.89 & -2190.27800479 & -17.78 & -0.13 & -2190.28780850 & -15.23 & 2.41 \\
\hline-2190.25172122 & -10.00 & 6.89 & -2190.26114668 & -7.20 & 9.69 & -2190.27097377 & -4.67 & 12.22 \\
\hline-2190.25295492 & -10.78 & 3.87 & -2190.26253042 & -8.07 & 6.58 & -2190.27247067 & -5.61 & 9.04 \\
\hline-2190.23067062 & 3.20 & 2.30 & -2190.24460951 & 3.18 & 2.28 & -2190.25844827 & 3.19 & 2.29 \\
\hline
\end{tabular}




\begin{tabular}{|c|c|c|c|c|c|c|c|c|}
\hline \multicolumn{3}{|c|}{ M06/basis2 (gas-phase) } & \multicolumn{3}{|c|}{ M06/basis2 (benzene) } & \multicolumn{3}{|c|}{ M06/basis2 (dichloromethane) } \\
\hline $\begin{array}{c}\mathbf{E}_{\mathrm{sp}} \\
{[\text { hartree] }}\end{array}$ & $\begin{array}{c}\Delta \mathbf{E}_{\mathrm{sp}} \\
{[\mathrm{kcal} / \mathrm{mol}]}\end{array}$ & $\begin{array}{c}\Delta \mathbf{G}_{\mathrm{sp}} \\
{[\mathrm{kcal} / \mathrm{mol}]}\end{array}$ & $\begin{array}{c}\mathbf{E}_{\mathrm{sp}} \\
\text { [hartree] }\end{array}$ & $\begin{array}{c}\Delta \mathbf{E}_{\mathrm{sp}} \\
{[\mathrm{kcal} / \mathrm{mol}]}\end{array}$ & $\begin{array}{c}\Delta \mathbf{G}_{\mathrm{sp}} \\
{[\mathrm{kcal} / \mathrm{mol}]}\end{array}$ & $\begin{array}{c}\mathbf{E}_{\mathrm{sp}} \\
\text { [hartree] }\end{array}$ & $\begin{array}{c}\Delta \mathbf{E}_{\mathrm{sp}} \\
{[\mathrm{kcal} / \mathrm{mol}]}\end{array}$ & $\begin{array}{c}\Delta \mathbf{G}_{\text {sp }} \\
{[\mathrm{kcal} / \mathrm{mol}]}\end{array}$ \\
\hline-2189.61868945 & 2.82 & 1.92 & -2189.63153238 & 2.78 & 1.88 & -2189.64428205 & 2.77 & 1.87 \\
\hline-2189.64208501 & -11.86 & 3.17 & -2189.65179262 & -9.93 & 5.10 & -2189.66163609 & -8.12 & 6.92 \\
\hline-2189.63689350 & -8.61 & 7.37 & -2189.64660039 & -6.67 & 9.30 & -2189.65648757 & -4.89 & 11.09 \\
\hline-2189.65166907 & -17.88 & -0.02 & -2189.66100874 & -15.72 & 2.14 & -2189.67040291 & -13.62 & 4.24 \\
\hline-2189.64443303 & -13.34 & 3.44 & -2189.65366358 & -11.11 & 5.67 & -2189.66288950 & -8.91 & 7.88 \\
\hline-2189.64839282 & -15.82 & -1.51 & -2189.65703366 & -13.22 & 1.09 & -2189.66551664 & -10.55 & 3.76 \\
\hline-2189.62317982 & 0.00 & 0.00 & -2189.63596323 & 0.00 & 0.00 & -2189.64869828 & 0.00 & 0.00 \\
\hline-2189.65056484 & -17.18 & 0.57 & -2189.65960454 & -14.84 & 2.92 & -2189.66908687 & -12.79 & 4.96 \\
\hline-2189.64747596 & -15.25 & 2.32 & -2189.65611393 & -12.64 & 4.92 & -2189.66549010 & -10.54 & 7.03 \\
\hline-2189.66041612 & -23.37 & -4.59 & -2189.66953851 & -21.07 & -2.29 & -2189.67893388 & -18.97 & -0.19 \\
\hline-2189.64460382 & -13.44 & 4.63 & -2189.65337605 & -10.93 & 7.15 & -2189.66255813 & -8.70 & 9.37 \\
\hline-2189.64911626 & -16.28 & -0.24 & -2189.65852984 & -14.16 & 1.88 & -2189.66849283 & -12.42 & 3.62 \\
\hline-2189.62035484 & 1.77 & 2.11 & -2189.63203463 & 2.47 & 2.80 & -2189.64405525 & 2.91 & 3.25 \\
\hline-2189.65034556 & -17.05 & -0.88 & -2189.65888326 & -14.38 & 1.78 & -2189.66769366 & -11.92 & 4.25 \\
\hline-2189.64489059 & -13.62 & 3.26 & -2189.65331300 & -10.89 & 6.00 & -2189.66218579 & -8.46 & 8.42 \\
\hline-2189.65727762 & -21.40 & -3.75 & -2189.66606128 & -18.89 & -1.24 & -2189.67518852 & -16.62 & 1.03 \\
\hline-2189.64237034 & -12.04 & 4.85 & -2189.65107060 & -9.48 & 7.41 & -2189.66020989 & -7.22 & 9.67 \\
\hline-2189.64377756 & -12.93 & 1.73 & -2189.65263598 & -10.46 & 4.19 & -2189.66189026 & -8.28 & 6.37 \\
\hline-2189.61868945 & 2.82 & 1.92 & -2189.63153238 & 2.78 & 1.88 & -2189.64428205 & 2.77 & 1.87 \\
\hline $\begin{array}{l}\mathrm{E} \ldots \ldots \ldots \ldots \text { gas } \\
\mathrm{G} \ldots \ldots \ldots \text { sum of } \\
\Delta \mathrm{E} \ldots \ldots \ldots \text { relativ } \\
\Delta \mathrm{G} \ldots \ldots \ldots \text { relativ } \\
\Delta \mathrm{G}_{\mathrm{cor}} \ldots \ldots \text { gas ph } \\
\mathrm{E}_{\mathrm{sp}} \ldots \ldots \ldots \text { single } \\
\Delta \mathrm{E}_{\mathrm{sp}} \ldots \ldots \text { relativ } \\
\Delta \mathrm{G}_{\mathrm{sp}} \ldots \ldots \text { relativ }\end{array}$ & $\begin{array}{l}\text { e electronic en } \\
\text { lectronic and } t \\
\text { lectronic ener } \\
\text { ree energy in } 1 \\
\text { e thermal corr } \\
\text { int electronic }\end{array}$ & $\begin{array}{l}\text { gy in hartree } \\
\text { ermal free ener } \\
\text { y in } \mathrm{kcal} / \mathrm{mol} \\
\mathrm{al} / \mathrm{mol} \text { with ba } \\
\text { tion to free en } \\
\text { eergy in gas ph }\end{array}$ & $\begin{array}{l}\text { ith basis } 1 \\
\text { gies with basis } 1 \\
\text { ith basis } 1 \\
\text { is } 1 \\
\text { rgy in } \mathrm{kcal} / \mathrm{mol} \text { obt } \\
\text { se or solution in ha }\end{array}$ & $\begin{array}{l}\text { ned with basis } \\
\text { ree with basis } 2\end{array}$ & $(\Delta \mathrm{G}=\Delta \mathrm{E}+\Delta$ & $\left.S_{\text {corr }}\right)$ & & \\
\hline
\end{tabular}




\section{Table S38. CM between vinyl sulfide and trans-dichloroethylene with catalyst $\mathrm{Ru}-4 \mathrm{~b}$ (ESMe, ZSMe in Figure 9)}

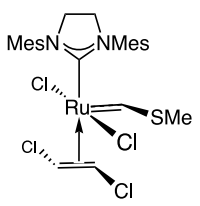

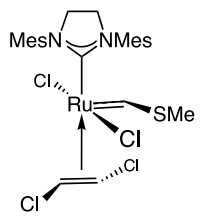

\begin{tabular}{|c|c|c|c|c|c|c|}
\hline & & \multicolumn{5}{|c|}{$\omega$ B97XD/basis1 (gas-phase) } \\
\hline \multicolumn{2}{|l|}{ pathway } & $\begin{array}{c}\mathbf{E} \\
\text { [hartree] }\end{array}$ & $\begin{array}{c}\Delta \mathbf{E} \\
{[\mathrm{kcal} / \mathrm{mol}]}\end{array}$ & $\begin{array}{c}\mathbf{G} \\
{[\text { hartree] }}\end{array}$ & $\begin{array}{c}\Delta \mathbf{G} \\
{[\mathrm{kcal} / \mathrm{mol}]}\end{array}$ & $\begin{array}{c}\Delta \mathbf{G}_{\mathrm{Corr}} \\
{[\mathrm{kcal} / \mathrm{mol}]}\end{array}$ \\
\hline \multirow{7}{*}{ ZSMe } & $14 e$ & -3415.02377863 & 0.00 & -3414.597217 & 0.00 & 0.00 \\
\hline & pc1 & -3415.04845529 & -15.48 & -3414.599665 & -1.54 & 13.95 \\
\hline & ts1 & -3415.03362636 & -6.18 & -3414.579987 & 10.81 & 16.99 \\
\hline & mcb & -3415.03964945 & -9.96 & -3414.583833 & 8.40 & 18.36 \\
\hline & ts2 & -3415.03079763 & -4.40 & -3414.576539 & 12.98 & 17.38 \\
\hline & pc2 & -3415.04538598 & -13.56 & -3414.593103 & 2.58 & 16.14 \\
\hline & $14 \mathrm{e}$ & -3415.02095429 & 1.77 & -3414.595828 & 0.87 & -0.90 \\
\hline \multirow{7}{*}{ ESMe } & $14 \mathrm{e}$ & -3415.02377863 & 0.00 & -3414.597217 & 0.00 & 0.00 \\
\hline & pc1 & -3415.04766087 & -14.99 & -3414.596610 & 0.38 & 15.37 \\
\hline & ts1 & -3415.03362716 & -6.18 & -3414.579091 & 11.37 & 17.55 \\
\hline & mcb & -3415.04035067 & -10.40 & -3414.586508 & 6.72 & 17.12 \\
\hline & ts2 & -3415.02868617 & -3.08 & -3414.573833 & 14.67 & 17.75 \\
\hline & pc2 & -3415.05338836 & -18.58 & -3414.598434 & -0.76 & 17.82 \\
\hline & $14 e$ & -3415.02081809 & 1.86 & -3414.594833 & 1.50 & -0.36 \\
\hline
\end{tabular}

$\omega \mathrm{B97XD/basis2} \mathrm{(gas-phase)}$

\begin{tabular}{|c|c|c|c|c|c|c|c|c|}
\hline $\begin{array}{c}\mathbf{E}_{\mathrm{sp}} \\
\text { [hartree] }\end{array}$ & $\begin{array}{c}\Delta \mathbf{E}_{\mathrm{sp}} \\
{[\mathrm{kcal} / \mathrm{mol}]}\end{array}$ & $\begin{array}{c}\Delta \mathbf{G}_{\mathrm{sp}} \\
{[\mathrm{kcal} / \mathrm{mol}]}\end{array}$ & $\begin{array}{c}\mathbf{E}_{\mathrm{sp}} \\
\text { [hartree] }\end{array}$ & $\begin{array}{c}\Delta \mathbf{E}_{\mathrm{sp}} \\
{[\mathrm{kcal} / \mathrm{mol}]}\end{array}$ & $\begin{array}{c}\Delta \mathbf{G}_{\text {sp }} \\
{[\mathrm{kcal} / \mathbf{m o l}]}\end{array}$ & $\begin{array}{c}\mathbf{E}_{\mathrm{sp}} \\
\text { [hartree] }\end{array}$ & $\begin{array}{c}\Delta \mathbf{E}_{\mathrm{sp}} \\
{[\mathrm{kcal} / \mathrm{mol}]}\end{array}$ & $\begin{array}{c}\Delta \mathbf{G}_{\mathrm{sp}} \\
{[\mathrm{kcal} / \mathrm{mol}]}\end{array}$ \\
\hline-3415.42811237 & 0.00 & 0.00 & -3415.44212799 & 0.00 & 0.00 & -3415.45653259 & 0.00 & 0.00 \\
\hline-3415.45213699 & -15.08 & -1.13 & -3415.46033551 & -11.43 & 2.52 & -3415.46887352 & -7.74 & 6.20 \\
\hline-3415.43588555 & -4.88 & 12.11 & -3415.44496581 & -1.78 & 15.21 & -3415.45466980 & 1.17 & 18.16 \\
\hline-3415.44119848 & -8.21 & 10.15 & -3415.45032583 & -5.14 & 13.21 & -3415.46014631 & -2.27 & 16.09 \\
\hline-3415.43296296 & -3.04 & 14.34 & -3415.44212487 & 0.00 & 17.38 & -3415.45214340 & 2.75 & 20.13 \\
\hline-3415.44853469 & -12.82 & 3.33 & -3415.45686865 & -9.25 & 6.89 & -3415.46559338 & -5.69 & 10.45 \\
\hline-3415.42685326 & 0.79 & -0.11 & -3415.44003909 & 1.31 & 0.41 & -3415.45391588 & 1.64 & 0.74 \\
\hline-3415.42811237 & 0.00 & 0.00 & -3415.44212799 & 0.00 & 0.00 & -3415.45653259 & 0.00 & 0.00 \\
\hline-3415.45119075 & -14.48 & 0.89 & -3415.45945533 & -10.87 & 4.49 & -3415.46801626 & -7.21 & 8.16 \\
\hline-3415.43608426 & -5.00 & 12.55 & -3415.44472214 & -1.63 & 15.93 & -3415.45387816 & 1.67 & 19.22 \\
\hline-3415.44214504 & -8.81 & 8.31 & -3415.45093303 & -5.53 & 11.59 & -3415.46027500 & -2.35 & 14.77 \\
\hline-3415.43118761 & -1.93 & 15.82 & -3415.43989232 & 1.40 & 19.16 & -3415.44930519 & 4.54 & 22.29 \\
\hline-3415.45671598 & -17.95 & -0.13 & -3415.46450733 & -14.04 & 3.77 & -3415.47242682 & -9.97 & 7.84 \\
\hline-3415.42570657 & 1.51 & 1.15 & -3415.43831473 & 2.39 & 2.03 & -3415.45147184 & 3.18 & 2.81 \\
\hline
\end{tabular}


Torker et al., Supporting Information, page S120

\begin{tabular}{|c|c|c|c|c|c|c|c|c|}
\hline \multicolumn{3}{|c|}{ M06/basis2 (gas-phase) } & \multicolumn{3}{|c|}{ M06/basis2 (benzene) } & \multicolumn{3}{|c|}{ M06/basis2 (dichloromethane) } \\
\hline $\begin{array}{c}\mathbf{E}_{\mathrm{sp}} \\
\text { [hartree] }\end{array}$ & $\begin{array}{c}\Delta \mathbf{E}_{\mathrm{sp}} \\
{[\mathrm{kcal} / \mathrm{mol}]}\end{array}$ & $\begin{array}{c}\Delta \mathbf{G}_{\mathrm{sp}} \\
{[\mathrm{kcal} / \mathrm{mol}]}\end{array}$ & $\begin{array}{c}\mathbf{E}_{\mathrm{sp}} \\
\text { [hartree] }\end{array}$ & $\begin{array}{c}\Delta \mathbf{E}_{\mathrm{sp}} \\
{[\mathrm{kcal} / \mathrm{mol}]}\end{array}$ & $\begin{array}{c}\Delta \mathbf{G}_{\mathrm{sp}} \\
{[\mathrm{kcal} / \mathrm{mol}]}\end{array}$ & $\begin{array}{c}\mathbf{E}_{\mathrm{sp}} \\
\text { [hartree] }\end{array}$ & $\begin{array}{c}\Delta \mathbf{E}_{\mathrm{sp}} \\
{[\mathrm{kcal} / \mathrm{mol}]}\end{array}$ & $\begin{array}{c}\Delta \mathbf{G}_{\text {sp }} \\
{[\mathrm{kcal} / \mathrm{mol}]}\end{array}$ \\
\hline-3414.79668742 & 0.00 & 0.00 & -3414.80912386 & 0.00 & 0.00 & -3414.82197532 & 0.00 & 0.00 \\
\hline-3414.82986152 & -20.82 & -6.87 & -3414.83720565 & -17.62 & -3.67 & -3414.84494770 & -14.42 & -0.47 \\
\hline-3414.81293749 & -10.20 & 6.79 & -3414.82108897 & -7.51 & 9.48 & -3414.82988833 & -4.97 & 12.03 \\
\hline-3414.81726944 & -12.92 & 5.44 & -3414.82546075 & -10.25 & 8.11 & -3414.83437304 & -7.78 & 10.58 \\
\hline-3414.81061078 & -8.74 & 8.64 & -3414.81878117 & -6.06 & 11.32 & -3414.82776000 & -3.63 & 13.75 \\
\hline-3414.82513555 & -17.85 & -1.71 & -3414.83261740 & -14.74 & 1.40 & -3414.84052645 & -11.64 & 4.50 \\
\hline-3414.79686442 & -0.11 & -1.01 & -3414.80854601 & 0.36 & -0.54 & -3414.82086670 & 0.70 & -0.21 \\
\hline-3414.79668742 & 0.00 & 0.00 & -3414.80912386 & 0.00 & 0.00 & -3414.82197532 & 0.00 & 0.00 \\
\hline-3414.82881187 & -20.16 & -4.79 & -3414.83625281 & -17.02 & -1.66 & -3414.84406030 & -13.86 & 1.51 \\
\hline-3414.81320874 & -10.37 & 7.19 & -3414.82096189 & -7.43 & 10.13 & -3414.82926743 & -4.58 & 12.98 \\
\hline-3414.81762911 & -13.14 & 3.98 & -3414.82550493 & -10.28 & 6.84 & -3414.83397620 & -7.53 & 9.59 \\
\hline-3414.80892683 & -7.68 & 10.07 & -3414.81669888 & -4.75 & 13.00 & -3414.82516208 & -2.00 & 15.75 \\
\hline-3414.83410273 & -23.48 & -5.66 & -3414.84112930 & -20.08 & -2.27 & -3414.84837120 & -16.56 & 1.25 \\
\hline-3414.79433684 & 1.48 & 1.11 & -3414.80550060 & 2.27 & 1.91 & -3414.81718571 & 3.01 & 2.64 \\
\hline $\begin{array}{l}\mathrm{E} \ldots \ldots \ldots \ldots \text { gas } \\
\mathrm{G} \ldots \ldots \ldots \ldots \text { sum of } \\
\Delta \mathrm{E} \ldots \ldots \ldots \text { relativ } \\
\Delta \mathrm{G} \ldots \ldots \ldots \text { relativ } \\
\Delta \mathrm{G}_{\mathrm{corr}} \ldots \ldots \text { gas ph} \\
\mathrm{E}_{\mathrm{sp}} \ldots \ldots \ldots \text { single } \\
\Delta \mathrm{E}_{\mathrm{sp}} \ldots \ldots \text { relativ } \\
\Delta \mathrm{G}_{\mathrm{sp}} \ldots \ldots \text { relativ }\end{array}$ & $\begin{array}{l}\text { e electronic en } \\
\text { lectronic and } t \\
\text { electronic ener } \\
\text { ree energy in } 1 \\
\text { e thermal corr } \\
\text { int electronic } \\
\text { ingle point ele }\end{array}$ & $\begin{array}{l}\text { gy in hartree } \\
\text { ermal free ener } \\
\text { y in kcal/mol v } \\
\text { al/mol with ba } \\
\text { tion to free en } \\
\text { ergy in gas ph } \\
\text { tronic energy i }\end{array}$ & $\begin{array}{l}\text { ith basis } 1 \\
\text { ies with basis } 1 \\
\text { th basis } 1 \\
\text { is } 1 \\
\text { gy in } \mathrm{kcal} / \mathrm{mol} \text { obta } \\
\text { se or solution in ha } \\
\text { gas phase or soluti }\end{array}$ & $\begin{array}{l}\text { ed with basis } \\
\text { in } \mathrm{kcal} / \mathrm{mol} \text {. }\end{array}$ & $\begin{array}{l}(\Delta \mathrm{G}=\Delta \mathrm{E}+\Delta \\
\mathrm{h} \text { basis } 2\end{array}$ & corr) & & \\
\hline
\end{tabular}


Table S39. Homodimerization of propene with catalyst Ru-4b (pathways EMe', ZMe' in Figure 10)<smiles>C=C[C@@](Cl)(C=CC)C1=NCCN1C</smiles>

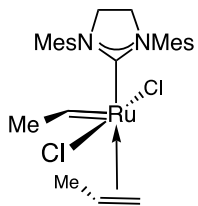

\begin{tabular}{|c|c|c|c|c|c|c|}
\hline & & \multicolumn{5}{|c|}{$\omega$ B97XD/basis1 (gas-phase) } \\
\hline \multicolumn{2}{|l|}{ pathway } & $\begin{array}{c}\mathbf{E} \\
\text { [hartree] }\end{array}$ & $\begin{array}{c}\Delta \mathrm{E} \\
{[\mathrm{kcal} / \mathrm{mol}]}\end{array}$ & $\begin{array}{c}\mathbf{G} \\
\text { [hartree] }\end{array}$ & $\begin{array}{c}\Delta \mathbf{G} \\
{[\mathrm{kcal} / \mathrm{mol}]}\end{array}$ & $\begin{array}{c}\Delta \mathbf{G}_{\text {Corr }} \\
{[\mathrm{kcal} / \mathrm{mol}]}\end{array}$ \\
\hline \multirow{7}{*}{ ZMe' } & $14 \mathrm{e}$ & -2137.00278047 & 0.00 & -2136.526563 & 0.00 & 0.00 \\
\hline & pc1 & -2137.03533585 & -20.43 & -2136.533971 & -4.65 & 15.78 \\
\hline & ts1 & -2137.03164549 & -18.11 & -2136.525788 & 0.49 & 18.60 \\
\hline & mcb & -2137.04027992 & -23.53 & -2136.534017 & -4.68 & 18.85 \\
\hline & ts 2 & -2137.02502735 & -13.96 & -2136.522057 & 2.83 & 16.79 \\
\hline & pc2 & -2137.02821140 & -15.96 & -2136.526621 & -0.04 & 15.92 \\
\hline & $14 \mathrm{e}$ & -2136.99252369 & 6.44 & -2136.518060 & 5.34 & -1.10 \\
\hline \multirow{7}{*}{$E M e^{\prime}$} & $14 \mathrm{e}$ & -2137.00278047 & 0.00 & -2136.526563 & 0.00 & 0.00 \\
\hline & pc1 & -2137.03540602 & -20.47 & -2136.534152 & -4.76 & 15.71 \\
\hline & ts1 & -2137.03239087 & -18.58 & -2136.526903 & -0.21 & 18.37 \\
\hline & mcb & -2137.04069952 & -23.79 & -2136.534702 & -5.11 & 18.69 \\
\hline & ts2 & -2137.02404650 & -13.34 & -2136.521855 & 2.95 & 16.30 \\
\hline & pc2 & -2137.02887407 & -16.37 & -2136.528612 & -1.29 & 15.09 \\
\hline & $14 \mathrm{e}$ & -2136.99412329 & 5.43 & -2136.518475 & 5.08 & -0.36 \\
\hline
\end{tabular}

$\omega \mathrm{B97XD/basis2} \mathrm{(gas-phase)}$

\begin{tabular}{|c|c|c|c|c|c|c|c|c|}
\hline $\begin{array}{c}\mathbf{E}_{\mathrm{sp}} \\
\text { [hartree] }\end{array}$ & $\begin{array}{c}\Delta \mathrm{E}_{\mathrm{sp}} \\
{[\mathrm{kcal} / \mathrm{mol}]}\end{array}$ & $\begin{array}{c}\Delta \mathrm{G}_{\mathrm{sp}} \\
{[\mathrm{kcal} / \mathrm{mol}]}\end{array}$ & $\begin{array}{c}\mathbf{E}_{\mathrm{sp}} \\
\text { [hartree] }\end{array}$ & $\begin{array}{c}\Delta \mathrm{E}_{\mathrm{sp}} \\
{[\mathrm{kcal} / \mathrm{mol}]}\end{array}$ & $\begin{array}{c}\Delta \mathbf{G}_{\mathrm{sp}} \\
{[\mathrm{kcal} / \mathrm{mol}]}\end{array}$ & $\begin{array}{c}\mathbf{E}_{\mathrm{sp}} \\
\text { [hartree] }\end{array}$ & $\begin{array}{c}\Delta \mathrm{E}_{\mathrm{sp}} \\
{[\mathrm{kcal} / \mathrm{mol}]}\end{array}$ & $\begin{array}{c}\Delta \mathbf{G}_{\mathrm{sp}} \\
{[\mathrm{kcal} / \mathrm{mol}]}\end{array}$ \\
\hline-2137.32152128 & 0.00 & 0.00 & -2137.33240770 & 0.00 & 0.00 & -2137.34375317 & 0.00 & 0.00 \\
\hline-2137.34869416 & -17.05 & -1.27 & -2137.35643864 & -15.08 & 0.70 & -2137.36439049 & -12.95 & 2.83 \\
\hline-2137.34531320 & -14.93 & 3.67 & -2137.35302923 & -12.94 & 5.66 & -2137.36109543 & -10.88 & 7.72 \\
\hline-2137.35404505 & -20.41 & -1.56 & -2137.36165250 & -18.35 & 0.50 & -2137.36973633 & -16.30 & 2.55 \\
\hline-2137.33846742 & -10.63 & 6.15 & -2137.34640389 & -8.78 & 8.00 & -2137.35475782 & -6.91 & 9.88 \\
\hline-2137.34143931 & -12.50 & 3.42 & -2137.34955432 & -10.76 & 5.16 & -2137.35809746 & -9.00 & 6.92 \\
\hline-2137.31099575 & 6.60 & 5.50 & -2137.32185047 & 6.62 & 5.52 & -2137.33309279 & 6.69 & 5.59 \\
\hline-2137.32152128 & 0.00 & 0.00 & -2137.33240770 & 0.00 & 0.00 & -2137.34375317 & 0.00 & 0.00 \\
\hline-2137.34874204 & -17.08 & -1.37 & -2137.35660263 & -15.18 & 0.53 & -2137.36476833 & -13.19 & 2.52 \\
\hline-2137.34597976 & -15.35 & 3.02 & -2137.35391959 & -13.50 & 4.87 & -2137.36240370 & -11.70 & 6.66 \\
\hline-2137.35428549 & -20.56 & -1.87 & -2137.36180576 & -18.45 & 0.24 & -2137.36976254 & -16.32 & 2.37 \\
\hline-2137.33740076 & -9.96 & 6.33 & -2137.34550721 & -8.22 & 8.08 & -2137.35411493 & -6.50 & 9.80 \\
\hline-2137.34222949 & -12.99 & 2.09 & -2137.35036476 & -11.27 & 3.82 & -2137.35892009 & -9.52 & 5.57 \\
\hline-2137.31253909 & 5.64 & 5.28 & -2137.32334545 & 5.69 & 5.33 & -2137.33454848 & 5.78 & 5.42 \\
\hline
\end{tabular}




\begin{tabular}{|c|c|c|c|c|c|c|c|c|}
\hline \multicolumn{3}{|c|}{ M06/basis2 (gas-phase) } & \multicolumn{3}{|c|}{ M06/basis2 (benzene) } & \multicolumn{3}{|c|}{ M06/basis2 (dichloromethane) } \\
\hline $\begin{array}{c}\mathbf{E}_{\mathrm{sp}} \\
\text { [hartree] }\end{array}$ & $\begin{array}{c}\Delta \mathbf{E}_{\mathrm{sp}} \\
{[\mathrm{kcal} / \mathrm{mol}]}\end{array}$ & $\begin{array}{c}\Delta \mathbf{G}_{\mathrm{sp}} \\
{[\mathrm{kcal} / \mathrm{mol}]}\end{array}$ & $\begin{array}{c}\mathbf{E}_{\mathrm{sp}} \\
\text { [hartree] }\end{array}$ & $\begin{array}{c}\Delta \mathbf{E}_{\mathrm{sp}} \\
{[\mathrm{kcal} / \mathrm{mol}]}\end{array}$ & $\begin{array}{c}\Delta \mathbf{G}_{\mathrm{sp}} \\
{[\mathrm{kcal} / \mathrm{mol}]}\end{array}$ & $\begin{array}{c}\mathbf{E}_{\mathrm{sp}} \\
\text { [hartree] }\end{array}$ & $\begin{array}{c}\Delta \mathbf{E}_{\mathrm{sp}} \\
{[\mathrm{kcal} / \mathrm{mol}]}\end{array}$ & $\begin{array}{c}\Delta \mathbf{G}_{\text {sp }} \\
{[\mathrm{kcal} / \mathrm{mol}]}\end{array}$ \\
\hline-2136.70872629 & 0.00 & 0.00 & -2136.71847514 & 0.00 & 0.00 & -2136.72865362 & 0.00 & 0.00 \\
\hline-2136.73670424 & -17.56 & -1.78 & -2136.74370357 & -15.83 & -0.05 & -2136.75097167 & -14.00 & 1.78 \\
\hline-2136.73334153 & -15.45 & 3.15 & -2136.74032010 & -13.71 & 4.89 & -2136.74770838 & -11.96 & 6.64 \\
\hline-2136.74200951 & -20.89 & -2.03 & -2136.74890523 & -19.10 & -0.24 & -2136.75632852 & -17.37 & 1.49 \\
\hline-2136.72775171 & -11.94 & 4.85 & -2136.73491625 & -10.32 & 6.47 & -2136.74254004 & -8.71 & 8.07 \\
\hline-2136.73111442 & -14.05 & 1.87 & -2136.73843519 & -12.53 & 3.40 & -2136.74621738 & -11.02 & 4.90 \\
\hline-2136.69853026 & 6.40 & 5.30 & -2136.70823477 & 6.43 & 5.33 & -2136.71832274 & 6.48 & 5.38 \\
\hline-2136.70872629 & 0.00 & 0.00 & -2136.71847514 & 0.00 & 0.00 & -2136.72865362 & 0.00 & 0.00 \\
\hline-2136.73725248 & -17.90 & -2.19 & -2136.74436647 & -16.25 & -0.54 & -2136.75184110 & -14.55 & 1.16 \\
\hline-2136.73455233 & -16.21 & 2.16 & -2136.74173157 & -14.59 & 3.77 & -2136.74949825 & -13.08 & 5.29 \\
\hline-2136.74262440 & -21.27 & -2.58 & -2136.74943711 & -19.43 & -0.74 & -2136.75674628 & -17.63 & 1.06 \\
\hline-2136.72611302 & -10.91 & 5.39 & -2136.73343193 & -9.39 & 6.91 & -2136.74128157 & -7.92 & 8.37 \\
\hline-2136.73181835 & -14.49 & 0.60 & -2136.73916180 & -12.98 & 2.11 & -2136.74695997 & -11.49 & 3.60 \\
\hline-2136.70024904 & 5.32 & 4.96 & -2136.70990909 & 5.38 & 5.02 & -2136.71996107 & 5.45 & 5.10 \\
\hline $\begin{array}{l}\mathrm{E} \ldots \ldots \ldots \ldots \text { gas } \\
\mathrm{G} \ldots \ldots \ldots \text { sum of } \\
\Delta \mathrm{E} \ldots \ldots \ldots \text { relativ } \\
\Delta \mathrm{G} \ldots \ldots \ldots \text { relativ } \\
\Delta \mathrm{G}_{\mathrm{corr}} \ldots \ldots \text { gas ph} \\
\mathrm{E}_{\mathrm{sp}} \ldots \ldots \ldots \text { single } \\
\Delta \mathrm{E}_{\mathrm{sp}} \ldots \ldots \text { relativ } \\
\Delta \mathrm{G}_{\mathrm{sp}} \ldots \ldots \text { relativ }\end{array}$ & $\begin{array}{l}\text { e electronic en } \\
\text { lectronic and } t \\
\text { electronic ener } \\
\text { ree energy in } 1 \\
\text { e thermal corre } \\
\text { int electronic } \\
\text { ingle point ele } \\
\text { single point fre }\end{array}$ & $\begin{array}{l}\text { gy in hartree } \\
\text { ermal free ener } \\
\text { y in kcal/mol v } \\
\text { al/mol with ba } \\
\text { tion to free en } \\
\text { hergy in gas ph } \\
\text { tronic energy } \mathrm{i} \\
\text { energy in gas }\end{array}$ & $\begin{array}{l}\text { ith basis } 1 \\
\text { ies with basis } 1 \\
\text { th basis } 1 \\
\text { is } 1 \\
\text { gy in } \mathrm{kcal} / \mathrm{mol} \text { obta } \\
\text { se or solution in ha } \\
\text { gas phase or soluti }\end{array}$ & $\begin{array}{l}\text { ned with basis } \\
\text { ree with basis? } \\
\text { in } \mathrm{kcal} / \mathrm{mol} v\end{array}$ & $\begin{array}{l}(\Delta \mathrm{G}=\Delta \mathrm{E}+\Delta \\
\mathrm{h} \text { basis } 2\end{array}$ & corr) & & \\
\hline
\end{tabular}


Table S40. ROCM with catalyst Ru-5b and styrene (pathways C1, CD1, D1 in Figure S23)

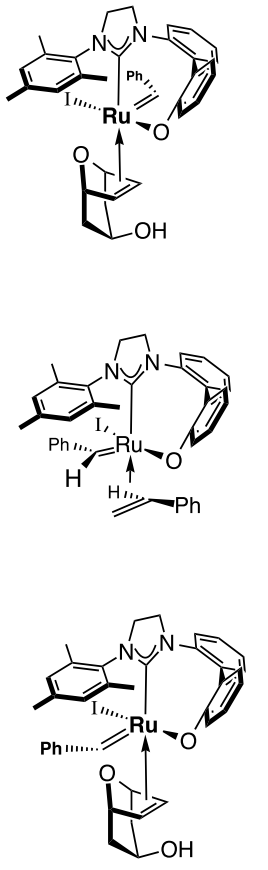

\begin{tabular}{|c|c|c|c|c|c|c|}
\hline & & \multicolumn{5}{|c|}{$\omega$ B97XD/basis1 (gas-phase) } \\
\hline \multicolumn{2}{|l|}{ pathway } & $\begin{array}{c}\Delta \mathbf{E} \\
\text { [hartree] }\end{array}$ & $\begin{array}{c}\Delta \mathbf{E} \\
{[\mathrm{kcal} / \mathrm{mol}]}\end{array}$ & $\begin{array}{c}\Delta \mathbf{G} \\
{[\text { hartree] }}\end{array}$ & $\begin{array}{c}\Delta \mathbf{G} \\
{[\mathrm{kcal} / \mathrm{mol}]}\end{array}$ & $\begin{array}{c}\Delta \mathbf{G}_{\mathrm{Corr}} \\
{[\mathrm{kcal} / \mathrm{mol}]}\end{array}$ \\
\hline \multirow{5}{*}{ C1 } & pc2 & -1912.61300977 & -28.16 & -1911.988363 & -9.37 & 18.79 \\
\hline & ts2 & -1912.59354798 & -15.95 & -1911.968534 & 3.08 & 19.02 \\
\hline & mcb & -1912.60040668 & -20.25 & -1911.974218 & -0.49 & 19.76 \\
\hline & ts1 & -1912.57845978 & -6.48 & -1911.954513 & 11.87 & 18.35 \\
\hline & pc1 & -1912.58807794 & -12.52 & -1911.964409 & 5.66 & 18.18 \\
\hline \multirow{7}{*}{ CD1 } & 5-endo & -1912.56147948 & 4.18 & -1911.966493 & 4.36 & 0.18 \\
\hline & pc-endo & -1912.59561073 & -17.24 & -1911.971142 & 1.44 & 18.68 \\
\hline & ts-endo & -1912.58833389 & -12.68 & -1911.959725 & 8.60 & 21.28 \\
\hline & mcb & -1912.60167959 & -21.05 & -1911.974164 & -0.46 & 20.59 \\
\hline & ts-exo & -1912.59200648 & -14.98 & -1911.967747 & 3.57 & 18.55 \\
\hline & pc-exo & -1912.60558074 & -23.50 & -1911.981068 & -4.79 & 18.71 \\
\hline & 5-exo & -1912.56813347 & 0.00 & -1911.973435 & 0.00 & 0.00 \\
\hline \multirow{5}{*}{ D1 } & pc1 & -1912.60010453 & -20.06 & -1911.979555 & -3.84 & 16.22 \\
\hline & ts1 & -1912.59220201 & -15.10 & -1911.967462 & 3.75 & 18.85 \\
\hline & mcb & -1912.60362439 & -22.27 & -1911.976811 & -2.12 & 20.15 \\
\hline & ts2 & -1912.58599361 & -11.21 & -1911.958897 & 9.12 & 20.33 \\
\hline & pc2 & -1912.59847761 & -19.04 & -1911.970789 & 1.66 & 20.70 \\
\hline
\end{tabular}

\begin{tabular}{|c|c|c|c|c|c|c|c|c|}
\hline \multicolumn{3}{|c|}{$\omega$ B97XD/basis2 (gas-phase) } & \multicolumn{3}{|c|}{$\omega \mathrm{B97XD/basis2} \mathrm{(benzene)}$} & \multicolumn{3}{|c|}{$\omega \mathrm{B97XD/basis2} \mathrm{(dichloromethane)}$} \\
\hline $\begin{array}{c}\Delta \mathbf{E} \\
\text { [hartree] }\end{array}$ & $\begin{array}{c}\Delta \mathbf{E} \\
{[\mathrm{kcal} / \mathrm{mol}]}\end{array}$ & $\begin{array}{c}\Delta \mathbf{G} \\
{[\mathrm{kcal} / \mathrm{mol}]}\end{array}$ & $\begin{array}{c}\Delta \mathbf{E} \\
\text { [hartree] }\end{array}$ & $\begin{array}{c}\Delta \mathrm{E} \\
{[\mathrm{kcal} / \mathrm{mol}]}\end{array}$ & $\begin{array}{c}\Delta \mathbf{G} \\
{[\mathrm{kcal} / \mathrm{mol}]}\end{array}$ & $\begin{array}{c}\Delta \mathbf{E} \\
\text { [hartree] }\end{array}$ & $\begin{array}{c}\Delta E \\
{[\mathrm{kcal} / \mathrm{mol}]}\end{array}$ & $\begin{array}{c}\Delta \mathbf{G} \\
{[\mathrm{kcal} / \mathrm{mol}]}\end{array}$ \\
\hline-1913.03297837 & -25.74 & -6.95 & -1913.04133113 & -22.96 & -4.16 & -1913.05019539 & -20.16 & -1.37 \\
\hline-1913.01507102 & -14.51 & 4.52 & -1913.02509736 & -12.77 & 6.25 & -1913.03586763 & -11.17 & 7.85 \\
\hline-1913.02251554 & -19.18 & 0.58 & -1913.03237411 & -17.34 & 2.42 & -1913.04290427 & -15.59 & 4.17 \\
\hline-1913.00045159 & -5.33 & 13.02 & -1913.01091617 & -3.87 & 14.48 & -1913.02228032 & -2.65 & 15.71 \\
\hline-1913.01006648 & -11.37 & 6.81 & -1913.01926545 & -9.11 & 9.07 & -1913.02899857 & -6.86 & 11.32 \\
\hline-1912.98554152 & 4.02 & 4.20 & -1912.99915025 & 3.51 & 3.69 & -1913.01388044 & 2.62 & 2.81 \\
\hline-1913.01418229 & -13.95 & 4.73 & -1913.02389323 & -12.02 & 6.67 & -1913.03423876 & -10.15 & 8.53 \\
\hline-1913.00765983 & -9.86 & 11.42 & -1913.01791688 & -8.27 & 13.01 & -1913.02847170 & -6.53 & 14.75 \\
\hline-1913.02186453 & -18.77 & 1.82 & -1913.03097822 & -16.46 & 4.13 & -1913.03995091 & -13.73 & 6.86 \\
\hline-1913.01105269 & -11.98 & 6.56 & -1913.02130609 & -10.39 & 8.16 & -1913.03186434 & -8.66 & 9.89 \\
\hline-1913.02391588 & -20.06 & -1.35 & -1913.03376241 & -18.21 & 0.50 & -1913.04363224 & -16.05 & 2.66 \\
\hline-1912.99195360 & 0.00 & 0.00 & -1913.00474554 & 0.00 & 0.00 & -1913.01806285 & 0.00 & 0.00 \\
\hline-1913.02124006 & -18.38 & -2.16 & -1913.03053892 & -16.19 & 0.04 & -1913.04014926 & -13.86 & 2.36 \\
\hline-1913.01442863 & -14.10 & 4.75 & -1913.02424466 & -12.24 & 6.62 & -1913.03458448 & -10.37 & 8.48 \\
\hline-1913.02552878 & -21.07 & -0.92 & -1913.03522066 & -19.12 & 1.03 & -1913.04587691 & -17.45 & 2.70 \\
\hline-1913.00714668 & -9.53 & 10.80 & -1913.01772004 & -8.14 & 12.19 & -1913.02968322 & -7.29 & 13.04 \\
\hline-1913.01834704 & -16.56 & 4.14 & -1913.02740585 & -14.22 & 6.48 & -1913.03765491 & -12.29 & 8.41 \\
\hline
\end{tabular}


Torker et al., Supporting Information, page S124

\begin{tabular}{|c|c|c|c|c|c|c|c|c|}
\hline \multicolumn{3}{|c|}{ M06/basis2 (gas-phase) } & \multicolumn{3}{|c|}{ M06/basis2 (benzene) } & \multicolumn{3}{|c|}{ M06/basis2 (dichloromethane) } \\
\hline $\begin{array}{c}\Delta \mathbf{E} \\
{[\text { hartree] }}\end{array}$ & $\begin{array}{c}\Delta \mathrm{E} \\
{[\mathrm{kcal} / \mathrm{mol}]}\end{array}$ & $\begin{array}{c}\Delta \mathbf{G} \\
{[\mathrm{kcal} / \mathrm{mol}]}\end{array}$ & $\begin{array}{c}\Delta \mathbf{E} \\
\text { [hartree] }\end{array}$ & $\begin{array}{c}\Delta \mathbf{E} \\
{[\mathrm{kcal} / \mathrm{mol}]}\end{array}$ & $\begin{array}{c}\Delta G \\
{[\mathrm{kcal} / \mathrm{mol}]}\end{array}$ & $\begin{array}{c}\Delta \mathbf{E} \\
\text { [hartree] }\end{array}$ & $\begin{array}{c}\Delta E \\
{[\mathrm{kcal} / \mathrm{mol}]}\end{array}$ & $\begin{array}{c}\Delta G \\
{[\mathrm{kcal} / \mathrm{mol}]}\end{array}$ \\
\hline-1912.19924391 & -24.91 & -6.12 & -1912.20661810 & -22.36 & -3.57 & -1912.21453415 & -19.84 & -1.05 \\
\hline-1912.17838736 & -11.82 & 7.20 & -1912.18735418 & -10.28 & 8.75 & -1912.19711834 & -8.91 & 10.11 \\
\hline-1912.18519704 & -16.09 & 3.67 & -1912.19406939 & -14.49 & 5.27 & -1912.20366566 & -13.02 & 6.74 \\
\hline-1912.16568588 & -3.85 & 14.50 & -1912.17505522 & -2.56 & 15.80 & -1912.18535960 & -1.53 & 16.82 \\
\hline-1912.17913644 & -12.29 & 5.89 & -1912.18735989 & -10.28 & 7.90 & -1912.19616680 & -8.31 & 9.86 \\
\hline-1912.15424046 & 3.33 & 3.51 & -1912.16633813 & 2.91 & 3.09 & -1912.17944976 & 2.18 & 2.36 \\
\hline-1912.18150023 & -13.77 & 4.91 & -1912.19016039 & -12.04 & 6.64 & -1912.19941087 & -10.35 & 8.33 \\
\hline-1912.17121386 & -7.32 & 13.96 & -1912.18040097 & -5.91 & 15.37 & -1912.18992283 & -4.40 & 16.88 \\
\hline-1912.18600989 & -16.60 & 3.99 & -1912.19425274 & -14.60 & 5.99 & -1912.20244429 & -12.25 & 8.34 \\
\hline-1912.17604626 & -10.35 & 8.20 & -1912.18525197 & -8.96 & 9.59 & -1912.19482315 & -7.47 & 11.08 \\
\hline-1912.18809164 & -17.91 & 0.80 & -1912.19693602 & -16.29 & 2.42 & -1912.20587636 & -14.41 & 4.30 \\
\hline-1912.15954919 & 0.00 & 0.00 & -1912.17097862 & 0.00 & 0.00 & -1912.18291667 & 0.00 & 0.00 \\
\hline-1912.18732047 & -17.43 & -1.20 & -1912.19563831 & -15.47 & 0.75 & -1912.20432225 & -13.43 & 2.79 \\
\hline-1912.17726473 & -11.12 & 7.73 & -1912.18607619 & -9.47 & 9.38 & -1912.19545977 & -7.87 & 10.98 \\
\hline-1912.18750292 & -17.54 & 2.61 & -1912.19624904 & -15.86 & 4.29 & -1912.20600762 & -14.49 & 5.66 \\
\hline-1912.17283521 & -8.34 & 11.99 & -1912.18223753 & -7.07 & 13.27 & -1912.19304684 & -6.36 & 13.97 \\
\hline-1912.18473998 & -15.81 & 4.89 & -1912.19270040 & -13.63 & 7.07 & -1912.20182486 & -11.87 & 8.84 \\
\hline
\end{tabular}


Table S41. ROCM with catalyst Ru-5b and styrene (pathways C2, CD2, D2 in Figure S23)

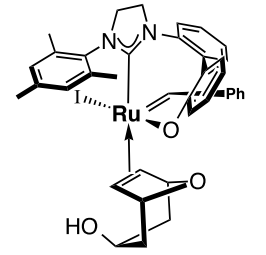<smiles>CCC1CCCC1</smiles>

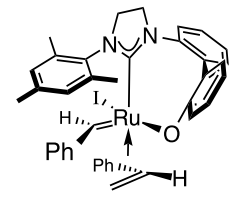

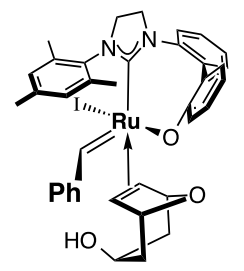

\begin{tabular}{|c|c|c|c|c|c|c|}
\hline \multirow{2}{*}{ pathway } & & \multicolumn{5}{|c|}{$\omega$ B97XD/basis1 (gas-phase) } \\
\hline & & $\begin{array}{c}\Delta \mathbf{E} \\
{[\text { hartree] }}\end{array}$ & $\begin{array}{c}\Delta E \\
{[\mathrm{kcal} / \mathrm{mol}]}\end{array}$ & $\begin{array}{c}\Delta \mathbf{G} \\
{[\text { [hartree] }}\end{array}$ & $\begin{array}{c}\Delta G \\
{[\mathrm{kcal} / \mathrm{mol}]}\end{array}$ & $\begin{array}{c}\Delta \mathbf{G}_{\text {Corr }} \\
{[\mathrm{kcal} / \mathbf{m o l}]}\end{array}$ \\
\hline \multirow{5}{*}{$\mathrm{C2}$} & pc2 & -1912.61133220 & -27.11 & -1911.987856 & -9.05 & 18.06 \\
\hline & ts2 & -1912.60063387 & -20.39 & -1911.975222 & -1.12 & 19.27 \\
\hline & mcb & -1912.60840213 & -25.27 & -1911.981812 & -5.26 & 20.01 \\
\hline & ts1 & -1912.58627340 & -11.38 & -1911.961659 & 7.39 & 18.77 \\
\hline & pc1 & -1912.59791678 & -18.69 & -1911.973751 & -0.20 & 18.49 \\
\hline \multirow{7}{*}{ CD2 } & 5-endo & -1912.56147948 & 4.18 & -1911.966493 & 4.36 & 0.18 \\
\hline & pc-endo & -1912.59932045 & -19.57 & -1911.973778 & -0.22 & 19.35 \\
\hline & ts-endo & -1912.58945161 & -13.38 & -1911.961562 & 7.45 & 20.83 \\
\hline & mcb & -1912.60354726 & -22.22 & -1911.976229 & -1.75 & 20.47 \\
\hline & ts-exo & -1912.59679715 & -17.99 & -1911.971438 & 1.25 & 19.24 \\
\hline & pc-exo & -1912.60139084 & -20.87 & -1911.978314 & -3.06 & 17.81 \\
\hline & 5-exo & -1912.56813347 & 0.00 & -1911.973435 & 0.00 & 0.00 \\
\hline \multirow{5}{*}{ D2 } & pc1 & -1912.60623345 & -23.91 & -1911.984522 & -6.96 & 16.95 \\
\hline & ts1 & -1912.59880303 & -19.25 & -1911.973016 & 0.26 & 19.51 \\
\hline & mcb & -1912.60964122 & -26.05 & -1911.982564 & -5.73 & 20.32 \\
\hline & ts2 & -1912.59224482 & -15.13 & -1911.965800 & 4.79 & 19.92 \\
\hline & pc2 & -1912.60073503 & -20.46 & -1911.978058 & -2.90 & 17.56 \\
\hline
\end{tabular}

\begin{tabular}{|c|c|c|c|c|c|c|c|c|}
\hline \multicolumn{3}{|c|}{$\omega$ B97XD/basis2 (gas-phase) } & \multicolumn{3}{|c|}{$\omega \mathrm{B97XD/basis2} \mathrm{(benzene)}$} & \multicolumn{3}{|c|}{$\omega \mathrm{B97XD/basis2} \mathrm{(dichloromethane)}$} \\
\hline $\begin{array}{c}\Delta \mathbf{E} \\
\text { [hartree] }\end{array}$ & $\begin{array}{c}\Delta \mathbf{E} \\
{[\mathrm{kcal} / \mathrm{mol}]}\end{array}$ & $\begin{array}{c}\Delta \mathbf{G} \\
{[\mathrm{kcal} / \mathrm{mol}]}\end{array}$ & $\begin{array}{c}\Delta \mathbf{E} \\
\text { [hartree] }\end{array}$ & $\begin{array}{c}\Delta \mathrm{E} \\
{[\mathrm{kcal} / \mathrm{mol}]}\end{array}$ & $\begin{array}{c}\Delta \mathbf{G} \\
{[\mathrm{kcal} / \mathrm{mol}]}\end{array}$ & $\begin{array}{c}\Delta \mathbf{E} \\
\text { [hartree] }\end{array}$ & $\begin{array}{c}\Delta E \\
{[\mathrm{kcal} / \mathrm{mol}]}\end{array}$ & $\begin{array}{c}\Delta \mathbf{G} \\
{[\mathrm{kcal} / \mathrm{mol}]}\end{array}$ \\
\hline-1913.03223925 & -25.28 & -7.22 & -1913.04118338 & -22.87 & -4.81 & -1913.05049028 & -20.35 & -2.29 \\
\hline-1913.02180664 & -18.73 & 0.54 & -1913.03135821 & -16.70 & 2.57 & -1913.04118922 & -14.51 & 4.76 \\
\hline-1913.03017556 & -23.98 & -3.97 & -1913.03965429 & -21.91 & -1.89 & -1913.04943932 & -19.69 & 0.32 \\
\hline-1913.00769428 & -9.88 & 8.90 & -1913.01807660 & -8.37 & 10.41 & -1913.02890195 & -6.80 & 11.97 \\
\hline-1913.01936890 & -17.20 & 1.29 & -1913.02855706 & -14.94 & 3.55 & -1913.03794086 & -12.47 & 6.02 \\
\hline-1912.98554152 & 4.02 & 4.20 & -1912.99915025 & 3.51 & 3.69 & -1913.01388044 & 2.62 & 2.81 \\
\hline-1913.01753042 & -16.05 & 3.31 & -1913.02790626 & -14.53 & 4.82 & -1913.03863653 & -12.91 & 6.44 \\
\hline-1913.00885436 & -10.61 & 10.22 & -1913.01880428 & -8.82 & 12.01 & -1913.02909425 & -6.92 & 13.91 \\
\hline-1913.02365184 & -19.89 & 0.58 & -1913.03281752 & -17.62 & 2.85 & -1913.04220434 & -15.15 & 5.32 \\
\hline-1913.01639518 & -15.34 & 3.90 & -1913.02571373 & -13.16 & 6.08 & -1913.03489022 & -10.56 & 8.68 \\
\hline-1913.01753042 & -16.05 & 1.76 & -1913.02790626 & -14.53 & 3.27 & -1913.03863653 & -12.91 & 4.90 \\
\hline-1912.99195360 & 0.00 & 0.00 & -1913.00474554 & 0.00 & 0.00 & -1913.01806285 & 0.00 & 0.00 \\
\hline-1913.02739414 & -22.24 & -5.29 & -1913.03606725 & -19.65 & -2.70 & -1913.04476064 & -16.75 & 0.20 \\
\hline-1913.02008828 & -17.65 & 1.85 & -1913.02967635 & -15.64 & 3.86 & -1913.03947228 & -13.43 & 6.07 \\
\hline-1913.03100899 & -24.51 & -4.19 & -1913.04036891 & -22.35 & -2.04 & -1913.05001613 & -20.05 & 0.27 \\
\hline-1913.01283597 & -13.10 & 6.82 & -1913.02306865 & -11.50 & 8.42 & -1913.03389928 & -9.94 & 9.98 \\
\hline-1913.02201308 & -18.86 & -1.31 & -1913.03201708 & -17.11 & 0.44 & -1913.04273920 & -15.48 & 2.07 \\
\hline
\end{tabular}


Torker et al., Supporting Information, page S126

\begin{tabular}{|c|c|c|c|c|c|c|c|c|}
\hline \multicolumn{3}{|c|}{ M06/basis2 (gas-phase) } & \multicolumn{3}{|c|}{ M06/basis2 (benzene) } & \multicolumn{3}{|c|}{ M06/basis2 (dichloromethane) } \\
\hline $\begin{array}{c}\Delta \mathbf{E} \\
{[\text { hartree] }}\end{array}$ & $\begin{array}{c}\Delta \mathbf{E} \\
{[\mathrm{kcal} / \mathrm{mol}]}\end{array}$ & $\begin{array}{c}\Delta \mathbf{G} \\
{[\mathrm{kcal} / \mathrm{mol}]}\end{array}$ & $\begin{array}{c}\Delta \mathbf{E} \\
{[\text { hartree] }}\end{array}$ & $\begin{array}{c}\Delta \mathbf{E} \\
{[\mathrm{kcal} / \mathrm{mol}]}\end{array}$ & $\begin{array}{c}\Delta \mathbf{G} \\
{[\mathrm{kcal} / \mathrm{mol}]}\end{array}$ & $\begin{array}{c}\Delta \mathbf{E} \\
{[\text { hartree] }}\end{array}$ & $\begin{array}{c}\Delta \mathbf{E} \\
{[\mathrm{kcal} / \mathrm{mol}]}\end{array}$ & $\begin{array}{c}\Delta \mathbf{G} \\
{[\mathrm{kcal} / \mathrm{mol}]}\end{array}$ \\
\hline-1912.19587489 & -22.79 & -4.74 & -1912.20383253 & -20.62 & -2.56 & -1912.21222335 & -18.39 & -0.33 \\
\hline-1912.18396803 & -15.32 & 3.95 & -1912.19252329 & -13.52 & 5.75 & -1912.20147718 & -11.65 & 7.63 \\
\hline-1912.19243952 & -20.64 & -0.63 & -1912.20097307 & -18.82 & 1.19 & -1912.20993141 & -16.95 & 3.06 \\
\hline-1912.17109798 & -7.25 & 11.53 & -1912.18037696 & -5.90 & 12.87 & -1912.19019241 & -4.57 & 14.21 \\
\hline-1912.18697677 & -17.21 & 1.28 & -1912.19514639 & -15.17 & 3.33 & -1912.20359206 & -12.97 & 5.52 \\
\hline-1912.15424046 & 3.33 & 3.51 & -1912.16633813 & 2.91 & 3.09 & -1912.17944976 & 2.18 & 2.36 \\
\hline-1912.18215626 & -14.19 & 5.17 & -1912.19145673 & -12.85 & 6.50 & -1912.20115657 & -11.45 & 7.91 \\
\hline-1912.17223775 & -7.96 & 12.87 & -1912.18121715 & -6.42 & 14.40 & -1912.19057248 & -4.80 & 16.02 \\
\hline-1912.18674958 & -17.07 & 3.40 & -1912.19506809 & -15.12 & 5.35 & -1912.20368082 & -13.03 & 7.44 \\
\hline-1912.17812620 & -11.66 & 7.58 & -1912.18654441 & -9.77 & 9.47 & -1912.19491304 & -7.53 & 11.71 \\
\hline-1912.18215626 & -14.19 & 3.62 & -1912.19145673 & -12.85 & 4.96 & -1912.20115657 & -11.45 & 6.36 \\
\hline-1912.15954919 & 0.00 & 0.00 & -1912.17097862 & 0.00 & 0.00 & -1912.18291667 & 0.00 & 0.00 \\
\hline-1912.19408366 & -21.67 & -4.72 & -1912.20179633 & -19.34 & -2.39 & -1912.20961604 & -16.75 & 0.20 \\
\hline-1912.18197439 & -14.07 & 5.44 & -1912.19054190 & -12.28 & 7.23 & -1912.19942798 & -10.36 & 9.15 \\
\hline-1912.19383559 & -21.52 & -1.20 & -1912.20222686 & -19.61 & 0.71 & -1912.21101373 & -17.63 & 2.69 \\
\hline-1912.17644411 & -10.60 & 9.32 & -1912.18553036 & -9.13 & 10.79 & -1912.19528345 & -7.76 & 12.16 \\
\hline-1912.18641668 & -16.86 & 0.70 & -1912.19526596 & -15.24 & 2.32 & -1912.20481080 & -13.74 & 3.82 \\
\hline
\end{tabular}


Table S42. ROCM with catalyst Ru-5b and styrene (pathways C3, CD3, D3 in Figure S23)

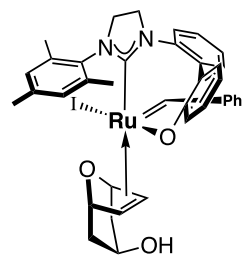

\begin{tabular}{|c|c|c|c|c|c|c|}
\hline pathway & & $\begin{array}{c}\Delta \mathbf{E} \\
{[\text { hartree] }}\end{array}$ & $\begin{array}{c}\Delta \mathrm{E} \\
{[\mathrm{kcal} / \mathrm{mol}]}\end{array}$ & $\begin{array}{c}\Delta \mathbf{G} \\
\text { [hartree] }\end{array}$ & $\begin{array}{c}\Delta \mathbf{G} \\
{[\mathrm{kcal} / \mathrm{mol}]}\end{array}$ & $\begin{array}{c}\Delta \mathbf{G}_{\text {Corr }} \\
{[\mathrm{kcal} / \mathrm{mol}]}\end{array}$ \\
\hline \multirow{5}{*}{$\mathrm{C3}$} & pc2 & -1912.62102815 & -33.19 & -1911.997021 & -14.80 & 18.39 \\
\hline & ts2 & -1912.60107534 & -20.67 & -1911.975554 & -1.33 & 19.34 \\
\hline & mcb & -1912.60934892 & -25.86 & -1911.982650 & -5.78 & 20.08 \\
\hline & ts1 & -1912.57903675 & -6.84 & -1911.955649 & 11.16 & 18.00 \\
\hline & pc1 & -1912.59791678 & -18.69 & -1911.973751 & -0.20 & 18.49 \\
\hline \multirow{7}{*}{ CD3 } & 5-endo & -1912.56147948 & 4.18 & -1911.966493 & 4.36 & 0.18 \\
\hline & pc-endo & -1912.59080659 & -14.23 & -1911.967289 & 3.86 & 18.08 \\
\hline & ts-endo & -1912.58307411 & -9.38 & -1911.955786 & 11.07 & 20.45 \\
\hline & mcb & -1912.59673110 & -17.95 & -1911.970422 & 1.89 & 19.84 \\
\hline & ts-exo & -1912.59056568 & -14.08 & -1911.963685 & 6.12 & 20.19 \\
\hline & pc-exo & -1912.60191762 & -21.20 & -1911.976310 & -1.80 & 19.40 \\
\hline & 5-exo & -1912.56813347 & 0.00 & -1911.973435 & 0.00 & 0.00 \\
\hline \multirow{5}{*}{ D3 } & pc1 & -1912.60623346 & -23.91 & -1911.984522 & -6.96 & 16.95 \\
\hline & ts1 & -1912.59213417 & -15.06 & -1911.969679 & 2.36 & 17.42 \\
\hline & mcb & -1912.60943469 & -25.92 & -1911.983164 & -6.11 & 19.81 \\
\hline & ts2 & -1912.59423974 & -16.38 & -1911.970000 & 2.16 & 18.54 \\
\hline & pc2 & -1912.61415812 & -28.88 & -1911.987429 & -8.78 & 20.10 \\
\hline
\end{tabular}

\begin{tabular}{|c|c|c|c|c|c|c|c|c|}
\hline \multicolumn{3}{|c|}{$\omega$ B97XD/basis2 (gas-phase) } & \multicolumn{3}{|c|}{$\omega \mathrm{B97XD/basis2} \mathrm{(benzene)}$} & \multicolumn{3}{|c|}{$\omega \mathrm{B97XD/basis2} \mathrm{(dichloromethane)}$} \\
\hline $\begin{array}{c}\Delta \mathbf{E} \\
{[\text { hartree] }}\end{array}$ & $\begin{array}{c}\Delta \mathbf{E} \\
{[\mathrm{kcal} / \mathrm{mol}]}\end{array}$ & $\begin{array}{c}\Delta \mathbf{G} \\
{[\mathrm{kcal} / \mathrm{mol}]}\end{array}$ & $\begin{array}{c}\Delta \mathbf{E} \\
\text { [hartree] }\end{array}$ & $\begin{array}{c}\Delta \mathbf{E} \\
{[\mathrm{kcal} / \mathrm{mol}]}\end{array}$ & $\begin{array}{c}\Delta \mathbf{G} \\
{[\mathrm{kcal} / \mathrm{mol}]}\end{array}$ & $\begin{array}{c}\Delta \mathbf{E} \\
\text { [hartree] }\end{array}$ & $\begin{array}{c}\Delta \mathbf{E} \\
{[\mathrm{kcal} / \mathrm{mol}]}\end{array}$ & $\begin{array}{c}\Delta \mathbf{G} \\
{[\mathrm{kcal} / \mathrm{mol}]}\end{array}$ \\
\hline-1913.04074527 & -30.62 & -12.23 & -1913.04855782 & -27.49 & -9.10 & -1913.05675538 & -24.28 & -5.89 \\
\hline-1913.02246240 & -19.14 & 0.20 & -1913.03309766 & -17.79 & 1.55 & -1913.04412876 & -16.36 & 2.99 \\
\hline-1913.03147772 & -24.80 & -4.72 & -1913.04165849 & -23.16 & -3.08 & -1913.05211922 & -21.37 & -1.29 \\
\hline-1913.00099938 & -5.68 & 12.33 & -1913.01172072 & -4.38 & 13.63 & -1913.02280658 & -2.98 & 15.03 \\
\hline-1913.01936889 & -17.20 & 1.29 & -1913.02855705 & -14.94 & 3.55 & -1913.03794085 & -12.47 & 6.02 \\
\hline-1912.98554152 & 4.02 & 4.20 & -1912.99915025 & 3.51 & 3.69 & -1913.01388044 & 2.62 & 2.81 \\
\hline-1913.00987367 & -11.25 & 6.84 & -1913.01972567 & -9.40 & 8.68 & -1913.03040801 & -7.75 & 10.34 \\
\hline-1913.00265307 & -6.71 & 13.74 & -1913.01319118 & -5.30 & 15.15 & -1913.02461352 & -4.11 & 16.34 \\
\hline-1913.01707283 & -15.76 & 4.07 & -1913.02683506 & -13.86 & 5.97 & -1913.03718407 & -12.00 & 7.84 \\
\hline-1913.01000690 & -11.33 & 8.87 & -1913.02036793 & -9.80 & 10.39 & -1913.03116372 & -8.22 & 11.97 \\
\hline-1913.02074547 & -18.07 & 1.33 & -1913.03038332 & -16.09 & 3.31 & -1913.04011925 & -13.84 & 5.56 \\
\hline-1912.99195360 & 0.00 & 0.00 & -1913.00474554 & 0.00 & 0.00 & -1913.01806285 & 0.00 & 0.00 \\
\hline-1913.02739418 & -22.24 & -5.29 & -1913.03606729 & -19.65 & -2.70 & -1913.04476069 & -16.75 & 0.20 \\
\hline-1913.01382318 & -13.72 & 3.69 & -1913.02428472 & -12.26 & 5.16 & -1913.03507598 & -10.68 & 6.74 \\
\hline-1913.03137429 & -24.74 & -4.92 & -1913.04160084 & -23.13 & -3.32 & -1913.05231984 & -21.50 & -1.68 \\
\hline-1913.01510996 & -14.53 & 4.01 & -1913.02610731 & -13.40 & 5.13 & -1913.03808671 & -12.57 & 5.97 \\
\hline-1913.03362323 & -26.15 & -6.05 & -1913.04262863 & -23.77 & -3.67 & -1913.05257054 & -21.65 & -1.55 \\
\hline
\end{tabular}


Torker et al., Supporting Information, page S128

\begin{tabular}{|c|c|c|c|c|c|c|c|c|}
\hline \multicolumn{3}{|c|}{ M06/basis2 (gas-phase) } & \multicolumn{3}{|c|}{ M06/basis2 (benzene) } & \multicolumn{3}{|c|}{ M06/basis2 (dichloromethane) } \\
\hline $\begin{array}{c}\Delta \mathbf{E} \\
\text { [hartree] }\end{array}$ & $\begin{array}{c}\Delta \mathbf{E} \\
{[\mathrm{kcal} / \mathrm{mol}]}\end{array}$ & $\begin{array}{c}\Delta \mathbf{G} \\
{[\mathrm{kcal} / \mathrm{mol}]}\end{array}$ & $\begin{array}{c}\Delta \mathbf{E} \\
\text { [hartree] }\end{array}$ & $\begin{array}{c}\Delta \mathbf{E} \\
{[\mathrm{kcal} / \mathrm{mol}]}\end{array}$ & $\begin{array}{c}\Delta \mathbf{G} \\
{[\mathrm{kcal} / \mathrm{mol}]}\end{array}$ & $\begin{array}{c}\Delta \mathbf{E} \\
\text { [hartree] }\end{array}$ & $\begin{array}{c}\Delta \mathbf{E} \\
{[\mathrm{kcal} / \mathrm{mol}]}\end{array}$ & $\begin{array}{c}\Delta \mathbf{G} \\
{[\mathrm{kcal} / \mathrm{mol}]}\end{array}$ \\
\hline-1912.20769990 & -30.22 & -11.82 & -1912.21457460 & -27.36 & -8.97 & -1912.22187252 & -24.45 & -6.05 \\
\hline-1912.18435136 & -15.56 & 3.78 & -1912.19384154 & -14.35 & 4.99 & -1912.20385709 & -13.14 & 6.20 \\
\hline-1912.19294306 & -20.95 & -0.87 & -1912.20209530 & -19.53 & 0.55 & -1912.21165353 & -18.03 & 2.05 \\
\hline-1912.16571015 & -3.87 & 14.14 & -1912.17531550 & -2.72 & 15.28 & -1912.18540344 & -1.56 & 16.44 \\
\hline-1912.18697669 & -17.21 & 1.28 & -1912.19514632 & -15.17 & 3.33 & -1912.20359198 & -12.97 & 5.52 \\
\hline-1912.15424046 & 3.33 & 3.51 & -1912.16633813 & 2.91 & 3.09 & -1912.17944976 & 2.18 & 2.36 \\
\hline-1912.17561679 & -10.08 & 8.00 & -1912.18443069 & -8.44 & 9.64 & -1912.19400936 & -6.96 & 11.12 \\
\hline-1912.16696739 & -4.65 & 15.80 & -1912.17646373 & -3.44 & 17.01 & -1912.18684065 & -2.46 & 17.99 \\
\hline-1912.18162598 & -13.85 & 5.98 & -1912.19049747 & -12.25 & 7.59 & -1912.19999058 & -10.71 & 9.12 \\
\hline-1912.17340401 & -8.69 & 11.50 & -1912.18273506 & -7.38 & 12.82 & -1912.19250690 & -6.02 & 14.18 \\
\hline-1912.18597742 & -16.58 & 2.81 & -1912.19469682 & -14.88 & 4.51 & -1912.20354742 & -12.95 & 6.45 \\
\hline-1912.15954919 & 0.00 & 0.00 & -1912.17097862 & 0.00 & 0.00 & -1912.18291667 & 0.00 & 0.00 \\
\hline-1912.19408370 & -21.67 & -4.72 & -1912.20179637 & -19.34 & -2.39 & -1912.20961609 & -16.75 & 0.20 \\
\hline-1912.17791144 & -11.52 & 5.90 & -1912.18725778 & -10.22 & 7.20 & -1912.19704019 & -8.86 & 8.55 \\
\hline-1912.19386924 & -21.54 & -1.72 & -1912.20302632 & -20.11 & -0.30 & -1912.21278373 & -18.74 & 1.07 \\
\hline-1912.17942939 & -12.48 & 6.06 & -1912.18920826 & -11.44 & 7.10 & -1912.20001884 & -10.73 & 7.81 \\
\hline-1912.19940858 & -25.01 & -4.91 & -1912.20732349 & -22.81 & -2.71 & -1912.21614999 & -20.85 & -0.75 \\
\hline
\end{tabular}


Table S43. ROCM with catalyst Ru-5b and styrene (pathways C4, CD4, D4 in Figure S23)

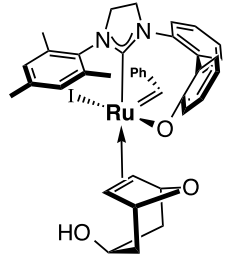

\begin{tabular}{|c|c|c|c|c|c|c|}
\hline \multicolumn{2}{|l|}{ pathway } & $\begin{array}{c}\Delta \mathbf{E} \\
{[\text { hartree] }}\end{array}$ & $\begin{array}{c}\Delta \mathbf{E} \\
{[\mathrm{kcal} / \mathrm{mol}]}\end{array}$ & $\begin{array}{c}\Delta \mathbf{G} \\
\text { [hartree] }\end{array}$ & $\begin{array}{c}\Delta \mathbf{G} \\
{[\mathrm{kcal} / \mathrm{mol}]}\end{array}$ & $\begin{array}{c}\Delta \mathbf{G}_{\text {Corr }} \\
{[\mathrm{kcal} / \mathrm{mol}]}\end{array}$ \\
\hline \multirow{5}{*}{$\mathrm{C4}$} & pc2 & -1912.61156132 & -27.25 & -1911.987457 & -8.80 & 18.45 \\
\hline & ts2 & -1912.59316510 & -15.71 & -1911.968069 & 3.37 & 19.07 \\
\hline & mcb & -1912.60390813 & -22.45 & -1911.979025 & -3.51 & 18.94 \\
\hline & ts1 & -1912.58156559 & -8.43 & -1911.957215 & 10.18 & 18.61 \\
\hline & pc1 & -1912.58807730 & -12.51 & -1911.965615 & 4.91 & 17.42 \\
\hline \multirow{7}{*}{ CD4 } & 5-endo & -1912.56147948 & 4.18 & -1911.966493 & 4.36 & 0.18 \\
\hline & pc-endo & -1912.59920809 & -19.50 & -1911.972708 & 0.46 & 19.96 \\
\hline & ts-endo & -1912.59034571 & -13.94 & -1911.963519 & 6.22 & 20.16 \\
\hline & mcb & -1912.60604010 & -23.79 & -1911.978239 & -3.01 & 20.77 \\
\hline & ts-exo & -1912.59693103 & -18.07 & -1911.971661 & 1.11 & 19.18 \\
\hline & pc-exo & -1912.60541135 & -23.39 & -1911.981790 & -5.24 & 18.15 \\
\hline & 5-exo & -1912.56813347 & 0.00 & -1911.973435 & 0.00 & 0.00 \\
\hline \multirow{5}{*}{ D4 } & pc1 & -1912.60015323 & -20.09 & -1911.979369 & -3.72 & 16.37 \\
\hline & ts1 & -1912.59431714 & -16.43 & -1911.971418 & 1.27 & 17.70 \\
\hline & mcb & -1912.60799556 & -25.01 & -1911.981796 & -5.25 & 19.77 \\
\hline & ts 2 & -1912.58927918 & -13.27 & -1911.963579 & 6.18 & 19.45 \\
\hline & pc2 & -1912.60697682 & -24.37 & -1911.980142 & -4.21 & 20.17 \\
\hline
\end{tabular}

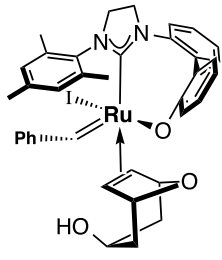

$\omega \mathrm{B97XD/basis2}$ (benzene)

\begin{tabular}{|c|c|c|c|c|c|c|c|c|}
\hline \multicolumn{3}{|c|}{$\omega$ B97XD/basis2 (gas-phase) } & \multicolumn{3}{|c|}{$\omega$ B97XD/basis2 (benzene) } & \multicolumn{3}{|c|}{$\omega$ B97XD/basis2 (dichloromethane) } \\
\hline $\begin{array}{c}\Delta \mathbf{E} \\
\text { [hartree] }\end{array}$ & $\begin{array}{c}\Delta \mathbf{E} \\
{[\mathrm{kcal} / \mathrm{mol}]}\end{array}$ & $\begin{array}{c}\Delta \mathbf{G} \\
{[\mathrm{kcal} / \mathrm{mol}]}\end{array}$ & $\begin{array}{c}\Delta \mathbf{E} \\
\text { [hartree] }\end{array}$ & $\begin{array}{c}\Delta \mathbf{E} \\
{[\mathrm{kcal} / \mathrm{mol}]}\end{array}$ & $\begin{array}{c}\Delta \mathbf{G} \\
{[\mathrm{kcal} / \mathrm{mol}]}\end{array}$ & $\begin{array}{c}\Delta \mathbf{E} \\
\text { [hartree] }\end{array}$ & $\begin{array}{c}\Delta \mathbf{E} \\
{[\mathrm{kcal} / \mathrm{mol}]}\end{array}$ & $\begin{array}{c}\Delta \mathbf{G} \\
{[\mathrm{kcal} / \mathrm{mol}]}\end{array}$ \\
\hline-1913.03263839 & -25.53 & -7.08 & -1913.04181579 & -23.26 & -4.81 & -1913.05161414 & -21.05 & -2.60 \\
\hline-1913.01458337 & -14.20 & 4.87 & -1913.02550728 & -13.03 & 6.05 & -1913.03740094 & -12.13 & 6.94 \\
\hline-1913.02663581 & -21.76 & -2.82 & -1913.03620951 & -19.74 & -0.80 & -1913.04618424 & -17.65 & 1.29 \\
\hline-1913.00350877 & -7.25 & 11.36 & -1913.01398289 & -5.80 & 12.81 & -1913.02551794 & -4.68 & 13.93 \\
\hline-1913.01006386 & -11.36 & 6.06 & -1913.01926706 & -9.11 & 8.31 & -1913.02900489 & -6.87 & 10.56 \\
\hline-1912.98554152 & 4.02 & 4.20 & -1912.99915025 & 3.51 & 3.69 & -1913.01388044 & 2.62 & 2.81 \\
\hline-1913.01762647 & -16.11 & 3.85 & -1913.02780313 & -14.47 & 5.49 & -1913.03822818 & -12.65 & 7.30 \\
\hline-1913.00898014 & -10.68 & 9.48 & -1913.01963604 & -9.34 & 10.82 & -1913.03039804 & -7.74 & 12.42 \\
\hline-1913.02552150 & -21.06 & -0.29 & -1913.03502158 & -19.00 & 1.77 & -1913.04422469 & -16.42 & 4.36 \\
\hline-1913.01623903 & -15.24 & 3.94 & -1913.02608108 & -13.39 & 5.80 & -1913.03556969 & -10.99 & 8.20 \\
\hline-1913.02362501 & -19.87 & -1.72 & -1913.03358361 & -18.10 & 0.05 & -1913.04339744 & -15.90 & 2.25 \\
\hline-1912.99195360 & 0.00 & 0.00 & -1913.00474554 & 0.00 & 0.00 & -1913.01806285 & 0.00 & 0.00 \\
\hline-1913.02127918 & -18.40 & -2.03 & -1913.03059133 & -16.22 & 0.15 & -1913.04020956 & -13.90 & 2.47 \\
\hline-1913.01614560 & -15.18 & 2.52 & -1913.02591762 & -13.29 & 4.41 & -1913.03609546 & -11.32 & 6.38 \\
\hline-1913.03005597 & -23.91 & -4.14 & -1913.03986259 & -22.04 & -2.27 & -1913.05043428 & -20.31 & -0.55 \\
\hline-1913.01079179 & -11.82 & 7.63 & -1913.02126055 & -10.36 & 9.09 & -1913.03282602 & -9.26 & 10.19 \\
\hline-1913.02779399 & -22.49 & -2.32 & -1913.03786945 & -20.79 & -0.62 & -1913.04904669 & -19.44 & 0.72 \\
\hline
\end{tabular}


Torker et al., Supporting Information, page S130

\begin{tabular}{|c|c|c|c|c|c|c|c|c|}
\hline \multicolumn{3}{|c|}{ M06/basis2 (gas-phase) } & \multicolumn{3}{|c|}{ M06/basis2 (benzene) } & \multicolumn{3}{|c|}{ M06/basis2 (dichloromethane) } \\
\hline $\begin{array}{c}\Delta \mathbf{E} \\
\text { [hartree] }\end{array}$ & $\begin{array}{c}\Delta \mathbf{E} \\
{[\mathrm{kcal} / \mathrm{mol}]}\end{array}$ & $\begin{array}{c}\Delta \mathbf{G} \\
{[\mathrm{kcal} / \mathrm{mol}]}\end{array}$ & $\begin{array}{c}\Delta \mathbf{E} \\
\text { [hartree] }\end{array}$ & $\begin{array}{c}\Delta \mathbf{E} \\
{[\mathrm{kcal} / \mathrm{mol}]}\end{array}$ & $\begin{array}{c}\Delta \mathbf{G} \\
{[\mathrm{kcal} / \mathrm{mol}]}\end{array}$ & $\begin{array}{c}\Delta \mathbf{E} \\
\text { [hartree] }\end{array}$ & $\begin{array}{c}\Delta \mathbf{E} \\
{[\mathrm{kcal} / \mathrm{mol}]}\end{array}$ & $\begin{array}{c}\Delta \mathbf{G} \\
{[\mathrm{kcal} / \mathrm{mol}]}\end{array}$ \\
\hline-1912.19860195 & -24.51 & -6.05 & -1912.20681046 & -22.48 & -4.03 & -1912.21566038 & -20.55 & -2.09 \\
\hline-1912.17856714 & -11.93 & 7.14 & -1912.18833717 & -10.89 & 8.18 & -1912.19910787 & -10.16 & 8.91 \\
\hline-1912.19040720 & -19.36 & -0.42 & -1912.19904988 & -17.61 & 1.33 & -1912.20817029 & -15.85 & 3.09 \\
\hline-1912.16884147 & -5.83 & 12.78 & -1912.17821460 & -4.54 & 14.07 & -1912.18864119 & -3.59 & 15.01 \\
\hline-1912.17915718 & -12.30 & 5.12 & -1912.18738337 & -10.29 & 7.13 & -1912.19619349 & -8.33 & 9.09 \\
\hline-1912.15424046 & 3.33 & 3.51 & -1912.16633813 & 2.91 & 3.09 & -1912.17944976 & 2.18 & 2.36 \\
\hline-1912.18207393 & -14.13 & 5.82 & -1912.19117386 & -12.67 & 7.28 & -1912.20057188 & -11.08 & 8.88 \\
\hline-1912.17133402 & -7.40 & 12.77 & -1912.18089313 & -6.22 & 13.94 & -1912.19065593 & -4.86 & 15.30 \\
\hline-1912.18841824 & -18.12 & 2.66 & -1912.19699698 & -16.33 & 4.45 & -1912.20541859 & -14.12 & 6.65 \\
\hline-1912.17856232 & -11.93 & 7.25 & -1912.18740725 & -10.31 & 8.87 & -1912.19604858 & -8.24 & 10.94 \\
\hline-1912.18779660 & -17.73 & 0.42 & -1912.19670511 & -16.14 & 2.01 & -1912.20558463 & -14.22 & 3.93 \\
\hline-1912.15954919 & 0.00 & 0.00 & -1912.17097862 & 0.00 & 0.00 & -1912.18291667 & 0.00 & 0.00 \\
\hline-1912.18736671 & -17.46 & -1.09 & -1912.19569553 & -15.51 & 0.86 & -1912.20438596 & -13.47 & 2.90 \\
\hline-1912.17937259 & -12.44 & 5.26 & -1912.18816971 & -10.79 & 6.91 & -1912.19745141 & -9.12 & 8.58 \\
\hline-1912.19324299 & -21.14 & -1.38 & -1912.20207565 & -19.51 & 0.25 & -1912.21172135 & -18.08 & 1.69 \\
\hline-1912.17575788 & -10.17 & 9.28 & -1912.18511578 & -8.87 & 10.58 & -1912.19557396 & -7.94 & 11.51 \\
\hline-1912.19368132 & -21.42 & -1.25 & -1912.20266326 & -19.88 & 0.28 & -1912.21270149 & -18.69 & 1.48 \\
\hline
\end{tabular}




\section{Energies and Gibbs Free Energies after optimization with BP86/basis $1_{D C M}$}

\section{Table S44. ROCM with catalyst Ru-4f and enol ether (pathways EPhos, ZPhos in Figure} 3)

\begin{tabular}{|c|c|c|c|c|c|c|c|}
\hline & & & & BP & /basis1 (DCM) & & \\
\hline & pathway & & $\begin{array}{c}\mathbf{E} \\
{[\text { hartree] }}\end{array}$ & $\begin{array}{c}\Delta \mathbf{E} \\
{[\mathrm{kcal} / \mathrm{mol}]}\end{array}$ & $\begin{array}{c}\mathbf{G} \\
{[\text { hartree] }}\end{array}$ & $\begin{array}{c}\Delta \mathbf{G} \\
{[\mathrm{kcal} / \mathrm{mol}]}\end{array}$ & $\begin{array}{c}\Delta \mathbf{G}_{\mathrm{Corr}} \\
{[\mathrm{kcal} / \mathrm{mol}]}\end{array}$ \\
\hline & & $14 \mathrm{e}$ & -2639.80030669 & 0.00 & -2639.203743 & 0.00 & 0.00 \\
\hline & & ts0d & -2639.79776624 & 1.59 & -2639.188338 & 9.67 & 8.07 \\
\hline & & ts0c & -2639.79760877 & 1.69 & -2639.185786 & 11.27 & 9.58 \\
\hline$.^{-19 y}-\mathrm{Cy}$ & & ts0b & -2639.79729468 & 1.89 & -2639.184317 & 12.19 & 10.30 \\
\hline & & ts0a & -2639.79662736 & 2.31 & -2639.184430 & 12.12 & 9.81 \\
\hline & ZPhos & ts0 & -2639.79538443 & 3.09 & -2639.179951 & 14.93 & 11.84 \\
\hline & & pc1 & -2639.81292412 & -7.92 & -2639.191193 & 7.88 & 15.79 \\
\hline & & ts1 & -2639.79388710 & 4.03 & -2639.171573 & 20.19 & 16.16 \\
\hline & & mcb & -2639.79802936 & 1.43 & -2639.176451 & 17.13 & 15.70 \\
\hline & & ts2 & -2639.78859754 & 7.35 & -2639.168013 & 22.42 & 15.07 \\
\hline & & pc2 & -2639.80434224 & -2.53 & -2639.183345 & 12.80 & 15.33 \\
\hline & & $14 e$ & -2639.80030669 & 0.00 & -2639.203743 & 0.00 & 0.00 \\
\hline${ }^{*} \mathrm{Cy}$ & & ts0d & -2639.79776624 & 1.59 & -2639.188338 & 9.67 & 8.07 \\
\hline & & ts0c & -2639.79760877 & 1.69 & -2639.185786 & 11.27 & 9.58 \\
\hline$H_{1 .}$ & & ts0b & -2639.79729468 & 1.89 & -2639.184317 & 12.19 & 10.30 \\
\hline & & ts0a & -2639.79662736 & 2.31 & -2639.184430 & 12.12 & 9.81 \\
\hline & EPhos & ts0 & -2639.79540393 & 3.08 & -2639.179461 & 15.24 & 12.16 \\
\hline & & pc1 & -2639.81285944 & -7.88 & -2639.191334 & 7.79 & 15.66 \\
\hline & & ts1 & -2639.79405168 & 3.93 & -2639.173563 & 18.94 & 15.01 \\
\hline & & mcb & -2639.79987433 & 0.27 & -2639.178770 & 15.67 & 15.40 \\
\hline & & ts2 & -2639.78617991 & 8.86 & -2639.166719 & 23.23 & 14.37 \\
\hline & & pc2 & -2639.80775947 & -4.68 & -2639.187473 & 10.21 & 14.89 \\
\hline
\end{tabular}

\begin{tabular}{|c|c|c|c|c|c|c|c|c|}
\hline \multicolumn{3}{|c|}{$\begin{array}{c}\text { BP86-D3BJ/Def2QZVP(gas phase) // } \\
\text { BP86/basis1(DCM) }\end{array}$} & \multicolumn{3}{|c|}{$\begin{array}{c}\text { BP86-D3BJ/Def2QZVP(benzene) // } \\
\text { BP86/basis1(DCM) }\end{array}$} & \multicolumn{3}{|c|}{$\begin{array}{c}\text { BP86-D3BJ/Def2QZVP(DCM) // } \\
\text { BP86/basis1(DCM) }\end{array}$} \\
\hline $\begin{array}{c}\mathbf{E}_{\mathrm{sp}} \\
{[\text { hartree] }}\end{array}$ & $\begin{array}{c}\Delta \mathbf{E}_{\mathrm{sp}} \\
{[\mathrm{kcal} / \mathrm{mol}]}\end{array}$ & $\begin{array}{c}\Delta \mathbf{G}_{\mathrm{sp}} \\
{[\mathrm{kcal} / \mathrm{mol}]}\end{array}$ & $\begin{array}{c}\mathbf{E}_{\mathrm{sp}} \\
{[\text { hartree] }}\end{array}$ & $\begin{array}{c}\Delta \mathbf{E}_{\mathrm{sp}} \\
{[\mathrm{kcal} / \mathrm{mol}]}\end{array}$ & $\begin{array}{c}\Delta \mathbf{G}_{\mathrm{sp}} \\
{[\mathrm{kcal} / \mathrm{mol}]}\end{array}$ & $\begin{array}{c}\mathbf{E}_{\mathrm{sp}} \\
{[\text { hartree] }}\end{array}$ & $\begin{array}{c}\Delta \mathbf{E}_{\mathrm{sp}} \\
{[\mathrm{kcal} / \mathrm{mol}]}\end{array}$ & $\begin{array}{c}\Delta \mathbf{G}_{\mathrm{sp}} \\
{[\mathrm{kcal} / \mathrm{mol}]}\end{array}$ \\
\hline-2640.65285110 & 0.00 & 0.00 & -2640.66647748 & 0.00 & 0.00 & -2640.67879272 & 0.00 & 0.00 \\
\hline-2640.65272804 & 0.08 & 8.15 & -2640.66498629 & 0.94 & 9.01 & -2640.68018182 & -0.87 & 7.20 \\
\hline-2640.65329448 & -0.28 & 9.30 & -2640.66683283 & -0.22 & 9.35 & -2640.68143959 & -1.66 & 7.91 \\
\hline-2640.65543854 & -1.62 & 8.68 & -2640.66941516 & -1.84 & 8.46 & -2640.68260824 & -2.39 & 7.91 \\
\hline-2640.65887017 & -3.78 & 6.03 & -2640.67190182 & -3.40 & 6.41 & -2640.68434984 & -3.49 & 6.32 \\
\hline-2640.66454938 & -7.34 & 4.50 & -2640.67596700 & -5.95 & 5.89 & -2640.68679717 & -5.02 & 6.82 \\
\hline-2640.68435205 & -19.77 & -3.97 & -2640.69282226 & -16.53 & -0.74 & -2640.70232266 & -14.77 & 1.03 \\
\hline-2640.66123972 & -5.26 & 10.89 & -2640.67128129 & -3.01 & 13.14 & -2640.68318496 & -2.76 & 13.40 \\
\hline-2640.66638851 & -8.49 & 7.20 & -2640.67649886 & -6.29 & 9.41 & -2640.68678118 & -5.01 & 10.68 \\
\hline-2640.66017154 & -4.59 & 10.48 & -2640.66981263 & -2.09 & 12.98 & -2640.68023436 & -0.90 & 14.17 \\
\hline-2640.68835078 & -22.28 & -6.94 & -2640.69660214 & -18.90 & -3.57 & -2640.70661529 & -17.46 & -2.13 \\
\hline
\end{tabular}


Torker et al., Supporting Information, page S132

\begin{tabular}{|c|c|c|c|c|c|c|c|c|}
\hline-2640.65285110 & 0.00 & 0.00 & -2640.66647748 & 0.00 & 0.00 & -2640.67879272 & 0.00 & 0.00 \\
\hline-2640.65272804 & 0.08 & 8.15 & -2640.66498629 & 0.94 & 9.01 & -2640.68018182 & -0.87 & 7.20 \\
\hline-2640.65329448 & -0.28 & 9.30 & -2640.66683283 & -0.22 & 9.35 & -2640.68143959 & -1.66 & 7.91 \\
\hline-2640.65543854 & -1.62 & 8.68 & -2640.66941516 & -1.84 & 8.46 & -2640.68260824 & -2.39 & 7.91 \\
\hline-2640.65887017 & -3.78 & 6.03 & -2640.67190182 & -3.40 & 6.41 & -2640.68434984 & -3.49 & 6.32 \\
\hline-2640.66463291 & -7.39 & 4.77 & -2640.67604020 & -6.00 & 6.16 & -2640.68685694 & -5.06 & 7.10 \\
\hline-2640.68399325 & -19.54 & -3.88 & -2640.69294708 & -16.61 & -0.95 & -2640.70188699 & -14.49 & 1.17 \\
\hline-2640.66111711 & -5.19 & 9.83 & -2640.67086263 & -2.75 & 12.26 & -2640.68332341 & -2.84 & 12.17 \\
\hline-2640.66812709 & -9.59 & 5.81 & -2640.67741466 & -6.86 & 8.54 & -2640.68915636 & -6.50 & 8.90 \\
\hline-2640.65603063 & -2.00 & 12.37 & -2640.66622471 & 0.16 & 14.53 & -2640.67680679 & 1.25 & 15.61 \\
\hline-2640.68562832 & -20.57 & -5.68 & -2640.69383920 & -17.17 & -2.28 & -2640.70313166 & -15.27 & -0.39 \\
\hline \\
\hline \multicolumn{9}{|c|}{$\begin{array}{l}\text { E ......... electronic energy in hartree with basis } 1 \text { in DCM } \\
\text { G ......... sum of electronic and thermal free energies with basis } 1\end{array}$} \\
\hline \multicolumn{9}{|c|}{$\Delta \mathrm{E} \ldots \ldots \ldots$ relative electronic energy in $\mathrm{kcal} / \mathrm{mol}$ with basis 1} \\
\hline \multicolumn{9}{|c|}{$\Delta \mathrm{G} \ldots \ldots \ldots$ relative free energy in $\mathrm{kcal} / \mathrm{mol}$ with basis 1} \\
\hline \multirow{2}{*}{\multicolumn{9}{|c|}{$\Delta \mathrm{G}_{\text {corr }} \ldots .$. thermal correction to free energy in $\mathrm{kcal} / \mathrm{mol}$ obtained with basis $1\left(\Delta \mathrm{G}=\Delta \mathrm{E}+\Delta \mathrm{G}_{\text {corr }}\right)$}} \\
\hline \multicolumn{8}{|c|}{$\mathrm{E}_{\mathrm{sp}} \ldots \ldots \ldots$ single point electronic energy in gas phase or solution in hartree with Def2QZVP } & \\
\hline \multicolumn{9}{|c|}{$\Delta \mathrm{E}_{\mathrm{sp}} \ldots \ldots$ relative single point electronic energy in gas phase or solution in $\mathrm{kcal} / \mathrm{mol}$ with Def2QZVP } \\
\hline
\end{tabular}

\begin{tabular}{|c|c|c|c|c|c|c|c|c|}
\hline \multicolumn{3}{|c|}{$\begin{array}{c}\text { PBE0-D3BJ/Def2QZVP(gas phase) // } \\
\text { BP86/basis1(DCM) }\end{array}$} & \multicolumn{3}{|c|}{$\begin{array}{c}\text { PBE0-D3BJ/Def2QZVP(benzene) // } \\
\text { BP86/basis1(DCM) }\end{array}$} & \multicolumn{3}{|c|}{$\begin{array}{c}\text { PBE0-D3BJ/Def2QZVP(DCM) // } \\
\text { BP86/basis1(DCM) }\end{array}$} \\
\hline $\begin{array}{c}\mathbf{E}_{\mathrm{sp}} \\
{[\text { hartree] }}\end{array}$ & $\begin{array}{c}\Delta \mathbf{E}_{\mathrm{sp}} \\
{[\mathrm{kcal} / \mathrm{mol}]}\end{array}$ & $\begin{array}{c}\Delta \mathbf{G}_{\mathrm{sp}} \\
{[\mathrm{kcal} / \mathrm{mol}]}\end{array}$ & $\begin{array}{c}\mathbf{E}_{\mathrm{sp}} \\
{[\text { hartree] }}\end{array}$ & $\begin{array}{c}\Delta \mathbf{E}_{\mathrm{sp}} \\
{[\mathrm{kcal} / \mathrm{mol}]}\end{array}$ & $\begin{array}{c}\Delta \mathbf{G}_{\text {sp }} \\
{[\mathrm{kcal} / \mathrm{mol}]}\end{array}$ & $\begin{array}{c}\mathbf{E}_{\mathrm{sp}} \\
{[\text { hartree] }}\end{array}$ & $\begin{array}{c}\Delta \mathbf{E}_{\mathrm{sp}} \\
{[\mathrm{kcal} / \mathrm{mol}]}\end{array}$ & $\begin{array}{c}\Delta \mathbf{G}_{\text {sp }} \\
{[\mathrm{kcal} / \mathrm{mol}]}\end{array}$ \\
\hline-2638.36177823 & 0.00 & 0.00 & -2638.37625894 & 0.00 & 0.00 & -2638.39125532 & 0.00 & 0.00 \\
\hline-2638.36125557 & 0.33 & 8.40 & -2638.37571626 & 0.34 & 8.41 & -2638.39219751 & -0.59 & 7.48 \\
\hline-2638.36252262 & -0.47 & 9.11 & -2638.37700587 & -0.47 & 9.11 & -2638.39335525 & -1.32 & 8.26 \\
\hline-2638.36429334 & -1.58 & 8.72 & -2638.37863651 & -1.49 & 8.81 & -2638.39473183 & -2.18 & 8.12 \\
\hline-2638.36664348 & -3.05 & 6.76 & -2638.38054632 & -2.69 & 7.12 & -2638.39581231 & -2.86 & 6.95 \\
\hline-2638.37118312 & -5.90 & 5.94 & -2638.38333377 & -4.44 & 7.40 & -2638.39640925 & -3.23 & 8.61 \\
\hline-2638.39368532 & -20.02 & -4.23 & -2638.40316902 & -16.89 & -1.09 & -2638.41310212 & -13.71 & 2.08 \\
\hline-2638.37523106 & -8.44 & 7.72 & -2638.38657754 & -6.48 & 9.68 & -2638.39862620 & -4.63 & 11.53 \\
\hline-2638.38123238 & -12.21 & 3.49 & -2638.39138895 & -9.49 & 6.20 & -2638.40355069 & -7.72 & 7.98 \\
\hline-2638.37139034 & -6.03 & 9.04 & -2638.38144062 & -3.25 & 11.82 & -2638.39336387 & -1.32 & 13.75 \\
\hline-2638.39030907 & -17.90 & -2.57 & -2638.39887028 & -14.19 & 1.14 & -2638.40997031 & -11.74 & 3.59 \\
\hline-2638.36177823 & 0.00 & 0.00 & -2638.37625894 & 0.00 & 0.00 & -2638.39125532 & 0.00 & 0.00 \\
\hline-2638.36125557 & 0.33 & 8.40 & -2638.37571626 & 0.34 & 8.41 & -2638.39219751 & -0.59 & 7.48 \\
\hline-2638.36252262 & -0.47 & 9.11 & -2638.37700587 & -0.47 & 9.11 & -2638.39335525 & -1.32 & 8.26 \\
\hline-2638.36429334 & -1.58 & 8.72 & -2638.37863651 & -1.49 & 8.81 & -2638.39473183 & -2.18 & 8.12 \\
\hline-2638.36664348 & -3.05 & 6.76 & -2638.38054632 & -2.69 & 7.12 & -2638.39581231 & -2.86 & 6.95 \\
\hline-2638.37123925 & -5.94 & 6.22 & -2638.38338950 & -4.47 & 7.69 & -2638.39644305 & -3.26 & 8.91 \\
\hline-2638.39343342 & -19.86 & -4.20 & -2638.40291917 & -16.73 & -1.07 & -2638.41305183 & -13.68 & 1.99 \\
\hline-2638.37503963 & -8.32 & 6.69 & -2638.38649483 & -6.42 & 8.59 & -2638.39862162 & -4.62 & 10.39 \\
\hline-2638.38192916 & -12.64 & 2.75 & -2638.39218067 & -9.99 & 5.41 & -2638.40467416 & -8.42 & 6.98 \\
\hline-2638.36672413 & -3.10 & 11.26 & -2638.37726785 & -0.63 & 13.74 & -2638.39042143 & 0.52 & 14.89 \\
\hline-2638.39067837 & -18.14 & -3.25 & -2638.39894752 & -14.24 & 0.65 & -2638.40912547 & -11.21 & 3.67 \\
\hline $\begin{array}{l}\mathrm{E} \ldots \ldots \ldots \ldots \text { electror } \\
\mathrm{G} \ldots \ldots \ldots \text { sum of } \\
\Delta \mathrm{E} \ldots \ldots \ldots \text { relative } \\
\Delta \mathrm{G} \ldots \ldots \ldots \text { relative } \\
\Delta \mathrm{G}_{\text {corr }} \ldots \ldots \text { therma } \\
\mathrm{E}_{\mathrm{sp}} \ldots \ldots \ldots \text { single } \\
\Delta \mathrm{E}_{\mathrm{sp}} \ldots \ldots \text { relative } \\
\Delta \mathrm{G}_{\mathrm{sp}} \ldots \ldots \text { relative }\end{array}$ & $\begin{array}{l}\text { c energy in har } \\
\text { lectronic and tl } \\
\text { lectronic ener } \\
\text { ree energy in } 1 \\
\text { orrection to fr } \\
\text { int electronic } \\
\text { ingle point ele } \\
\text { ingle point fre }\end{array}$ & $\begin{array}{l}\text { ee with basis } \\
\text { rmal free ener } \\
\text { in kcal/mol w } \\
\text { al/mol with ba } \\
\text { energy in kca } \\
\text { ergy in gas ph } \\
\text { ronic energy i }\end{array}$ & $\begin{array}{l}\text { in DCM } \\
\text { ies with basis } 1 \\
\text { th basis } 1 \\
\text { is } 1 \\
\text { /mol obtained with } \\
\text { se or solution in ha } \\
\text { gas phase or soluti }\end{array}$ & $\begin{array}{l}\text { asis } 1(\Delta \mathrm{G}=\Delta \\
\text { ee with Def2C } \\
\text { in } \mathrm{kcal} / \mathrm{mol}\end{array}$ & $\begin{array}{l}\left.+\Delta \mathrm{G}_{\text {corr }}\right) \\
\text { ZVP } \\
\text { th Def2QZVP }\end{array}$ & & & \\
\hline
\end{tabular}




\begin{tabular}{|c|c|c|c|c|c|c|c|c|}
\hline \multicolumn{3}{|c|}{$\begin{array}{c}\omega \mathrm{B97XD} / \text { Def2QZVP(gas phase) } / / \\
\text { BP86/basis1(DCM) }\end{array}$} & \multicolumn{3}{|c|}{$\begin{array}{c}\omega \mathrm{B97XD/Def2QZVP(benzene)} / / \\
\text { BP86/basis1(DCM) }\end{array}$} & \multicolumn{3}{|c|}{$\begin{array}{c}\omega \text { B97XD/Def2QZVP(DCM) // } \\
\text { BP86/basis1(DCM) }\end{array}$} \\
\hline $\begin{array}{c}\mathbf{E}_{\mathrm{sp}} \\
{[\text { hartree] }}\end{array}$ & $\begin{array}{c}\Delta \mathbf{E}_{\mathrm{sp}} \\
{[\mathrm{kcal} / \mathrm{mol}]} \\
\end{array}$ & $\begin{array}{c}\Delta \mathbf{G}_{\mathrm{sp}} \\
{[\mathrm{kcal} / \mathrm{mol}]}\end{array}$ & $\begin{array}{c}\mathbf{E}_{\mathrm{sp}} \\
{[\text { hartree] }} \\
\end{array}$ & $\begin{array}{c}\Delta \mathbf{E}_{\mathrm{sp}} \\
{[\mathrm{kcal} / \mathrm{mol}]} \\
\end{array}$ & $\begin{array}{c}\Delta \mathbf{G}_{\text {sp }} \\
{[\mathrm{kcal} / \mathrm{mol}]}\end{array}$ & $\begin{array}{c}\mathbf{E}_{\mathrm{sp}} \\
{[\text { hartree] }}\end{array}$ & $\begin{array}{c}\Delta \mathbf{E}_{\mathrm{sp}} \\
{[\mathrm{kcal} / \mathrm{mol}]} \\
\end{array}$ & $\begin{array}{c}\Delta \mathbf{G}_{\text {sp }} \\
{[\mathrm{kcal} / \mathrm{mol}]}\end{array}$ \\
\hline-2639.89037381 & 0.00 & 0.00 & -2639.90493335 & 0.00 & 0.00 & -2639.92114174 & 0.00 & 0.00 \\
\hline-2639.88980122 & 0.36 & 8.43 & -2639.90512082 & -0.12 & 7.95 & -2639.92174103 & -0.38 & 7.70 \\
\hline-2639.89099678 & -0.39 & 9.18 & -2639.90629145 & -0.85 & 8.72 & -2639.92257522 & -0.90 & 8.68 \\
\hline-2639.89271829 & -1.47 & 8.83 & -2639.90785388 & -1.83 & 8.47 & -2639.92390434 & -1.73 & 8.57 \\
\hline-2639.89520295 & -3.03 & 6.78 & -2639.90982104 & -3.07 & 6.74 & -2639.92511534 & -2.49 & 7.32 \\
\hline-2639.89993126 & -6.00 & 5.84 & -2639.91257765 & -4.80 & 7.04 & -2639.92566190 & -2.84 & 9.00 \\
\hline-2639.91976298 & -18.44 & -2.65 & -2639.92976654 & -15.58 & 0.21 & -2639.93932471 & -11.41 & 4.38 \\
\hline-2639.90186505 & -7.21 & 8.95 & -2639.91362514 & -5.45 & 10.70 & -2639.92713067 & -3.76 & 12.40 \\
\hline-2639.90654956 & -10.15 & 5.55 & -2639.91754085 & -7.91 & 7.79 & -2639.92925351 & -5.09 & 10.61 \\
\hline-2639.89786469 & -4.70 & 10.37 & -2639.90801188 & -1.93 & 13.14 & -2639.92085006 & 0.18 & 15.26 \\
\hline-2639.91403111 & -14.85 & 0.49 & -2639.92287229 & -11.26 & 4.08 & -2639.93442259 & -8.33 & 7.00 \\
\hline-2639.89037381 & 0.00 & 0.00 & -2639.90493335 & 0.00 & 0.00 & -2639.92114174 & 0.00 & 0.00 \\
\hline-2639.88980122 & 0.36 & 8.43 & -2639.90512082 & -0.12 & 7.95 & -2639.92174103 & -0.38 & 7.70 \\
\hline-2639.89099678 & -0.39 & 9.18 & -2639.90629145 & -0.85 & 8.72 & -2639.92257522 & -0.90 & 8.68 \\
\hline-2639.89271829 & -1.47 & 8.83 & -2639.90785388 & -1.83 & 8.47 & -2639.92390434 & -1.73 & 8.57 \\
\hline-2639.89520295 & -3.03 & 6.78 & -2639.90982104 & -3.07 & 6.74 & -2639.92511534 & -2.49 & 7.32 \\
\hline-2639.89994932 & -6.01 & 6.15 & -2639.91258651 & -4.80 & 7.36 & -2639.92568420 & -2.85 & 9.31 \\
\hline-2639.91966481 & -18.38 & -2.72 & -2639.92963568 & -15.50 & 0.16 & -2639.93922762 & -11.35 & 4.31 \\
\hline-2639.90163343 & -7.07 & 7.95 & -2639.91275638 & -4.91 & 10.10 & -2639.92655339 & -3.40 & 11.62 \\
\hline-2639.90704037 & -10.46 & 4.94 & -2639.91828878 & -8.38 & 7.02 & -2639.93004680 & -5.59 & 9.81 \\
\hline-2639.89359013 & -2.02 & 12.35 & -2639.90456674 & 0.23 & 14.60 & -2639.91724818 & 2.44 & 16.81 \\
\hline-2639.91780105 & -17.21 & -2.32 & -2639.92681092 & -13.73 & 1.16 & -2639.93697180 & -9.93 & 4.95 \\
\hline $\begin{array}{l}\mathrm{E} \ldots \ldots \ldots \ldots \text { electro } \\
\mathrm{G} \ldots \ldots \ldots . \text { sum of } \\
\Delta \mathrm{E} \ldots \ldots \ldots \text { relative } \\
\Delta \mathrm{G} \ldots \ldots \ldots \text { relative } \\
\Delta \mathrm{G}_{\mathrm{corr}} \ldots \ldots \text { therma } \\
\mathrm{E}_{\mathrm{sp}} \ldots \ldots \ldots \text { single } \\
\Delta \mathrm{E}_{\mathrm{sp}} \ldots \ldots \text { relative } \\
\Delta \mathrm{G}_{\mathrm{sp}} \ldots \ldots . \text { relative }\end{array}$ & $\begin{array}{l}\text { c energy in ha } \\
\text { lectronic and } t \\
\text { electronic ener } \\
\text { ree energy in } 1 \\
\text { correction to } \mathrm{fr} \\
\text { int electronic } \\
\text { single point ele }\end{array}$ & $\begin{array}{l}\text { ree with basis } \\
\text { ermal free ene } \\
\text { y in } \mathrm{kcal} / \mathrm{mol} \text { v } \\
\mathrm{al} / \mathrm{mol} \text { with bo } \\
\text { e energy in } \mathrm{kc} \\
\text { hergy in gas ph } \\
\text { tronic energy } \mathrm{i}\end{array}$ & $\begin{array}{l}\text { in DCM } \\
\text { ies with basis } 1 \\
\text { th basis } 1 \\
\text { is } 1 \\
\text { /mol obtained with } \\
\text { se or solution in ha } \\
\text { gas phase or soluti }\end{array}$ & $\begin{array}{l}\text { asis } 1(\Delta G=\Delta \\
\text { ree with Def } 2 \\
\text { in } \mathrm{kcal} / \mathrm{mol}\end{array}$ & $\begin{array}{l}\left.+\Delta \mathrm{G}_{\text {corr }}\right) \\
\text { ZVP } \\
\text { th Def } 2 \mathrm{QZVP}\end{array}$ & & & \\
\hline
\end{tabular}

\begin{tabular}{|c|c|c|c|c|c|c|c|c|}
\hline \multicolumn{3}{|c|}{$\begin{array}{c}\text { M06/Def2QZVP(gas phase) // } \\
\text { BP86/basis1(DCM) }\end{array}$} & \multicolumn{3}{|c|}{$\begin{array}{c}\text { M06/Def2QZVP(benzene) // } \\
\text { BP86/basis1(DCM) }\end{array}$} & \multicolumn{3}{|c|}{$\begin{array}{c}\text { M06/Def2QZVP(DCM) // } \\
\text { BP86/basis1(DCM) }\end{array}$} \\
\hline $\begin{array}{c}\mathbf{E}_{\mathrm{sp}} \\
{[\text { hartree] }}\end{array}$ & $\begin{array}{c}\Delta \mathbf{E}_{\mathrm{sp}} \\
{[\mathrm{kcal} / \mathrm{mol}]}\end{array}$ & $\begin{array}{c}\Delta \mathbf{G}_{\mathrm{sp}} \\
{[\mathrm{kcal} / \mathrm{mol}]}\end{array}$ & $\begin{array}{c}\mathbf{E}_{\mathrm{sp}} \\
{[\text { hartree] }}\end{array}$ & $\begin{array}{c}\Delta \mathbf{E}_{\mathrm{sp}} \\
{[\mathrm{kcal} / \mathrm{mol}]}\end{array}$ & $\begin{array}{c}\Delta \mathbf{G}_{\text {sp }} \\
{[\mathrm{kcal} / \mathrm{mol}]}\end{array}$ & $\begin{array}{c}\mathbf{E}_{\mathrm{sp}} \\
{[\text { hartree] }}\end{array}$ & $\begin{array}{c}\Delta \mathbf{E}_{\mathrm{sp}} \\
{[\mathrm{kcal} / \mathrm{mol}]}\end{array}$ & $\begin{array}{c}\Delta \mathbf{G}_{\mathrm{sp}} \\
{[\mathrm{kcal} / \mathrm{mol}]}\end{array}$ \\
\hline-2639.25823945 & 0.00 & 0.00 & -2639.27249926 & 0.00 & 0.00 & -2639.28651678 & 0.00 & 0.00 \\
\hline-2639.25759452 & 0.40 & 8.48 & -2639.27142932 & 0.67 & 8.74 & -2639.28690012 & -0.24 & 7.83 \\
\hline-2639.25859376 & -0.22 & 9.35 & -2639.27245558 & 0.03 & 9.60 & -2639.28793034 & -0.89 & 8.69 \\
\hline-2639.26021503 & -1.24 & 9.06 & -2639.27399141 & -0.94 & 9.36 & -2639.28931635 & -1.76 & 8.54 \\
\hline-2639.26181141 & -2.24 & 7.57 & -2639.27519687 & -1.69 & 8.12 & -2639.28983705 & -2.08 & 7.73 \\
\hline-2639.26769084 & -5.93 & 5.91 & -2639.27922716 & -4.22 & 7.62 & -2639.29150107 & -3.13 & 8.71 \\
\hline-2639.29454391 & -22.78 & -6.99 & -2639.30387289 & -19.69 & -3.89 & -2639.31374195 & -17.08 & -1.29 \\
\hline-2639.27378819 & -9.76 & 6.40 & -2639.28472131 & -7.67 & 8.49 & -2639.29701128 & -6.59 & 9.57 \\
\hline-2639.27800884 & -12.41 & 3.29 & -2639.28968588 & -10.78 & 4.91 & -2639.30141650 & -9.35 & 6.35 \\
\hline-2639.27089405 & -7.94 & 7.13 & -2639.28106871 & -5.38 & 9.70 & -2639.29231828 & -3.64 & 11.43 \\
\hline-2639.28761313 & -18.43 & -3.10 & -2639.29605486 & -14.78 & 0.55 & -2639.30583225 & -12.12 & 3.21 \\
\hline
\end{tabular}




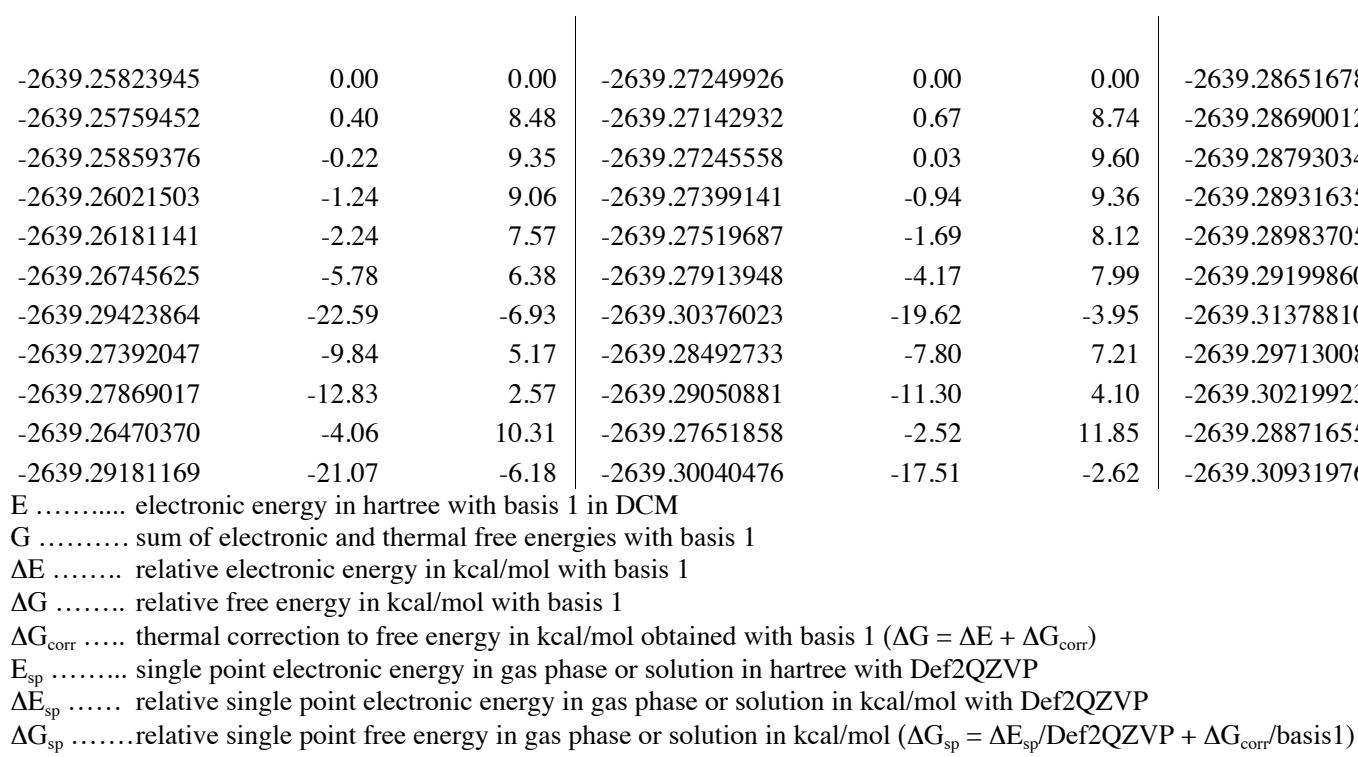


Table S45. ROCM with catalyst Ru-4b and enol ether (pathways ENHC, ZNHC in Figure 3)
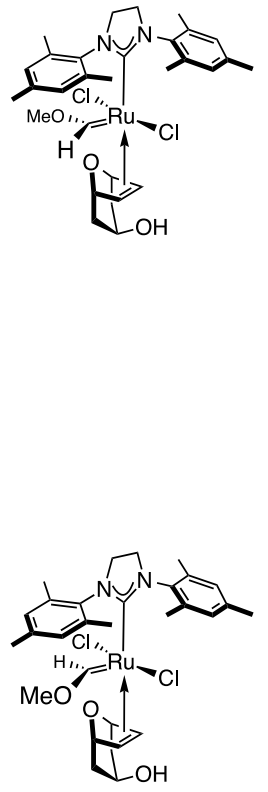

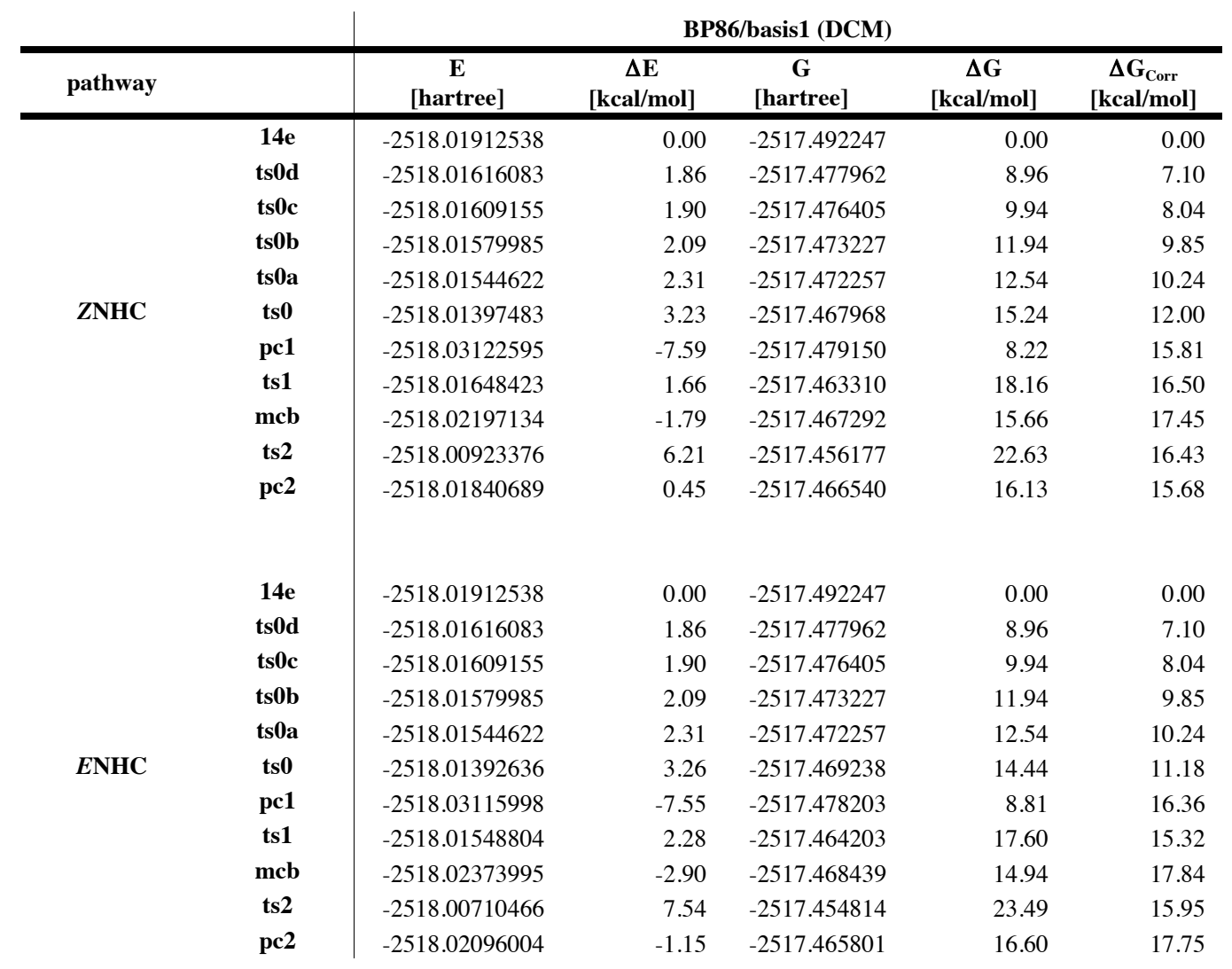

BP86-D3BJ/Def2QZVP(gas phase) // BP86/basis1(DCM)

\begin{tabular}{|c|c|c|c|c|c|c|c|c|}
\hline \multicolumn{3}{|c|}{ BP86/basis1(DCM) } & \multicolumn{3}{|c|}{ BP86/basis1(DCM) } & \multicolumn{3}{|c|}{ BP86/basis1(DCM) } \\
\hline $\begin{array}{c}\mathbf{E}_{\mathrm{sp}} \\
\text { [hartree] }\end{array}$ & $\begin{array}{c}\Delta \mathbf{E}_{\mathrm{sp}} \\
{[\mathrm{kcal} / \mathrm{mol}]}\end{array}$ & $\begin{array}{c}\Delta \mathbf{G}_{\mathrm{sp}} \\
{[\mathrm{kcal} / \mathrm{mol}]}\end{array}$ & $\begin{array}{c}\mathbf{E}_{\mathrm{sp}} \\
\text { [hartree] }\end{array}$ & $\begin{array}{c}\Delta \mathbf{E}_{\mathrm{sp}} \\
{[\mathrm{kcal} / \mathrm{mol}]}\end{array}$ & $\begin{array}{c}\Delta \mathbf{G}_{\mathrm{sp}} \\
{[\mathrm{kcal} / \mathrm{mol}]}\end{array}$ & $\begin{array}{c}\mathbf{E}_{\mathrm{sp}} \\
\text { [hartree] }\end{array}$ & $\begin{array}{c}\Delta \mathbf{E}_{\mathrm{sp}} \\
{[\mathrm{kcal} / \mathrm{mol}]}\end{array}$ & $\begin{array}{c}\Delta \mathbf{G}_{\mathrm{sp}} \\
{[\mathrm{kcal} / \mathrm{mol}]}\end{array}$ \\
\hline-2518.91211309 & 0.00 & 0.00 & -2518.92670159 & 0.00 & 0.00 & -2518.94082304 & 0.00 & 0.00 \\
\hline-2518.91193751 & 0.11 & 7.21 & -2518.92597300 & 0.46 & 7.56 & -2518.94246696 & -1.03 & 6.07 \\
\hline-2518.91344797 & -0.84 & 7.20 & -2518.92742509 & -0.45 & 7.58 & -2518.94376452 & -1.85 & 6.19 \\
\hline-2518.91317626 & -0.67 & 9.18 & -2518.92865219 & -1.22 & 8.62 & -2518.94531092 & -2.82 & 7.03 \\
\hline-2518.91793107 & -3.65 & 6.58 & -2518.93136201 & -2.92 & 7.31 & -2518.94696566 & -3.85 & 6.38 \\
\hline-2518.92472578 & -7.91 & 4.09 & -2518.93750687 & -6.78 & 5.22 & -2518.94999578 & -5.76 & 6.25 \\
\hline-2518.94559475 & -21.01 & -5.20 & -2518.95613817 & -18.47 & -2.66 & -2518.96715165 & -16.52 & -0.71 \\
\hline-2518.92828058 & -10.15 & 6.36 & -2518.93964999 & -8.13 & 8.38 & -2518.95188178 & -6.94 & 9.56 \\
\hline-2518.93679570 & -15.49 & 1.96 & -2518.94730620 & -12.93 & 4.52 & -2518.95848169 & -11.08 & 6.36 \\
\hline-2518.92731794 & -9.54 & 6.89 & -2518.93773105 & -6.92 & 9.51 & -2518.95020559 & -5.89 & 10.54 \\
\hline-2518.94666057 & -21.68 & -6.00 & -2518.95719099 & -19.13 & -3.45 & -2518.96731670 & -16.63 & -0.94 \\
\hline
\end{tabular}


Torker et al., Supporting Information, page S136

\begin{tabular}{|c|c|c|c|c|c|c|c|c|}
\hline-2518.91211309 & 0.00 & 0.00 & -2518.92670159 & 0.00 & 0.00 & -2518.94082304 & 0.00 & 0.00 \\
\hline-2518.91193751 & 0.11 & 7.21 & -2518.92597300 & 0.46 & 7.56 & -2518.94246696 & -1.03 & 6.07 \\
\hline-2518.91344797 & -0.84 & 7.20 & -2518.92742509 & -0.45 & 7.58 & -2518.94376452 & -1.85 & 6.19 \\
\hline-2518.91317626 & -0.67 & 9.18 & -2518.92865219 & -1.22 & 8.62 & -2518.94531092 & -2.82 & 7.03 \\
\hline-2518.91793107 & -3.65 & 6.58 & -2518.93136201 & -2.92 & 7.31 & -2518.94696566 & -3.85 & 6.38 \\
\hline-2518.92469714 & -7.90 & 3.28 & -2518.93742921 & -6.73 & 4.44 & -2518.94991403 & -5.70 & 5.47 \\
\hline-2518.94520104 & -20.76 & -4.40 & -2518.95620912 & -18.52 & -2.15 & -2518.96717545 & -16.54 & -0.17 \\
\hline-2518.92688521 & -9.27 & 6.05 & -2518.93783597 & -6.99 & 8.33 & -2518.95071213 & -6.21 & 9.11 \\
\hline-2518.93731382 & -15.81 & 2.02 & -2518.94848449 & -13.67 & 4.17 & -2518.95979735 & -11.91 & 5.93 \\
\hline-2518.92480399 & -7.96 & 7.98 & -2518.93565735 & -5.62 & 10.33 & -2518.94906494 & -5.17 & 10.77 \\
\hline-2518.94314885 & -19.48 & -1.73 & -2518.95271992 & -16.33 & 1.42 & -2518.96222049 & -13.43 & 4.32 \\
\hline \\
\hline \multicolumn{9}{|c|}{...... sum of electronic and thermal free energies with basis 1} \\
\hline \multicolumn{9}{|c|}{$\Delta \mathrm{E}$........ relative electronic energy in $\mathrm{kcal} / \mathrm{mol}$ with basis 1} \\
\hline \multicolumn{9}{|c|}{$\Delta \mathrm{G} \ldots \ldots \ldots$ relative free energy in $\mathrm{kcal} / \mathrm{mol}$ with basis 1} \\
\hline \multicolumn{9}{|c|}{$\begin{array}{l}\Delta \mathrm{G}_{\text {corr }} \ldots . . \text { gas phase thermal correction to free energy in } \mathrm{kcal} / \mathrm{mol} \text { obtained with basis } 1\left(\Delta \mathrm{G}=\Delta \mathrm{E}+\Delta \mathrm{G}_{\text {corr }}\right) \\
\mathrm{E} \ldots \ldots \ldots \text { single point electronic energy in gas phase or solution in hartree with basis } 2\end{array}$} \\
\hline \multicolumn{9}{|c|}{$\mathrm{E}_{\mathrm{sp}} \ldots \ldots \ldots$ single point electronic energy in gas phase or solution in hartree with basis 2} \\
\hline \multicolumn{9}{|c|}{$\Delta \mathrm{E}_{\mathrm{sp}} \ldots \ldots$ relative single point electronic energy in gas phase or solution in $\mathrm{kcal} / \mathrm{mol}$ with basis 2} \\
\hline
\end{tabular}

PBE0-D3BJ/Def2QZVP(gas phase) // BP86/basis1(DCM)

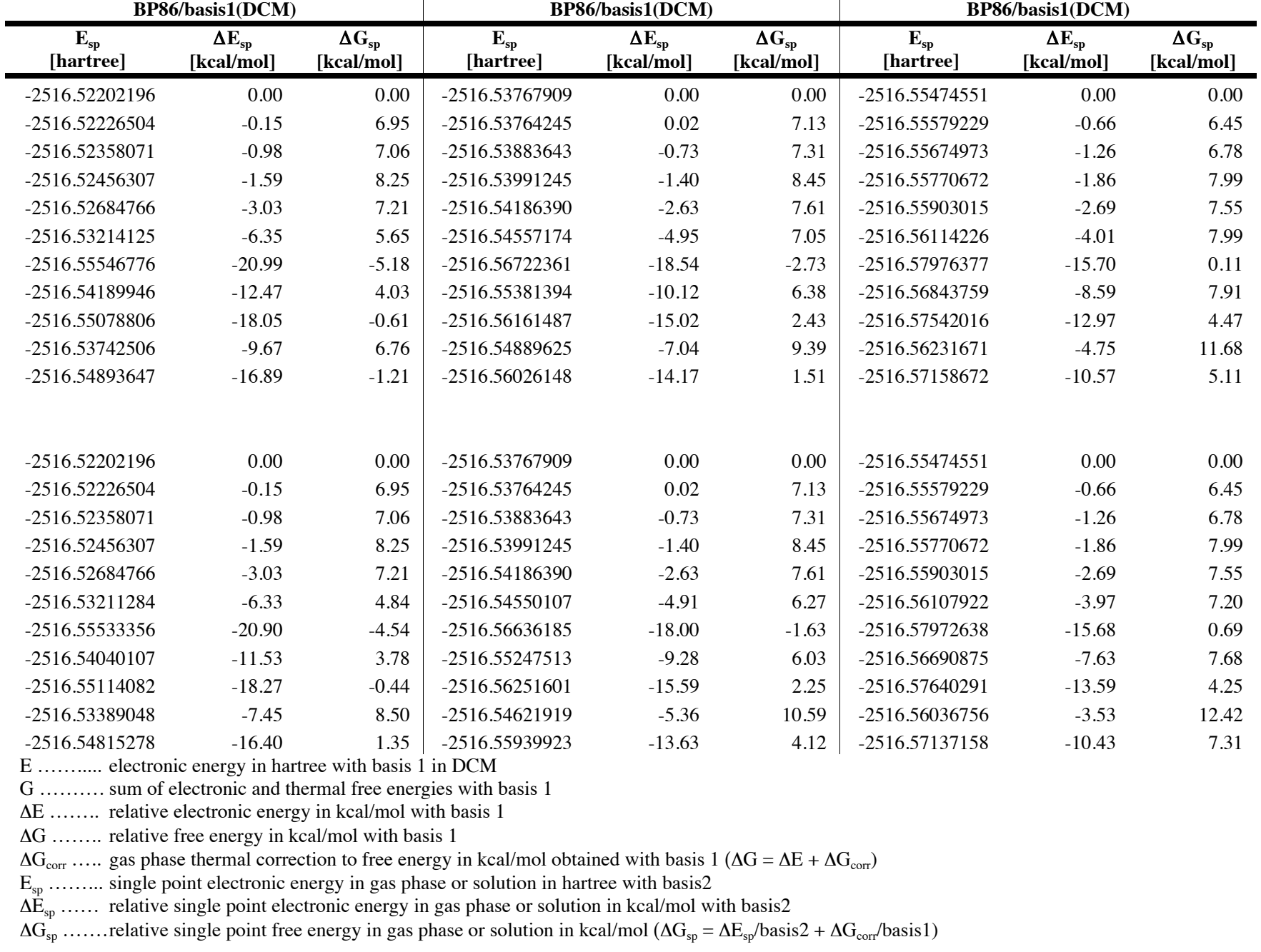

\begin{tabular}{l|l} 
PBE0-D3BJ/Def2QZVP(benzene) // & PBE0-D3BJ/Def2QZVP(DCM) //
\end{tabular} BP86/basis1(DCM) ${ }^{155}$ 199 .11 7.91 


\begin{tabular}{|c|c|c|c|c|c|c|c|c|}
\hline \multicolumn{3}{|c|}{$\begin{array}{c}\omega \mathrm{B97XD} / \text { Def2QZVP(gas phase) } / / \\
\text { BP86/basis1(DCM) }\end{array}$} & \multicolumn{3}{|c|}{$\begin{array}{c}\text { wB97XD/Def2QZVP(benzene) } / / \\
\text { BP86/basis1(DCM) }\end{array}$} & \multicolumn{3}{|c|}{$\begin{array}{c}\omega \mathrm{B97XD} / \mathrm{Def2QZVP}(\mathrm{DCM}) / / \\
\text { BP86/basis1(DCM) }\end{array}$} \\
\hline $\begin{array}{c}\mathbf{E}_{\mathrm{sp}} \\
{[\text { hartree] }}\end{array}$ & $\begin{array}{c}\Delta \mathbf{E}_{\mathrm{sp}} \\
{[\mathrm{kcal} / \mathrm{mol}]}\end{array}$ & $\begin{array}{c}\Delta \mathbf{G}_{\mathrm{sp}} \\
{[\mathrm{kcal} / \mathrm{mol}]}\end{array}$ & $\begin{array}{c}\mathbf{E}_{\mathrm{sp}} \\
\text { [hartree] }\end{array}$ & $\begin{array}{c}\Delta \mathbf{E}_{\mathrm{sp}} \\
{[\mathrm{kcal} / \mathrm{mol}]}\end{array}$ & $\begin{array}{c}\Delta \mathbf{G}_{\mathrm{sp}} \\
{[\mathrm{kcal} / \mathrm{mol}]}\end{array}$ & $\begin{array}{c}\mathbf{E}_{\mathrm{sp}} \\
\text { [hartree] }\end{array}$ & $\begin{array}{c}\Delta \mathbf{E}_{\mathrm{sp}} \\
{[\mathrm{kcal} / \mathrm{mol}]}\end{array}$ & $\begin{array}{c}\Delta \mathbf{G}_{\mathrm{sp}} \\
{[\mathrm{kcal} / \mathrm{mol}]}\end{array}$ \\
\hline-2518.06687520 & 0.00 & 0.00 & -2518.08321194 & 0.00 & 0.00 & -2518.10051863 & 0.00 & 0.00 \\
\hline-2518.06705070 & -0.11 & 6.99 & -2518.08308446 & 0.08 & 7.18 & -2518.10184600 & -0.83 & 6.27 \\
\hline-2518.06826820 & -0.87 & 7.16 & -2518.08418143 & -0.61 & 7.43 & -2518.10278501 & -1.42 & 6.62 \\
\hline-2518.06932182 & -1.54 & 8.31 & -2518.08530946 & -1.32 & 8.53 & -2518.10395821 & -2.16 & 7.69 \\
\hline-2518.07148222 & -2.89 & 7.34 & -2518.08714846 & -2.47 & 7.77 & -2518.10540603 & -3.07 & 7.17 \\
\hline-2518.07735141 & -6.57 & 5.43 & -2518.09187641 & -5.44 & 6.57 & -2518.10600730 & -3.44 & 8.56 \\
\hline-2518.09859809 & -19.91 & -4.09 & -2518.11073989 & -17.27 & -1.46 & -2518.12405138 & -14.77 & 1.04 \\
\hline-2518.08458295 & -11.11 & 5.39 & -2518.09754187 & -8.99 & 7.51 & -2518.11186962 & -7.12 & 9.38 \\
\hline-2518.09259204 & -16.14 & 1.31 & -2518.10462115 & -13.43 & 4.01 & -2518.11780320 & -10.85 & 6.60 \\
\hline-2518.08062287 & -8.63 & 7.80 & -2518.09285201 & -6.05 & 10.38 & -2518.10626597 & -3.61 & 12.82 \\
\hline-2518.08978048 & -14.37 & 1.31 & -2518.10060858 & -10.92 & 4.76 & -2518.11304135 & -7.86 & 7.82 \\
\hline-2518.06687520 & 0.00 & 0.00 & -2518.08321194 & 0.00 & 0.00 & -2518.10051863 & 0.00 & 0.00 \\
\hline-2518.06705070 & -0.11 & 6.99 & -2518.08308446 & 0.08 & 7.18 & -2518.10184600 & -0.83 & 6.27 \\
\hline-2518.06826820 & -0.87 & 7.16 & -2518.08418143 & -0.61 & 7.43 & -2518.10278501 & -1.42 & 6.62 \\
\hline-2518.06932182 & -1.54 & 8.31 & -2518.08530946 & -1.32 & 8.53 & -2518.10395821 & -2.16 & 7.69 \\
\hline-2518.07148222 & -2.89 & 7.34 & -2518.08714846 & -2.47 & 7.77 & -2518.10540603 & -3.07 & 7.17 \\
\hline-2518.07733393 & -6.56 & 4.61 & -2518.09181924 & -5.40 & 5.77 & -2518.10592567 & -3.39 & 7.78 \\
\hline-2518.09848439 & -19.84 & -3.47 & -2518.11063746 & -17.21 & -0.85 & -2518.12401295 & -14.74 & 1.62 \\
\hline-2518.08305995 & -10.16 & 5.16 & -2518.09618488 & -8.14 & 7.17 & -2518.11035205 & -6.17 & 9.14 \\
\hline-2518.09269047 & -16.20 & 1.64 & -2518.10516717 & -13.78 & 4.06 & -2518.11846112 & -11.26 & 6.58 \\
\hline-2518.07785394 & -6.89 & 9.06 & -2518.09013780 & -4.35 & 11.60 & -2518.10526066 & -2.98 & 12.97 \\
\hline-2518.09283863 & -16.29 & 1.45 & -2518.10347941 & -12.72 & 5.03 & -2518.11654626 & -10.06 & 7.69 \\
\hline $\begin{array}{l}\mathrm{E} \ldots \ldots \ldots \ldots \text { electron } \\
\mathrm{G} \ldots \ldots \ldots \text {. } \ldots \text { sum of } \\
\Delta \mathrm{E} \ldots \ldots \ldots \text { relative } \\
\Delta \mathrm{G} \ldots \ldots \ldots \text { relative } \\
\Delta \mathrm{G}_{\text {corr }} \ldots \ldots \text { thermal } \\
\mathrm{E}_{\mathrm{sp}} \ldots \ldots \ldots \text { single } \mathrm{p} \\
\Delta \mathrm{E}_{\mathrm{sp}} \ldots \ldots \text { relative } \\
\Delta \mathrm{G}_{\mathrm{s}} \ldots \ldots . \text { relative }\end{array}$ & $\begin{array}{l}\text { c energy in ha } \\
\text { lectronic and } t \\
\text { lectronic ener } \\
\text { ree energy in } \\
\text { orrection to } \mathrm{ft} \\
\text { int electronic } \\
\text { ingle point ele }\end{array}$ & $\begin{array}{l}\text { ee with basis } \\
\text { rmal free ene } \\
\text { in } \mathrm{kcal} / \mathrm{mol} \\
\mathrm{al} / \mathrm{mol} \text { with ba } \\
\text { energy in } \mathrm{kc} \\
\text { ergy in gas ph }\end{array}$ & $\begin{array}{l}\text { in DCM } \\
\text { ies with basis } 1 \\
\text { th basis } 1 \\
\text { is } 1 \\
\text { mol obtained with } \\
\text { se or solution in har }\end{array}$ & asis $1(\Delta G=\Delta$ & $\begin{array}{l}\left.+\Delta \mathrm{G}_{\text {corr }}\right) \\
\mathrm{NP}\end{array}$ & & & \\
\hline
\end{tabular}

\begin{tabular}{|c|c|c|c|c|c|c|c|c|}
\hline \multicolumn{3}{|c|}{$\begin{array}{c}\text { M06/Def2QZVP(gas phase) } / / \\
\text { BP86/basis1(DCM) }\end{array}$} & \multicolumn{3}{|c|}{$\begin{array}{c}\text { M06/Def2QZVP(benzene) // } \\
\text { BP86/basis1(DCM) }\end{array}$} & \multicolumn{3}{|c|}{$\begin{array}{c}\text { M06/Def2QZVP(DCM) // } \\
\text { BP86/basis1(DCM) }\end{array}$} \\
\hline $\begin{array}{c}\mathbf{E}_{\mathrm{sp}} \\
\text { [hartree] }\end{array}$ & $\begin{array}{c}\Delta \mathbf{E}_{\mathrm{sp}} \\
{[\mathrm{kcal} / \mathrm{mol}]}\end{array}$ & $\begin{array}{c}\Delta \mathbf{G}_{\mathrm{sp}} \\
{[\mathrm{kcal} / \mathrm{mol}]}\end{array}$ & $\begin{array}{c}\mathbf{E}_{\mathrm{sp}} \\
{[\text { hartree] }}\end{array}$ & $\begin{array}{c}\Delta \mathbf{E}_{\mathrm{sp}} \\
{[\mathrm{kcal} / \mathrm{mol}]}\end{array}$ & $\underset{[\mathrm{kcal} / \mathrm{mol}]}{\Delta \mathbf{G}_{\mathrm{sp}}}$ & $\begin{array}{c}\mathbf{E}_{\mathrm{sp}} \\
\text { [hartree] }\end{array}$ & $\begin{array}{c}\Delta \mathbf{E}_{\mathrm{sp}} \\
{[\mathrm{kcal} / \mathrm{mol}]}\end{array}$ & $\begin{array}{c}\Delta \mathbf{G}_{\mathrm{sp}} \\
{[\mathrm{kcal} / \mathrm{mol}]}\end{array}$ \\
\hline-2517.41387638 & 0.00 & 0.00 & -2517.42967144 & 0.00 & 0.00 & -2517.44506551 & 0.00 & 0.00 \\
\hline-2517.41314256 & 0.46 & 7.56 & -2517.42841035 & 0.79 & 7.90 & -2517.44541772 & -0.22 & 6.88 \\
\hline-2517.41401336 & -0.09 & 7.95 & -2517.42923758 & 0.27 & 8.31 & -2517.44612771 & -0.67 & 7.37 \\
\hline-2517.41460615 & -0.46 & 9.39 & -2517.42996134 & -0.18 & 9.67 & -2517.44768727 & -1.65 & 8.20 \\
\hline-2517.41659177 & -1.70 & 8.53 & -2517.43159355 & -1.21 & 9.03 & -2517.44784088 & -1.74 & 8.49 \\
\hline-2517.42249719 & -5.41 & 6.59 & -2517.43581296 & -3.85 & 8.15 & -2517.45051495 & -3.42 & 8.58 \\
\hline-2517.44912662 & -22.12 & -6.31 & -2517.46079043 & -19.53 & -3.72 & -2517.47380865 & -18.04 & -2.22 \\
\hline-2517.43286614 & -11.92 & 4.58 & -2517.44506066 & -9.66 & 6.84 & -2517.45932354 & -8.95 & 7.55 \\
\hline-2517.44151833 & -17.35 & 0.10 & -2517.45270461 & -14.45 & 2.99 & -2517.46609984 & -13.20 & 4.25 \\
\hline-2517.43069888 & -10.56 & 5.87 & -2517.44146282 & -7.40 & 9.03 & -2517.45395366 & -5.58 & 10.85 \\
\hline-2517.44155788 & -17.37 & -1.69 & -2517.45190264 & -13.95 & 1.73 & -2517.46358951 & -11.62 & 4.06 \\
\hline
\end{tabular}




\section{Images of transition states}

TSs for ROCM of 2 with Ru-4f and Ru-4b and enol ether (pathways EPhos, ZPhos, ENHC, ZNHC in Figure 3)

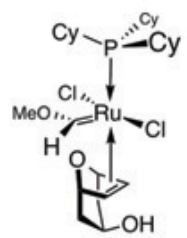

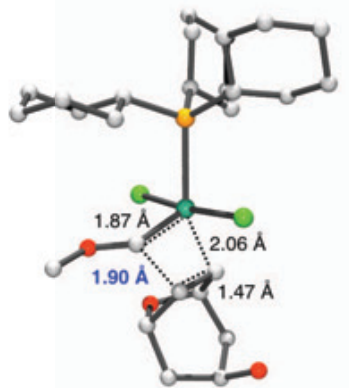

ts $1_{\text {ZPhos }}$

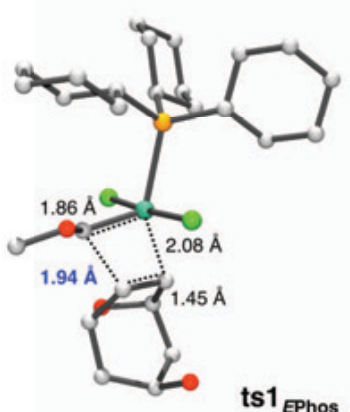

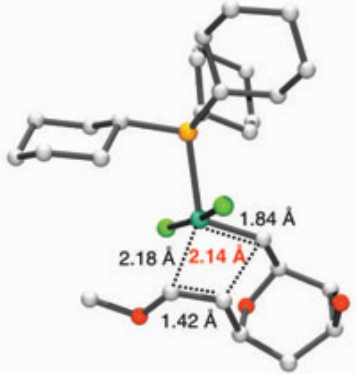

ts2 ZPhos

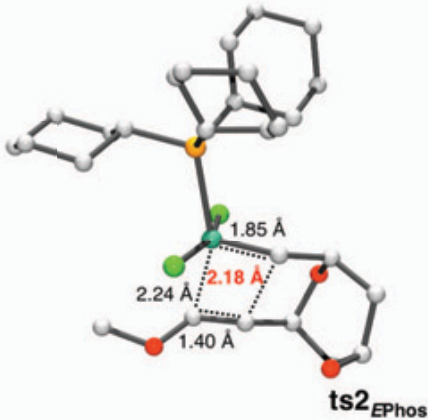

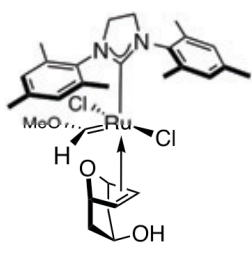

ZNHC

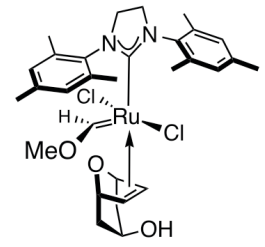

ENHC

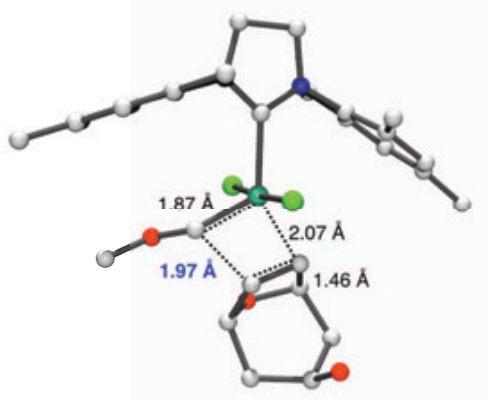

ts $1_{\text {ZNHC }}$

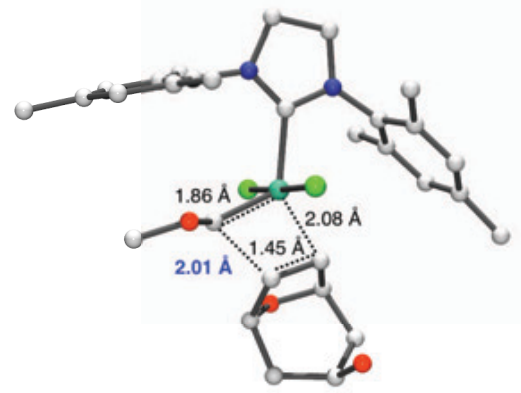

ts $1_{\text {ENHC }}$
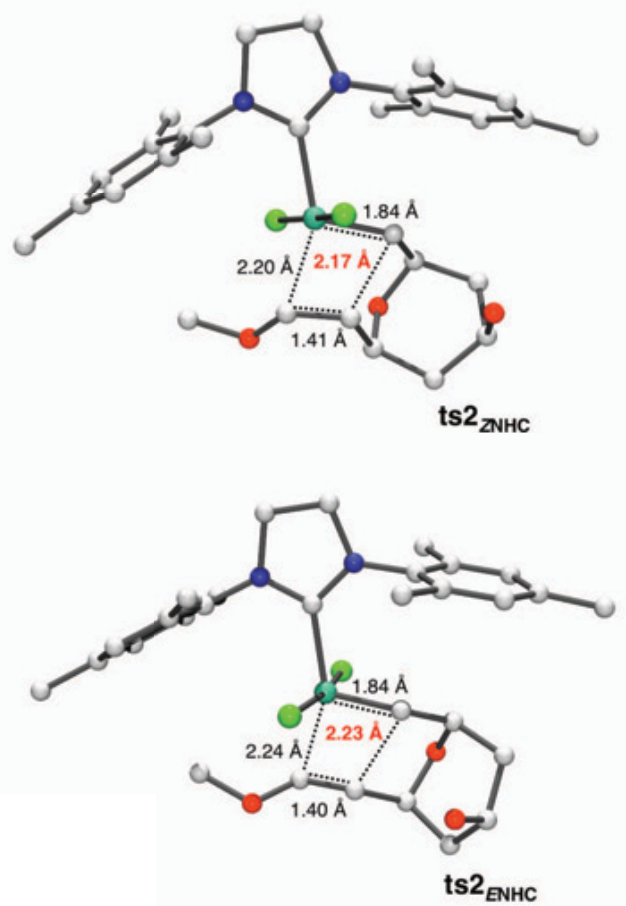
TSs for ROCM of 2 with catalyst Ru-5b and enol ether (pathways $E R u-5 b, Z R u-5 b$; A1B4 in Figure S22)

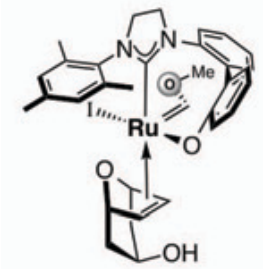

A1

$=\mathbf{Z R u}-\mathbf{5 b}$

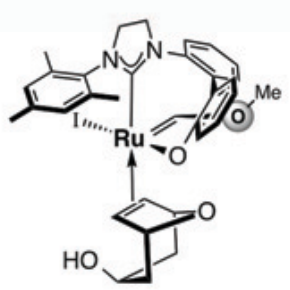

A2

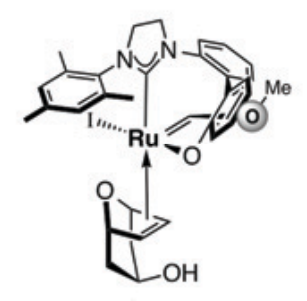

A3

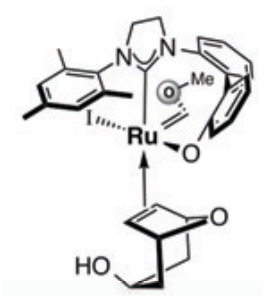

A4

$=E R u-5 b$
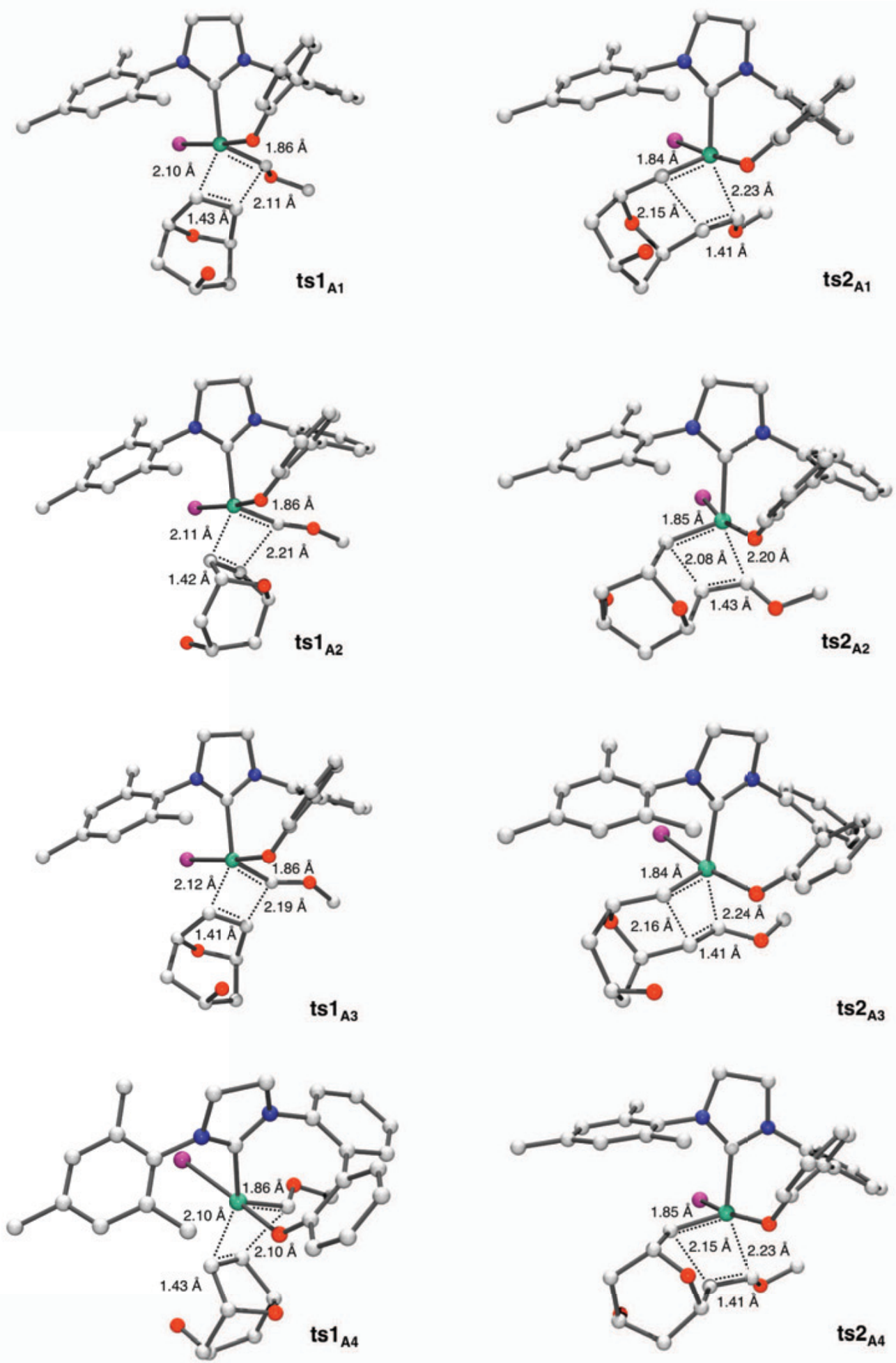


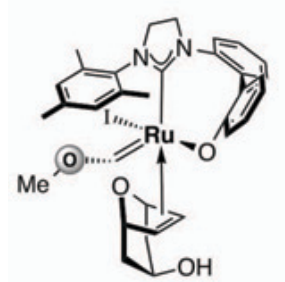

B1

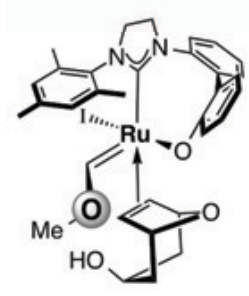

B2

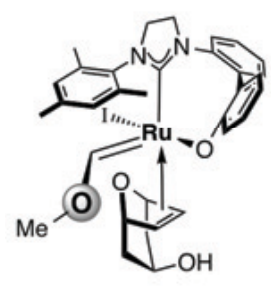

B3

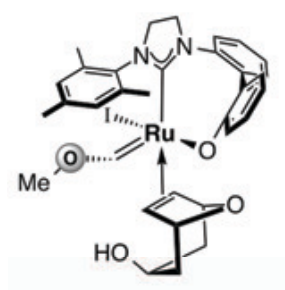

B4
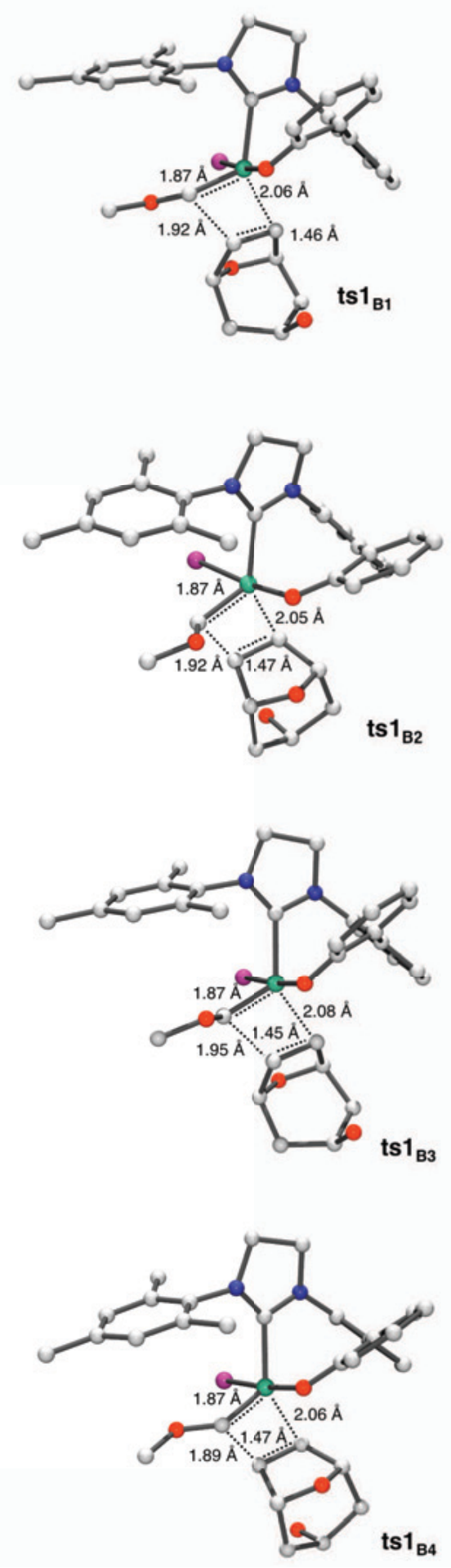
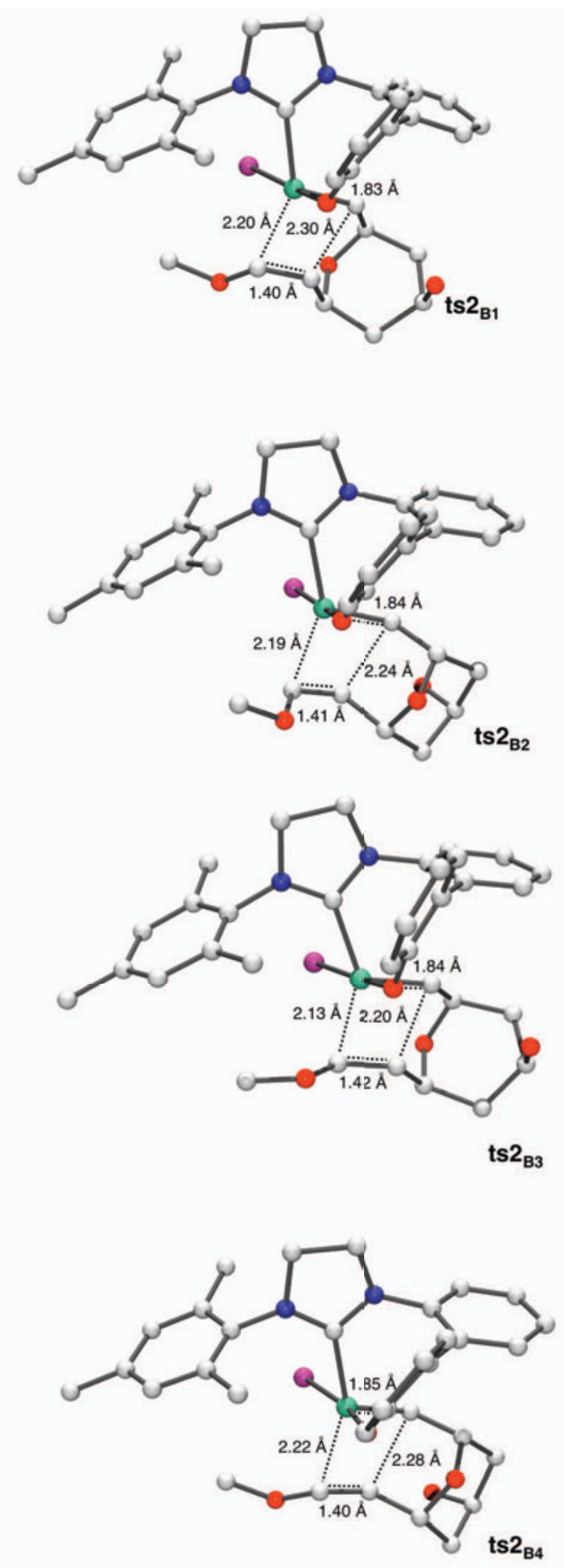
TSs for nonproductive OM of enol ether with catalyst Ru-5b (pathways AB1-AB4 in Figure S22)

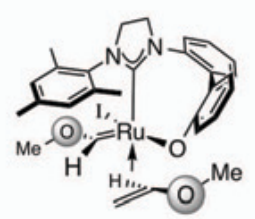

AB1

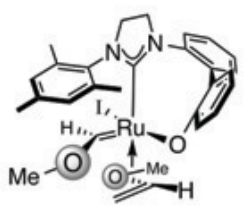

AB2

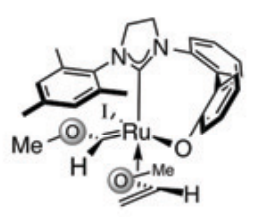

AB3

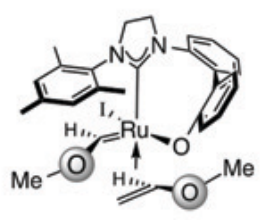

AB4
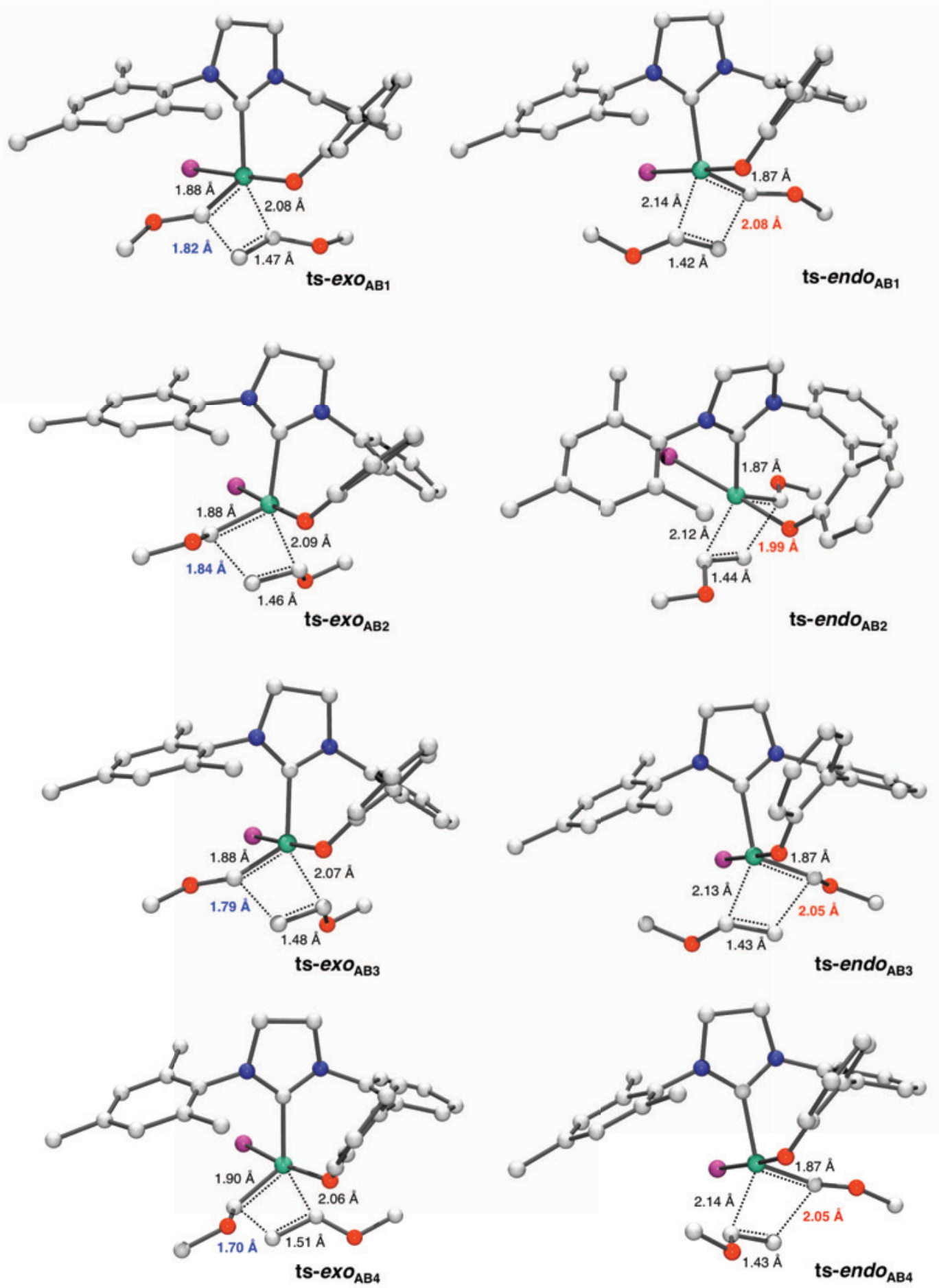
TSs for CM between propene and acrylonitrile with $\mathrm{Ru}-4 \mathrm{~b}$ (pathways $E M e, Z M e, E C N$, ZCN in Figure 7)

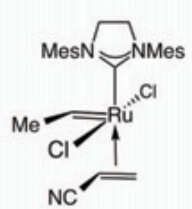

ZMe

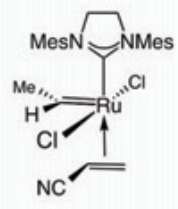

EMe

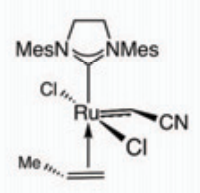

$\mathrm{Me}-\mathrm{CN}$<smiles>CC=C[C@@](Cl)(C1=NCCN1C)C(C#N)=C(C)C#N</smiles>

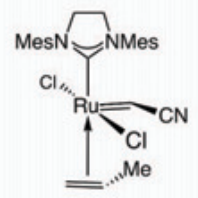

ECN
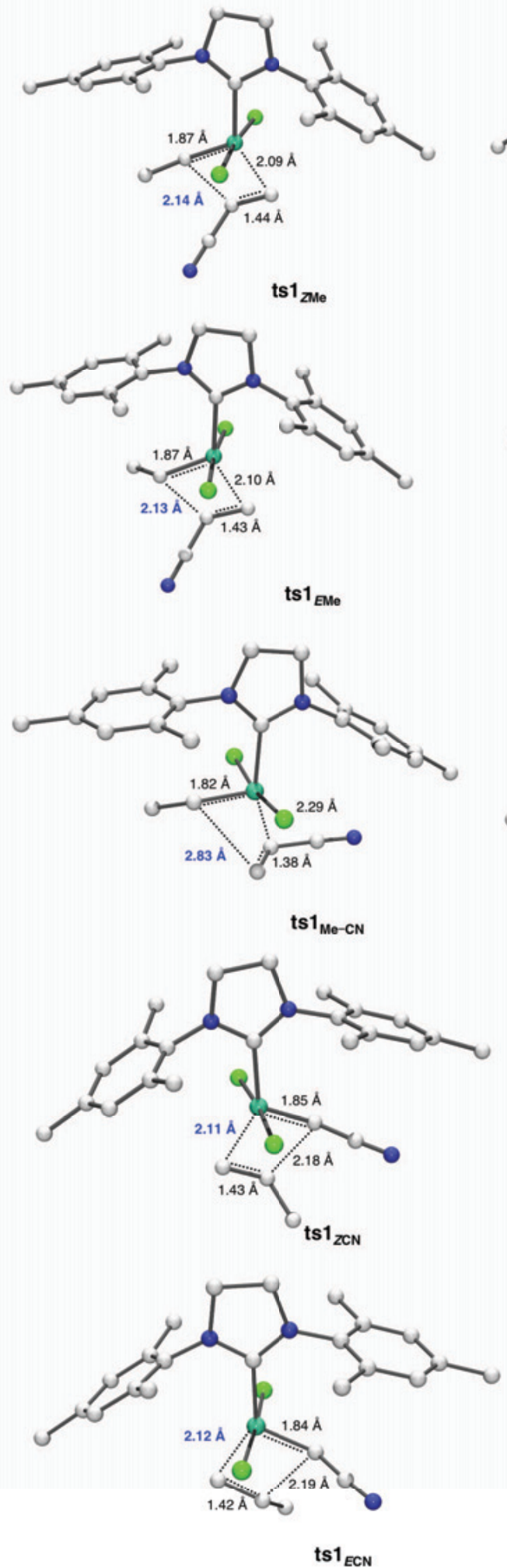
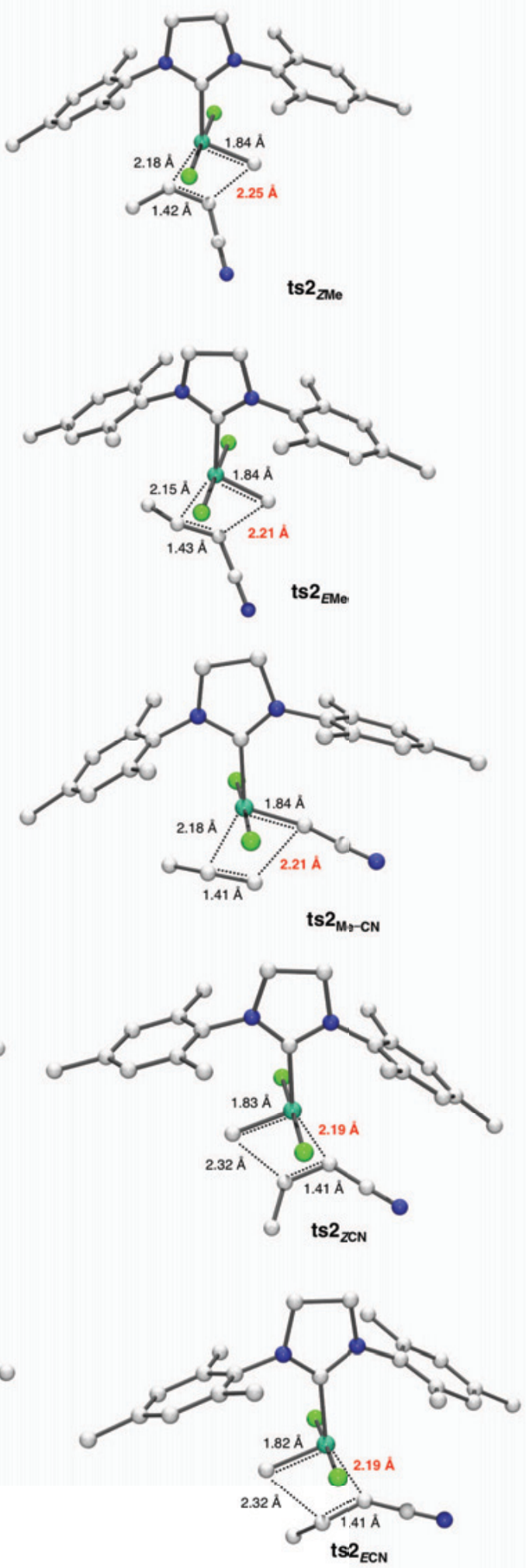
TSs for CM between methyl vinyl sulfide and trans-dichloroethylene (pathways ESMe, ZSMe in Figure 9) and homodimerization of propene with catalyst $\mathrm{Ru}-4 \mathrm{~b}$ (pathways $E M e^{\prime}, Z M e '$ in Figure 10)

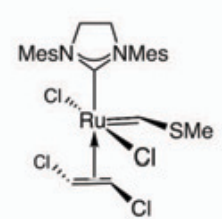

ZSMe

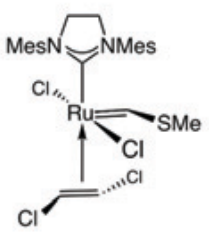

ESMe
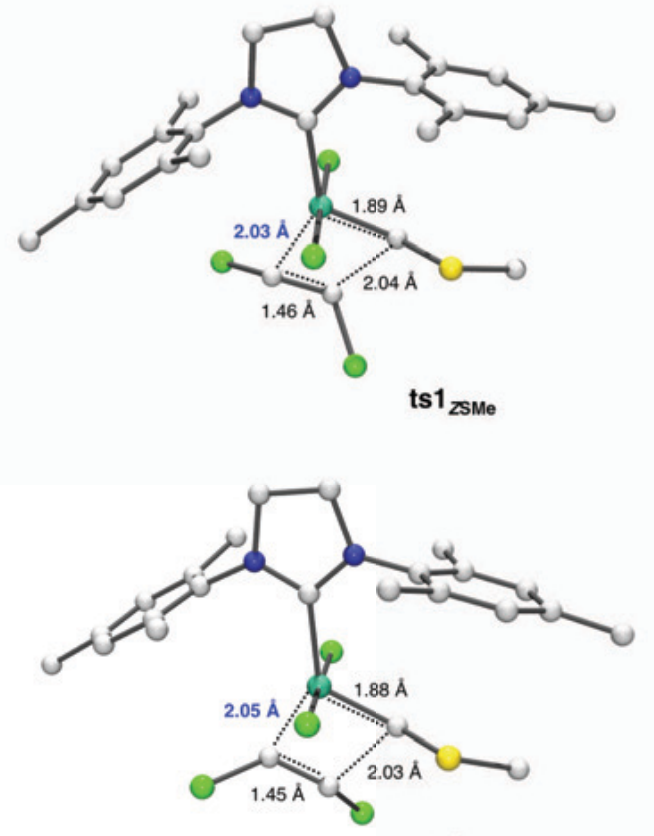

ts $1_{\text {ESMe }}$

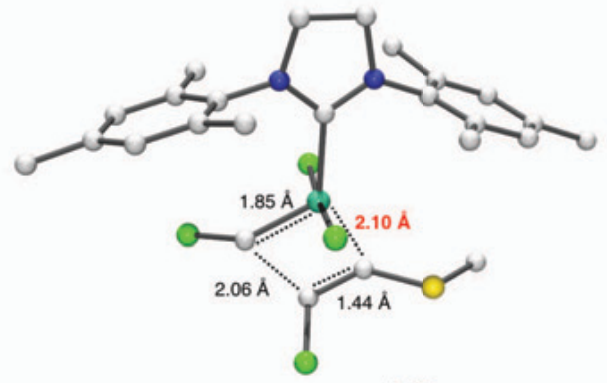

ts2 2 zsme

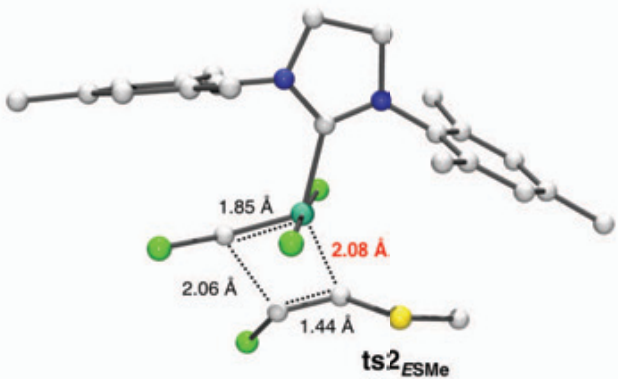

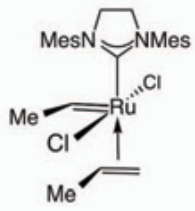

ZMe'

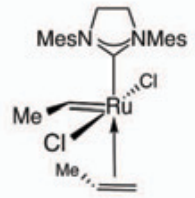

EMe'

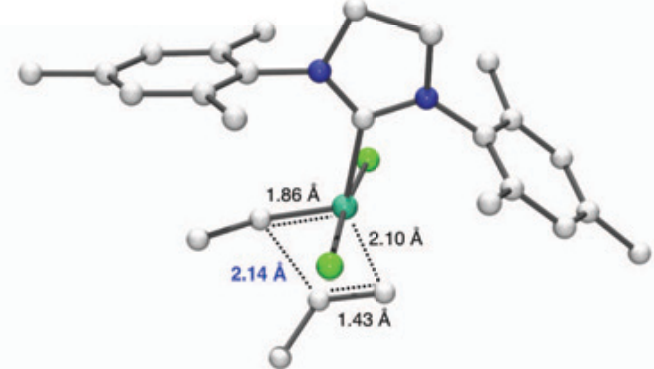

ts $_{\text {zMe' }}$

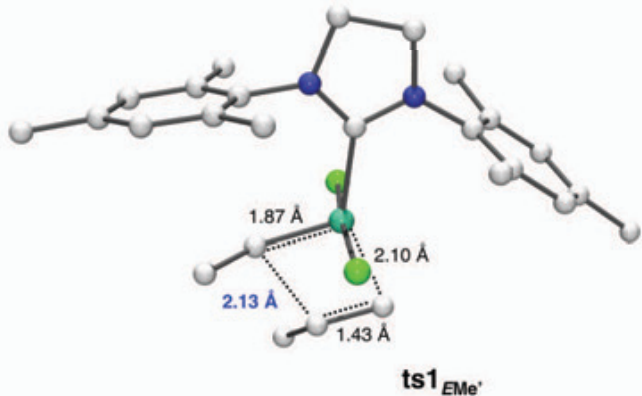

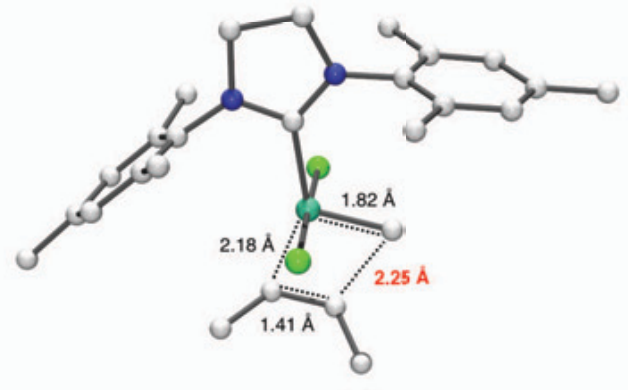

ts2 ${ }_{\text {ZMe' }}$

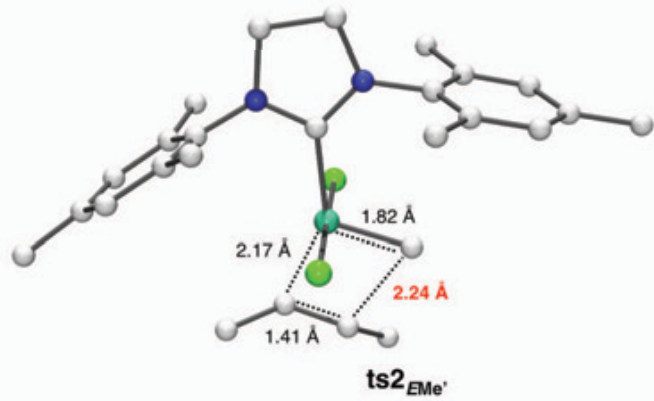


TSs for ROCM of 2 with catalyst Ru-5b and styrene (pathways C1-D4 in Figure S23)

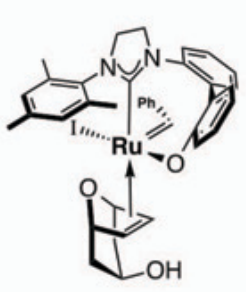

C1

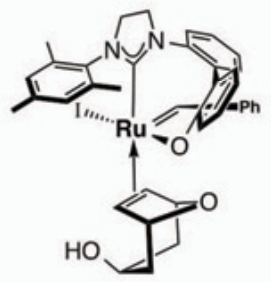

C2

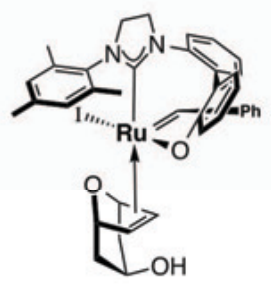

C3

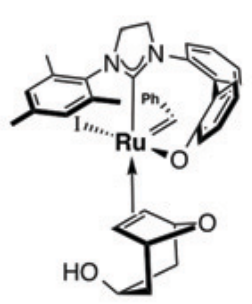

C4
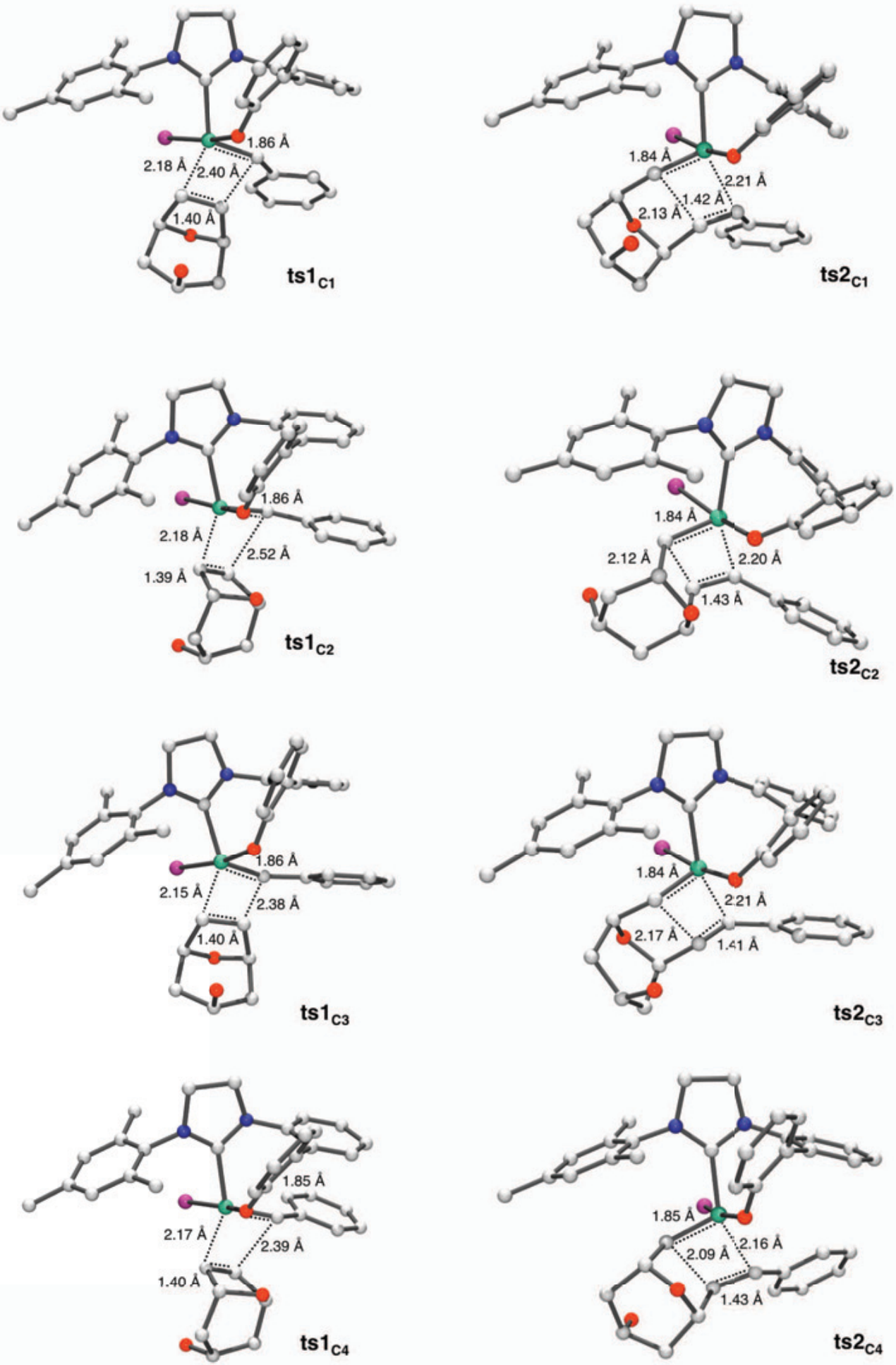


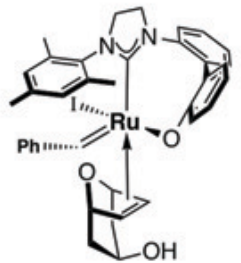

D1

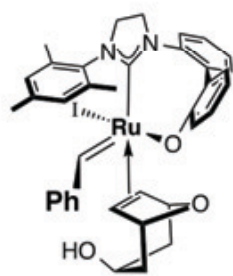

D2

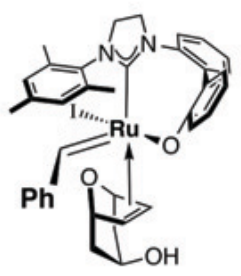

favored

D3

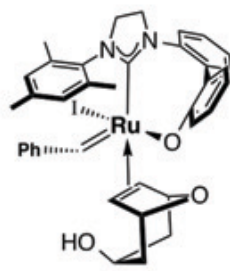

D4
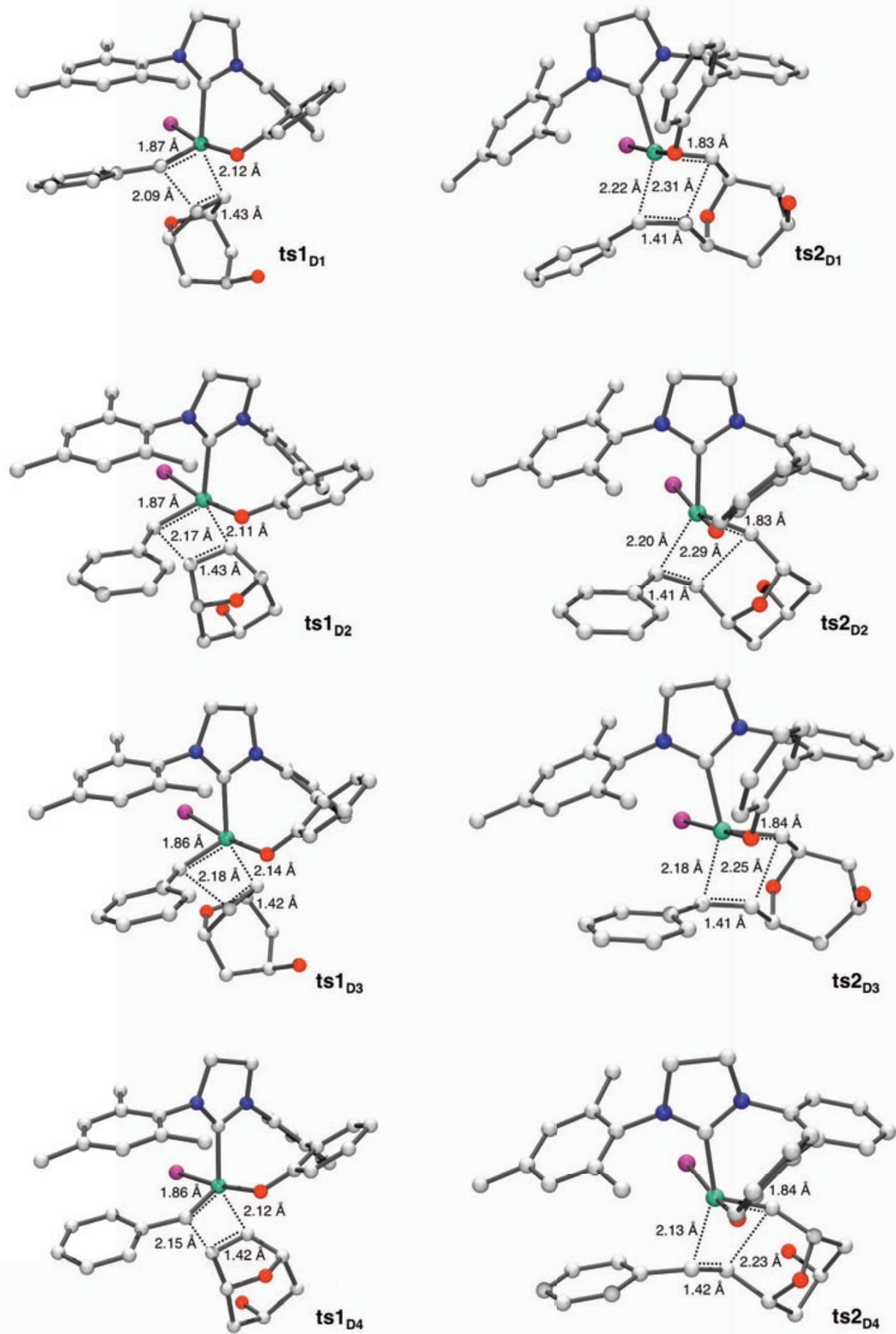
TSs for nonproductive OM of styrene with catalyst Ru-5b (pathways CD1-CD4 in Figure S23)
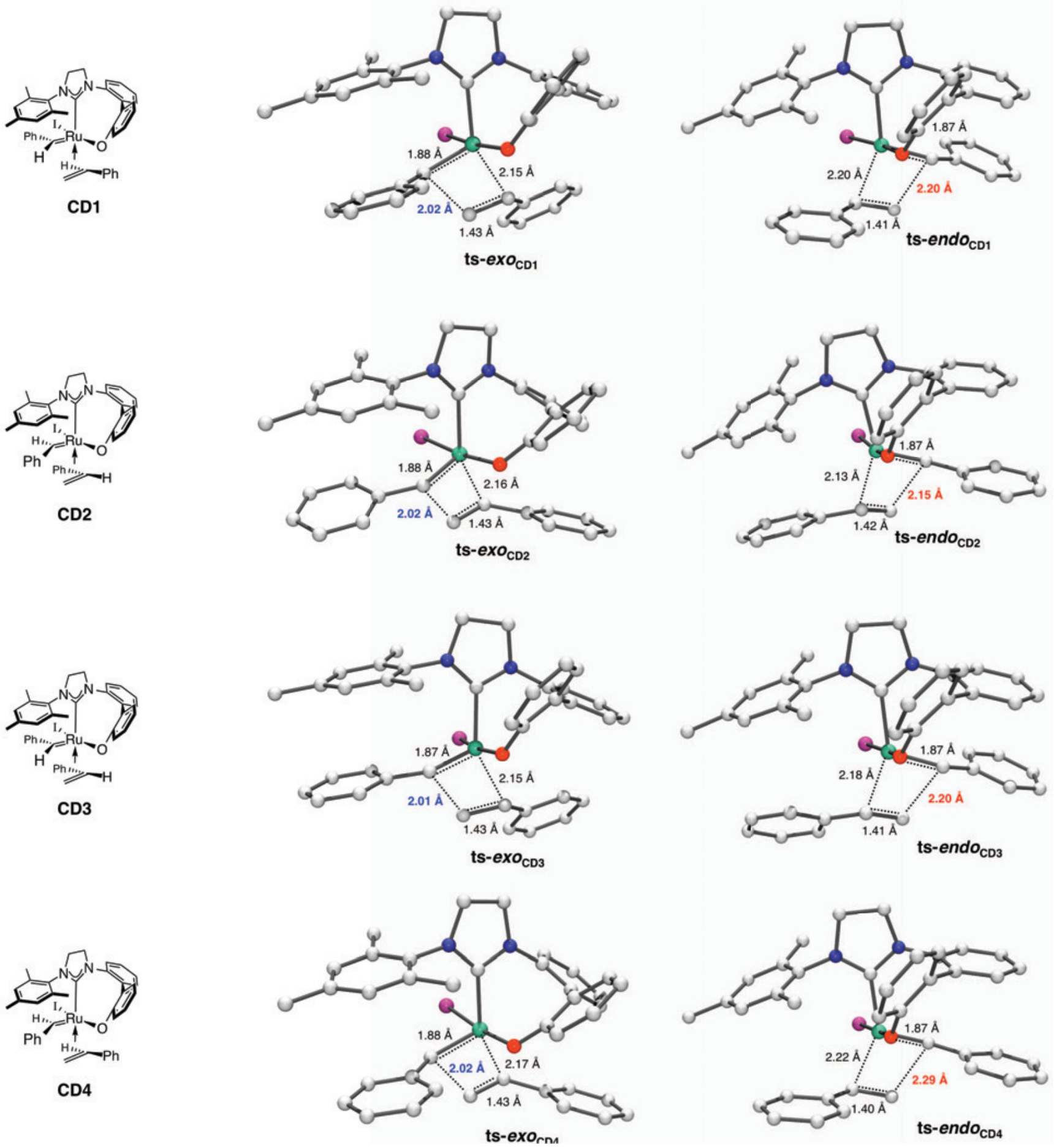